\title{
Metabolic Regulation of Antiviral Immune Responses in Macrophages
}

By

Duale Ahmed, M.Sc.

A thesis submitted to the Faculty of Graduate and Postdoctoral Affairs

in partial fulfillment of the requirements for the degree of

Doctor of Philosophy

in

Biology

Carleton University

Ottawa, Ontario

(C) 2020

Duale Ahmed 


\section{ABSTRACT}

It is increasingly clear that there is an intimate link between cellular metabolism and macrophage function. Several studies in the last decade have established that alterations in cellular metabolism both drive and regulate macrophage function, allowing them to mount a functional immune response against viruses by promoting viral resistance, antigen presentation and the production of inflammatory and antiviral cytokines. As a result, metabolic reprogramming of macrophages allows them to adapt the spectrum and magnitude of their response depending on the microenvironment, stimuli, and stage of infection. Yet, it is unclear how different viral ligands alter metabolism to induce specific immune responses and if specific metabolic components can act as rheostats to amplify these responses based on the microenvironment.

The central hypothesis of my thesis is that cellular metabolism is crucial to regulating antiviral immune responses. Specifically, I hypothesized that differential mitochondrial reprogramming allows macrophages to modulate ligand-specific effector response. Moreover, mitochondria further function as rheostats, fine-tuning immune responses based on the microenvironment.

First, I used publicly available microarray datasets to develop a metabolic signature associated with early IFN- $\alpha$ responses in mouse BMMs and human MDMs. $>500$ metabolic genes related to cellular bioenergetics, cellular redox status, amino acid, and lipid metabolism were identified. Next, I show that TLR3 and TLR4 engagement in mouse BMMs drive differential ETC remodeling, linked to differential mitochondrial activity and subsequent ROS generation, to support ligand-specific inflammatory and antiviral profiles. Furthermore, when exploring different types of TLR3 engagement, based on ligand length, they trigger distinct inflammatory and antiviral 
programs, due to unique regulatory mechanisms surrounding HIF-1 $\alpha$ function as well as altered ETC architecture and function, respectively.

The aim of this thesis is to gain a more thorough understanding of the critical role of cellular metabolism in regulating macrophage antiviral responses. A systematic understanding of these critical processes to regulating effector function under non-diseased conditions can provide critical insights into the dysregulation of metabolic processes during chronic viral infections. The data presented may be the foundation towards the development of new antiviral therapeutics by targeting selective mitochondrial components. 


\section{DEDICATION}

\section{To HODAN NALAYEH \\ Your legacy lives on through us!}

To all the dream chasers in the world, keep chasing! 


\section{PREFACE}

This thesis "Metabolic Regulation of Antiviral Immune Responses in Macrophages" is comprised of four chapters adapted from research manuscripts. Chapters 2 (Mediators of Inflammation, 2018) and 3 (Scientific Reports, 2019) have been published. Chapter 4 (Journal of Immunology, 2020) has been submitted for publication and Chapter 5 is in preparation for submission at Frontiers of Immunology. D.A. is the primary co-author on each of these chapters. The authors contributions for each chapter are as follows:

\section{Chapter 2}

This chapter is a finished manuscript published at Mediators of Inflammation in 2018 and is a collaborative work of D.A., Jaworski, A., Roy, D., Willmore, W., Golshani, A., and Cassol, E. entitled "Transcriptional profiling suggests extensive metabolic rewiring of human and mouse macrophages during early interferon alpha responses." D.A. is the main contributor, having curated the dataset and conducted the analysis, aided by A.J, D.R., W.W., and E.C. A.G. and E.C. supervising the research. All contributors contributed to the writing and editing of the manuscript.

\section{Chapter 3}

This chapter is a completed manuscript published at Scientific Reports in 2019 and is a collaborative work of D.A., Roy, D., Jaworski, A., Edwards, A., Abizaid, A., Kumar, A., Golshani, A., and Cassol, E. entitled "Differential remodeling of the electron transport chain is required to support TLR3 and TLR4 signaling and cytokine production in macrophages." A.G. and E.C supervised the work in this chapter. D.A. performed all of the experiments, with D.R. assisting and D.A. and E.C. designing the experiments. A.J., A.E. and A.A. helped procured the mouse bone marrow progenitors used in this chapter. D.A., D.R., A.K., and E.C. assisted in the analysis and interpretation. All co-authors contributed to the writing and critiquing of the manuscript. 


\section{Chapter 4}

This chapter is a completed manuscript submitted at Journal of Immunology in 2020 and is a collaborative work of D.A., Humphrey, A., Roy, D., Sheridan, M-E, Jaworski, A., Edwards, A., Donner, J., Abizaid, A., Willmore, W., Kumar, A., Golshani, A., and Cassol, E. entitled "HIF$1 \alpha$ regulation of pro-inflammatory cytokine production following TLR3 engagement is dependent on viral nucleic acid length and glucose availability." D.A. is the main contributor of this experiment, conducting all of the experiments with the help of A.H., D.R., M.E.S, and J.D, supervised by A.G. and E.C. The experiments were designed by D.A. and E.C. A.J., A.E. and A.A. helped harvested the mouse bone marrow progenitor cells used in this study. D.A., A.H., D.R., M.E.S., J.D., W.W., A.K. and E.C. participated in the analysis and interpreting of the data. All contributors on this chapter assisted in the careful writing of this manuscript.

\section{Chapter 5}

This chapter is a manuscript in preparation to be submitted at Frontiers of Immunology and is a collaborative work of D.A., Humphrey, A., Roy, D., Sheridan, M-E, Versey, Z., Jaworski, A., Edwards, A., Abizaid, A., Kumar, A., Golshani, A., and Cassol, E. entitled "dsRNA strand length dictates the manner of mitochondrial reprogramming and ROS-activated antiviral response during TLR3 engagement." D.A. is the primary co-author, conducting all of the experiments of this chapter with support from A.H., D.R., M.E.S, and Z.V, with supervision from A.G. and E.C. The experiments of this chapter were designed by D.A. and E.C., and mouse bone marrow progenitors were collected A.J., A.E., and A.A. D.A., A.H., D.R., M.E.S., Z.V., A.K. and E.C. contributed to the analysis and interpreting of the data. All co-authors on this chapter assisted in the creation of this manuscript. 


\section{ACKNOWLEDGEMENTS}

There is an old African proverb that says, "It takes a village to raise a child." One person's success is not only due to their skills and expertise, but the infrastructure that surrounds them. The completion of this Ph.D. would not have been possible with the numerous pillars that held me up. Firstly, I would like to thank Dr. Edana Cassol for giving me the opportunity to work under her supervision. There is no doubt that this Ph.D. experience can be considered one of the more "unique" experiences that one could go through. There has been lots of ups, lots of downs, lots of relocations and readjustments. But through it all, we were able to find a way to make it work. I've worked harder during this Ph.D. than I ever have for anything in my life and even though some of that can be attributed to circumstances out of our control, you pushed me to be the best that I could be. There is absolutely no way I would be in the position that I am in today without your guidance. I am a better person because I was a part of your lab. I am a better person because of what we went through. And like Super Saiyans, what doesn't kill you can only make you stronger!

Next, I would like to extend my appreciation to my advisory committee for their guidance through this Ph.D.: Dr. Ashok Kumar, Dr. Bruce McKay, and especially Dr. Ashkan Golshani. When I had to switch labs in the middle of this process, you did not hesitate to help and lend a hand. You all had a hand in pushing me to be better, and this would not be possible without you!

To my fellow and former lab mates, Mary-Elizabeth Sheridan, Adam Kemp, Allan Humphrey, David Roy, Zoya Versey: Thank you for your help and support throughout our time together. You all also played a part in this, and for that I am forever grateful. To the undergraduate students that I worked alongside during my time in the lab: Garrett, Aleksandra, Morgan, Melinda, Olivia, Claudia, Marwa, Kamaara, Mara, Catherine, James, Dan, Millie, Kylen, Emma, Romanah, and Emily: Thank you all for helping me with my research and know that you all have taught me 
something even though it was my job to teach you. To all the people I have worked alongside within the Biology and Health Sciences departments over the years: Mohsen, Bahram, Katayoun, Kristina, Dan, Dominique, Eddy, Emily, Tom, Taylor, Nathalie, Houman, Anna, Thet, Krishna, Ziggy, Hailey, Lilian, Waleska, and Carole: Thank you for providing a welcoming and friendly environment. And to the thousands of undergraduate students, I had the pleasure of TAing: Thank you! You have made me a better person and a better teacher!

Lastly, but certainly not least, I would not be the person that I am today without the support of my closest family and friends. Hooyo and Aabo, you brought me into this world, nurtured me and raised me to become someone who can accomplished anything that they set their mind to. While life was not always easy for us, you still gave everything you had to make sure that my three brothers and I never felt those hardships. Hopefully, I have and will continue to live up to the name you have bestowed onto me. To the rest of my support system: My brothers Mohamed, Mubarak, and Mukhtar; my cousins Kombe and Samia; my aunt Muna and my friends Hussein, Brandon, Vernon, Frank, and Allison: Thank you for being there for me whenever I needed you. To the members of my family that I have not mentioned (and there is a lot of you!), I am grateful for you as I would not be here without you. You are the foundation from which I prosper! Without you, I would fail!

The completion of this Ph.D. is a monumental achievement in my life. This is a cumulation of the buckets of blood, sweat and tears that I have put into this project. But I still have a lot to give and a long way to go before I have reached my full potential. What I do know is that without all of you, I would not have gotten this far. And whatever I end up doing next, I do with all of you propelling me forward. And because of each and every one of you: I'm Ph.Ding it! How 'bout that! Now, heeeeerrrre's what I want to talk to you about... 


\section{TABLE OF CONTENTS}

Page

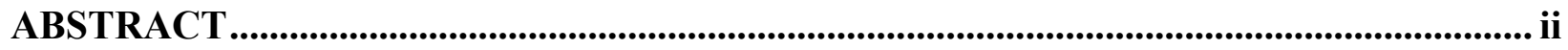

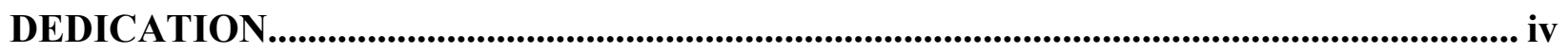

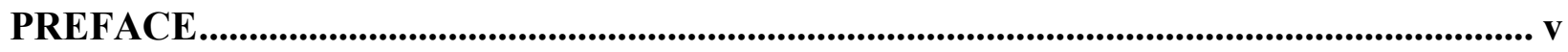

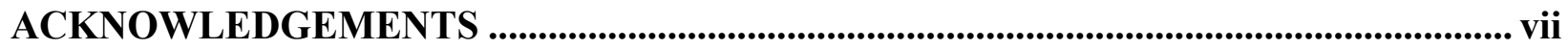

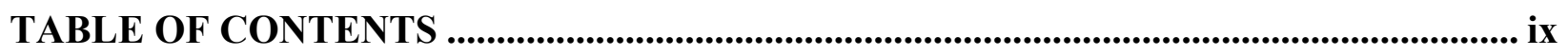

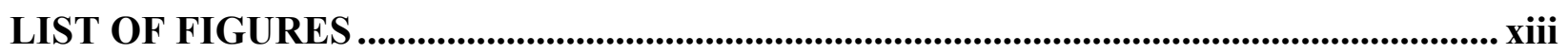

LIST OF SUPPLEMENTAL FIGURES ..........................................................................

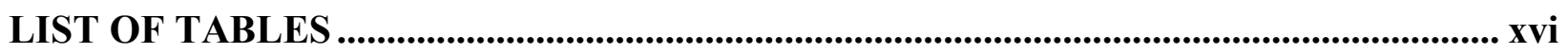

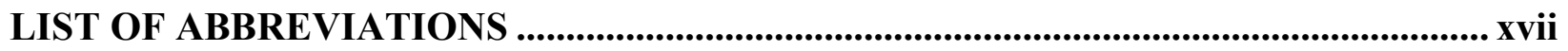

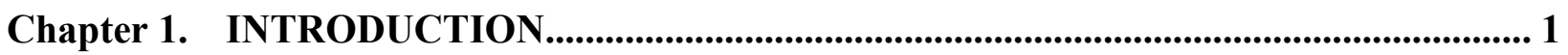

1.1. Macrophages and the Mononuclear Phagocyte System .......................................... 2

1.2. Host Pathogen Recognition by Macrophages .................................................... 4

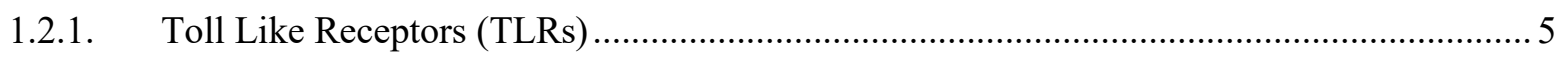

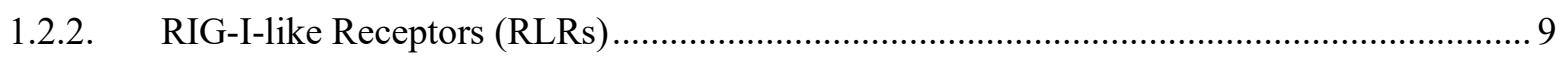

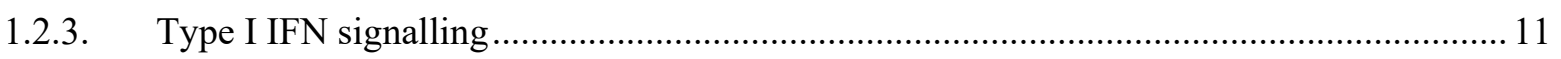

1.3. Mitochondria: Master Regulator of Cell Function .................................................. 14

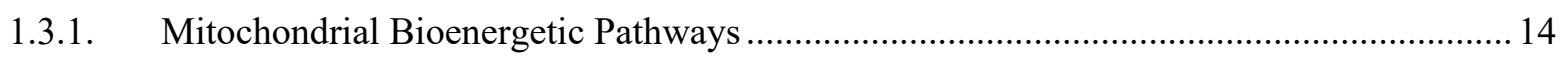

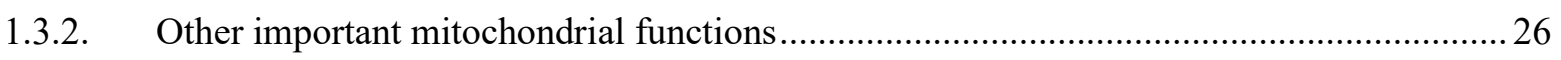

1.4. Metabolic Regulation of Macrophages .................................................................... 26

1.4.1. Metabolic Reprogramming of LPS engagement of TLR4 …............................................ 30

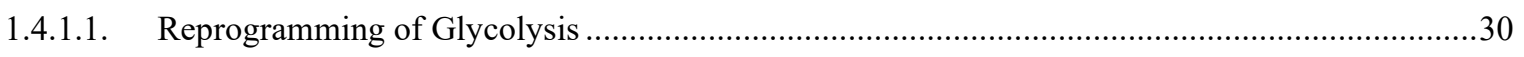

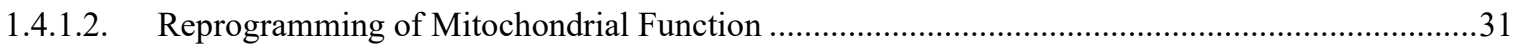

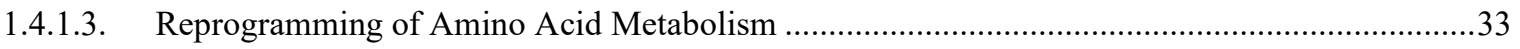

1.4.1.4. Reprogramming of Lipid Metabolism....................................................................................... 34

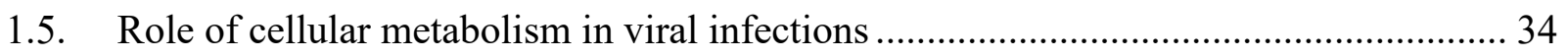

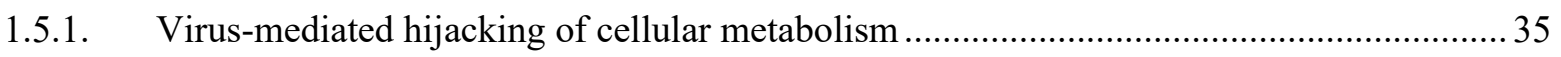

1.5.2. The regulation of antiviral immune responses by cellular metabolism............................. 37

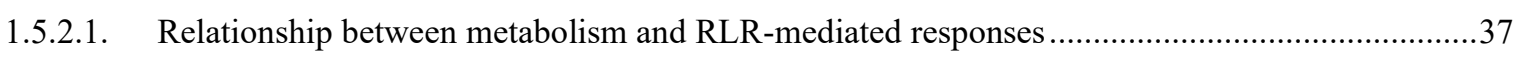

1.5.2.2. TLR3-mediated responses depend on altered metabolic flux ................................................... 38

1.5.2.3. The metabolic reprogramming induced by other viral TLRs ......................................................39

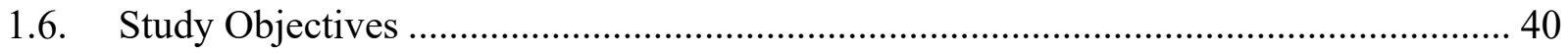




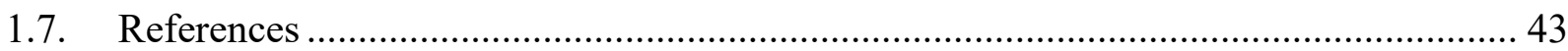

Chapter 2. Transcriptional profiling suggests extensive metabolic rewiring of human and mouse macrophages during early interferon alpha responses .......................................... 62

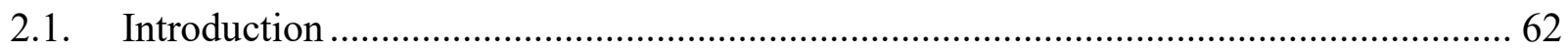

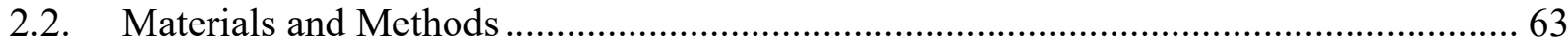

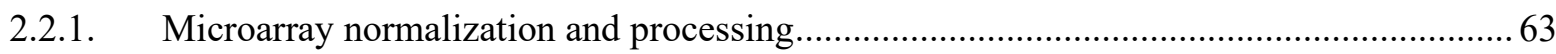

2.2.2. The identification of significant metabolic genes in IFN-stimulated macrophages (BMM \&

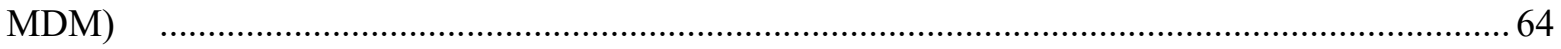

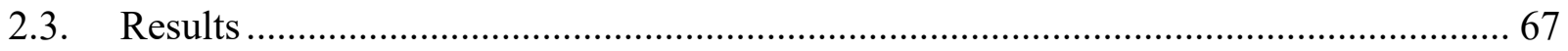

2.3.1. Type I IFN-stimulation of mouse BMM and human MDM is associated with enrichment of

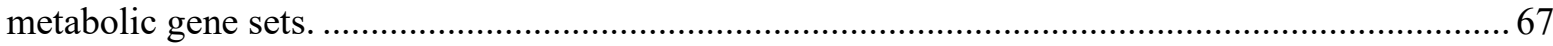

2.3.2. Metabolic genes represent important classifers of early type I IFN responses in BMM and MDM

2.3.3. Short term IFN- $\alpha$ stimulation alters genes associated with energy metabolism in BMM and

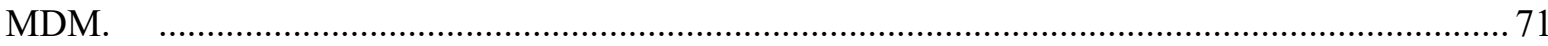

2.3.4. IFN- $\alpha$ stimulated BMM and MDM show signs of alterations in genes associated with redox regulation.

2.3.5. Early type I IFN responses are associated with alterations in genes associated with cAMP and cGMP production.

2.3.6. Short term IFN- $\alpha$ stimulation is associated with alterations in tryptophan and branchedchain amino acid catabolism in BMM and MDM.

2.3.7. IFN- $\alpha$ is associated with altered lipid metabolism in BMM and MDM.

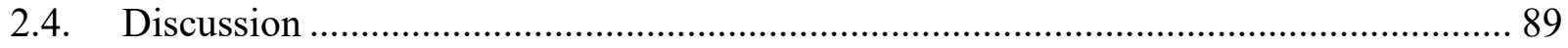

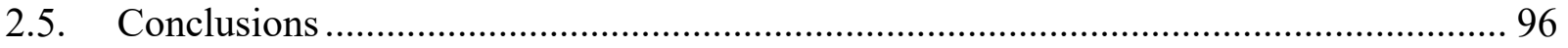

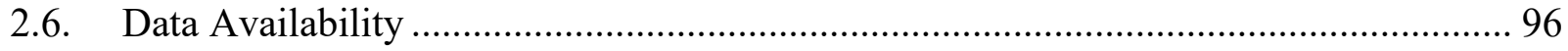

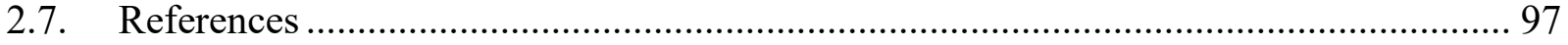

2.8. Chapter 2 Supplemental Tables ....................................................................... 105

Chapter 3. Differential remodeling of the electron transport chain is required to support TLR3 and TLR4 signaling and cytokine production in macrophages ............................. 182

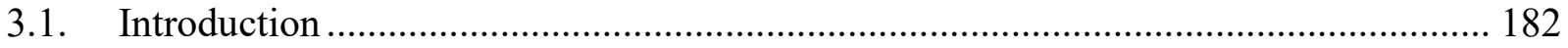

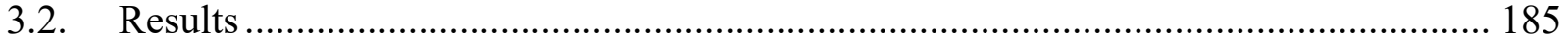

3.2.1. Differential production of pro-inflammatory and antiviral cytokines in PIC- and LPS-

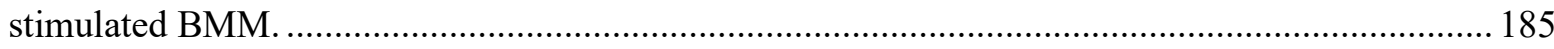

3.2.2. BMM stimulated with low versus high concentrations of PIC differ in their ability to ramp

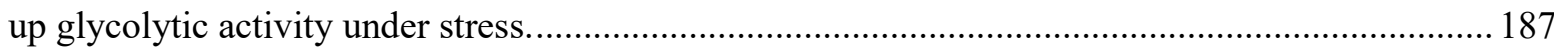

3.2.3. Maintenance of OXPHOS activity is an important for PIC but not LPS stimulation....... 189 
3.2.4. Reduced glucose availability is associated with increased inflammatory and antiviral cytokine production in PIC- but not LPS-stimulated BMM

3.2.5. Reduced glucose availability is associated with altered mitochondrial membrane potential and ETC complex expression following TLR engagement.

3.2.6. Flux through the ETC chain is required for inflammatory and antiviral cytokine production following TLR engagement. 198

3.2.7. Mitochondrial and cytosolic ROS accumulate in PIC stimulated BMM under low glucose conditions. 201

3.2.8. Mitochondrial and cellular ROS play a central role in TLR3 and TLR4 associated cytokine

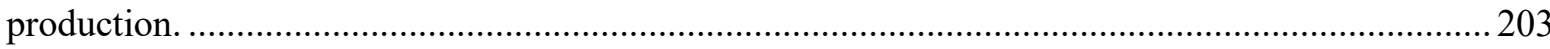

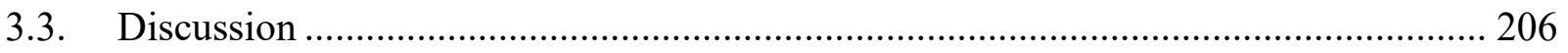

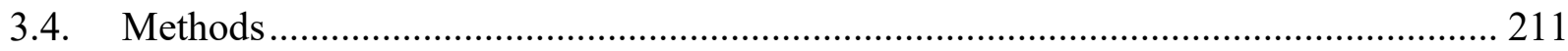

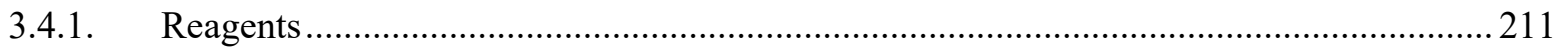

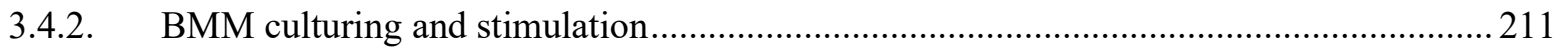

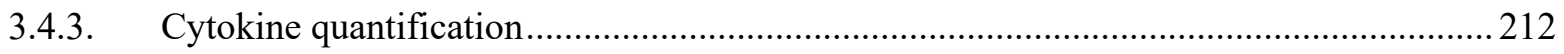

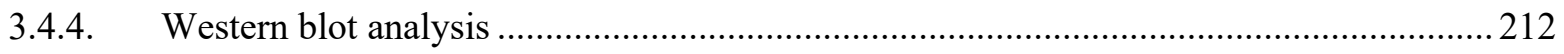

3.4.5. Assessment of mitochondrial function by flow cytometry …...................................... 212

3.4.6. Quantification of cellular hydrogen peroxide production ............................................. 213

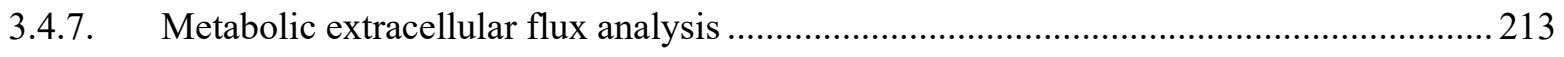

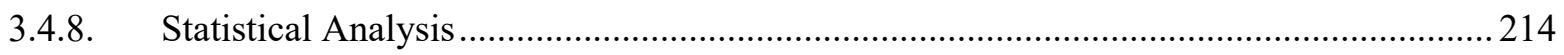

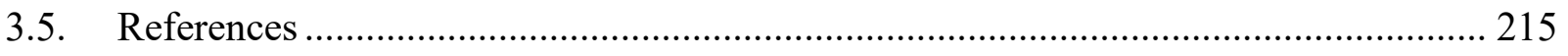

Chapter 4. HIF-1 $\alpha$ regulation of pro-inflammatory cytokine production following TLR3 engagement is dependent on viral nucleic acid length and glucose availability................. 220

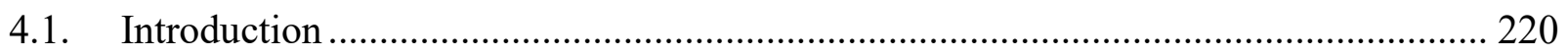

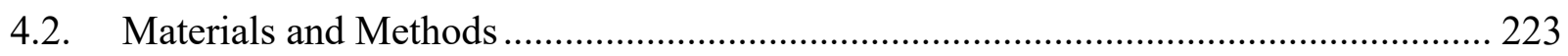

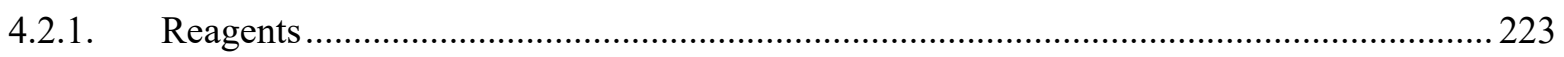

4.2.2. Isolation, differentiation, and treatment of bone marrow-derived macrophages (BMM). 223

4.2.3. Quantification of Cytokine Production in Culture Supernatants .................................... 224

4.2.4. Evaluation of protein expression in total BMM lysates................................................ 224

4.2.5. Evaluation of Mitochondrial Membrane Potential, Mitochondrial ROS and Total Cytosolic

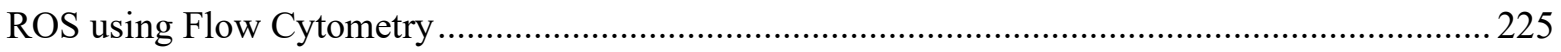

4.2.6. Characterization of Energy Metabolism of BMM ….................................................... 226

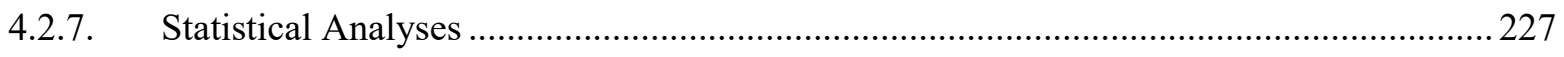

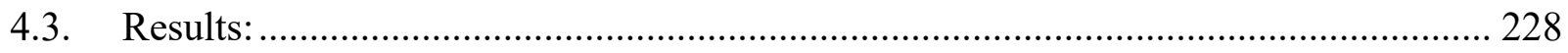

4.3.1. HIF- $1 \alpha$ is induced following TLR3 engagement and is regulated by glucose availability 228

4.3.2. Stimulation of BMM with LMW Poly(I:C) does not alter glycolytic activity................. 231 
4.3.3. Preventing HIF-1 dimerization blocks pro-inflammatory cytokine production via inhibition of NF- $\mathrm{KB}$ activation

4.3.4. PKM2 dimerization is important for HIF-1 $\alpha$ - and NF- $\kappa B$-driven inflammatory responses following TLR3 engagement

4.3.5. Cytosolic and mitochondrial ROS differentially regulate HMW and LMW inflammatory cytokine production

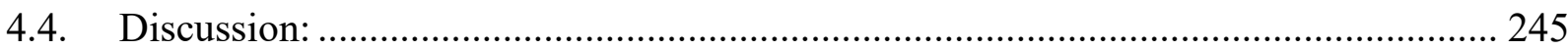

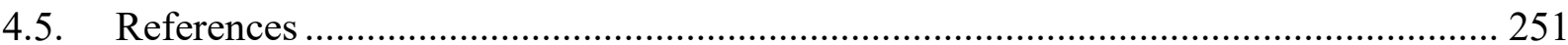

Chapter 5. dsRNA strand length dictates the manner of mitochondrial reprogramming and ROS-activated antiviral response during TLR3 engagement ....................................... 259

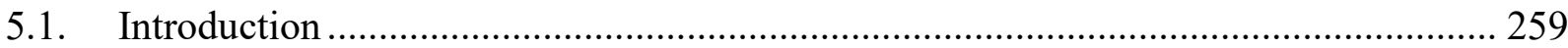

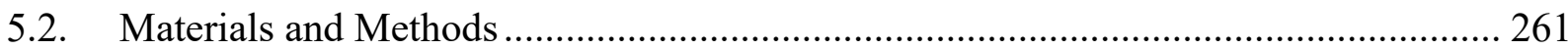

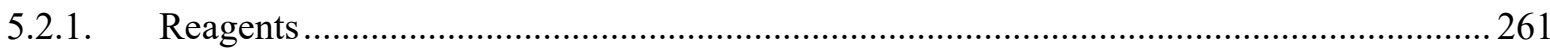

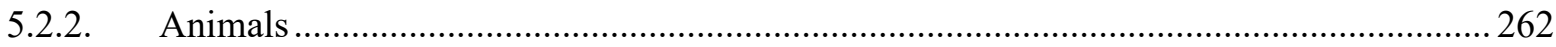

5.2.3. Culture and Treatment of Mouse Bone Marrow-derived Macrophages (BMMs) ............262

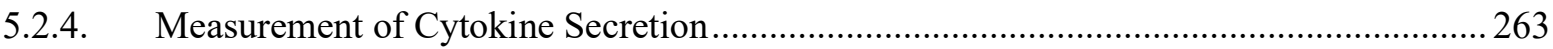

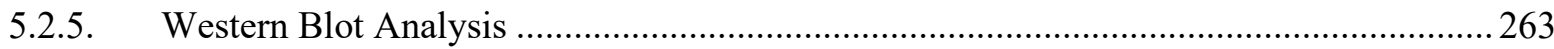

5.2.6. Assessment of Mitochondrial Function using Flow Cytometry.......................................263

5.2.7. Measurement of Cellular $\mathrm{H}_{2} \mathrm{O}_{2}$ Production by fluorescence spectrometry ...................... 264

5.2.8. Characterization of Energy Metabolism of BMMs.......................................................... 264

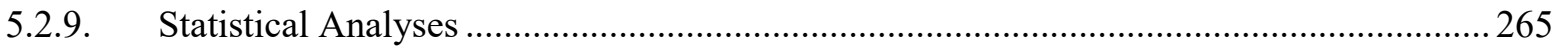

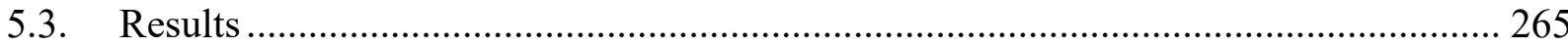

5.3.1. TLR3 associated type I IFN responses are amplified under low glucose conditions........ 265

5.3.2. Low glucose conditions are associated with increased cellular respiration to compensate

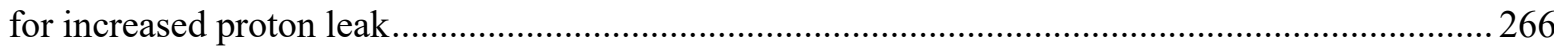

5.3.3. The amplification of type I IFN responses in low glucose conditions is driven by increased

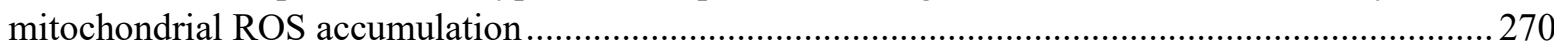

5.3.4. Complex I expression is differentially modulated by HMW vs. LMW Poly(I:C)........... 271

5.3.5. Mitochondrial ROS from Complexes I and III differentially drive type I IFN responses following stimulation with HMW or LMW Poly(I:C) ........................................................... 275

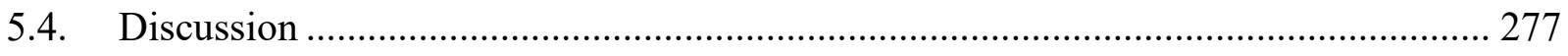

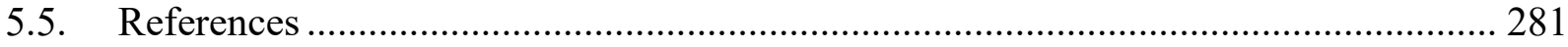

Chapter 6. CONCLUSIONS AND PRESPECTIVES .................................................. 286

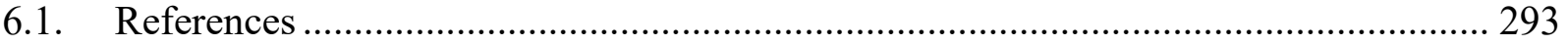




\section{LIST OF FIGURES}

Page

\section{Chapter 1 Figures:}

Figure 1.1: A summary of the bacterial and viral PAMPs that are detected by Toll-like

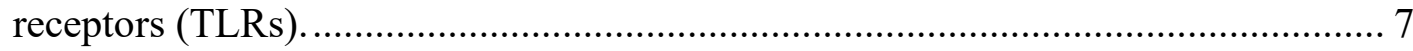

Figure 1.2: TLR3 (viral dsRNA) vs TLR4 (bacterial LPS) signalling dynamics. .................... 8

Figure 1.3: Overview of RLR signalling................................................................ 10

Figure 1.4: Traditional type I IFN signalling cascade.................................................... 13

Figure 1.5: Mitochondria are the central hubs for innate immune responses. ........................ 15

Figure 1.6: The importance of Glycolysis and the TCA cycle intermediates as building blocks

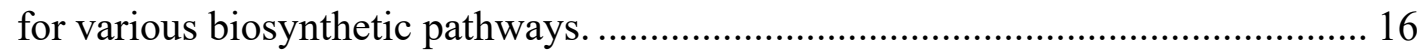

Figure 1.7: Methods of $\mathrm{NAD}^{+} / \mathrm{NADH}$ transport into the mitochondria. ............................... 19

Figure 1.8: Oxidative phosphorylation is coupled with the electron transport chain to generate

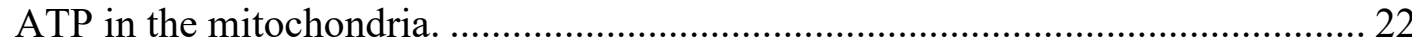

Figure 1.9: The Mitochondrial Bioenergetic Pathways. ........................................................ 25

Figure 1.10: The differences between M1 and M2 macrophages........................................ 29

\section{Chapter 2 Figures:}

Figure 2.1: Short term IFN- $\alpha$ stimulation is associated with altered expression of metabolic genes in human monocyte-derived macrophages (MDM) and mouse bone marrow-

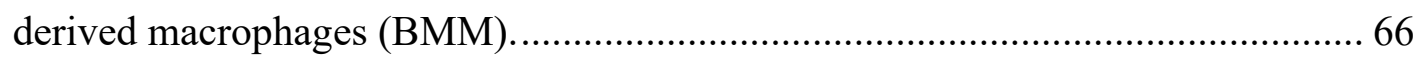

Figure 2.2: Metabolic genes are top classifiers of IFN- $\alpha$ stimulation in BMM and MDM. ..... 69

Figure 2.3: Genes associated with bioenergetic processes are differentially expressed in mouse BMM and human MDM following IFN- $\alpha$ stimulation. ................................... 72

Figure 2.4: IFN- $\alpha$ stimulation of MDM is associated with increased expression of genes associated with ROS production and antioxidant responses. .............................. 76

Figure 2.5: Type I IFN responses are associated with altered cAMP and cGMP production in BMM and MDM.

Figure 2.6: Tryptophan and branched-chain amino acid catabolism is altered in BMM and MDM following short-term IFN- $\alpha$ treatment.

Figure 2.7: Expression of genes associated with lipid metabolism were differentially modulated in IFN- $\alpha$ stimulated BMM compared MDM.

\section{Chapter 3 Figures:}

Figure 3.1: High, but not low, concentrations of Poly(I:C) are associated with proinflammatory cytokine production.

Figure 3.2: Macrophages activated using higher concentrations of Poly(I:C) are functioning near their maximum glycolytic capacity.

Figure 3.3: Poly(I:C) stimulation is linked to low sustained levels of oxidative phosphorylation (OXPHOS). 
Figure 3.4: Low glucose conditions are associated with increased IRF activation and increased type I IFN production.

Figure 3.5: Poly(I:C) activation is linked to altered mitochondrial activity under low glucose conditions.

Figure 3.6: Targeting ETC activity reduces type I IFN-mediated responses during Poly(I:C) activation.

Figure 3.7: Poly(I:C) activation promotes mitochondrial ROS production and accumulation.202

Figure 3.8: Type I IFN production can be inhibited by altering mtROS generation during Poly(I:C) activation. 204

\section{Chapter 4 Figures:}

Figure 4.1: HIF-1 $\alpha$ differentially accumulates in BMM following stimulation with HMW vs.

LMW Poly(I:C) in low and high glucose.

Figure 4.2: HMW Poly(I:C) stimulation is associated with increased glycolytic demands compared to LMW.

Figure 4.3: Low glucose conditions increased NF- $\kappa \mathrm{B}$ activation and pro-inflammatory cytokine production.

Figure 4.4: Targeting HIF-1 dimerization blunts pro-inflammatory but not anti-inflammatory cytokine expression.

Figure 4.5: Maintenance of PKM2 in tetramer form reduces inflammatory cytokine production under low glucose conditions by reducing HIF-1 $\alpha$ accumulation and NF- $\kappa \mathrm{B}$ activation. 242

Figure 4.6: HMW- and LMW-mediated inflammatory cytokine production is blunted by mitochondrial ROS scavenging.

Figure 4.7: NAC differentially alters HIF-1 $\alpha$ accumulation and completely inhibits inflammatory responses following HMW and LMW.

\section{Chapter 5 Figures:}

Figure 5.1: Low glucose conditions are associated with amplified antiviral signalling and subsequent cytokine production.

Figure 5.2: Increased proton leak drives boost in respiration under low glucose conditions. . 268

Figure 5.3: HMW and LMW activation is linked to elevated mitochondrial ROS and loss of mitochondrial antioxidant response under low glucose conditions.

Figure 5.4: Scavenging ROS blunts Poly(I:C)-mediated type I IFN production.... 273

Figure 5.5: dsRNA length-dependent TLR3 engagement determines the degree of Complex I expression under low glucose conditions.

Figure 5.6: Preventing the production of Complex I generated mtROS can unlock HMWmediated IFN production via increased IRF3/7 activation.

\section{Chapter 6 Figures:}

Figure 6.1: A summary of the findings pertaining to the role of cellular metabolism in TLR3mediated responses. 


\section{LIST OF SUPPLEMENTAL FIGURES}

Chapter 2 Supplemental Figures:

Page

Supplemental Figure S2.1: Study workflow of BMM and MDM differentiation and IFN- $\alpha$

stimulation.

Supplemental Figure S2.2: IFN- $\alpha$ is associated with differential enrichment of metabolic

pathways in mouse BMM compared to human MDM 70

Supplemental Figure S2.3: IFN responses are associated with altered bioenergetic profiles in mouse and human macrophages. 73

Supplemental Figure S2.4: BMM and MDM express redox-related genes following short term

IFN- $\alpha$ stimulation. 77

Supplemental Figure S2.5: IFN- $\alpha$ is associated with altered expression of genes that regulate nucleotide metabolism and $\mathrm{cAMP} / \mathrm{cGMP}$ ratios. 80

Supplemental Figure S2.6: Short term IFN- $\alpha$ stimulation is associated with alterations in tryptophan and branched chain amino acid catabolism.

Supplemental Figure S2.7: Altered expression of genes associated with lipid metabolism is a key feature of Type I IFN responses. 88

Chapter 3 Supplemental Figures:

Supplemental Figure S3.1: PIC activation is associated with increased OXPHOS function under low glucose conditions. 194

Supplemental Figure S3.2: TRIF and TRAF6 expression during PIC activation is not affected by glucose levels.

Supplemental Figure S3.3: Targeting ETC function in BMM leads to complete loss of type I

IFN responses. 200

Supplemental Figure S3.4: Targeting mtROS leads to loss of type I IFN production. 205

Chapter 4 Supplemental Figures:

Supplemental Figure S4.1: HIF-1 $\alpha$ expression is activated under glucose and/or oxygen deprivation conditions. 230

Supplemental Figure S4.2: Acriflavine treatment does not affect HIF- $1 \alpha$ accumulation during HMW or LMW activation. 235

Supplemental Figure S4.3: HIF-1 $\alpha$ does not directly regulate IL-10 expression. 238

Chapter 5 Supplemental Figures:

Supplemental Figure S5.1: TLR3 engagement does not alter mitochondrial mass. 269 


\section{LIST OF TABLES}

Chapter 2 Tables:

Page

Supplemental Table S2.1: A list of all metabolic genes identified in the BMM dataset. ........ 105

Supplemental Table S2.2: A list of all metabolic genes identified in the MDM dataset. ........ 119

Supplemental Table S2.3: A list of significantly altered gene sets identified by GSEA in the

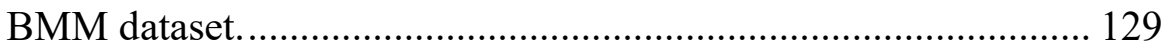

Supplemental Table S2.4: A list of significantly altered gene sets identified by GSEA in the MDM dataset.... 


\section{LIST OF ABBREVIATIONS}

25-HC

2-DG

ACLY

ACR

ADP

AMP

AMPK

APC

ATG

ATP

BCAA

BCAT

BCKDH

BCL2

BMM

BSA

CARD

CCR5

CD 25-hydroxycholesterol

2-deoxyglucose

ATP citrate lyase

Acriflavine

Adenosine diphosphate

Adenosine monophosphate

AMP-activated protein kinase

Antigen-presenting cells

Autophagy related protein

Adenosine triphosphate

Branched-chain amino acid

BCAA aminotransferase

Branched-chain alpha-keto acid dehydrogenase

B cell lymphoma 2

Mouse bone marrow-derived macrophage

Bovine serum albumin

Caspase active recruitment domain

C-C chemokine receptor type 5

Cluster of differentiation 
CH25H

CoA

CpG

CPT

CXCL10

DAMP

DAVID

DC

DMEM

DMSO

DNA

Drp1

dsRNA

ECAR

EHMN

ERK

ETC

FA

FAD/FADH 2
Cholesterol 25-hydroxylase

Coenzyme A

Cytosine-Guanine regions in DNA

Carnitine palmitoyltransferase

C-X-C motif chemokine ligand 10

Danger-associated molecular pattern

Database for Annotation, Visualization, and Integrated Discovery

Dendritic cell

Dulbecco's Modified Eagle Medium

Dimethylsulfoxide

Deoxyribonucleic acid

Dynamin-related protein 1

Double-stranded ribonucleic acid

Extracellular acidification rate

Edinburgh Human Metabolic Network

Extracellular signal-regulated kinase

Electron transport chain

Fatty acid

Flavin adenine dinucleotide 
FAO

FBS

FC

FCCP

FDR

FIH-I

G3P

G6PD

GAPDH

GAS

GEO

GLUT

GPD

GPS

GPX4

GSEA

$\mathrm{H}_{2} \mathrm{O}_{2}$

HCMV

HCV
Fatty acid oxidation

Fetal bovine serum

Fold change

Carbonyl cyanide 4-(trifluoromethoxy)phenylhydrazone

False discovery rate

Factor inhibiting HIF-I

Glycerol 3-phosphate

Glucose 6-phosphate dehydrogenase

Glyceraldehyde 3-phosphate dehydrogenase

Interferon- $\gamma$-associated site

Gene expression omnibus

Glucose transporter

Glycerol 3-phosphate dehydrogenase

Glycerol phosphate shuttle

Glutathione peroxidase 4

Gene set enrichment analysis

Hydrogen peroxide

Human cytomegalovirus

Hepatitis C virus 


\begin{tabular}{|c|c|}
\hline HG & High glucose \\
\hline HIF-1a & Hypoxic-inducible factor- $1 \alpha$ \\
\hline HIV & Human immunodeficiency virus \\
\hline HSV & Herpes simplex virus \\
\hline IAV & Influenza A virus \\
\hline IDH & Isocitrate dehydrogenase \\
\hline IFN & Interferon \\
\hline IFNAR & Interferon- $\alpha$ receptor \\
\hline IKK & IкB kinase \\
\hline IL & Interleukin \\
\hline iNOS & inducible NO synthase \\
\hline IRAK & Interleukin-1 receptor associated kinase \\
\hline IRF & Interferon regulatory factor \\
\hline IRG1 & Immunoresponsive gene 1 \\
\hline ISG & Interferon stimulating gene \\
\hline ISGF3 & Interferon-stimulated gene factor 3 complex \\
\hline ISRE & Interferon-stimulated responsive element \\
\hline ІкВ & Nuclear factor of kappa light polypeptide gene enhancer in B-cells inhibitor \\
\hline JAK & Janus activated kinase \\
\hline
\end{tabular}


KEGG

LDH

LG

LPS

LXR

MAPK

MAVS

M-CSF

MDA5

MDM

MEF

MFI

MFN

MMP

MPC

mRNA

MSigDB

MT

mtDNA
Kyoto Encyclopedia of Genes and Genomes

Lactate dehydrogenase

Low glucose

Lipopolysaccharide

Liver X receptor

Mitogen-activated protein kinase

Mitochondrial antiviral signaling protein

Macrophage-colony stimulating factor

Melanoma differentiation-associated protein 5

Human monocyte derived macrophage

Mouse embryonic fibroblasts

Mean fluorescence intensity

Mitofusin

Mitochondrial membrane potential

Mitochondrial pyruvate carrier

Messenger ribonucleic acid

Molecular Signatures Database

MitoTEMPO

Mitochondrial DNA 
mTOR

mtROS

MyD88

NAC

$\mathbf{N A D}^{+} / \mathbf{N A D H}$

$\mathbf{N A D P}^{+} / \mathbf{N A D P H}$

NCBI

NEMO

NFAT

NF-кB

NK

NLRP3

NO

NOX

NRF2

OCR

O-GIcNAc

OM

OPA1
Mechanistic target of rapamycin

Mitochondrial reactive oxygen species

Myeloid differentiation primary response- 88

$\mathrm{N}$-acetylcysteine

Nicotinamide adenine dinucleotide

Nicotinamide adenine dinucleotide phosphate

National center for biotechnology information

NF-kappa-B essential modulator

Nuclear factor of activated T cells

Nuclear factor of kappa light polypeptide gene enhancer in B-cells

Natural Killer cell

NOD-like receptor family pyrin domain containing 3

Nitric Oxide

NADPH oxidase

NF-E2-related factor 2

Oxygen consumption rate

O-linked $\beta$-N-acetylglucosamine

Oligomycin

Optic atrophy 1 
OXPHOS

PAMP

PBS

PCA

PDH

PDK

PenStrep

PER

PFKFB

PHD

PI3K

PKM2

Poly(I:C)/PIC

PPP

PRR

R837

R848

RET

RF
Oxidative phosphorylation

Pathogen-associated molecular pattern

Phosphate buffered saline

Principal component analysis

Pyruvate dehydrogenase

PDH kinase

Penicillin/Streptomycin

Proton efflux rate

6-phosphofructo-2-kinase/fructose-2,6-biphosphatase

Prolyl hydroxylase

Phosphoinositide 3-kinase

Pyruvate kinase muscle isozyme 2

Polyinosinic:polycytidylic acid

Pentose phosphate pathway

Pathogen Recognition Receptor

Imiquimod

Resiquimod

Reverse electron transport

Random forest 


\begin{tabular}{|c|c|}
\hline RIG-I & Retinoic acid-inducible gene-I \\
\hline RISP & Rieske iron-sulfur protein \\
\hline RLR & RIG-I-like receptors \\
\hline RNA & Ribonucleic acid \\
\hline ROS & Reactive oxygen species \\
\hline ROT/AA & Rotenone + Antimycin A \\
\hline RSV & Respiratory syncytial virus \\
\hline S1QEL & Suppressors of site $I_{Q}$ Electron Leak \\
\hline S3QEL & Suppressors of site $\mathrm{III}_{\mathrm{Q}}$ Electron Leak \\
\hline SDH & Succinate dehydrogenase \\
\hline shRNA & short hairpin RNA \\
\hline SiRNA & Small interfering RNA \\
\hline SOD & Superoxide dismutase \\
\hline SPHK2 & Sphingosine kinase 2 \\
\hline SRC & Spare respiratory capacity \\
\hline SREBP & Sterol regulatory element-binding protein \\
\hline ssRNA & Single-stranded ribonucleic acid \\
\hline STAT & Signal transducer and activator of transcription \\
\hline STING & Stimulator of type I interferon genes \\
\hline
\end{tabular}


TBK1

TBS

TCA

TGF- $\beta$

TIR

TLR

TMRM

TNF

TRAF

TRAM

TRIF

TYK2

UCP

$\mathbf{U Q / U Q H} 2$

VIP
TANK-binding kinase 1

Tris buffered saline

Tricarboxylic acid cycle

Transforming growth factor- $\beta$

Toll/Interleukin-1 receptor

Toll-like receptor

Tetramethylrhodamine

Tumour necrosis factor

TNF receptor-associated factor

Translocating chain-associated membrane protein

Toll/Interleukin-1 receptor-domain-containing adapter-inducing IFN- $\beta$

Tyrosine kinase 2

Uncoupling protein

Ubiquinone/Ubiquinol

Variable Importance in Projection 


\section{Chapter 1. INTRODUCTION}

The immune system consists of a variety of cells and complex biological processes that protect the host organism against foreign agents (1). It is made up of two interconnected subsystems: the innate and adaptive immune systems. The innate immune system provides the first line of defense against pathogens by undertaking nonspecific defense mechanisms against foreign microorganisms (1-3). This first line acts quickly to prevent the spread of the infection via the sensing of conserved components and the initiation of inflammation (1-3). They also phagocytose and destroy local invaders, secrete antibacterial and antiviral molecules, and recruit other cells to the site of infection through the release of chemokines (1-3). The innate immune system is comprised of a diverse set of cells capable of exerting tissue-protective mechanisms in their fight against pathogens, such as neutrophils, dendritic cells (DCs), natural killer (NK) cells, monocytes and macrophages $(1,3)$. DCs, monocytes, macrophages, known as antigen-presenting cells (APCs), can engulf the pathogen and present an antigen to activate adaptive immune cells (2).

Once activated, the adaptive immune response provides an antigen-specific response against pathogens $(1,4)$. Adaptive immune cells include $\mathrm{B}$ cells and $\mathrm{T}$ cells, named based on the initial tissue of discovery: the bursa of Fabricius of birds and the human thymus respectively (1, 4). B cells are responsible for producing antigen-specific antibodies that can bind to the pathogen and facilitate their recognition by APCs, triggering phagocytosis via $\mathrm{Fc}$ receptor recognition (1). T cells can trigger the death of infected cells, activate B cells, and secrete cytokines to attract other immune cells to the site of infection $(1,4)$. After the initial infection, adaptive immunity maintains immunological memory against the defeated pathogen, leading to a quicker and heightened response upon secondary exposure $(1,4)$. Together, the innate and adaptive systems provide host defense against a vast number of organisms of bacterial, viral, or fungal origin. 


\subsection{Macrophages and the Mononuclear Phagocyte System}

Macrophages were first discovered by Ilya Metchnikoff in 1882, who was later awarded the Nobel Prize in Physiology or Medicine in 1908 for this discovery $(5,6)$. Van Furth et al. $(7,8)$ proposed that macrophages are derived from bone marrow progenitors, which differentiate into circulating monocytes. However, recent work has found that macrophages can originate from at least three different progenitors that give rise to macrophages at different stages of fetal development and adulthood: Yolk sac-derived macrophages, fetal liver-derived macrophages, and bone marrow-derived macrophages (9-11). Macrophages originating from yolk sac progenitors are initially developed during the embryonic phase prior to fetal monocyte development, circumventing the monocytic intermediary stage (12). These cells are maintained locally and persist throughout the life $(5,6)$. Yolk sac-derived macrophages make up the majority of tissueresident macrophages found in the body. Examples include heart macrophages, alveolar macrophages of the lung, microglia of the brain, Kupffer cells of the liver and Langerhans cells of the skin (10-12). Later in development, hematopoietic stem cells seed the fetal liver and their progenitors also lead to the development of tissue-resident macrophages excluding microglia, which only originate from the yolk sac (6). The third known macrophage lineage is the welldiscussed bone marrow progenitors (6). Cellular descendants of this lineage give rise to circulating monocytes in adults, which are newly recruited into inflamed tissue microenvironments during infection and stress. A small number also maintain tissue-resident populations under conditions of homeostasis $(5,6)$. As a result, macrophages populations can arise through different origins which may be a contributing factor to the plasticity of this cell type.

Circulating monocytes can be classified based on specific surface marker expression in mice and humans. In mice, circulating monocytes are categorized based on Ly6C expression (13). 
Ly $6 \mathrm{C}^{+}$monocytes are inflammatory cells, infiltrating tissues in response to damage or infection and differentiate into macrophages (14). Conversely, Ly6C $\mathrm{C}^{-}$monocytes do not enter tissues and survey the vascular system to clear damaged endothelial cells $(14,15)$. Human circulating monocytes are separated into three distinct classes based on the expression of Cluster of differentiation (CD)-14 and CD16 (14, 16-19). Similar to $\mathrm{Ly}^{+} \mathrm{C}^{+}$monocytes in mice, $\mathrm{CD} 14^{++} \mathrm{CD} 16^{-}$monocytes are recruited to inflamed tissues and they differentiate into inflammatory macrophages $(14,19)$. $\mathrm{CD} 14^{+} \mathrm{CD} 16^{++}$monocytes shared functions similar to mice LyC6 ${ }^{-}$cells, in that they patrol the vascular system and contribute to wound healing (16-18). CD14 ${ }^{++} \mathrm{CD} 16^{+}$ monocytes are intermediary monocytes, performing the functions of the other two classes $(17,18)$.

Once in the tissue, resident tissue macrophages maintain their ability to detect microorganisms and cellular debris while adapting suppressive strategies to prevent hyperactivation and associated cellular damage due to the constant exposure to microbes and cellular debris (20). When these immunomodulatory mechanisms are absent, macrophage hyper-activation can lead to severe inflammation and tissue damage (21-23). As a result, macrophages display regional heterogeneity depending on the specific tissue microenvironment (24). For example, alveolar macrophages in the lung express higher levels of pathogen recognition receptors (PRRs) and scavenger receptors to clear pathogens and inhaled environmental particles (25). Yet, these cells are relatively anergic due to high levels of Transforming growth factor- $\beta$ (TGF- $\beta$ ) expression, critical for the development and differentiation of alveolar macrophages (26). Another regional tissue macrophage, Kupffer cells of the liver, possess heightened scavenger and bactericidal activities (27), yet are highly sensitive to entering a state of hypo-responsiveness due to stimuli overexposure (28). Intestinal macrophages are also inflammatory anergic due to TGF- $\beta$-driven inhibition of NF- $\mathrm{kB}$-associated inflammation $(29,30)$, yet retain their scavenger and bactericidal 
activities $(29,31)$. This highlights the immense pressure that a macrophage's microenvironment places on its function and the versatility of the macrophage to adapt its function to its environment.

Macrophages perform a diverse set of roles that are central to a proper immune response. During early infection, this includes the ability to phagocytose microorganisms or pathogenic components, to produce pro-inflammatory cytokines that can help recruit and activate other innate and adaptive immune cells, and the processing and presenting of pathogen-specific antigens to further activate adaptive immune cells. To promote the resolution of the inflammatory process, macrophages expressed anti-inflammatory cytokines to quell inflammation and take up apoptotic debris to help facilitate tissue remodeling and repair $(24,32-34)$. This establishes the macrophages as an invaluable link between the innate and adaptive immune systems. This adaptability of macrophages to facilitate a wide range of response is governed by the metabolic re-programming that occurs due to specific pathogen recognition and the resulting cellular microenvironment (35).

\subsection{Host Pathogen Recognition by Macrophages}

Macrophages survey their tissue microenvironment for pathogens, microbial components/proteins or host biomolecules by recognizing conserved molecular motifs known as pathogen-associated molecular patterns (PAMPs) or host components released from damaged or dying cells, that otherwise would not be present in the extracellular space, known as dangerassociated molecular patterns (DAMPs) $(1,36)$. PAMPs and DAMPs are detected by specific extracellular and intracellular PRRs $(1,36)$. Several classes of PRRs have been identified that sense viruses, among the most important are Toll-like receptors (TLRs) and Retinoic acidinducible gene I (RIG-I)-like receptors (RLRs) $(37,38)$. 


\subsubsection{Toll Like Receptors (TLRs)}

The best characterized class of PRRs are TLRs. Each member is a single-pass type I membrane protein with a $\mathrm{N}$-terminal ectodomain of leucine-rich repeats and a cytosolic $\mathrm{C}$-terminal Toll/Interleukin-1 receptor (TIR) domain (37). Mammals possess up to 12 distinct TLRs that can be categorized by their ligand specificity and by their location (Figure 1.1). Bacterial PAMPs are detected by surface TLRs such as TLR1/2/6 (lipoproteins from Gram-positive bacteria), TLR4 (lipopolysaccharide [LPS] of Gram-negative bacteria), and TLR5 (Flagellin) (39-44) (Figure 1.1). Viral PAMPs are detected by TLRs that mainly reside in endosomes including TLR3 (dsRNA), and TLR7/8 (ssRNA) (45-47) (Figure 1.1). TLR9 can detect both bacterial and viral unmethylated CpG DNA motifs $(48,49)$ (Figure 1.1).

PAMP engagement results in TLR dimerization and the initiation of downstream signalling either through Myeloid differentiation primary response 88 (MyD88) or TIR-domain-containing adapter-inducing interferon- $\beta$ (TRIF) (50). All TLRs except TLR3 utilized MyD88-dependent signalling, which recruit Interleukin (IL)-1 receptor associated kinase (IRAK) proteins to activate Tumour necrosis factor (TNF) receptor-associated factor 6 (TRAF6) (2) (Figure 1.2). TRAF6 then drives the activation of the Nuclear factor of kappa light polypeptide gene enhancer in B-cells (NF$\kappa \mathrm{B})$ inhibitor $(\mathrm{I} \kappa \mathrm{B})$ kinase (IKK) complex, which subsequently phosphorylates $\mathrm{I} \kappa \mathrm{B} \alpha$. This results

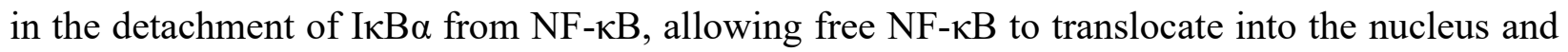
induce the expression of inflammatory cytokines (51). To maintain this activation state, NF- $\kappa \mathrm{B}$ also directly suppresses the expression of other gene targets, such as Fos and the early growth response protein (EGR), as well as up-regulate NF-kB-induced transcription factors, including Myc and Interferon regulatory factor 1 (IRF1) (52). Both TLR3 and TLR4 employ the TRIFdependent pathway (2) (Figure 1.2). For TLR3, TRIF triggers both the activation of TRAF6- 
mediated NF-KB signalling and TANK-binding kinase 1 (TBK1)/IKKe-mediated IRF3/7 signalling (50). Conversely, TRIF activation during TLR4 engagement only results in the activation of TBK1/IKK $\varepsilon$-mediated IRF3/7 signalling (53) (Figure 1.2). The phosphorylation and dimerization of IRF3 and IRF7 triggers the expression of type I interferons (IFNs), a family of antiviral cytokines $(50,54)$. 

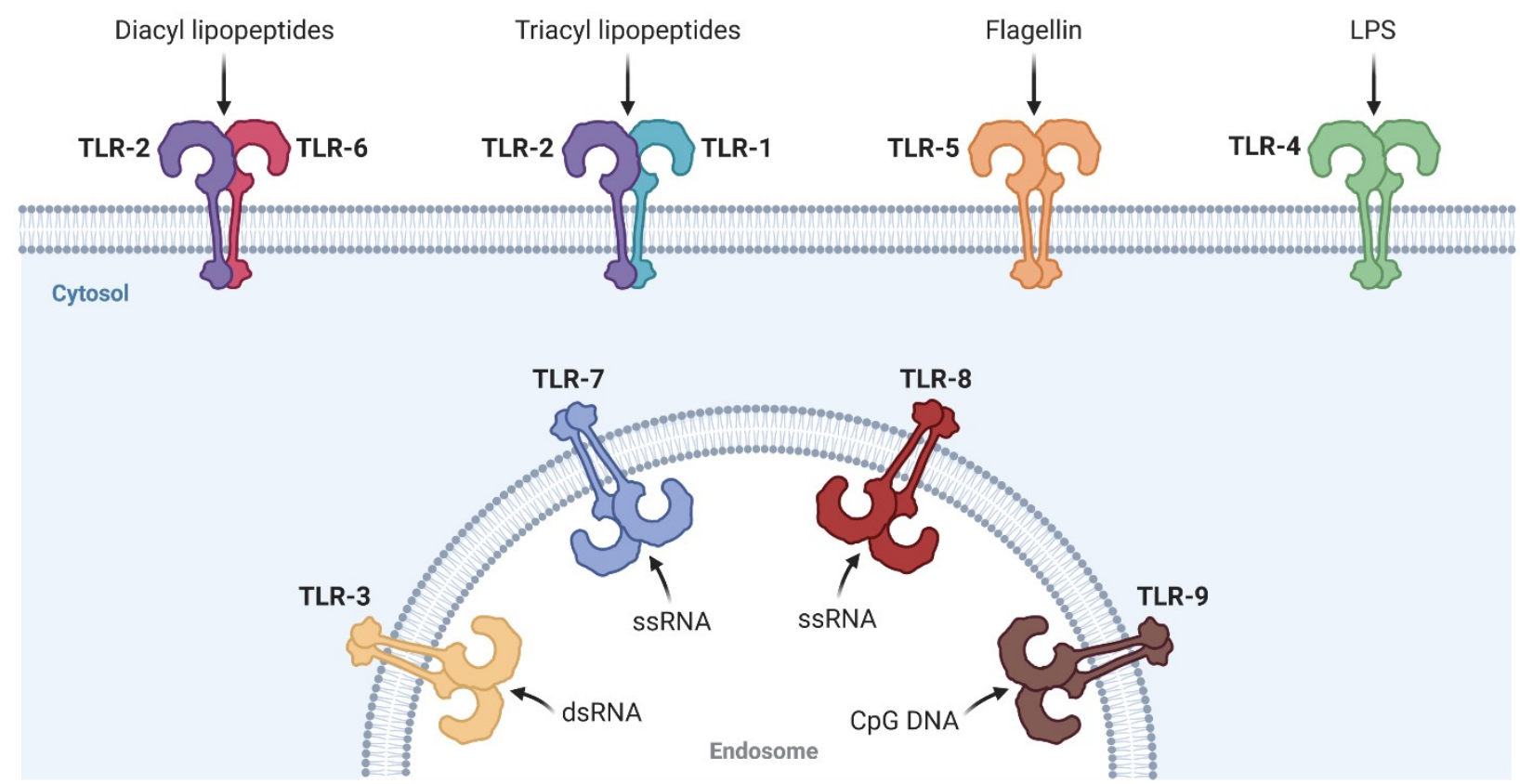

Figure 1.1: A summary of the bacterial and viral PAMPs that are detected by Toll-like receptors (TLRs). Bacterial-sensing TLRs are located along the surface of plasma membrane whereas, RNA/DNA-sensing TLRs are primarily located in the endosomes. Image created with BioRender.com. 


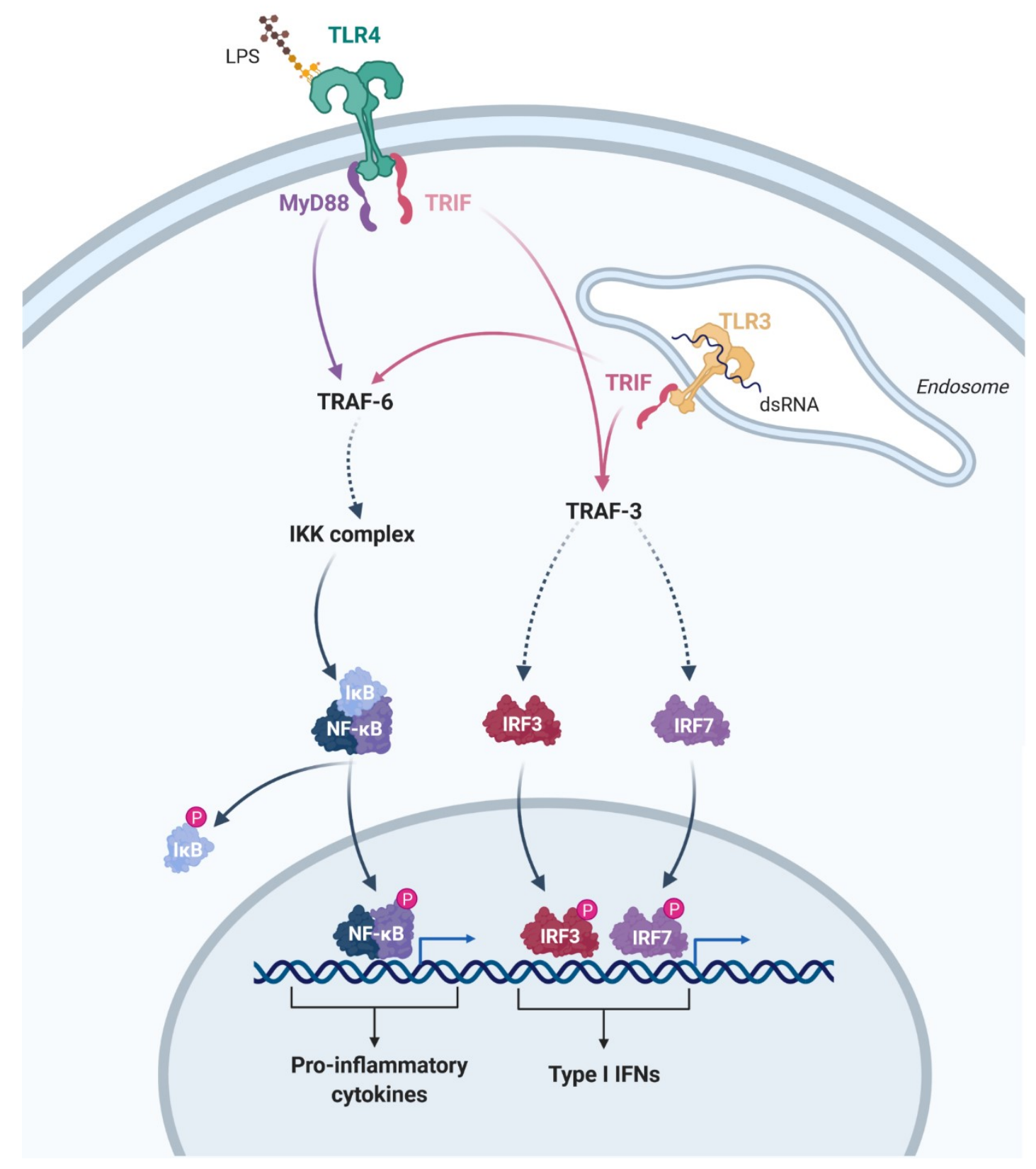

Figure 1.2: TLR3 (viral dsRNA) vs TLR4 (bacterial LPS) signaling dynamics. TLR4 induces MyD88 and TRIF activation to support the production of pro-inflammatory cytokine and Type I IFNs, respectively. Conversely, TLR3-mediated TRIF activation results in the activation of the same pro-inflammatory and IFN cascades. Image created with BioRender.com. 


\subsubsection{RIG-I-like Receptors (RLRS)}

RLRs are a family of DExD/H box RNA helicases found in the cytosol and are responsible for the detection of cytosolic viral dsRNA (55). These receptors possess a C-terminal domain and catalytic helicase core responsible for binding viral RNA, accompanied by N-terminal caspase active recruitment domains (CARD) which are essential for downstream signalling (55-57). There are two major receptors responsible for triggering effector responses: RIG-I and Melanoma differentiation-associated protein 5 (MDA5). These receptors detect dsRNA of specific lengths. RIG-I recognize short RNA strands preferentially $(<1 \mathrm{~kb})$ whereas MDA5 prefers longer RNA strands $(>2 \mathrm{~kb})(58,59)$ (Figure 1.3). This differs from the TLR family, where one receptor (TLR3) is responsible for dsRNA engagement irrespective of length (46).

Upon engagement, both RIG-I and MDA5 form filaments along their preferred dsRNA strands, leading to the polymerization of their respective CARD domains (60-63). These oligomeric formations facilitate the recruitment of the mitochondrial antiviral signaling protein (MAVS), forming the MAVS signalosome along the outer membrane of mitochondria $(61,64-67)$ (Figure 1.3). Additional MAVS proteins are then recruited to the signalosome site, leading to signal amplification (67). The MAVS signalosome drives the activation of NF- $\mathrm{B}$-mediated inflammation and the IRF3/7-driven antiviral response, via the recruitment of the IKK complex and TBK1/IKKe respectively (56) (Figure 1.3). 


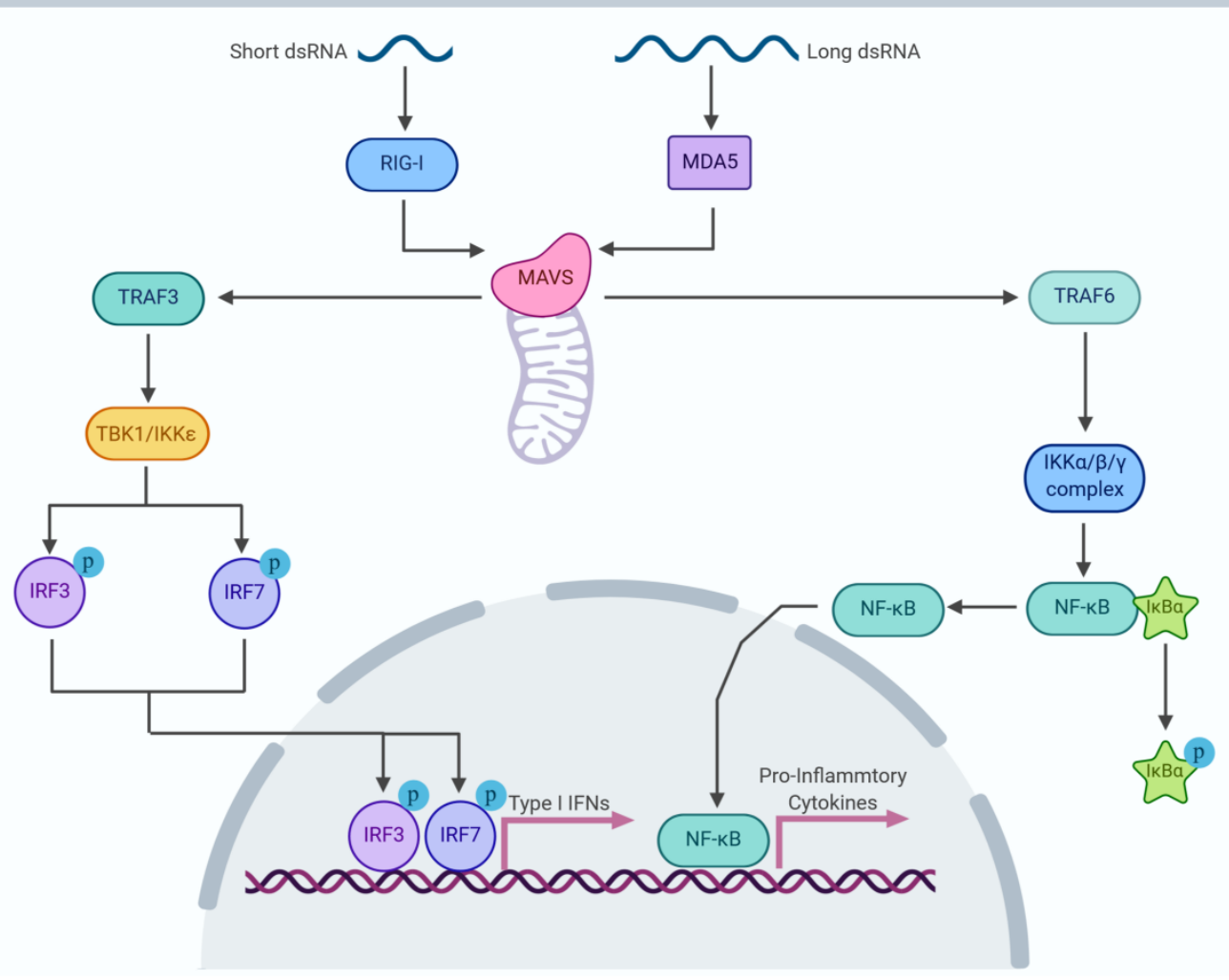

Figure 1.3: Overview of RLR signaling. RIG-I and MDA5 detect dsRNA of different lengths before engaging MAVS, initiating NF- $\kappa$ B and IRF signaling. The activation of NF- $\kappa$ B results in pro-inflammatory cytokine production while IRF3/7 activation leads to type I IFNs production. Image created with BioRender.com. 


\subsubsection{Type I IFN signalling}

The one commonality between TLR and RLR signalling is the induction of type I IFNs, triggered by IRF3/7 signalling. Type I IFNs are the largest class of IFNs, consisting of IFN- $\delta$, IFN$\varepsilon$, IFN- $\kappa$, IFN- $\tau$, IFN- $\omega$, IFN- $\beta$, and 13 different subtypes of IFN- $\alpha(68,69)$. They act in an autocrine and paracrine manner to induce antiviral states in infected and bystander cells in the local microenvironment (Figure 1.4). This state is associated with the upregulation of IFN-stimulating genes (ISGs) and expression of antiviral proteins, which prevent further viral propagation and replication. Type I IFNs also regulate antigen presentation and phagocytic activity to support adaptive immune system activation, which is critical to the locating and killing of infected cells (70-72).

Type I IFNs interact with either IFN- $\alpha$ receptor 1 or 2 (IFNAR1/2) leading to IFNAR dimerization (73) (Figure 1.4). This results in the autophosphorylation and activation of the Janus activated kinase (JAK) proteins, JAK1 and Tyrosine kinase 2 (TYK2), which are constitutively associated with IFNAR1 and IFNAR2 respectively (73). Both proteins activate the signal transducer and activator of transcription (STAT) proteins STAT1 and STAT2, leading to their subsequent nuclear translocation (74). Two different mechanisms exist resulting in ISG expression. First, STAT1 and STAT2 can bind to IRF9 to form the IFN-stimulated gene factor 3 complex (ISGF3) which recognizes the IFN-stimulated responsive elements (ISREs) resulting in ISG expression (73) (Figure 1.4). Second, phosphorylated STAT proteins can form homo- or heterodimers that are capable of binding to IFN- $\gamma$-associated sites (GAS) elements leading to ISG expression (75). ISGs can have an ISRE site and/or a GAS site, thus a combination of different STAT complex formations may be required for the expression of $>1000$ known ISGs and therefore facilitating specific immune responses (76) (Figure 1.4). In addition to the traditional JAK-STAT 
pathway, IFNAR engagement can also activate the phosphoinositide 3-kinase (PI3K)-AktMechanistic target of rapamycin (mTOR) pathway, critical for the transcription and translation of ISGs as well as the phosphorylation of STAT proteins (77-79). In addition, this pathway is responsible for the expression of genes related to energy metabolism in activated immune cells such as Hypoxic-inducible factor-1 $\alpha$ (HIF-1 $\alpha)$ and pyruvate kinase muscle isozyme 2 (PKM2) (8082). 


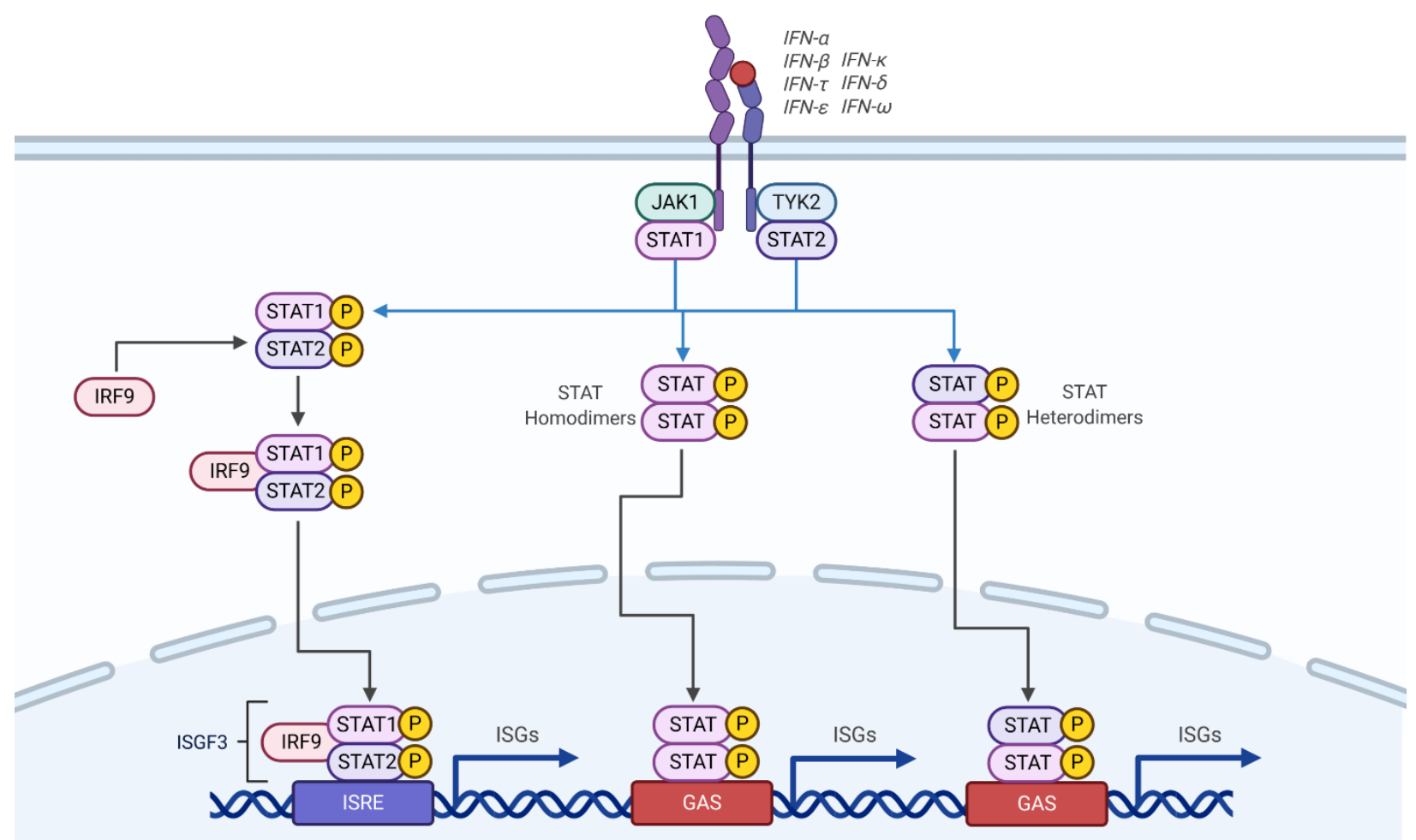

Figure 1.4: Traditional type I IFN signaling cascade. Type I IFNs act through IFNAR1 and IFNAR2, leading to the activation of JAK proteins JAK1 and TYK2. They, in turn, phosphorylate STAT family of transcription factors. STAT proteins can either form homo- or heterodimers and activate GAS-mediated ISG expression. In addition, STAT1 and STAT2 interact with IRF9 to form the ISGF3 complex and drive ISRE-mediated ISG expression. Image created with BioRender.com. 


\subsection{Mitochondria: Master Regulator of Cell Function}

Mitochondria are the central hub for several metabolic processes and play a critical role in modulating diverse cellular functions. These functions include catabolizing nutrients for energy, producing biosynthetic precursors for macromolecules (e.g., nucleotides, proteins, lipids), maintaining redox balance, and regulating apoptosis (83-85) (Figure 1.5). Here, I will outline these mitochondrial-associated processes and highlight their importance in regulating immune function.

\subsubsection{Mitochondrial Bioenergetic Pathways}

Glucose is the primary source of energy to the mitochondrion $(86,87)$. Its breakdown via glycolysis involves a ten-enzyme pathway and nets 2 pyruvate, 2 nicotinamide adenine dinucleotide $\left(\mathrm{NAD}^{+}\right.$or $\left.\mathrm{NADH}\right)$ and 2 ATP molecules per glucose molecule $(86,87)$. Other sugars, such as fructose and galactose, can feed into this pathway at various points, providing a focal point for the breakdown of sugars in cells (88) (Figure 1.6). The intermediates produced by this pathway also serves as metabolites for several different pathways critical to immune function. For example, naïve DCs build up their glycogen stores by taking up extracellular glucose and initially use these stores to support DC function in the early activation phase (89) (Figure 1.6). The pentose phosphate pathway (PPP) (responsible for nucleotide and NAD phosphate (NADPH) synthesis) has been linked to T cell activation and its need for NADPH $(90,91)$ (Figure 1.6). Glucose-derived amino acids, such as serine, are also used during $\mathrm{T}$ cell activation to drive their proliferation due to its link to de novo purine biosynthesis (92) (Figure 1.6). This highlights the fundamental role of glycolysis in cell function. 


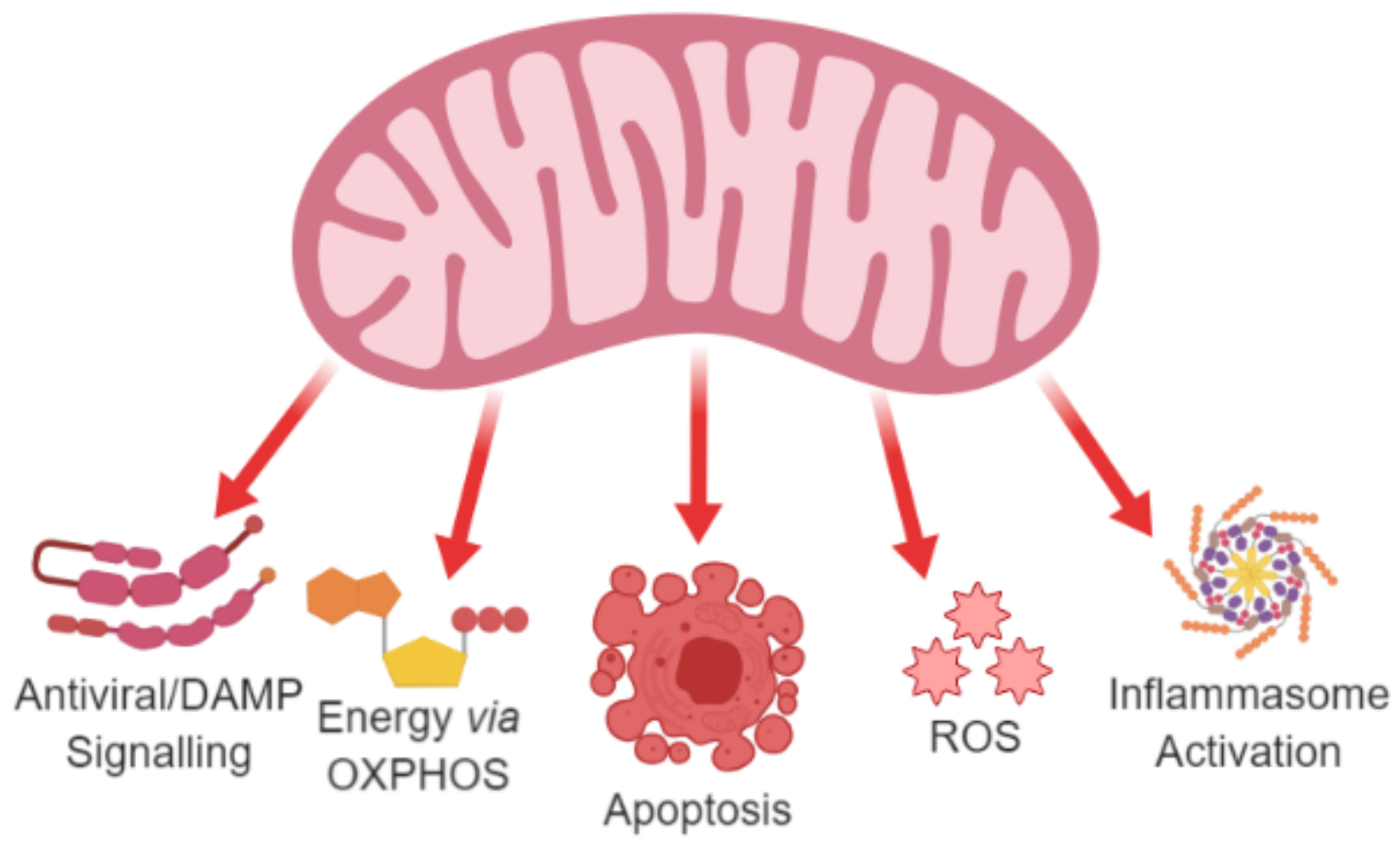

Figure 1.5: Mitochondria are the central hubs for innate immune responses. Mitochondria play a central role in several metabolic and immune processes such as functioning as scaffold for antiviral signaling, energy production via oxidative phosphorylation (OXPHOS), as well as mediate apoptosis and facilitates inflammation through regulation mitochondrial reactive oxygen species (ROS) production and inflammasome activation. Image created with BioRender.com. 


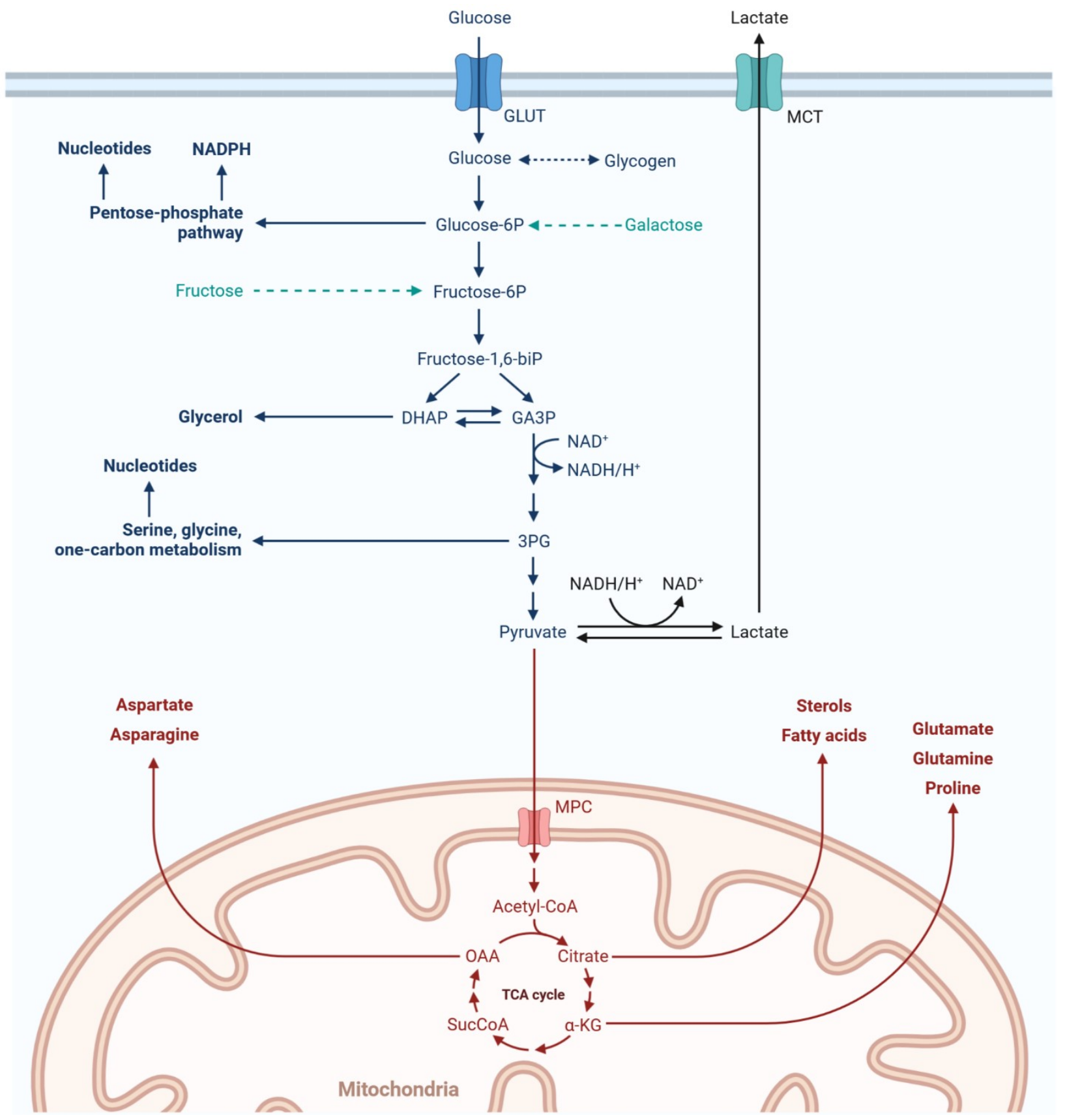

Figure 1.6: The importance of Glycolysis and the TCA cycle intermediates as building blocks for various biosynthetic pathways. Glucose and the other sugars that feed into its catalytic pathway (fructose and galactose) are processed and can be used in the generation of nucleotides, NADPH, glycerol, serine, and glycine. TCA cycle metabolites can be used to produce sterols, fatty acids, and a variety of amino acids. Image created with BioRender.com. 
Glycolytic-derived pyruvate can be further metabolized by anaerobic or aerobic means. While initially discovered in bacteria, mammalian cells can process pyruvate in the absence of oxygen in a process known as lactic acid fermentation (88). In this process, pyruvate is converted into lactate by lactate dehydrogenase $(\mathrm{LDH})$, providing a rapid and anaerobic means of $\mathrm{NAD}^{+}$ recycling to maintain this cycle of rapid glycolytic-derived ATP production $(86,88)$ (Figure 1.6). Inflammatory immune cells produce large amounts of lactate as a consequence of their need for rapid energy production (93-96). Interestingly, emerging evidence suggests that lactate possesses immunosuppressive properties to control inflammation and may serve as a feedback loop to regulate these responses $(97,98)$.

Pyruvate is differentially consumed under aerobic conditions leading to slower, yet more efficient ATP production. First, it is transported across the mitochondrial membranes by the mitochondrial pyruvate carrier (MPC) before it is consumed by the pyruvate dehydrogenase (PDH) protein complex, producing acetyl-CoA and NADH $(86,99)$ (Figure 1.6). Acetyl-CoA enters the tricarboxylic acid (TCA) cycle, and through a 10-step cycle, generates three NADH molecules and one flavin adenine dinucleotide $\left(\mathrm{FADH}_{2}\right)$ molecule $(86,88)$. TCA intermediates are also used to synthesize several macromolecules. Citrate can be transported out of mitochondria where it is used for lipid synthesis, via ATP citrate lyase (ACLY), which under inflammatory conditions assists in the production in prostaglandins, nitric oxide (NO), and reactive oxygen species (ROS) (100-102) (Figure 1.6). Both oxaloacetate and $\alpha$-ketoglutarate serve as the backbone for the generation of amino acids such as aspartate, asparagine, glutamine, and arginine $(86,88)$ (Figure 1.6). Other TCA metabolites are critical to regulating other cellular functions. Succinate accumulation can protect key inflammatory and metabolic transcription factor, HIF-1 $\alpha$, from degradation (103). Conversely, $\alpha$-ketoglutarate can boost prolyl hydroxylase (PHD) activity, responsible for targeting 
HIF-1 $\alpha$ for degradation (104). In addition, $\alpha$-ketoglutarate is a key substrate to regulating epigenetic modifications (105). This demonstrates the TCA cycle's position as a central metabolic network regulating immune function.

In addition to providing pyruvate to mitochondria, glycolysis also generates NADH that are transferred into mitochondria via two different "electron shuttles" which transport electrons from cytosolic $\mathrm{NADH}$ across the membranes to accepting mitochondrial $\mathrm{NAD}^{+}(86,88)$ (Figure 1.7). As a vital energy source, maintaining adequate levels of NADH is vital to mounting a functional inflammatory response $(106,107)$. The first shuttle, the malate-aspartate shuttle, transfers electrons from glycolytic NADH to cytosolic oxaloacetate to generate malate $(86,88)$ (Figure 1.7). Once in mitochondria, malate is re-oxidized by mitochondrial $\mathrm{NAD}^{+}$to form oxaloacetate and NADH. Oxaloacetate is recycled, via its conversion to aspartate, and transported to the cytosol $(86,88)$ (Figure 1.7). Inflammatory macrophages utilized this shuttle to replenish cytosolic $\mathrm{NAD}^{+}$to maintain the rapid glycolytic ATP production (108). The second shuttle, the glycerol phosphate shuttle (GPS), cytosolic glycerol 3-phosphate (G3P) dehydrogenase 1 (GPD1) reduces dihydroxyacetone using cytosolic NADH, to form G3P (Figure 1.7). G3P readily transverse the outer mitochondrial membrane where it is re-oxidized by mitochondrial GPD2, which reduces FAD within the inner mitochondrial membrane $(86,88)$ (Figure 1.7). GPD2-/macrophages produce less inflammatory mediators (IL-6 and IL-1 $\beta$ ) due to reduced glycolytic activity (109). 


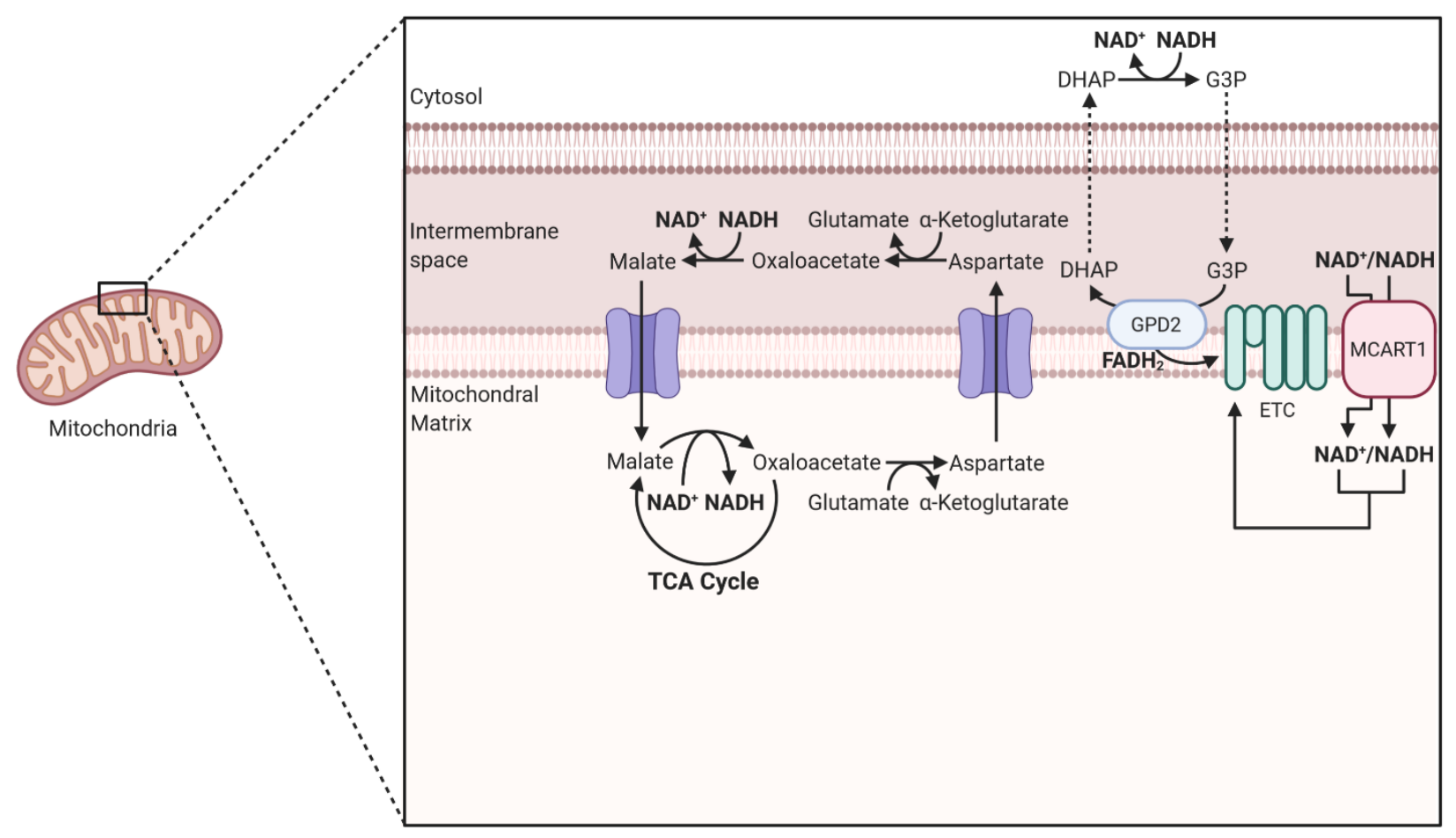

Figure 1.7: Methods of $\mathrm{NAD}^{+} / \mathrm{NADH}$ transport into the mitochondria. $\mathrm{NAD}^{+} / \mathrm{NADH}$ can be transported into mitochondria via three methods: The malate-aspartate shuttle, the glycerol phosphate shuttle and the newly discovered SLC25A51/MCART1 transporter. Image created with BioRender.com. 
It has long been believed that the inner mitochondrial membrane was impermeable to $\mathrm{NAD}^{+} / \mathrm{NADH}$ and thus cytosolic $\mathrm{NAD}^{+} / \mathrm{NADH}$ could only enter mitochondria via these "shuttles" $(86,88)$. Recently, two independent groups have identified a mitochondrial $\mathrm{NAD}^{+} / \mathrm{NADH}$ transporter (SLC25A51/MCART1), providing a direct mechanism of glycolytic NADH to enter mitochondria $(110,111)$. More work is required to determine which mechanism is the primary means of $\mathrm{NAD}^{+} / \mathrm{NADH}$ transfer or if specific pathways are important under certain conditions.

The generated NADH molecules from these pathways then enter the mitochondrial process called oxidative phosphorylation (OXPHOS) (99). NADH molecules are shuttled into the electron transport chain (ETC), a series of protein complexes that transfer electrons through a series of electron donors embedded within the inner mitochondrial membrane $(86,99)$ (Figure 1.8). NADH is oxidized by Complex I (NADH ubiquinone oxidoreductase) and the electrons are passed to the electron carrier ubiquinone (UQ) forming ubiquinol $\left(\mathrm{UQH}_{2}\right)(86,99)$. The energy generated by the electron transfer is used by Complex I to transport four protons into the mitochondrial intermembrane space (Figure 1.8). Complex II (Succinate dehydrogenase [SDH]) is a parallel electron transfer pathway, linked to the TCA cycle. Succinate oxidation leads to the reduction of FAD which transfers its electrons to $\mathrm{UQ}$ forming $\mathrm{UQH}_{2}(86,99)$ (Figure 1.8). However, Complex II does not participate in the shuttling of protons out of mitochondria and thus generates less energy compared to Complex I. The reduced $\mathrm{UQH}_{2}$ then passes the electrons onto Complex III (Coenzyme Q: cytochrome c-oxidoreductase), where it is shuttled to cytochrome c and results in the translocation of four protons out of the mitochondrial matrix (Figure 1.8). Cytochrome $\mathrm{c}$ interacts with Complex IV (Cytochrome c oxidase), transferring electrons to molecular oxygen, producing water $(86,99)$. Another four protons are shuttled into the intermembrane space by Complex IV (Figure 1.8). The shuttling of these protons across the mitochondrial inner membrane creates a 
proton gradient, which is used by Complex V (ATP synthase) to generate ATP (Figure 1.8). In short, Complex V transports three protons back into the mitochondrial matrix and uses the energy of that transfer to create a single ATP molecule (Figure 1.8). In total, under aerobic conditions, one glucose molecule can generate 36 ATP molecules compared to 2 ATP molecules from anaerobic glycolysis, making OXPHOS the more efficient means of producing cellular energy (86, 99).

To further increase the efficiency of electron flow and limit electron leakage, Complexes I, III, and IV can also form a series of ETC supercomplexes (112). These complexes work with the mitochondrial uncoupling protein family (UCP) to reduce mitochondrial membrane potential (MMP) by funneling protons back into the mitochondria, reduce the electron leakage and prevent ROS production $(112,113)$. These higher-level structures are critical to maximize energy efficiency of the organelle. Interestingly, under inflammatory conditions (e.g., LPS activation), macrophages undergo ETC supercomplex disassembly, which increases mitochondrial ROS (mtROS) production/release and activates NOD-like receptor family pyrin domain containing 3 (NLRP3) inflammasome (114). 


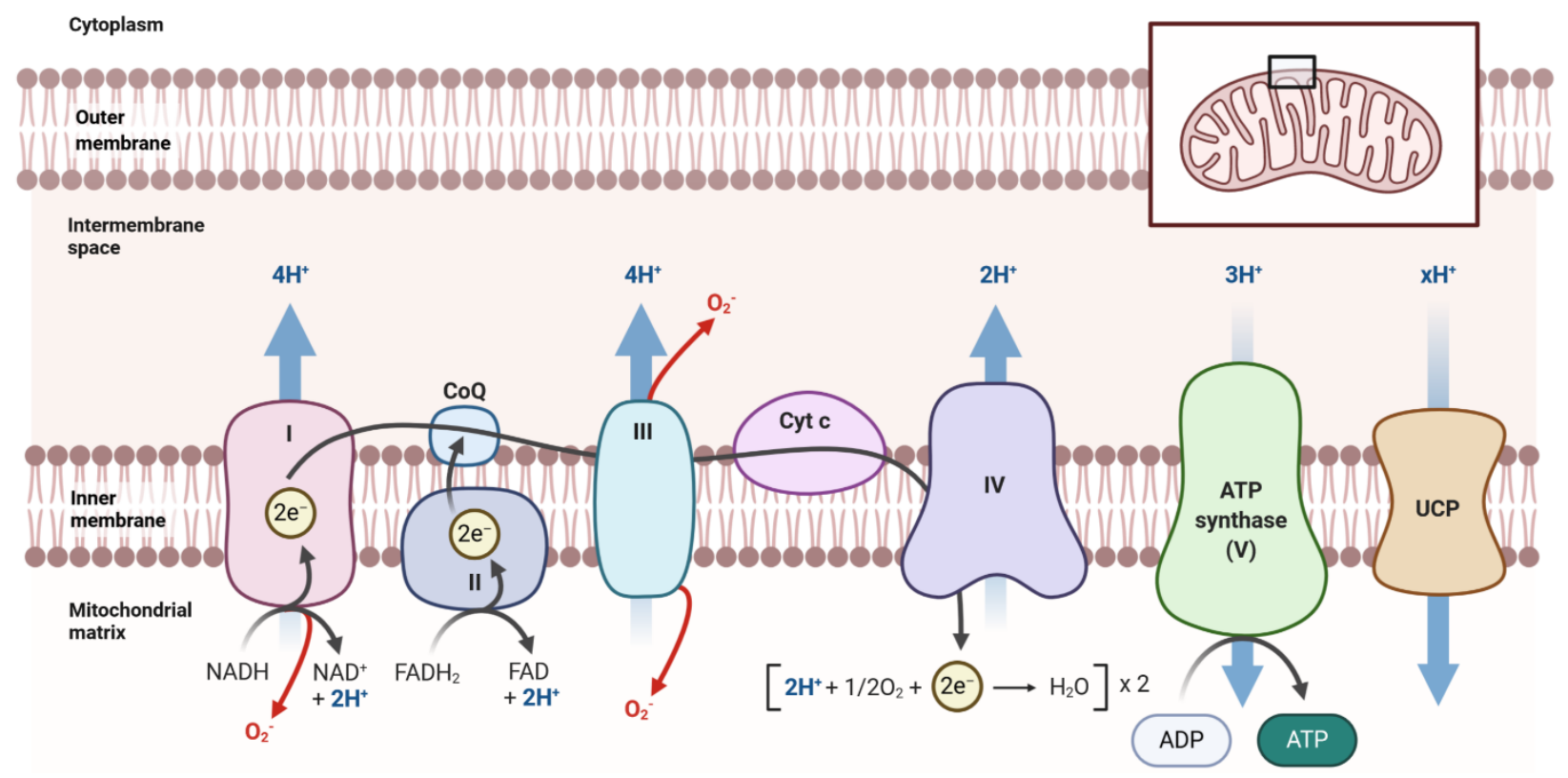

Figure 1.8: Oxidative phosphorylation is coupled with the electron transport chain to generate ATP in the mitochondria. Located in the inner mitochondrial membrane, electrons from NADH are transferred across the four complexes of the ETC. The electron movement is coupled with the pumping of protons out of the mitochondrial matrix into the intermembrane space, where ATP synthase (Complex V) transports the protons back into the matrix, using the energy created to produce ATP. Uncoupling proteins are capable of transporting protons back into the matrix absent of ATP production. Image created with BioRender.com. 
There are alternative energy pathways that make use of OXPHOS without any input from glycolysis (Figure 1.9). Fatty acid (FA) oxidation (FAO) breaks down FAs to produce acetyl-CoA (99) (Figure 1.9). However, before they can be broken down, they are transported into mitochondria using the carnitine shuttle. Long chain fatty acid-CoA ligase attaches coenzyme A (CoA) to free FAs to facilitate transport (99). The resulting Acyl-CoA is transferred onto carnitine by the outer mitochondrial membrane-embedded carnitine palmitoyltransferase (CPT) 1 (99). Carnitine-acylcarnitine translocase relocates acyl-carnitine into mitochondria where CPT2, located on the inner mitochondrial membrane, separates carnitine and the attached FA (99). The released carnitine is then transported back to the cytosol to continue the cycle. Once in the mitochondrial matrix, acyl-CoA undergoes $\beta$-oxidation by cleaving two carbons from the acyl group to form acetyl-CoA and the shorter acyl-CoA (99). This cycle is continued until the final cycle yields two acetyl-CoA molecules. Each cycle of $\beta$-oxidation yields 1 NADH and acetyl-CoA molecule which feeds into OXPHOS and TCA cycle, respectively (Figure 1.9). This results in 14 ATP molecules per cycle $(86,99)$. This alternative energy pathway is mainly utilized during antiinflammatory and antiviral responses (115-117). In addition, the overexpression of a constitutively active CPT1 mutant led to reduced inflammation and improved insulin sensitivity in RAW 264.7 macrophages. (118).

Another mitochondrial bioenergetic pathway is glutaminolysis, or the breakdown of glutamine (Figure 1.9). Glutamine is transported into mitochondria where it converted into glutamate $(86,88,99)$. The subsequent glutamate is directed into the TCA cycle, via its transformation into $\alpha$-ketoglutarate $(86,88,99)$ (Figure 1.9). This pathway generates one NADH molecule, in addition to the $2 \mathrm{NADH}$ produced due to $\alpha$-ketoglutarate's insertion into the TCA 
cycle $(86,88,99)$ (Figure 1.9). In addition, this pathway is used under inflammatory conditions to replenish citrate and itaconate levels in the cell (108).

Lastly, the catabolism of branched-chain amino acids (BCAAs), such as leucine, isoleucine, and valine, is another source of energy into mitochondria $(86,88,99)$ (Figure 1.9). Broken down by the branched-chain alpha-keto acid dehydrogenase (BCKDH) complex, BCAAs are converted into various acyl-CoA derivatives which eventually result in acetyl-CoA and/or succinyl-CoA generation that enter the TCA cycle $(86,88,99)$ (Figure 1.9). This process directly nets $1 \mathrm{NADH}$ plus either 1 or $3 \mathrm{NADH}$ due to succinyl-CoA or acetyl-CoA entry into the TCA cycle $(86,88,99)$. This pathway is critical to macrophage effector function. Intestinal macrophages with a selective knockout of BCAA transporter CD98hc were found to be more prone to apoptosis (119). Further, the cytosolic BCAA aminotransferase (BCAT1) is upregulated during antibacterial responses to provide additional fuel to mount an immune response $(120,121)$. Interestingly, this is independent of its role in BCAA catabolism as leucine levels were unchanged, suggesting a possible moonlighting role for BCAT1 (120). This assortment of energetic pathways to support cellular activation and function provides the cell with some level of flexibility in tissue microenvironments with varied levels of nutrients available and/or under conditions of stress such as infections where nutrients are rapidly consumed. 


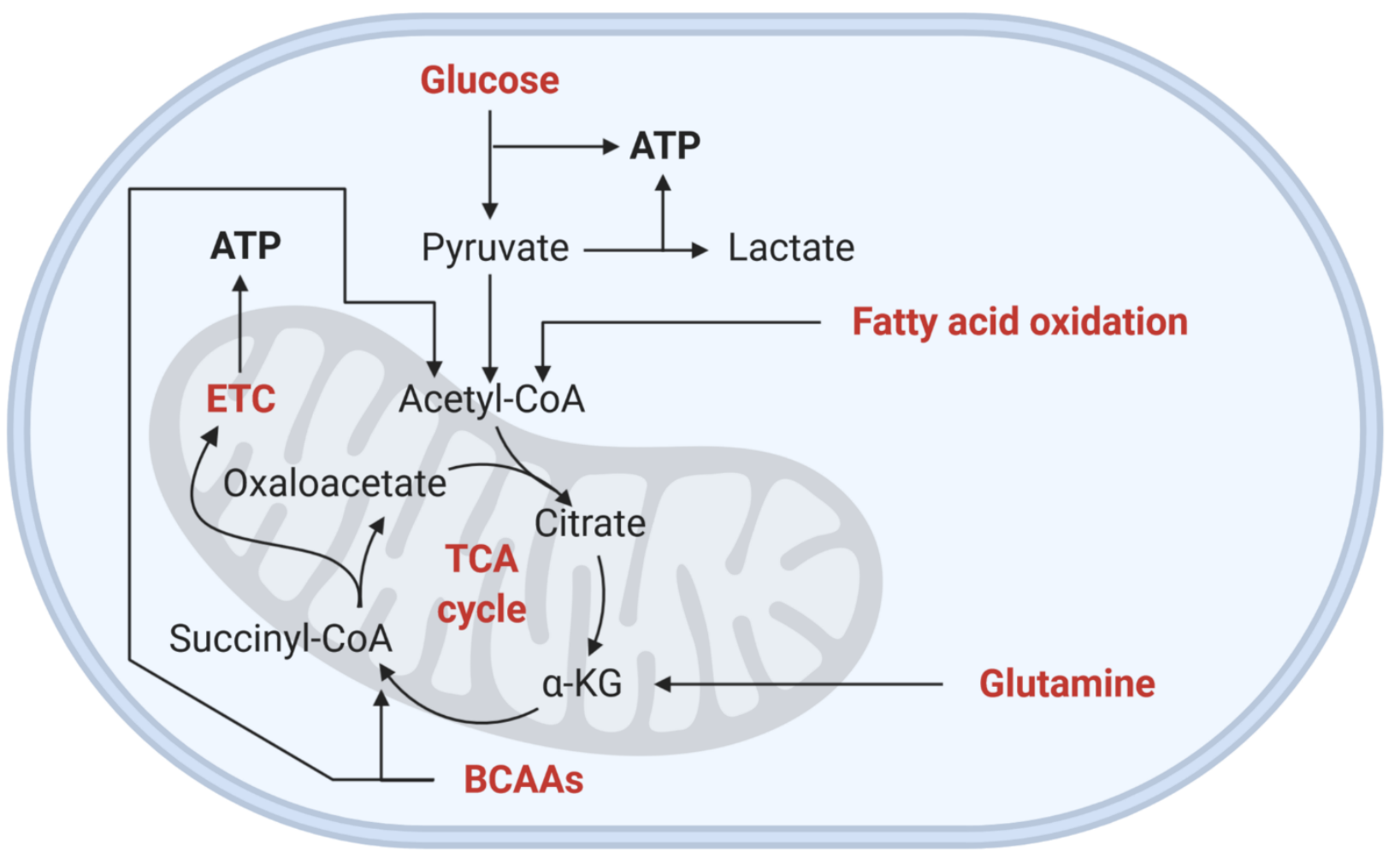

Figure 1.9: The Mitochondrial Bioenergetic Pathways. Several different bioenergetic pathways feed into mitochondria to produce ATP. While glycolysis can rapidly generate ATP, pyruvate directed into mitochondria produces more ATP per glucose molecule. Fatty acid oxidation, glutamine, and branched-chain amino acids (BCAAs) can feed into the TCA cycle, leading to the generation of ATP via oxidative phosphorylation. Image created with BioRender.com. 


\subsubsection{Other important mitochondrial functions}

In addition to being the powerhouse of the cell, mitochondria produce several other bioactive molecules, which regulate cell function. Among the most potent of these are mtROS, which are generated as a by-product of electron flux through the ETC (Voet et al., 2016; Berg et al., 2008; Spinelli \& Haigis, 2018) (Figure 1.8). ROS are generated because of electron leakage from Complexes I and III $(86,99)$ (Voet et al., 2016; Spinelli \& Haigis, 2018). Initially thought to be detrimental to cells, it is now recognized that low levels of ROS regulate several cellular processes including cell proliferation, differentiation, and migration $(122,123)$. In plasmacytoid DCs, mitochondrial superoxide is a key driver of antiviral responses via increased MAVS protein expression (124). Mitochondrial-derived hydrogen peroxide $\left(\mathrm{H}_{2} \mathrm{O}_{2}\right)$ has been also shown to support antiviral and inflammatory responses through the activation of redox-sensitive NF- $\mathrm{kB}$ and IRF signalling (125-128). Further, mtROS contributes to NLRP3 inflammasome activation (114) and drive the transcription of inflammatory genes (129) (Figure 1.5). For the most part, the damaging effects of ROS on mitochondria is limited by the presence of antioxidant proteins (86,

$88,99)$. However, if the redox balance is lost, accumulating ROS trigger the intrinsic apoptosis pathway via activation of the B cell lymphoma 2 (BCL2) protein family (130). Damaged mitochondria released mitochondrial DNA (mtDNA) as a danger signal and recent evidence has pointed to this being a key promoter of inflammation, via PRR and NLRP3 activation (131-133).

\subsection{Metabolic Regulation of Macrophages}

The last decade of research has placed a spotlight on the central role that cellular metabolism plays in controlling the magnitude and specificity of the innate immune response (35, 83). It is well recognized that cellular metabolism provides the necessary biosynthetic and bioenergetic requirements to support effector function and survival. Consistent with these findings, 
initial work done by Warburg and colleagues, demonstrated that activated leukocytes and cancer cells were metabolically similar, in that they favoured glycolysis as a primary energy producer over the more efficient OXPHOS (134-136). This was done to provide essential metabolic precursors quickly for rapid cell division and function (Figure 1.6). However, an increasing number of studies from researchers in the field have shown it is so much more complicated and interesting than that. Metabolic enzymes, metabolites and bioactive molecules also play a central role in regulating gene transcription, epigenetics and signalling cascades, which contribute to immune cell differentiation, maturation, and activation (103, 137-139).

Macrophages are highly plastic and heterogeneous cells that are responsible for a wide range of responses along the inflammatory spectrum (140). Macrophages initiate and suppress inflammation and as a result it has been customary to bifurcate activated cells based on those primary functions (140). The M1 state, induced by inflammatory ligands LPS and IFN- $\gamma$, drives a pro-inflammatory state by producing inflammatory cytokines (TNF- $\alpha$, IL-1 $\beta$, IL-6) to attract other immune cells to the infection site to target and kill microorganisms (93, 94) (Figure 1.10). Conversely, M2 macrophages, due to IL-4 and IL-10 activation, generate anti-inflammatory cytokines (IL-10, TGF- $\beta$ ), clearing cellular debris to quell inflammation and drive tissue remodeling and repair (5) (Figure 1.10). M2 macrophages can be further divided into three separate sub-phenotypes based on function: M2a, M2b, M2c $(141,142)$. M2a macrophages arises in response to IL-4 and are involved in the T helper 2 cell-type immune response; M2b macrophages are induced in response to immune complexes and TLR and IL-1 receptor agonists and regulate the magnitude of immune responses; M2c macrophages arises in response to IL-10 or TGF- $\beta$ and are involved in tissue remodeling $(141,142)$. 
Interestingly, these activation states are associated with distinct metabolic profiles driven in part by differential mitochondrial function (140). Supported by the Warburg effect, M1 macrophages are heavily reliant on glycolysis as its primary energy source (134-136) (Figure 1.9). Mitochondria are reprogrammed from an ATP-producing to a ROS-generating organelle via reduced SDH activity, driving reverse electron transport (RET), a buildup of electrons at Complex I of the ETC and subsequent ROS generation (143). The altered SDH activity leads to succinate accumulation, which inhibits PHDs and results in the stabilization of HIF-1 $\alpha$, responsible for the upregulation of glycolytic and inflammatory genes $(103,144)$. Alternatively, M2 macrophages are dependent on OXPHOS and FAO as their primary energy sources, mediated by nutrient-sensing AMP-activated protein kinase (AMPK) activation $(145,146)$ (Figure 1.9). The plasticity of macrophages allows them to adapt their phenotype based on their microenvironment, but this ability is dependent on the degree of mitochondrial impairment in the cell $(147,148)$, highlighting the importance of mitochondria as the dynamic regulator of proper innate immune function. 


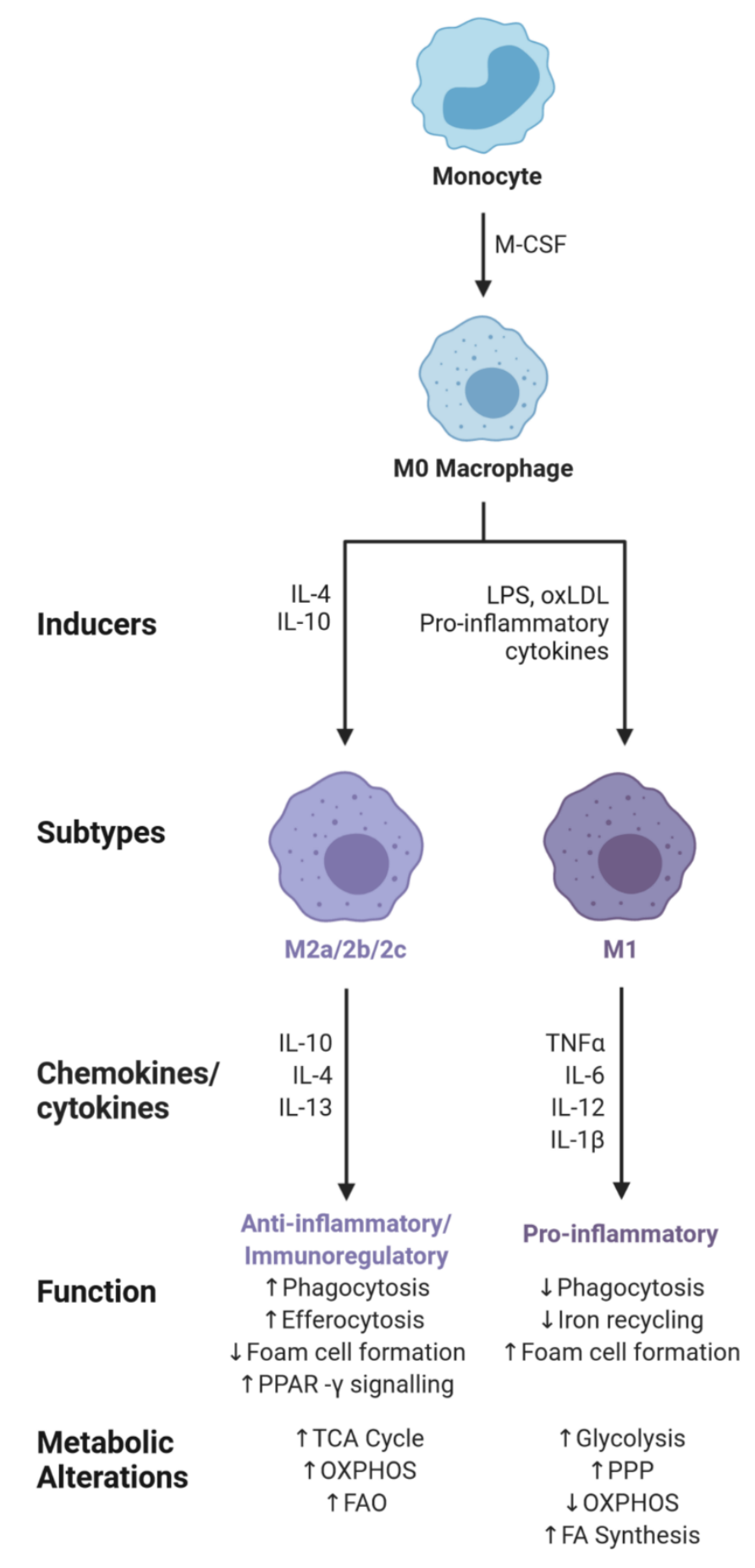

Figure 1.10: The differences between $M 1$ and $M 2$ macrophages. M1 and M2 are functionally distant macrophage phenotypes. While M1 macrophages are pro-inflammatory in nature, M2 macrophages are considered anti-inflammatory. These divergent functions are associated with differential metabolic re-programming which supports their respective functions. M1 macrophages rely on glycolysis for ATP production, whereas M2 macrophages are fueled by OXPHOS and FAO. Image created with BioRender.com. 


\subsubsection{Metabolic Reprogramming of LPS engagement of TLR4}

LPS stimulation is thought to induce an M1 profile and has been widely used to model changes in cellular metabolism across inflammatory cells. However, it is increasingly recognized that the metabolic alterations associated with LPS stimulation are not observed in all inflammatory conditions. A plethora of studies have outlined that diverse pro-inflammatory and antiinflammatory stimuli possess their own distinct metabolic profiles and suggest that the M1/M2 dichotomy is more of a spectrum of macrophages activation states with varying degrees of metabolic reprogramming (32).

\subsubsection{Reprogramming of Glycolysis}

A key feature of LPS-associated metabolic reprogramming is an increased reliance on glycolysis, mediated by HIF-1 $\alpha$ and NF- $\mathrm{KB}(93,149,150)$. This reprogramming starts within minutes of receiving the stimuli by increasing glucose influx through Glucose transporter (GLUT)1 and GLUT6 $(150,151)$. This influx is required to initiate inflammatory responses (152). To handle the increased influx of glucose, glycolytic genes (e.g. LDHA, PDK1, PFKFB3) are upregulated to quickly metabolize glucose to pyruvate and funnel pyruvate towards the production of lactate, and not acetyl-CoA $(93,153,154)$. Interestingly, these glycolytic enzymes also participate in secondary "moon-lighting" roles to facilitate inflammation. For instance, Glyceraldehyde 3-phosphate dehydrogenase (GAPDH) is recruited to the mRNAs of inflammatory genes such as IFN- $\gamma$ and TNF- $\alpha$ and suppresses their translation under resting conditions (155157). Following LPS activation, GAPDH malonylation results in its dissociation from these mRNAs, leading to inflammatory cytokine expression (157). Similarly, PKM2 traditionally functions as a tetramer in glycolysis (158). However, under inflammatory conditions, PKM2 is converted into its dimer form and translocates to the nucleus to co-activate HIF-1 $\alpha$ and STAT3, 
boosting IL-1 $\beta$ and IL-6 levels respectively (Palsson-McDermott et al., 2015; Shirai et al., 2016). This increased dependence on glycolysis for energy leads to rapid ATP and NADH production from the pathway $(93,106)$.

The intermediary molecules of glycolysis are also used as precursors to biomolecules that serve critical roles in regulating LPS-mediated inflammation. For example, PPP gene expression (e.g. G6PD) and activity is elevated in LPS-stimulated mouse bone marrow-derived macrophages (BMM) $(108,159)$. The PPP is a major producer of the cofactor NADPH, a critical substrate for ROS-producing NADPH oxidase (NOX) and various enzymes involved in energy metabolism, cholesterol, and FA synthesis (108). Further, in its dephosphorylated form, NADH serves as the main source of energy transport throughout the macrophage. High levels of $\mathrm{NAD}^{+}$are also consumed during inflammatory responses to protect against excess ROS-associated DNA damage (107). To maintain a sufficient supply of $\mathrm{NAD}^{+}$, two separate NAD-related pathways are activated during LPS activation: Tryptophan-driven de novo $\mathrm{NAD}^{+}$synthesis (160) and $\mathrm{NAD}^{+}$salvaging pathway $(106,107)$. Both pathways allow for the sustaining of Warburg metabolism during LPS activation. Finally, glycolytic-derived NADH is funnelled into mitochondria using the GPS, to fuel various vital mitochondrial reactions supporting IL-6 and IL-1 $\beta$ production (109).

\subsubsection{Reprogramming of Mitochondrial Function}

Another central feature of LPS engagement is the multi-faceted impairment of OXPHOS activity. During LPS stimulation, two breaks occur along the TCA cycle: at isocitrate dehydrogenase (IDH) and SDH (108). LPS-induced IFN- $\beta$ downregulates the expression of IDH via IL-10 production (161). Itaconate is capable of inducing IFN- $\beta$ production, creating a feedforward mechanism to quell inflammation (162). The resulting break at IDH forces citrate into two directions. First, citrate is transported out of mitochondria to facilitate FA and prostaglandin 
biosynthesis via increased activity of mitochondrial citrate carrier and ACLY $(101,163)$. Second, citrate is directed towards immunoresponsive gene 1 (IRG1), responsible for producing antibacterial and immunosuppressive itaconate $(161,164,165)$. Itaconate targets several different proteins including NF-E2-related factor 2 (NRF2) (responsible for blocking IL-6 and IL-1 $\beta$ production), and $\mathrm{SDH}(162,164,166-169)$. This decrease in SDH activity leads to succinate accumulation and the inhibition of PHDs, stabilizing HIF-1 $\alpha(103,164,168)$. However, some SDH activity is required to drive the RET phenomenon, funnelling electrons to Complex I and ROS generation and further stabilizing HIF-1 $\alpha$ by inactivating the iron cofactor of PHDs $(114,143$, 170-172). This can be blocked with the use diabetic drug Metformin, which inhibits Complex I activity and boosts IL-10 expression (173). It also blocks glucose uptake and glycolytic activity to further quell the inflammatory response (174). Other TCA metabolites, such as fumarate and $\alpha$ ketoglutarate, also play a role in driving epigenetic reprograming to augment TNF- $\alpha$ and IL-6 production (175).

In addition to the repurposing of the TCA cycle, the ETC undergoes a considerable rearrangement during LPS activation. Activated macrophages force ETC supercomplex disassembly via Optic atrophy 1 (OPA1) downregulation, facilitating the increased reliance on glycolytic energy and amplified inflammatory response observed in LPS-activated macrophages $(114,176)$. This disassembly leads to increased mtROS-mediated NLRP3 activation $(114,177)$. The conversion of mitochondria from an energy-producing organelle to a ROS-generating organelle augments the bactericidal activity of macrophages as they are recruited to bacteriacontaining phagosomes to facilitate bacteria killing (170). Furthermore, Complex III-derived $\mathrm{H}_{2} \mathrm{O}_{2}$ drives disulfide bond formation within the IKK complex, activating it and driving NF-KB translation $(128,178)$. To prevent hyper-inflammation, UCP2 reduces MMP to lessen mtROS 
levels (179-181). Although the mitochondrion is not used in its traditional role as a central energy hub, ETC activity still is vital to LPS-driven effector functions.

\subsubsection{Reprogramming of Amino Acid Metabolism}

Amino acids metabolism serves diverse roles during LPS activation. This includes the catabolism of glutamine, arginine, serine, and leucine. In LPS-stimulated macrophages, glutamine is broken down, via glutaminolysis, and funnelled into the TCA cycle as $\alpha$-ketoglutarate (103, 108). This is done to replenish the carbons lost due to the various diversions of citrate and further drive succinate accumulation $(103,108)$. Arginine transport is upregulated during LPS activation, facilitating NO production by the inducible NO synthase (iNOS) (182-184). The molecule NO is a powerful bactericide and inhibitor of OXPHOS activity, both of which further enable the proinflammatory state $(183,184)$. Additionally, NO inhibits TCA enzyme aconitase and PDH, blocking glucose-derived carbon flow through the TCA cycle (185). The other product of the iNOS reaction, citrulline, is then directed towards the feedforward aspartate-arginosuccinate shunt, to continuously generate arginine for iNOS-derived NO production (108). Glucose-derived serine and serine uptake is critical for IL-1 $\beta$ expression $(186,187)$. Serine is used to generate glycine, which is required for glutathione synthesis as well as to fuel the epigenetic modification of proinflammatory genes such as IL-1 $\beta(186,187)$. BCAAs (e.g. leucine, valine, isoleucine) are also used to maintain the inflammatory state, with increased uptake and expression of BCAT1 (121, 188). The loss of BCAT1 resulted in reduced itaconate and IRG1 levels due to decreased glycolytic activity $(120,121)$. Interestingly, this occurs independent to its native function by driving NRF2mediated antioxidant response and glucose-derived TCA metabolite production (120). This further demonstrates the complicated networks of pathways integral to driving effector function. 


\subsubsection{Reprogramming of Lipid Metabolism}

Lipid metabolism plays a critical role in regulating LPS-driven responses in macrophages. LPS-stimulated macrophages diminish cholesterol biosynthesis and promotes cholesterol ester formation in a type I IFN-dependent manner (189-191). Autocrine IFN- $\beta$ signalling also diverts excess cholesterol towards Cholesterol 25-hydroxylase (CH25H)-mediated 25-hydroxycholesterol (25-HC) production (189). This removal of cholesterol and the reprogramming of its lipid membrane composition, mediated by MyD88-dependent NRF2 and Sterol regulatory elementbinding protein (SREBP) signalling, increases membrane fluidity and improves phagocytotic capabilities $(190,192)$. 25 -HC provides resistance against the binding of cholesterol-dependent cytolysins to cellular membranes (193). Moreover, the production of 25-HC also reduces SREBP2driven translation, responsible for the expression of cholesterol biosynthetic genes (189). Its counterpart, SREBP1, is upregulated in the later stages of activation phase to drive antiinflammatory FA production in a Liver X receptor (LXR)-dependent manner (194). Another late expressing protein, Sphingosine kinase 2 (SPHK2) is degraded within 6hrs of activation to

promote inflammation before its induction $24 \mathrm{hrs}$ post-stimulation to quell inflammation in a Stimulator of type I interferon genes (STING)- and NF-kB-dependent manner $(195,196)$.

\subsection{Role of cellular metabolism in viral infections}

The role of cellular metabolism in regulating macrophage effector function is not limited to antibacterial responses. Recent evidence has indicated that antiviral responses are supported by a rewiring of metabolic networks (197). In addition, viruses attempt to manipulate these cellular networks to favour viral replication and expansion and subvert the host immune response (197, 198). A better understanding of this complex interplay is required to elucidate their individual contributions as well as their cumulative effect on macrophage metabolism during viral infections. 


\subsubsection{Virus-mediated hijacking of cellular metabolism}

Viruses are obligate intracellular microorganisms that use the host cell machinery to support their life cycle. To do this, they hijack the host cell's metabolism to build the macromolecules required to promote viral replication and assembly as well as to support the survival of infected cells (197). Rapid viral replication depends on an immense amount of energy and requires rapid and robust energy production (198). One key metabolic pathway that provides rapid energy and the production of macromolecule precursors is glycolysis. Human cytomegaloviruses (HCMV) increase glucose uptake and consumption in infected cells by promoting the expression of GLUT4, which has three times higher glucose affinity than GLUT1 (199). This increases glucose-derived TCA metabolite flux, providing the building blocks to facilitate inflammation (200). Metabolomic analysis of Influenza A (IAV)-infected macrophages revealed that the virus upregulates glycolytic-derived citrate and succinate to drive FA synthesis, for viral envelope assembly, and HIF-1 $\alpha$ accumulation respectively (201). IAV is not the only virus that upregulates HIF-1 $\alpha$. Both the Human immunodeficiency virus (HIV) and Hepatitis C (HCV) increased HIF-1 $\alpha$ levels to boost glycolysis (202-206). During HIV infection, HIF-1 $\alpha$ expression is elevated in both infected and bystander cells to promote a pro-inflammatory state and viral replication (207). Alternatively, Adenovirus and the Herpes simplex virus (HSV) upregulate glycolysis to drive intermediates to PPP and boost nucleotide synthesis to support viral genome replication $(208-210)$.

In addition to altered glucose metabolism, viruses employ strategies to altered mitochondrial function to support viral replication. For instance, Dengue virus infection is associated with increased mtROS and blocked mitochondrial fusion, which drives mitochondrial damage and DNA release leading to a dependency on glycolysis and the production of 
biomolecular precursors required for viral replication (211-213). Conversely, Hepatitis B infection increases Dynamin-related protein 1 (DRP1) activation while promoting mitofusin 2 (MFN2) degradation, favouring mitochondrial fission (214). HCV expresses several proteins that alter mitochondrial function by either reducing the levels of Complex IV subunits $(215,216)$, inhibiting Complex I activity to induce mtROS $(217,218)$, or by altering mitochondrial fusion/fission (219). These processes force the cell to rely on glycolysis for rapid energy production. Interestingly, HIV1 selectively infects high energy CD4+ T cells irrespective of their activation phenotype (220). Yet, HIV-infected macrophages that survive the initial infection have reduced ATP production, suggesting differential cell type machinery adaptation to ensure viral survival (221).

Viral replication is an energy intensive process and as a result requires access to multiple bioenergetic pathways for viral propagation. Adenovirus and HCV infections cause increased glutaminolysis and glutamine dependence to support robust viral replication and cell growth (208, 222). In addition, HIV-infected macrophages have an increased reliance of glutamine as an energy source (221). Vaccinia viral infection preferentially increase intracellular glutamine levels as it is the primary energy source during viral replication (223). This dependency on glutamine for energy has the added benefit of maintaining TCA cycle metabolites to generate amino acids for Vaccinia viral protein synthesis (223). Dengue-infected cells increase FAO via AMPK activation (224). Other viruses preferentially use lipids to support viral replication and assembly. For example, HIV infection promotes FA and cholesterol biosynthesis to support viral propagation (225). HCMV also induces lipogenesis via SREBP1 and SREBP2 upregulation (226). Interestingly, a Dengue component protein recruits fatty acid synthase to sites of viral particle replication and stimulates its activity (227). Targeting de novo fatty acid biosynthesis during vaccinia infection reduced viral load (228). HCV infection boost lipid metabolism by activating SREBP protein translation and 
increasing its proteolytic cleavage into its mature form (229-232). As a result, host immune cells must enact defense strategies to counteract the re-programming driven by viruses and central to these strategies is the ability of cellular metabolism to manipulate the degree and magnitude of the host response.

\subsubsection{The regulation of antiviral immune responses by cellular metabolism}

In response to viral infection, macrophage employ countermeasures against the co-opting of cell machinery by the virus (197). Central to these responses is the revamping of metabolic networks, such as glycolysis, OXPHOS, amino acid metabolism and lipid metabolism. These alterations play critical roles in supporting viral resistance, antigen presentation and the production of inflammatory and antiviral mediators $(76,233-236)$.

\subsubsection{Relationship between metabolism and RLR-mediated responses}

The best characterized viral PPR pathway shown to reprogram cellular metabolism in macrophages are the cytosolic RLRs. This is likely given their intimate link between the signalling of these receptors and the mitochondria. The MAVS protein participates in several interactions which propel RLR-mediated responses. MAVS undergoes O-linked $\beta$-N-acetylglucosamine (OGlcNAc) modifications via O-GlcNAc transferase, leading to MAVS ubiquitination and MAVS aggregation (237). A component of Complex IV (COX5B) directly interacts with MAVS, preventing MAVS aggregation, ROS production and ultimately suppressing antiviral responses (238). This provides a direct link between the homeostatic functions of the ETC and MAVSmediated signalling. MAVS also interacts with glycolytic protein hexokinase 2 (HK2) natively (97). Upon RLR activation, this interaction is lost resulting in reduced glycolytic activity (97). This is because lactate naturally binds to MAVS and prevents its aggregation and subsequent RLRmediated activation (97) (Zhang et al., 2019). While glycolysis suppresses RLR activation, 
increased PFKFB3 is required for type I IFN induction (236), suggesting some glycolytic activity is required for RLR-mediated antiviral responses.

There are other mechanisms by which mitochondria have been shown to regulate RLRmediated responses independent of its signalling dynamics. Examples include the mitochondrial fusion proteins MFN1 and MFN2, which are critical to eliciting type I IFN and pro-inflammatory cytokine expression following RLR engagement. Whereas MFN1 facilitates RLR signalling, MFN2 maintains MMP $(212,239)$. The latter is crucial to maintaining OXPHOS activity, which is required for ROS-mediated potentiation of RLR signalling $(240,241)$. To prevent hyperactivation, Autophagy related 5 (ATG5)-mediated autophagy of dysfunctional mitochondria, a large source of mtROS during RLR activation, limits the degree of RLR activation (240).

\subsubsection{TLR3-mediated responses depend on altered metabolic flux}

In response to dsRNA, TLR3 engagement propels an antiviral immune response. However, little is known regarding the role of cellular metabolism in regulating these responses in macrophages. One key regulator that has been identified is ROS. To et al. (242) have shown that NOX2-generated ROS facilitates the expression of IFN- $\beta$, IL-1 $\beta$, TNF- $\alpha$, and IL-6 at supraphysiological concentrations of the artificial dsRNA ligand Polyinosinic:polycytidylic acid (Poly(I:C) or PIC). This is essential for the activation of NF-kB, IRF3 and STAT1 signalling (243). This is accompanied by reduced Complex I-associated ATP production, partly linked to a loss ETC supercomplex assembly which forces a reliance on glycolysis $(114,176)$. This dependency on glycolysis is reliant on PFKFB3 activity, which supports phagocytotic activity (236). The immunosuppressive itaconate can block type I IFN induction during Poly(I:C) activation as a feedback mechanism to limit overactivation (169). In addition, proteomic analysis has directly connected the expression of 18 different proteins to the proper activation of TYK2, a member of 
the JAK-STAT signalling pathway (244). These proteins are linked to glucose metabolism (e.g. TIM) as well as FA synthesis (e.g. ACLY, ACSL4) and degradation (e.g. ACSL4) (163, 244). This suggests that TLR-driven metabolic re-programming is partly due to autocrine type I IFN signalling.

As shown in Grunert et al. (244), lipid metabolism is important to TLR3-mediated responses. Activated macrophages have significantly lower levels of saturated and unsaturated long-chain FAs as well as cholesterol and thus rely on lipid import rather than de novo synthesis regulated by SREBP (235). This is partly to a funneling of cholesterol towards the generation of 25-HC, which replaces cholesterol in the plasma membrane, reducing viral entry (245). To further assist in preventing viral entry, TRIF-induced type I IFN signalling promote a reprogramming of lipid membrane composition (192).

\subsubsection{The metabolic reprogramming induced by other viral TLRs}

Other viral-sensing TLRs are considerably less studied in the context of immunometabolism, with most of the work done on these TLR ligands conducted as an accessory to either global TLR studies or used as a comparison to TLR4-focused studies. The ssRNA-sensing TLR7/8 possess a few artificial ligands capable of initiating their activation, including Imidazoquinolinones (Imiquimod/R837, Resiquimod/R848, CL097) (246-248). While NOX2derived ROS is produced during TLR7 activation, unlike TLR3, ROS represses cytokine production and the use of apocynin (NOX2 inhibitor) enhances cytokine secretion in a MyD88dependent manner (242). In accordance with this, proteomic analysis of R848 treated macrophages found 27 differentially expressed protein, including several antioxidant proteins (SOD2, GPX, PRDX1/6) (178). Yet, imidazoquinolinone engagement also inhibits Complex I activity, leading 
to increased ROS and ROS-mediated NLRP3 activation, suggesting that mtROS, but not cytosolic ROS, may be key in modulating TLR7/8 responses $(249,250)$.

Similar to other viral ligand receptors, activation of TLR7/8 alters macrophage lipid metabolism to protect the cell against infection. This occurs via MyD88-dependent lipid membrane re-composition by NRF2 and SREBP, which facilitates inflammatory cytokine production (192, 251). Yet, uncontrolled TLR7/8-mediated inflammation can result in foam cell formation due to excess cellular lipid accumulation (252). A key modulator of this homeostatic balance is SPHK2, which is degraded in the early phase of activation but induced in the late phase to quell inflammation (195). Another immunosuppressive molecule produced during TLR7/8 activation is itaconate, which assists in preventing hyper-inflammation (167).

Very little is known regarding the role of cellular metabolism in regulating the activation of the DNA-sensing TLR9. Like TLR3 and TLR4, TLR9 activation with CpG oligonucleotides has been shown to induce ETC supercomplex disassembly and increase dependency on glycolysis for energy $(176,253)$. Yet, TLR9-activated macrophages also require other energy sources for proper activation. $\mathrm{CpG}$ activation reduce glucose input into the TCA cycle to favour carbons from glutaminolysis (115). Furthermore, TCA metabolites are shunted towards de novo lipid biosynthesis via increased CPT1A and ACLY activity $(115,251)$. In addition to their incorporation into the plasma membrane akin to other MyD88-dependent TLRs (TLR3/7/8) (192), newly generated lipids are used to increase FAO's energy contribution (115).

\subsection{Study Objectives}

The literature presented above demonstrates that like other inflammatory responses, the ability of macrophages to mount an effective antiviral response depends on metabolic re- 
programming of critical pathways. Yet, there are several gaps in the literature that yet have not been addressed. Initial assumptions in the literature were that TLR4-mediated responses in macrophages were representative for all TLR-mediated responses. However, based on limited data available, the metabolic reprogramming associated with TLR3-medaited responses differ from LPS activation. In addition, there has yet to be a systematic characterization of which metabolic pathways are associated with type I IFN responses and which metabolic pathways contribute to specific aspects of the response. Moreover, it is currently unknown if specific metabolic proteins or pathways are capable of functioning as rheostats, capable of fine-tuning individual components of the response and regulate its magnitude. For example, increased glycolysis in macrophages is associated with increase inflammation yet it has not been elucidated if the altered expression of specific proteins controls the magnitude of the inflammation and can it be done without altering other aspects of the response. In this vein, this thesis endeavours to build a better understanding of the role of cellular metabolism in regulating antiviral responses.

Despite activating the same family of transcription factors, TLR-associated antibacterial and antiviral responses produce distinct inflammatory and antiviral responses. While the role of cellular metabolism in driving antibacterial responses, such as LPS, has been well-established (93, $96,98,103,143,144,173,254-256)$, there has yet to be a comprehensive characterization of the metabolic processes that are altered during TLR-associated antiviral responses and their specific role in regulating effector function during these responses. In addition, there is limited knowledge as to the direct contribution of type I IFNs in the metabolic reprogramming of macrophages, which are induced by antibacterial and antiviral responses. Therefore, my thesis aims to show that changes to cellular metabolism are key drivers of the antiviral function of macrophages. To 
accomplish this, this project has mainly focus on IFN- $\alpha$ as well as two vital, yet divergent producers of type I IFNs: LPS and Poly(I:C). The aims of this thesis are as follows:

1. The systematic characterization of metabolic processes associated with type I IFNmediated responses in macrophages

2. Examine the differences in mitochondrial re-programming in TLR3- and TLR4-mediated responses

3. Investigate the differential responses of Poly(I:C) of different strand lengths in macrophages

A. Assess the inflammatory profiles of Poly(I:C)-mediated responses based on strand length

B. Probe the differential mitochondrial re-programming of Poly(I:C)-mediated responses based on strand length 


\subsection{References}

1. Murphy, K. M. 2011. Janeway's Immunobiology. Taylor \& Francis Group.

2. Akira, S., S. Uematsu, and O. Takeuchi. 2006. Pathogen Recognition and Innate Immunity. Cell 124: 783-801.

3. Gasteiger, G., A. D'Osualdo, D. A. Schubert, A. Weber, E. M. Bruscia, and D. Hartl. 2017. Cellular innate immunity: An old game with new players. J Innate Immun 9: 111-125.

4. Bonilla, F. A., and H. C. Oettgen. 2010. Adaptive immunity. J Allergy Clin Immunol 125: S33-S40.

5. Wynn, T. A., A. Chawla, and J. W. Pollard. 2013. Origins and Hallmarks of Macrophages: Development, Homeostasis, and Disease. Nature 496: 445-455.

6. Theret, M., R. Mounier, and F. Rossi. 2019. The origins and non-canonical functions of macrophages in development and regeneration. Development 146: dev156000.

7. van Furth, R., and Z. A. Cohn. 1968. The origin and kinetics of mononuclear phagocytes. J Exp Med 128: 415-435.

8. van Furth, R., Z. A. Cohn, J. G. Hirsch, J. H. Humphrey, W. G. Spector, and H. L. Langevoort. 1972. The mononuclear phagocyte system: a new classification of macrophages, monocytes, and their precursor cells. Bull World Health Organ 46: 845-852.

9. Schulz, C., E. Gomez Perdiguero, L. Chorro, H. Szabo-Rogers, N. Cagnard, K. Kierdorf, M. Prinz, B. Wu, S. E. Jacobsen, J. W. Pollard, J. Frampton, K. J. Liu, and F. Geissmann. 2012. A lineage of myeloid cells independent of Myb and hematopoietic stem cells. Science 336: 86-90.

10. Ginhoux, F., M. Greter, M. Leboeuf, S. Nandi, P. See, S. Gokhan, M. F. Mehler, S. J. Conway, L. G. Ng, E. R. Stanley, I. M. Samokhvalov, and M. Merad. 2010. Fate mapping analysis reveals that adult microglia derive from primitive macrophages. Science 330: 841845.

11. Hoeffel, G., Y. Wang, M. Greter, P. See, P. Teo, B. Malleret, M. Leboeuf, D. Low, G. Oller, F. Almeida, S. H. Y. Choy, M. Grisotto, L. Renia, S. J. Conway, E. R. Stanley, J. K. Y. Chan, L.-G. Ng, I. M. Samokhvalov, M. Merad, and F. Ginhoux. 2012. Adult Langerhans cells derive predominantly from embryonic fetal liver monocytes with a minor contribution of yolk sac-derived macrophages. J Exp Med 209: 1167-1181.

12. Hoeffel, G., and F. Ginhoux. 2015. Ontogeny of tissue-resident macrophages. Front Immunol 6: 486.

13. Yona, S., K.-W. Kim, Y. Wolf, A. Mildner, D. Varol, M. Breker, D. Strauss-Ayali, S. Viukov, M. Guilliams, and A. Misharin. 2013. Fate mapping reveals origins and dynamics of monocytes and tissue macrophages under homeostasis. Immunity 38: 79-91.

14. Geissmann, F., S. Jung, and D. R. Littman. 2003. Blood Monocytes Consist of Two Principal Subsets with Distinct Migratory Properties. Immunity 19: 71-82.

15. Carlin, L. M., E. G. Stamatiades, C. Auffray, R. N. Hanna, L. Glover, G. Vizcay-Barrena, C. C. Hedrick, H. T. Cook, S. Diebold, and F. Geissmann. 2013. Nr4a1-dependent Ly6Clow monocytes monitor endothelial cells and orchestrate their disposal. Cell 153: $362-375$.

16. Cros, J., N. Cagnard, K. Woollard, N. Patey, S.-Y. Zhang, B. Senechal, A. Puel, S. K. Biswas, D. Moshous, C. Picard, J.-P. Jais, D. D'Cruz, J.-L. Casanova, C. Trouillet, and F. Geissmann. 2010. Human CD14dim Monocytes Patrol and Sense Nucleic Acids and Viruses via TLR7 and TLR8 Receptors. Immunity 33: 375-386. 
17. Belge, K.-U., F. Dayyani, A. Horelt, M. Siedlar, M. Frankenberger, B. Frankenberger, T. Espevik, and L. Ziegler-Heitbrock. 2002. The Proinflammatory CD14+CD16+DR++ Monocytes Are a Major Source of TNF. The Journal of Immunology 168: 3536-3542.

18. Ziegler-Heitbrock, L. 2007. The CD14+ CD16+ blood monocytes: their role in infection and inflammation. Journal of Leukocyte Biology 81: 584-592.

19. Ziegler-Heitbrock, L., P. Ancuta, S. Crowe, M. Dalod, V. Grau, D. N. Hart, P. J. M. Leenen, Y.-J. Liu, G. MacPherson, G. J. Randolph, J. Scherberich, J. Schmitz, K. Shortman, S. Sozzani, H. Strobl, M. Zembala, J. M. Austyn, and M. B. Lutz. 2010. Nomenclature of monocytes and dendritic cells in blood. Blood 116: e74.

20. Varol, C., A. Mildner, and S. Jung. 2015. Macrophages: Development and Tissue Specialization. Annual Review of Immunology 33: 643-675.

21. Toth, C. A., and D. P. Thomas. 1992. Liver endocytosis and Kupffer cells. Hepatology 16: 255-266.

22. Tate, M. D., D. L. Pickett, N. van Rooijen, A. G. Brooks, and P. C. Reading. 2010. Critical Role of Airway Macrophages in Modulating Disease Severity during Influenza Virus Infection of Mice. Journal of Virology 84: 7569-7580.

23. Kühl, A. A., U. Erben, L. I. Kredel, and B. Siegmund. 2015. Diversity of Intestinal Macrophages in Inflammatory Bowel Diseases. Frontiers in Immunology 6: 613.

24. Ross, J. A., and M. J. Auger. 2002. The biology of the macrophage. In The macrophage, 2nd ed. B. Burke, and C. E. Lewis, eds. Oxford University Press, New York, New York. $1-57$.

25. Gordon, S., and P. R. Taylor. 2005. Monocyte and macrophage heterogeneity. Nat Rev Immunol 5: 953-964.

26. Yu, X., A. Buttgereit, I. Lelios, S. G. Utz, D. Cansever, B. Becher, and M. Greter. 2017. The cytokine TGF- $\beta$ promotes the development and homeostasis of alveolar macrophages. Immunity 47: 903-912 e904.

27. Naito, M., G. Hasegawa, Y. Ebe, and T. Yamamoto. 2004. Differentiation and function of Kupffer cells. Medical Electron Microscopy 37: 16-28.

28. Biswas, S. K., and E. Lopez-Collazo. 2009. Endotoxin tolerance: new mechanisms, molecules and clinical significance. Trends in Immunology 30: 475-487.

29. Smythies, L. E., R. Shen, D. Bimczok, L. Novak, R. H. Clements, D. E. Eckhoff, P. Bouchard, M. D. George, W. K. Hu, S. Dandekar, and P. D. Smith. 2010. Inflammation Anergy in Human Intestinal Macrophages Is Due to Smad-induced IкB $\alpha$ Expression and NF- $\mathrm{kB}$ Inactivation. Journal of Biological Chemistry 285: 19593-19604.

30. Zigmond, E., C. Varol, J. Farache, E. Elmaliah, Ansuman T. Satpathy, G. Friedlander, M. Mack, N. Shpigel, Ivo G. Boneca, Kenneth M. Murphy, G. Shakhar, Z. Halpern, and S. Jung. 2012. Ly6Chi Monocytes in the Inflamed Colon Give Rise to Proinflammatory Effector Cells and Migratory Antigen-Presenting Cells. Immunity 37: 1076-1090.

31. Schenk, M., and C. Mueller. 2007. Adaptations of intestinal macrophages to an antigenrich environment. Semin Immunol 19: 84-93.

32. Mosser, D. M., and J. P. Edwards. 2008. Exploring the full spectrum of macrophage activation. Nat Rev Immunol 8: 958-969.

33. Fadok, V. A., D. L. Bratton, A. Konowal, P. W. Freed, J. Y. Westcott, and P. M. Henson. 1998. Macrophages that have ingested apoptotic cells in vitro inhibit proinflammatory cytokine production through autocrine/paracrine mechanisms involving TGF-beta, PGE2, and PAF. Journal of Clinical Investigation 101: 890-898. 
34. Fadok, V. A., P. P. McDonald, D. L. Bratton, and P. M. Henson. 1998. Regulation of macrophage cytokine production by phagocytosis of apoptotic and post-apoptotic cells. Biochem Soc Trans 26: 653-656.

35. Murray, P. J., J. Rathmell, and E. Pearce. 2015. SnapShot: Immunometabolism. Cell Metabolism 22: 190-190.e191.

36. Bianchi, M. E. 2007. DAMPs, PAMPs and alarmins: all we need to know about danger. $J$. Leukoc. Biol. 81: 1-5.

37. Fitzgerald, K. A., and J. C. Kagan. 2020. Toll-like receptors and the control of immunity. Cell 180: 1044-1066.

38. Reikine, S., J. B. Nguyen, and Y. Modis. 2014. Pattern recognition and signaling mechanisms of RIG-I and MDA5. Front Immunol 5: 342-342.

39. Takeuchi, O., K. Hoshino, T. Kawai, H. Sanjo, H. Takada, T. Ogawa, K. Takeda, and S. Akira. 1999. Differential roles of TLR2 and TLR4 in recognition of gram-negative and gram-positive bacterial cell wall components. Immunity 11: 443-451.

40. Takeuchi, O., S. Sato, T. Horiuchi, K. Hoshino, K. Takeda, Z. Dong, R. L. Modlin, and S. Akira. 2002. Cutting edge: Role of Toll-like receptor 1 in mediating immune response to microbial lipoproteins. J Immunol 169: 10-14.

41. Kang, J. Y., X. Nan, M. S. Jin, S.-J. Youn, Y. H. Ryu, S. Mah, S. H. Han, H. Lee, S.-G. Paik, and J.-O. Lee. 2009. Recognition of lipopeptide patterns by Toll-like receptor 2-Tolllike receptor 6 heterodimer. Immunity 31: 873-884.

42. Poltorak, A., X. He, I. Smirnova, M.-Y. Liu, C. Van Huffel, X. Du, D. Birdwell, E. Alejos, M. Silva, and C. Galanos. 1998. Defective LPS signaling in $\mathrm{C} 3 \mathrm{H} / \mathrm{HeJ}$ and C57BL/10ScCr mice: mutations in Tlr4 gene. Science 282: 2085-2088.

43. Shimazu, R., S. Akashi, H. Ogata, Y. Nagai, K. Fukudome, K. Miyake, and M. Kimoto. 1999. MD-2, a molecule that confers lipopolysaccharide responsiveness on Toll-like receptor 4. J Exp Med 189: 1777-1782.

44. Hayashi, F., K. D. Smith, A. Ozinsky, T. R. Hawn, C. Y. Eugene, D. R. Goodlett, J. K. Eng, S. Akira, D. M. Underhill, and A. Aderem. 2001. The innate immune response to bacterial flagellin is mediated by Toll-like receptor 5. Nature 410: 1099-1103.

45. Heil, F., H. Hemmi, H. Hochrein, F. Ampenberger, C. Kirschning, S. Akira, G. Lipford, H. Wagner, and S. Bauer. 2004. Species-specific recognition of single-stranded RNA via Toll-like receptor 7 and 8. Science 303: 1526-1529.

46. Alexopoulou, L., A. C. Holt, R. Medzhitov, and R. A. Flavell. 2001. Recognition of double-stranded RNA and activation of NF-[kappa]B by Toll-like receptor 3. Nature 413: 732-738.

47. Lund, J. M., L. Alexopoulou, A. Sato, M. Karow, N. C. Adams, N. W. Gale, A. Iwasaki, and R. A. Flavell. 2004. Recognition of single-stranded RNA viruses by Toll-like receptor 7. Proc Natl Acad Sci U S A 101: 5598-5603.

48. Lund, J., A. Sato, S. Akira, R. Medzhitov, and A. Iwasaki. 2003. Toll-like receptor 9mediated recognition of herpes simplex virus-2 by plasmacytoid dendritic cells. J Exp Med 198: 513-520.

49. Hemmi, H., O. Takeuchi, T. Kawai, T. Kaisho, S. Sato, H. Sanjo, M. Matsumoto, K. Hoshino, H. Wagner, K. Takeda, and S. Akira. 2000. A Toll-like receptor recognizes bacterial DNA. Nature 408: 740-745.

50. Kawai, T., and S. Akira. 2010. The role of pattern-recognition receptors in innate immunity: update on Toll-like receptors. Nat Immunol 11: 373-384. 
51. Bhoj, V. G., and Z. J. Chen. 2009. Ubiquitylation in innate and adaptive immunity. Nature 458: 430-437.

52. Zhao, M., J. Joy, W. Zhou, S. De, W. H. I. Wood, K. G. Becker, H. Ji, and R. Sen. 2018. Transcriptional outcomes and kinetic patterning of gene expression in response to NF- $\mathrm{KB}$ activation. PLOS Biol 16.

53. Hacker, H., and M. Karin. 2006. Regulation and function of IKK and IKK-related kinases. Sci STKE 2006: re13.

54. Taniguchi, T., K. Ogasawara, A. Takaoka, and N. Tanaka. 2001. IRF family of transcription factors as regulators of host defense. Annu Rev Immunol 19: 623-655.

55. Yoneyama, M., M. Kikuchi, K. Matsumoto, T. Imaizumi, M. Miyagishi, K. Taira, E. Foy, Y.-M. Loo, M. Gale, and S. Akira. 2005. Shared and unique functions of the DExD/H-box helicases RIG-I, MDA5, and LGP2 in antiviral innate immunity. $J$ Immunol 175: 28512858.

56. Yoneyama, M., M. Kikuchi, T. Natsukawa, N. Shinobu, T. Imaizumi, M. Miyagishi, K. Taira, S. Akira, and T. Fujita. 2004. The RNA helicase RIG-I has an essential function in double-stranded RNA-induced innate antiviral responses. Nat Immunol 5: 730-737.

57. Saito, T., R. Hirai, Y.-M. Loo, D. Owen, C. L. Johnson, S. C. Sinha, S. Akira, T. Fujita, and M. Gale. 2007. Regulation of innate antiviral defenses through a shared repressor domain in RIG-I and LGP2. Proc Natl Acad Sci U S A 104: 582-587.

58. Kato, H., O. Takeuchi, S. Sato, M. Yoneyama, M. Yamamoto, K. Matsui, S. Uematsu, A. Jung, T. Kawai, K. J. Ishii, O. Yamaguchi, K. Otsu, T. Tsujimura, C.-S. Koh, C. Reis e Sousa, Y. Matsuura, T. Fujita, and S. Akira. 2006. Differential roles of MDA5 and RIG-I helicases in the recognition of RNA viruses. Nature 441: 101-105.

59. Kato, H., O. Takeuchi, E. Mikamo-Satoh, R. Hirai, T. Kawai, K. Matsushita, A. Hiiragi, T. S. Dermody, T. Fujita, and S. Akira. 2008. Length-dependent recognition of doublestranded ribonucleic acids by Retinoic acid-inducible gene-I and Melanoma differentiation-associated gene 5. J Exp Med 205: 1601-1610.

60. Peisley, A., C. Lin, B. Wu, M. Orme-Johnson, M. Liu, T. Walz, and S. Hur. 2011. Cooperative assembly and dynamic disassembly of MDA5 filaments for viral dsRNA recognition. Proc Natl Acad Sci U S A 108: 21010-21015.

61. Berke, I. C., and Y. Modis. 2012. MDA5 cooperatively forms dimers and ATP-sensitive filaments upon binding double-stranded RNA. EMBO J 31: 1714-1726.

62. Kowalinski, E., T. Lunardi, A. A. McCarthy, J. Louber, J. Brunel, B. Grigorov, D. Gerlier, and S. Cusack. 2011. Structural basis for the activation of innate immune patternrecognition receptor RIG-I by viral RNA. Cell 147: 423-435.

63. Jiang, F., A. Ramanathan, M. T. Miller, G.-Q. Tang, M. Gale, S. S. Patel, and J. Marcotrigiano. 2011. Structural basis of RNA recognition and activation by innate immune receptor RIG-I. Nature 479: 423-427.

64. Peisley, A., B. Wu, H. Xu, Z. J. Chen, and S. Hur. 2014. Structural basis for ubiquitinmediated antiviral signal activation by RIG-I. Nature 509: 110-114.

65. Seth, R. B., L. Sun, C.-K. Ea, and Z. J. Chen. 2005. Identification and characterization of MAVS, a mitochondrial antiviral signaling protein that a ctivates NF- $\kappa B$ and IRF3. Cell 122: 669-682.

66. Kawai, T., K. Takahashi, S. Sato, C. Coban, H. Kumar, H. Kato, K. J. Ishii, O. Takeuchi, and S. Akira. 2005. IPS-1, an adaptor triggering RIG-I- and MDA5-mediated type I interferon induction. Nat Immunol 6: 981-988. 
67. Hou, F., L. Sun, H. Zheng, B. Skaug, Q.-X. Jiang, and Z. J. Chen. 2011. MAVS forms functional prion-like aggregates to activate and propagate antiviral innate immune response. Cell 146: 448-461.

68. Pestka, S., C. D. Krause, and M. R. Walter. 1987. Interferons, interferon-like cytokines, and their receptors. Immunol. Rev. 202 8-32.

69. Pestka, S. 1997. The human interferon-alpha species and hybrid proteins. Semin Oncol 24: S9-4-S9-17.

70. Ivashkiv, L. B., and L. T. Donlin. 2014. Regulation of type I interferon responses. Nat Rev Immunol 14: 36-49.

71. Trinchieri, G. 2010. Type I interferon: friend or foe? J. Exp. Med. 207: 2053-2063.

72. Stark, G. R., I. M. Kerr, B. R. G. Williams, R. H. Silverman, and R. D. Schreiber. 1998. How cells respond to interferons. Annu. Rev. Biochem. 67: 227-264.

73. Ihle, J. N. 1995. Cytokine receptor signalling. Nature 377: 591-594.

74. Darnell, J., I. Kerr, and G. Stark. 1994. Jak-STAT pathways and transcriptional activation in response to IFNs and other extracellular signaling proteins. Science 264: 1415-1421.

75. Nakaya, T., M. Sato, N. Hata, M. Asagiri, H. Suemori, S. Noguchi, N. Tanaka, and T. Taniguchi. 2001. Gene Induction Pathways Mediated by Distinct IRFs during Viral Infection. Biochem Biophys Res Commun 283: 1150-1156.

76. Schoggins, J. W., and C. M. Rice. 2011. Interferon-stimulated genes and their antiviral effector functions. Curr. Opin. Virol. 1: 519-525.

77. Lekmine, F., S. Uddin, A. Sassano, S. Parmar, S. M. Brachmann, B. Majchrzak, N. Sonenberg, N. Hay, E. N. Fish, and L. C. Platanias. 2003. Activation of the p70 S6 Kinase and Phosphorylation of the 4E-BP1 Repressor of mRNA Translation by Type I Interferons. J. Biol. Chem. 278: 27772-27780.

78. Uddin, S., B. Majchrzak, P.-C. Wang, S. Modi, M. K. Khan, E. N. Fish, and L. C. Platanias. 2000. Interferon-Dependent Activation of the Serine Kinase PI 3'-Kinase Requires Engagement of the IRS Pathway but Not the Stat Pathway. Biochem. Biophys. Res. Commun. 270: 158-162.

79. Huang, J., and Brendan D. Manning. 2009. A complex interplay between Akt, TSC2 and the two mTOR complexes. Biochemical Society Transactions 37: 217-222.

80. Sun, Q., X. Chen, J. Ma, H. Peng, F. Wang, X. Zha, Y. Wang, Y. Jing, H. Yang, R. Chen, L. Chang, Y. Zhang, J. Goto, H. Onda, T. Chen, M.-R. Wang, Y. Lu, H. You, D. Kwiatkowski, and H. Zhang. 2011. Mammalian target of rapamycin up-regulation of pyruvate kinase isoenzyme type M2 is critical for aerobic glycolysis and tumor growth. Proceedings of the National Academy of Sciences 108: 4129-4134.

81. Dang, Eric V., J. Barbi, H.-Y. Yang, D. Jinasena, H. Yu, Y. Zheng, Z. Bordman, J. Fu, Y. Kim, H.-R. Yen, W. Luo, K. Zeller, L. Shimoda, Suzanne L. Topalian, Gregg L. Semenza, Chi V. Dang, Drew M. Pardoll, and F. Pan. 2011. Control of TH17/Treg Balance by Hypoxia-Inducible Factor 1. Cell 146: 772-784.

82. Shi, L. Z., R. Wang, G. Huang, P. Vogel, G. Neale, D. R. Green, and H. Chi. 2011. HIF1 $\alpha-$ dependent glycolytic pathway orchestrates a metabolic checkpoint for the differentiation of TH17 and Treg cells. J Exp Med 208: 1367-1376.

83. O'Neill, L. A. J., R. J. Kishton, and J. Rathmell. 2016. A guide to immunometabolism for immunologists. Nature Reviews Immunology 16: 553-565. 
84. Angajala, A., S. Lim, J. B. Phillips, J.-H. Kim, C. Yates, Z. You, and M. Tan. 2018. Diverse roles of mitochondria in immune responses: Novel insights into immuno-metabolism. Front Immunol 9: 1605.

85. Arnoult, D., F. Soares, I. Tattoli, and S. E. Girardin. 2011. Mitochondria in innate immunity. EMBO Rep 12: 901-910.

86. Voet, D., J. G. Voet, and C. W. Pratt. 2016. Fundamentals of biochemistry: life at the molecular level. John Wiley \& Sons.

87. Tanner, L. B., A. G. Goglia, M. H. Wei, T. Sehgal, L. R. Parsons, J. O. Park, E. White, J. E. Toettcher, and J. D. Rabinowitz. 2018. Four key steps control glycolytic flux in Mammalian cells. Cell Syst 7: 49-62.e48.

88. Berg, J. M., J. L. Tymoczko, and L. Stryer. 2008. Biochemistry. Macmillan.

89. Thwe, P. M., L. R. Pelgrom, R. Cooper, S. Beauchamp, J. A. Reisz, A. D'Alessandro, B. Everts, and E. Amiel. 2017. Cell-intrinsic glycogen metabolism supports early glycolytic reprogramming required for Dendritic cell immune responses. Cell Metab 26: 558567.e555.

90. Ghergurovich, J. M., J. C. García-Cañaveras, J. Wang, E. Schmidt, Z. Zhang, T. TeSlaa, H. Patel, L. Chen, E. C. Britt, M. Piqueras-Nebot, M. C. Gomez-Cabrera, A. Lahoz, J. Fan, U. H. Beier, H. Kim, and J. D. Rabinowitz. 2020. A small molecule G6PD inhibitor reveals immune dependence on pentose phosphate pathway. Nat Chem Biol 16: 731-739.

91. Wang, R., C. P. Dillon, L. Z. Shi, S. Milasta, R. Carter, D. Finkelstein, L. L. McCormick, P. Fitzgerald, H. Chi, and J. Munger. 2011. The transcription factor Myc controls metabolic reprogramming upon T lymphocyte activation. Immunity 35: 871-882.

92. Ma, E. H., G. Bantug, T. Griss, S. Condotta, R. M. Johnson, B. Samborska, N. Mainolfi, V. Suri, H. Guak, M. L. Balmer, M. J. Verway, T. C. Raissi, H. Tsui, G. Boukhaled, S. Henriques da Costa, C. Frezza, C. M. Krawczyk, A. Friedman, M. Manfredi, M. J. Richer, C. Hess, and R. G. Jones. 2017. Serine is an essential metabolite for effector T cell expansion. Cell Metabolism 25: 345-357.

93. Rodríguez-Prados, J., P. G. Través, J. Cuenca, D. Rico, J. Aragonés, P. Martín-Sanz, M. Cascante, and L. Boscá. 2010. Substrate fate in activated macrophages: A comparison between innate, classic, and alternative activation. J Immunol 185: 605-614.

94. Vazquez, A., J. Liu, Y. Zhou, and Z. N. Oltvai. 2010. Catabolic efficiency of aerobic glycolysis: The Warburg effect revisited. BMC Syst. Biol. 4: 1-9.

95. Wang, T., H. Liu, G. Lian, S.-Y. Zhang, X. Wang, and C. Jiang. 2017. HIF1 $\alpha$-induced glycolysis metabolism is essential to the activation of inflammatory macrophages. Mediators Inflamm 2017: 10.

96. Aki, T., T. Funakoshi, K. Noritake, K. Unuma, and K. Uemura. 2020. Extracellular glucose is crucially involved in the fate decision of LPS-stimulated RAW264.7 murine macrophage cells. Scientific Reports 10: 10581.

97. Zhang, W., G. Wang, Z. G. Xu, H. Tu, F. Hu, J. Dai, Y. Chang, Y. Chen, Y. Lu, H. Zeng, Z. Cai, F. Han, C. Xu, G. Jin, L. Sun, B. S. Pan, S. W. Lai, C. C. Hsu, J. Xu, Z. Z. Chen, H. Y. Li, P. Seth, J. Hu, X. Zhang, H. Li, and H. K. Lin. 2019. Lactate is a natural suppressor of RLR signaling by targeting MAVS. Cell 178: 176-189.e115.

98. Errea, A., D. Cayet, P. Marchetti, C. Tang, J. Kluza, S. Offermanns, J.-C. Sirard, and M. Rumbo. 2016. Lactate Inhibits the Pro-Inflammatory Response and Metabolic Reprogramming in Murine Macrophages in a GPR81-Independent Manner. PLoS ONE 11: e0163694. 
99. Spinelli, J. B., and M. C. Haigis. 2018. The multifaceted contributions of mitochondria to cellular metabolism. Nat Cell Biol 20: 745-754.

100. Williams, N. C., and L. A. J. O'Neill. 2018. A role for the Krebs cycle intermediate Citrate in metabolic reprogramming in innate immunity and inflammation. Front Immunol 9: 141.

101. Infantino, V., P. Convertini, L. Cucci, Maria A. Panaro, Maria A. Di Noia, R. Calvello, F. Palmieri, and V. Iacobazzi. 2011. The mitochondrial citrate carrier: a new player in inflammation. Biochem. J. 438: 433-436.

102. Ratter, J. M., H. M. M. Rooijackers, G. J. Hooiveld, A. G. M. Hijmans, B. E. de Galan, C. J. Tack, and R. Stienstra. 2018. In vitro and in vivo effects of Lactate on metabolism and cytokine production of Human primary PBMCs and onocytes. Front Immunol 9: 2564.

103. Tannahill, G. M., A. M. Curtis, J. Adamik, E. M. Palsson-McDermott, A. F. McGettrick, G. Goel, C. Frezza, N. J. Bernard, B. Kelly, N. H. Foley, L. Zheng, A. Gardet, Z. Tong, S. S. Jany, S. C. Corr, M. Haneklaus, B. E. Caffrey, K. Pierce, S. Walmsley, F. C. Beasley, E. Cummins, V. Nizet, M. Whyte, C. T. Taylor, H. Lin, S. L. Masters, E. Gottlieb, V. P. Kelly, C. Clish, P. E. Auron, R. J. Xavier, and L. A. J. O/'Neill. 2013. Succinate is an inflammatory signal that induces IL-1 $\beta$ through HIF-1 $\alpha$. Nature 496: 238-242.

104. Tennant, D. A., C. Frezza, E. D. MacKenzie, Q. D. Nguyen, L. Zheng, M. A. Selak, D. L. Roberts, C. Dive, D. G. Watson, and E. O. Aboagye. 2009. Reactivating HIF prolyl hydroxylases under hypoxia results in metabolic catastrophe and cell death. Oncogene 28: 4009-4021.

105. Liu, P.-S., H. Wang, X. Li, T. Chao, T. Teav, S. Christen, G. Di Conza, W.-C. Cheng, C.H. Chou, and M. Vavakova. 2017. $\alpha$-ketoglutarate orchestrates macrophage activation through metabolic and epigenetic reprogramming. Nature Immunol 18: 985-994.

106. Liu, T. F., C. M. Brown, M. El Gazzar, L. McPhail, P. Millet, A. Rao, V. T. Vachharajani, B. K. Yoza, and C. E. McCall. 2012. Fueling the flame: bioenergy couples metabolism and inflammation. J Leukoc Biol 92: 499-507.

107. Cameron, A. M., A. Castoldi, D. E. Sanin, L. J. Flachsmann, C. S. Field, D. J. Puleston, R. L. Kyle, A. E. Patterson, F. Hässler, J. M. Buescher, B. Kelly, E. L. Pearce, and E. J. Pearce. 2019. Inflammatory macrophage dependence on NAD+ salvage is a consequence of reactive oxygen species-mediated DNA damage. Nature Immunology 20: 420-432.

108. Jha, A. K., S. C.-C. Huang, A. Sergushichev, V. Lampropoulou, Y. Ivanova, E. Loginicheva, K. Chmielewski, K. M. Stewart, J. Ashall, B. Everts, E. J. Pearce, E. M. Driggers, and M. N. Artyomov. 2015. Network Integration of Parallel Metabolic and Transcriptional Data Reveals Metabolic Modules that Regulate Macrophage Polarization. Immunity 42: 419-430.

109. Langston, P. K., A. Nambu, J. Jung, M. Shibata, H. I. Aksoylar, J. Lei, P. Xu, M. T. Doan, H. Jiang, M. R. MacArthur, X. Gao, Y. Kong, E. T. Chouchani, J. W. Locasale, N. W. Snyder, and T. Horng. 2019. Glycerol phosphate shuttle enzyme GPD2 regulates macrophage inflammatory responses. Nat Immunol 20: 1186-1195.

110. Kory, N., J. Uit de Bos, S. van der Rijt, N. Jankovic, M. Güra, N. Arp, I. A. Pena, G. Prakash, S. H. Chan, T. Kunchok, C. A. Lewis, and D. M. Sabatini. 2020. MCART1/SLC25A51 is required for mitochondrial NAD transport. Sci Adv 6.

111. Luongo, T. S., J. M. Eller, M. J. Lu, M. Niere, F. Raith, C. Perry, M. R. Bornstein, P. Oliphint, L. Wang, M. R. McReynolds, M. E. Migaud, J. D. Rabinowitz, F. B. Johnson, K. Johnsson, M. Ziegler, X. A. Cambronne, and J. A. Baur. 2020. SLC25A51 is a mammalian mitochondrial $\mathrm{NAD}(+)$ transporter. Nature 2020: 1-10. 
112. Acín-Pérez, R., P. Fernández-Silva, M. L. Peleato, A. Pérez-Martos, and J. A. Enriquez. 2008. Respiratory active mitochondrial supercomplexes. Mol Cell 32: 529-539.

113. Garlid, K. D., M. Jabůrek, P. Ježek, and M. Vařecha. 2000. How do uncoupling proteins uncouple? Biochim Biophys Acta 1459: 383-389.

114. Garaude, J., R. Acín-Pérez, S. Martínez-Cano, M. Enamorado, M. Ugolini, E. NistalVillán, S. Hervás-Stubbs, P. Pelegrín, L. E. Sander, J. A. Enríquez, and D. Sancho. 2016. Mitochondrial respiratory-chain adaptations in macrophages contribute to antibacterial host defense. Nat Immunol 17: 1037-1045.

115. Liu, M., R. S. O’Connor, S. Trefely, K. Graham, N. W. Snyder, and G. L. Beatty. 2019. Metabolic rewiring of macrophages by $\mathrm{CpG}$ potentiates clearance of cancer cells and overcomes tumor-expressed CD47-mediated 'don't-eat-me' signal. Nature Immunology 20: $265-275$.

116. Vats, D., L. Mukundan, J. I. Odegaard, L. Zhang, K. L. Smith, C. R. Morel, R. A. Wagner, D. R. Greaves, P. J. Murray, and A. Chawla. 2006. Oxidative metabolism and PGC-1beta attenuate macrophage-mediated inflammation. Cell Metab 4: 13-24.

117. Wang, T., J. F. Fahrmann, H. Lee, Y.-J. Li, S. C. Tripathi, C. Yue, C. Zhang, V. Lifshitz, J. Song, and Y. Yuan. 2018. JAK/STAT3-regulated fatty acid $\beta$-oxidation is critical for breast cancer stem cell self-renewal and chemoresistance. Cell Metab 27: 136-150.

118. Malandrino, M. I., R. Fucho, M. Weber, M. Calderon-Dominguez, J. F. Mir, L. Valcarcel, X. Escoté, M. Gómez-Serrano, B. Peral, L. Salvadó, S. Fernández-Veledo, N. Casals, M. Vázquez-Carrera, F. Villarroya, J. J. Vendrell, D. Serra, and L. Herrero. 2015. Enhanced fatty acid oxidation in adipocytes and macrophages reduces lipid-induced triglyceride accumulation and inflammation. Am J Physiol Endocrinol Metab 308: E756-769.

119. Wuggenig, P., B. Kaya, H. Melhem, C. K. Ayata, I. B. D. C. I. Swiss, P. Hruz, A. E. Sayan, H. Tsumura, M. Ito, J. Roux, and J. H. Niess. 2020. Loss of the branched-chain amino acid transporter CD98hc alters the development of colonic macrophages in mice. Commun Biol 3: 130.

120. Ko, J. H., A. Olona, A. E. Papathanassiu, N. Buang, K. S. Park, A. S. H. Costa, C. Mauro, C. Frezza, and J. Behmoaras. 2020. BCAT1 affects mitochondrial metabolism independently of leucine transamination in activated human macrophages. J Cell Sci 2020: jcs.247957.

121. Papathanassiu, A. E., J.-H. Ko, M. Imprialou, M. Bagnati, P. K. Srivastava, H. A. Vu, D. Cucchi, S. P. McAdoo, E. A. Ananieva, C. Mauro, and J. Behmoaras. 2017. BCAT1 controls metabolic reprogramming in activated human macrophages and is associated with inflammatory diseases. Nat Commun 8: 16040.

122. Nathan, C., and A. Cunningham-Bussel. 2013. Beyond oxidative stress: an immunologist's guide to reactive oxygen species. Nature Reviews Immunology 13: 349.

123. Holmström, K. M., and T. Finkel. 2014. Cellular mechanisms and physiological consequences of redox-dependent signalling. Nature Reviews Molecular Cell Biology 15: 411.

124. Agod, Z., T. Fekete, M. M. Budai, A. Varga, A. Szabo, H. Moon, I. Boldogh, T. Biro, A. Lanyi, A. Bacsi, and K. Pazmandi. 2017. Regulation of type I interferon responses by mitochondria-derived reactive oxygen species in plasmacytoid dendritic cells. Redox Biol 13: 633-645.

125. Takada, Y., A. Mukhopadhyay, G. C. Kundu, G. H. Mahabeleshwar, S. Singh, and B. B.

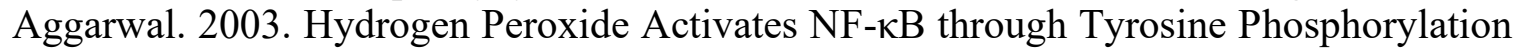




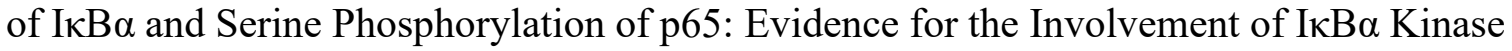
and Syk Protein-Tyrosine Kinase. J Biol Chem 278: 24233-24241.

126. Indukuri, H., S. M. Castro, S.-M. Liao, L. A. Feeney, M. Dorsch, A. J. Coyle, R. P. Garofalo, A. R. Brasier, and A. Casola. 2006. Ikke regulates viral-induced interferon regulatory factor-3 activation via a redox-sensitive pathway. Virology 353: 155-165.

127. Gonzalez-Dosal, R., K. A. Horan, S. H. Rahbek, H. Ichijo, Z. J. Chen, J. J. Mieyal, R. Hartmann, and S. R. Paludan. 2011. HSV infection induces production of ROS, which potentiate signaling from pattern recognition receptors: Role for S-glutathionylation of TRAF3 and 6. PLOS Pathog 7: e1002250.

128. Herb, M., A. Gluschko, K. Wiegmann, A. Farid, A. Wolf, O. Utermöhlen, O. Krut, M. Krönke, and M. Schramm. 2019. Mitochondrial reactive oxygen species enable proinflammatory signaling through disulfide linkage of NEMO. Sci Signal 12: eaar5926.

129. Bell, E. L., T. A. Klimova, J. Eisenbart, C. T. Moraes, M. P. Murphy, G. R. S. Budinger, and N. S. Chandel. 2007. The Qo site of the mitochondrial complex III is required for the transduction of hypoxic signaling via reactive oxygen species production. J Cell Biol 177: 1029-1036.

130. Kale, J., E. J. Osterlund, and D. W. Andrews. 2018. BCL-2 family proteins: changing partners in the dance towards death. Cell Death Differ 25: 65-80.

131. Zhang, Q., M. Raoof, Y. Chen, Y. Sumi, T. Sursal, W. Junger, K. Brohi, K. Itagaki, and C. J. Hauser. 2010. Circulating mitochondrial DAMPs cause inflammatory responses to injury. Nature 464: 104.

132. Zhang, Q., K. Itagaki, and C. J. Hauser. 2010. Mitochondrial DNA is released by shock and activates neutrophils via p38 map kinase. Shock 34: 55-59.

133. Nakahira, K., J. A. Haspel, V. A. K. Rathinam, S.-J. Lee, T. Dolinay, H. C. Lam, J. A. Englert, M. Rabinovitch, M. Cernadas, and H. P. Kim. 2011. Autophagy proteins regulate innate immune responses by inhibiting the release of mitochondrial DNA mediated by the NALP3 inflammasome. Nat Immunol 12: 222-230.

134. Warburg, O. 1956. On the origin of cancer cells. Science 123: 309-314.

135. Warburg, O., K. Gawehn, and A. W. Geissler. 1958. Metabolism of leukocytes. $Z$ Naturforsch B 13b: 515-516.

136. Deberardinis, R. J., N. Sayed, D. Ditsworth, and C. B. Thompson. 2008. Brick by brick: metabolism and tumor cell growth. Curr Opin Genet Dev 18: 54-61.

137. Kesarwani, P., A. K. Murali, A. A. Al-Khami, and S. Mehrotra. 2013. Redox regulation of T-cell function: from molecular mechanisms to significance in human health and disease. Antioxid. Redox Signal. 18: 1497-1534.

138. Wang, H., Y. Hsieh, W. Cheng, C. Lin, Y. Lin, S. Yang, C. Chen, Y. Izumiya, J. Yu, H. Kung, and W. Wang. 2014. JMJD5 regulates PKM2 nuclear translocation and reprograms HIF-1 $\alpha$-mediated glucose metabolism. Proc. Natl. Acad. Sci. U. S. A 111: 279-284.

139. Wang, H., H. Flach, M. Onizawa, L. Wei, M. T. McManus, and A. Weiss. 2014. Negative regulation of Hifla expression and $\mathrm{TH} 17$ differentiation by the hypoxia-regulated microRNA miR-210. Nat. Immunol. 15: 393-401.

140. Murray, Peter J., Judith E. Allen, Subhra K. Biswas, Edward A. Fisher, Derek W. Gilroy, S. Goerdt, S. Gordon, John A. Hamilton, Lionel B. Ivashkiv, T. Lawrence, M. Locati, A. Mantovani, Fernando O. Martinez, J.-L. Mege, David M. Mosser, G. Natoli, Jeroen P. Saeij, Joachim L. Schultze, Kari A. Shirey, A. Sica, J. Suttles, I. Udalova, Jo A. 
van Ginderachter, Stefanie N. Vogel, and Thomas A. Wynn. 2014. Macrophage Activation and Polarization: Nomenclature and Experimental Guidelines. Immunity 41: 14-20.

141. Mantovani, A., A. Sica, S. Sozzani, P. Allavena, A. Vecchi, and M. Locati. 2004. The chemokine system in diverse forms of macrophage activation and polarization. Trends in Immunology 25: 677-686.

142. Gordon, S. 2003. Alternative activation of macrophages. Nat Rev Immunol 3: 23-35.

143. Mills, E. L., B. Kelly, A. Logan, A. S. H. Costa, M. Varma, C. E. Bryant, P. Tourlomousis, J. H. M. Däbritz, E. Gottlieb, I. Latorre, S. C. Corr, G. McManus, D. Ryan, H. T. Jacobs, M. Szibor, R. J. Xavier, T. Braun, C. Frezza, M. P. Murphy, and L. A. O’Neill. 2016. Succinate dehydrogenase supports metabolic repurposing of mitochondria to drive inflammatory macrophages. Cell 167: 457-470.e413.

144. Palsson-McDermott, E. M., A. M. Curtis, G. Goel, M. A. R. Lauterbach, F. J. Sheedy, L. E. Gleeson, M. W. M. van den Bosch, S. R. Quinn, R. Domingo-Fernandez, D. G. W. Johnston, J.-k. Jiang, W. J. Israelsen, J. Keane, C. Thomas, C. Clish, M. Vander Heiden, R. J. Xavier, and L. A. J. O’Neill. 2015. Pyruvate kinase M2 regulates Hif-1 $\alpha$ activity and IL-1 $\beta$ induction and is a critical determinant of the warburg effect in LPS-activated macrophages. Cell Metab. 21: 65-80.

145. Sag, D., D. Carling, R. D. Stout, and J. Suttles. 2008. Adenosine 5'-monophosphateactivated protein kinase promotes macrophage polarization to an anti-inflammatory functional phenotype. Journal of immunology (Baltimore, Md. : 1950) 181: 8633-8641.

146. Steinberg, G. R., and J. D. Schertzer. 2014. AMPK promotes macrophage fatty acid oxidative metabolism to mitigate inflammation: implications for diabetes and cardiovascular disease. Immunology \& Cell Biology 92: 340-345.

147. Stout, R. D., C. Jiang, B. Matta, I. Tietzel, S. K. Watkins, and J. Suttles. 2005. Macrophages Sequentially Change Their Functional Phenotype in Response to Changes in Microenvironmental Influences. The Journal of Immunology 175: 342-349.

148. Van den Bossche, J., J. Baardman, Natasja A. Otto, S. van der Velden, Annette E. Neele, Susan M. van den Berg, R. Luque-Martin, H.-J. Chen, Marieke C. S. Boshuizen, M. Ahmed, Marten A. Hoeksema, Alex F. de Vos, and Menno P. J. de Winther. 2016. Mitochondrial Dysfunction Prevents Repolarization of Inflammatory Macrophages. Cell Rep 17: 684-696.

149. Cramer, T., Y. Yamanishi, B. E. Clausen, I. Förster, R. Pawlinski, N. Mackman, V. H. Haase, R. Jaenisch, M. Corr, V. Nizet, G. S. Firestein, H.-P. Gerber, N. Ferrara, and R. S. Johnson. 2003. HIF-1 $\alpha$ is essential for myeloid cell-mediated inflammation. Cell 112: 645657.

150. Maedera, S., T. Mizuno, H. Ishiguro, T. Ito, T. Soga, and H. Kusuhara. 2019. GLUT6 is a lysosomal transporter that is regulated by inflammatory stimuli and modulates glycolysis in macrophages. FEBS Lett 593: 195-208.

151. Fukuzumi, M., H. Shinomiya, Y. Shimizu, K. Ohishi, and S. Utsumi. 1996. Endotoxininduced enhancement of glucose influx into murine peritoneal macrophages via GLUT1. Infection and Immunity 64: 108-112.

152. Varanasi, S. K., D. Donohoe, U. Jaggi, and B. T. Rouse. 2017. Manipulating Glucose Metabolism during Different Stages of Viral Pathogenesis Can Have either Detrimental or Beneficial Effects. J Immunol.

153. Liu, L., Y. Lu, J. Martinez, Y. Bi, G. Lian, T. Wang, S. Milasta, J. Wang, M. Yang, G. Liu, D. R. Green, and R. Wang. 2016. Proinflammatory signal suppresses proliferation and 
shifts macrophage metabolism from Myc-dependent to HIF1 $\alpha$-dependent. Proc Natl Acad Sci US A 113: 1564.

154. Tan, Z., N. Xie, H. Cui, D. R. Moellering, E. Abraham, V. J. Thannickal, and G. Liu. 2015. Pyruvate Dehydrogenase Kinase 1 Participates in Macrophage Polarization via Regulating Glucose Metabolism. J Immunol 194: 6082.

155. Chang, C. H., J. D. Curtis, L. B. Maggi, B. Faubert, A. V. Villarino, D. O'sullivan, S. C. Huang, G. L. van der Windt, J. Blagih, J. Qiu, J. D. Weber, E. J. Pearce, R. G. Jones, and E. L. Pearce. 2013. Posttranscriptional control of $\mathrm{T}$ cell effector function by aerobic glycolysis. Cell 153.

156. Blagih, J., F. Coulombe, Emma E. Vincent, F. Dupuy, G. Galicia-Vázquez, E. Yurchenko, Thomas C. Raissi, Gerritje J. W. van der Windt, B. Viollet, Erika L. Pearce, J. Pelletier, Ciriaco A. Piccirillo, Connie M. Krawczyk, M. Divangahi, and Russell G. Jones. 2015. The Energy Sensor AMPK Regulates T Cell Metabolic Adaptation and Effector Responses In Vivo. Immunity 42: 41-54.

157. Galván-Peña, S., R. G. Carroll, C. Newman, E. C. Hinchy, E. Palsson-McDermott, E. K. Robinson, S. Covarrubias, A. Nadin, A. M. James, M. Haneklaus, S. Carpenter, V. P. Kelly, M. P. Murphy, L. K. Modis, and L. A. O'Neill. 2019. Malonylation of GAPDH is an inflammatory signal in macrophages. Nature Commun 10: 338-338.

158. Zhang, Z., X. Deng, Y. Liu, Y. Liu, L. Sun, and F. Chen. 2019. PKM2, function and expression and regulation. Cell Biosci 9: 52.

159. Ham, M., J.-W. Lee, A. H. Choi, H. Jang, G. Choi, J. Park, C. Kozuka, D. D. Sears, H. Masuzaki, and J. B. Kim. 2013. Macrophage glucose-6-phosphate dehydrogenase stimulates proinflammatory responses with oxidative stress. Mol Cell Biol 33: 2425-2435.

160. Minhas, P. S., L. Liu, P. K. Moon, A. U. Joshi, C. Dove, S. Mhatre, K. Contrepois, Q. Wang, B. A. Lee, M. Coronado, D. Bernstein, M. P. Snyder, M. Migaud, R. Majeti, D. Mochly-Rosen, J. D. Rabinowitz, and K. I. Andreasson. 2019. Macrophage de novo $\mathrm{NAD}(+)$ synthesis specifies immune function in aging and inflammation. Nat Immunol 20: 50-63.

161. De Souza, D. P., A. Achuthan, M. K. Lee, K. J. Binger, M.-C. Lee, S. Davidson, D. L. Tull, M. J. McConville, A. D. Cook, A. J. Murphy, J. A. Hamilton, and A. J. Fleetwood. 2019. Autocrine IFN-I inhibits isocitrate dehydrogenase in the TCA cycle of LPS-stimulated macrophages. The Journal of clinical investigation 129: 4239-4244.

162. Swain, A., M. Bambouskova, H. Kim, P. S. Andhey, D. Duncan, K. Auclair, V. Chubukov, D. M. Simons, T. P. Roddy, K. M. Stewart, and M. N. Artyomov. 2020. Comparative evaluation of itaconate and its derivatives reveals divergent inflammasome and type I interferon regulation in macrophages. Nat Metab 2: 594-602.

163. Infantino, V., V. Iacobazzi, F. Palmieri, and A. Menga. 2013. ATP-citrate lyase is essential for macrophage inflammatory response. Biochem. Biophys. Res. Commun. 440: 105-111.

164. Lampropoulou, V., A. Sergushichev, M. Bambouskova, S. Nair, E. E. Vincent, E. Loginicheva, L. Cervantes-Barragan, X. Ma, S. C.-C. Huang, T. Griss, C. J. Weinheimer, S. Khader, G. J. Randolph, E. J. Pearce, R. G. Jones, A. Diwan, M. S. Diamond, and M. N. Artyomov. 2016. Itaconate Links Inhibition of Succinate Dehydrogenase with Macrophage Metabolic Remodeling and Regulation of Inflammation. Cell Metab. 24: 158-166.

165. Michelucci, A., T. Cordes, J. Ghelfi, A. Pailot, N. Reiling, O. Goldmann, T. Binz, A. Wegner, A. Tallam, A. Rausell, M. Buttini, C. L. Linster, E. Medina, R. Balling, and K. Hiller. 2013. Immune-responsive gene 1 protein links metabolism to immunity by 
catalyzing itaconic acid production. Proceedings of the National Academy of Sciences 110: 7820 .

166. Kobayashi, E. H., T. Suzuki, R. Funayama, T. Nagashima, M. Hayashi, H. Sekine, N. Tanaka, T. Moriguchi, H. Motohashi, K. Nakayama, and M. Yamamoto. 2016. Nrf2 suppresses macrophage inflammatory response by blocking proinflammatory cytokine transcription. Nat Commun 7: 11624.

167. Bambouskova, M., L. Gorvel, V. Lampropoulou, A. Sergushichev, E. Loginicheva, K. Johnson, D. Korenfeld, M. E. Mathyer, H. Kim, L.-H. Huang, D. Duncan, H. Bregman, A. Keskin, A. Santeford, R. S. Apte, R. Sehgal, B. Johnson, G. K. Amarasinghe, M. P. Soares, T. Satoh, S. Akira, T. Hai, C. de Guzman Strong, K. Auclair, T. P. Roddy, S. A. Biller, M. Jovanovic, E. Klechevsky, K. M. Stewart, G. J. Randolph, and M. N. Artyomov. 2018. Electrophilic properties of itaconate and derivatives regulate the IкB $\zeta-\mathrm{ATF} 3$ inflammatory axis. Nature 556: 501-504.

168. Cordes, T., M. Wallace, A. Michelucci, A. S. Divakaruni, S. C. Sapcariu, C. Sousa, H. Koseki, P. Cabrales, A. N. Murphy, K. Hiller, and C. M. Metallo. 2016. Immunoresponsive Gene 1 and Itaconate Inhibit Succinate Dehydrogenase to Modulate Intracellular Succinate Levels. J Biol Chem 291: 14274-14284.

169. Mills, E. L., D. G. Ryan, H. A. Prag, D. Dikovskaya, D. Menon, Z. Zaslona, M. P. Jedrychowski, A. S. H. Costa, M. Higgins, E. Hams, J. Szpyt, M. C. Runtsch, M. S. King, J. F. McGouran, R. Fischer, B. M. Kessler, A. F. McGettrick, M. M. Hughes, R. G. Carroll, L. M. Booty, E. V. Knatko, P. J. Meakin, M. L. J. Ashford, L. K. Modis, G. Brunori, D. C. Sévin, P. G. Fallon, S. T. Caldwell, E. R. S. Kunji, E. T. Chouchani, C. Frezza, A. T. Dinkova-Kostova, R. C. Hartley, M. P. Murphy, and L. A. O'Neill. 2018. Itaconate is an anti-inflammatory metabolite that activates Nrf2 via alkylation of KEAP1. Nature 556: 113-117.

170. West, A. P., I. E. Brodsky, C. Rahner, D. K. Woo, H. Erdjument-Bromage, P. Tempst, M. C. Walsh, Y. Choi, G. S. Shadel, and S. Ghosh. 2011. TLR signalling augments macrophage bactericidal activity through mitochondrial ROS. Nature 472: 476-480.

171. Chua, Y. L., E. Dufour, E. P. Dassa, P. Rustin, H. T. Jacobs, C. T. Taylor, and T. Hagen. 2010. Stabilization of Hypoxia-inducible factor- $1 \alpha$ protein in hypoxia occurs independently of mitochondrial reactive oxygen species production. $J$ Biol Chem 285: 31277-31284.

172. Brüne, B., and J. Zhou. 2007. Hypoxia-inducible factor-1 $\alpha$ under the control of Nitric oxide. Methods Enzymol 435: 463-478.

173. Kelly, B., G. M. Tannahill, M. P. Murphy, and L. A. J. O'Neill. 2015. Metformin Inhibits the Production of Reactive Oxygen Species from NADH:Ubiquinone Oxidoreductase to Limit Induction of Interleukin-1 $\beta$ (IL-1 $\beta$ ) and Boosts Interleukin-10 (IL-10) in Lipopolysaccharide (LPS)-activated Macrophages. J Biol Chem 290: 20348-20359.

174. Ip, W. K. E., N. Hoshi, D. S. Shouval, S. Snapper, and R. Medzhitov. 2017. Antiinflammatory effect of IL-10 mediated by metabolic reprogramming of macrophages. Science 356: 513-519.

175. Arts, R. J. W., B. Novakovic, R. Ter Horst, A. Carvalho, S. Bekkering, E. Lachmandas, F. Rodrigues, R. Silvestre, S.-C. Cheng, S.-Y. Wang, E. Habibi, L. G. Gonçalves, I. Mesquita, C. Cunha, A. van Laarhoven, F. L. van de Veerdonk, D. L. Williams, J. W. M. van der Meer, C. Logie, L. A. O'Neill, C. A. Dinarello, N. P. Riksen, R. van Crevel, C. Clish, R. A. Notebaart, L. A. B. Joosten, H. G. Stunnenberg, R. J. Xavier, and M. G. Netea. 2016. 
Glutaminolysis and fumarate accumulation integrate immunometabolic and epigenetic programs in trained immunity. Cell Metab 24: 807-819.

176. Su, Y., T. P. Miettinen, L. Mu, E. Mirek, S. R. Manalis, T. G. Anthony, H. Sesaki, and J. Chen. 2020. Disassembly of ETC Complexes Drives Macrophage Inflammatory Responses by Reprogramming Cellular Metabolism and Translation [Manuscirpt submitted for publication]. Cell Rep Available at SSRN: https://ssrn.com/abstract=3611881: 1-54.

177. Zhou, R., A. S. Yazdi, P. Menu, and J. Tschopp. 2011. A role for mitochondria in NLRP3 inflammasome activation. Nature 469: 221-225.

178. Rakkola, R., S. Matikainen, and T. A. Nyman. 2007. Proteome analysis of human macrophages reveals the upregulation of manganese-containing superoxide dismutase after toll-like receptor activation. Proteomics 7: 378-384.

179. Emre, Y., C. Hurtaud, T. Nübel, F. Criscuolo, D. Ricquier, and A.-M. Cassard-Doulcier. 2007. Mitochondria contribute to LPS-induced MAPK activation via uncoupling protein UCP2 in macrophages. Biochemical Journal 402: 271-278.

180. Kizaki, T., K. Suzuki, Y. Hitomi, N. Taniguchi, D. Saitoh, K. Watanabe, K. Onoé, N. K. Day, R. A. Good, and H. Ohno. 2002. Uncoupling protein 2 plays an important role in nitric oxide production of lipopolysaccharide-stimulated macrophages. Proceedings of the National Academy of Sciences 99: 9392-9397.

181. Arsenijevic, D., H. Onuma, C. Pecqueur, S. Raimbault, B. S. Manning, B. Miroux, E. Couplan, M.-C. Alves-Guerra, M. Goubern, R. Surwit, F. Bouillaud, D. Richard, S. Collins, and D. Ricquier. 2000. Disruption of the uncoupling protein-2 gene in mice reveals a role in immunity and reactive oxygen species production. Nat Genet 26: 435-439.

182. Dreißig, M. D. M., R. Hammermann, J. Mössner, M. Göthert, and K. Racké. 2000. In rat alveolar macrophages lipopolysaccharides exert divergent effects on the transport of the cationic amino acids L-arginine and L-ornithine. Naunyn Schmiedebergs Arch Pharmacol 361: 621-628.

183. Sun, C., L. Sun, H. Ma, J. Peng, Y. Zhen, K. Duan, G. Liu, W. Ding, and Y. Zhao. 2012. The phenotype and functional alterations of macrophages in mice with hyperglycemia for long term. J Cell Physiol. 227: 1670-1679.

184. Everts, B., E. Amiel, G. J. W. van der Windt, T. C. Freitas, R. Chott, K. E. Yarasheski, E. L. Pearce, and E. J. Pearce. 2012. Commitment to glycolysis sustains survival of NOproducing inflammatory dendritic cells. Blood 120: 1422-1431.

185. Palmieri, E. M., C. McGinity, D. A. Wink, and D. W. McVicar. 2020. Nitric oxide in macrophage immunometabolism: Hiding in plain sight. Metabolites 10: 429.

186. Rodriguez, A. E., G. S. Ducker, L. K. Billingham, C. A. Martinez, N. Mainolfi, V. Suri, A. Friedman, M. G. Manfredi, S. E. Weinberg, J. D. Rabinowitz, and N. S. Chandel. 2019. Serine metabolism supports macrophage IL-1 $\beta$ production. Cell Metab 29: 10031011.e1004.

187. Yu, W., Z. Wang, K. Zhang, Z. Chi, T. Xu, D. Jiang, S. Chen, W. Li, X. Yang, X. Zhang, Y. Wu, and D. Wang. 2019. One-Carbon metabolism supports S-Adenosylmethionine and histone methylation to drive inflammatory macrophages. Mol Cell 75: 1147-1160.e1145.

188. Yoon, B. R., Y.-J. Oh, S. W. Kang, E. B. Lee, and W.-W. Lee. 2018. Role of SLC7A5 in metabolic reprogramming of Human monocyte/macrophage immune responses. Front Immunol 9: 53. 
189. Dang, E. V., J. G. McDonald, D. W. Russell, and J. G. Cyster. 2017. Oxysterol Restraint of Cholesterol Synthesis Prevents AIM2 Inflammasome Activation. Cell 171: 10571071.e1011.

190. Araldi, E., M. Fernández-Fuertes, A. Canfrán-Duque, W. Tang, G. W. Cline, J. MadrigalMatute, J. S. Pober, M. A. Lasunción, D. Wu, C. Fernández-Hernando, and Y. Suárez. 2017. Lanosterol Modulates TLR4-Mediated Innate Immune Responses in Macrophages. Cell Reports 19: 2743-2755.

191. Lopes-Virella, M. F., R. L. Klein, and H. C. Stevenson. 1987. Low density lipoprotein metabolism in human macrophages stimulated with microbial or microbial-related products. Arteriosclerosis 7: 176-184.

192. Hsieh, W.-Y., Q. D. Zhou, A. G. York, K. J. Williams, P. O. Scumpia, E. B. Kronenberger, X. P. Hoi, B. Su, X. Chi, V. L. Bui, E. Khialeeva, A. Kaplan, Y. M. Son, A. S. Divakaruni, J. Sun, S. T. Smale, R. A. Flavell, and S. J. Bensinger. 2020. Toll-Like Receptors Induce Signal-Specific Reprogramming of the Macrophage Lipidome. Cell Metabolism 32: 128143.e125.

193. Zhou, Q. D., X. Chi, M. S. Lee, W. Y. Hsieh, J. J. Mkrtchyan, A.-C. Feng, C. He, A. G. York, V. L. Bui, and E. B. Kronenberger. 2020. Interferon-mediated reprogramming of membrane cholesterol to evade bacterial toxins. Nat Immunol: 1-10.

194. Oishi, Y., N. J. Spann, V. M. Link, E. D. Muse, T. Strid, C. Edillor, M. J. Kolar, T. Matsuzaka, S. Hayakawa, J. Tao, M. U. Kaikkonen, A. F. Carlin, M. T. Lam, I. Manabe, H. Shimano, A. Saghatelian, and C. K. Glass. 2017. SREBP1 contributes to resolution of pro-inflammatory TLR4 signaling by reprogramming fatty acid metabolism. Cell Metab 25: 412-427.

195. Weigert, A., A. von Knethen, D. Thomas, I. Faria, D. Namgaladze, E. Zezina, D. Fuhrmann, A. Petcherski, D. M. z. Heringdorf, H. H. Radeke, and B. Brüne. 2019. Sphingosine kinase 2 is a negative regulator of inflammatory macrophage activation. Biochimica et Biophysica Acta (BBA) - Molecular and Cell Biology of Lipids 1864: 12351246.

196. Joshi, J. C., B. Joshi, I. Rochford, S. Rayees, M. Z. Akhter, S. Baweja, K. R. Chava, M. Tauseef, H. Abdelkarim, V. Natarajan, V. Gaponenko, and D. Mehta. 2020. SPHK2generated S1P in $\mathrm{CD} 11 \mathrm{~b}(+)$ macrophages blocks STING to suppress the inflammatory function of alveolar macrophages. Cell Rep 30: 4096-4109.e4095.

197. Fritsch, S. D., and T. Weichhart. 2016. Effects of Interferons and Viruses on Metabolism. Front. Immunol. 7: 630.

198. Zhang, S., J. Carriere, X. Lin, N. Xie, and P. Feng. 2018. Interplay between cellular metabolism and cytokine responses during viral infection. Viruses 10: 521.

199. Yu, Y., T. G. Maguire, and J. C. Alwine. 2011. Human cytomegalovirus activates glucose transporter 4 expression to increase glucose uptake during infection. J Virol 85: 1573-1580.

200. Munger, J., B. D. Bennett, A. Parikh, X. J. Feng, J. McArdle, H. A. Rabitz, T. Shenk, and J. D. Rabinowitz. 2008. Systems-level metabolic flux profiling identifies fatty acid synthesis as a target for antiviral therapy. Nat Biotechnol 26.

201. Ritter, J. B., A. S. Wahl, S. Freund, Y. Genzel, and U. Reichl. 2010. Metabolic effects of influenza virus infection in cultured animal cells: intra- and extracellular metabolite profiling. BMC Syst Biol 4.

202. Deshmane, S. L., S. Amini, S. Sen, K. Khalili, and B. E. Sawaya. 2011. Regulation of the HIV-1 promoter by HIF-1 $\alpha$ and Vpr proteins. Virol J 8: 477. 
203. Ripoli, M., A. D'Aprile, G. Quarato, M. Sarasin-Filipowicz, J. Gouttenoire, R. Scrima, O. Cela, D. Boffoli, M. H. Heim, D. Moradpour, N. Capitanio, and C. Piccoli. 2010. Hepatitis $\mathrm{C}$ virus-linked mitochondrial dysfunction promotes Hypoxia-inducible factor $1 \alpha$-mediated glycolytic adaptation. $J$ Virol 84: 647-660.

204. Palmer, C. S., T. Hussain, G. Duette, T. J. Weller, M. Ostrowski, I. Sada-Ovalle, and S. M. Crowe. 2016. Regulators of Glucose Metabolism in CD4+ and CD8+ T Cells. International Reviews of Immunology 35: 477-488.

205. Palmer, C. S., G. A. Duette, M. C. E. Wagner, D. C. Henstridge, S. Saleh, C. Pereira, J. Zhou, D. Simar, S. R. Lewin, M. Ostrowski, J. M. McCune, and S. M. Crowe. 2017. Metabolically active CD4+ T cells expressing Glut1 and OX40 preferentially harbor HIV during in vitro infection. Febs Letters 591: 3319-3332.

206. Anzinger, J. J., T. R. Butterfield, M. Gouillou, J. M. McCune, S. M. Crowe, and C. S. Palmer. 2018. Glut1 Expression Level on Inflammatory Monocytes is Associated With Markers of Cardiovascular Disease Risk in HIV-Infected Individuals. J Acquir Immune Defic Syndr 77.

207. Duette, G., P. Pereyra Gerber, J. Rubione, P. S. Perez, A. L. Landay, S. M. Crowe, Z. Liao, K. W. Witwer, M. P. Holgado, J. Salido, J. Geffner, O. Sued, C. S. Palmer, and M. Ostrowski. 2018. Induction of HIF-1 $\alpha$ by HIV-1 infection in CD4(+) T cells promotes viral replication and drives extracellular vesicle-mediated inflammation. mBio 9: e00757-00718.

208. Thai, M., S. K. Thaker, J. Feng, Y. Du, H. Hu, T. T. Wu, T. G. Graeber, D. Braas, and H. R. Christofk. 2015. MYC-induced reprogramming of glutamine catabolism supports optimal virus replication. Nature communications 6: 1-9.

209. Vastag, L., E. Koyuncy, S. L. Grady, T. E. Shenk, and J. D. Rabinowitz. 2011. Divergent effects of human cytomegalovirus and herpes simplex virus-1 on cellular metabolism. PLoS Pathog. 7: e1002124.

210. Grady, S. L., J. G. Purdy, J. D. Rabinowitz, and T. Shenk. 2013. Argininosuccinate synthetase 1 depletion produces a metabolic state conducive to herpes simplex virus 1 infection. Proc Natl Acad Sci U S A 110.

211. Sun, B., K. B. Sundström, J. J. Chew, P. Bist, E. S. Gan, H. C. Tan, K. C. Goh, T. Chawla, C. K. Tang, and E. E. Ooi. 2017. Dengue virus activates cGAS through the release of mitochondrial DNA. Sci Rep 7: 1-8.

212. Yu, C.-Y., J.-J. Liang, J.-K. Li, Y.-L. Lee, B.-L. Chang, C.-I. Su, W.-J. Huang, M. M. C. Lai, and Y.-L. Lin. 2015. Dengue virus impairs mitochondrial fusion by cleaving mitofusins. PLoS pathog 11: e1005350.

213. Chatel-Chaix, L., M. Cortese, I. Romero-Brey, S. Bender, C. J. Neufeldt, W. Fischl, P. Scaturro, N. Schieber, Y. Schwab, and B. Fischer. 2016. Dengue virus perturbs mitochondrial morphodynamics to dampen innate immune responses. Cell Host Microbe 20: 342-356.

214. Kim, S.-J., M. Khan, J. Quan, A. Till, S. Subramani, and A. Siddiqui. 2013. Hepatitis B virus disrupts mitochondrial dynamics: induces fission and mitophagy to attenuate apoptosis. PLoS Pathog 9: e1003722.

215. Peng, Y.-T., P. Chen, R.-Y. Ouyang, and L. Song. 2015. Multifaceted role of prohibitin in cell survival and apoptosis. Apoptosis 20: 1135-1149.

216. Tsutsumi, T., M. Matsuda, H. Aizaki, K. Moriya, H. Miyoshi, H. Fujie, Y. Shintani, H. Yotsuyanagi, T. Miyamura, T. Suzuki, and K. Koike. 2009. Proteomics analysis of 
mitochondrial proteins reveals overexpression of a mitochondrial protein chaperon, prohibitin, in cells expressing hepatitis C virus core protein. Hepatology 50: 378-386.

217. Deng, L., T. Adachi, K. Kitayama, Y. Bungyoku, S. Kitazawa, S. Ishido, I. Shoji, and H. Hotta. 2008. Hepatitis $\mathrm{C}$ virus infection induces apoptosis through a Bax-triggered, mitochondrion-mediated, Caspase 3-dependent pathway. J Virol 82: 10375.

218. Korenaga, M., T. Wang, Y. Li, L. A. Showalter, T. Chan, J. Sun, and S. A. Weinman. 2005. Hepatitis $\mathrm{C}$ virus core protein inhibits mitochondrial electron transport and increases reactive oxygen species (ROS) production. J Biol Chem 280: 37481-37488.

219. Nomura-Takigawa, Y., M. Nagano-Fujii, L. Deng, S. Kitazawa, S. Ishido, K. Sada, and H. Hotta. 2006. Non-structural protein $4 \mathrm{~A}$ of Hepatitis $\mathrm{C}$ virus accumulates on mitochondria and renders the cells prone to undergoing mitochondria-mediated apoptosis. J Gen Virol 87: 1935-1945.

220. Valle-Casuso, J. C., M. Angin, S. Volant, C. Passaes, V. Monceaux, A. Mikhailova, K. Bourdic, V. Avettand-Fenoel, F. Boufassa, M. Sitbon, O. Lambotte, M. I. Thoulouze, M. Müller-Trutwin, N. Chomont, and A. Sáez-Cirión. 2019. Cellular metabolism is a major determinant of HIV-1 reservoir seeding in CD4(+) T cells and offers an opportunity to tackle infection. Cell Metab 29: 611-626 e615.

221. Castellano, P., L. Prevedel, S. Valdebenito, and E. A. Eugenin. 2019. HIV infection and latency induce a unique metabolic signature in human macrophages. Sci Rep 9: 3941.

222. Lévy, P. L., S. Duponchel, H. Eischeid, J. Molle, M. Michelet, G. Diserens, M. Vermathen, P. Vermathen, J. F. Dufour, and H. P. Dienes. 2017. Hepatitis C virus infection triggers a tumor-like glutamine metabolism. Hepatology 65: 789-803.

223. Fontaine, K. A., R. Camarda, and M. Lagunoff. 2014. Vaccinia virus requires glutamine but not glucose for efficient replication. Journal of virology 88: 4366-4374.

224. Jordan, T. X., and G. Randall. 2017. Dengue virus activates the AMP kinase-mTOR axis to stimulate a proviral lipophagy. J Virol 91.

225. Rasheed, S., J. S. Yan, A. Lau, and A. S. Chan. 2008. HIV Replication Enhances Production of Free Fatty Acids, Low Density Lipoproteins and Many Key Proteins Involved in Lipid Metabolism: A Proteomics Study. PLOS ONE 3: e3003.

226. Yu, Y., T. G. Maguire, and J. C. Alwine. 2012. Human cytomegalovirus infection induces adipocyte-like lipogenesis through activation of sterol regulatory element binding protein 1. J Virol 86: 2942-2949.

227. Heaton, N. S., R. Perera, K. L. Berger, S. Khadka, D. J. LaCount, R. J. Kuhn, and G. Randall. 2010. Dengue virus nonstructural protein 3 redistributes fatty acid synthase to sites of viral replication and increases cellular fatty acid synthesis. Proceedings of the National Academy of Sciences 107: 17345-17350.

228. Greseth, M. D., and P. Traktman. 2014. De novo Fatty Acid Biosynthesis Contributes Significantly to Establishment of a Bioenergetically Favorable Environment for Vaccinia Virus Infection. PLOS Pathogens 10: e1004021.

229. Perlemuter, G., A. Sabile, P. Letteron, G. Vona, A. Topilco, Y. Chrétien, K. Koike, D. Pessayre, J. Chapman, and G. Barba. 2002. Hepatitis C virus core protein inhibits microsomal triglyceride transfer protein activity and very low density lipoprotein secretion: a model of viral-related steatosis. FASEB J 16: 185-194.

230. Moriishi, K., R. Mochizuki, K. Moriya, H. Miyamoto, Y. Mori, T. Abe, S. Murata, K. Tanaka, T. Miyamura, and T. Suzuki. 2007. Critical role of PA28y in hepatitis C virus- 
associated steatogenesis and hepatocarcinogenesis. Proc Natl Acad Sci U S A 104: 16611666.

231. Oem, J.-K., C. Jackel-Cram, Y.-P. Li, Y. Zhou, J. Zhong, H. Shimano, L. A. Babiuk, and Q. Liu. 2008. Activation of sterol regulatory element-binding protein 1c and fatty acid synthase transcription by hepatitis C virus non-structural protein 2. J Gen Virol 89: 1225.

232. Waris, G., D. J. Felmlee, F. Negro, and A. Siddiqui. 2007. Hepatitis C virus induces proteolytic cleavage of Sterol Regulatory Element Binding Proteins and stimulates their phosphorylation via oxidative stress. J Virol 81: 8122.

233. de Veer, M. J., M. Holko, M. Frevel, E. Walker, S. Der, J. M. Paranjape, R. H. Silverman, and B. R. G. Williams. 2001. Functional classification of interferon-stimulated genes identified using microarrays. J. Leukoc. Biol. 69: 912-920.

234. Honda, K., A. Takaoka, and T. Taniguchi. 2006. Type I Inteferon Gene Induction by the Interferon Regulatory Factor Family of Transcription Factors. Immunity 25: 349-360.

235. York, Autumn G., Kevin J. Williams, Joseph P. Argus, Quan D. Zhou, G. Brar, L. Vergnes, Elizabeth E. Gray, A. Zhen, Nicholas C. Wu, Douglas H. Yamada, Cameron R. Cunningham, Elizabeth J. Tarling, Moses Q. Wilks, D. Casero, David H. Gray, Amy K. Yu, Eric S. Wang, David G. Brooks, R. Sun, Scott G. Kitchen, T.-T. Wu, K. Reue, Daniel B. Stetson, and Steven J. Bensinger. 2015. Limiting Cholesterol Biosynthetic Flux Spontaneously Engages Type I IFN Signaling. Cell 163: 1716-1729.

236. Jiang, H., H. Shi, M. Sun, Y. Wang, Q. Meng, P. Guo, Y. Cao, J. Chen, X. Gao, E. Li, and J. Liu. 2016. PFKFB3-Driven Macrophage Glycolytic Metabolism Is a Crucial Component of Innate Antiviral Defense. J. Immunol. 197: 2880-2890.

237. Li, T., X. Li, K. S. Attri, C. Liu, L. Li, L. E. Herring, J. M. Asara, Y. L. Lei, P. K. Singh, C. Gao, and H. Wen. 2018. O-GlcNAc Transferase Links Glucose Metabolism to MAVSMediated Antiviral Innate Immunity. Cell Host \& Microbe 24: 791-803.e796.

238. Zhao, Y., X. Sun, X. Nie, L. Sun, T.-s. Tang, D. Chen, and Q. Sun. 2012. COX5B Regulates MAVS-mediated Antiviral Signaling through Interaction with ATG5 and Repressing ROS Production. PLOS Pathogens 8: e1003086.

239. Koshiba, T., K. Yasukawa, Y. Yanagi, and S.-i. Kawabata. 2011. Mitochondrial membrane potential is required for MAVS-mediated antiviral signaling. Sci Signal 4: ra7.

240. Tal, M. C., M. Sasai, H. K. Lee, B. Yordy, G. S. Shadel, and A. Iwasaki. 2009. Absence of autophagy results in reactive oxygen species-dependent amplification of RLR signaling. Proc Natl Acad Sci U S A 106: 2770.

241. Yoshizumi, T., H. Imamura, T. Taku, T. Kuroki, A. Kawaguchi, K. Ishikawa, K. Nakada, and T. Koshiba. 2017. RLR-mediated antiviral innate immunity requires oxidative phosphorylation activity. Sci Rep 7: 5379.

242. To, E. E., R. Vlahos, R. Luong, M. L. Halls, P. C. Reading, P. T. King, C. Chan, G. R. Drummond, C. G. Sobey, B. R. S. Broughton, M. R. Starkey, R. van der Sluis, S. R. Lewin, S. Bozinovski, L. A. J. O’Neill, T. Quach, C. J. H. Porter, D. A. Brooks, J. J. O’Leary, and S. Selemidis. 2017. Endosomal NOX2 oxidase exacerbates virus pathogenicity and is a target for antiviral therapy. Nature Communications 8: 69.

243. Yang, C.-S., J.-J. Kim, S. J. Lee, J. H. Hwang, C.-H. Lee, M.-S. Lee, and E.-K. Jo. 2013. TLR3-triggered reactive oxygen species contribute to inflammatory responses by activating Signal transducer and activator of transcription-1. J Immunol 190: 6368.

244. Grunert, T., N. R. Leitner, M. Marchetti-Deschmann, I. Miller, B. Wallner, M. Radwan, C. Vogl, T. Kolbe, D. Kratky, M. Gemeiner, G. Allmaier, M. Müller, and B. Strobl. 2011. A 
comparative proteome analysis links tyrosine kinase 2 (Tyk2) to the regulation of cellular glucose and lipid metabolism in response to poly(I:C). J. Proteomics 74: 2866-2880.

245. Blanc, M., Wei Y. Hsieh, Kevin A. Robertson, Kai A. Kropp, T. Forster, G. Shui, P. Lacaze, S. Watterson, Samantha J. Griffiths, Nathanael J. Spann, A. Meljon, S. Talbot, K. Krishnan, Douglas F. Covey, Markus R. Wenk, M. Craigon, Z. Ruzsics, J. Haas, A. Angulo, William J. Griffiths, Christopher K. Glass, Y. Wang, and P. Ghazal. 2013. The Transcription Factor STAT-1 Couples Macrophage Synthesis of 25-Hydroxycholesterol to the Interferon Antiviral Response. Immunity 38: 106-118.

246. Jurk, M., F. Heil, J. Vollmer, C. Schetter, A. M. Krieg, H. Wagner, G. Lipford, and S. Bauer. 2002. Human TLR7 or TLR8 independently confer responsiveness to the antiviral compound R-848. Nat Immunol 3: 499-499.

247. Lee, J., T.-H. Chuang, V. Redecke, L. She, P. M. Pitha, D. A. Carson, E. Raz, and H. B. Cottam. 2003. Molecular basis for the immunostimulatory activity of guanine nucleoside analogs: Activation of Toll-like receptor 7. Proc Natl Acad Sci U S A 100: 6646.

248. Gorden, K. B., K. S. Gorski, S. J. Gibson, R. M. Kedl, W. C. Kieper, X. Qiu, M. A. Tomai, S. S. Alkan, and J. P. Vasilakos. 2005. Synthetic TLR agonists reveal functional differences between human TLR7 and TLR8. J Immunol 174: 1259-1268.

249. Patoli, D., F. Mignotte, V. Deckert, A. Dusuel, A. Dumont, A. Rieu, A. Jalil, K. Van Dongen, T. Bourgeois, T. Gautier, C. Magnani, N. Le Guern, S. Mandard, J. Bastin, F. Djouadi, C. Schaeffer, N. Guillaumot, M. Narce, M. Nguyen, J. Guy, A. Dargent, J.-P. Quenot, M. Rialland, D. Masson, J. Auwerx, L. Lagrost, and C. Thomas. 2020. Inhibition of mitophagy drives macrophage activation and antibacterial defense during sepsis. The Journal of Clinical Investigation 130.

250. Groß, Christina J., R. Mishra, Katharina S. Schneider, G. Médard, J. Wettmarshausen, Daniela C. Dittlein, H. Shi, O. Gorka, P.-A. Koenig, S. Fromm, G. Magnani, T. Ćiković, L. Hartjes, J. Smollich, Avril A. B. Robertson, Matthew A. Cooper, M. Schmidt-Supprian, M. Schuster, K. Schroder, P. Broz, C. Traidl-Hoffmann, B. Beutler, B. Kuster, J. Ruland, S. Schneider, F. Perocchi, and O. Groß. 2016. K+ Efflux-Independent NLRP3 Inflammasome Activation by Small Molecules Targeting Mitochondria. Immunity 45: 761773.

251. Carroll, R. G., Z. Zasłona, S. Galván-Peña, E. L. Koppe, D. C. Sévin, S. Angiari, M. Triantafilou, K. Triantafilou, L. K. Modis, and L. A. O'Neill. 2018. An unexpected link between fatty acid synthase and cholesterol synthesis in proinflammatory macrophage activation. Journal of Biological Chemistry 293: 5509-5521.

252. Bernard, M. A., X. Han, S. Inderbitzin, I. Agbim, H. Zhao, H. Koziel, and S. D. Tachado. 2014. HIV-Derived ssRNA Binds to TLR8 to Induce Inflammation-Driven Macrophage Foam Cell Formation. PLOS ONE 9: e104039.

253. Fensterheim, B., J. Bohannon, Y. Guo, and E. Sherwood. 2016. MyD88-associated Tolllike receptor ligands prime macrophage metabolism for infection. The Journal of Immunology 196: 203.224.

254. Frede, S., C. Stockmann, P. Freitag, and J. Fandrey. 2006. Bacterial lipopolysaccharide induces HIF-1 activation in human monocytes via p44/42 MAPK and NF-kappaB. Biochem J 396: 517-527.

255. Meiser, J., L. Krämer, S. C. Sapcariu, N. Battello, J. Ghelfi, A. F. D'Herouel, A. Skupin, and K. Hiller. 2016. Pro-inflammatory Macrophages Sustain Pyruvate Oxidation through 
Pyruvate Dehydrogenase for the Synthesis of Itaconate and to Enable Cytokine Expression. J. Biol. Chem. 291: 3932-3946.

256. Al-Shabany, A., A. Moody, A. Foey, and R. Billington. 2016. Intracellular NAD(+) levels are associated with LPS-induced TNF- $\alpha$ release in pro-inflammatory macrophages. Biosci. Rep. 36: e00301. 


\section{Chapter 2. Transcriptional profiling suggests extensive metabolic rewiring of human and mouse macrophages during early interferon alpha responses (Ahmed et al., 2018; Published in Mediators of Inflammation)}

\subsection{Introduction}

Type I interferons (IFN) (IFN- $\alpha$ and IFN- $\beta$ ) play a seminal role in anti-viral, anti-bacterial, and anti-tumour responses and act as critical regulators of innate and adaptive immune responses (1-5). These pleiotropic cytokines are produced following engagement of pattern recognition receptors and signal through the ubiquitously expressed transmembrane IFN- $\alpha$ receptor (IFNAR), composed of IFNAR1 and IFNAR2 subunits (5-7). Cellular responses to type I IFN are cell- and context-dependent and vary during the course of an immune responses (8-11). The variability in these responses is due, in part, to the cumulative effects of JAK-STAT, the p38 MAP kinase, the MAP kinase kinase/ERK/MAPK signal-interacting kinase and the phosphatidylinositol 3-kinase (PI3K)/AKT/mammalian target of rapamycin (mTOR) signaling pathways (11-13).

Emerging evidence suggests cellular metabolism plays a critical role in regulating and finetuning immune function (14-16). Alterations in cellular bioenergetics, amino acid and lipid metabolism have been shown to affect cytokine production, signaling protein activity and cell differentiation (17-19). In macrophages, stimulation with type I IFNs, has been shown to increase glycolytic flux, inhibit sterol biosynthesis, shift lipid metabolism from de novo synthesis to lipid import, and increase tryptophan catabolism (20-25). This metabolic reprogramming is required to mount functional antiviral responses and has been shown to regulate antigen presentation, inflammatory mediator production, phagocytosis efficiency and intracellular killing $(26,27)$.

Recent studies suggest metabolic adaptions in macrophages occur at the molecular level (i.e., gene expression) very early during the process activation and functional polarization (28-30). In lipopolysaccharide (LPS) stimulated macrophages, cellular activation undergoes stages of time- 
resolved metabolic reprogramming into initiation-, early- and amplification-phases (28). To date, there has been no systematic characterization of metabolic reprogramming associated with type I IFN responses, particularly when examined as a function of time. In the study, I used transcriptional profiling to evaluate global changes in metabolic gene expression following short term IFN- $\alpha$ stimulation ( $<4$ hours) in two well-characterized primary macrophage model systems: mouse bone marrow-derived macrophages (BMM) and human monocyte derived macrophages (MDM). Our findings provide a systematic understanding of altered metabolic genes associated with early IFN- $\alpha$ responses in BMM and MDM and identify potential metabolic mechanisms that may contribute to initial establishment of antiviral immune responses.

\subsection{Materials and Methods}

\subsubsection{Microarray normalization and processing}

Microarray datasets were extracted from the Gene Expression Omnibus (GEO) repository from National Center for Biotechnology Information (NCBI). Datasets were identified using the search terms: "Macrophage Interferon" and "Type I Interferon" (31). A total of 10 datasets were identified using these search criteria. Two studies were identified that assessed short-term stimulation ( $<4$ hours) in either mouse bone marrow-derived macrophages (BMM) or human monocyte-derived macrophages (MDM). The selected BMM dataset (32) differentiated bone marrow cells in DMEM media with $10 \mathrm{ng} / \mathrm{mL}$ of Macrophage-Colony Stimulating Factor (MCSF), 10\% FBS, 1\% penicillin/streptomycin for seven days before replacing the media on day six. BMM were stimulated with 62U/mL IFN- $\alpha$ (approximately $1 \mathrm{ng} / \mathrm{ml}$ ) for 2.5 hours prior to cell lysis and RNA extraction (Supplemental Figure S2.1). The selected MDM dataset (33) isolated monocytes via adherence (4 hours) and differentiated cells in DMEM media with $2 \mathrm{mM}$ Lglutamine, $100 \mathrm{U} / \mathrm{mL}$ penicillin, $100 \mu \mathrm{g} / \mathrm{mL}$ streptomycin and $10 \%$ FCS for 7 days. After differentiation, MDM were treated with $10 \mathrm{ng} / \mathrm{mL}$ IFN- $\alpha$ for 4 hours prior to cell lysis and RNA 
extraction (Supplemental Figure S2.1). Raw gene expression data was normalized by median centering and preprocessing was performed in dChip (34). Probes were excluded from the analysis when present calls $(\mathrm{P}<0.05)$ were identified in less than $20 \%$ of samples.

\subsubsection{The identification of significant metabolic genes in IFN-stimulated macrophages (BMM \& MDM)}

Metabolic genes were identified using the MetScape plugin in Cytoscape (35-37). These genes were identified by mapping Entrez Gene IDs to metabolic pathways found in the Kyoto Encyclopedia of Genes and Genomes (KEGG) and Edinburgh Human Metabolic Network (EHMN) databases. Manual curation was performed to identify metabolic genes that did not map to KEGG or EHMN pathways. Fold change (FC) analyses, p-values, and false discovery rates (FDR) were calculated in R. Differentially expressed genes were defined as a $-1.2 \leq \mathrm{FC} \geq 1.2$, pvalue $\leq 0.05$ and FDR $\leq 0.1$. This fold change cut-off was selected to be inclusive of small differences in gene expression. Biologically relevant alterations in metabolic gene expression were identified by combining FC with pathway and network analyses. Classification analyses including Principal Component Analysis (PCA), Partial Least Squares-Discriminant Analysis (PLS-DA), Random Forest (RF) and unsupervised hierarchical clustering were performed in MetaboAnalyst (38). Gene Set Enrichment Analysis (GSEA) (39) was performed using the hallmark gene sets (H; $\mathrm{n}=50)$, the curated gene sets $(\mathrm{C} 2 ; \mathrm{n}=4731)$ and the $\mathrm{GO}$ gene sets $(\mathrm{C} 5 ; \mathrm{n}=5917)$ from Molecular Signatures Database (MSigDB) version 6.0. Significant enrichment was defined as a $p \leq 0.05$ and FDR $<0.25$. Metabolic pathway enrichment and topology analysis was performed in MetaboAnalyst. Significant enrichment of metabolic data sets was defined with $\mathrm{p} \leq 0.05$. Metabolic network annotation and analysis was performed in Cytoscape and the Database for Annotation, Visualization, and Integrated Discovery (DAVID) $(40,41)$. The workflow is shown in Figure 2.1A. 

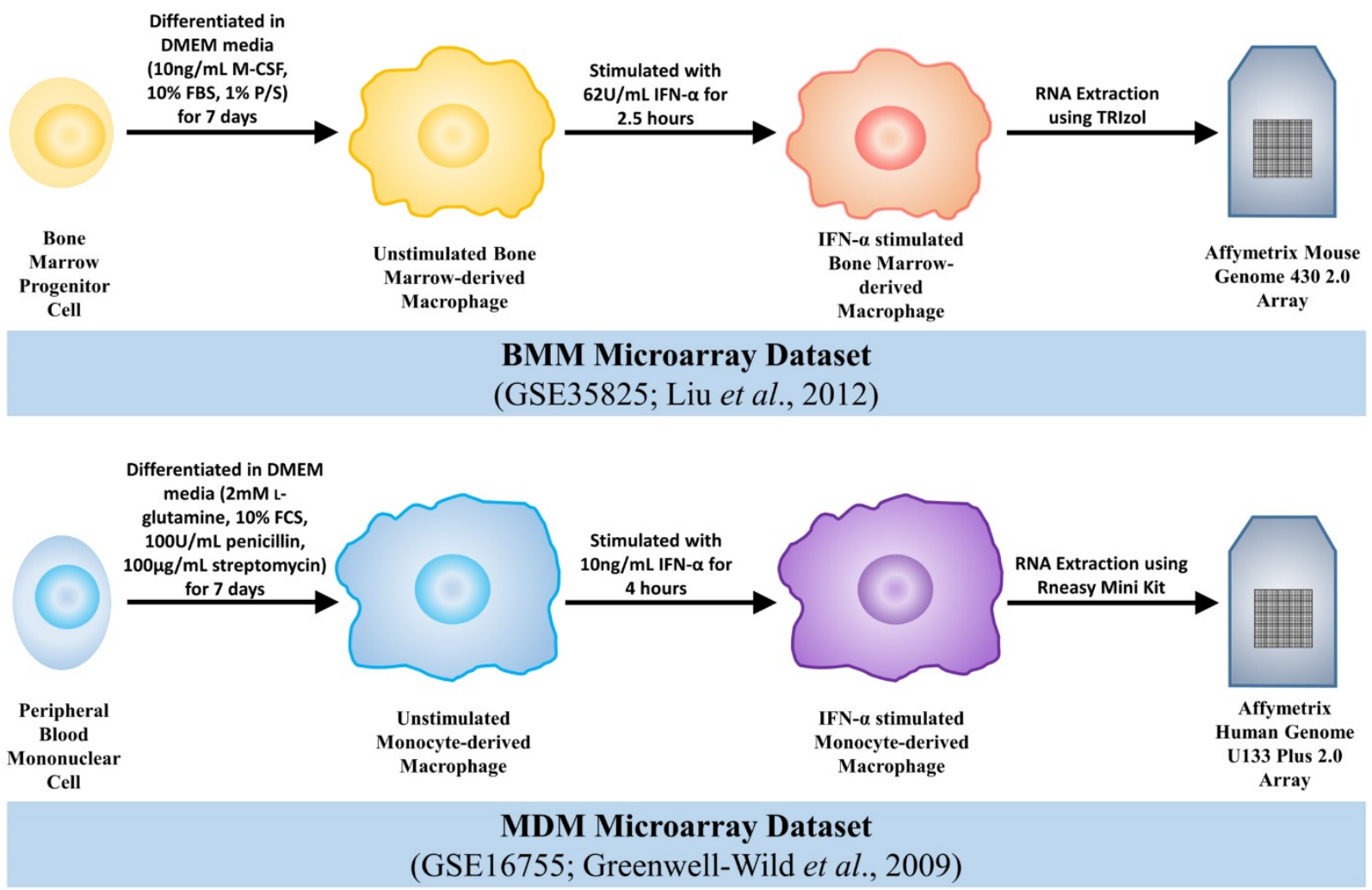

Supplemental Figure S2.1: Study workflow of BMM and MDM differentiation and IFN- $\alpha$ stimulation. Mouse bone marrow cells and human monocytes were differentiated for 7 days into BMM and MDM, respectively. Cells were then stimulated with IFN- $\alpha$ for less than 4 hours. RNA was extracted from total cells and prepared for microarray analysis. 

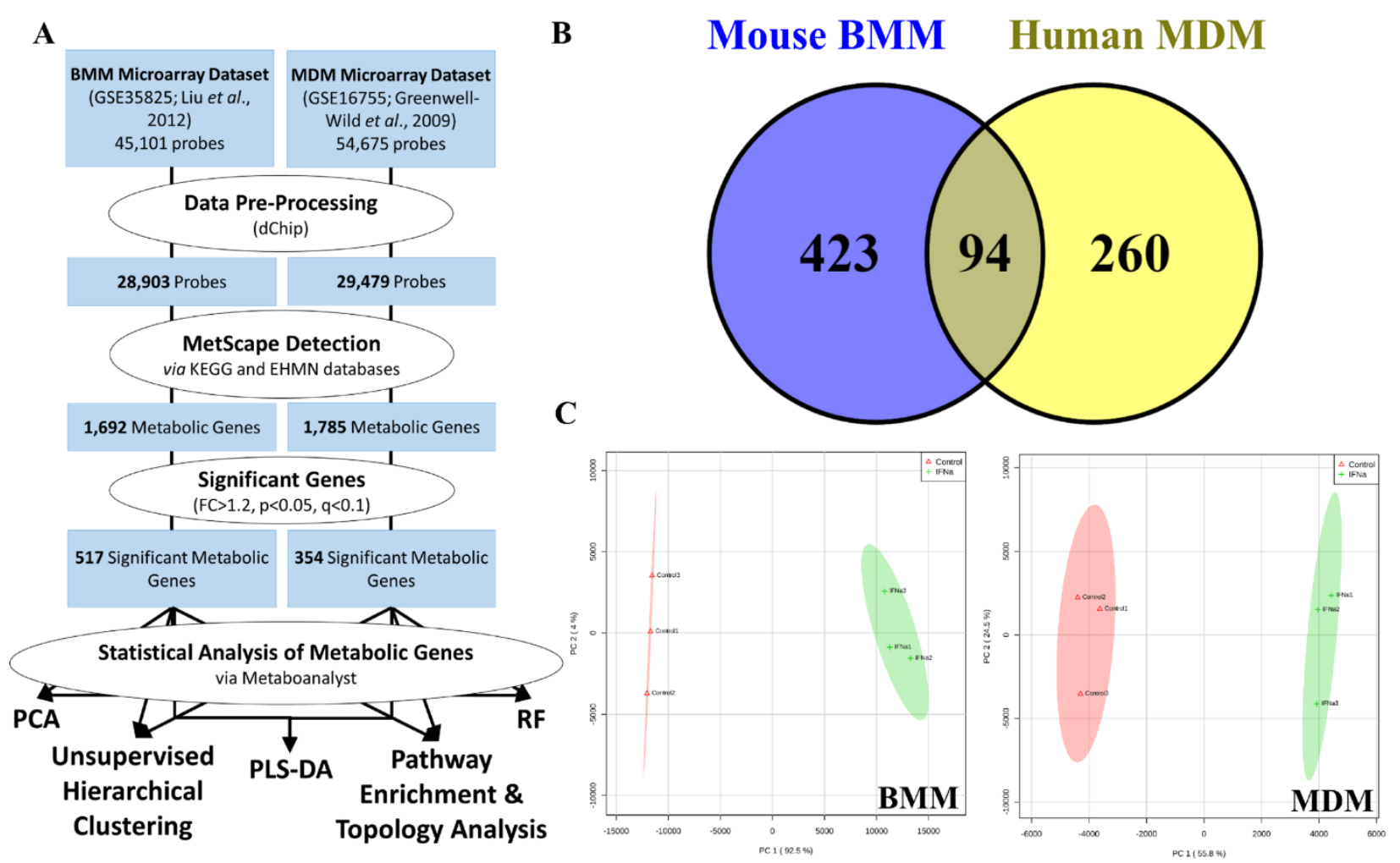

Figure 2.1: Short term IFN- $\alpha$ stimulation is associated with altered expression of metabolic genes in human monocyte-derived macrophages (MDM) and mouse bone marrow-derived macrophages (BMM). (A) Workflow used to identify differentially expressed metabolic genes in IFN- $\alpha$-stimulated mouse BMM (2.5 hours) and human MDM (4 hours). Metabolic genes were identified in MetScape using the Kyoto Encyclopedia of Genes and Genomes (KEGG) and the Edinburgh Human Metabolic Network (EHMN) databases. (B) Venn diagram showing the number of metabolic genes common to the BMM (Yellow) and MDM (Blue) datasets (FC $>1.2, \mathrm{p}<0.05$, FDR $<0.10$ ). (C) Principal component analysis (PCA) of metabolic gene sets from BMM (left) and MDM (right) following IFN- $\alpha$ stimulation $(n=517$ and 354 metabolic genes in the BMM and MDM datasets, respectively). 


\subsection{Results}

\subsubsection{Type I IFN-stimulation of mouse BMM and human MDM is associated with enrichment of metabolic gene sets.}

Of the $>45,000$ unique probe sets analyzed across the two datasets, 28,903 and 29,479 probes were identified as present in the BMM and MDM datasets, respectively. In total, 7,338 genes were differentially expressed in IFN- $\alpha$ stimulated BMM compared to unstimulated controls (Supplemental Table S2.1). Conversely, 3,804 genes were differentially expressed in IFN- $\alpha$ stimulated MDM compared to controls (Supplemental Table S2.2). GSEA identified significantly enriched gene sets within each dataset. Two hundred and eighty-five gene sets were enriched in BMM distributed across three main functional categories including immune signaling and function $(n=151)$, cellular metabolism $(n=40)$ and other biological states and processes $(n=94)$. Enriched metabolic processes in BMM included glycolysis and gluconeogenesis, the regulation of NO biosynthetic process, and tryptophan, arginine, and proline metabolism (Supplemental Table S2.3). In MDM, GSEA identified 948 enriched gene sets (immune signaling and function: $\mathrm{n}=360$, cellular metabolism: $n=107$, other biological states and processes: $n=481$ ). Enriched metabolic processes included reactive oxygen species (ROS) metabolism and biosynthesis, tryptophan metabolism, regulation of steroid biosynthetic process and regulation of oxidoreductase activity (Supplemental Table S2.4).

\subsubsection{Metabolic genes represent important classifiers of early type I IFN responses in BMM and MDM}

Given the significant enrichment of metabolic gene sets in GSEA analysis, I used MetScape to identify all metabolic genes detected across datasets. Of the $>1600$ metabolic genes identified, 517 and 354 were altered following short term IFN- $\alpha$ stimulation of BMM and MDM, respectively $(-1.2 \leq \mathrm{FC} \geq 1.2$, p-value $\leq 0.05, \mathrm{FDR} \leq 0.1)$. Ninety-four genes were altered in both datasets with 
the same directionality (either up or down regulated) (Figure 2.1B) including genes associated with cellular bioenergetics (PFKFB3, PDP), tryptophan metabolism (KMO, WARS), nucleotide metabolism (NT5C3,CNP) and lipid metabolism (SPTLC2, AGPAT5, SQLE, SOAT1). PCA showed a clear separation between the control and IFN- $\alpha$ treated samples in both BMM and MDM based on the metabolic gene subset (Figure 2.1C). Similarly, random forest analysis classified control and IFN- $\alpha$-stimulated cells with $100 \%$ predictive accuracy using metabolic genes. Variable importance in projection (VIP) analysis identified genes involved in nucleotide degradation ( $P N P$, $A M P D 3)$ and lipid metabolism (ETNK1, HMGCS1) as top classifiers in IFN- $\alpha$ stimulation in BMM. Top classifiers in MDM included genes associated with glycolysis (PFKFB3), tryptophan catabolism $(K Y N U)$ and reactive oxygen species production (GCH1, SOD2) (Figures $2.2 \mathrm{C}$ and 2.2D). Only EIF2AK2, NAMPT, NT5C3 and the ubiquitin-related gene USP18 overlapped as top classifiers across datasets. EIF2AK2 is involved in mRNA translation and inflammasome activation (42), NAMPT is a key NAD+ producing gene (43) and NT5C3 is an antiviral pyrimidine nucleotidase (44).

Pathway enrichment and topology analysis identified enrichment in purine, pyrimidine, inositol phosphate, and branched-chain amino acid metabolism in addition to lysine degradation in both BMM and MDM. Metabolic pathways uniquely enriched in BMM included arginine and proline metabolism, steroid biosynthesis, sphingolipid and glycerophospholipid metabolism. (Figure 2.2A, Supplemental Figure S2.2). Metabolic pathways uniquely enriched in MDM included amino sugar and nucleotide sugar metabolism, nicotinate and nicotinamide metabolism, galactose metabolism and fatty acid (FA) metabolism (Figure 2.2B, Supplemental Figure S2.2). 

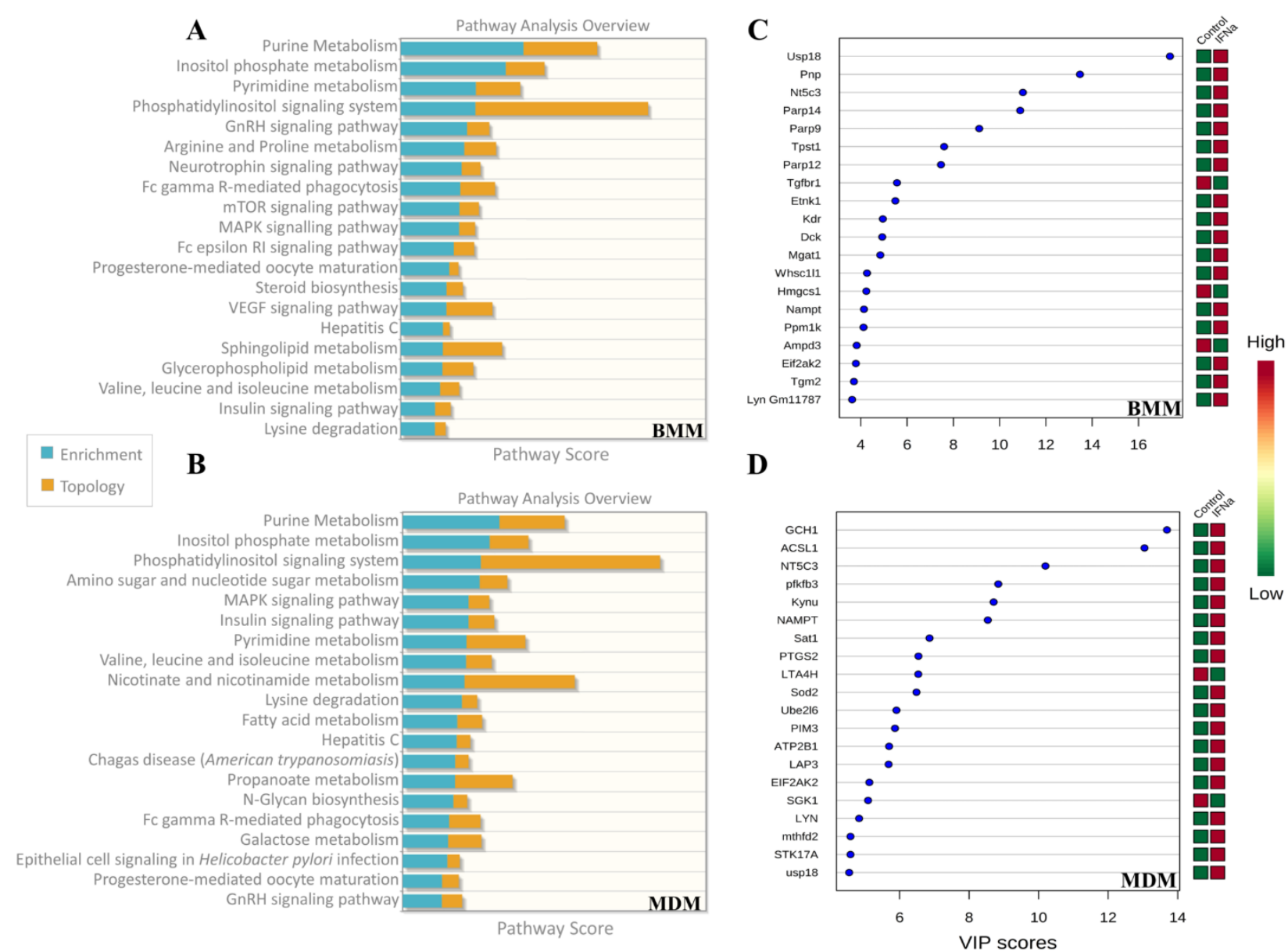

D

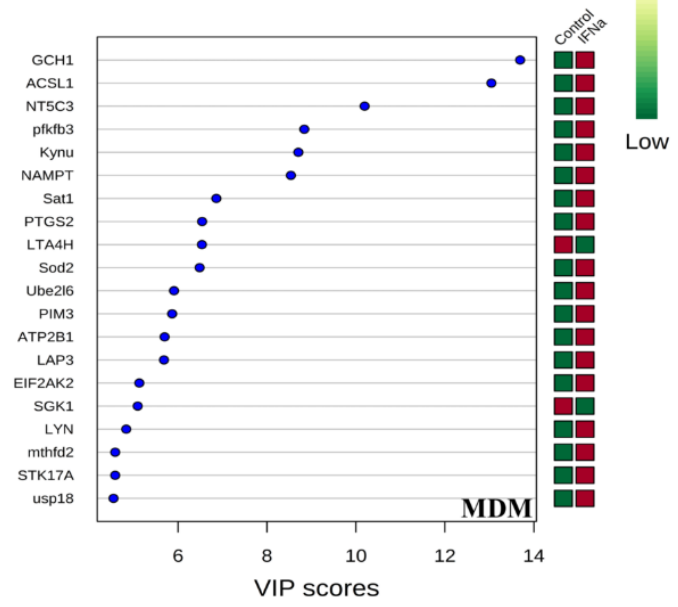

Figure 2.2: Metabolic genes are top classifiers of IFN- $\alpha$ stimulation in BMM and MDM. (A) Pathway enrichment and topology analysis of mouse BMM and human MDM following IFN- $\alpha$ stimulation $(\mathrm{P}<0.05)$. Analyses were performed using all metabolic genes. The blue bars represent enrichment analysis. The yellow bars represent topology scores. (B) Top metabolic classifiers of IFN- $\alpha$ stimulation were identified using Variable Importance in Projection (VIP) Scores based on PLS-DA models $(\mathrm{P}<0.05)$. Analyses were performed using all metabolic genes $(\mathrm{P}<0.05)$. Red and green in the heat map represent up and down regulation of gene expression, respectively. 


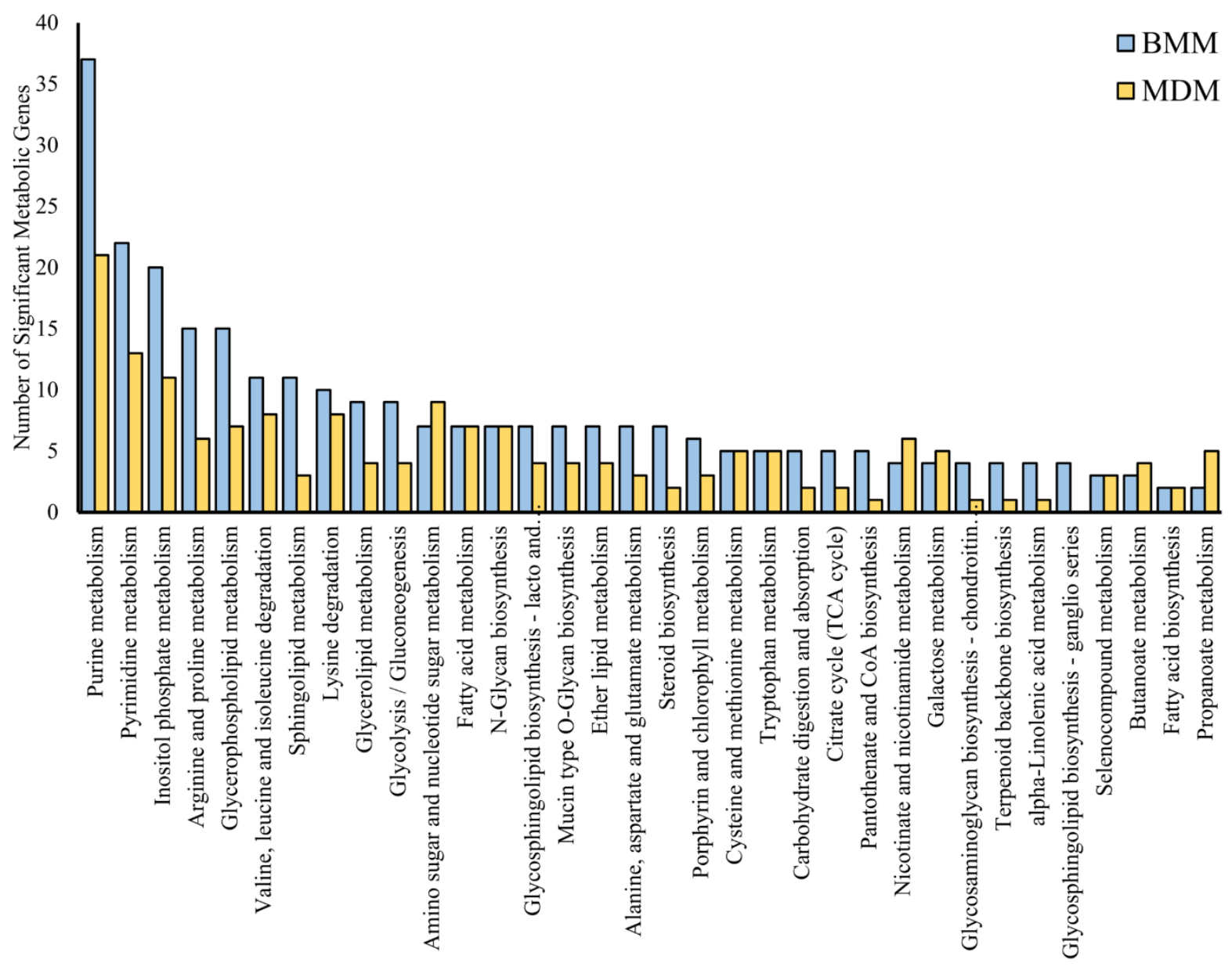

Supplemental Figure S2.2: IFN-a is associated with differential enrichment of metabolic pathways in mouse BMM compared to human MDM. Metabolite set enrichment analysis (MSEA) was performed in Metaboanalyst using metabolic gene datasets. Pathways shown were significantly enriched $(\mathrm{P}<0.05)$ in either IFN-stimulated BMM, MDM or both. Yellow and blue represent enrichment scores in BMM and MDM, respectively (Error bars = s.e.m.; $\mathrm{n}=3$ ). 


\subsubsection{Short term IFN-a stimulation alters genes associated with energy metabolism in BMM and MDM.}

To better functionally characterize differential gene expression in IFN- $\alpha$ stimulated BMM and MDM, altered genes $(-1.2 \leq \mathrm{FC} \geq 1.2$, p-value $\leq 0.05$, FDR $\leq 0.1)$ were mapped to metabolic pathways and networks. Consistent with the literature (20), short-term IFN- $\alpha$ stimulation of BMM was associated with an upregulation of glycolytic genes (HK2, HK3, PGM2, PFKP, PFKFB3, INSR) compared to unstimulated controls (Figure 2.3, Supplemental Figure S2.3). Key genes involved in pyruvate metabolism were also altered in BMM following IFN- $\alpha$ stimulation. Whereas pyruvate dehydrogenase kinase $3(P D K 3)$ was upregulated, pyruvate dehydrogenase phosphatase $1(P D P 1)$ and dihydrolipoamide dehydrogenase $(D L D)$ were downregulated. These alterations may affect the activity of the pyruvate dehydrogenase complex $(P D H)$ and increase lactate production. Consistent with these findings, lactate dehydrogenase $\mathrm{D}(L D H D)$ was also upregulated in IFN- $\alpha$ stimulated BMM. Interestingly, IFN- $\alpha$-stimulated BMMs also upregulated levels of isocitrate dehydrogenase $(I D H 3 A)$ and the downregulation of $D L D$ and dihydrolipoamide Ssuccinyltransferase (DLST) expression. DLD and DLST are key components of oxoglutarate dehydrogenase complex (OGDC) and play an important role in converting 2-oxoglutarate to succinyl-CoA. Along the succinate-fumarate-malate axis, succinate dehydrogenase complex subunit A $(S D H A)$ was downregulated in stimulated compared to unstimulated BMM. SDHA is the major catalytic subunit of the succinate-ubiquinone oxidoreductase. Altered SDHA expression may also have significant effects on oxidative phosphorylation (OXPHOS). 


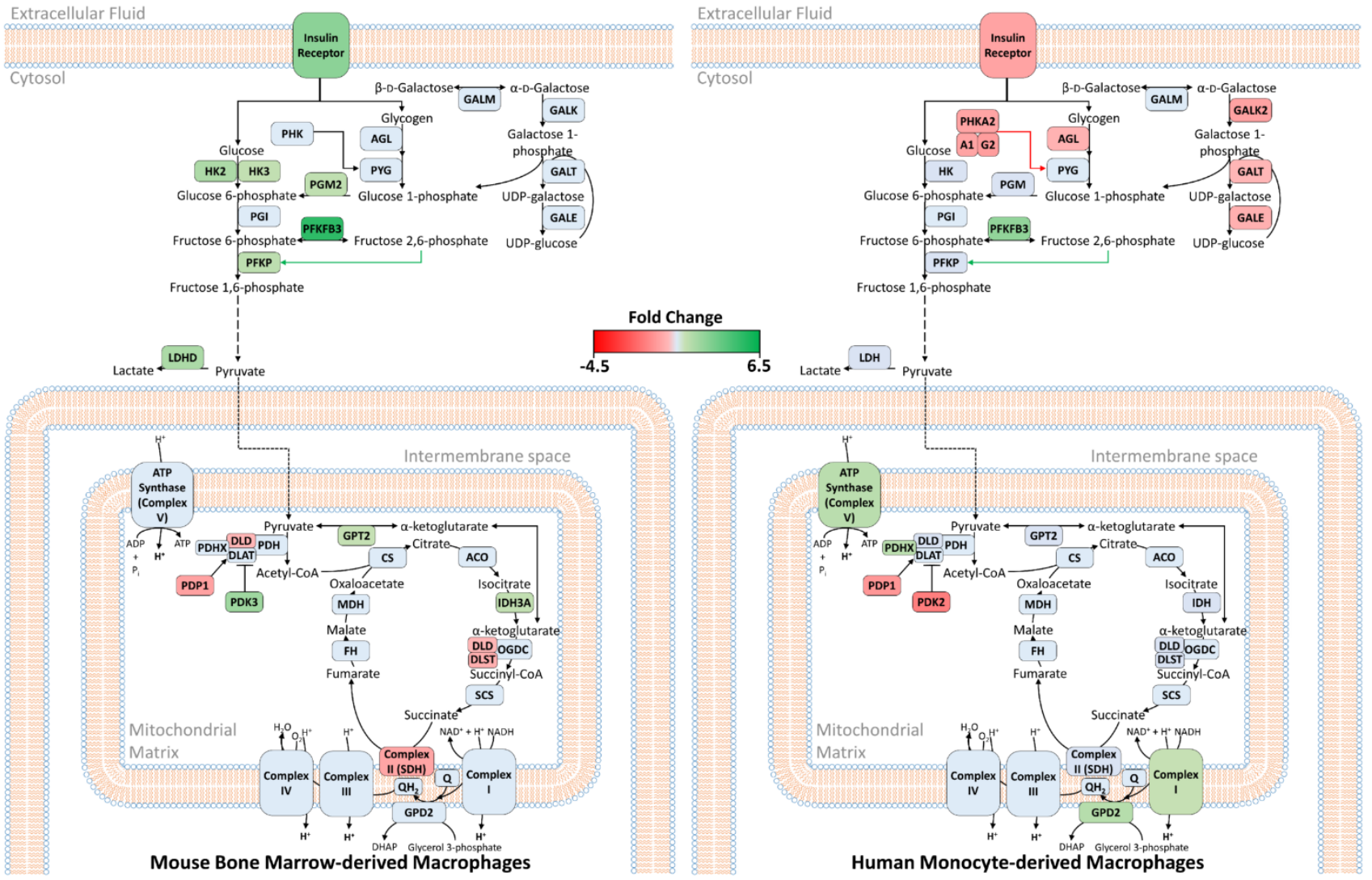

Figure 2.3: Genes associated with bioenergetic processes are differentially expressed in mouse BMM and human MDM following IFN- $\alpha$ stimulation. Significantly altered $(-1.2 \leq$ FC $\geq 1.2$, $p$-value $\leq 0.05$, FDR $\leq 0.1$ ) metabolic genes involved in energy production were mapped to their respective pathways using MetScape and DAVID. Green and red represent genes that have been significantly upregulated or downregulated, respectively. Blue represents genes that were not altered following IFN- $\alpha$ stimulation. 
Mouse BMM
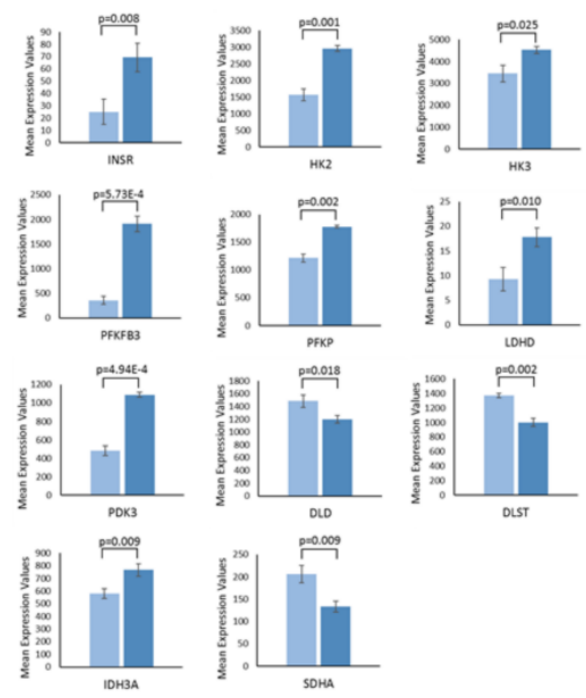
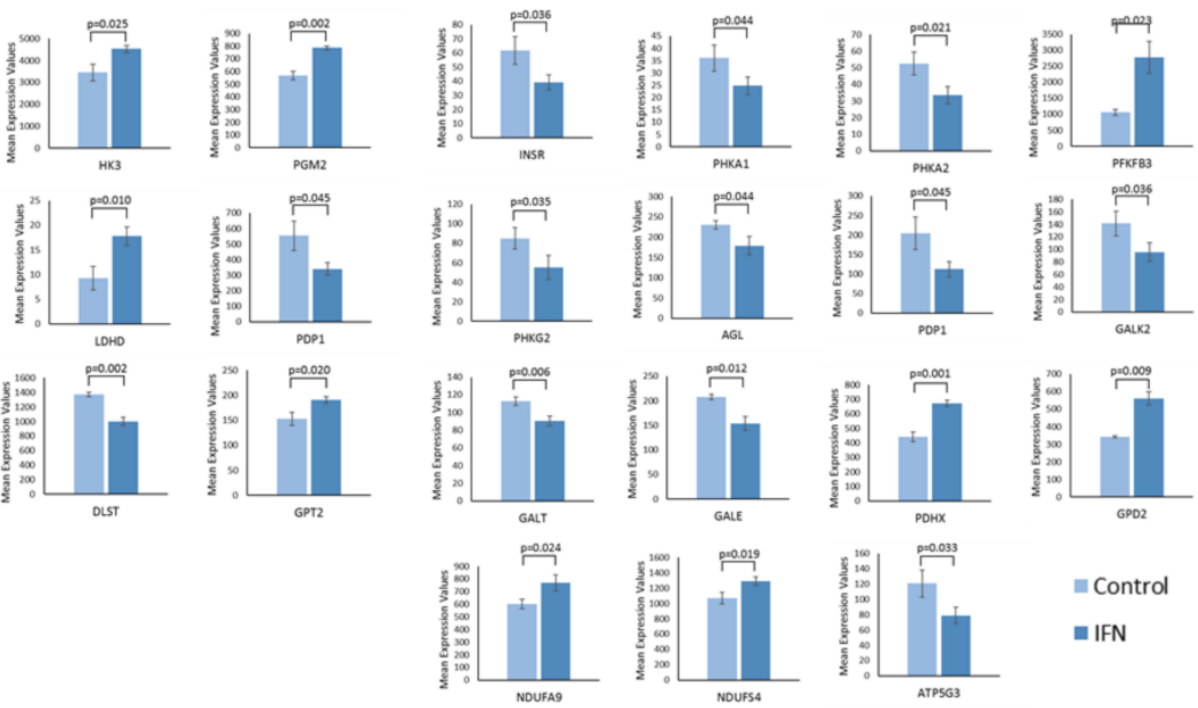

incontrol

a IFN

Supplemental Figure S2.3: IFN responses are associated with altered bioenergetic profiles in mouse and human macrophages. The bar plots show significantly altered genes associated with bioenergetics pathways $(\mathrm{FC}>1.2, \mathrm{p}<0.05$, FDR $<0.10)$. Light blue and dark blue represent expression levels in unstimulated (control) and IFN- $\alpha$ treated macrophages, respectively (Error bars $=$ s.e.m.; $\mathrm{n}=3$ ). 
Short term IFN- $\alpha$ stimulation of MDM was not associated with significant alterations in glycolytic genes or genes linked to lactate production (Figure 2.3, Supplemental Figure S2.3). Alternatively, stimulation was associated with the downregulation of genes associated with the conversion of galactose to glucose (GALK2, GALT, GALE) and glycogen breakdown (INSR, PHKA1, PHKA2, PHKG2, AGL). Early responses in MDM were associated with increased levels of phosphofructokinase (PFK) activator $P F K F B 3$, which assists in the production of pyruvate from glucose and pyruvate dehydrogenase complex component $\mathrm{X}(P D H X)$, which may facilitate acetylCoA production from pyruvate. IFN- $\alpha$ was also associated with the upregulation of genes associated with OXPHOS including genes from Complex I and V of the electron transport chain (NDUFA9, NDUFS4, ATP5G3) and the glycerol phosphate shuttle (glycerol 3-phosphate dehydrogenase $2[G P D 2])$. Collectively, these results suggest early changes in energy metabolism may play an important role in the initiation of antiviral responses in both BMM and MDM.

\subsubsection{IFN- $\alpha$ stimulated BMM and MDM show signs of alterations in genes associated with redox regulation.}

Given the link between energy metabolism and ROS metabolism (45-47), I examined alterations between early IFN- $\alpha$ responses and genes linked to cellular redox status (oxidant and antioxidant genes). In BMM, IFN- $\alpha$ short term stimulation was associated with altered expression of genes associated with the nitric oxide cycle including the upregulation of argininosuccinate synthetase 1 (ASS1) and nitric oxide synthase 1 (NOS1), and down-regulation of arginase 2 (ARG2)

expression, which may favour flux of arginine towards NO production (Figure 2.4, Supplemental Figure S2.4). Early IFN- $\alpha$ responses in BMM were also associated with the downregulation of genes associated with the antioxidant response (superoxide dismutase 2 (SOD2), glutamatecysteine ligase, catalytic subunit $(G C L C)$, NAD kinase $(N A D K)$, thioredoxin reductase 1 and 3 
$(T X N R D 1, T X N R D 3)$ and the upregulation of thioredoxin interacting protein (TXNIP), which inhibits the antioxidant activity of thioredoxin $(48,49)$.

Alternatively, IFN- $\alpha$ stimulation of MDM was associated with the upregulation of antioxidant genes including $S O D 2$ and myeloperoxidase $(M P O)$ as well as genes associated with glutathione production (glutamate-cysteine ligase $(G L C M)$ and $N A D K)$ (Figure 2.4, Supplemental Figure S2.4). Short term IFN- $\alpha$ was also associated with upregulation of TXNIP, glutaredoxin (GLRX), and thioredoxin 1 (TXNI). These alterations may help regulate electron linkage and subsequent ROS production associated with the upregulation of genes associated with OXPHOS in these cells (Figures 2.3 and 2.4). 


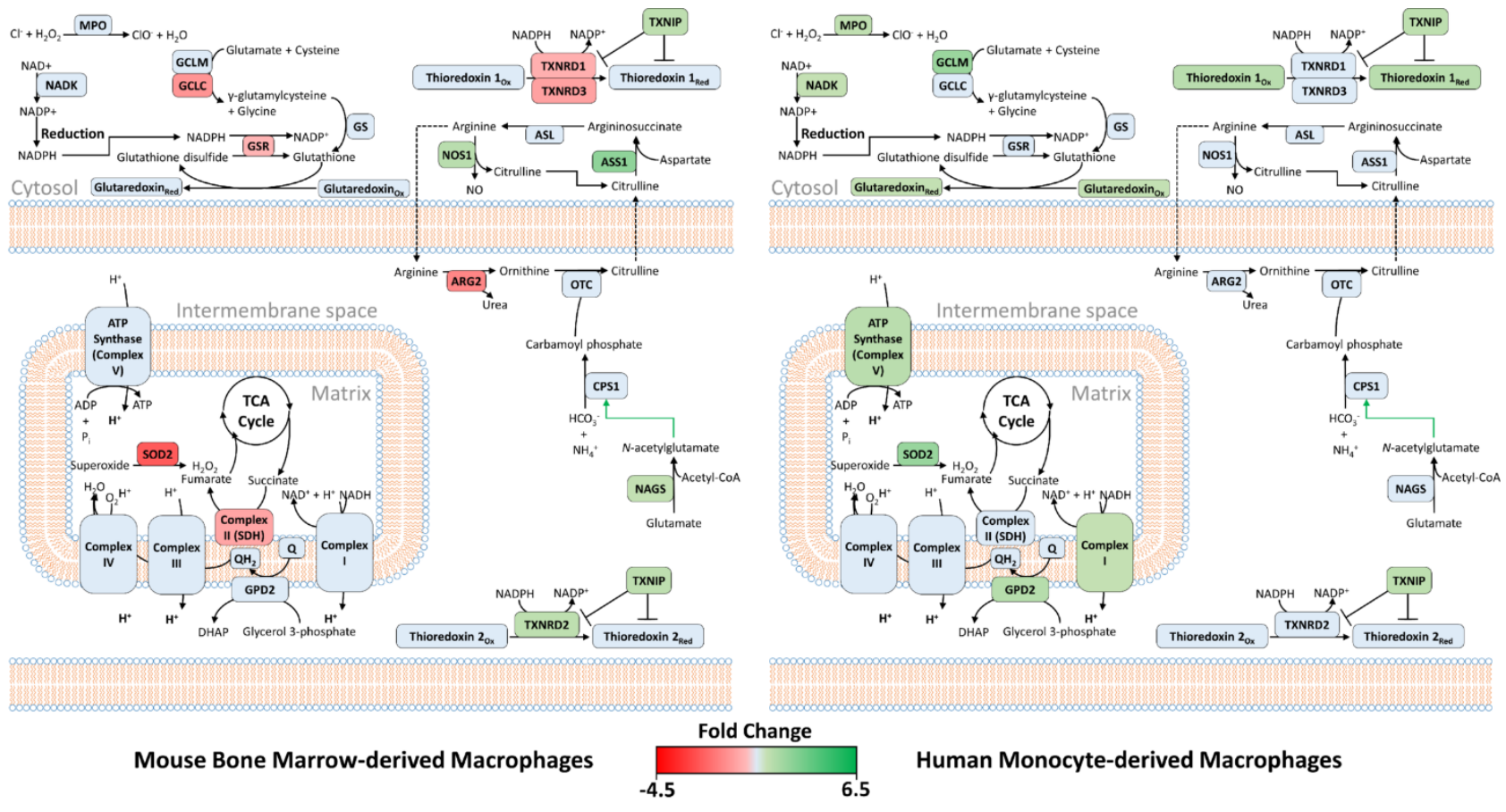

Figure 2.4: IFN- $\alpha$ stimulation of MDM is associated with increased expression of genes associated with ROS production and antioxidant responses. Differentially expressed metabolic genes $(-1.2 \leq \mathrm{FC} \geq 1.2$, $\mathrm{p}$-value $\leq 0.05$, FDR $\leq 0.1)$ were mapped to pathways associated with cellular redox status using MetScape and DAVID. Green and red represent genes that have been significantly upregulated or downregulated, respectively. Blue represents genes that were not altered following IFN- $\alpha$ stimulation. 
Mouse BMM
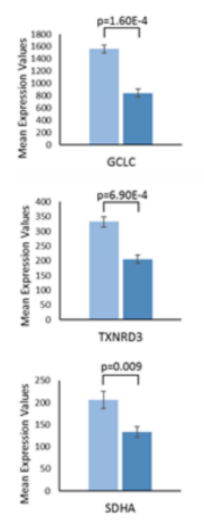
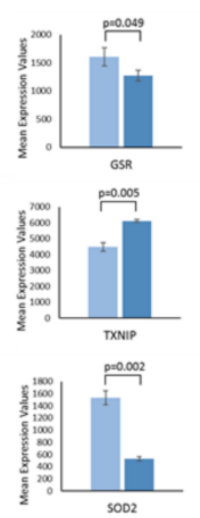
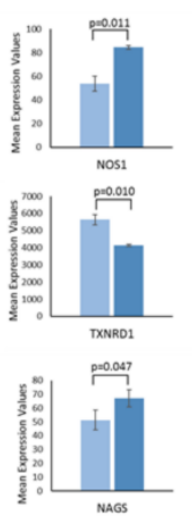
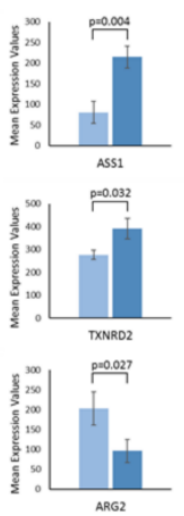
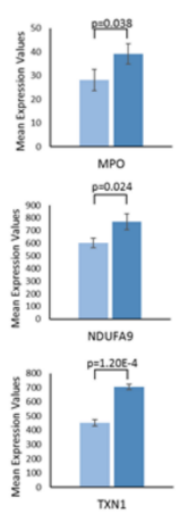

Human MDM
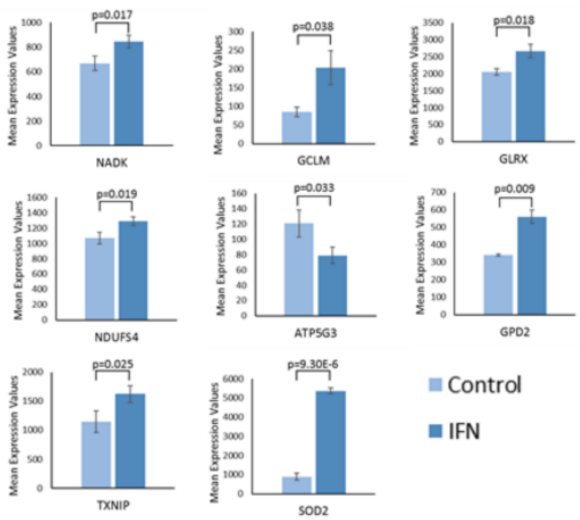

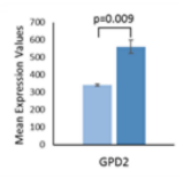

Control

Supplemental Figure S2.4: BMM and MDM express redox-related genes following short term IFN- $\alpha$ stimulation. The bar plots show significantly altered genes associated with cellular redox pathways $(\mathrm{FC}>1.2, \mathrm{p}<0.05, \mathrm{FDR}<0.10)$. Light blue and dark blue represent expression levels in unstimulated (control) and IFN- $\alpha$ treated macrophages, respectively (Error bars = s.e.m.; n = 3). 


\subsubsection{Early type I IFN responses are associated with alterations in genes associated with cAMP and cGMP production.}

Given the enrichment of gene sets associated with nucleotide metabolism in both data sets, I examined the specific effects on short term IFN- $\alpha$ stimulation on purine and pyrimidine metabolism. In BMM, short-term stimulation was associated with the downregulation of amidophosphoribosyltransferase (PPAT) and UMP synthetase (UMPS) (Figure 2.5, Supplemental Figure S2.5). These enzymes play a central role in ribose 5-phosphate incorporation during de novo purine and pyrimidine synthesis, which may represent an antiviral mechanism. At the level of purine degradation, IFN- $\alpha$ was associated with an upregulation of purine nucleoside phosphorylase $(P N P)$, guanine deaminase $(G D A)$, xanthine dehydrogenase $(X D H)$, ectonucleoside triphosphate diphosphohydrolase 2 and 5 (ENTPD2, ENTPD5) suggesting increased degradation. Interestingly, IFN responses were also associated with alterations in genes that regulate cyclic guanine monophosphate (cGMP)/GMP and cyclic adenosine monophosphate (cAMP)/AMP ratios

(Figure 2.5, Supplemental Figure S2.5). Four phosphodiesterases (PDE4D, PDE7A, PDE7B, $P D E 8 B)$ and two adenylate cyclases $(A D C Y 2, A D C Y 4)$ were upregulated and adenylate kinase $(A D K)$ and AMP deaminase $3(A M P D 3)$ were downregulated in stimulated $v s$. unstimulated cells. These profiles suggest IFN- $\alpha$-activated BMM may accumulate both AMP and cAMP. IFN- $\alpha$ stimulation of BMM was also associated with the upregulation of guanylate kinase $(G U K)$ and down-regulation of phosphodiesterase 1B $(P D E 1 B)$, suggesting these cells may favour cGMP production 


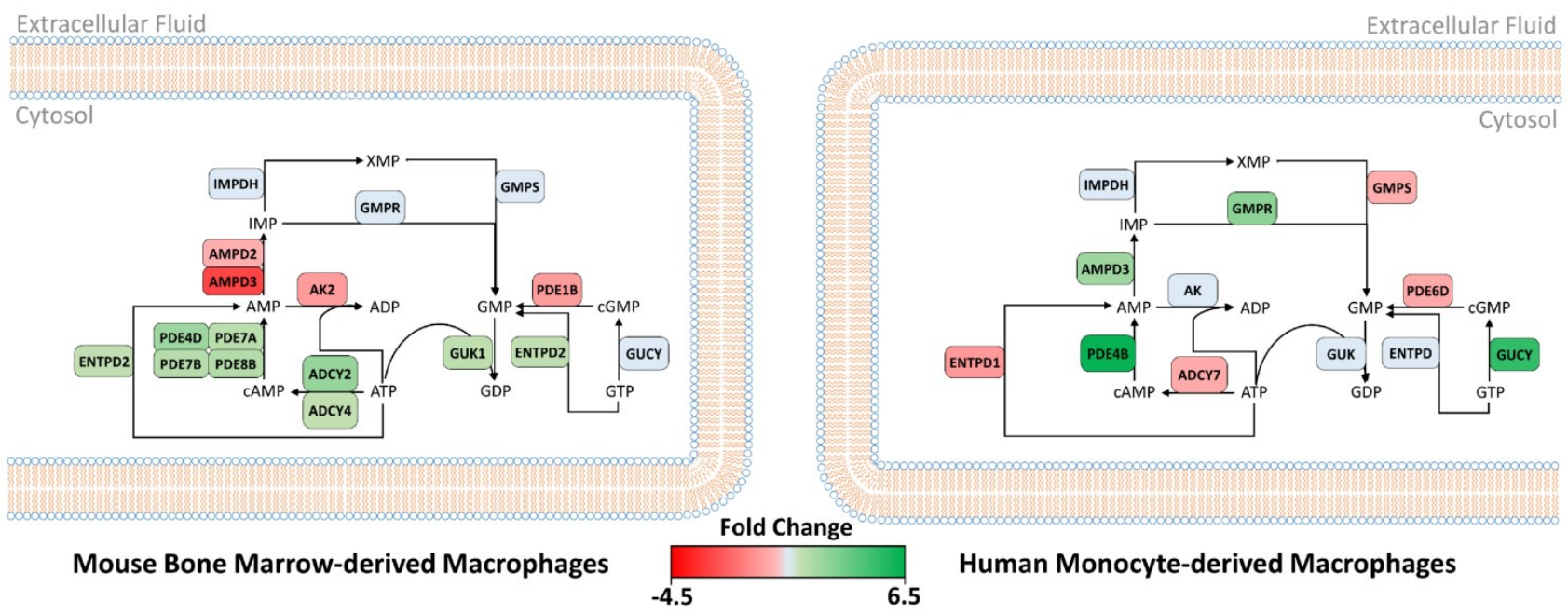

Figure 2.5: Type I IFN responses are associated with altered cAMP and cGMP production in BMM and MDM. Metabolic genes identified as significantly altered $(-1.2 \leq \mathrm{FC} \geq 1.2$, $\mathrm{p}$-value $\leq 0.05$, FDR $\leq 0.1$ ) were mapped to pathways associated with AMP and GMP production using MetScape and DAVID. Green and red represent genes that have been significantly upregulated or downregulated, respectively. Blue represents genes that were not altered following IFN- $\alpha$ stimulation. 
Mouse BMM
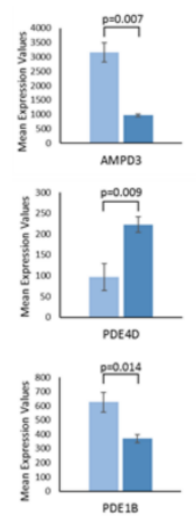
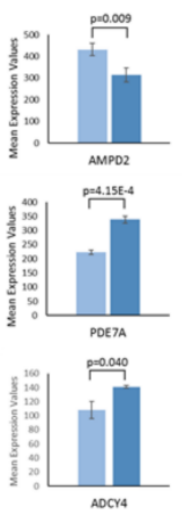
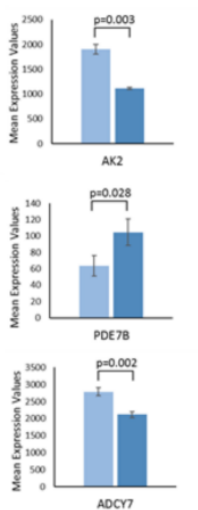
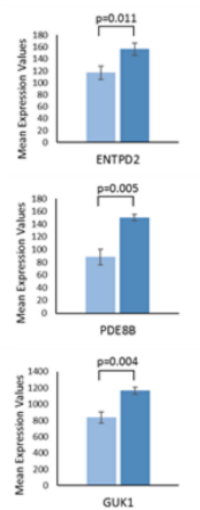

Human MDM
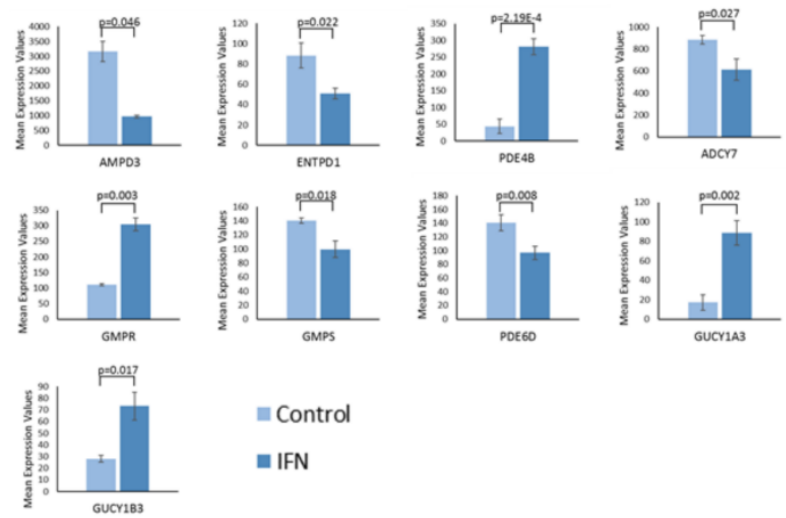

= Control

a IFN

Supplemental Figure S2.5: IFN- $\alpha$ is associated with altered expression of genes that regulate nucleotide metabolism and cAMP/cGMP ratios. The bar plots show significantly altered genes associated with nucleotide metabolism $(\mathrm{FC}>1.2, \mathrm{p}<0.05$, FDR $<0.10)$. Light blue and dark blue represent expression levels in unstimulated (control) and IFN- $\alpha$ treated macrophages, respectively (Error bars = s.e.m.; $\mathrm{n}=3$ ). 
In MDM, IFN- $\alpha$ stimulation was not associated with significant changes in genes associated with de novo purine synthesis. However, IFN- $\alpha$ was associated with the downregulation of carbamoyl-phosphate synthetase 2, aspartate transcarbamylase and dihydroorotase $(C A D)$, a protein responsible for the first three enzymatic steps of pyrimidine biosynthesis pathway (Figure 2.5, Supplemental Figure S2.5). Genes involved in nucleoside production within the purine and pyrimidine degradation pathways, such as GMP reductase (GMPR), AMPD3, 5 '-nucleotidase, cytosolic II (NT5C2), adenosine deaminase $(A D A)$, are found to be up-regulated in MDM which suggest an increase in nucleotide salvaging (Figure 2.5, Supplemental Figure S2.5). At the level of cGMP/GMP and cAMP/AMP regulation, the downregulation of $P D E 6 D$ and adenylate cyclase $7(A D C Y 7)$ as well as the upregulation of $P D E 4 B$ and two soluble forms of guanylate cyclase (GUCY1A3, GUCY1B3) suggest a shift towards cGMP and AMP production. Varied expression of these bioactive nucleotides that function as intracellular secondary messengers may contribute to early type I IFN responses.

\subsubsection{Short term IFN- $\alpha$ stimulation is associated with alterations in tryptophan and branched- chain amino acid catabolism in BMM and MDM.}

Consistent with the literature $(50,51)$, alterations in genes associated with tryptophan and branched-chain amino acid catabolism were pronounced in early IFN- $\alpha$ responses. Both BMMs and MDMs exhibited a pronounced up-regulation of genes associated with tryptophan catabolism via the kynurenine pathway (Figure 2.6, Supplemental Figure S2.6). While IFN- $\alpha$ stimulated BMM upregulated tryptophan 2,3-dioxygenase (TDO2) and $K M O$, stimulation of MDM was associated with the upregulation of $K M O$ and kynureninase $(K Y N U)$. This shift in tryptophan catabolism was accompanied by the upregulation of nicotinamide phosphoribosyltransferase $(N A M P T)$, suggesting an increased flux of tryptophan towards $\mathrm{NAD}^{+}$production. 


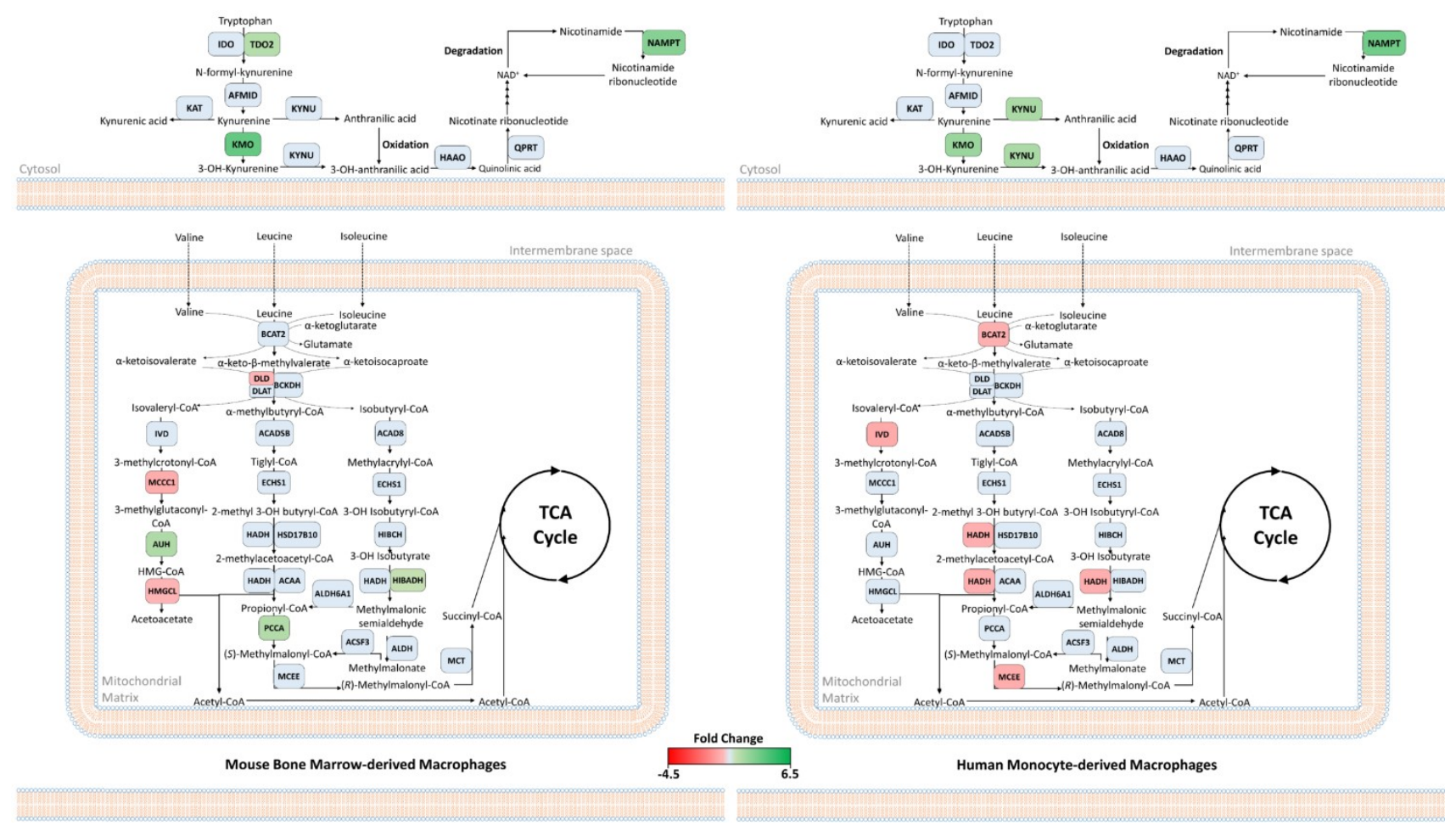

Figure 2.6: Tryptophan and branched-chain amino acid catabolism is altered in BMM and MDM following short-term IFN- $\alpha$ treatment. Metabolic genes altered in IFN- $\alpha$ stimulated cells compared to controls $(-1.2 \leq \mathrm{FC} \geq 1.2$, $\mathrm{p}$-value $\leq 0.05$, FDR $\leq 0.1)$ were mapped to amino acid metabolism pathways using MetScape and DAVID. Green and red represent genes that have been significantly upregulated or downregulated, respectively. Blue represents genes that were not altered following IFN- $\alpha$ stimulation. 


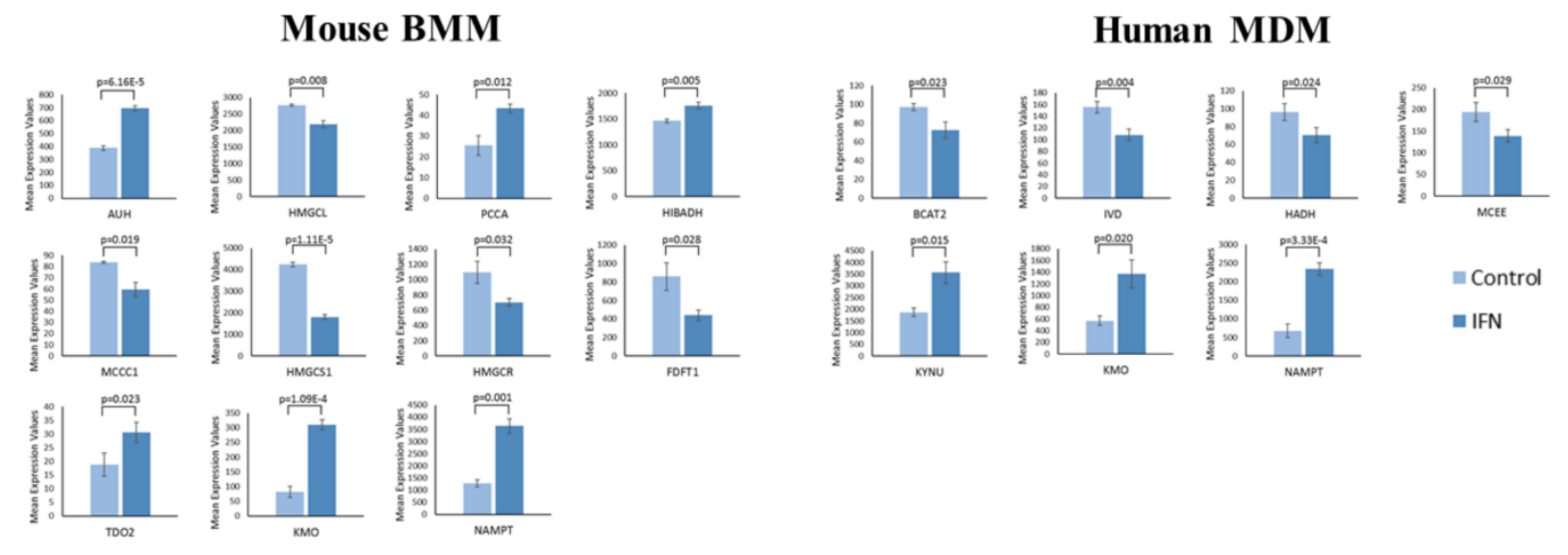

Supplemental Figure S2.6: Short term IFN- $\alpha$ stimulation is associated with alterations in tryptophan and branched chain amino acid catabolism. The bar plots show significantly altered genes associated with tryptophan and branched chain amino acid metabolism $(\mathrm{FC}>1.2, \mathrm{p}<0.05$, FDR $<0.10$ ). Light blue and dark blue represent expression levels in unstimulated (control) and IFN- $\alpha$ treated macrophages, respectively (Error bars $=$ s.e.m.; $n=3$ ). 
Both IFN- $\alpha$-activated BMM and MDM showed altered expression of genes associated with branched-chain amino acid (Figure 2.6, Supplemental Figure S2.6). In BMMs, IFN- $\alpha$ stimulation leads to the upregulation of genes associated with isoleucine (propionyl-CoA carboxylase; $P P C A$, 3-ketoacyl-CoA thiolase 1A and 2; ACAA1A/2) and valine (3-hydroxyisobutyrate dehydrogenase; $H I B A D H)$ catabolism. Upregulation of AU-RNA binding/methylglutaconyl-CoA hydratase $(A U H)$ and the downregulation of methylcrotonoyl-CoA carboxylase 1 (MCCC1) and 3hydroxymethyl-3-methylglutaryl-CoA lyase $(H M G C L)$ suggest decreased leucine catabolism in BMM. However, AUH may be functioning in its secondary role in promoting mRNA degradation (52). In MDM, IFN- $\alpha$ stimulation was associated with a downregulation of multiple genes associated with branched chain amino acids catabolism including branched-chain aminotransferase 2 (BCAT2), isovaleryl-CoA dehydrogenase (IVD), hydroxyacyl-CoA dehydrogenase $(H A D H)$, and methylmalonyl-CoA epimerase (MCEE). Together, this indicates that alterations in branched-chain amino acid catabolism may be key to driver of early IFN- $\alpha$ responses in primary macrophage systems.

\subsubsection{IFN- $\alpha$ is associated with altered lipid metabolism in BMM and MDM.}

Lipid metabolism has been shown to play an important role in antiviral responses in BMM and MDM (53). Several studies have reported alterations in cholesterol metabolism during IFN and antiviral responses $(22,23,54)$. Here, I also identified alterations in genes associated with phospholipid and sphingolipid metabolism and FA biosynthesis following short-term IFN responses. Consistent with previous studies, short term IFN- $\alpha$ stimulation of BMM and MDM was associated with the downregulation of genes associated with de novo cholesterol synthesis (Figure 2.7, Supplemental Figure S2.7). In BMM, IFN- $\alpha$ was associated with the downregulation of genes involved in mevalonate synthesis (HMGCS1, HMGCR), lanosterol synthesis (FDFT1, SQLE, LSS) 
and cholesterol synthesis (CYP51, MSMO1, HSD17B7, SC5D). It was also associated with the downregulation of genes associated with cholesterol ester formation and the upregulation of carboxyl ester lipase $(\mathrm{CEL})$, cholesterol 25-hydroxylase $(\mathrm{CH} 25 \mathrm{H})$ and sterol 27-hydroxylase (CYP27A1). In MDM, IFN- $\alpha$ stimulation was associated with decreased levels of SQLE and sterol O-acyltransferase 1 (SOATI) and increased levels of $\mathrm{CH} 25 \mathrm{H}$ (Figure 2.7, Supplemental Figure S2.7). SQLE catalyzes the first oxygenation step in sterol biosynthesis and is thought to be a ratelimiting enzyme of this process (55).

At the level of phospholipid and sphingolipid metabolism, IFN- $\alpha$ treated BMM upregulated phospholipid phosphatase $2(P L P P 2)$ and neutral ceramidase (ASAH2) and downregulated sphingolipid kinases (SPHK2, CERK) suggesting a shift away from phosphorylated sphingolipids to sphingosine in acute IFN responses (Figure 2.7, Supplemental Figure S2.7). Stimulation of BMM was also associated with the upregulation of 1-acylglycerol-3-phosphate O-acyltransferase 1 (AGPATl) and phosphatidate cytidylyltransferase 1 (CDS1), which may increase cytidine diphosphate (CDP)-diacylglycerol production, a precursor for phosphatidylinositol, phosphatidylglycerol, and cardiolipin synthesis. Phosphatidylinositol is a minor component on the cytosolic side of cell membranes and cardiolipin is an important component of the inner mitochondrial membrane (56). Consistent with these findings, IFN- $\alpha$ stimulation was associated with the upregulation of three different phospholipase A2 (PLA2G2D, PLA2G4A, PLA2G16) genes and the downregulation of two phospholipase $\mathrm{D}(P L D 1, P L D 2)$ genes. These genes cleave phosphatidylcholine and phosphatidylethanolamine, which represent the major phospholipids found in mammalian membranes. The relative ratio of these lipids to one another within the cell membrane has significant implications on membrane integrity (56). In MDM, PLA2G4A and sphingomyelin synthase 1 ( $S G M S 1)$ were upregulated and lysophospholipid acyltransferase 
(LPCAT4) and PLA2G15 were downregulated following IFN- $\alpha$ stimulation (Figure 2.7, Supplemental Figure S2.7). IFN- $\alpha$ stimulation was also associated with the downregulation of AGPAT5, choline kinase alpha (CHKA) and ethanolamine kinase 1 (ETNK1), which play an important role in the synthesis of phosphatidylglycerol, phosphatidylcholine and phosphotidylethanolamine. Collectively, these alterations suggest IFN- $\alpha$ responses may alter the composition of the plasma and mitochondrial membranes of BMM and MDM as part of early type I IFN responses.

Finally, at the level of fatty acid synthesis, IFN- $\alpha$ stimulation of BMM was also associated with the downregulation of FA synthase $(F A S)$ and FA desaturase $1(F A D S 1)$ as well as the upregulation of carnitine palmitoyltransferase 1A (CPT1A). Alternatively, in MDM, IFN- $\alpha$ was associated with the upregulation of long-chain fatty acid (LCFA)-producing aldehyde dehydrogenase 3b1 (ALDH3B1) and three acyl-CoA synthetase long-chain genes (ACSL1, ACSL5, $A C S L 6)$ and the downregulation of carbonyl reductase 4 (CBR4), acetyl-CoA carboxylase- $\alpha$ $(A C A C A)$, mitochondrial 3-oxoacyl-ACP synthase (OXSM), trimethylysine hydrolase $\varepsilon(T M L H E)$ and hydroxyacyl-CoA dehydrogenase $(H A D H)$. Differences in fatty acid metabolism may indicate differential dependencies of BMM and MDM on $\beta$-oxidation for energy production. 


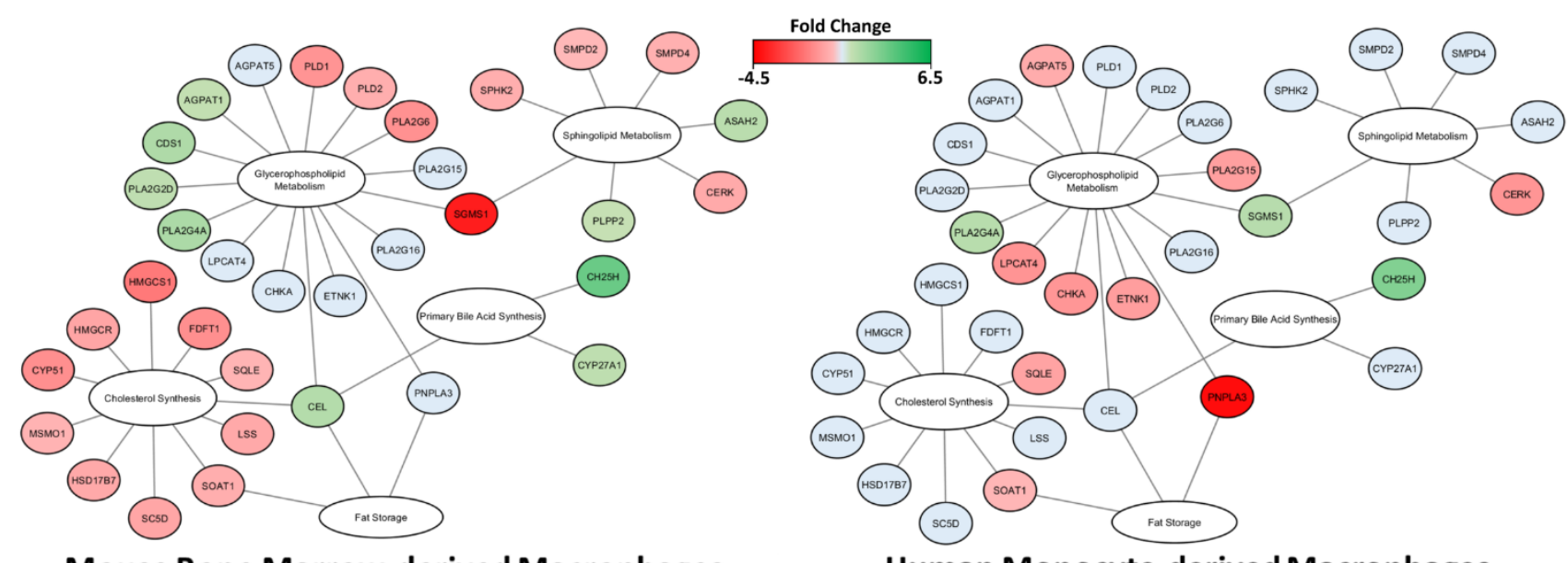

Mouse Bone Marrow-derived Macrophages

Figure 2.7: Expression of genes associated with lipid metabolism were differentially modulated in IFN-a stimulated BMM compared MDM. Differentially expressed metabolic genes $(-1.2 \leq \mathrm{FC} \geq 1.2$, p-value $\leq 0.05$, FDR $\leq 0.1)$ involved in cholesterol metabolism and phospholipid and sphingolipid synthesis were mapped using MetScape and DAVID. Green and red represent genes that have been significantly upregulated or downregulated, respectively. Blue represents genes that were not altered following IFN- $\alpha$ stimulation. 


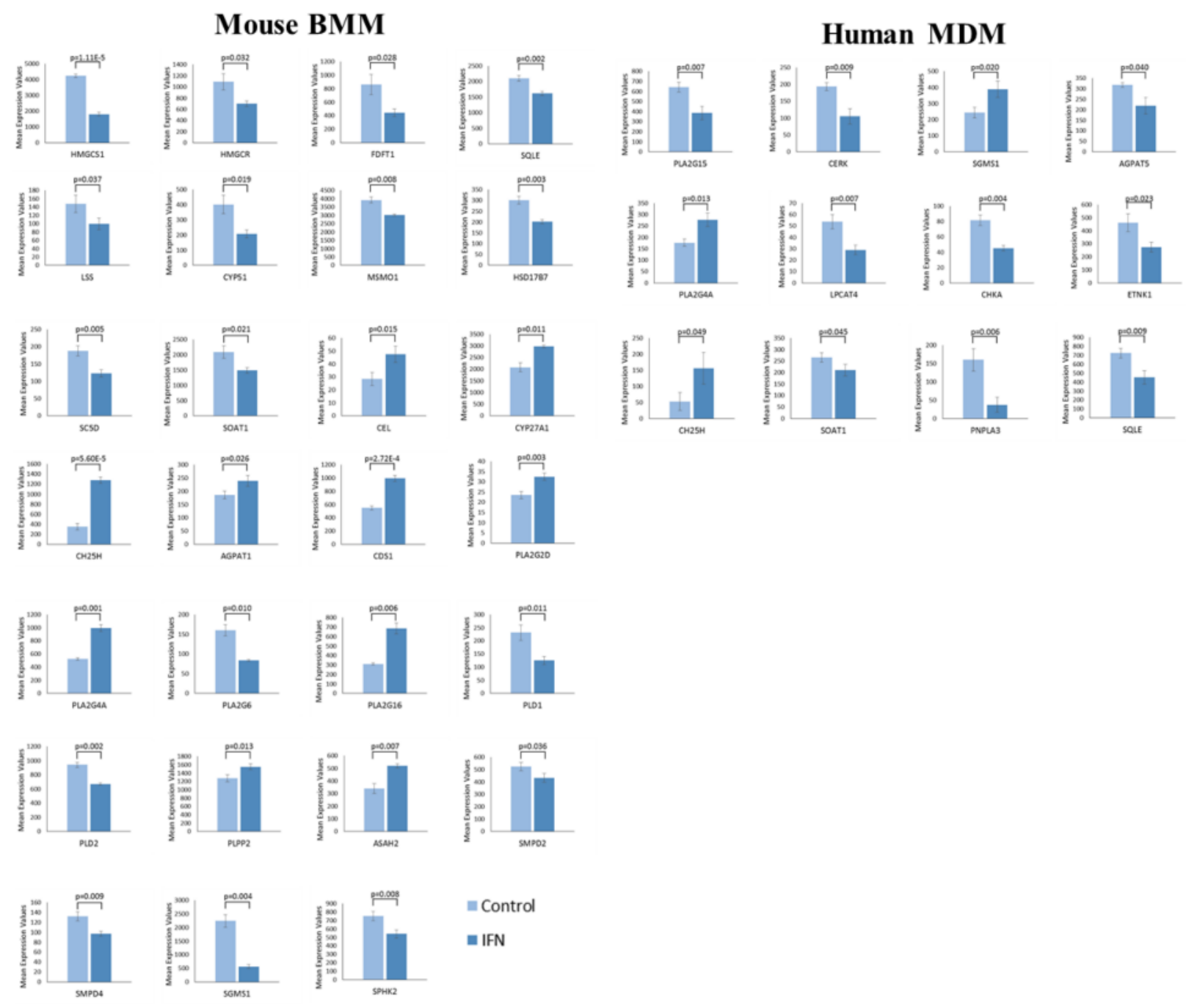

Supplemental Figure S2.7: Altered expression of genes associated with lipid metabolism is a key feature of Type I IFN responses. The bar plots show significantly altered genes associated with lipid metabolism $(\mathrm{FC}>1.2, \mathrm{p}<0.05, \mathrm{FDR}<0.10)$. Light blue and dark blue represent expression levels in unstimulated (control) and IFN- $\alpha$ treated macrophages, respectively (Error bars = s.e.m.; $\mathrm{n}=3)$. 


\subsection{Discussion}

In the current study, I used publicly available transcriptional profiling datasets to develop metabolic gene signatures associated with short term IFN- $\alpha$ stimulation in mouse and human macrophage models. Enrichment analysis, pathway mapping and network construction identified alterations in central metabolic pathways in early type I IFN responses including glycolysis, oxidative phosphorylation, redox regulation, nucleotide metabolism, amino acid catabolism, and lipid metabolism. BMM had increased expression of genes associated with aerobic glycolysis, nitric oxide production, branched-chain amino acid metabolism and fatty acid $\beta$-oxidation as well as decreased expression of genes associated with cholesterol biosynthesis. MDM had increased expression of genes associated with increased OXPHOS activity and antioxidant production and decreased expression of genes associated with branched-chain amino acid catabolism and fatty acid $\beta$-oxidation. While the current study only examines alterations in gene expression, these findings suggest that metabolic rewiring, at the level of transcription, is a key feature of early type I IFN responses. Future studies are required to validate the identified gene signatures and to validate the biological relevance of these alterations during early antiviral immune responses.

A number of studies have reported increased aerobic glycolysis and reduced oxidative phosphorylation in macrophages following activation with inflammatory stimuli (57-60). Consistent with the literature, short term IFN- $\alpha$ stimulation of BMM was associated with increased expression of genes associated with glycolysis (HK2, HK3, PGM2, PFKP, PFKFB3, INSR) and lactate production $(L D H D)$ and decreased expression of genes associated with pyruvate production $(P D K 3, P D P 1, D L D)$ and flux through the TCA cycle (DLD, DLST, $S D H A)$. In addition to meeting bioenergetic requirements of the cells, these alterations may increase intracellular levels of bioactive metabolites such as D-lactate, $\alpha$-ketoglutarate, and succinate. Lactate accumulation in 
the microenvironment has been shown to suppress cytokine production and migration of human cytotoxic $\mathrm{T}$ cells $(61,62)$. Similarly, $\alpha$-ketoglutarate has been shown to quell inflammatory processes by suppressing NF-kB-mediated inflammatory pathways (63). Succinate can also modulate inflammatory cytokine production. A recent study found that succinate stabilizes HIF$1 \alpha$ expression in LPS-activated macrophages (14), which facilitates HIF-1 $\alpha$ transport into the nucleus where it induces the expression of glycolytic targets such as $L D H A, H K 2$, and PKM2, as well as inflammatory genes such as $I L 1 B(64,65)$. Inhibition or decreased expression of succinate dehydrogenase (SDH) has been shown to promote IL-10 expression and to repurpose the mitochondria for ROS production (19). While functional studies are required to validate these profiles, our findings suggest bioenergetic reprogramming of BMM may represent a feedforward mechanism that may contribute to the immunomodulatory properties of type I IFNs during early antiviral immune responses.

Unlike BMM, short term IFN- $\alpha$ stimulation of MDM was associated with the upregulation of a range of genes associated with the electron transport chain, which may favour OXPHOS for energy production. While OXPHOS provides more ATP per glucose molecule compared to aerobic glycolysis, energy and metabolic precursor production occurs more slowly. Further, IFN$\alpha$ stimulation of MDM was associated with the downregulation of genes associated with the Leloir pathway (GALK2, GALT, GALE), which is responsible for the conversion of galactose to glucose. Recent studies have shown that $\mathrm{T}$ cells, but not $\mathrm{B}$ cells, can be activated and proliferate in presence of galactose when glucose is absent $(66,67)$. However, unlike activation in glucose rich environments, $\mathrm{T}$ cells in galactose are forced to rely on OXPHOS for energy production, which occurs at significantly slower rates (66). This reliance on galactose also results in suboptimal IFN$\gamma$ and IL-2 production suggesting galactose should only be used when no other energy substrate is 
available (66). Thus, decreased expression of genes associated with the Leloir pathway in MDM may represent a means by which cells can improve the efficiency of energy production while maintaining functional immune responses.

Transcriptional profiling also identified redox regulation as a key feature of early IFN- $\alpha$ responses in mouse and human primary macrophage models. In BMM, IFN- $\alpha$ reprogramming was associated with increased expression of genes associated with nitric oxide (NO) production ( $A S S 1$, $N O S 1, N A G S)$ and decreased antioxidants (SOD2, GCLC, TXNRD1, TXNRD3). NO is a potent antimicrobial molecule that has been shown to modulate cellular metabolism (68-71) and immune function (72). ASS1 and ASL are part of the aspartate-argininosuccinate shunt, which recycles citrulline to resynthesize arginine for prolonged NO production $(73,74)$. In M1 macrophages, increased expression of ASS1 and the subsequent increased flux through this shunt has been shown to replenish TCA cycle intermediates following decreased $I D H$ and $S D H$ gene expression (73). Unlike BMM, IFN- $\alpha$ stimulated MDM had increased expression of genes associated with OXPHOS and reactive oxygen species (ROS) production. ROS are also potent antimicrobial molecules, capable of killing intracellular pathogens (75). The matched upregulation of antioxidant genes (e.g., glutathione, glutaredoxin, thioredoxin) in conjunction with ROS, likely reflects a protective mechanism to limit any associated cellular damage. Interestingly, a recent study found that the reducing nature of glutathione can prime $\mathrm{T}$ cell inflammatory responses by promoting mTOR-activated metabolic reprogramming (76). It is currently unclear if similar priming occurs in macrophages. Collectively, our data suggest mouse BMM and human MDM may adopt differential metabolic strategies to mount intracellular antimicrobial responses during acute IFN responses. 
Pathway mapping and network reconstruction identified IFN- $\alpha$-associated alterations in nucleotide metabolism. In both BMM and MDM, IFN- $\alpha$ stimulation was associated with a downregulation of genes associated with de novo pyrimidine biosynthesis. A number of viruses including human cytomegalovirus and herpes simplex viruses require de novo pyrimidine synthesis for propagation and survival $(77,78)$. Furthermore, inhibitors of de novo pyrimidine biosynthesis have broad antiviral effects against RNA, DNA, and retroviruses such as influenza A, hepatitis C, human adenovirus, and human immunodeficiency virus (HIV) $(79,80)$. In MDM, IFN- $\alpha$ stimulation was also associated with increased expression of genes associated with purine and pyrimidine degradation pathways and nucleotide salvaging. The induction of nucleotide degradation pathways may act as a counterstrategy against viral-driven nucleotide biosynthesis (81). Moreover, the activation of nucleotide salvaging pathways may allow the cell to recycle degraded bases and nucleosides and produce nucleotides to maintain cellular function. Interestingly, IFN- $\alpha$ responses were also associated with alterations in genes that regulate cyclic guanine monophosphate (cGMP)/GMP and cyclic adenosine monophosphate (cAMP)/AMP ratios. Cyclic nucleotide second messengers, including cAMP and cGMP, are potent secondary messengers that contribute to the regulation of a variety of cellular processes including metabolism (82). cAMP has been shown to suppress innate immune function including inflammatory cytokine production, cell adhesion, phagocytosis, and intracellular killing $(83,84)$. Additionally, the cAMP axis plays an important role in antimicrobial defense as many microbes have evolved virulenceenhancing strategies that exploit this pathway (85-87). In BMM, LPS responses are associated with low levels of cAMP and cGMP accumulation, which inhibit inflammatory cytokine production $(88,89)$. Thus, alterations in (cAMP)/AMP and (cGMP)/GMP ratio may play an 
important role in regulating inflammatory, antimicrobial and metabolism responses in acute type I IFN responses.

Alterations in genes associated with tryptophan and branched-chain amino acid catabolism were pronounced in early IFN responses. Consistent with previous studies $(50,90,91)$, IFN- $\alpha$ stimulation was associated with increased levels of genes associated with tryptophan catabolism. While I did not observe alterations in $I D O 1$ expression, $T D O 2, K M O$ and $K Y N U$ were increased in both BMM and MDM. Many studies have shown that tryptophan catabolism (via IDO activation) represents a potent antiviral immune response (91-93). Our study suggests downstream enzymes of the kynurenine pathway may also contribute to this phenotype. Interestingly, I also found that $N A M P T$ was upregulated following acute IFN- $\alpha$ stimulation, suggesting tryptophan may be directed towards $\mathrm{NAD}^{+}$salvaging. NAMPT plays an important role in regulating glycolytic flux, phagocytic activity and TNF- $\alpha$ production in LPS-stimulated macrophages and may also contribute to IFN responses $(94,95)$. At the level of branched-chain amino acid metabolism, BMM had increased expression of genes associated with branched-chain amino acid catabolism. Conversely, MDM downregulated genes associated with this pathway. Catabolic products of branched chain amino acid metabolism feed into the TCA cycle contributing to the production of succinyl-CoA and acetyl-CoA (96). Previous studies have shown that branched-chain amino acid availability is critical for lymphocyte proliferation and M1 macrophage activation, but little is known regarding the role of these amino acids in regulating immune responses $(97,98)$. Thus, upregulation of branched chain amino acid catabolism in BMM may compensate for the loss of OXPHOS activity, which is not required in MDM.

Lipid metabolism has been shown to play an important role in antiviral responses in BMM and $\operatorname{MDM}(23,24,53)$. Consistent with the literature, altered gene expression in BMM and MDM 
suggest these cells may downregulate de novo cholesterol synthesis and shunt available free cholesterol towards the production of oxysterols including 25-hydroxycholesterol and 27hydroxycholesterol. The role of cholesterol flux in antiviral responses has been described previously $(22,23,54)$. Increased cholesterol levels help facilitate the entry of the dengue virus during the early phases of infection, which is reduced by the presence of oxysterols such as 25 hydroxycholesterol $(22,99-101)$. Macrophages can also counteract this demand by switching away from de novo synthesis towards lipid import (23). Limiting flux through the cholesterol biosynthetic pathway induces a STING-mediated type I IFN response, which can be attenuated by exogenous free cholesterol $(23,54)$. I also observed significant alterations in genes associated with phospholipid, sphingolipid, and FA metabolism. Lipid membrane composition can play a critical role in the antiviral capabilities of immune cells. Sphingolipids and phosphatidylserine have been shown to function as receptors for polyomavirus, HIV, and vesicular stomatitis virus (VSV) (102104). Altering the lipid composition of the plasma membrane is a vital protective strategy against viral entry by altering the potential interaction sites for viruses $(105,106)$. Thus, altering the membrane lipid composition may be a critical feature of metabolic reprogramming in early antiviral responses.

Collectively, our study provides critical new insights into the molecular underpinnings of metabolic reprogramming associated with short term IFN- $\alpha$ responses in mouse BMM and human MDM. This is the first study to systematically characterize changes in metabolic gene expression using transcriptional profiling in this context. However, I acknowledge certain limitations of this study. The current study only evaluates gene expression profiles via microarray. Validation and functional testing are required to understand the biological relevance of these findings. While both BMM and MDM were stimulated with short term IFN- $\alpha$, I cannot exclude the possibility that some 
of the reported differences may reflect the length of time in stimulant (2.5h vs. $4 \mathrm{~h})$. Preliminary studies from our laboratory suggest metabolic profiles in stimulated BMM are similar within a 26-hour window. However, future time-course studies are required to examine how metabolic signatures change in BMM and MDM over short and long term IFN- $\alpha$ stimulation. Culture conditions and differentiation protocols may also affect metabolic profiles in BMM vs. MDM. This limitation should be an important consideration across all studies examining relationships between immune and metabolic processes in vitro, whether in humans or in mice. Careful consideration of the model system may be required depending on downstream applications of the findings. Meta-analyses of transcriptional data sets may represent a powerful tool to identify metabolic signatures that are consistently altered across different studies using different models, time points, culture conditions etc. To minimize these effects, only studies performed in high glucose DMEM (plus glutamine and sodium pyruvate) with 10\% FBS were selected for analyses. Finally, both BMM and MDM were analyzed using Affymetrix technologies' microarray chips. However, I cannot exclude the possibility that the reported differences may be affected by the microarray used. Despite these limitations, I strongly believe this comparative study provides important new insights into metabolic processes that contribute to IFN responses in mouse and human macrophages. I believe that the power of an untargeted approach such as transcriptional profiling is to systematically characterize these differences, which may have important implications on effector function depending on the local microenvironment. In the future, more targeted studies are required to evaluate the effects of these gene expression profiles on protein expression and functional metabolic and immune responses. 


\subsection{Conclusions}

In summary, this study identified a variety of metabolic pathways altered following short term IFN- $\alpha$ stimulation in mouse and human macrophage systems. This may have important implications for the initiation of early antiviral immune responses, including the induction of the specific antimicrobial and immunomodulatory functions of IFN- $\alpha$. While functional studies are required to clearly elucidate the relationships between this metabolic reprogramming and effector function, it is clear that transcriptional regulation of metabolic processes is a key feature of early type I IFN responses. An in-depth understanding of this early reprogramming may lead to the development of targeted therapeutics that regulate and fine tune specific type I IFN effector function.

\subsection{Data Availability}

Gene expression data used in this study have been previously published in the GEO $\begin{array}{llll}\text { database } & \text { under } & \text { accession } & \text { numbers }\end{array}$ (https://www.ncbi.nlm.nih.gov/geo/query/acc.cgi?acc=GSE16755) and GSE35825 (https://www.ncbi.nlm.nih.gov/geo/query/acc.cgi?acc=GSE35825) 


\subsection{References}

1. Isaacs, A., and J. Lindenmann. 1957. Virus Interference. I. The Interferon. Proc. R. Soc. Lond. B. Biol. Sci. 147: 258-267.

2. Isaacs, A., J. Lindenmann, and R. C. Valentine. 1957. Virus Interference. II. Some Properties of Interferon. Proc. R. Soc. Lond. B. Biol. Sci. 147: 268-273.

3. Pestka, S., C. D. Krause, and M. R. Walter. 1987. Interferons, interferon-like cytokines, and their receptors. Immunol. Rev. 202 8-32.

4. Trinchieri, G. 2010. Type I interferon: friend or foe? J. Exp. Med. 207: 2053-2063.

5. Samuel, C. E. 2001. Antiviral Actions of Interferons. Clin. Microbiol. Rev. 14: 778-809.

6. Stark, G. R., I. M. Kerr, B. R. G. Williams, R. H. Silverman, and R. D. Schreiber. 1998. How cells respond to interferons. Annu. Rev. Biochem. 67: 227-264.

7. Platanias, L. C. 2005. Mechanisms of type-I- and type-II-interferon-mediated signalling. Nat. Rev. Immunol. 5: 375-386.

8. Ivashkiv, L. B., and L. T. Donlin. 2014. Regulation of type I interferon responses. Nat. Rev. Immunol. 14: 36-49.

9. Arimoto, K. I., S. Lochte, S. A. Stoner, C. Burkart, Y. Zhang, S. Miyauchi, S. Wilmes, J.B. Fan, J. J. Heinisch, Z. Li, M. Yan, S. Pellegrini, F. Colland, J. Piehler, and D.-E. Zhang. 2017. STAT2 is an essential adaptor in USP18-mediated suppression of type I interferon signaling. Nat. Struct. Mol. Biol. 24: 279-289.

10. Schneider, W. M., M. D. Chevillotte, and C. M. Rice. 2014. Interferon-Stimulated Genes: A Complex Web of Host Defenses. Annu. Rev. Immunol. 32: 513-545.

11. Schoggins, J. W., and C. M. Rice. 2011. Interferon-stimulated genes and their antiviral effector functions. Curr. Opin. Virol. 1: 519-525.

12. Bianchi, M. E. 2007. DAMPs, PAMPs and alarmins: all we need to know about danger. J. Leukoc. Biol. 81: 1-5.

13. Iannello, A., O. Debbeche, E. Martin, L. H. Attalah, S. Samarani, and A. Ahmad. 2006. Viral strategies for evading antiviral cellular immune responses of the host. J. Leukoc. Biol. 79: 16-35.

14. Tannahill, G. M., A. M. Curtis, J. Adamik, E. M. Palsson-McDermott, A. F. McGettrick, G. Goel, C. Frezza, N. J. Bernard, B. Kelly, N. H. Foley, L. Zheng, A. Gardet, Z. Tong, S. S. Jany, S. C. Corr, M. Haneklaus, B. E. Caffrey, K. Pierce, S. Walmsley, F. C. Beasley, E. Cummins, V. Nizet, M. Whyte, C. T. Taylor, H. Lin, S. L. Masters, E. Gottlieb, V. P. Kelly, C. Clish, P. E. Auron, R. J. Xavier, and L. A. J. O/'Neill. 2013. Succinate is an inflammatory signal that induces IL-1 $\beta$ through HIF-1 $\alpha$. Nature 496: 238242.

15. Errea, A., D. Cayet, P. Marchetti, C. Tang, J. Kluza, S. Offermanns, J.-C. Sirard, and M. Rumbo. 2016. Lactate Inhibits the Pro-Inflammatory Response and Metabolic Reprogramming in Murine Macrophages in a GPR81-Independent Manner. PLoS ONE 11: e0163694.

16. Infantino, V., V. Iacobazzi, F. Palmieri, and A. Menga. 2013. ATP-citrate lyase is essential for macrophage inflammatory response. Biochem. Biophys. Res. Commun. 440: 105-111.

17. Kesarwani, P., A. K. Murali, A. A. Al-Khami, and S. Mehrotra. 2013. Redox regulation of T-cell function: from molecular mechanisms to significance in human health and disease. Antioxid. Redox Signal. 18: 1497-1534. 
18. Wang, H., H. Flach, M. Onizawa, L. Wei, M. T. McManus, and A. Weiss. 2014. Negative regulation of Hifla expression and TH17 differentiation by the hypoxiaregulated microRNA miR-210. Nat. Immunol. 15: 393-401.

19. Mills, E. L., B. Kelly, A. Logan, A. S. H. Costa, M. Varma, C. E. Bryant, P. Tourlomousis, J. H. M. Däbritz, E. Gottlieb, I. Latorre, S. C. Corr, G. McManus, D. Ryan, H. T. Jacobs, M. Szibor, R. J. Xavier, T. Braun, C. Frezza, M. P. Murphy, and L. A. O’Neill. 2016. Succinate Dehydrogenase Supports Metabolic Repurposing of Mitochondria to Drive Inflammatory Macrophages. Cell 167: 457-470.e413.

20. Jiang, H., H. Shi, M. Sun, Y. Wang, Q. Meng, P. Guo, Y. Cao, J. Chen, X. Gao, E. Li, and J. Liu. 2016. PFKFB3-Driven Macrophage Glycolytic Metabolism Is a Crucial Component of Innate Antiviral Defense. J. Immunol. 197: 2880-2890.

21. Blanc, M., W. Y. Hsieh, K. A. Robertson, S. Watterson, G. Shui, P. Lacaze, M. Khondoker, P. Dickinson, G. Sing, S. Rodríguez-Martín, P. Phelan, T. Forster, B. Strobl, M. Müller, R. Riemersma, T. Osborne, M. R. Wenk, A. Angulo, and P. Ghazal. 2011. Host Defense against Viral Infection Involves Interferon Mediated Down-Regulation of Sterol Biosynthesis. PLoS Biol 9: e1000598.

22. Blanc, M., Wei Y. Hsieh, Kevin A. Robertson, Kai A. Kropp, T. Forster, G. Shui, P. Lacaze, S. Watterson, Samantha J. Griffiths, Nathanael J. Spann, A. Meljon, S. Talbot, K. Krishnan, Douglas F. Covey, Markus R. Wenk, M. Craigon, Z. Ruzsics, J. Haas, A. Angulo, William J. Griffiths, Christopher K. Glass, Y. Wang, and P. Ghazal. 2013. The Transcription Factor STAT-1 Couples Macrophage Synthesis of 25-Hydroxycholesterol to the Interferon Antiviral Response. Immunity 38: 106-118.

23. York, Autumn G., Kevin J. Williams, Joseph P. Argus, Quan D. Zhou, G. Brar, L. Vergnes, Elizabeth E. Gray, A. Zhen, Nicholas C. Wu, Douglas H. Yamada, Cameron R. Cunningham, Elizabeth J. Tarling, Moses Q. Wilks, D. Casero, David H. Gray, Amy K. Yu, Eric S. Wang, David G. Brooks, R. Sun, Scott G. Kitchen, T.-T. Wu, K. Reue, Daniel B. Stetson, and Steven J. Bensinger. 2015. Limiting Cholesterol Biosynthetic Flux Spontaneously Engages Type I IFN Signaling. Cell 163: 1716-1729.

24. Boshuizen, M. C. S., M. A. Hoeksema, A. E. Neele, S. van der Velden, A. A. J. Hamers, J. Van den Bossche, E. Lutgens, and M. P. J. de Winther. 2016. Interferon- $\beta$ promotes macrophage foam cell formation by altering both cholesterol influx and efflux mechanisms. Cytokine 77: 220-226.

25. Maneglier, B., C. Rogez-Kreuz, O. Spreux-Varoquaux, B. Malleret, P. Thérond, B. Samah, I. Drouet, D. Dormont, C. Advenier, and P. Clayette. 2007. Comparative effects of two type I interferons, human IFN- $\alpha$ and ovine IFN- $\tau$ on indoleamine-2,3-dioxygenase in primary cultures of human macrophages. Fundam. Clin. Pharmacol. 21: 29-34.

26. Acosta-Iborra, B., A. Elorza, I. M. Olazabal, N. B. Martín-Cofreces, S. Martin-Puig, M. Miró, M. J. Calzada, J. Aragonés, F. Sánchez-Madrid, and M. O. Landázuri. 2009. Macrophage Oxygen Sensing Modulates Antigen Presentation and Phagocytic Functions Involving IFN- $\gamma$ Production through the HIF-1 $\alpha$ Transcription Factor. J. Immunol. 182: 3155-3164.

27. Peyssonnaux, C., V. Datta, T. Cramer, A. Doedens, E. A. Theodorakis, R. L. Gallo, N. Hurtado-Ziola, V. Nizet, and R. S. Johnson. 2005. HIF-1 $\alpha$ expression regulates the bactericidal capacity of phagocytes. J. Clin. Invest. 115: 1806-1815. 
28. Nagy, C., and A. Haschemi. 2015. Time and Demand are Two Critical Dimensions of Immunometabolism: The Process of Macrophage Activation and the Pentose Phosphate Pathway. Frontiers in Immunology 6: 164.

29. Kelly, B., and L. A. J. O'Neill. 2015. Metabolic reprogramming in macrophages and dendritic cells in innate immunity. Cell Res. 25: 771-784.

30. O'Neill, L. A. J., and E. J. Pearce. 2016. Immunometabolism governs dendritic cell and macrophage function. J. Exp. Med. 213: 15-23.

31. Barrett, T., S. E. Wilhite, P. Ledoux, C. Evangelista, I. F. Kim, M. Tomashevsky, K. A. Marshall, K. H. Phillippy, P. M. Sherman, M. Holko, A. Yefanov, H. Lee, N. Zhang, C. L. Robertson, N. Serova, S. Davis, and A. Soboleva. 2013. NCBI GEO: archive for functional genomics data sets-update. Nucleic Acids Res. 41: D991-D995.

32. Liu, S.-Y., D. J. Sanchez, R. Aliyari, S. Lu, and G. Cheng. 2012. Systematic identification of type I and type II interferon-induced antiviral factors. Proc. Natl. Acad. Sci. U. S. A. 109: 4239-4244.

33. Greenwell-Wild, T., N. Vázquez, W. Jin, Z. Rangel, P. J. Munson, and S. M. Wahl. 2009. Interleukin-27 inhibition of HIV-1 involves an intermediate induction of type I interferon. Blood 114: 1864-1874.

34. Li, C., and W. H. Wong. 2001. Model-based analysis of oligonucleotide arrays: Expression index computation and outlier detection. Proc. Natl. Acad. Sci. U. S. A. 98: 31-36.

35. Gao, J., V. G. Tarcea, A. Karnovsky, B. R. Mirel, T. E. Weymouth, C. W. Beecher, J. D. Cavalcoli, B. D. Athey, G. S. Omenn, C. F. Burant, and H. V. Jagadish. 2010. Metscape: a Cytoscape plug-in for visualizing and interpreting metabolomic data in the context of human metabolic networks. Bioinformatics 26: 971-973.

36. Karnovsky, A., T. Weymouth, T. Hull, V. G. Tarcea, G. Scardoni, C. Laudanna, M. A. Sartor, K. A. Stringer, H. V. Jagadish, C. Burant, B. Athey, and G. S. Omenn. 2012. Metscape 2 bioinformatics tool for the analysis and visualization of metabolomics and gene expression data. Bioinformatics 28: 373-380.

37. Cline, M. S., M. Smoot, E. Cerami, A. Kuchinsky, N. Landys, C. Workman, R. Christmas, I. Avila-Campilo, M. Creech, B. Gross, K. Hanspers, R. Isserlin, R. Kelley, S. Killcoyne, S. Lotia, S. Maere, J. Morris, K. Ono, V. Pavlovic, A. R. Pico, A. Vailaya, P.L. Wang, A. Adler, B. R. Conklin, L. Hood, M. Kuiper, C. Sander, I. Schmulevich, B. Schwikowski, G. J. Warner, T. Ideker, and G. D. Bader. 2007. Integration of biological networks and gene expression data using Cytoscape. Nat. Protocols 2: 2366-2382.

38. Xia, J., I. V. Sinelnikov, B. Han, and D. S. Wishart. 2015. MetaboAnalyst 3.0-making metabolomics more meaningful. Nucleic Acids Res. 43: W251-257.

39. Subramanian, A., P. Tamayo, V. K. Mootha, S. Mukherjee, B. L. Ebert, M. A. Gillette, A. Paulovich, S. L. Pomeroy, T. R. Golub, E. S. Lander, and J. P. Mesirov. 2005. Gene set enrichment analysis: A knowledge-based approach for interpreting genome-wide expression profiles. Prod. Natl. Acad. Sci. U.S.A. 102: 15545-15550.

40. Huang, D. W., B. T. Sherman, X. Zheng, J. Yang, T. Imamichi, R. Stephens, and R. A. Lempicki. 2009. Extracting Biological Meaning from Large Gene Lists with DAVID. In Curr. Protoc. Bioinformatics. John Wiley and Sons, Inc. 1-13.

41. Huang, D. W., B. T. Sherman, and R. A. Lempicki. 2009. Systematic and integrative analysis of large gene lists using DAVID bioinformatics resources. Nat. Protocols 4: 4457. 
42. Lu, B., T. Nakamura, K. Inouye, J. Li, Y. Tang, P. Lundbäck, S. I. Valdes-Ferrer, P. S. Olofsson, T. Kalb, J. Roth, Y. Zou, H. Erlandsson-Harris, H. Yang, J. P. Y. Ting, H. Wang, U. Andersson, D. J. Antoine, S. S. Chavan, G. S. Hotamisligil, and K. J. Tracey. 2012. Novel role of PKR in inflammasome activation and HMGB1 release. Nature 488: 670.

43. Garten, A., S. Petzold, A. Körner, S.-i. Imai, and W. Kiess. 2009. Nampt: linking NAD biology, metabolism and cancer. Trends in Endocrinology \& Metabolism 20: 130-138.

44. Schoggins, J. W., S. J. Wilson, M. Panis, M. Y. Murphy, C. T. Jones, P. Bieniasz, and C. M. Rice. 2011. A diverse range of gene products are effectors of the type I interferon antiviral response. Nature 472: 481-485.

45. Liemburg-Apers, D. C., P. H. G. M. Willems, W. J. H. Koopman, and S. Grefte. 2015. Interactions between mitochondrial reactive oxygen species and cellular glucose metabolism. Arch. Toxicol. 89: 1209-1226.

46. Quijano, C., M. Trujillo, L. Castro, and A. Trostchansky. 2016. Interplay between oxidant species and energy metabolism. Redox Biol. 8: 28-42.

47. Kang, S. W., S. Lee, and E. K. Lee. 2015. ROS and energy metabolism in cancer cells: alliance for fast growth. Arch. Pharm. Res. 38: 338-345.

48. Hwang, J., H.-W. Suh, Y. H. Jeon, E. Hwang, L. T. Nguyen, J. Yeom, S.-G. Lee, C. Lee, K. J. Kim, B. S. Kang, J.-O. Jeong, T.-K. Oh, I. Choi, J.-O. Lee, and M. H. Kim. 2014. The structural basis for the negative regulation of thioredoxin by thioredoxin-interacting protein. Nat. Commun. 5: 2958.

49. Watanabe, R., H. Nakamura, H. Masutani, and J. Yodoi. 2010. Anti-oxidative, anticancer and anti-inflammatory actions by thioredoxin 1 and thioredoxin-binding protein-2. Pharmacol. Ther. 127: 261-270.

50. Mellor, A. L., and D. H. Munn. 1999. Tryptophan catabolism and T-cell tolerance: immunosuppression by starvation? Immunol. Today 20: 469-473.

51. Boasso, A., A. W. Hardy, S. A. Anderson, M. J. Dolan, and G. M. Shearer. 2008. HIVInduced Type I Interferon and Tryptophan Catabolism Drive T Cell Dysfunction Despite Phenotypic Activation. PLoS ONE 3: e2961.

52. Nakagawa, J., H. Waldner, S. Meyer-Monard, J. Hofsteenge, P. Jenö, and C. Moroni. 1995. AUH, a gene encoding an AU-specific RNA binding protein with intrinsic enoylCoA hydratase activity. Proc. Natl. Acad. Sci. U. S. A. 92: 2051-2055.

53. Coulombe, F., J. Jaworska, M. Verway, F. Tzelepis, A. Massoud, J. Gillard, G. Wong, G. Kobinger, Z. Xing, C. Couture, P. Joubert, Jörg H. Fritz, William S. Powell, and M. Divangahi. 2014. Targeted Prostaglandin E2 Inhibition Enhances Antiviral Immunity through Induction of Type I Interferon and Apoptosis in Macrophages. Immunity 40: 554568.

54. Blanc, M., W. Y. Hsieh, K. A. Robertson, S. Watterson, G. Shui, P. Lacaze, M. Khondoker, P. Dickinson, G. Sing, and S. Rodriguez-Martin. 2011. Host defense against viral infection involves interferon mediated down-regulation of sterol biosynthesis. PLoS Biol 9: e1000598.

55. Chugh, A., A. Ray, and J. B. Gupta. 2003. Squalene epoxidase as hypocholesterolemic drug target revisited. Prog. Lipid Res. 42: 37-50.

56. van Meer, G., D. R. Voelker, and G. W. Feigenson. 2008. Membrane lipids: where they are and how they behave. Nat. Rev. Mol. Cell Biol. 9: 112-124. 
57. Rodríguez-Prados, J., P. G. Través, J. Cuenca, D. Rico, J. Aragonés, P. Martín-Sanz, M. Cascante, and L. Boscá. 2010. Substrate Fate in Activated Macrophages: A Comparison between Innate, Classic, and Alternative Activation. J. Immunol. 185: 605-614.

58. Vazquez, A., J. Liu, Y. Zhou, and Z. N. Oltvai. 2010. Catabolic efficiency of aerobic glycolysis: The Warburg effect revisited. BMC Syst. Biol. 4: 1-9.

59. Imtiyaz, H. Z., and M. C. Simon. 2010. Hypoxia-inducible factors as essential regulators of inflammation. Curr. Top. Microbiol. Immunol. 345: 105-120.

60. Infantino, V., P. Convertini, L. Cucci, Maria A. Panaro, Maria A. Di Noia, R. Calvello, F. Palmieri, and V. Iacobazzi. 2011. The mitochondrial citrate carrier: a new player in inflammation. Biochem. J. 438: 433-436.

61. Fischer, K., P. Hoffmann, S. Voelkl, N. Meidenbauer, J. Ammer, M. Edinger, E. Gottfried, S. Schwarz, G. Rothe, S. Hoves, K. Renner, B. Timischl, A. Mackensen, L. Kunz-Schughart, R. Andreesen, S. W. Krause, and M. Kreutz. 2007. Inhibitory effect of tumor cell-derived lactic acid on human T cells. Blood 109: 3812.

62. Haas, R., J. Smith, V. Rocher-Ros, S. Nadkarni, T. Montero-Melendez, F. D’Acquisto, E. J. Bland, M. Bombardieri, C. Pitzalis, M. Perretti, F. M. Marelli-Berg, and C. Mauro. 2015. Lactate Regulates Metabolic and Pro-inflammatory Circuits in Control of T Cell Migration and Effector Functions. PLoS Biol. 13: e1002202.

63. He, L., H. Li, N. Huang, X. Zhou, J. Tian, T. Li, J. Wu, Y. Tian, Y. Yin, and K. Yao. 2017. Alpha-ketoglutarate suppresses the NF-kappaB-mediated inflammatory pathway and enhances the PXR-regulated detoxification pathway. Oncotarget: 1-15.

64. Luo, W., H. Hu, R. Chang, J. Zhong, M. Knabel, R. O'Meally, Robert N. Cole, A. Pandey, and Gregg L. Semenza. 2011. Pyruvate Kinase M2 Is a PHD3-Stimulated Coactivator for Hypoxia-Inducible Factor 1. Cell 145: 732-744.

65. Luo, W., and G. L. Semenza. 2011. Pyruvate kinase M2 regulates glucose metabolism by functioning as a coactivator for hypoxia-inducible factor 1 in cancer cells. Oncotarget 2: 551-556.

66. Chang, C.-H., J. D. Curtis, L. B. Maggi, B. Faubert, A. V. Villarino, D. O'Sullivan, S. C.C. Huang, G. J. W. van der Windt, J. Blagih, J. Qiu, J. D. Weber, E. J. Pearce, R. G. Jones, and E. L. Pearce. 2013. Posttranscriptional Control of T Cell Effector Function by Aerobic Glycolysis. Cell 153: 1239-1251.

67. Milasta, S., Christopher P. Dillon, Oliver E. Sturm, Katherine C. Verbist, Taylor L. Brewer, G. Quarato, Scott A. Brown, S. Frase, Laura J. Janke, S. S. Perry, Paul G. Thomas, and Douglas R. Green. 2016. Apoptosis-Inducing-Factor-Dependent Mitochondrial Function Is Required for T Cell but Not B Cell Function. Immunity 44: 88102.

68. Drapier, J. C., and J. B. Hibbs. 1988. Differentiation of murine macrophages to express nonspecific cytotoxicity for tumor cells results in L-arginine-dependent inhibition of mitochondrial iron-sulfur enzymes in the macrophage effector cells. J. Immunol. 140: 2829-2838.

69. Clementi, E., G. C. Brown, M. Feelisch, and S. Moncada. 1998. Persistent inhibition of cell respiration by nitric oxide: Crucial role of S-nitrosylation of mitochondrial complex I and protective action of glutathione. Proc. Natl. Acad. Sci. U.S.A. 95: 7631-7636.

70. Cleeter, M. W. J., J. M. Cooper, V. M. Darley-Usmar, S. Moncada, and A. H. V. Schapira. 1994. Reversible inhibition of cytochrome c oxidase, the terminal enzyme of 
the mitochondrial respiratory chain, by nitric oxide. Implications for neurodegenerative diseases. FEBS Lett. 345: 50-54.

71. Doulias, P.-T., M. Tenopoulou, J. L. Greene, K. Raju, and H. Ischiropoulos. 2013. Nitric oxide regulates mitochondrial fatty acid metabolism through reversible protein Snitrosylation. Sci. Signal. 6: rs1.

72. Herbst, S., U. E. Schaible, and B. E. Schneider. 2011. Interferon Gamma Activated Macrophages Kill Mycobacteria by Nitric Oxide Induced Apoptosis. PLoS ONE 6: e19105.

73. Jha, Abhishek K., Stanley C.-C. Huang, A. Sergushichev, V. Lampropoulou, Y. Ivanova, E. Loginicheva, K. Chmielewski, Kelly M. Stewart, J. Ashall, B. Everts, Edward J. Pearce, Edward M. Driggers, and Maxim N. Artyomov. 2015. Network Integration of Parallel Metabolic and Transcriptional Data Reveals Metabolic Modules that Regulate Macrophage Polarization. Immunity 42: 419-430.

74. El Kasmi, K. C., and K. R. Stenmark. 2015. Contribution of metabolic reprogramming to macrophage plasticity and function. Seminars in Immunology 27: 267-275.

75. West, A. P., I. E. Brodsky, C. Rahner, D. K. Woo, H. Erdjument-Bromage, P. Tempst, M. C. Walsh, Y. Choi, G. S. Shadel, and S. Ghosh. 2011. TLR signalling augments macrophage bactericidal activity through mitochondrial ROS. Nature 472: 476-480.

76. Mak, T. W., M. Grusdat, G. S. Duncan, C. Dostert, Y. Nonnenmacher, M. Cox, C. Binsfeld, Z. Hao, A. Brüstle, M. Itsumi, C. Jäger, Y. Chen, O. Pinkenburg, B. Camara, M. Ollert, C. Bindslev-Jensen, V. Vasiliou, C. Gorrini, P. A. Lang, M. Lohoff, I. S. Harris, K. Hiller, and D. Brenner. 2017. Glutathione Primes T Cell Metabolism for Inflammation. Immunity 46: 675-689.

77. DeVito, S. R., E. Ortiz-Riaño, L. Martínez-Sobrido, and J. Munger. 2014. Cytomegalovirus-mediated activation of pyrimidine biosynthesis drives UDP-sugar synthesis to support viral protein glycosylation. Proc. Natl. Acad. Sci. U. S. A. 111: $18019-18024$.

78. Vastag, L., E. Koyuncu, S. L. Grady, T. E. Shenk, and J. D. Rabinowitz. 2011. Divergent Effects of Human Cytomegalovirus and Herpes Simplex Virus-1 on Cellular Metabolism. PLoS Pathog. 7: e1002124.

79. Hoffmann, H.-H., A. Kunz, V. A. Simon, P. Palese, and M. L. Shaw. 2011. Broadspectrum antiviral that interferes with de novo pyrimidine biosynthesis. Proc. Natl. Acad. Sci. U. S. A. 108: 5777-5782.

80. Lucas-Hourani, M., D. Dauzonne, P. Jorda, G. Cousin, A. Lupan, O. Helynck, G. Caignard, G. Janvier, G. André-Leroux, S. Khiar, N. Escriou, P. Desprès, Y. Jacob, H. Munier-Lehmann, F. Tangy, and P.-O. Vidalain. 2013. Inhibition of Pyrimidine Biosynthesis Pathway Suppresses Viral Growth through Innate Immunity. PLOS Pathog. 9: e1003678.

81. Gavegnano, C., E. M. Kennedy, B. Kim, and R. F. Schinazi. 2012. The Impact of Macrophage Nucleotide Pools on HIV-1 Reverse Transcription, Viral Replication, and the Development of Novel Antiviral Agents. Mol Biol Int. 2012: 625983.

82. Tasken, K., B. S. Skalhegg, K. A. Tasken, R. Solberg, H. K. Knutsen, F. O. Levy, M. Sandberg, S. Orstavik, T. Larsen, A. K. Johansen, T. Vang, H. P. Schrader, N. T. Reinton, K. M. Torgersen, V. Hansson, and T. Jahnsen. 1997. Structure, function, and regulation of human cAMP-dependent protein kinases. Adv. Second Messenger Phosphoprotein Res. 31: 191-204. 
83. Serezani, C. H., M. N. Ballinger, D. M. Aronoff, and M. Peters-Golden. 2008. Cyclic AMP: master regulator of innate immune cell function. Am. J. Respir. Cell Mol. Biol. 39: 127-132.

84. Jin, S. L. C., L. Lan, M. Zoudilova, and M. Conti. 2005. Specific Role of Phosphodiesterase 4B in Lipopolysaccharide-Induced Signaling in Mouse Macrophages. J. Immunol. 175: 1523-1531.

85. Rickman, L., C. Scott, D. M. Hunt, T. Hutchinson, M. C. Menéndez, R. Whalan, J. Hinds, M. J. Colston, J. Green, and R. S. Buxton. 2005. A member of the cAMP receptor protein family of transcription regulators in Mycobacterium tuberculosis is required for virulence in mice and controls transcription of the rpfA gene coding for a resuscitation promoting factor. Mol. Microbiol. 56: 1274-1286.

86. Smith, R. S., M. C. Wolfgang, and S. Lory. 2004. An Adenylate Cyclase-Controlled Signaling Network Regulates Pseudomonas aeruginosa Virulence in a Mouse Model of Acute Pneumonia. Infect. Immun. 72: 1677-1684.

87. Zhan, L., Y. Han, L. Yang, J. Geng, Y. Li, H. Gao, Z. Guo, W. Fan, G. Li, L. Zhang, C. Qin, D. Zhou, and R. Yang. 2008. The Cyclic AMP Receptor Protein, CRP, Is Required for Both Virulence and Expression of the Minimal CRP Regulon in Yersinia pestis Biovar microtus. Infect. Immun. 76: 5028-5037.

88. Connelly, L., A. T. Jacobs, M. Palacios-Callender, S. Moncada, and A. J. Hobbs. 2003. Macrophage Endothelial Nitric-oxide Synthase Autoregulates Cellular Activation and Pro-inflammatory Protein Expression. J. Biol. Chem. 278: 26480-26487.

89. Endres, S., H. J. Fülle, B. Sinha, D. Stoll, C. A. Dinarello, R. Gerzer, and P. C. Weber. 1991. Cyclic nucleotides differentially regulate the synthesis of tumour necrosis factoralpha and interleukin-1 beta by human mononuclear cells. Immunology 72: 56-60.

90. Adams, O., K. Besken, C. Oberdörfer, C. R. MacKenzie, O. Takikawa, and W. Däubener. 2004. Role of Indoleamine-2,3-Dioxygenase in Alpha/Beta and Gamma InterferonMediated Antiviral Effects against Herpes Simplex Virus Infections. J. Virol. 78: 26322636.

91. Taylor, M. W., and G. S. Feng. 1991. Relationship between interferon-gamma, indoleamine 2,3-dioxygenase, and tryptophan catabolism. FASEB J. 5: 2516-2522.

92. Mellor, A. L., and D. H. Munn. 2004. IDO expression by dendritic cells: tolerance and tryptophan catabolism. Nat. Rev. Immunol. 4: 762-774.

93. Obojes, K., O. Andres, K. S. Kim, W. Däubener, and J. Schneider-Schaulies. 2005. Indoleamine 2,3-Dioxygenase Mediates Cell Type-Specific Anti-Measles Virus Activity of Gamma Interferon. J. Virol. 79: 7768-7776.

94. Venter, G., F. T. J. J. Oerlemans, M. Willemse, M. Wijers, J. A. M. Fransen, and B. Wieringa. 2014. NAMPT-Mediated Salvage Synthesis of NAD+ Controls Morphofunctional Changes of Macrophages. PLoS ONE 9: e97378.

95. Al-Shabany, A., A. Moody, A. Foey, and R. Billington. 2016. Intracellular NAD(+) levels are associated with LPS-induced TNF- $\alpha$ release in pro-inflammatory macrophages. Biosci. Rep. 36: e00301.

96. Harper, A. E., R. H. Miller, and K. P. Block. 1984. Branched-Chain Amino Acid Metabolism. Annu. Rev. Nutr. 4: 409-454.

97. Skaper, S. D., D. P. Molden, and J. E. Seegmiller. 1976. Maple syrup urine disease: branched-chain amino acid concentrations and metabolism in cultured human lymphoblasts. Biochem Genet 14: 527-539. 
98. Meiser, J., L. Krämer, S. C. Sapcariu, N. Battello, J. Ghelfi, A. F. D'Herouel, A. Skupin, and K. Hiller. 2016. Pro-inflammatory Macrophages Sustain Pyruvate Oxidation through Pyruvate Dehydrogenase for the Synthesis of Itaconate and to Enable Cytokine Expression. J. Biol. Chem. 291: 3932-3946.

99. Soto-Acosta, R., C. Mosso, M. Cervantes-Salazar, H. Puerta-Guardo, F. Medina, L. Favari, J. E. Ludert, and R. M. del Angel. 2013. The increase in cholesterol levels at early stages after dengue virus infection correlates with an augment in LDL particle uptake and HMG-CoA reductase activity. Virology 442: 132-147.

100. Park, K., and A. L. Scott. 2010. Cholesterol 25-hydroxylase production by dendritic cells and macrophages is regulated by type I interferons. J. Leukoc. Biol. 88: 1081-1087.

101. Liu, S.-Y., R. Aliyari, K. Chikere, G. Li, Matthew D. Marsden, Jennifer K. Smith, O. Pernet, H. Guo, R. Nusbaum, Jerome A. Zack, Alexander N. Freiberg, L. Su, B. Lee, and G. Cheng. 2013. Interferon-Inducible Cholesterol-25-Hydroxylase Broadly Inhibits Viral Entry by Production of 25-Hydroxycholesterol. Immunity 38: 92-105.

102. Rawat, S. S., M. Viard, S. A. Gallo, R. Blumenthal, and A. Puri. 2006. Sphingolipids, cholesterol, and HIV-1: A paradigm in viral fusion. Glycoconj. J. 23: 189-197.

103. Smith, A. E., H. Lilie, and A. Helenius. 2003. Ganglioside-dependent cell attachment and endocytosis of murine polyomavirus-like particles. FEBS Lett. 555: 199-203.

104. Ewers, H., W. Romer, A. E. Smith, K. Bacia, S. Dmitrieff, W. Chai, R. Mancini, J. Kartenbeck, V. Chambon, L. Berland, A. Oppenheim, G. Schwarzmann, T. Feizi, P. Schwille, P. Sens, A. Helenius, and L. Johannes. 2010. GM1 structure determines SV40induced membrane invagination and infection. Nat. Cell Biol. 12: 11-18.

105. Merino-Ramos, T., Á. Vázquez-Calvo, J. Casas, F. Sobrino, J.-C. Saiz, and M. A. MartínAcebes. 2016. Modification of the Host Cell Lipid Metabolism Induced by Hypolipidemic Drugs Targeting the Acetyl Coenzyme A Carboxylase Impairs West Nile Virus Replication. Antimicrob. Agents Chemother. 60: 307-315.

106. Pezacki, J. P., S. M. Sagan, A. M. Tonary, Y. Rouleau, S. Bélanger, L. Supekova, and A. I. Su. 2009. Transcriptional profiling of the effects of 25-hydroxycholesterol on human hepatocyte metabolism and the antiviral state it conveys against the hepatitis $\mathrm{C}$ virus. BMC Chem. Biol. 9: 2. 


\subsection{Chapter 2 Supplemental Tables}

\section{Supplemental Table S2.1: A list of all metabolic genes identified in the BMM dataset.}

\begin{tabular}{|c|c|c|c|c|c|}
\hline $\begin{array}{l}\text { Entrez } \\
\text { Gene } \\
\text { ID }\end{array}$ & Gene Symbol & Gene Title & $\begin{array}{l}\text { Fold } \\
\text { Change }\end{array}$ & P-value & FDR \\
\hline 210106 & Papd7 & PAP associated domain containing 7 & 6.763807 & 4.38E-07 & 0.000139 \\
\hline 17308 & Mgat1 & mannoside acetylglucosaminyltransferase 1 & 2.781795 & $6.01 \mathrm{E}-07$ & 0.000139 \\
\hline 70052 & Prpf4 & $\begin{array}{l}\text { PRP4 pre-mRNA processing factor } 4 \text { homolog } \\
\text { (yeast) }\end{array}$ & 3.405339 & $1.72 \mathrm{E}-06$ & 0.000266 \\
\hline 20449 & St8sia1 & $\begin{array}{l}\text { ST8 alpha-N-acetyl-neuraminide alpha-2,8- } \\
\text { sialyltransferase } 1\end{array}$ & 7.746754 & 4.42E-06 & 0.000433 \\
\hline 22375 & Wars & tryptophanyl-tRNA synthetase & 2.38714 & 8.39E-06 & 0.000535 \\
\hline 53625 & B3gnt2 & $\begin{array}{l}\text { UDP-GlcNAc:betaGal beta-1,3-N- } \\
\text { acetylglucosaminyltransferase } 2 / / / \mathrm{COMM} \text { domain } \\
\text { containing } 1\end{array}$ & 2.102979 & $9.27 \mathrm{E}-06$ & 0.000548 \\
\hline 16542 & $\mathrm{Kdr}$ & kinase insert domain protein receptor & 6.182894 & $9.46 \mathrm{E}-06$ & 0.000551 \\
\hline 208715 & Hmgcs 1 & 3-hydroxy-3-methylglutaryl-Coenzyme A synthase 1 & -2.33769 & $1.11 \mathrm{E}-05$ & 0.000571 \\
\hline 20620 & Plk2 & polo-like kinase 2 & 2.603204 & $1.94 \mathrm{E}-05$ & 0.00081 \\
\hline 12799 & Cnp & 2',3'-cyclic nucleotide 3' phosphodiesterase & 2.532145 & $2.70 \mathrm{E}-05$ & 0.000958 \\
\hline 17423 & Ndst2 & $\begin{array}{l}\text { N-deacetylase/N-sulfotransferase (heparan } \\
\text { glucosaminyl) } 2\end{array}$ & 1.793242 & $2.93 \mathrm{E}-05$ & 0.000996 \\
\hline 18472 & Pafah1b1 & $\begin{array}{l}\text { platelet-activating factor acetylhydrolase, isoform } \\
1 \mathrm{~b} \text {, subunit } 1\end{array}$ & -1.26278 & $3.26 \mathrm{E}-05$ & 0.001045 \\
\hline 22021 & Tpst1 & protein-tyrosine sulfotransferase 1 & 7.198429 & $3.54 \mathrm{E}-05$ & 0.001081 \\
\hline 15369 & Hmox 2 & heme oxygenase (decycling) 2 & 1.460464 & 3.74E-05 & 0.001105 \\
\hline 14538 & Gcnt2 & $\begin{array}{l}\text { glucosaminyl (N-acetyl) transferase 2, I-branching } \\
\text { enzyme }\end{array}$ & 2.982574 & $3.86 \mathrm{E}-05$ & 0.001119 \\
\hline 18950 & Pnp & purine-nucleoside phosphorylase & 2.239241 & 4.27E-05 & 0.001165 \\
\hline 11992 & Auh & $\begin{array}{l}\text { AU RNA binding protein/enoyl-coenzyme A } \\
\text { hydratase }\end{array}$ & 1.789546 & $6.16 \mathrm{E}-05$ & 0.001438 \\
\hline 22247 & Umps & uridine monophosphate synthetase & -1.69624 & $8.01 \mathrm{E}-05$ & 0.001639 \\
\hline 71978 & Ppp2r2a & protein phosphatase 2 , regulatory subunit $\mathrm{B}$, alpha & 1.525692 & $8.16 \mathrm{E}-05$ & 0.001653 \\
\hline 22217 & Usp12 & $\begin{array}{l}\text { predicted gene, } 19496 / / / \text { ubiquitin specific peptidase } \\
12\end{array}$ & 3.421452 & $8.18 \mathrm{E}-05$ & 0.001654 \\
\hline 16922 & Phyh & phytanoyl-CoA hydroxylase & 1.557676 & $9.08 \mathrm{E}-05$ & 0.001734 \\
\hline 22224 & Usp10 & ubiquitin specific peptidase 10 & -1.60565 & $9.73 \mathrm{E}-05$ & 0.001786 \\
\hline 26433 & Plod3 & procollagen-lysine, 2-oxoglutarate 5-dioxygenase 3 & 1.635048 & 0.000101 & 0.00181 \\
\hline 76630 & Stambpl1 & STAM binding protein like 1 & 2.262333 & 0.000107 & 0.001853 \\
\hline 98256 & Kmo & $\begin{array}{l}\text { kynurenine 3-monooxygenase (kynurenine 3- } \\
\text { hydroxylase) }\end{array}$ & 3.808386 & 0.000109 & 0.001868 \\
\hline 99929 & Tiparp & TCDD-inducible poly(ADP-ribose) polymerase & 3.036925 & 0.00012 & 0.001969 \\
\hline 104418 & Dgkz & diacylglycerol kinase zeta & -1.31347 & 0.000126 & 0.002016 \\
\hline 235584 & Dusp7 & dual specificity phosphatase 7 & -2.27693 & 0.00015 & 0.002225 \\
\hline 17096 & Lyn; Gm11787 & $\begin{array}{l}\text { Yamaguchi sarcoma viral (v-yes-1) oncogene } \\
\text { homolog }\end{array}$ & 1.693171 & 0.000157 & 0.002285 \\
\hline 14629 & Gclc & Glutamate-cysteine ligase, catalytic subunit & -1.8552 & 0.00016 & 0.002305 \\
\hline 243771 & Parp12 & poly (ADP-ribose) polymerase family, member 12 & 3.268517 & 0.00021 & 0.002647 \\
\hline 11669 & Aldh2 & aldehyde dehydrogenase 2 , mitochondrial & -2.70208 & 0.000215 & 0.002674 \\
\hline 14231 & Fkbp7 & FK506 binding protein 7 & 2.225479 & 0.000217 & 0.002686 \\
\hline
\end{tabular}




\begin{tabular}{|c|c|c|c|c|c|}
\hline 12988 & Csk & c-src tyrosine kinase & 1.650648 & 0.000222 & 0.002715 \\
\hline 14534 & Kat2a & $\mathrm{K}($ lysine $)$ acetyltransferase $2 \mathrm{~A}$ & 1.410931 & 0.000239 & 0.002809 \\
\hline 74596 & Cds1 & CDP-diacylglycerol synthase 1 & 1.8141 & 0.000272 & 0.002963 \\
\hline 14081 & Acsl1 & acyl-CoA synthetase long-chain family member 1 & -1.40739 & 0.000279 & 0.002995 \\
\hline 74167 & Nudt9 & $\begin{array}{l}\text { nudix (nucleoside diphosphate linked moiety X)-type } \\
\text { motif } 9\end{array}$ & 1.507661 & 0.000304 & 0.003097 \\
\hline 18715 & Pim2 & proviral integration site 2 & -1.5358 & 0.000321 & 0.003161 \\
\hline 17330 & Minpp1 & $\begin{array}{l}\text { multiple inositol polyphosphate histidine } \\
\text { phosphatase } 1\end{array}$ & 1.670051 & 0.000342 & 0.003234 \\
\hline 110157 & Raf1 & v-raf-leukemia viral oncogene 1 & 1.307271 & 0.000349 & 0.003258 \\
\hline 26410 & Map3k8 & mitogen-activated protein kinase kinase kinase 8 & 3.134357 & 0.000351 & 0.003264 \\
\hline 13548 & Dyrk1a & $\begin{array}{l}\text { dual-specificity tyrosine-(Y)-phosphorylation } \\
\text { regulated kinase 1a }\end{array}$ & -1.51587 & 0.000384 & 0.003366 \\
\hline 22234 & Ugcg & UDP-glucose ceramide glucosyltransferase & 2.437817 & 0.000413 & 0.003444 \\
\hline 18583 & Pde7a & phosphodiesterase 7A & 1.515764 & 0.000416 & 0.003451 \\
\hline 26408 & Map3k5 & mitogen-activated protein kinase kinase kinase 5 & 1.417154 & 0.000432 & 0.003491 \\
\hline 268470 & Ube $2 z$ & ubiquitin-conjugating enzyme E2Z (putative) & -1.50424 & 0.000451 & 0.003537 \\
\hline 217127 & Kat7 & $\mathrm{K}$ (lysine) acetyltransferase 7 & -1.34363 & 0.000459 & 0.003555 \\
\hline 69922 & Vrk2 & vaccinia related kinase 2 & 1.675036 & 0.000462 & 0.00356 \\
\hline 69241 & Polr2d & polymerase (RNA) II (DNA directed) polypeptide D & -1.4936 & 0.000462 & 0.003562 \\
\hline 236900 & Pdk3 & pyruvate dehydrogenase kinase, isoenzyme 3 & 2.262069 & 0.000494 & 0.003629 \\
\hline 17769 & Mthfr & 5,10-methylenetetrahydrofolate reductase & 3.793631 & 0.000494 & 0.003629 \\
\hline 24110 & Usp18 & ubiquitin specific peptidase 18 & 46.3677 & 0.000504 & 0.003649 \\
\hline 211347 & Pank3 & pantothenate kinase 3 & -1.3012 & 0.000515 & 0.003671 \\
\hline 83813 & Tnk1 & tyrosine kinase, non-receptor, 1 & 1.483272 & 0.000521 & 0.003682 \\
\hline 72349 & Dusp3 & $\begin{array}{l}\text { dual specificity phosphatase } 3 \text { (vaccinia virus } \\
\text { phosphatase VH1-related) }\end{array}$ & -1.3074 & 0.000523 & 0.003685 \\
\hline 18707 & Pik3cd & $\begin{array}{l}\text { phosphatidylinositol 3-kinase catalytic delta } \\
\text { polypeptide }\end{array}$ & 1.998752 & 0.000535 & 0.003707 \\
\hline 213452 & Dstyk & dual serine/threonine and tyrosine protein kinase & -1.43624 & 0.000537 & 0.00371 \\
\hline 14645 & Glul & glutamate-ammonia ligase (glutamine synthetase) & -1.20573 & 0.000543 & 0.003721 \\
\hline 19108 & Prkx & protein kinase, $\mathrm{X}$-linked & 2.008997 & 0.000544 & 0.003723 \\
\hline 214424 & Parp16 & poly (ADP-ribose) polymerase family, member 16 & -1.98746 & 0.000552 & 0.003737 \\
\hline 67618 & Aasdhppt & $\begin{array}{l}\text { aminoadipate-semialdehyde dehydrogenase- } \\
\text { phosphopantetheinyl transferase }\end{array}$ & -1.21136 & 0.000558 & 0.003748 \\
\hline 22214 & Ube2h & ubiquitin-conjugating enzyme E2H & -1.42323 & 0.00056 & 0.003751 \\
\hline 170768 & Pfkfb3 & $\begin{array}{l}\text { 6-phosphofructo-2-kinase/fructose-2,6- } \\
\text { biphosphatase } 3\end{array}$ & 5.291241 & 0.000574 & 0.003773 \\
\hline 103534 & Mgat4b & $\begin{array}{l}\text { mannoside acetylglucosaminyltransferase } 4, \\
\text { isoenzyme B }\end{array}$ & -1.50731 & 0.000577 & 0.003779 \\
\hline 20779 & $\mathrm{Src}$ & Rous sarcoma oncogene & -1.54227 & 0.000579 & 0.003782 \\
\hline 26420 & Mapk9 & mitogen-activated protein kinase 9 & 1.32252 & 0.000583 & 0.003788 \\
\hline 66878 & Riok3 & RIO kinase 3 & -1.27173 & 0.0006 & 0.003814 \\
\hline 56375 & B4galt4 & $\begin{array}{l}\text { UDP-Gal:betaGlcNAc beta } 1,4- \\
\text { galactosyltransferase, polypeptide } 4\end{array}$ & 4.302791 & 0.000621 & 0.003844 \\
\hline 69737 & $\mathrm{Ttl}$ & tubulin tyrosine ligase & -1.85121 & 0.000639 & 0.003871 \\
\hline 73914 & Irak3 & interleukin-1 receptor-associated kinase 3 & -2.11207 & 0.000648 & 0.003883 \\
\hline 30955 & Pik3cg & $\begin{array}{l}\text { phosphoinositide-3-kinase, catalytic, gamma } \\
\text { polypeptide }\end{array}$ & -1.50705 & 0.000666 & 0.003906 \\
\hline
\end{tabular}




\begin{tabular}{|c|c|c|c|c|c|}
\hline 11982 & Atp10a & ATPase, class V, type $10 \mathrm{~A}$ & 3.81068 & 0.000691 & 0.003964 \\
\hline 77976 & Nuak1 & NUAK family, SNF1-like kinase, 1 & -15.2322 & 0.000714 & 0.004014 \\
\hline 104015 & Synj1 & synaptojanin 1 & 2.199326 & 0.000736 & 0.004059 \\
\hline 104458 & Rars & arginyl-tRNA synthetase & 1.303931 & 0.000764 & 0.004138 \\
\hline 217837 & Itpk1 & inositol 1,3,4-triphosphate 5/6 kinase & 1.52582 & 0.000802 & 0.004239 \\
\hline 73086 & Rps6ka5 & ribosomal protein S6 kinase, polypeptide 5 & -1.66255 & 0.00081 & 0.00426 \\
\hline 207839 & Galnt6 & $\begin{array}{l}\text { UDP-N-acetyl-alpha-D-galactosamine:polypeptide } \\
\text { N-acetylgalactosaminyltransferase } 6\end{array}$ & -1.72417 & 0.000888 & 0.004453 \\
\hline 20112 & Rps6ka2 & ribosomal protein S6 kinase, polypeptide 2 & -1.5991 & 0.000918 & 0.004523 \\
\hline 330260 & Pon2 & paraoxonase 2 & -1.20562 & 0.000926 & 0.00454 \\
\hline 20448 & St6galnac4 & $\begin{array}{l}\text { ST6 (alpha-N-acetyl-neuraminyl-2,3-beta-galactosyl- } \\
\text { 1,3)-N-acetylgalactosaminide alpha-2,6- } \\
\text { sialyltransferase } 4\end{array}$ & 2.002204 & 0.000926 & 0.004541 \\
\hline 20768 & Sephs2 & selenophosphate synthetase 2 & -1.83655 & 0.000927 & 0.004543 \\
\hline 80285 & Parp9 & poly (ADP-ribose) polymerase family, member 9 & 5.357382 & 0.000951 & 0.004596 \\
\hline 12894 & Cpt1a & carnitine palmitoyltransferase 1a, liver & 1.239826 & 0.000999 & 0.004699 \\
\hline 50877 & Neu3 & neuraminidase 3 & 2.789321 & 0.001017 & 0.004736 \\
\hline 17768 & Mthfd2 & $\begin{array}{l}\text { methylenetetrahydrofolate dehydrogenase (NAD+ } \\
\text { dependent), methenyltetrahydrofolate cyclohydrolase }\end{array}$ & 2.173051 & 0.001023 & 0.004747 \\
\hline 101187 & Parp11 & poly (ADP-ribose) polymerase family, member 11 & 2.375364 & 0.001096 & 0.004889 \\
\hline 67956 & $\mathrm{Kmt5a}$ & SET domain containing (lysine methyltransferase) 8 & -1.98859 & 0.001102 & 0.004901 \\
\hline 67045 & Riok2 & RIO kinase 2 (yeast) & -1.59731 & 0.001122 & 0.004936 \\
\hline 24086 & Tlk2 & tousled-like kinase 2 (Arabidopsis) & 2.46983 & 0.00117 & 0.005022 \\
\hline 19267 & Ptpre & protein tyrosine phosphatase, receptor type, E & -2.10887 & 0.001185 & 0.005047 \\
\hline 234135 & Whsc111 & $\begin{array}{l}\text { Wolf-Hirschhorn syndrome candidate 1-like } 1 \\
\text { (human) }\end{array}$ & 2.092489 & 0.001185 & 0.005048 \\
\hline 68603 & Pmvk & phosphomevalonate kinase & 1.578973 & 0.001191 & 0.005057 \\
\hline 27399 & Ip6k1 & inositol hexaphosphate kinase 1 & -1.32241 & 0.001218 & 0.005103 \\
\hline 547253 & Parp14 & poly (ADP-ribose) polymerase family, member 14 & 5.081282 & 0.001221 & 0.005107 \\
\hline 12566 & $\mathrm{Cdk} 2$ & cyclin-dependent kinase 2 & -1.33454 & 0.001225 & 0.005114 \\
\hline 170755 & Sgk3 & serum/glucocorticoid regulated kinase 3 & 2.03164 & 0.001233 & 0.005127 \\
\hline 18099 & Nlk & nemo like kinase & -1.34664 & 0.001239 & 0.005137 \\
\hline 327951 & Cyb5d1 & cytochrome b5 domain containing 1 & -1.55873 & 0.001269 & 0.005184 \\
\hline 15277 & $\mathrm{Hk} 2$ & hexokinase 2 & 1.893444 & 0.001304 & 0.005238 \\
\hline 14537 & Gent1 & glucosaminyl (N-acetyl) transferase 1 , core 2 & 1.721606 & 0.00131 & 0.005247 \\
\hline 101540 & $\operatorname{Prkd} 2$ & protein kinase D2 & -1.49341 & 0.001359 & 0.00532 \\
\hline 59027 & Nampt & nicotinamide phosphoribosyltransferase & 2.861968 & 0.001374 & 0.00534 \\
\hline 19042 & Ppmla & $\begin{array}{l}\text { protein phosphatase } 1 \mathrm{~A} \text {, magnesium dependent, } \\
\text { alpha isoform }\end{array}$ & -1.36846 & 0.001379 & 0.005348 \\
\hline 14528 & Gch1 & GTP cyclohydrolase 1 & 2.090721 & 0.001389 & 0.005362 \\
\hline 18783 & Pla2g4a & $\begin{array}{l}\text { phospholipase A2, group IVA (cytosolic, calcium- } \\
\text { dependent) }\end{array}$ & 1.901651 & 0.001434 & 0.005423 \\
\hline 16891 & Lipg & lipase, endothelial & 3.397824 & 0.001464 & 0.005462 \\
\hline 11545 & Parp1 & poly (ADP-ribose) polymerase family, member 1 & -1.87172 & 0.001465 & 0.005464 \\
\hline 110119 & Mpi & mannose phosphate isomerase & 1.714154 & 0.001481 & 0.005485 \\
\hline 107823 & Whsc1 & Wolf-Hirschhorn syndrome candidate 1 (human) & -2.17186 & 0.001539 & 0.005557 \\
\hline
\end{tabular}




\begin{tabular}{|c|c|c|c|c|c|}
\hline 20773 & Sptlc2 & $\begin{array}{l}\text { serine palmitoyltransferase, long chain base subunit } \\
2\end{array}$ & 2.274875 & 0.001543 & 0.005562 \\
\hline 70750 & Kdsr & 3-ketodihydrosphingosine reductase & -1.24223 & 0.001546 & 0.005565 \\
\hline 74568 & Mlkl & mixed lineage kinase domain-like & 5.262045 & 0.001548 & 0.005568 \\
\hline 269181 & Mgat4a & $\begin{array}{l}\text { mannoside acetylglucosaminyltransferase } 4 \text {, } \\
\text { isoenzyme A }\end{array}$ & 1.762276 & 0.001558 & 0.00558 \\
\hline 18970 & Polb & polymerase (DNA directed), beta & 1.597279 & 0.001559 & 0.005581 \\
\hline 30940 & Usp25 & ubiquitin specific peptidase 25 & 1.731778 & 0.00159 & 0.005617 \\
\hline 21871 & Atp6v0a2 & ATPase, $\mathrm{H}+$ transporting, lysosomal V0 subunit A2 & 1.647803 & 0.001595 & 0.005623 \\
\hline 18519 & Kat $2 b$ & $\mathrm{~K}$ (lysine) acetyltransferase 2B & 1.582017 & 0.001605 & 0.005635 \\
\hline 81601 & Kat5 & $\mathrm{K}$ (lysine) acetyltransferase 5 & -1.34364 & 0.001632 & 0.005677 \\
\hline 17772 & Mtm1 & X-linked myotubular myopathy gene 1 & -1.8719 & 0.001746 & 0.005841 \\
\hline 107569 & $\mathrm{Nt} 5 \mathrm{c} 3$ & 5'-nucleotidase, cytosolic III & 7.718086 & 0.001753 & 0.005851 \\
\hline 78920 & Dlst & $\begin{array}{l}\text { dihydrolipoamide S-succinyltransferase (E2 } \\
\text { component of 2-oxo-glutarate complex) }\end{array}$ & -1.37118 & 0.001766 & 0.005868 \\
\hline 73699 & Ppp2r1b & protein phosphatase 2 , regulatory subunit $A$, beta & -1.43771 & 0.00179 & 0.005901 \\
\hline 19088 & Prkar2b & $\begin{array}{l}\text { protein kinase, cAMP dependent regulatory, type II } \\
\text { beta }\end{array}$ & -1.48713 & 0.001817 & 0.005937 \\
\hline 71340 & Riok1 & RIO kinase 1 (yeast) & -1.56169 & 0.001826 & 0.005949 \\
\hline 11513 & Adcy7 & adenylate cyclase 7 & -1.31653 & 0.00186 & 0.005994 \\
\hline 18706 & Pik3ca & $\begin{array}{l}\text { phosphatidylinositol 3-kinase, catalytic, alpha } \\
\text { polypeptide }\end{array}$ & -2.12379 & 0.001893 & 0.006035 \\
\hline 13178 & Dck & deoxycytidine kinase & 11.01118 & 0.001899 & 0.006043 \\
\hline 233011 & Itpkc & inositol 1,4,5-trisphosphate 3-kinase C & -4.10654 & 0.001935 & 0.006087 \\
\hline 20775 & Sqle & squalene epoxidase & -1.29846 & 0.001957 & 0.006114 \\
\hline 381406 & Trp53rka & RIKEN cDNA 2810408M09 gene & -2.92393 & 0.001957 & 0.006115 \\
\hline 14660 & Gls & glutaminase & -1.45952 & 0.001986 & 0.006149 \\
\hline 93765 & $\begin{array}{l}\text { Ube2n; } \\
\text { Mcg1038069 }\end{array}$ & ubiquitin-conjugating enzyme E2N & -1.22274 & 0.002048 & 0.006221 \\
\hline 22390 & Weel & WEE 1 homolog 1 (S. pombe) & -1.96865 & 0.002095 & 0.006275 \\
\hline 19277 & Ptpro & protein tyrosine phosphatase, receptor type, $\mathrm{O}$ & 1.285696 & 0.002115 & 0.006298 \\
\hline 56421 & Pfkp & phosphofructokinase, platelet & 1.462618 & 0.002132 & 0.006316 \\
\hline 20656 & Sod2 & superoxide dismutase 2 , mitochondrial & -2.8823 & 0.00215 & 0.006335 \\
\hline 58193 & Extl2 & exostoses (multiple)-like 2 & -1.38527 & 0.002151 & 0.006336 \\
\hline 232533 & Stk381 & serine/threonine kinase 38 like & 1.854298 & 0.002206 & 0.006395 \\
\hline 11432 & Acp2 & acid phosphatase 2 , lysosomal & -1.3285 & 0.002216 & 0.006405 \\
\hline 20963 & Syk & spleen tyrosine kinase & -1.42526 & 0.002216 & 0.006405 \\
\hline 75320 & Etnk1 & ethanolamine kinase 1 & 3.421538 & 0.002237 & 0.006427 \\
\hline 26406 & Map3k3 & mitogen-activated protein kinase kinase kinase 3 & -1.37786 & 0.002266 & 0.006456 \\
\hline 56361 & Pus1 & pseudouridine synthase 1 & -1.37161 & 0.002278 & 0.006468 \\
\hline 14048 & Eya1 & eyes absent 1 homolog (Drosophila) & -1.91034 & 0.002301 & 0.006491 \\
\hline 104831 & Ptpn23 & protein tyrosine phosphatase, non-receptor type 23 & -1.72145 & 0.002312 & 0.006502 \\
\hline 14718 & Got1 & glutamate oxaloacetate transaminase 1 , soluble & 1.318871 & 0.002335 & 0.006525 \\
\hline 29869 & Ulk2 & unc-51 like kinase 2 & -1.51079 & 0.002343 & 0.006532 \\
\hline 269941 & Chsy1 & chondroitin sulfate synthase 1 & -1.64039 & 0.002352 & 0.006541 \\
\hline 72157 & Pgm2 & phosphoglucomutase 2 & 1.383666 & 0.002362 & 0.00655 \\
\hline
\end{tabular}




\begin{tabular}{|c|c|c|c|c|c|}
\hline 12572 & Cdk7 & cyclin-dependent kinase 7 & -1.21154 & 0.0024 & 0.006586 \\
\hline 18806 & Pld2 & phospholipase D2 & -1.40494 & 0.002402 & 0.006588 \\
\hline 252870 & Usp7 & ubiquitin specific peptidase 7 & 1.35695 & 0.002403 & 0.006589 \\
\hline 11479 & Acvrlb & activin A receptor, type 1B & -1.24857 & 0.002459 & 0.006683 \\
\hline 243382 & Ppmlk & protein phosphatase $1 \mathrm{~K}$ (PP2C domain containing) & 11.89657 & 0.002546 & 0.006822 \\
\hline 432486 & Gnptab & $\begin{array}{l}\text { N-acetylglucosamine-1-phosphate transferase, alpha } \\
\text { and beta subunits }\end{array}$ & 1.337376 & 0.0026 & 0.006907 \\
\hline 20975 & Synj2 & synaptojanin 2 & 1.564419 & 0.002607 & 0.006918 \\
\hline 18712 & Pim1 & proviral integration site 1 & 1.447229 & 0.002717 & 0.007086 \\
\hline 72535 & Aldh $1 \mathrm{~b} 1$ & aldehyde dehydrogenase 1 family, member B1 & 7.98456 & 0.002724 & 0.007098 \\
\hline 245841 & Polr2h & polymerase (RNA) II (DNA directed) polypeptide H & -1.50153 & 0.002733 & 0.00711 \\
\hline 18975 & Polg & polymerase (DNA directed), gamma & -1.21695 & 0.00277 & 0.007166 \\
\hline 67092 & Gatm & $\begin{array}{l}\text { glycine amidinotransferase (L-arginine:glycine } \\
\text { amidinotransferase) }\end{array}$ & 1.556465 & 0.002804 & 0.007216 \\
\hline 381113 & Cdk14 & cyclin-dependent kinase-like 4 & 1.689056 & 0.002856 & 0.007291 \\
\hline 18720 & Pip5k1a & $\begin{array}{l}\text { phosphatidylinositol-4-phosphate 5-kinase, type } 1 \\
\text { alpha }\end{array}$ & -1.58835 & 0.002857 & 0.007293 \\
\hline 16068 & Il18bp & interleukin 18 binding protein & 3.267926 & 0.002899 & 0.007352 \\
\hline 18557 & Cdk18 & cyclin-dependent kinase 18 & -1.63624 & 0.002907 & 0.007364 \\
\hline 12651 & Chkb & choline kinase beta & -1.32428 & 0.002939 & 0.007409 \\
\hline 192195 & Ash11 & ash1 (absent, small, or homeotic)-like (Drosophila) & -1.25729 & 0.002976 & 0.007461 \\
\hline 19260 & Ptpn22 & $\begin{array}{l}\text { protein tyrosine phosphatase, non-receptor type } 22 \\
\text { (lymphoid) }\end{array}$ & -1.6213 & 0.003011 & 0.007509 \\
\hline 235587 & Parp3 & poly (ADP-ribose) polymerase family, member 3 & 1.32592 & 0.003018 & 0.007519 \\
\hline 16330 & Inpp $5 b$ & inositol polyphosphate-5-phosphatase B & 2.015754 & 0.003185 & 0.007751 \\
\hline 70974 & Pgm211 & phosphoglucomutase 2-like 1 & -1.76471 & 0.003221 & 0.007799 \\
\hline 13728 & Mark2 & MAP/microtubule affinity-regulating kinase 2 & -1.28083 & 0.003289 & 0.00789 \\
\hline 11637 & Ak2 & adenylate kinase 2 & -1.70646 & 0.003326 & 0.007938 \\
\hline 15490 & Hsd17b7 & hydroxysteroid (17-beta) dehydrogenase 7 & -1.48856 & 0.003339 & 0.007955 \\
\hline 18451 & P4hal & $\begin{array}{l}\text { Procollagen-proline, 2-oxoglutarate 4-dioxygenase } \\
\text { (proline 4-hydroxylase), alpha } 1 \text { polypeptide }\end{array}$ & 3.933435 & 0.003411 & 0.008047 \\
\hline 18782 & Pla2g2d & phospholipase A2, group IID & 1.383774 & 0.003418 & 0.008056 \\
\hline 69051 & Pycr2 & pyrroline-5-carboxylate reductase family, member 2 & -1.28045 & 0.003447 & 0.008093 \\
\hline 11898 & Ass1; Gm5424 & argininosuccinate synthetase 1 & 2.675768 & 0.003508 & 0.008169 \\
\hline 208449 & Sgms1 & sphingomyelin synthase 1 & -3.97643 & 0.003558 & 0.008231 \\
\hline 54667 & Atp8b2 & ATPase, class I, type 8B, member 2 & 1.542026 & 0.003566 & 0.008241 \\
\hline 18797 & Plcb3 & phospholipase C, beta 3 & 1.319991 & 0.003591 & 0.008271 \\
\hline 74112 & Usp16 & ubiquitin specific peptidase 16 & -1.75364 & 0.00361 & 0.008294 \\
\hline 235441 & Usp3 & ubiquitin specific peptidase 3 & -1.30355 & 0.003623 & 0.008309 \\
\hline 19106 & Eif2ak2 & $\begin{array}{l}\text { eukaryotic translation initiation factor 2-alpha kinase } \\
2\end{array}$ & 7.0627 & 0.003644 & 0.008335 \\
\hline 17961 & Nat2 & $\begin{array}{l}\text { N-acetyltransferase } 2 \text { (arylamine N- } \\
\text { acetyltransferase) }\end{array}$ & 1.871224 & 0.003658 & 0.008352 \\
\hline 100198 & H6pd & $\begin{array}{l}\text { Hexose-6-phosphate dehydrogenase (glucose 1- } \\
\text { dehydrogenase) }\end{array}$ & -1.40004 & 0.0037 & 0.008405 \\
\hline 56335 & Mettl3 & methyltransferase like 3 & -1.5812 & 0.00377 & 0.008498 \\
\hline 20873 & Plk4 & polo-like kinase 4 & -2.12422 & 0.003814 & 0.008554 \\
\hline 170789 & Acot8 & acyl-CoA thioesterase 8 & 1.219281 & 0.003969 & 0.008751 \\
\hline
\end{tabular}




\begin{tabular}{|c|c|c|c|c|c|}
\hline 217057 & Ptrh2 & peptidyl-tRNA hydrolase 2 & -1.65671 & 0.004074 & 0.00888 \\
\hline 50772 & Mapk6 & mitogen-activated protein kinase 6 & -1.46808 & 0.004085 & 0.008894 \\
\hline 74205 & Acsl3 & acyl-CoA synthetase long-chain family member 3 & -1.86005 & 0.004162 & 0.008987 \\
\hline 60525 & Acss 2 & acyl-CoA synthetase short-chain family member 2 & 1.563718 & 0.004216 & 0.009051 \\
\hline 11352 & Abl2 & $\begin{array}{l}\text { v-abl Abelson murine leukemia viral oncogene } 2 \\
\text { (arg, Abelson-related gene) }\end{array}$ & 2.210896 & 0.004326 & 0.009179 \\
\hline 12892 & Cpox & coproporphyrinogen oxidase & 1.315867 & 0.004342 & 0.009197 \\
\hline 16452 & Jak2 & Janus kinase 2 & 1.880227 & 0.004349 & 0.009206 \\
\hline 14923 & Guk1 & guanylate kinase 1 & 1.392089 & 0.004354 & 0.009211 \\
\hline 18105 & $\mathrm{Nqo} 2$ & $\mathrm{NAD}(\mathrm{P}) \mathrm{H}$ dehydrogenase, quinone 2 & 1.44201 & 0.004478 & 0.009352 \\
\hline 16453 & Jak3 & Janus kinase 3 & 1.373527 & 0.004486 & 0.009361 \\
\hline 19053 & Ppp2cb & $\begin{array}{l}\text { protein phosphatase } 2 \text { (formerly } 2 \mathrm{~A} \text { ), catalytic } \\
\text { subunit, beta isoform }\end{array}$ & -1.27934 & 0.004539 & 0.00942 \\
\hline 14431 & Gamt & guanidinoacetate methyltransferase & 1.289126 & 0.004568 & 0.009451 \\
\hline 235293 & Sc5d & $\begin{array}{l}\text { sterol-C5-desaturase (fungal ERG3, delta-5- } \\
\text { desaturase) homolog (S. cerevisae) }\end{array}$ & -1.52971 & 0.004788 & 0.009688 \\
\hline 69870 & Polr3gl & $\begin{array}{l}\text { polymerase (RNA) III (DNA directed) polypeptide G } \\
\text { like }\end{array}$ & 1.259242 & 0.004789 & 0.00969 \\
\hline 244349 & Kat6a & $\mathrm{K}($ lysine $)$ acetyltransferase $6 \mathrm{~A}$ & 1.615962 & 0.004857 & 0.00976 \\
\hline 17309 & Mgat3 & mannoside acetylglucosaminyltransferase 3 & 1.330662 & 0.004871 & 0.009774 \\
\hline 264064 & Cdk8 & cyclin-dependent kinase 8 & -1.50981 & 0.004879 & 0.009783 \\
\hline 107435 & Hat1 & histone aminotransferase 1 & 1.288675 & 0.004927 & 0.009833 \\
\hline 19052 & Ppp2ca & $\begin{array}{l}\text { protein phosphatase } 2 \text { (formerly } 2 \mathrm{~A} \text { ), catalytic } \\
\text { subunit, alpha isoform }\end{array}$ & -1.2089 & 0.005039 & 0.009945 \\
\hline 218461 & Pde $8 b$ & phosphodiesterase $8 \mathrm{~B}$ & 1.707761 & 0.005151 & 0.010056 \\
\hline 110279 & Bcr & breakpoint cluster region & 1.42383 & 0.005189 & 0.010093 \\
\hline 52552 & Parp8 & poly (ADP-ribose) polymerase family, member 8 & 2.366188 & 0.00527 & 0.010171 \\
\hline 58875 & Hibadh & 3-hydroxyisobutyrate dehydrogenase & 1.200547 & 0.005296 & 0.010196 \\
\hline 67418 & Ppil4 & peptidylprolyl isomerase (cyclophilin)-like 4 & -1.79395 & 0.005399 & 0.010293 \\
\hline 16798 & Lats1 & large tumor suppressor & -1.37085 & 0.005434 & 0.010325 \\
\hline 13000 & Csnk2a2 & casein kinase 2, alpha prime polypeptide & -1.2341 & 0.005472 & 0.01036 \\
\hline 67292 & Pigc & $\begin{array}{l}\text { phosphatidylinositol glycan anchor biosynthesis, } \\
\text { class C }\end{array}$ & -1.36049 & 0.005475 & 0.010363 \\
\hline 18107 & Nmt1 & N-myristoyltransferase 1 & -1.31844 & 0.005477 & 0.010365 \\
\hline 67800 & Dgat2 & diacylglycerol O-acyltransferase 2 & 1.733422 & 0.005488 & 0.010375 \\
\hline 108148 & Galnt2 & $\begin{array}{l}\text { UDP-N-acetyl-alpha-D-galactosamine:polypeptide } \\
\text { N-acetylgalactosaminyltransferase } 2\end{array}$ & 1.277501 & 0.005584 & 0.010462 \\
\hline 14158 & Fer & fer (fms/fps related) protein kinase, testis specific 2 & 1.304868 & 0.005591 & 0.010469 \\
\hline 17691 & Sik1 & salt inducible kinase 1 & 3.079703 & 0.005631 & 0.010504 \\
\hline 217119 & Xylt2 & xylosyltransferase II & -1.41727 & 0.005744 & 0.010604 \\
\hline 74178 & Stk40 & serine/threonine kinase 40 & -2.01727 & 0.005823 & 0.010673 \\
\hline 56791 & Ube216 & ubiquitin-conjugating enzyme E2L 6 & 5.899447 & 0.005833 & 0.010681 \\
\hline 14544 & Gda & guanine deaminase & 1.29685 & 0.005845 & 0.010692 \\
\hline 216136 & Ilvbl & ilvB (bacterial acetolactate synthase)-like & -2.48152 & 0.005957 & 0.010787 \\
\hline 19248 & Ptpn12 & protein tyrosine phosphatase, non-receptor type 12 & -1.87284 & 0.005972 & 0.0108 \\
\hline 56626 & Poll & polymerase (DNA directed), lambda & 1.245762 & 0.005978 & 0.010804 \\
\hline 74340 & Ahcyl2 & S-adenosylhomocysteine hydrolase-like 2 & 1.329344 & 0.005992 & 0.010816 \\
\hline
\end{tabular}




\begin{tabular}{|c|c|c|c|c|c|}
\hline 14583 & Gfpt1 & glutamine fructose-6-phosphate transaminase 1 & -1.25349 & 0.005994 & 0.010818 \\
\hline 70425 & Csnk1g3 & casein kinase 1 , gamma 3 & -1.7595 & 0.006028 & 0.010846 \\
\hline 17289 & Mertk & c-mer proto-oncogene tyrosine kinase & 1.773021 & 0.0061 & 0.010905 \\
\hline 71472 & Usp19 & ubiquitin specific peptidase 19 & -1.33552 & 0.0062 & 0.010987 \\
\hline 69635 & Dapk1 & death associated protein kinase 1 & -1.35424 & 0.006242 & 0.011021 \\
\hline 223775 & Pim3 & proviral integration site 3 & -1.29374 & 0.006338 & 0.011096 \\
\hline 18004 & Nek1 & $\begin{array}{l}\text { NIMA (never in mitosis gene a)-related expressed } \\
\text { kinase } 1\end{array}$ & 1.260282 & 0.006397 & 0.011143 \\
\hline 20443 & St3gal4 & ST3 beta-galactoside alpha-2,3-sialyltransferase 4 & 1.534809 & 0.006399 & 0.011144 \\
\hline 22436 & Xdh & xanthine dehydrogenase & 1.295806 & 0.006497 & 0.011219 \\
\hline 21812 & Tgfbrl & transforming growth factor, beta receptor I & -1.85882 & 0.006515 & 0.011234 \\
\hline 12499 & Entpd5 & ectonucleoside triphosphate diphosphohydrolase 5 & -1.23394 & 0.00654 & 0.011252 \\
\hline 14360 & Fyn & Fyn proto-oncogene & 1.296731 & 0.006567 & 0.011273 \\
\hline 98432 & Phlpp1 & $\begin{array}{l}\text { PH domain and leucine rich repeat protein } \\
\text { phosphatase } 1\end{array}$ & 1.780875 & 0.006604 & 0.011301 \\
\hline 14229 & Fkbp5 & FK506 binding protein 5 & 1.247204 & 0.006609 & 0.011305 \\
\hline 21951 & Tnks & $\begin{array}{l}\text { tankyrase, TRF1-interacting ankyrin-related ADP- } \\
\text { ribose polymerase }\end{array}$ & 1.853836 & 0.006612 & 0.011307 \\
\hline 71701 & Pnpt1 & polyribonucleotide nucleotidyltransferase 1 & 3.584633 & 0.006785 & 0.011437 \\
\hline 224860 & Plcl2 & phospholipase C-like 2 & 1.538249 & 0.006856 & 0.011489 \\
\hline 11350 & Abl1 & c-abl oncogene 1 , non-receptor tyrosine kinase & -1.78831 & 0.006862 & 0.011493 \\
\hline 50776 & Polg2 & $\begin{array}{l}\text { polymerase (DNA directed), gamma 2, accessory } \\
\text { subunit }\end{array}$ & 1.445073 & 0.00688 & 0.011506 \\
\hline 18700 & Piga & $\begin{array}{l}\text { phosphatidylinositol glycan anchor biosynthesis, } \\
\text { class A }\end{array}$ & -1.53693 & 0.006906 & 0.011525 \\
\hline 54447 & Asah2 & $\mathrm{N}$-acylsphingosine amidohydrolase 2 & 1.530461 & 0.00703 & 0.011615 \\
\hline 11717 & Ampd3 & adenosine monophosphate deaminase 3 & -3.25208 & 0.007069 & 0.011642 \\
\hline 56294 & Ptpn9 & protein tyrosine phosphatase, non-receptor type 9 & -1.28777 & 0.007296 & 0.0118 \\
\hline 15117 & Has2 & hyaluronan synthase 2 & -2.73741 & 0.007395 & 0.011867 \\
\hline 54613 & St3gal6 & ST3 beta-galactoside alpha-2,3-sialyltransferase 6 & 1.908463 & 0.007412 & 0.011879 \\
\hline 208266 & Dot11 & $\begin{array}{l}\text { DOT1-like, histone H3 methyltransferase (S. } \\
\text { cerevisiae) }\end{array}$ & -2.32878 & 0.007419 & 0.011883 \\
\hline 17169 & Mark3 & MAP/microtubule affinity-regulating kinase 3 & -1.31985 & 0.007497 & 0.011935 \\
\hline 223753 & Cerk & ceramide kinase & -1.46525 & 0.007526 & 0.011954 \\
\hline 22210 & Ube2b & ubiquitin-conjugating enzyme E2B & -1.30514 & 0.007542 & 0.011965 \\
\hline 56632 & Sphk2 & sphingosine kinase 2 & -1.39043 & 0.00755 & 0.01197 \\
\hline 244418 & D8Ertd82e & DNA segment, Chr 8, ERATO Doi 82, expressed & -4.86986 & 0.007601 & 0.012003 \\
\hline 13436 & Dnmt3b & DNA methyltransferase 3B & -1.79897 & 0.007714 & 0.012076 \\
\hline 74841 & Usp38 & ubiquitin specific peptidase 38 & -1.31434 & 0.007775 & 0.012114 \\
\hline 15356 & $\mathrm{Hmgcl}$ & 3-hydroxy-3-methylglutaryl-Coenzyme A lyase & -1.26188 & 0.007782 & 0.012119 \\
\hline 21817 & Tgm2 & transglutaminase $2, \mathrm{C}$ polypeptide & 1.547159 & 0.007805 & 0.012134 \\
\hline 230661 & Tesk2 & testis-specific kinase 2 & -3.44003 & 0.00784 & 0.012155 \\
\hline 56336 & B4galt5 & $\begin{array}{l}\text { UDP-Gal:betaGlcNAc beta } 1,4- \\
\text { galactosyltransferase, polypeptide } 5\end{array}$ & 1.967004 & 0.007846 & 0.012159 \\
\hline 171210 & Acot2 & acyl-CoA thioesterase 2 & -1.23567 & 0.007986 & 0.012246 \\
\hline 16337 & Insr & insulin receptor & 2.760092 & 0.008067 & 0.012295 \\
\hline 320119 & Rps6kc1 & ribosomal protein S6 kinase polypeptide 1 & 1.564504 & 0.00808 & 0.012303 \\
\hline
\end{tabular}




\begin{tabular}{|c|c|c|c|c|c|}
\hline 228005 & Ppig & peptidyl-prolyl isomerase G (cyclophilin G) & -2.20418 & 0.008082 & 0.012304 \\
\hline 66234 & Msmo1 & methylsterol monoxygenase 1 & -1.29911 & 0.008121 & 0.012328 \\
\hline 12795 & Plk3 & polo-like kinase 3 & -3.51263 & 0.008144 & 0.012341 \\
\hline 20454 & St3gal5 & ST3 beta-galactoside alpha-2,3-sialyltransferase 5 & -1.76047 & 0.008153 & 0.012347 \\
\hline 17161 & Maoa & monoamine oxidase $\mathrm{A}$ & 1.283626 & 0.00819 & 0.012369 \\
\hline 76367 & Trp53rkb & transformation related protein 53 regulating kinase & -1.43414 & 0.008233 & 0.012395 \\
\hline 105689 & Mycbp2 & MYC binding protein 2 & 1.577058 & 0.008307 & 0.012444 \\
\hline 26384 & Gnpda1 & glucosamine-6-phosphate deaminase 1 & -1.35069 & 0.008386 & 0.012497 \\
\hline 14426 & Galnt4 & $\begin{array}{l}\text { UDP-N-acetyl-alpha-D-galactosamine:polypeptide } \\
\mathrm{N} \text {-acetylgalactosaminyltransferase } 4\end{array}$ & -1.56396 & 0.008572 & 0.012635 \\
\hline 237898 & Usp32 & ubiquitin specific peptidase 32 & -1.47249 & 0.008607 & 0.012661 \\
\hline 20019 & Polr1a & polymerase (RNA) I polypeptide A & -1.3512 & 0.008645 & 0.012688 \\
\hline 67834 & Idh3a & isocitrate dehydrogenase $3(\mathrm{NAD}+$ ) alpha & 1.31945 & 0.00882 & 0.012815 \\
\hline 19043 & Ppmlb & $\begin{array}{l}\text { protein phosphatase 1B, magnesium dependent, beta } \\
\text { isoform }\end{array}$ & -1.76458 & 0.008938 & 0.012899 \\
\hline 18984 & Por & P450 (cytochrome) oxidoreductase & -1.32617 & 0.008943 & 0.012902 \\
\hline 238871 & Pde4d & 0 & 2.305055 & 0.009013 & 0.012951 \\
\hline 66945 & Sdha & $\begin{array}{l}\text { Succinate dehydrogenase complex, subunit A, } \\
\text { flavoprotein }(\mathrm{Fp})\end{array}$ & -1.54835 & 0.00907 & 0.01299 \\
\hline 319594 & Hiflan & hypoxia-inducible factor 1 , alpha subunit inhibitor & -1.45904 & 0.009076 & 0.012995 \\
\hline 227292 & Ctdsp1 & $\begin{array}{l}\text { CTD (carboxy-terminal domain, RNA polymerase II, } \\
\text { polypeptide A) small phosphatase } 1\end{array}$ & -1.23869 & 0.009102 & 0.013013 \\
\hline 19766 & Ripk1 & $\begin{array}{l}\text { receptor (TNFRSF)-interacting serine-threonine } \\
\text { kinase } 1\end{array}$ & 1.261217 & 0.009232 & 0.013102 \\
\hline 14479 & Usp15 & ubiquitin specific peptidase 15 & 1.577083 & 0.009239 & 0.013107 \\
\hline 109674 & Ampd2 & adenosine monophosphate deaminase 2 & -1.37284 & 0.009281 & 0.013136 \\
\hline 15258 & Hipk2 & homeodomain interacting protein kinase 2 & -1.72615 & 0.009461 & 0.013256 \\
\hline 218271 & B4galt7 & $\begin{array}{l}\text { xylosylprotein beta1,4-galactosyltransferase, } \\
\text { polypeptide } 7 \text { (galactosyltransferase I) }\end{array}$ & -1.39371 & 0.009532 & 0.013303 \\
\hline 15368 & Hmox 1 & heme oxygenase (decycling) 1 & -1.3231 & 0.009602 & 0.013349 \\
\hline 74137 & Nuak2 & NUAK family, SNF1-like kinase, 2 & 1.3503 & 0.009725 & 0.013429 \\
\hline 140780 & Bmp2k & BMP2 inducible kinase & 1.74302 & 0.009781 & 0.013464 \\
\hline 77626 & Smpd4 & sphingomyelin phosphodiesterase 4 & -1.35765 & 0.009781 & 0.013465 \\
\hline 53892 & Ppm1d & $\begin{array}{l}\text { protein phosphatase 1D magnesium-dependent, delta } \\
\text { isoform }\end{array}$ & -1.77334 & 0.009861 & 0.013516 \\
\hline 75292 & Prkd3 & protein kinase D3 & 1.25541 & 0.009889 & 0.013534 \\
\hline 14254 & Flt1 & FMS-like tyrosine kinase 1 & 1.624418 & 0.009931 & 0.01356 \\
\hline 52815 & Ldhd & lactate dehydrogenase $\mathrm{D}$ & 1.913965 & 0.010086 & 0.013657 \\
\hline 53357 & Pla2g6 & phospholipase A2, group VI & -1.9055 & 0.010119 & 0.013678 \\
\hline 76800 & Usp42 & ubiquitin specific peptidase 42 & 2.144848 & 0.010122 & 0.013679 \\
\hline 11980 & Atp8a1 & $\begin{array}{l}\text { ATPase, aminophospholipid transporter (APLT), } \\
\text { class I, type 8A, member } 1\end{array}$ & 1.596596 & 0.010126 & 0.013682 \\
\hline 140499 & Ube2j2 & ubiquitin-conjugating enzyme E2J 2 & -1.55329 & 0.010189 & 0.01372 \\
\hline 50493 & Txnrdl & thioredoxin reductase 1 & -1.36409 & 0.010229 & 0.013745 \\
\hline 67440 & Mtpap & mitochondrial poly(A) polymerase & -1.26068 & 0.010242 & 0.013753 \\
\hline 19023 & Ppef2 & $\begin{array}{l}\text { protein phosphatase, EF hand calcium-binding } \\
\text { domain } 2\end{array}$ & 1.639406 & 0.010482 & 0.013898 \\
\hline 235574 & Atp2c1 & ATPase, $\mathrm{Ca}++$-sequestering & -2.09949 & 0.010528 & 0.013925 \\
\hline
\end{tabular}




\begin{tabular}{|c|c|c|c|c|c|}
\hline 76267 & Fads1 & fatty acid desaturase 1 & -1.27061 & 0.010544 & 0.013934 \\
\hline 26939 & Polr3e & polymerase (RNA) III (DNA directed) polypeptide E & -1.51073 & 0.010694 & 0.014022 \\
\hline 23797 & Akt3 & thymoma viral proto-oncogene 3 & 1.371375 & 0.010754 & 0.014057 \\
\hline 382985 & $\operatorname{Rrm} 2 \mathrm{~b}$ & ribonucleotide reductase M2 B (TP53 inducible) & 1.374619 & 0.010819 & 0.014094 \\
\hline 23972 & Papss2 & 3'-phosphoadenosine 5'-phosphosulfate synthase 2 & -1.25264 & 0.010852 & 0.014113 \\
\hline 12496 & Entpd2 & ectonucleoside triphosphate diphosphohydrolase 2 & 1.341714 & 0.010914 & 0.014149 \\
\hline 216965 & Taok1 & TAO kinase 1 & -1.6692 & 0.010933 & 0.014159 \\
\hline 18805 & Pld1 & phospholipase D1 & -1.84578 & 0.010957 & 0.014173 \\
\hline 223722 & Mcat & malonyl CoA:ACP acyltransferase (mitochondrial) & 1.414553 & 0.011164 & 0.014289 \\
\hline 17347 & Mknk2 & MAP kinase-interacting serine/threonine kinase 2 & -1.36049 & 0.011173 & 0.014294 \\
\hline 244650 & Phlpp2 & $\begin{array}{l}\text { PH domain and leucine rich repeat protein } \\
\text { phosphatase } 2\end{array}$ & 1.237588 & 0.011291 & 0.014359 \\
\hline 104086 & Cyp27a1 & $\begin{array}{l}\text { cytochrome P450, family } 27 \text {, subfamily a, } \\
\text { polypeptide } 1\end{array}$ & 1.426482 & 0.011308 & 0.014368 \\
\hline 18578 & $\operatorname{Pde} 4 \mathrm{~b}$ & phosphodiesterase 4B, cAMP specific & -3.08994 & 0.011327 & 0.014379 \\
\hline 18125 & Nos1 & nitric oxide synthase 1 , neuronal & 1.568898 & 0.011379 & 0.014406 \\
\hline 26403 & Map3k11 & mitogen-activated protein kinase kinase kinase 11 & -2.27656 & 0.011438 & 0.014438 \\
\hline 22367 & Vrk1 & vaccinia related kinase 1 & 1.434406 & 0.011641 & 0.014547 \\
\hline 330177 & Taok3 & TAO kinase 3 & 1.658102 & 0.011685 & 0.01457 \\
\hline 94192 & C1galt1 & $\begin{array}{l}\text { core } 1 \text { synthase, glycoprotein-N-acetylgalactosamine } \\
\text { 3-beta-galactosyltransferase, } 1\end{array}$ & 1.219714 & 0.011886 & 0.014675 \\
\hline 19063 & Ppt1 & palmitoyl-protein thioesterase 1 & 1.216261 & 0.011903 & 0.014683 \\
\hline 327942 & Pigl & $\begin{array}{l}\text { phosphatidylinositol glycan anchor biosynthesis, } \\
\text { class L }\end{array}$ & -1.86476 & 0.011942 & 0.014703 \\
\hline 59028 & Rcl1 & RNA terminal phosphate cyclase-like 1 & -1.41172 & 0.012008 & 0.014737 \\
\hline 110821 & Pcca & $\begin{array}{l}\text { propionyl-Coenzyme A carboxylase, alpha } \\
\text { polypeptide }\end{array}$ & 1.704841 & 0.012016 & 0.014741 \\
\hline 12747 & Clk1 & CDC-like kinase 1 & -2.09933 & 0.012207 & 0.014838 \\
\hline 27369 & Dguok & deoxyguanosine kinase & 1.328536 & 0.01221 & 0.014839 \\
\hline 218294 & $\mathrm{Cdc} 14 \mathrm{~b}$ & CDC14 cell division cycle 14B & -1.50503 & 0.012272 & 0.01487 \\
\hline 18601 & Padi3 & peptidyl arginine deiminase, type III & -1.45729 & 0.012301 & 0.014884 \\
\hline 50523 & Lats2 & large tumor suppressor 2 & -1.53141 & 0.012463 & 0.015003 \\
\hline 11477 & Acvr1 & activin A receptor, type 1 & 1.254986 & 0.012613 & 0.015111 \\
\hline 50784 & Plpp2 & phosphatidic acid phosphatase type $2 \mathrm{C}$ & 1.21339 & 0.012616 & 0.015113 \\
\hline 30941 & Usp21 & ubiquitin specific peptidase 21 & 1.313759 & 0.012679 & 0.015158 \\
\hline 216150 & $\mathrm{Cdc} 34$ & cell division cycle 34 & -1.2241 & 0.012899 & 0.015314 \\
\hline 19057 & Ppp3cc & $\begin{array}{l}\text { protein phosphatase 3, catalytic subunit, gamma } \\
\text { isoform }\end{array}$ & -1.47316 & 0.012922 & 0.015331 \\
\hline 66136 & Znrd1 & zinc ribbon domain containing, 1 & 1.227041 & 0.013034 & 0.015409 \\
\hline 108083 & Pip4k2b & $\begin{array}{l}\text { phosphatidylinositol-5-phosphate 4-kinase, type II, } \\
\text { beta }\end{array}$ & -1.46747 & 0.013344 & 0.015626 \\
\hline 67005 & Polr3k & polymerase (RNA) III (DNA directed) polypeptide K & -1.21192 & 0.01338 & 0.015651 \\
\hline 67897 & Rnmt & RNA (guanine-7-) methyltransferase & -1.96504 & 0.013416 & 0.015676 \\
\hline 22245 & Uck1 & uridine-cytidine kinase 1 & -1.25283 & 0.01356 & 0.015775 \\
\hline 63953 & Dusp10 & dual specificity phosphatase 10 & 1.687271 & 0.013629 & 0.015822 \\
\hline 18574 & Pdelb & phosphodiesterase 1B, Ca2+-calmodulin dependent & -1.69355 & 0.013755 & 0.015907 \\
\hline 57295 & Icmt & isoprenylcysteine carboxyl methyltransferase & -1.53956 & 0.01403 & 0.016092 \\
\hline
\end{tabular}




\begin{tabular}{|c|c|c|c|c|c|}
\hline 26409 & Map3k7 & mitogen-activated protein kinase kinase kinase 7 & -1.20091 & 0.014046 & 0.016102 \\
\hline 105278 & $\mathrm{Cdk} 20$ & cyclin-dependent kinase 20 & -1.6885 & 0.014187 & 0.016196 \\
\hline 17346 & Mknk1 & MAP kinase-interacting serine/threonine kinase 1 & -1.56621 & 0.014199 & 0.016204 \\
\hline 75735 & Pank1 & pantothenate kinase 1 & -1.29244 & 0.014302 & 0.016271 \\
\hline 69181 & Dyrk2 & $\begin{array}{l}\text { dual-specificity tyrosine-(Y)-phosphorylation } \\
\text { regulated kinase } 2\end{array}$ & -1.40152 & 0.01435 & 0.016303 \\
\hline 16329 & Inpp1 & inositol polyphosphate-1-phosphatase & 2.871469 & 0.014416 & 0.016346 \\
\hline 20111 & Rps6ka1 & ribosomal protein S6 kinase polypeptide 1 & -1.29241 & 0.014715 & 0.016538 \\
\hline 66812 & Ppcdc & phosphopantothenoylcysteine decarboxylase & 1.39982 & 0.014803 & 0.016594 \\
\hline 59047 & Pnkp & polynucleotide kinase 3 '- phosphatase & -1.38291 & 0.014864 & 0.016633 \\
\hline 20874 & Slk & STE20-like kinase & -1.45548 & 0.014916 & 0.016666 \\
\hline 20868 & Stk10 & serine/threonine kinase 10 & -1.52357 & 0.014939 & 0.01668 \\
\hline 12613 & Cel & carboxyl ester lipase & 1.673997 & 0.014952 & 0.016688 \\
\hline 70661 & Sik3 & SIK family kinase 3 & 1.41369 & 0.015102 & 0.016783 \\
\hline 23939 & Mapk7 & mitogen-activated protein kinase 7 & -2.63017 & 0.015265 & 0.016884 \\
\hline 51789 & Tnk2 & tyrosine kinase, non-receptor, 2 & 1.352419 & 0.015663 & 0.017167 \\
\hline 22213 & Ube2g2 & ubiquitin-conjugating enzyme E2G 2 & -1.32789 & 0.015878 & 0.017317 \\
\hline 18607 & Pdpk1 & 3-phosphoinositide dependent protein kinase 1 & -1.6214 & 0.015882 & 0.01732 \\
\hline 59031 & Chst12 & carbohydrate sulfotransferase 12 & -1.4617 & 0.016088 & 0.017463 \\
\hline 217734 & Pomt2 & protein-O-mannosyltransferase 2 & 1.436724 & 0.016463 & 0.017719 \\
\hline 19229 & Ptk2b & PTK2 protein tyrosine kinase 2 beta & 1.300954 & 0.016476 & 0.017728 \\
\hline 20425 & Shmt1 & serine hydroxymethyltransferase 1 (soluble) & 1.533136 & 0.016707 & 0.017884 \\
\hline 12660 & Chka & choline kinase alpha & -1.6828 & 0.016945 & 0.018043 \\
\hline 15531 & Ndst1 & $\begin{array}{l}\text { N-deacetylase/N-sulfotransferase (heparan } \\
\text { glucosaminyl) } 1\end{array}$ & -1.221 & 0.017104 & 0.018148 \\
\hline 269180 & Inpp 4a & inositol polyphosphate-4-phosphatase, type I & -1.26696 & 0.017126 & 0.018163 \\
\hline 74493 & Tnks2 & $\begin{array}{l}\text { tankyrase, TRF1-interacting ankyrin-related ADP- } \\
\text { ribose polymerase } 2\end{array}$ & -1.26016 & 0.017165 & 0.018188 \\
\hline 60507 & Qtrt1 & queuine tRNA-ribosyltransferase 1 & -1.3732 & 0.017348 & 0.018308 \\
\hline 70008 & Ace2 & $\begin{array}{l}\text { angiotensin I converting enzyme (peptidyl- } \\
\text { dipeptidase A) } 2\end{array}$ & -2.90032 & 0.017391 & 0.018336 \\
\hline 74414 & Polr3c & polymerase (RNA) III (DNA directed) polypeptide C & 1.266901 & 0.017584 & 0.018462 \\
\hline 192185 & Nadk & NAD kinase & -1.27237 & 0.017658 & 0.018509 \\
\hline 399510 & Map4k5 & $\begin{array}{l}\text { mitogen-activated protein kinase kinase kinase } \\
\text { kinase } 5\end{array}$ & -1.68361 & 0.017727 & 0.018554 \\
\hline 192656 & Ripk2 & $\begin{array}{l}\text { receptor (TNFRSF)-interacting serine-threonine } \\
\text { kinase } 2\end{array}$ & 1.595815 & 0.018076 & 0.018776 \\
\hline 18218 & Dusp8 & dual specificity phosphatase 8 & -2.81691 & 0.018349 & 0.018947 \\
\hline 26405 & Map3k2 & mitogen-activated protein kinase kinase kinase 2 & -1.65601 & 0.018523 & 0.019055 \\
\hline 13382 & Dld & dihydrolipoamide dehydrogenase & -1.23751 & 0.018609 & 0.019109 \\
\hline 15162 & Hck & hemopoietic cell kinase & 1.260567 & 0.018667 & 0.019144 \\
\hline 68082 & Dusp19 & dual specificity phosphatase 19 & 1.218342 & 0.018736 & 0.019187 \\
\hline 67655 & Ctdp1 & $\begin{array}{l}\text { CTD (carboxy-terminal domain, RNA polymerase II, } \\
\text { polypeptide A) phosphatase, subunit } 1\end{array}$ & 1.394635 & 0.018741 & 0.01919 \\
\hline 17156 & Man1a2 & mannosidase, alpha, class $1 \mathrm{~A}$, member 2 & 1.266249 & 0.018747 & 0.019194 \\
\hline 231327 & Ppat & phosphoribosyl pyrophosphate amidotransferase & -1.28172 & 0.019061 & 0.019385 \\
\hline 72039 & Mcce1 & methylcrotonoyl-Coenzyme A carboxylase 1 (alpha) & -1.40604 & 0.019189 & 0.019462 \\
\hline
\end{tabular}




\begin{tabular}{|c|c|c|c|c|c|}
\hline 13121 & Cyp51 & cytochrome P450, family 51 & -1.93693 & 0.019259 & 0.019505 \\
\hline 64580 & Ndst4 & $\begin{array}{l}\text { N-deacetylase/N-sulfotransferase (heparin } \\
\text { glucosaminyl) } 4\end{array}$ & -1.71606 & 0.019409 & 0.019594 \\
\hline 108960 & Irak2 & interleukin-1 receptor-associated kinase 2 & -1.47996 & 0.019806 & 0.01983 \\
\hline 108079 & Prkaa2 & $\begin{array}{l}\text { protein kinase, AMP-activated, alpha } 2 \text { catalytic } \\
\text { subunit }\end{array}$ & 1.859203 & 0.019835 & 0.019847 \\
\hline 78783 & Brpf1 & bromodomain and PHD finger containing, 1 & -1.4743 & 0.019837 & 0.019848 \\
\hline 26363 & Btd & biotinidase & -1.25829 & 0.020051 & 0.019972 \\
\hline 109979 & Art3 & ADP-ribosyltransferase 3 & 2.756145 & 0.02009 & 0.019995 \\
\hline 20810 & Srm & spermidine synthase & -1.24836 & 0.020133 & 0.02002 \\
\hline 66590 & Farsa & phenylalanyl-tRNA synthetase, alpha subunit & 1.243331 & 0.02014 & 0.020024 \\
\hline 108682 & Gpt2 & $\begin{array}{l}\text { glutamic pyruvate transaminase (alanine } \\
\text { aminotransferase) } 2\end{array}$ & 1.248706 & 0.020187 & 0.020051 \\
\hline 67486 & Polr3g & polymerase (RNA) III (DNA directed) polypeptide G & -1.83334 & 0.02033 & 0.020134 \\
\hline 13807 & Eno2 & enolase 2, gamma neuronal & -3.29195 & 0.020462 & 0.02021 \\
\hline 330010 & Tt1110 & tubulin tyrosine ligase-like family, member 10 & 1.309144 & 0.020568 & 0.02027 \\
\hline 235344 & Sik2 & salt inducible kinase 2 & -1.3557 & 0.020587 & 0.020281 \\
\hline 56542 & Ick & intestinal cell kinase & -1.27013 & 0.020674 & 0.02033 \\
\hline 24018 & Rngtt & RNA guanylyltransferase and 5'-phosphatase & -1.25735 & 0.02133 & 0.020697 \\
\hline 13666 & Eif2ak3 & $\begin{array}{l}\text { eukaryotic translation initiation factor } 2 \text { alpha kinase } \\
3\end{array}$ & -1.83592 & 0.021498 & 0.020789 \\
\hline 70686 & Dusp16 & dual specificity phosphatase 16 & -1.50198 & 0.021515 & 0.020799 \\
\hline 20652 & Soat1 & 0 & -1.4004 & 0.021599 & 0.020845 \\
\hline 19134 & Prpf4b & $\begin{array}{l}\text { PRP4 pre-mRNA processing factor } 4 \text { homolog B } \\
\text { (yeast) }\end{array}$ & -1.38407 & 0.021736 & 0.020919 \\
\hline 14859 & Gsta3 & glutathione S-transferase, alpha 3 & -1.66324 & 0.021832 & 0.020971 \\
\hline 109232 & Sccpdh & saccharopine dehydrogenase (putative) & -1.31675 & 0.021886 & 0.021 \\
\hline 11655 & Alas1 & aminolevulinic acid synthase 1 & -1.28258 & 0.021964 & 0.021042 \\
\hline 69740 & Dph5 & DPH5 homolog (S. cerevisiae) & -1.52944 & 0.022093 & 0.021111 \\
\hline 64436 & Inpp5e & inositol polyphosphate-5-phosphatase E & -1.41245 & 0.022351 & 0.021249 \\
\hline 16818 & Lck & lymphocyte protein tyrosine kinase & 2.599679 & 0.023085 & 0.021631 \\
\hline 13026 & Pcyt1a & $\begin{array}{l}\text { phosphate cytidylyltransferase } 1 \text {, choline, alpha } \\
\text { isoform }\end{array}$ & 1.350674 & 0.023118 & 0.021649 \\
\hline 56720 & Tdo2 & tryptophan 2,3-dioxygenase & 1.629909 & 0.023142 & 0.021661 \\
\hline 66848 & Fuca2 & fucosidase, alpha-L- 2, plasma & -1.46667 & 0.02319 & 0.021685 \\
\hline 13435 & Dnmt3a & DNA methyltransferase 3A & -1.35289 & 0.023242 & 0.021712 \\
\hline 328572 & Ep300 & E1A binding protein $\mathrm{p} 300$ & 1.246388 & 0.02332 & 0.021752 \\
\hline 67980 & Gnpda2 & glucosamine-6-phosphate deaminase 2 & 1.549614 & 0.02335 & 0.021767 \\
\hline 22241 & Ulk1 & unc-51 like kinase 1 & -2.146 & 0.023422 & 0.021804 \\
\hline 218232 & Ptpde1 & protein tyrosine phosphatase domain containing 1 & 1.681793 & 0.023442 & 0.021814 \\
\hline 71742 & Ulk3 & unc-51-like kinase 3 & -1.39685 & 0.023599 & 0.021893 \\
\hline 110960 & Tars & threonyl-tRNA synthetase & -1.21244 & 0.02374 & 0.021964 \\
\hline 237459 & Cdk17 & cyclin-dependent kinase 17 & -1.61204 & 0.023915 & 0.022052 \\
\hline 170707 & Usp48 & ubiquitin specific peptidase 48 & -1.28824 & 0.024102 & 0.022153 \\
\hline 26921 & Map4k4 & $\begin{array}{l}\text { mitogen-activated protein kinase kinase kinase } \\
\text { kinase } 4\end{array}$ & -4.64958 & 0.024257 & 0.022239 \\
\hline 11652 & Akt2 & thymoma viral proto-oncogene 2 & -1.26628 & 0.024321 & 0.022275 \\
\hline
\end{tabular}




\begin{tabular}{|c|c|c|c|c|c|}
\hline 19255 & Ptpn2 & protein tyrosine phosphatase, non-receptor type 2 & 1.279237 & 0.024441 & 0.022341 \\
\hline 27103 & Eif2ak4 & $\begin{array}{l}\text { eukaryotic translation initiation factor } 2 \text { alpha kinase } \\
4\end{array}$ & -1.40149 & 0.02466 & 0.022467 \\
\hline 53418 & B4galt2 & $\begin{array}{l}\text { coiled-coil domain containing 24///UDP- } \\
\text { Gal:betaGlcNAc beta 1,4- galactosyltransferase, } \\
\text { polypeptide } 2\end{array}$ & 1.551705 & 0.025354 & 0.022861 \\
\hline 53859 & 1700028N14Rik & RIKEN cDNA 1700028 N14 gene & -1.58298 & 0.025508 & 0.022948 \\
\hline 98267 & Stk17b & serine/threonine kinase $17 \mathrm{~b}$ (apoptosis-inducing) & -2.01955 & 0.025615 & 0.023007 \\
\hline 212032 & Hk3 & hexokinase 3 & 1.311769 & 0.025732 & 0.023072 \\
\hline 55979 & Agpat1 & $\begin{array}{l}\text { 1-acylglycerol-3-phosphate O-acyltransferase } 1 \\
\text { (lysophosphatidic acid acyltransferase, alpha) }\end{array}$ & 1.283884 & 0.025892 & 0.023161 \\
\hline 20442 & St3gal1 & ST3 beta-galactoside alpha-2,3-sialyltransferase 1 & -1.33836 & 0.026443 & 0.023463 \\
\hline 15159 & Hecs & holocytochrome c synthetase & -1.47077 & 0.026605 & 0.023551 \\
\hline 269252 & Gtf3c4 & general transcription factor IIIC, polypeptide 4 & -1.39156 & 0.026685 & 0.023594 \\
\hline 214897 & Csnk1g1 & casein kinase 1 , gamma 1 & -1.5867 & 0.026797 & 0.023654 \\
\hline 27052 & Aoah & acyloxyacyl hydrolase & -4.60746 & 0.026899 & 0.023709 \\
\hline 11847 & Arg2 & arginase type II & -2.1052 & 0.027218 & 0.02388 \\
\hline 18181 & Nrf1 & nuclear respiratory factor 1 & -1.21371 & 0.027414 & 0.023984 \\
\hline 14137 & Fdft1 & farnesyl diphosphate farnesyl transferase 1 & -1.95442 & 0.027698 & 0.024133 \\
\hline 29863 & Pde $7 b$ & phosphodiesterase 7B & 1.646263 & 0.027714 & 0.024142 \\
\hline 546071 & Mast3 & microtubule associated serine/threonine kinase 3 & -1.98685 & 0.028145 & 0.024366 \\
\hline 14104 & Fasn & fatty acid synthase & -1.40158 & 0.028175 & 0.024382 \\
\hline 18753 & Prkcd & protein kinase $\mathrm{C}$, delta & -2.88952 & 0.028658 & 0.02463 \\
\hline 26362 & Axl & AXL receptor tyrosine kinase & 1.709075 & 0.028998 & 0.024802 \\
\hline 78943 & Ern1 & endoplasmic reticulum (ER) to nucleus signalling 1 & 1.392665 & 0.029091 & 0.02485 \\
\hline 216578 & $\begin{array}{l}\text { Papolg; } \\
\text { LOC553090 }\end{array}$ & predicted gene, 19887///uncharacterized LOC553090 & -1.88766 & 0.029503 & 0.025056 \\
\hline 26447 & Poli & polymerase (DNA directed), iota & 1.391949 & 0.029567 & 0.025088 \\
\hline 59125 & Nek7 & $\begin{array}{l}\text { NIMA (never in mitosis gene a)-related expressed } \\
\text { kinase } 7\end{array}$ & 1.359472 & 0.029634 & 0.025121 \\
\hline 20016 & Polr1c & polymerase (RNA) I polypeptide C & -1.26527 & 0.029718 & 0.025163 \\
\hline 230809 & Pdik11 & PDLIM1 interacting kinase 1 like & -1.40269 & 0.029792 & 0.0252 \\
\hline 69718 & Ipmk & inositol polyphosphate multikinase & -1.26203 & 0.029828 & 0.025217 \\
\hline 17164 & Mapkapk2 & MAP kinase-activated protein kinase 2 & -1.49365 & 0.030583 & 0.025586 \\
\hline 76187 & Adhfe 1 & alcohol dehydrogenase, iron containing, 1 & -1.7943 & 0.03059 & 0.02559 \\
\hline 67128 & Ube2g1 & ubiquitin-conjugating enzyme E2G 1 & -1.3979 & 0.031169 & 0.0259 \\
\hline 76055 & Mgea5 & meningioma expressed antigen 5 (hyaluronidase) & -1.45901 & 0.031508 & 0.026081 \\
\hline 15357 & Hmgcr & 3-hydroxy-3-methylglutaryl-Coenzyme A reductase & -1.55728 & 0.031846 & 0.026259 \\
\hline 26462 & Txnrd2 & thioredoxin reductase 2 & 1.414386 & 0.032448 & 0.026573 \\
\hline 71773 & Ugt2b1 & $\begin{array}{l}\text { UDP glucuronosyltransferase } 2 \text { family, polypeptide } \\
\text { B1 }\end{array}$ & 1.322566 & 0.03304 & 0.026877 \\
\hline 240505 & Cdc42bpg & CDC42 binding protein kinase gamma (DMPK-like) & -1.41541 & 0.033329 & 0.027025 \\
\hline 18717 & Pip5k1c & $\begin{array}{l}\text { phosphatidylinositol-4-phosphate 5-kinase, type } 1 \\
\text { gamma }\end{array}$ & -1.42534 & 0.033331 & 0.027026 \\
\hline 18803 & Plcg1 & phospholipase $\mathrm{C}$, gamma 1 & -1.48674 & 0.033525 & 0.027124 \\
\hline 103135 & Pan2 & $\begin{array}{l}\text { PAN2 polyA specific ribonuclease subunit homolog } \\
\text { (S. cerevisiae) }\end{array}$ & -1.25014 & 0.033675 & 0.0272 \\
\hline
\end{tabular}




\begin{tabular}{|c|c|c|c|c|c|}
\hline 19878 & Rock2 & $\begin{array}{l}\text { Rho-associated coiled-coil containing protein kinase } \\
2\end{array}$ & -1.79832 & 0.033724 & 0.027224 \\
\hline 76500 & Ip6k2 & inositol hexaphosphate kinase 2 & -1.52614 & 0.034196 & 0.027461 \\
\hline 117592 & B3galt6 & $\begin{array}{l}\text { UDP-Gal:betaGal beta 1,3-galactosyltransferase, } \\
\text { polypeptide } 6\end{array}$ & -1.44205 & 0.034373 & 0.027549 \\
\hline 320951 & Pisd & phosphatidylserine decarboxylase & -1.21276 & 0.034497 & 0.02761 \\
\hline 216134 & Pdxk & pyridoxal (pyridoxine, vitamin B6) kinase & -1.22717 & 0.034647 & 0.027684 \\
\hline 14194 & Fh1 & fumarate hydratase 1 & 1.227958 & 0.034754 & 0.027737 \\
\hline 20598 & Smpd2 & sphingomyelin phosphodiesterase 2 , neutral & -1.20759 & 0.035528 & 0.028115 \\
\hline 235626 & Setd2 & SET domain containing 2 & -1.29035 & 0.035709 & 0.028202 \\
\hline 56318 & Acpp & acid phosphatase, prostate & 1.27834 & 0.035804 & 0.028248 \\
\hline 11790 & Speg & SPEG complex locus & -1.29609 & 0.035875 & 0.028282 \\
\hline 19211 & Pten & phosphatase and tensin homolog & -1.56514 & 0.03604 & 0.028361 \\
\hline 76179 & Usp31 & ubiquitin specific peptidase 31 & -1.70549 & 0.036284 & 0.028478 \\
\hline 16987 & Lss & lanosterol synthase & -1.48188 & 0.036924 & 0.02878 \\
\hline 77697 & Mmab & $\begin{array}{l}\text { methylmalonic aciduria (cobalamin deficiency) type } \\
\text { B homolog (human) }\end{array}$ & -1.4977 & 0.037195 & 0.028907 \\
\hline 52123 & Agpat5 & $\begin{array}{l}\text { 1-acylglycerol-3-phosphate O-acyltransferase } 5 \\
\text { (lysophosphatidic acid acyltransferase, epsilon) }\end{array}$ & -1.21852 & 0.037262 & 0.028938 \\
\hline 67333 & Stk35 & serine/threonine kinase 35 & -1.98887 & 0.03764 & 0.029113 \\
\hline 110350 & 2610019N06Rik & $\begin{array}{l}\text { uncharacterized LOC100505161///RIKEN cDNA } \\
\text { 2610019N06 gene }\end{array}$ & -3.08925 & 0.038307 & 0.029419 \\
\hline 269614 & Pank4 & pantothenate kinase 4 & -1.37862 & 0.038512 & 0.029513 \\
\hline 18711 & Pikfyve & phosphoinositide kinase, FYVE finger containing & 1.483283 & 0.038925 & 0.029699 \\
\hline 52538 & Acaa2 & $\begin{array}{l}\text { acetyl-Coenzyme A acyltransferase } 2 \text { (mitochondrial } \\
\text { 3-oxoacyl-Coenzyme A thiolase) }\end{array}$ & 1.234894 & 0.0391 & 0.029778 \\
\hline 57813 & $\mathrm{Tk} 2$ & thymidine kinase 2 , mitochondrial & -1.24443 & 0.03962 & 0.030029 \\
\hline 113868 & Acaa1a & acetyl-Coenzyme A acyltransferase 1A & 1.209673 & 0.039994 & 0.03021 \\
\hline 104110 & Adcy 4 & adenylate cyclase 4 & 1.302206 & 0.040024 & 0.030225 \\
\hline 20817 & Srpk2 & serine/arginine-rich protein specific kinase 2 & -1.39881 & 0.040589 & 0.030496 \\
\hline 55936 & Ctps2 & cytidine 5'-triphosphate synthase 2 & -1.54353 & 0.040658 & 0.030529 \\
\hline 12571 & Cdk6 & cyclin-dependent kinase 6 & 1.473677 & 0.04112 & 0.030749 \\
\hline 225997 & Trpm6 & $\begin{array}{l}\text { transient receptor potential cation channel, subfamily } \\
\mathrm{M} \text {, member } 6\end{array}$ & 1.28623 & 0.041195 & 0.030785 \\
\hline 53376 & Usp2 & ubiquitin specific peptidase 2 & 1.327027 & 0.041224 & 0.030798 \\
\hline 19271 & Ptprj & protein tyrosine phosphatase, receptor type, J & -1.20434 & 0.041543 & 0.030949 \\
\hline 78894 & Aacs & acetoacetyl-CoA synthetase & -1.31949 & 0.042598 & 0.03144 \\
\hline 100756 & Usp30 & ubiquitin specific peptidase 30 & -1.20304 & 0.043095 & 0.031669 \\
\hline 210044 & Adcy 2 & adenylate cyclase 2 & 2.480524 & 0.04354 & 0.031872 \\
\hline 26419 & Mapk8 & mitogen-activated protein kinase 8 & -1.7321 & 0.043732 & 0.031959 \\
\hline 69562 & $\mathrm{Cdk} 13$ & cyclin-dependent kinase 13 & -1.45115 & 0.044658 & 0.032374 \\
\hline 56737 & Alg2 & $\begin{array}{l}\text { asparagine-linked glycosylation } 2 \text { (alpha-1,3- } \\
\text { mannosyltransferase) }\end{array}$ & -1.99622 & 0.044719 & 0.032401 \\
\hline 109880 & Braf & Braf transforming gene & -1.49128 & 0.044809 & 0.032441 \\
\hline 13885 & Gm2904; Esd & esterase $\mathrm{D} /$ formylglutathione hydrolase & -2.15361 & 0.044847 & 0.032458 \\
\hline 381511 & Pdp1 & $\begin{array}{l}\text { pyruvate dehyrogenase phosphatase catalytic subunit } \\
1\end{array}$ & -1.6266 & 0.045118 & 0.032579 \\
\hline 93683 & Glce & glucuronyl C5-epimerase & -1.28658 & 0.045532 & 0.032761 \\
\hline
\end{tabular}




\begin{tabular}{|r|l|l|r|r|r|}
15257 & Hipk1 & homeodomain interacting protein kinase 1 & -1.23139 & 0.046056 & 0.03299 \\
\hline 26401 & Map3k1 & mitogen-activated protein kinase kinase kinase 1 & 1.286126 & 0.046773 & 0.033301 \\
\hline 22138 & Ttn & titin & -1.49931 & 0.047029 & 0.033411 \\
\hline 217214 & Nags & N-acetylglutamate synthase & 1.304931 & 0.047036 & 0.033414 \\
\hline 76295 & Atp11b & ATPase, class VI, type 11B & 1.343552 & 0.047218 & 0.033492 \\
\hline 243085 & Ugt2b35 & $\begin{array}{l}\text { UDP glucuronosyltransferase 2 family, polypeptide } \\
\text { B35 }\end{array}$ & 1.328672 & 0.047271 & 0.033515 \\
\hline 28169 & Agpat3 & 1-acylglycerol-3-phosphate O-acyltransferase 3 & -1.34678 & 0.047552 & 0.033635 \\
\hline 19714 & Rev31 & $\begin{array}{l}\text { REV3-like, catalytic subunit of DNA polymerase } \\
\text { zeta RAD54 like (S. cerevisiae) }\end{array}$ & 1.849724 & 0.049033 & 0.034272 \\
\hline 14782 & Gsr & glutathione reductase & -1.26175 & 0.049253 & 0.034365 \\
\hline 19280 & Ptprs & protein tyrosine phosphatase, receptor type, S & 1.289708 & 0.049669 & 0.034541 \\
\hline
\end{tabular}




\section{Supplemental Table S2.2: A list of all metabolic genes identified in the MDM dataset.}

\begin{tabular}{|c|c|c|c|c|c|}
\hline $\begin{array}{l}\text { Entrez } \\
\text { Gene } \\
\text { ID }\end{array}$ & Gene Symbol & Gene Title & $\begin{array}{l}\text { Fold } \\
\text { Change }\end{array}$ & P-value & FDR \\
\hline 3717 & Jak2 & Janus kinase 2 & 3.176442 & $1.89 \mathrm{E}-05$ & 0.009626 \\
\hline 5533 & PPP3CC & $\begin{array}{l}\text { protein phosphatase } 3 \text { (formerly } 2 \mathrm{~B} \text { ), catalytic subunit, } \\
\text { gamma isoform }\end{array}$ & 2.236797 & $3.69 \mathrm{E}-05$ & 0.009626 \\
\hline 11011 & TLK2 & tousled-like kinase 2 & 2.723902 & $5.34 \mathrm{E}-05$ & 0.009626 \\
\hline 64761 & PARP12 & poly (ADP-ribose) polymerase family, member 12 & 2.268545 & $5.51 \mathrm{E}-05$ & 0.009626 \\
\hline 23032 & usp33 & ubiquitin specific peptidase 33 & 1.424304 & $8.84 \mathrm{E}-05$ & 0.012347 \\
\hline 5775 & PTPN4 & $\begin{array}{l}\text { protein tyrosine phosphatase, non-receptor type } 4 \\
\text { (megakaryocyte) }\end{array}$ & -2.01923 & 0.000127 & 0.014743 \\
\hline 5142 & $\mathrm{Pde} 4 \mathrm{~b}$ & $\begin{array}{l}\text { phosphodiesterase 4B, cAMP-specific } \\
\text { (phosphodiesterase E4 dunce homolog, Drosophila) }\end{array}$ & 6.353167 & 0.000219 & 0.019543 \\
\hline 8972 & LOC642103 & similar to Maltase-glucoamylase, intestinal & 4.678397 & 0.000227 & 0.019862 \\
\hline 2180 & ACSL1 & acyl-CoA synthetase long-chain family member 1 & 2.276471 & 0.0003 & 0.022328 \\
\hline 10135 & NAMPT & nicotinamide phosphoribosyltransferase & 3.449473 & 0.000333 & 0.023236 \\
\hline 2710 & GK3P & glycerol kinase 3 pseudogene; glycerol kinase & 1.73406 & 0.0004 & 0.024744 \\
\hline 10114 & Hipk3 & homeodomain interacting protein kinase 3 & 1.428001 & 0.000437 & 0.025458 \\
\hline 3631 & INPP4A & inositol polyphosphate-4-phosphatase, type I, $107 \mathrm{kDa}$ & -2.21237 & 0.000534 & 0.02713 \\
\hline 1540 & CYLD & cylindromatosis (turban tumor syndrome) & 2.37934 & 0.000579 & 0.027773 \\
\hline 1545 & CYP1B1 & cytochrome P450, family 1 , subfamily B, polypeptide 1 & 1.536851 & 0.000617 & 0.028272 \\
\hline 5168 & ENPP2 & ectonucleotide pyrophosphatase/phosphodiesterase 2 & 4.555246 & 0.000659 & 0.02876 \\
\hline 5610 & EIF2AK2 & eukaryotic translation initiation factor 2-alpha kinase 2 & 3.172761 & 0.000766 & 0.029828 \\
\hline 80146 & UXS1 & UDP-glucuronate decarboxylase 1 & 1.726443 & 0.000832 & 0.030379 \\
\hline 6793 & STK10 & serine/threonine kinase 10 & -1.50535 & 0.000952 & 0.031219 \\
\hline 7326 & ube2g1 & $\begin{array}{l}\text { ubiquitin-conjugating enzyme E2G } 1 \text { (UBC7 homolog, } \\
\text { yeast) }\end{array}$ & -1.46646 & 0.001118 & 0.032139 \\
\hline 8050 & PDHX & Pyruvate dehydrogenase complex, component X & 1.515724 & 0.001146 & 0.03227 \\
\hline 1847 & dusp5 & dual specificity phosphatase 5 & 3.340293 & 0.001176 & 0.032408 \\
\hline 57205 & ATP10D & ATPase, class V, type 10D & -1.77633 & 0.001202 & 0.032521 \\
\hline 84446 & Brsk1 & BR serine/threonine kinase 1 & 1.901541 & 0.001206 & 0.032539 \\
\hline 3735 & KARS & lysyl-tRNA synthetase & 1.388079 & 0.001277 & 0.032828 \\
\hline 9334 & B4galt5 & $\begin{array}{l}\text { UDP-Gal:betaGlcNAc beta 1,4- galactosyltransferase, } \\
\text { polypeptide } 5\end{array}$ & 3.402661 & 0.001314 & 0.032966 \\
\hline 51056 & LAP3 & leucine aminopeptidase 3 & 1.775856 & 0.001383 & 0.033211 \\
\hline 57194 & ATP10A & ATPase, class V, type 10A & 4.50366 & 0.001433 & 0.033375 \\
\hline 4329 & Aldh6a1 & aldehyde dehydrogenase 6 family, member A1 & -1.38328 & 0.001481 & 0.033524 \\
\hline 1845 & DUSP3 & dual specificity phosphatase 3 & 1.37165 & 0.00153 & 0.033666 \\
\hline 1358 & Cpa2 & carboxypeptidase A2 (pancreatic) & 2.552861 & 0.001559 & 0.033748 \\
\hline 644 & BLVRA & biliverdin reductase $\mathrm{A}$ & 1.543476 & 0.001573 & 0.033786 \\
\hline 5566 & PRKACA & protein kinase, cAMP-dependent, catalytic, alpha & -1.28369 & 0.001634 & 0.033945 \\
\hline 2643 & $\mathrm{GCH} 1$ & GTP cyclohydrolase 1 & 22.51212 & 0.001689 & 0.034081 \\
\hline 4507 & $\mathrm{mtaP}$ & methylthioadenosine phosphorylase & -1.44946 & 0.00171 & 0.034132 \\
\hline 5170 & PDPK1 & 3-phosphoinositide dependent protein kinase-1 & -1.86312 & 0.001931 & 0.035481 \\
\hline 8767 & RIPK2 & receptor-interacting serine-threonine kinase 2 & 2.66792 & 0.001976 & 0.035733 \\
\hline
\end{tabular}




\begin{tabular}{|c|c|c|c|c|c|}
\hline 4067 & LYN & $\begin{array}{l}\text { v-yes-1 Yamaguchi sarcoma viral related oncogene } \\
\text { homolog }\end{array}$ & 2.093221 & 0.001989 & 0.035802 \\
\hline 57134 & MAN1C1 & mannosidase, alpha, class $1 \mathrm{C}$, member 1 & -1.58222 & 0.00208 & 0.036278 \\
\hline 2982 & GUCY1A3 & guanylate cyclase 1 , soluble, alpha 3 & 5.151509 & 0.002185 & 0.036792 \\
\hline 6732 & srpk1 & SFRS protein kinase 1 & -1.27224 & 0.002248 & 0.037084 \\
\hline 83666 & PARP9 & poly (ADP-ribose) polymerase family, member 9 & 2.333172 & 0.002315 & 0.037381 \\
\hline 23522 & MYST4 & $\begin{array}{l}\text { MYST histone acetyltransferase (monocytic leukemia) } \\
4\end{array}$ & -1.62314 & 0.002325 & 0.037426 \\
\hline 9360 & PPIG & peptidylprolyl isomerase $\mathrm{G}$ (cyclophilin G) & -1.58321 & 0.002369 & 0.037616 \\
\hline 7324 & UBE2E1 & $\begin{array}{l}\text { ubiquitin-conjugating enzyme E2E } 1 \text { (UBC4/5 } \\
\text { homolog, yeast) }\end{array}$ & 1.442897 & 0.002653 & 0.038894 \\
\hline 23235 & sik2 & salt-inducible kinase 2 & -1.78666 & 0.002712 & 0.039135 \\
\hline 7453 & wars & tryptophanyl-tRNA synthetase & 1.658944 & 0.002906 & 0.039878 \\
\hline 2766 & gmpr & guanosine monophosphate reductase & 2.749062 & 0.003 & 0.040213 \\
\hline 5156 & pdgfra & $\begin{array}{l}\text { platelet-derived growth factor receptor, alpha } \\
\text { polypeptide }\end{array}$ & 1.588831 & 0.003062 & 0.040429 \\
\hline 9517 & Sptlc2 & serine palmitoyltransferase, long chain base subunit 2 & 1.875558 & 0.003371 & 0.041397 \\
\hline 1786 & dnmt1 & DNA (cytosine-5-)-methyltransferase 1 & -1.60668 & 0.003465 & 0.041666 \\
\hline 7803 & ptp4a1 & protein tyrosine phosphatase type IVA, member 1 & 1.242832 & 0.003508 & 0.041786 \\
\hline 440275 & EIF2AK4 & eukaryotic translation initiation factor 2 alpha kinase 4 & -1.49734 & 0.003768 & 0.042463 \\
\hline 8396 & PIP4K2B & phosphatidylinositol-5-phosphate 4-kinase, type II, beta & -2.13808 & 0.003948 & 0.042888 \\
\hline 3712 & ivd & isovaleryl Coenzyme A dehydrogenase & -1.43586 & 0.00395 & 0.042893 \\
\hline 79567 & fam65a & family with sequence similarity 65 , member $\mathrm{A}$ & -1.35494 & 0.004017 & 0.043045 \\
\hline 53944 & CSNK1G1 & casein kinase 1 , gamma 1 & 1.408734 & 0.004065 & 0.04315 \\
\hline 2713 & GK3P & glycerol kinase 3 pseudogene; glycerol kinase & 1.619607 & 0.004097 & 0.04322 \\
\hline 1119 & CHKA & choline kinase alpha & -1.81576 & 0.004109 & 0.043245 \\
\hline 7321 & UBE2D1 & $\begin{array}{l}\text { ubiquitin-conjugating enzyme E2D } 1 \text { (UBC4/5 } \\
\text { homolog, yeast) }\end{array}$ & 1.479857 & 0.004119 & 0.043268 \\
\hline 7375 & USP4 & ubiquitin specific peptidase 4 (proto-oncogene) & -1.71882 & 0.004201 & 0.043439 \\
\hline 2530 & fut8 & fucosyltransferase 8 (alpha $(1,6)$ fucosyltransferase) & -1.6802 & 0.004283 & 0.043606 \\
\hline 11274 & usp 18 & ubiquitin specific peptidase 18 & 13.47735 & 0.004391 & 0.043818 \\
\hline 9246 & Ube216 & ubiquitin-conjugating enzyme E2L 6 & 2.006129 & 0.004572 & 0.044157 \\
\hline 7328 & Ube2h & $\begin{array}{l}\text { ubiquitin-conjugating enzyme E2H (UBC8 homolog, } \\
\text { yeast) }\end{array}$ & 1.954939 & 0.00463 & 0.04426 \\
\hline 9958 & USP15 & ubiquitin specific peptidase 15 & 1.552271 & 0.004717 & 0.044412 \\
\hline 57695 & USP37 & ubiquitin specific peptidase 37 & -1.71885 & 0.004861 & 0.044653 \\
\hline 415116 & PIM3 & pim-3 oncogene & 2.069768 & 0.004865 & 0.04466 \\
\hline 7329 & UBE2I & $\begin{array}{l}\text { ubiquitin-conjugating enzyme E2I (UBC9 homolog, } \\
\text { yeast) }\end{array}$ & -1.83281 & 0.004876 & 0.044678 \\
\hline 9262 & STK17B & serine/threonine kinase $17 \mathrm{~b}$ & 2.270061 & 0.004916 & 0.044742 \\
\hline 84196 & usp48 & ubiquitin specific peptidase 48 & -1.58416 & 0.005055 & 0.044961 \\
\hline 51805 & COQ3 & $\begin{array}{l}\text { coenzyme Q3 homolog, methyltransferase (S. } \\
\text { cerevisiae) }\end{array}$ & -1.44833 & 0.00514 & 0.045088 \\
\hline 84930 & MASTL & microtubule associated serine/threonine kinase-like & 4.491827 & 0.005216 & 0.0452 \\
\hline 1267 & CNP & 2',3'-cyclic nucleotide 3' phosphodiesterase & 1.982498 & 0.005324 & 0.045355 \\
\hline 64324 & NSD1 & nuclear receptor binding SET domain protein 1 & -1.98816 & 0.005375 & 0.045427 \\
\hline 91 & ACVR1B & activin A receptor, type IB & 2.03274 & 0.005493 & 0.045587 \\
\hline 4145 & matK & megakaryocyte-associated tyrosine kinase & -1.22476 & 0.005519 & 0.04562 \\
\hline
\end{tabular}




\begin{tabular}{|c|c|c|c|c|c|}
\hline 7046 & $\operatorname{tgfbr} 1$ & transforming growth factor, beta receptor 1 & -5.35032 & 0.005529 & 0.045635 \\
\hline 10007 & gnpda1 & glucosamine-6-phosphate deaminase 1 & -1.83086 & 0.005583 & 0.045705 \\
\hline 2592 & galT & galactose-1-phosphate uridylyltransferase & -1.2438 & 0.005607 & 0.045736 \\
\hline 7320 & ube $2 b$ & ubiquitin-conjugating enzyme E2B (RAD6 homolog) & 1.272066 & 0.00581 & 0.04599 \\
\hline 23463 & icmt & isoprenylcysteine carboxyl methyltransferase & -1.30094 & 0.005846 & 0.046033 \\
\hline 1387 & CREBBP & CREB binding protein & 1.704567 & 0.00591 & 0.046109 \\
\hline 29943 & PADI1 & peptidyl arginine deiminase, type I & 1.552521 & 0.00611 & 0.046339 \\
\hline 80222 & TARS2 & threonyl-tRNA synthetase 2 , mitochondrial (putative) & -1.7581 & 0.006229 & 0.046469 \\
\hline 5058 & Pak1 & p21 protein $(\mathrm{Cdc} 42 / \mathrm{Rac})$-activated kinase 1 & -1.76388 & 0.006339 & 0.046586 \\
\hline 6872 & TAF1 & $\begin{array}{l}\text { TAF1 RNA polymerase II, TATA box binding protein } \\
\text { (TBP)-associated factor, } 250 \mathrm{kDa}\end{array}$ & -1.63093 & 0.006344 & 0.046591 \\
\hline 2648 & KAT2A & $\mathrm{K}$ (lysine) acetyltransferase $2 \mathrm{~A}$ & -1.57085 & 0.006436 & 0.046686 \\
\hline 80339 & pnpla3 & patatin-like phospholipase domain containing 3 & -4.29169 & 0.006518 & 0.046769 \\
\hline 84132 & Usp42 & ubiquitin specific peptidase 42 & 1.307377 & 0.006593 & 0.046843 \\
\hline 490 & ATP2B1 & ATPase, $\mathrm{Ca}++$ transporting, plasma membrane 1 & 4.468336 & 0.00664 & 0.046888 \\
\hline 80854 & setd7 & SET domain containing (lysine methyltransferase) 7 & -1.51194 & 0.006834 & 0.047071 \\
\hline 10038 & PARP2 & poly (ADP-ribose) polymerase 2 & -1.50724 & 0.006898 & 0.047129 \\
\hline 79834 & SGK269 & NKF3 kinase family member & 4.019939 & 0.006993 & 0.047214 \\
\hline 5520 & Ppp2r2a & $\begin{array}{l}\text { protein phosphatase } 2 \text { (formerly } 2 \mathrm{~A} \text { ), regulatory subunit } \\
\mathrm{B} \text {, alpha isoform }\end{array}$ & 1.885083 & 0.007159 & 0.047358 \\
\hline 9263 & STK17A & serine/threonine kinase $17 \mathrm{a}$ & 4.099909 & 0.007166 & 0.047364 \\
\hline 254531 & lpcat4 & lysophosphatidylcholine acyltransferase 4 & -1.86205 & 0.007173 & 0.04737 \\
\hline 11044 & papd7 & polymerase (DNA directed) sigma & 1.799687 & 0.007213 & 0.047403 \\
\hline 1854 & dut & deoxyuridine triphosphatase & -1.53256 & 0.007214 & 0.047404 \\
\hline 224 & aldh3a2 & aldehyde dehydrogenase 3 family, member A2 & -1.68795 & 0.00725 & 0.047434 \\
\hline 5128 & Cdk17 & PCTAIRE protein kinase 2 & 1.88957 & 0.007422 & 0.047574 \\
\hline 8459 & TPST2 & tyrosylprotein sulfotransferase 2 & -1.49668 & 0.007562 & 0.047683 \\
\hline 23659 & PLA2G15 & phospholipase A2, group XV & -1.66229 & 0.007635 & 0.047739 \\
\hline 55781 & RIOK2 & RIO kinase 2 (yeast) & -2.06321 & 0.007713 & 0.047798 \\
\hline 8737 & RIPK1 & $\begin{array}{l}\text { receptor (TNFRSF)-interacting serine-threonine kinase } \\
1\end{array}$ & 2.134155 & 0.007726 & 0.047807 \\
\hline 9100 & USP10 & ubiquitin specific peptidase 10 & -1.25337 & 0.008011 & 0.048012 \\
\hline 6850 & SYK & spleen tyrosine kinase & -2.6376 & 0.008158 & 0.048112 \\
\hline 22928 & SEPHS2 & selenophosphate synthetase 2 & -1.28463 & 0.008195 & 0.048138 \\
\hline 146057 & TTBK2 & tau tubulin kinase 2 & -1.87784 & 0.008232 & 0.048162 \\
\hline 5780 & Ptpn9 & protein tyrosine phosphatase, non-receptor type 9 & -1.3253 & 0.008278 & 0.048192 \\
\hline 55589 & bmp2k & BMP2 inducible kinase & 1.288683 & 0.008296 & 0.048204 \\
\hline 80025 & PANK2 & pantothenate kinase 2 & 1.453979 & 0.008353 & 0.048241 \\
\hline 5147 & PDE6D & phosphodiesterase 6D, cGMP-specific, rod, delta & -1.45298 & 0.00839 & 0.048265 \\
\hline 2683 & B4GALT1 & $\begin{array}{l}\text { UDP-Gal:betaGlcNAc beta 1,4- galactosyltransferase, } \\
\text { polypeptide } 1\end{array}$ & 2.401377 & 0.008517 & 0.048345 \\
\hline 54926 & ube2r2 & ubiquitin-conjugating enzyme E2R 2 & -1.3505 & 0.008526 & 0.048351 \\
\hline 1849 & Dusp7 & dual specificity phosphatase 7 & -3.18086 & 0.008576 & 0.048382 \\
\hline 2820 & Gpd2 & glycerol-3-phosphate dehydrogenase 2 (mitochondrial) & 1.636963 & 0.008589 & 0.04839 \\
\hline 1788 & DNMT3A & DNA (cytosine-5-)-methyltransferase 3 alpha & -1.31588 & 0.008628 & 0.048414 \\
\hline
\end{tabular}




\begin{tabular}{|c|c|c|c|c|c|}
\hline 26191 & PTPN22 & $\begin{array}{l}\text { protein tyrosine phosphatase, non-receptor type } 22 \\
\text { (lymphoid) }\end{array}$ & -2.23273 & 0.00871 & 0.048464 \\
\hline 9953 & Hs3st3b1 & heparan sulfate (glucosamine) 3-O-sulfotransferase 3B1 & 7.626608 & 0.008723 & 0.048471 \\
\hline 65264 & Ube2z & ubiquitin-conjugating enzyme E2Z & 1.688868 & 0.008724 & 0.048472 \\
\hline 2181 & ACSL3 & acyl-CoA synthetase long-chain family member 3 & -1.37523 & 0.008938 & 0.048597 \\
\hline 3718 & JAK3 & Janus kinase 3 & 1.859792 & 0.008953 & 0.048606 \\
\hline 5770 & ptpn1 & protein tyrosine phosphatase, non-receptor type 1 & 1.675984 & 0.009007 & 0.048636 \\
\hline 5771 & PTPN2 & protein tyrosine phosphatase, non-receptor type 2 & 1.869886 & 0.009115 & 0.048697 \\
\hline 6713 & sqlE & squalene epoxidase & -1.60303 & 0.009123 & 0.048701 \\
\hline 64132 & Xylt2 & xylosyltransferase II & -1.52725 & 0.009178 & 0.048731 \\
\hline 26750 & RPS6KC1 & ribosomal protein S6 kinase, $52 \mathrm{kDa}$, polypeptide 1 & 1.527532 & 0.009302 & 0.048798 \\
\hline 51251 & NT5C3 & 5'-nucleotidase, cytosolic III & 10.33578 & 0.009329 & 0.048812 \\
\hline 31 & ACACA & acetyl-Coenzyme A carboxylase alpha & -1.67031 & 0.009371 & 0.048835 \\
\hline 64781 & CERK & ceramide kinase & -1.84446 & 0.009492 & 0.048898 \\
\hline 79858 & Nek11 & NIMA (never in mitosis gene a)- related kinase 11 & -1.43052 & 0.009579 & 0.048942 \\
\hline 5332 & Plcb4 & phospholipase $\mathrm{C}$, beta 4 & -2.89589 & 0.009661 & 0.048983 \\
\hline 57097 & parp11 & poly (ADP-ribose) polymerase family, member 11 & 1.963593 & 0.009679 & 0.048992 \\
\hline 30849 & PIK3R4 & phosphoinositide-3-kinase, regulatory subunit 4 & -1.60315 & 0.00984 & 0.049174 \\
\hline 8658 & tnks & $\begin{array}{l}\text { tankyrase, TRF1-interacting ankyrin-related ADP- } \\
\text { ribose polymerase }\end{array}$ & -2.05439 & 0.009897 & 0.049237 \\
\hline 51465 & UBE2J1 & $\begin{array}{l}\text { ubiquitin-conjugating enzyme E2, J1 (UBC6 homolog, } \\
\text { yeast) }\end{array}$ & 1.266213 & 0.009992 & 0.049341 \\
\hline 5494 & PPM1A & $\begin{array}{l}\text { protein phosphatase } 1 \mathrm{~A} \text { (formerly } 2 \mathrm{C} \text { ), magnesium- } \\
\text { dependent, alpha isoform }\end{array}$ & -1.38216 & 0.010231 & 0.049597 \\
\hline 1152 & $\mathrm{CKB}$ & creatine kinase, brain & 18.102 & 0.010332 & 0.049701 \\
\hline 23387 & SIK3 & serine/threonine-protein kinase QSK & 2.07963 & 0.010426 & 0.049797 \\
\hline 8540 & AGPS & alkylglycerone phosphate synthase & -1.95406 & 0.010556 & 0.049929 \\
\hline 7048 & Tgfbr2 & transforming growth factor, beta receptor II $(70 / 80 \mathrm{kDa})$ & -1.58661 & 0.010738 & 0.050108 \\
\hline 2241 & FER & $\begin{array}{l}\text { FER tyrosine kinase [Source:HGNC } \\
\text { Symbol;Acc:HGNC:3655] }\end{array}$ & -1.51166 & 0.010749 & 0.050118 \\
\hline 535 & ATP6V0A1 & ATPase, $\mathrm{H}+$ transporting, lysosomal V0 subunit al & -1.34507 & 0.010767 & 0.050136 \\
\hline 2872 & MKNK2 & MAP kinase interacting serine/threonine kinase 2 & -1.99963 & 0.010787 & 0.050155 \\
\hline 124454 & Ears2 & glutamyl-tRNA synthetase 2 , mitochondrial (putative) & -1.51881 & 0.010853 & 0.050218 \\
\hline 8576 & STK16 & serine/threonine kinase 16 & -1.23471 & 0.010856 & 0.050221 \\
\hline 247 & ALOX15B & arachidonate 15-lipoxygenase, type B & 1.669457 & 0.010954 & 0.050342 \\
\hline 528 & ATP6V1C1 & $\begin{array}{l}\text { ATPase, } \mathrm{H}+\text { transporting, lysosomal } 42 \mathrm{kDa}, \mathrm{V} 1 \text { subunit } \\
\mathrm{C} 1\end{array}$ & -1.36537 & 0.011361 & 0.05103 \\
\hline 6484 & ST3GAL4 & ST3 beta-galactoside alpha-2,3-sialyltransferase 4 & 1.48017 & 0.011478 & 0.051221 \\
\hline 5778 & PTPN7 & protein tyrosine phosphatase, non-receptor type 7 & -1.29849 & 0.011706 & 0.051589 \\
\hline 51611 & DPH5 & DPH5 homolog (S. cerevisiae) & -2.41781 & 0.011867 & 0.051844 \\
\hline 5287 & PIK3C2B & phosphoinositide-3-kinase, class 2 , beta polypeptide & -1.55396 & 0.012067 & 0.052152 \\
\hline 2582 & galE & UDP-galactose-4-epimerase & -1.35184 & 0.012427 & 0.052694 \\
\hline 152926 & Ppm1k & protein phosphatase $1 \mathrm{~K}$ ( $\mathrm{PP} 2 \mathrm{C}$ domain containing) & 10.06714 & 0.012508 & 0.052812 \\
\hline 4122 & MAN2A2 & mannosidase, alpha, class $2 \mathrm{~A}$, member 2 & -1.37047 & 0.012552 & 0.052876 \\
\hline 1890 & Tymp & thymidine phosphorylase & 1.79696 & 0.012712 & 0.053107 \\
\hline 87178 & Pnpt1 & polyribonucleotide nucleotidyltransferase 1 & 5.452124 & 0.013163 & 0.053738 \\
\hline
\end{tabular}




\begin{tabular}{|c|c|c|c|c|c|}
\hline 5321 & pla2g4a & $\begin{array}{l}\text { phospholipase A2, group IVA (cytosolic, calcium- } \\
\text { dependent) }\end{array}$ & 1.565368 & 0.013297 & 0.053919 \\
\hline 55683 & KIAA1310 & KIAA1310 & -2.33025 & 0.013491 & 0.054179 \\
\hline 8731 & rnmt & RNA (guanine-7-) methyltransferase & -1.5279 & 0.013565 & 0.054276 \\
\hline 10068 & IL18BP & interleukin 18 binding protein & -1.27637 & 0.013601 & 0.054323 \\
\hline 5980 & REV3L & $\begin{array}{l}\text { REV3-like, catalytic subunit of DNA polymerase zeta } \\
\text { (yeast) }\end{array}$ & -1.93195 & 0.013633 & 0.054365 \\
\hline 4047 & LSS & $\begin{array}{l}\text { lanosterol synthase (2,3-oxidosqualene-lanosterol } \\
\text { cyclase) }\end{array}$ & 1.553256 & 0.013695 & 0.054446 \\
\hline 993 & $\mathrm{CDC} 25 \mathrm{~A}$ & cell division cycle 25 homolog A (S. pombe) & -2.48417 & 0.013822 & 0.054609 \\
\hline 2651 & GCNT2 & $\begin{array}{l}\text { glucosaminyl (N-acetyl) transferase 2, I-branching } \\
\text { enzyme (I blood group) }\end{array}$ & 1.984682 & 0.013827 & 0.054616 \\
\hline 1457 & Csnk2a1 & $\begin{array}{l}\text { casein kinase 2, alpha } 1 \text { polypeptide pseudogene; casein } \\
\text { kinase } 2 \text {, alpha } 1 \text { polypeptide }\end{array}$ & 1.38179 & 0.013919 & 0.054733 \\
\hline 9128 & PRPF4 & PRP4 pre-mRNA processing factor 4 homolog (yeast) & -1.42448 & 0.013991 & 0.054823 \\
\hline 7226 & TRPM2 & $\begin{array}{l}\text { transient receptor potential cation channel, subfamily } \\
\mathrm{M}, \text { member } 2\end{array}$ & -1.29721 & 0.014127 & 0.054994 \\
\hline 5859 & QARS & glutaminyl-tRNA synthetase & -1.25667 & 0.01416 & 0.055035 \\
\hline 8790 & TNNI3K & $\begin{array}{l}\text { TNNI3 interacting kinase; fucose-1-phosphate } \\
\text { guanylyltransferase }\end{array}$ & -1.51773 & 0.014362 & 0.055282 \\
\hline 2590 & GALNT2 & $\begin{array}{l}\text { UDP-N-acetyl-alpha-D-galactosamine:polypeptide N- } \\
\text { acetylgalactosaminyltransferase } 2 \text { (GalNAc-T2) }\end{array}$ & 1.386324 & 0.014649 & 0.055626 \\
\hline 51086 & TNNI3K & $\begin{array}{l}\text { TNNI3 interacting kinase; fucose-1-phosphate } \\
\text { guanylyltransferase }\end{array}$ & -2.05154 & 0.014677 & 0.055658 \\
\hline 51728 & POLR3K & $\begin{array}{l}\text { polymerase (RNA) III (DNA directed) polypeptide K, } \\
12.3 \mathrm{kDa}\end{array}$ & -1.32289 & 0.014793 & 0.055794 \\
\hline 100 & ada & adenosine deaminase & 4.346509 & 0.014832 & 0.05584 \\
\hline 57602 & usp36 & ubiquitin specific peptidase 36 & -1.69339 & 0.014873 & 0.055888 \\
\hline 55703 & Polr3b & polymerase (RNA) III (DNA directed) polypeptide B & -1.49881 & 0.014967 & 0.055996 \\
\hline 5292 & Pim1 & pim-1 oncogene & 2.956011 & 0.015008 & 0.056043 \\
\hline 8942 & Kynu & kynureninase (L-kynurenine hydrolase) & 1.903991 & 0.015012 & 0.056047 \\
\hline 6733 & SRPK2 & SFRS protein kinase 2 & -2.02216 & 0.015372 & 0.056452 \\
\hline 8202 & NCOA3 & nuclear receptor coactivator 3 & 1.412453 & 0.015377 & 0.056458 \\
\hline 4215 & MAP3K3 & mitogen-activated protein kinase kinase kinase 3 & -1.49813 & 0.015415 & 0.0565 \\
\hline 11072 & Dusp14 & dual specificity phosphatase 14 & -1.26343 & 0.015518 & 0.056613 \\
\hline 55033 & FKBP14 & FK506 binding protein $14,22 \mathrm{kDa}$ & -1.41888 & 0.015546 & 0.056643 \\
\hline 7334 & UBE2N & $\begin{array}{l}\text { ubiquitin-conjugating enzyme E2N (UBC13 homolog, } \\
\text { yeast) }\end{array}$ & 1.221237 & 0.015598 & 0.0567 \\
\hline 9583 & entpd4 & ectonucleoside triphosphate diphosphohydrolase 4 & -1.52141 & 0.015776 & 0.056891 \\
\hline 23305 & Acsl6 & acyl-CoA synthetase long-chain family member 6 & 2.395987 & 0.015854 & 0.056973 \\
\hline 6790 & AURKA & aurora kinase A; aurora kinase A pseudogene 1 & -1.54599 & 0.015932 & 0.057056 \\
\hline 6714 & Src & $\begin{array}{l}\text { V-src sarcoma (Schmidt-Ruppin A-2) viral oncogene } \\
\text { homolog (avian) }\end{array}$ & 3.263054 & 0.015965 & 0.05709 \\
\hline 8460 & TPST1 & tyrosylprotein sulfotransferase 1 & -1.5048 & 0.016073 & 0.057203 \\
\hline 3707 & Itpkb & inositol 1,4,5-trisphosphate 3-kinase B & -2.29458 & 0.016157 & 0.05729 \\
\hline 10667 & FARS2 & phenylalanyl-tRNA synthetase 2 , mitochondrial & -1.44212 & 0.01637 & 0.05751 \\
\hline 8394 & PIP5K1A & phosphatidylinositol-4-phosphate 5-kinase, type I, alpha & 1.611574 & 0.016474 & 0.057617 \\
\hline 3611 & ILK & integrin-linked kinase & -1.23012 & 0.016648 & 0.057793 \\
\hline 57486 & NLN & neurolysin (metallopeptidase M3 family) & -1.41486 & 0.017075 & 0.058213 \\
\hline 5599 & mapk8 & mitogen-activated protein kinase 8 & 1.562214 & 0.0171 & 0.058237 \\
\hline
\end{tabular}




\begin{tabular}{|c|c|c|c|c|c|}
\hline 2983 & gucy 1 b3 & guanylate cyclase 1 , soluble, beta 3 & 2.612677 & 0.017124 & 0.05826 \\
\hline 23759 & ppil2 & peptidylprolyl isomerase (cyclophilin)-like 2 & -1.35352 & 0.017437 & 0.058557 \\
\hline 4234 & mettl1 & methyltransferase like 1 & 1.420605 & 0.017631 & 0.058738 \\
\hline 65220 & $\operatorname{nadK}$ & NAD kinase & 1.261872 & 0.017681 & 0.058784 \\
\hline 4117 & mak & male germ cell-associated kinase & 3.065857 & 0.017695 & 0.058797 \\
\hline 8833 & GMPS & guanine monphosphate synthetase & -1.41322 & 0.017726 & 0.058825 \\
\hline 3636 & INPPL1 & inositol polyphosphate phosphatase-like 1 & -1.35187 & 0.018204 & 0.059255 \\
\hline 84105 & pcbd2 & $\begin{array}{l}\text { pterin- } 4 \text { alpha-carbinolamine dehydratase/dimerization } \\
\text { cofactor of hepatocyte nuclear factor } 1 \text { alpha (TCF1) } 2\end{array}$ & -1.50775 & 0.018399 & 0.059425 \\
\hline 790 & cad & $\begin{array}{l}\text { carbamoyl-phosphate synthetase } 2 \text {, aspartate } \\
\text { transcarbamylase, and dihydroorotase }\end{array}$ & -1.78901 & 0.018415 & 0.059439 \\
\hline 23043 & TNIK & TRAF2 and NCK interacting kinase & 1.857638 & 0.018442 & 0.059462 \\
\hline 18 & ABAT & 4-aminobutyrate aminotransferase & -1.77407 & 0.018497 & 0.05951 \\
\hline 661 & Polr3d & $\begin{array}{l}\text { polymerase (RNA) III (DNA directed) polypeptide D, } \\
44 \mathrm{kDa}\end{array}$ & 1.637942 & 0.018614 & 0.05961 \\
\hline 4724 & NDUFS4 & $\begin{array}{l}\text { NADH dehydrogenase (ubiquinone) Fe-S protein } 4, \\
18 \mathrm{kDa} \text { (NADH-coenzyme Q reductase) }\end{array}$ & 1.203269 & 0.018836 & 0.059798 \\
\hline 22929 & sephs1 & $\begin{array}{l}\text { selenophosphate synthetase } 1 \text {; similar to } \\
\text { selenophosphate synthetase } 1\end{array}$ & -1.39607 & 0.018984 & 0.059921 \\
\hline 84532 & acss 1 & acyl-CoA synthetase short-chain family member 1 & -2.31876 & 0.019061 & 0.059985 \\
\hline 5832 & ALDH18A1 & aldehyde dehydrogenase 18 family, member A1 & -1.65869 & 0.019124 & 0.060037 \\
\hline 58497 & PRUNE & prune homolog (Drosophila) & -1.22962 & 0.019162 & 0.060068 \\
\hline 6648 & Sod2 & superoxide dismutase 2 , mitochondrial & 2.435665 & 0.019162 & 0.060068 \\
\hline 9957 & Hs3st1 & heparan sulfate (glucosamine) 3-O-sulfotransferase 1 & -5.66164 & 0.019181 & 0.060084 \\
\hline 54995 & OXSM & 3-oxoacyl-ACP synthase, mitochondrial & -1.22026 & 0.019398 & 0.060408 \\
\hline 8564 & $\mathrm{kmo}$ & $\begin{array}{l}\text { kynurenine 3-monooxygenase (kynurenine 3- } \\
\text { hydroxylase) }\end{array}$ & 2.401636 & 0.019693 & 0.060842 \\
\hline 22978 & NT5C2 & 5'-nucleotidase, cytosolic II & 1.224539 & 0.019845 & 0.061065 \\
\hline 259230 & sgms1 & sphingomyelin synthase 1 & 1.590344 & 0.019848 & 0.06107 \\
\hline 3613 & Impa2 & inositol(myo)-1(or 4)-monophosphatase 2 & -1.69186 & 0.020022 & 0.06132 \\
\hline 4140 & Mark3 & MAP/microtubule affinity-regulating kinase 3 & 1.503244 & 0.020566 & 0.062092 \\
\hline 84869 & cbr4 & carbonyl reductase 4 & -2.94871 & 0.020758 & 0.062359 \\
\hline 5594 & MAPK1 & mitogen-activated protein kinase 1 & -1.26245 & 0.020847 & 0.062482 \\
\hline 5256 & PHKA2 & phosphorylase kinase, alpha 2 (liver) & -1.56911 & 0.021261 & 0.063045 \\
\hline 55904 & MLL5 & $\begin{array}{l}\text { myeloid/lymphoid or mixed-lineage leukemia } 5 \\
\text { (trithorax homolog, Drosophila) }\end{array}$ & -1.32216 & 0.021357 & 0.063174 \\
\hline 953 & ENTPD1 & ectonucleoside triphosphate diphosphohydrolase 1 & -1.73678 & 0.021949 & 0.063957 \\
\hline 80896 & NPL & $\begin{array}{l}\mathrm{N} \text {-acetylneuraminate pyruvate lyase } \\
\text { (dihydrodipicolinate synthase) }\end{array}$ & -1.36642 & 0.022212 & 0.064297 \\
\hline 10087 & COL4A3BP & $\begin{array}{l}\text { collagen, type IV, alpha } 3 \text { (Goodpasture antigen) } \\
\text { binding protein }\end{array}$ & -1.8092 & 0.022266 & 0.064367 \\
\hline 613 & bcr & breakpoint cluster region & -1.25381 & 0.022293 & 0.064402 \\
\hline 4258 & mgst2 & microsomal glutathione S-transferase 2 & -1.31713 & 0.022528 & 0.0647 \\
\hline 5286 & pik3c2a & phosphoinositide-3-kinase, class 2, alpha polypeptide & -1.33913 & 0.023357 & 0.065728 \\
\hline 5209 & pfkfb3 & 6-phosphofructo-2-kinase/fructose-2,6-biphosphatase 3 & 2.616625 & 0.023382 & 0.065759 \\
\hline 587 & BCAT2 & branched chain aminotransferase 2 , mitochondrial & -1.33749 & 0.023408 & 0.065789 \\
\hline 64064 & OXCT2 & 3-oxoacid CoA transferase 2 & -1.36343 & 0.023429 & 0.065815 \\
\hline 4704 & NDUFA9 & $\begin{array}{l}\text { NADH dehydrogenase (ubiquinone) } 1 \text { alpha } \\
\text { subcomplex, } 9,39 \mathrm{kDa}\end{array}$ & 1.276696 & 0.02372 & 0.066165 \\
\hline
\end{tabular}




\begin{tabular}{|c|c|c|c|c|c|}
\hline 55500 & etnk1 & ethanolamine kinase 1 & -1.6816 & 0.023819 & 0.066282 \\
\hline 6446 & SGK1 & serum/glucocorticoid regulated kinase 1 & -1.57205 & 0.023852 & 0.066322 \\
\hline 10623 & POLR3C & $\begin{array}{l}\text { polymerase (RNA) III (DNA directed) polypeptide C } \\
(62 \mathrm{kD})\end{array}$ & 1.665438 & 0.023889 & 0.066366 \\
\hline 622 & BDH1 & 3-hydroxybutyrate dehydrogenase, type 1 & -1.30987 & 0.02401 & 0.066508 \\
\hline 5294 & PIK3CG & $\begin{array}{l}\text { phosphoinositide-3-kinase, catalytic, gamma } \\
\text { polypeptide }\end{array}$ & -1.67238 & 0.024139 & 0.066659 \\
\hline 26873 & oplaH & 5-oxoprolinase (ATP-hydrolysing) & -1.46179 & 0.024261 & 0.066802 \\
\hline 4247 & mgat2 & $\begin{array}{l}\text { mannosyl (alpha-1,6-)-glycoprotein beta-1,2-N- } \\
\text { acetylglucosaminyltransferase }\end{array}$ & 1.287292 & 0.024618 & 0.067214 \\
\hline 2762 & Gmds & GDP-mannose 4,6-dehydratase & -1.53845 & 0.024623 & 0.067219 \\
\hline 3033 & hadh & hydroxyacyl-Coenzyme A dehydrogenase & -1.36393 & 0.024722 & 0.067333 \\
\hline 3480 & IGF1R & insulin-like growth factor 1 receptor & -2.01693 & 0.024777 & 0.067395 \\
\hline 54434 & SSH1 & slingshot homolog 1 (Drosophila) & -1.25296 & 0.024956 & 0.067597 \\
\hline 58190 & Ctdsp1 & $\begin{array}{l}\text { CTD (carboxy-terminal domain, RNA polymerase II, } \\
\text { polypeptide A) small phosphatase } 1\end{array}$ & -1.76237 & 0.024983 & 0.067627 \\
\hline 4548 & $\mathrm{mtr}$ & $\begin{array}{l}\text { 5-methyltetrahydrofolate-homocysteine } \\
\text { methyltransferase }\end{array}$ & -1.53185 & 0.025219 & 0.067891 \\
\hline 84875 & PARP10 & poly (ADP-ribose) polymerase family, member 10 & 1.927591 & 0.025306 & 0.067987 \\
\hline 54986 & Ulk4 & unc-51-like kinase 4 (C. elegans) & -1.88954 & 0.025775 & 0.06861 \\
\hline 7327 & UBE2G2 & $\begin{array}{l}\text { ubiquitin-conjugating enzyme E2G } 2 \text { (UBC7 homolog, } \\
\text { yeast) }\end{array}$ & -1.49683 & 0.025857 & 0.068718 \\
\hline 5442 & Polrmt & polymerase (RNA) mitochondrial (DNA directed) & -1.76879 & 0.026139 & 0.069085 \\
\hline 4217 & MAP3K5 & mitogen-activated protein kinase kinase kinase 5 & 1.563246 & 0.02615 & 0.069099 \\
\hline 1432 & Mapk14 & mitogen-activated protein kinase 14 & -1.39769 & 0.026276 & 0.069262 \\
\hline 11201 & poli & polymerase (DNA directed) iota & -1.55778 & 0.026736 & 0.069892 \\
\hline 113 & ADCY7 & adenylate cyclase 7 & -1.44613 & 0.027021 & 0.070277 \\
\hline 158 & ADSL & adenylosuccinate lyase & -1.2012 & 0.027719 & 0.071203 \\
\hline 79813 & EHMT1 & euchromatic histone-lysine N-methyltransferase 1 & -1.54112 & 0.027811 & 0.071324 \\
\hline 5048 & Pafah1b1 & $\begin{array}{l}\text { platelet-activating factor acetylhydrolase, isoform Ib, } \\
\text { subunit } 1 \text { ( } 45 \mathrm{kDa})\end{array}$ & 1.348842 & 0.027858 & 0.071385 \\
\hline 8899 & prpf $4 b$ & $\begin{array}{l}\text { similar to hCG1820375; PRP4 pre-mRNA processing } \\
\text { factor } 4 \text { homolog B (yeast) }\end{array}$ & 1.485916 & 0.027954 & 0.07151 \\
\hline 3163 & Hmox 2 & heme oxygenase (decycling) 2 & -1.29953 & 0.028171 & 0.071791 \\
\hline 11329 & stk38 & serine/threonine kinase 38 & -1.66133 & 0.028668 & 0.072427 \\
\hline 157285 & SGK223 & homolog of rat pragma of Rnd2 & -3.02345 & 0.028912 & 0.072735 \\
\hline 84693 & MCEE & methylmalonyl CoA epimerase & -1.39746 & 0.028982 & 0.072822 \\
\hline 5422 & polA1 & polymerase (DNA directed), alpha 1, catalytic subunit & -1.43995 & 0.029435 & 0.073386 \\
\hline 5351 & PLOD1 & procollagen-lysine 1, 2-oxoglutarate 5-dioxygenase 1 & -1.2086 & 0.029781 & 0.073811 \\
\hline 6120 & rpe & rcRPE; ribulose-5-phosphate-3-epimerase & -1.30809 & 0.029829 & 0.07387 \\
\hline 3985 & LIMK2 & LIM domain kinase 2 & 4.58606 & 0.029932 & 0.073995 \\
\hline 23031 & MAST3 & microtubule associated serine/threonine kinase 3 & -3.41994 & 0.031091 & 0.075375 \\
\hline 27005 & USP21 & ubiquitin specific peptidase 21 & -1.54586 & 0.031269 & 0.075582 \\
\hline 4836 & NMT1 & $\mathrm{N}$-myristoyltransferase 1 & -1.53829 & 0.031634 & 0.076003 \\
\hline 23178 & PASK & PAS domain containing serine/threonine kinase & -2.03636 & 0.031739 & 0.076123 \\
\hline 80824 & DUSP16 & dual specificity phosphatase 16 & 1.930353 & 0.032305 & 0.076763 \\
\hline 79087 & Alg12 & $\begin{array}{l}\text { asparagine-linked glycosylation 12, alpha-1,6- } \\
\text { mannosyltransferase homolog (S. cerevisiae) }\end{array}$ & -2.11336 & 0.032463 & 0.07694 \\
\hline
\end{tabular}




\begin{tabular}{|c|c|c|c|c|c|}
\hline 55217 & TMLHE & trimethyllysine hydroxylase, epsilon & -2.11672 & 0.032768 & 0.077279 \\
\hline 2185 & PTK2B & PTK2B protein tyrosine kinase 2 beta & 1.732137 & 0.032899 & 0.077424 \\
\hline 518 & ATP5G3 & $\begin{array}{l}\text { ATP synthase, } \mathrm{H}+\text { transporting, mitochondrial F0 } \\
\text { complex, subunit } \mathrm{C} 3 \text { (subunit 9) }\end{array}$ & -1.53326 & 0.033335 & 0.077899 \\
\hline 9736 & USP34 & ubiquitin specific peptidase 34 & -1.75486 & 0.03368 & 0.078272 \\
\hline 8702 & b4galt4 & $\begin{array}{l}\text { UDP-Gal:betaGlcNAc beta 1,4- galactosyltransferase, } \\
\text { polypeptide } 4\end{array}$ & 1.261925 & 0.033711 & 0.078305 \\
\hline 55284 & UBE2W & ubiquitin-conjugating enzyme E2W (putative) & -1.55397 & 0.033782 & 0.078381 \\
\hline 8569 & mknk1 & MAP kinase interacting serine/threonine kinase 1 & -1.23959 & 0.034079 & 0.078697 \\
\hline 84340 & GFM2 & G elongation factor, mitochondrial 2 & -1.27849 & 0.034378 & 0.079013 \\
\hline 23395 & Lars2 & leucyl-tRNA synthetase 2 , mitochondrial & -1.4847 & 0.035084 & 0.079745 \\
\hline 2650 & GCNT1 & $\begin{array}{l}\text { glucosaminyl (N-acetyl) transferase } 1 \text {, core } 2 \text { (beta-1,6- } \\
\text { N-acetylglucosaminyltransferase) }\end{array}$ & -1.8049 & 0.0351 & 0.079762 \\
\hline 5261 & phkg2 & phosphorylase kinase, gamma 2 (testis) & -1.53901 & 0.035113 & 0.079775 \\
\hline 54956 & PARP16 & poly (ADP-ribose) polymerase family, member 16 & -1.23941 & 0.035229 & 0.079894 \\
\hline 6303 & Sat1 & spermidine/spermine N1-acetyltransferase 1 & 1.320878 & 0.035497 & 0.080167 \\
\hline 2585 & Galk2 & galactokinase 2 & -1.47591 & 0.03609 & 0.080762 \\
\hline 2591 & GALNT3 & $\begin{array}{l}\text { UDP-N-acetyl-alpha-D-galactosamine:polypeptide N- } \\
\text { acetylgalactosaminyltransferase } 3 \text { (GalNAc-T3) }\end{array}$ & 2.230769 & 0.036289 & 0.080959 \\
\hline 9101 & usp8 & ubiquitin specific peptidase 8 & -1.23924 & 0.036409 & 0.081077 \\
\hline 3643 & INSR & insulin receptor & -1.57718 & 0.036616 & 0.081281 \\
\hline 3551 & IKBKB & $\begin{array}{l}\text { inhibitor of kappa light polypeptide gene enhancer in B- } \\
\text { cells, kinase beta }\end{array}$ & -1.2703 & 0.036968 & 0.081624 \\
\hline 5091 & $\mathrm{Pc}$ & pyruvate carboxylase & -1.30897 & 0.037448 & 0.082085 \\
\hline 952 & $\operatorname{cd} 38$ & CD38 molecule & 15.3799 & 0.037492 & 0.082127 \\
\hline 29110 & Tbk1 & TANK-binding kinase 1 & 1.504834 & 0.037581 & 0.082212 \\
\hline 4353 & MPO & myeloperoxidase & 1.387249 & 0.037759 & 0.082381 \\
\hline 2730 & GCLM & Glutamate-cysteine ligase, modifier subunit & 2.376054 & 0.038101 & 0.082702 \\
\hline 8073 & ptp4a2 & protein tyrosine phosphatase type IVA, member 2 & -1.41321 & 0.038377 & 0.082959 \\
\hline 126410 & cyp4f22 & $\begin{array}{l}\text { cytochrome P450, family } 4 \text {, subfamily F, polypeptide } \\
22\end{array}$ & 1.805861 & 0.03847 & 0.083046 \\
\hline 54625 & PARP14 & poly (ADP-ribose) polymerase family, member 14 & 3.521972 & 0.038508 & 0.083081 \\
\hline 23228 & PLCL2 & phospholipase C-like 2 & -1.46176 & 0.039218 & 0.083731 \\
\hline 6391 & C1orf192 & chromosome 1 open reading frame 192 & -1.34913 & 0.039999 & 0.08443 \\
\hline 5589 & prkcsh & protein kinase $\mathrm{C}$ substrate $80 \mathrm{~K}-\mathrm{H}$ & -1.21846 & 0.040041 & 0.084467 \\
\hline 219333 & USP12 & ubiquitin specific peptidase 12 & 3.511667 & 0.040047 & 0.084473 \\
\hline 51703 & ACSL5 & acyl-CoA synthetase long-chain family member 5 & 1.309798 & 0.040066 & 0.084489 \\
\hline 55326 & AGPAT5 & $\begin{array}{l}\text { 1-acylglycerol-3-phosphate O-acyltransferase } 5 \\
\text { (lysophosphatidic acid acyltransferase, epsilon) }\end{array}$ & -1.44594 & 0.040105 & 0.084523 \\
\hline 132160 & PPM1M & protein phosphatase $1 \mathrm{M}$ ( $\mathrm{PP} 2 \mathrm{C}$ domain containing) & -1.25275 & 0.040237 & 0.084639 \\
\hline 83931 & Stk40 & serine/threonine kinase 40 & 1.278941 & 0.040325 & 0.084717 \\
\hline 25 & ABL1 & c-abl oncogene 1 , receptor tyrosine kinase & -1.31386 & 0.04041 & 0.084791 \\
\hline 11221 & Dusp10 & dual specificity phosphatase 10 & -1.6521 & 0.040579 & 0.084939 \\
\hline 7465 & wee1 & WEE1 homolog (S. pombe) & -2.99546 & 0.040715 & 0.085057 \\
\hline 58508 & MLL3 & myeloid/lymphoid or mixed-lineage leukemia 3 & -1.4705 & 0.040784 & 0.085117 \\
\hline 128 & ADH5 & $\begin{array}{l}\text { alcohol dehydrogenase } 5 \text { (class III), chi polypeptide, } \\
\text { pseudogene } 4 \text {; alcohol dehydrogenase } 5 \text { (class III), chi } \\
\text { polypeptide }\end{array}$ & -1.40619 & 0.041129 & 0.085414 \\
\hline
\end{tabular}




\begin{tabular}{|c|c|c|c|c|c|}
\hline 262 & AMD1 & adenosylmethionine decarboxylase 1 & 1.367343 & 0.041176 & 0.085454 \\
\hline 5164 & PDK2 & pyruvate dehydrogenase kinase, isozyme 2 & -2.23529 & 0.041471 & 0.085706 \\
\hline 10905 & MAN1A2 & mannosidase, alpha, class $1 \mathrm{~A}$, member 2 & -1.52329 & 0.04198 & 0.086135 \\
\hline 54961 & Ssh3 & slingshot homolog 3 (Drosophila) & -1.63321 & 0.042517 & 0.08658 \\
\hline 3176 & HNMT & histamine N-methyltransferase & -2.30044 & 0.042609 & 0.086656 \\
\hline 51380 & CSAD & cysteine sulfinic acid decarboxylase & -1.44705 & 0.042761 & 0.08678 \\
\hline 8648 & NCOA1 & nuclear receptor coactivator 1 & 1.246658 & 0.042894 & 0.086889 \\
\hline 984 & Cdk11b & $\begin{array}{l}\text { similar to cell division cycle 2-like } 1 \text { (PITSLRE } \\
\text { proteins); cell division cycle 2-like } 1 \text { (PITSLRE } \\
\text { proteins); cell division cycle 2-like } 2 \text { (PITSLRE } \\
\text { proteins) }\end{array}$ & -1.29588 & 0.043237 & 0.087167 \\
\hline 221 & Aldh3b1 & aldehyde dehydrogenase 3 family, member B1 & 1.21895 & 0.043787 & 0.087608 \\
\hline 5255 & PHKA1 & $\begin{array}{l}\text { phosphorylase kinase, alpha } 1 \text { pseudogene } 1 ; \\
\text { phosphorylase kinase, alpha } 1 \text { (muscle) }\end{array}$ & -1.45131 & 0.044104 & 0.087859 \\
\hline 178 & agl & amylo-1, 6-glucosidase, 4-alpha-glucanotransferase & -1.28695 & 0.044421 & 0.088107 \\
\hline 25778 & DSTYK & dual serine/threonine and tyrosine protein kinase & 2.031911 & 0.044696 & 0.088322 \\
\hline 57396 & clk4 & CDC-like kinase 4 & 1.311973 & 0.044732 & 0.088349 \\
\hline 64802 & NMNAT1 & nicotinamide nucleotide adenylyltransferase 1 & -1.42073 & 0.044864 & 0.088452 \\
\hline 54704 & Pdp1 & pyruvate dehyrogenase phosphatase catalytic subunit 1 & -1.81725 & 0.044979 & 0.088541 \\
\hline 3656 & IRAK2 & interleukin-1 receptor-associated kinase 2 & 3.849269 & 0.045149 & 0.088671 \\
\hline 6675 & UAP1 & UDP-N-acteylglucosamine pyrophosphorylase 1 & 1.319292 & 0.045355 & 0.088829 \\
\hline 6646 & soat1 & sterol O-acyltransferase 1 & -1.26465 & 0.045401 & 0.088864 \\
\hline 5210 & Pfkfb4 & 6-phosphofructo-2-kinase/fructose-2,6-biphosphatase 4 & -2.24328 & 0.045581 & 0.089 \\
\hline 272 & AMPD3 & adenosine monophosphate deaminase (isoform E) & 2.223332 & 0.045904 & 0.089244 \\
\hline 79731 & NARS2 & $\begin{array}{l}\text { asparaginyl-tRNA synthetase } 2 \text {, mitochondrial } \\
\text { (putative) }\end{array}$ & -1.56073 & 0.046124 & 0.089408 \\
\hline 8408 & ULK1 & unc-51-like kinase 1 (C. elegans) & -1.46332 & 0.046278 & 0.089523 \\
\hline 4820 & NKTR & natural killer-tumor recognition sequence & -1.48559 & 0.046542 & 0.089719 \\
\hline 25930 & Ptpn23 & protein tyrosine phosphatase, non-receptor type 23 & -1.50376 & 0.046709 & 0.089841 \\
\hline 158067 & c9orf98 & chromosome 9 open reading frame 98 & 1.715008 & 0.046851 & 0.089946 \\
\hline 1263 & plk3 & polo-like kinase 3 (Drosophila) & -1.47058 & 0.046899 & 0.089981 \\
\hline 10477 & Ube2e3 & $\begin{array}{l}\text { ubiquitin-conjugating enzyme E2E } 3 \text { (UBC4/5 } \\
\text { homolog, yeast) }\end{array}$ & -1.33503 & 0.047054 & 0.090094 \\
\hline 5728 & PTENP1 & $\begin{array}{l}\text { phosphatase and tensin homolog; phosphatase and } \\
\text { tensin homolog pseudogene } 1\end{array}$ & -1.28449 & 0.047283 & 0.09026 \\
\hline 2050 & EPHB4 & EPH receptor B4 & -1.60532 & 0.047391 & 0.090338 \\
\hline 9448 & MAP4K4 & mitogen-activated protein kinase kinase kinase kinase 4 & 1.523996 & 0.047778 & 0.090617 \\
\hline 10000 & Akt3 & $\begin{array}{l}\text { v-akt murine thymoma viral oncogene homolog } 3 \\
\text { (protein kinase B, gamma) }\end{array}$ & 1.62333 & 0.04792 & 0.090718 \\
\hline 64895 & PAPOLG & poly(A) polymerase gamma & -1.99907 & 0.048332 & 0.09101 \\
\hline 5279 & PIGC & phosphatidylinositol glycan anchor biosynthesis, class C & -1.82392 & 0.048389 & 0.09105 \\
\hline 5515 & PPP2CA & $\begin{array}{l}\text { protein phosphatase } 2 \text { (formerly } 2 \mathrm{~A} \text { ), catalytic subunit, } \\
\text { alpha isoform }\end{array}$ & 1.21001 & 0.048486 & 0.091117 \\
\hline 3055 & HCK & hemopoietic cell kinase & 1.778428 & 0.048637 & 0.091223 \\
\hline 85464 & $\mathrm{SSH} 2$ & slingshot homolog 2 (Drosophila) & -1.81624 & 0.04936 & 0.091724 \\
\hline 2047 & ephb1 & EPH receptor B1 & -1.81499 & 0.049381 & 0.091738 \\
\hline 10797 & mthfd 2 & $\begin{array}{l}\text { methylenetetrahydrofolate dehydrogenase (NADP+ } \\
\text { dependent) 2, methenyltetrahydrofolate cyclohydrolase }\end{array}$ & 1.554809 & 0.049397 & 0.091749 \\
\hline
\end{tabular}




\begin{tabular}{|c|c|c|c|c|c|}
\hline 4707 & NDUFB1 & $\begin{array}{l}\text { NADH dehydrogenase (ubiquinone) } 1 \text { beta subcomplex, } \\
1,7 \mathrm{kDa}\end{array}$ & -1.34528 & 0.0496 & 0.091888 \\
\hline 1371 & CPOX & coproporphyrinogen oxidase & -1.29513 & 0.04964 & 0.091915 \\
\hline 160851 & DGKH & diacylglycerol kinase, eta & 2.068368 & 0.049845 & 0.092055 \\
\hline
\end{tabular}




\section{Supplemental Table S2.3: A list of significantly altered gene sets identified by GSEA in the BMM dataset.}

\begin{tabular}{|c|c|c|c|c|c|c|}
\hline Filter & NAME & SIZE & ES & NES & $\begin{array}{l}\text { NOM p- } \\
\text { val }\end{array}$ & $\begin{array}{l}\text { FDR q- } \\
\text { val }\end{array}$ \\
\hline 1 & GO ALCOHOL CATABOLIC PROCESS & 38 & -0.606 & -1.842 & 0 & 0.008027 \\
\hline 1 & $\begin{array}{l}\text { GO_DICARBOXYLIC_ACID_METABOLIC_PROCE } \\
\text { SS }\end{array}$ & 71 & -0.478 & -1.648 & 0 & 0.046959 \\
\hline 1 & GO_GLYCOSAMINOGLYCAN_BINDING & 106 & -0.468 & -1.682 & 0 & 0.036145 \\
\hline 1 & GO_SMALL_MOLECULE_CATABOLIC_PROCESS & 211 & -0.403 & -1.606 & 0 & 0.063664 \\
\hline 1 & $\begin{array}{l}\text { KEGG_GLYCOSPHINGOLIPID_BIOSYNTHESIS_L } \\
\text { ACTO_AND_NEOLACTO_SERIES }\end{array}$ & 16 & -0.831 & -2.071 & 0 & 2.87E-04 \\
\hline 1 & $\begin{array}{l}\text { GO_ORGANIC_HYDROXY_COMPOUND_CATAB } \\
\text { OLIC_PROCESS }\end{array}$ & 43 & -0.584 & -1.795 & 0.001845 & 0.013283 \\
\hline 1 & $\begin{array}{l}\text { GO_ORGANONITROGEN_COMPOUND_CATAB } \\
\text { OLIC_PROCESS }\end{array}$ & 202 & -0.387 & -1.536 & 0.001866 & 0.100556 \\
\hline 1 & GO_CARBOHYDRATE_BINDING & 140 & -0.393 & -1.517 & 0.001992 & 0.112226 \\
\hline 1 & $\begin{array}{l}\text { GO_POSITIVE_REGULATION_OF_REACTIVE_OX } \\
\text { YGEN_SPECIES_BIOSYNTHETIC_PROCESS }\end{array}$ & 36 & -0.55 & -1.67 & 0.002004 & 0.039486 \\
\hline 1 & KEGG_GALACTOSE_METABOLISM & 21 & -0.689 & -1.836 & 0.002028 & 0.008425 \\
\hline 1 & $\begin{array}{l}\text { GO_REGULATION_OF_NITRIC_OXIDE_BIOSYNT } \\
\text { HETIC_PROCESS }\end{array}$ & 39 & -0.563 & -1.734 & 0.002062 & 0.023622 \\
\hline 1 & KEGG_TRYPTOPHAN_METABOLISM & 23 & -0.702 & -1.921 & 0.003984 & 0.003121 \\
\hline 1 & $\begin{array}{l}\text { GO_POSITIVE_REGULATION_OF_MAPK_CASCA } \\
\text { DE }\end{array}$ & 284 & -0.342 & -1.416 & 0.007233 & 0.186908 \\
\hline 1 & $\begin{array}{l}\text { GO_REGULATION_OF_REACTIVE_OXYGEN_SPE } \\
\text { CIES_BIOSYNTHETIC_PROCESS }\end{array}$ & 49 & -0.502 & -1.619 & 0.007648 & 0.058081 \\
\hline 1 & KEGG_GLYCEROLIPID_METABOLISM & 33 & -0.579 & -1.707 & 0.007843 & 0.029353 \\
\hline 1 & KEGG_HISTIDINE_METABOLISM & 17 & -0.641 & -1.646 & 0.00813 & 0.047722 \\
\hline 1 & BIOCARTA_BIOPEPTIDES_PATHWAY & 35 & -0.531 & -1.611 & 0.01002 & 0.061531 \\
\hline 1 & GO_LIPASE_ACTIVITY & 64 & -0.47 & -1.586 & 0.014056 & 0.072478 \\
\hline 1 & $\begin{array}{l}\text { GO_GLYCOSPHINGOLIPID_METABOLIC_PROCE } \\
\text { SS }\end{array}$ & 51 & -0.496 & -1.592 & 0.015625 & 0.069857 \\
\hline 1 & GO_SERINE_HYDROLASE_ACTIVITY & 93 & -0.407 & -1.48 & 0.019268 & 0.138648 \\
\hline 1 & GO_MEMBRANE_LIPID_CATABOLIC_PROCESS & 17 & -0.616 & -1.597 & 0.020619 & 0.067574 \\
\hline
\end{tabular}

\begin{tabular}{|l|c|}
\hline \multicolumn{2}{|c|}{ Legend } \\
\hline 1 & Immune signaling and function \\
\hline 2 & Cellular metabolism \\
\hline NAME & Other biological states and processes \\
\hline SIZE & Name of gene set \\
\hline ES & $\begin{array}{c}\text { Enrichment Score for gene set (Degree to } \\
\text { which this gene set is overrepresented at the } \\
\text { top or bottom of the ranked lit of genes) }\end{array}$ \\
\hline NES & Normalized enrichment score \\
\hline NOM p-val & Nominal p-value \\
\hline FDR q-val & $\begin{array}{c}\text { False discovery rate (corrected for multiple } \\
\text { testing) }\end{array}$ \\
\hline
\end{tabular}




\begin{tabular}{|c|c|c|c|c|c|c|c|}
\hline 1 & KEGG_BETA_ALANINE_METABOLISM & 16 & -0.644 & -1.597 & 0.021552 & 0.067672 & 22 \\
\hline 1 & GO_SPHINGOLIPID_METABOLIC_PROCESS & 97 & -0.391 & -1.407 & 0.022857 & 0.19404 & 23 \\
\hline 1 & $\begin{array}{l}\text { KEGG_ARGININE_AND_PROLINE_METABOLIS } \\
\mathrm{M}\end{array}$ & 34 & -0.519 & -1.542 & 0.022965 & 0.097655 & 24 \\
\hline 1 & GO_MEMBRANE_LIPID_METABOLIC_PROCESS & 132 & -0.364 & -1.362 & 0.026718 & 0.232762 & 25 \\
\hline 1 & GO_CELLULAR_LIPID_CATABOLIC_PROCESS & 100 & -0.398 & -1.436 & 0.028681 & 0.170066 & 26 \\
\hline 1 & BIOCARTA_CERAMIDE_PATHWAY & 21 & -0.565 & -1.492 & 0.030426 & 0.130386 & 27 \\
\hline 1 & GO CARBOHYDRATE PHOSPHORYLATION & 18 & -0.599 & -1.554 & 0.030928 & 0.090754 & 28 \\
\hline 1 & $\begin{array}{l}\text { GO_ORGANOPHOSPHATE_CATABOLIC_PROCE } \\
\text { SS }\end{array}$ & 80 & -0.403 & -1.406 & 0.035714 & 0.1943 & 29 \\
\hline 1 & GO_PHOSPHOLIPASE_ACTIVITY & 53 & -0.446 & -1.439 & 0.037475 & 0.167423 & 30 \\
\hline 1 & GO_L_ASCORBIC_ACID_BINDING & 17 & -0.611 & -1.568 & 0.037694 & 0.082579 & 31 \\
\hline 1 & $\begin{array}{l}\text { GO_CELLULAR_AMINO_ACID_CATABOLIC_PR } \\
\text { OCESS }\end{array}$ & 65 & -0.415 & -1.401 & 0.037924 & 0.198854 & 32 \\
\hline 1 & $\begin{array}{l}\text { GO_BENZENE_CONTAINING_COMPOUND_ME } \\
\text { TABOLIC_PROCESS }\end{array}$ & 15 & -0.647 & -1.615 & 0.038217 & 0.059969 & 33 \\
\hline 1 & $\begin{array}{l}\text { GO_TETRAHYDROFOLATE_METABOLIC_PROCE } \\
\text { SS }\end{array}$ & 16 & -0.619 & -1.555 & 0.039175 & 0.089808 & 34 \\
\hline 1 & $\begin{array}{l}\text { KEGG_NICOTINATE_AND_NICOTINAMIDE_ME } \\
\text { TABOLISM }\end{array}$ & 16 & -0.59 & -1.517 & 0.041667 & 0.112188 & 35 \\
\hline 1 & $\begin{array}{l}\text { GO_RNA_PHOSPHODIESTER_BOND_HYDROLY } \\
\text { SIS_EXONUCLEOLYTIC }\end{array}$ & 26 & -0.535 & -1.49 & 0.04251 & 0.131591 & 36 \\
\hline 1 & $\begin{array}{l}\text { GO_PYRIMIDINE_NUCLEOSIDE_METABOLIC_P } \\
\text { ROCESS }\end{array}$ & 31 & -0.513 & -1.493 & 0.044444 & 0.129418 & 37 \\
\hline 1 & REACTOME_PYRIMIDINE_METABOLISM & 17 & -0.577 & -1.493 & 0.044625 & 0.129332 & 38 \\
\hline 1 & KEGG_GLYCOLYSIS_GLUCONEOGENESIS & 39 & -0.486 & -1.454 & 0.045161 & 0.157203 & 39 \\
\hline 1 & GO_PHOSPHOLIPASE_C_ACTIVITY & 18 & -0.602 & -1.559 & 0.048523 & 0.087738 & 40 \\
\hline 2 & GO_CCR_CHEMOKINE_RECEPTOR_BINDING & 15 & -0.813 & -2.012 & 0 & $8.16 \mathrm{E}-04$ & 1 \\
\hline 2 & $\begin{array}{l}\text { GO_CELLULAR_RESPONSE_TO_CYTOKINE_STI } \\
\text { MULUS }\end{array}$ & 379 & -0.514 & -2.213 & 0 & 2.25E-05 & 2 \\
\hline 2 & $\begin{array}{l}\text { GO_CELLULAR_RESPONSE_TO_INTERFERON_G } \\
\text { AMMA }\end{array}$ & 64 & -0.687 & -2.288 & 0 & 0 & 3 \\
\hline 2 & GO_CELLULAR_RESPONSE_TO_VIRUS & 16 & -0.76 & -1.875 & 0 & 0.005497 & 4 \\
\hline 2 & GO_CHEMOKINE_ACTIVITY & 23 & -0.687 & -1.91 & 0 & 0.003569 & 5 \\
\hline 2 & GO_CHEMOKINE_RECEPTOR_BINDING & 31 & -0.671 & -1.967 & 0 & 0.001607 & 6 \\
\hline 2 & GO CYTOKINE ACTIVITY & 86 & -0.572 & -2.016 & 0 & 7.73E-04 & 7 \\
\hline
\end{tabular}




\begin{tabular}{|c|c|c|c|c|c|c|}
\hline 2 & $\begin{array}{l}\text { GO_CYTOKINE_MEDIATED_SIGNALING_PATH } \\
\text { WAY }\end{array}$ & 277 & -0.553 & -2.286 & 0 & 0 \\
\hline 2 & GO_CYTOKINE_RECEPTOR_BINDING & 145 & -0.462 & -1.772 & 0 & 0.016825 \\
\hline 2 & $\begin{array}{l}\text { GO_DEFENSE_RESPONSE_TO_OTHER_ORGANI } \\
\text { SM }\end{array}$ & 233 & -0.529 & -2.145 & 0 & 7.15E-05 \\
\hline 2 & GO_DEFENSE_RESPONSE_TO_VIRUS & 91 & -0.726 & -2.581 & 0 & 0 \\
\hline 2 & GO_IMMUNE_EFFECTOR_PROCESS & 288 & -0.444 & -1.831 & 0 & 0.008851 \\
\hline 2 & GO_INFLAMMATORY_RESPONSE & 260 & -0.421 & -1.729 & 0 & 0.024451 \\
\hline 2 & GO_INNATE_IMMUNE_RESPONSE & 298 & -0.551 & -2.316 & 0 & 0 \\
\hline 2 & $\begin{array}{l}\text { GO_INTERFERON_GAMMA_MEDIATED_SIGNA } \\
\text { LING_PATHWAY }\end{array}$ & 41 & -0.717 & -2.181 & 0 & 3.36E-05 \\
\hline 2 & $\begin{array}{l}\text { GO_INTRACELLULAR_RECEPTOR_SIGNALING_ } \\
\text { PATHWAY }\end{array}$ & 112 & -0.439 & -1.616 & 0 & 0.05963 \\
\hline 2 & GO_LEUKOCYTE_ACTIVATION & 283 & -0.349 & -1.45 & 0 & 0.159365 \\
\hline 2 & GO_LYMPHOCYTE_CHEMOTAXIS & 18 & -0.723 & -1.863 & 0 & 0.006418 \\
\hline 2 & $\begin{array}{l}\text { GO_NEGATIVE_REGULATION_OF_IMMUNE_SY } \\
\text { STEM_PROCESS }\end{array}$ & 225 & -0.408 & -1.654 & 0 & 0.044845 \\
\hline 2 & $\begin{array}{l}\text { GO_NEGATIVE_REGULATION_OF_LOCOMOTIO } \\
\mathrm{N}\end{array}$ & 175 & -0.388 & -1.52 & 0 & 0.110171 \\
\hline 2 & $\begin{array}{l}\text { GO_NEGATIVE_REGULATION_OF_TYPE_I_INTE } \\
\text { RFERON_PRODUCTION }\end{array}$ & 28 & -0.675 & -1.915 & 0 & 0.003356 \\
\hline 2 & $\begin{array}{l}\text { GO_NEGATIVE_REGULATION_OF_VIRAL_GEN } \\
\text { OME_REPLICATION }\end{array}$ & 30 & -0.717 & -2.102 & 0 & $1.60 \mathrm{E}-04$ \\
\hline 2 & $\begin{array}{l}\text { GO_NEGATIVE_REGULATION_OF_VIRAL_PROC } \\
\text { ESS }\end{array}$ & 60 & -0.642 & -2.157 & 0 & $6.44 \mathrm{E}-05$ \\
\hline 2 & GO_POSITIVE_REGULATION_OF_CHEMOTAXIS & 78 & -0.516 & -1.8 & 0 & 0.01257 \\
\hline 2 & $\begin{array}{l}\text { GO_POSITIVE_REGULATION_OF_DEFENSE_RES } \\
\text { PONSE }\end{array}$ & 241 & -0.354 & -1.448 & 0 & 0.160176 \\
\hline 2 & $\begin{array}{l}\text { GO_POSITIVE_REGULATION_OF_INTERFERON } \\
\text { _ALPHA_PRODUCTION }\end{array}$ & 15 & -0.831 & -2.064 & 0 & 3.25E-04 \\
\hline 2 & $\begin{array}{l}\text { GO_POSITIVE_REGULATION_OF_LEUKOCYTE_ } \\
\text { CHEMOTAXIS }\end{array}$ & 54 & -0.586 & -1.939 & 0 & 0.002453 \\
\hline 2 & $\begin{array}{l}\text { GO_POSITIVE_REGULATION_OF_LEUKOCYTE_ } \\
\text { MIGRATION }\end{array}$ & 72 & -0.538 & -1.821 & 0 & 0.009989 \\
\hline 2 & $\begin{array}{l}\text { GO_POSITIVE_REGULATION_OF_RESPONSE_T } \\
\text { O_EXTERNAL_STIMULUS }\end{array}$ & 186 & -0.4 & -1.596 & 0 & 0.06794 \\
\hline 2 & $\begin{array}{l}\text { GO_REGULATION_OF_CYTOKINE_PRODUCTIO } \\
\mathrm{N}\end{array}$ & 354 & -0.373 & -1.592 & 0 & 0.0699 \\
\hline 2 & GO_REGULATION_OF_DEFENSE_RESPONSE & 475 & -0.378 & -1.642 & 0 & 0.048973 \\
\hline
\end{tabular}




\begin{tabular}{|c|c|c|c|c|c|c|c|}
\hline 2 & $\begin{array}{l}\text { GO_REGULATION_OF_IMMUNE_EFFECTOR_P } \\
\text { ROCESS }\end{array}$ & 251 & -0.401 & -1.657 & 0 & 0.043913 & 32 \\
\hline 2 & $\begin{array}{l}\text { GO_REGULATION_OF_INNATE_IMMUNE_RES } \\
\text { PONSE }\end{array}$ & 243 & -0.421 & -1.719 & 0 & 0.026798 & 33 \\
\hline 2 & $\begin{array}{l}\text { GO_REGULATION_OF_INTERFERON_ALPHA_P } \\
\text { RODUCTION }\end{array}$ & 18 & -0.843 & -2.196 & 0 & $2.20 \mathrm{E}-05$ & 34 \\
\hline 2 & $\begin{array}{l}\text { GO_REGULATION_OF_LEUKOCYTE_APOPTOTI } \\
\text { C_PROCESS }\end{array}$ & 54 & -0.594 & -1.937 & 0 & 0.002539 & 35 \\
\hline 2 & $\begin{array}{l}\text { GO_REGULATION_OF_LEUKOCYTE_CHEMOTA } \\
\text { XIS }\end{array}$ & 60 & -0.595 & -1.969 & 0 & 0.001556 & 36 \\
\hline 2 & $\begin{array}{l}\text { GO_REGULATION_OF_LEUKOCYTE_MIGRATIO } \\
\mathrm{N}\end{array}$ & 97 & -0.51 & -1.837 & 0 & 0.008325 & 37 \\
\hline 2 & $\begin{array}{l}\text { GO_REGULATION_OF_LEUKOCYTE_PROLIFERA } \\
\text { TION }\end{array}$ & 122 & -0.417 & -1.569 & 0 & 0.08232 & 38 \\
\hline 2 & $\begin{array}{l}\text { GO_REGULATION_OF_LYMPHOCYTE_MIGRATI } \\
\text { ON }\end{array}$ & 26 & -0.703 & -2.02 & 0 & 7.27E-04 & 39 \\
\hline 2 & GO_REGULATION_OF_STAT_CASCADE & 79 & -0.495 & -1.748 & 0 & 0.020825 & 40 \\
\hline 2 & $\begin{array}{l}\text { GO_REGULATION_OF_TYPE_I_INTERFERON_M } \\
\text { EDIATED_SIGNALING_PATHWAY }\end{array}$ & 20 & -0.725 & -1.916 & 0 & 0.00333 & 41 \\
\hline 2 & $\begin{array}{l}\text { GO_REGULATION_OF_TYPE_I_INTERFERON_P } \\
\text { RODUCTION }\end{array}$ & 89 & -0.515 & -1.849 & 0 & 0.007535 & 42 \\
\hline 2 & $\begin{array}{l}\text { GO_REGULATION_OF_VIRAL_ENTRY_INTO_H } \\
\text { OST_CELL }\end{array}$ & 20 & -0.703 & -1.86 & 0 & 0.006584 & 43 \\
\hline 2 & $\begin{array}{l}\text { GO_REGULATION_OF_VIRAL_GENOME_REPLI } \\
\text { CATION }\end{array}$ & 50 & -0.62 & -2.009 & 0 & $8.72 \mathrm{E}-04$ & 44 \\
\hline 2 & GO_RESPONSE_TO_CYTOKINE & 459 & -0.488 & -2.117 & 0 & $1.24 \mathrm{E}-04$ & 45 \\
\hline 2 & GO_RESPONSE_TO_INTERFERON_ALPHA & 17 & -0.823 & -2.107 & 0 & $1.36 \mathrm{E}-04$ & 46 \\
\hline 2 & GO_RESPONSE_TO_INTERFERON_GAMMA & 78 & -0.664 & -2.324 & 0 & 0 & 47 \\
\hline 2 & $\begin{array}{l}\text { GO_RESPONSE_TO_TUMOR_NECROSIS_FACT } \\
\text { OR }\end{array}$ & 148 & -0.439 & -1.688 & 0 & 0.034537 & 48 \\
\hline 2 & GO_RESPONSE_TO_TYPE_I_INTERFERON & 38 & -0.876 & -2.635 & 0 & 0 & 49 \\
\hline 2 & GO_RESPONSE_TO_VIRUS & 148 & -0.65 & -2.491 & 0 & 0 & 50 \\
\hline 2 & GO_STAT_CASCADE & 35 & -0.658 & -1.995 & 0 & 0.001071 & 51 \\
\hline 2 & HALLMARK_ALLOGRAFT_REJECTION & 139 & -0.522 & -2.003 & 0 & 9.45E-04 & 52 \\
\hline 2 & HALLMARK_IL2_STAT5_SIGNALING & 152 & -0.45 & -1.757 & 0 & 0.019207 & 53 \\
\hline 2 & HALLMARK_IL6_JAK_STAT3_SIGNALING & 72 & -0.593 & -2.037 & 0 & $5.35 \mathrm{E}-04$ & 54 \\
\hline 2 & HALLMARK_INFLAMMATORY_RESPONSE & 156 & -0.442 & -1.721 & 0 & 0.026341 & 55 \\
\hline 2 & HALLMARK_INTERFERON_ALPHA_RESPONSE & 74 & -0.903 & -3.124 & 0 & 0 & 56 \\
\hline
\end{tabular}




\begin{tabular}{|c|c|c|c|c|c|c|c|}
\hline 2 & HALLMARK_INTERFERON_GAMMA_RESPONSE & 159 & -0.826 & -3.203 & 0 & 0 & 57 \\
\hline 2 & KEGG_CHEMOKINE_SIGNALING_PATHWAY & 140 & -0.425 & -1.641 & 0 & 0.04959 & 58 \\
\hline 2 & $\begin{array}{l}\text { KEGG_CYTOKINE_CYTOKINE_RECEPTOR_INTER } \\
\text { ACTION }\end{array}$ & 131 & -0.518 & -1.954 & 0 & 0.001986 & 59 \\
\hline 2 & KEGG_CYTOSOLIC_DNA_SENSING_PATHWAY & 36 & -0.653 & -1.95 & 0 & 0.002077 & 60 \\
\hline 2 & KEGG_JAK_STAT_SIGNALING_PATHWAY & 94 & -0.516 & -1.85 & 0 & 0.007426 & 61 \\
\hline 2 & $\begin{array}{l}\text { KEGG_TOLL_LIKE_RECEPTOR_SIGNALING_PAT } \\
\text { HWAY }\end{array}$ & 81 & -0.492 & -1.733 & 0 & 0.023771 & 62 \\
\hline 2 & $\begin{array}{l}\text { REACTOME_ANTIVIRAL_MECHANISM_BY_IFN } \\
\text { STIMULATED_GENES }\end{array}$ & 57 & -0.619 & -2.027 & 0 & $6.22 \mathrm{E}-04$ & 63 \\
\hline 2 & $\begin{array}{l}\text { REACTOME_CYTOKINE_SIGNALING_IN_IMMU } \\
\text { NE_SYSTEM }\end{array}$ & 196 & -0.611 & -2.438 & 0 & 0 & 64 \\
\hline 2 & $\begin{array}{l}\text { REACTOME_INTERFERON_ALPHA_BETA_SIGN } \\
\text { ALING }\end{array}$ & 37 & -0.867 & -2.645 & 0 & 0 & 65 \\
\hline 2 & $\begin{array}{l}\text { REACTOME_INTERFERON_GAMMA_SIGNALIN } \\
\text { G }\end{array}$ & 38 & -0.729 & -2.219 & 0 & $1.39 \mathrm{E}-05$ & 66 \\
\hline 2 & REACTOME_INTERFERON_SIGNALING & 109 & -0.717 & -2.602 & 0 & 0 & 67 \\
\hline 2 & $\begin{array}{l}\text { REACTOME_NEGATIVE_REGULATORS_OF_RIG } \\
\text { _I_MDA5_SIGNALING }\end{array}$ & 22 & -0.698 & -1.891 & 0 & 0.004576 & 68 \\
\hline 2 & $\begin{array}{l}\text { REACTOME_RIG_I_MDA5_MEDIATED_INDUCT } \\
\text { ION_OF_IFN_ALPHA_BETA_PATHWAYS }\end{array}$ & 46 & -0.588 & -1.85 & 0 & 0.007426 & 69 \\
\hline 2 & GO_LEUKOCYTE_DIFFERENTIATION & 199 & -0.376 & -1.478 & 0.001852 & 0.139835 & 70 \\
\hline 2 & $\begin{array}{l}\text { GO_NEGATIVE_REGULATION_OF_CYTOKINE_P } \\
\text { RODUCTION }\end{array}$ & 129 & -0.425 & -1.59 & 0.001916 & 0.071031 & 71 \\
\hline 2 & GO_CELL_CHEMOTAXIS & 106 & -0.406 & -1.488 & 0.001931 & 0.132835 & 72 \\
\hline 2 & REACTOME_INNATE_IMMUNE_SYSTEM & 161 & -0.405 & -1.574 & 0.001946 & 0.079098 & 73 \\
\hline 2 & $\begin{array}{l}\text { GO_REGULATION_OF_RESPONSE_TO_CYTOKI } \\
\text { NE_STIMULUS }\end{array}$ & 99 & -0.458 & -1.645 & 0.001961 & 0.047782 & 74 \\
\hline 2 & GO_REGULATION_OF_CHEMOTAXIS & 111 & -0.462 & -1.7 & 0.00198 & 0.03133 & 75 \\
\hline 2 & GO_LEUKOCYTE_PROLIFERATION & 52 & -0.531 & -1.716 & 0.001996 & 0.027287 & 76 \\
\hline 2 & $\begin{array}{l}\text { GO_POSITIVE_REGULATION_OF_INTERFERON } \\
\text { _BETA_PRODUCTION }\end{array}$ & 28 & -0.634 & -1.808 & 0.002016 & 0.011516 & 77 \\
\hline 2 & GO_NATURAL_KILLER_CELL_ACTIVATION & 24 & -0.653 & -1.814 & 0.002033 & 0.010719 & 78 \\
\hline 2 & BIOCARTA_IL10_PATHWAY & 16 & -0.711 & -1.769 & 0.002045 & 0.017192 & 79 \\
\hline 2 & $\begin{array}{l}\text { GO_POSITIVE_REGULATION_OF_STAT_CASCA } \\
\text { DE }\end{array}$ & 38 & -0.571 & -1.733 & 0.002058 & 0.02379 & 80 \\
\hline 2 & GO_MHC_PROTEIN_BINDING & 17 & -0.747 & -1.912 & 0.002114 & 0.003434 & 81 \\
\hline
\end{tabular}




\begin{tabular}{|c|c|c|c|c|c|c|c|}
\hline 2 & GO_RESPONSE_TO_INTERFERON_BETA & 17 & -0.707 & -1.825 & 0.002128 & 0.009489 & 82 \\
\hline 2 & $\begin{array}{l}\text { GO_POSITIVE_REGULATION_OF_CYTOKINE_PR } \\
\text { ODUCTION }\end{array}$ & 235 & -0.359 & -1.47 & 0.003448 & 0.145288 & 83 \\
\hline 2 & $\begin{array}{l}\text { GO_RESPONSE_TO_MOLECULE_OF_BACTERIA } \\
\text { L_ORIGIN }\end{array}$ & 225 & -0.355 & -1.455 & 0.003534 & 0.156641 & 84 \\
\hline 2 & $\begin{array}{l}\text { GO_NEGATIVE_REGULATION_OF_IMMUNE_EF } \\
\text { FECTOR_PROCESS }\end{array}$ & 61 & -0.496 & -1.677 & 0.003774 & 0.037419 & 85 \\
\hline 2 & GO_LYMPHOCYTE_MIGRATION & 22 & -0.717 & -1.911 & 0.004016 & 0.003462 & 86 \\
\hline 2 & $\begin{array}{l}\text { GO_NEGATIVE_REGULATION_OF_T_CELL_PRO } \\
\text { LIFERATION }\end{array}$ & 27 & -0.612 & -1.772 & 0.00409 & 0.016805 & 87 \\
\hline 2 & $\begin{array}{l}\text { GO_NEGATIVE_REGULATION_OF_LEUKOCYTE } \\
\text { _MEDIATED_IMMUNITY }\end{array}$ & 29 & -0.62 & -1.778 & 0.004149 & 0.015867 & 88 \\
\hline 2 & $\begin{array}{l}\text { REACTOME_IMMUNOREGULATORY_INTERACT } \\
\text { IONS_BETWEEN_A_LYMPHOID_AND_A_NON_ } \\
\text { LYMPHOID_CELL }\end{array}$ & 28 & -0.623 & -1.786 & 0.004211 & 0.01465 & 89 \\
\hline 2 & $\begin{array}{l}\text { GO_NEGATIVE_REGULATION_OF_INTERFERO } \\
\text { N_GAMMA_PRODUCTION }\end{array}$ & 18 & -0.682 & -1.766 & 0.004255 & 0.017619 & 90 \\
\hline 2 & $\begin{array}{l}\text { GO_NEGATIVE_REGULATION_OF_LEUKOCYTE } \\
\text { _APOPTOTIC_PROCESS }\end{array}$ & 29 & -0.619 & -1.763 & 0.004255 & 0.018136 & 91 \\
\hline 2 & $\begin{array}{l}\text { GO_NEGATIVE_REGULATION_OF_INNATE_IM } \\
\text { MUNE_RESPONSE }\end{array}$ & 19 & -0.669 & -1.731 & 0.004329 & 0.024205 & 92 \\
\hline 2 & GO_IMMUNE_SYSTEM_DEVELOPMENT & 390 & -0.32 & -1.375 & 0.005525 & 0.21944 & 93 \\
\hline 2 & $\begin{array}{l}\text { GO_REGULATION_OF_T_CELL_APOPTOTIC_PR } \\
\text { OCESS }\end{array}$ & 21 & -0.622 & -1.628 & 0.005929 & 0.055051 & 94 \\
\hline 2 & $\begin{array}{l}\text { GO_CHEMOKINE_MEDIATED_SIGNALING_PAT } \\
\text { HWAY }\end{array}$ & 35 & -0.592 & -1.774 & 0.006024 & 0.016461 & 95 \\
\hline 2 & $\begin{array}{l}\text { GO_REGULATION_OF_LYMPHOCYTE_APOPTO } \\
\text { TIC_PROCESS }\end{array}$ & 37 & -0.553 & -1.657 & 0.006186 & 0.043854 & 96 \\
\hline 2 & KEGG_PRIMARY_IMMUNODEFICIENCY & 24 & -0.625 & -1.716 & 0.006772 & 0.027187 & 97 \\
\hline 2 & GO_MYELOID_LEUKOCYTE_ACTIVATION & 72 & -0.443 & -1.531 & 0.007619 & 0.1038 & 98 \\
\hline 2 & $\begin{array}{l}\text { GO_POSITIVE_REGULATION_OF_TYPE_IIINTER } \\
\text { FERON_PRODUCTION }\end{array}$ & 61 & -0.48 & -1.605 & 0.007663 & 0.064268 & 99 \\
\hline 2 & $\begin{array}{l}\text { GO_POSITIVE_REGULATION_OF_LEUKOCYTE_ } \\
\text { PROLIFERATION }\end{array}$ & 83 & -0.44 & -1.556 & 0.007905 & 0.089419 & 100 \\
\hline 2 & GO_ANTIGEN_BINDING & 28 & -0.583 & -1.655 & 0.008032 & 0.044545 & 101 \\
\hline 2 & $\begin{array}{l}\text { GO_REGULATION_OF_TYROSINE_PHOSPHORY } \\
\text { LATION_OF_STAT_PROTEIN }\end{array}$ & 37 & -0.546 & -1.664 & 0.008048 & 0.041646 & 102 \\
\hline 2 & $\begin{array}{l}\text { GO_REGULATION_OF_RESPONSE_TO_INTERFE } \\
\text { RON_GAMMA }\end{array}$ & 17 & -0.649 & -1.687 & 0.008065 & 0.034773 & 103 \\
\hline
\end{tabular}




\begin{tabular}{|c|c|c|c|c|c|c|c|}
\hline 2 & $\begin{array}{l}\text { KEGG_INTESTINAL_IMMUNE_NETWORK_FOR } \\
\text { _IGA_PRODUCTION }\end{array}$ & 25 & -0.645 & -1.759 & 0.008351 & 0.018745 & 104 \\
\hline 2 & GO_RESPONSE_TO_EXOGENOUS_DSRNA & 21 & -0.633 & -1.684 & 0.008602 & 0.035416 & 105 \\
\hline 2 & KEGG_SYSTEMIC_LUPUS_ERYTHEMATOSUS & 37 & -0.516 & -1.592 & 0.009785 & 0.069762 & 106 \\
\hline 2 & GO_LEUKOCYTE_CHEMOTAXIS & 74 & -0.43 & -1.492 & 0.011765 & 0.130324 & 107 \\
\hline 2 & $\begin{array}{l}\text { GO_REGULATION_OF_T_CELL_PROLIFERATIO } \\
\mathrm{N}\end{array}$ & 81 & -0.434 & -1.52 & 0.012097 & 0.11046 & 108 \\
\hline 2 & GO_RESPONSE_TO_INTERLEUKIN_4 & 20 & -0.656 & -1.758 & 0.012146 & 0.018826 & 109 \\
\hline 2 & $\begin{array}{l}\text { GO_NEGATIVE_REGULATION_OF_LEUKOCYTE } \\
\text { _PROLIFERATION }\end{array}$ & 38 & -0.532 & -1.6 & 0.012195 & 0.066222 & 110 \\
\hline 2 & $\begin{array}{l}\text { GO_CELLULAR_RESPONSE_TO_INTERLEUKIN_ } \\
4\end{array}$ & 18 & -0.644 & -1.651 & 0.01227 & 0.045868 & 111 \\
\hline 2 & $\begin{array}{l}\text { GO_NEGATIVE_REGULATION_OF_CYTOKINE_B } \\
\text { IOSYNTHETIC_PROCESS }\end{array}$ & 19 & -0.638 & -1.642 & 0.01227 & 0.048993 & 112 \\
\hline 2 & GO_MONOCYTE_CHEMOTAXIS & 18 & -0.668 & -1.694 & 0.012632 & 0.032854 & 113 \\
\hline 2 & $\begin{array}{l}\text { GO_POSITIVE_REGULATION_OF_INFLAMMAT } \\
\text { ORY_RESPONSE }\end{array}$ & 66 & -0.451 & -1.524 & 0.013208 & 0.107306 & 114 \\
\hline 2 & $\begin{array}{l}\text { GO_REGULATION_OF_LEUKOCYTE_MEDIATED } \\
\text { _IMMUNITY }\end{array}$ & 98 & -0.411 & -1.483 & 0.013834 & 0.137092 & 115 \\
\hline 2 & $\begin{array}{l}\text { REACTOME_ANTIGEN_PROCESSING_CROSS_P } \\
\text { RESENTATION }\end{array}$ & 61 & -0.467 & -1.548 & 0.014141 & 0.093798 & 116 \\
\hline 2 & $\begin{array}{l}\text { GO_CYTOPLASMIC_PATTERN_RECOGNITION_ } \\
\text { RECEPTOR_SIGNALING_PATHWAY }\end{array}$ & 22 & -0.598 & -1.606 & 0.014228 & 0.063783 & 117 \\
\hline 2 & $\begin{array}{l}\text { GO_POSITIVE_REGULATION_OF_LEUKOCYTE_ } \\
\text { APOPTOTIC_PROCESS }\end{array}$ & 17 & -0.658 & -1.68 & 0.014463 & 0.036618 & 118 \\
\hline 2 & GO_ZYMOGEN_ACTIVATION & 74 & -0.451 & -1.552 & 0.015209 & 0.091755 & 119 \\
\hline 2 & $\begin{array}{l}\text { REACTOME_CHEMOKINE_RECEPTORS_BIND_C } \\
\text { HEMOKINES }\end{array}$ & 31 & -0.576 & -1.651 & 0.015842 & 0.045838 & 120 \\
\hline 2 & GO_REGULATION_OF_T_CELL_MIGRATION & 15 & -0.692 & -1.708 & 0.016598 & 0.029133 & 121 \\
\hline 2 & GO_DENDRITIC_CELL_DIFFERENTIATION & 21 & -0.589 & -1.613 & 0.017167 & 0.060817 & 122 \\
\hline 2 & $\begin{array}{l}\text { GO_ANTIGEN_PROCESSING_AND_PRESENTATI } \\
\text { ON_OF_PEPTIDE_ANTIGEN_VIA_MHC_CLASS_ } \\
\text { I }\end{array}$ & 74 & -0.425 & -1.466 & 0.017375 & 0.148547 & 123 \\
\hline 2 & GO_LEUKOCYTE_MIGRATION & 174 & -0.348 & -1.374 & 0.019724 & 0.220618 & 124 \\
\hline 2 & $\begin{array}{l}\text { KEGG_NOD_LIKE_RECEPTOR_SIGNALING_PAT } \\
\text { HWAY }\end{array}$ & 41 & -0.495 & -1.531 & 0.020367 & 0.103558 & 125 \\
\hline 2 & $\begin{array}{l}\text { GO_REGULATION_OF_INTERFERON_BETA_PR } \\
\text { ODUCTION }\end{array}$ & 37 & -0.538 & -1.623 & 0.021277 & 0.05672 & 126 \\
\hline
\end{tabular}




\begin{tabular}{|c|c|c|c|c|c|c|c|}
\hline 2 & $\begin{array}{l}\text { GO_REGULATION_OF_PRODUCTION_OF_MOL } \\
\text { ECULAR_MEDIATOR_OF_IMMUNE_RESPONSE }\end{array}$ & 60 & -0.426 & -1.428 & 0.021782 & 0.176893 & 127 \\
\hline 2 & GO_REGULATION_OF_CELL_KILLING & 32 & -0.543 & -1.575 & 0.022358 & 0.078728 & 128 \\
\hline 2 & GO_LYMPHOCYTE_DIFFERENTIATION & 142 & -0.358 & -1.367 & 0.02277 & 0.227237 & 129 \\
\hline 2 & GO_LYMPHOCYTE_ACTIVATION & 233 & -0.338 & -1.359 & 0.023214 & 0.235489 & 130 \\
\hline 2 & $\begin{array}{l}\text { GO_REGULATION_OF_INTERFERON_GAMMA_ } \\
\text { PRODUCTION }\end{array}$ & 54 & -0.448 & -1.462 & 0.023529 & 0.151276 & 131 \\
\hline 2 & GO_RESPONSE_TO_PROTOZOAN & 16 & -0.636 & -1.599 & 0.023861 & 0.067067 & 132 \\
\hline 2 & $\begin{array}{l}\text { GO_REGULATION_OF_DEFENSE_RESPONSE_T } \\
\text { O_VIRUS }\end{array}$ & 113 & -0.384 & -1.407 & 0.025225 & 0.193971 & 133 \\
\hline 2 & $\begin{array}{l}\text { REACTOME_CLASS_I_MHC_MEDIATED_ANTIG } \\
\text { EN_PROCESSING_PRESENTATION }\end{array}$ & 192 & -0.342 & -1.348 & 0.025688 & 0.244188 & 134 \\
\hline 2 & GO_MYELOID_CELL_DIFFERENTIATION & 129 & -0.373 & -1.408 & 0.026769 & 0.193629 & 135 \\
\hline 2 & $\begin{array}{l}\text { GO_NEGATIVE_REGULATION_OF_IMMUNE_R } \\
\text { ESPONSE }\end{array}$ & 67 & -0.421 & -1.431 & 0.027613 & 0.174147 & 136 \\
\hline 2 & $\begin{array}{l}\text { GO_POSITIVE_REGULATION_OF_VIRAL_GENO } \\
\text { ME_REPLICATION }\end{array}$ & 25 & -0.548 & -1.532 & 0.029228 & 0.103297 & 137 \\
\hline 2 & BIOCARTA_IL2_PATHWAY & 19 & -0.601 & -1.567 & 0.029644 & 0.082852 & 138 \\
\hline 2 & $\begin{array}{l}\text { GO_NEGATIVE_REGULATION_OF_LYMPHOCYT } \\
\text { E_MEDIATED_IMMUNITY }\end{array}$ & 19 & -0.603 & -1.551 & 0.031513 & 0.092303 & 139 \\
\hline 2 & $\begin{array}{l}\text { GO_REGULATION_OF_TYROSINE_PHOSPHORY } \\
\text { LATION_OF_STAT3_PROTEIN }\end{array}$ & 28 & -0.536 & -1.537 & 0.033126 & 0.100497 & 140 \\
\hline 2 & GO_B_CELL_PROLIFERATION & 17 & -0.625 & -1.542 & 0.035197 & 0.09744 & 141 \\
\hline 2 & $\begin{array}{l}\text { KEGG_RIG_I_LIKE_RECEPTOR_SIGNALING_PAT } \\
\text { HWAY }\end{array}$ & 41 & -0.485 & -1.498 & 0.035565 & 0.125655 & 142 \\
\hline 2 & $\begin{array}{l}\text { GO_POSITIVE_REGULATION_OF_TOLL_LIKE_R } \\
\text { ECEPTOR_SIGNALING_PATHWAY }\end{array}$ & 17 & -0.611 & -1.608 & 0.035941 & 0.062976 & 143 \\
\hline 2 & $\begin{array}{l}\text { GO_REGULATION_OF_TOLL_LIKE_RECEPTOR_ } \\
\text { SIGNALING_PATHWAY }\end{array}$ & 36 & -0.487 & -1.469 & 0.03626 & 0.146389 & 144 \\
\hline 2 & GO_RESPONSE_TO_INTERLEUKIN_1 & 72 & -0.401 & -1.38 & 0.038095 & 0.21606 & 145 \\
\hline 2 & $\begin{array}{l}\text { GO_REGULATION_OF_LEUKOCYTE_MEDIATED } \\
\text { _CYTOTOXICITY }\end{array}$ & 27 & -0.529 & -1.485 & 0.038544 & 0.135237 & 146 \\
\hline 2 & BIOCARTA_IL6_PATHWAY & 22 & -0.568 & -1.51 & 0.04251 & 0.117262 & 147 \\
\hline 2 & $\begin{array}{l}\text { GO_PATTERN_RECOGNITION_RECEPTOR_SIGN } \\
\text { ALING_PATHWAY }\end{array}$ & 80 & -0.396 & -1.379 & 0.046422 & 0.216607 & 148 \\
\hline 2 & GO_HUMORAL_IMMUNE_RESPONSE & 65 & -0.422 & -1.42 & 0.046967 & 0.183324 & 149 \\
\hline 2 & GO_MYELOID_LEUKOCYTE_DIFFERENTIATION & 66 & -0.412 & -1.399 & 0.047337 & 0.200921 & 150 \\
\hline
\end{tabular}




\begin{tabular}{|c|c|c|c|c|c|c|c|}
\hline 2 & $\begin{array}{l}\text { GO_REGULATION_OF_CYTOKINE_PRODUCTIO } \\
\text { N_INVOLVED_IN_IMMUNE_RESPONSE }\end{array}$ & 39 & -0.463 & -1.436 & 0.048924 & 0.17031 & 151 \\
\hline 3 & GO_CELL_CELL_SIGNALING & 367 & -0.347 & -1.477 & 0 & 0.140528 & 1 \\
\hline 3 & GO_CELL_SURFACE & 426 & -0.327 & -1.418 & 0 & 0.185459 & 2 \\
\hline 3 & $\begin{array}{l}\text { GO_CELLULAR_RESPONSE_TO_GROWTH_HOR } \\
\text { MONE_STIMULUS }\end{array}$ & 15 & -0.711 & -1.77 & 0 & 0.017127 & 3 \\
\hline 3 & $\begin{array}{l}\text { GO_EXTERNAL_SIDE_OF_PLASMA_MEMBRAN } \\
\text { E }\end{array}$ & 124 & -0.463 & -1.728 & 0 & 0.024653 & 4 \\
\hline 3 & $\begin{array}{l}\text { GO_NAD_ADP_RIBOSYLTRANSFERASE_ACTIVIT } \\
\mathrm{Y}\end{array}$ & 23 & -0.741 & -1.987 & 0 & 0.001233 & 5 \\
\hline 3 & $\begin{array}{l}\text { GO_NEGATIVE_REGULATION_OF_MULTI_ORG } \\
\text { ANISM_PROCESS }\end{array}$ & 97 & -0.533 & -1.915 & 0 & 0.003347 & 6 \\
\hline 3 & $\begin{array}{l}\text { GO_POSITIVE_REGULATION_OF_ERK1_AND_E } \\
\text { RK2_CASCADE }\end{array}$ & 93 & -0.479 & -1.725 & 0 & 0.025321 & 7 \\
\hline 3 & $\begin{array}{l}\text { GO_POSITIVE_REGULATION_OF_LYMPHOCYTE } \\
\text { _MIGRATION }\end{array}$ & 18 & -0.741 & -1.901 & 0 & 0.004012 & 8 \\
\hline 3 & $\begin{array}{l}\text { GO_REGULATION_OF_ERK1_AND_ERK2_CASC } \\
\text { ADE }\end{array}$ & 143 & -0.42 & -1.621 & 0 & 0.057411 & 9 \\
\hline 3 & $\begin{array}{l}\text { GO_REGULATION_OF_MULTI_ORGANISM_PR } \\
\text { OCESS }\end{array}$ & 309 & -0.389 & -1.631 & 0 & 0.05388 & 10 \\
\hline 3 & $\begin{array}{l}\text { GO_REGULATION_OF_SYMBIOSIS_ENCOMPAS } \\
\text { SING_MUTUALISM_THROUGH_PARASITISM }\end{array}$ & 152 & -0.487 & -1.89 & 0 & 0.004616 & 11 \\
\hline 3 & GO_RESPONSE_TO_BIOTIC_STIMULUS & 485 & -0.408 & -1.771 & 0 & 0.016975 & 12 \\
\hline 3 & GO_SIDE_OF_MEMBRANE & 247 & -0.383 & -1.568 & 0 & 0.082382 & 13 \\
\hline 3 & GO_SULFUR_COMPOUND_BINDING & 146 & -0.411 & -1.568 & 0 & 0.08247 & 14 \\
\hline 3 & $\begin{array}{l}\text { GO_TRANSFERASE_ACTIVITY_TRANSFERRING_ } \\
\text { GLYCOSYL_GROUPS }\end{array}$ & 173 & -0.413 & -1.608 & 0 & 0.063202 & 15 \\
\hline 3 & $\begin{array}{l}\text { GO_TRANSFERASE_ACTIVITY_TRANSFERRING_ } \\
\text { PENTOSYL_GROUPS }\end{array}$ & 37 & -0.593 & -1.793 & 0 & 0.013542 & 16 \\
\hline 3 & $\begin{array}{l}\text { GO_TRANSMEMBRANE_RECEPTOR_PROTEIN_ } \\
\text { TYROSINE_KINASE_ACTIVITY }\end{array}$ & 36 & -0.639 & -1.891 & 0 & 0.00457 & 17 \\
\hline 3 & HALLMARK_KRAS_SIGNALING_UP & 130 & -0.44 & -1.639 & 0 & 0.05033 & 18 \\
\hline 3 & $\begin{array}{l}\text { REACTOME_TRAF6_MEDIATED_IRF7_ACTIVATI } \\
\text { ON }\end{array}$ & 16 & -0.831 & -2.101 & 0 & $1.59 \mathrm{E}-04$ & 19 \\
\hline 3 & $\begin{array}{l}\text { GO_G_PROTEIN_COUPLED_RECEPTOR_BINDI } \\
\text { NG }\end{array}$ & 139 & -0.416 & -1.6 & 0.001812 & 0.066462 & 20 \\
\hline 3 & GO_HEPARIN_BINDING & 86 & -0.517 & -1.846 & 0.001883 & 0.007678 & 21 \\
\hline 3 & GO_GLIOGENESIS & 117 & -0.411 & -1.521 & 0.001927 & 0.109681 & 22 \\
\hline 3 & REACTOME_G_ALPHA_I_SIGNALLING_EVENTS & 94 & -0.451 & -1.624 & 0.001927 & 0.056553 & 23 \\
\hline
\end{tabular}




\begin{tabular}{|c|c|c|c|c|c|c|c|}
\hline 3 & $\begin{array}{l}\text { GO_POSITIVE_REGULATION_OF_CALCIUM_IO } \\
\text { N_TRANSPORT }\end{array}$ & 64 & -0.466 & -1.601 & 0.001946 & 0.065902 & 24 \\
\hline 3 & $\begin{array}{l}\text { GO_TRANSMEMBRANE_RECEPTOR_PROTEIN_ } \\
\text { KINASE_ACTIVITY }\end{array}$ & 49 & -0.554 & -1.77 & 0.001972 & 0.017076 & 25 \\
\hline 3 & $\begin{array}{l}\text { GO_CALCIUM_CHANNEL_REGULATOR_ACTIVI } \\
\text { TY }\end{array}$ & 25 & -0.652 & -1.793 & 0.002012 & 0.013469 & 26 \\
\hline 3 & GO_REGULATION_OF_CELL_CELL_ADHESION & 226 & -0.364 & -1.472 & 0.003906 & 0.144601 & 27 \\
\hline 3 & $\begin{array}{l}\text { GO_ACTIVATION_OF_CYSTEINE_TYPE_ENDOP } \\
\text { EPTIDASE_ACTIVITY }\end{array}$ & 68 & -0.477 & -1.619 & 0.003937 & 0.058349 & 28 \\
\hline 3 & $\begin{array}{l}\text { GO_POSITIVE_REGULATION_OF_RESPONSE_T } \\
\text { O_WOUNDING }\end{array}$ & 98 & -0.461 & -1.67 & 0.004032 & 0.039444 & 29 \\
\hline 3 & HALLMARK_APOPTOSIS & 139 & -0.416 & -1.578 & 0.00404 & 0.07712 & 30 \\
\hline 3 & $\begin{array}{l}\text { GO_POSITIVE_REGULATION_OF_LOCOMOTIO } \\
\mathrm{N}\end{array}$ & 281 & -0.348 & -1.444 & 0.005376 & 0.163865 & 31 \\
\hline 3 & GO_ENDOPEPTIDASE_ACTIVITY & 226 & -0.363 & -1.468 & 0.005671 & 0.146402 & 32 \\
\hline 3 & GO_DOUBLE_STRANDED_RNA_BINDING & 51 & -0.511 & -1.67 & 0.006036 & 0.039463 & 33 \\
\hline 3 & $\begin{array}{l}\text { GO_EXONUCLEASE_ACTIVITY_ACTIVE_WITH_E } \\
\text { ITHER_RIBO_OR_DEOXYRIBONUCLEIC_ACIDS_- } \\
\text { AND_PRODUCING_5_PHOSPHOMONOESTERS }\end{array}$ & 35 & -0.565 & -1.686 & 0.006036 & 0.035029 & 34 \\
\hline 3 & $\begin{array}{l}\text { REACTOME_GROWTH_HORMONE_RECEPTOR } \\
\text { _SIGNALING }\end{array}$ & 18 & -0.697 & -1.785 & 0.006061 & 0.014728 & 35 \\
\hline 3 & GO_RESPONSE_TO_VITAMIN & 76 & -0.463 & -1.597 & 0.006085 & 0.067904 & 36 \\
\hline 3 & REACTOME_ER_PHAGOSOME_PATHWAY & 51 & -0.509 & -1.652 & 0.006198 & 0.045652 & 37 \\
\hline 3 & GO_FIBRONECTIN_BINDING & 18 & -0.67 & -1.745 & 0.006438 & 0.021313 & 38 \\
\hline 3 & $\begin{array}{l}\text { GO_NEGATIVE_REGULATION_OF_CELL_CELL_ } \\
\text { ADHESION }\end{array}$ & 84 & -0.425 & -1.515 & 0.007843 & 0.113539 & 39 \\
\hline 3 & GO_ORGANIC_ACID_BINDING & 122 & -0.413 & -1.546 & 0.009398 & 0.094693 & 40 \\
\hline 3 & $\begin{array}{l}\text { GO_REGULATION_OF_HOMOTYPIC_CELL_CELL } \\
\text { _ADHESION }\end{array}$ & 182 & -0.349 & -1.381 & 0.00947 & 0.215539 & 41 \\
\hline 3 & GO_TAXIS & 267 & -0.33 & -1.35 & 0.009488 & 0.242767 & 42 \\
\hline 3 & GO_PEPTIDYL_TYROSINE_MODIFICATION & 122 & -0.389 & -1.46 & 0.00956 & 0.15343 & 43 \\
\hline 3 & $\begin{array}{l}\text { GO_MORPHOGENESIS_OF_A_BRANCHING_ST } \\
\text { RUCTURE }\end{array}$ & 104 & -0.408 & -1.508 & 0.009579 & 0.118869 & 44 \\
\hline 3 & GO_RESPONSE_TO_MECHANICAL_STIMULUS & 138 & -0.385 & -1.456 & 0.01 & 0.156269 & 45 \\
\hline 3 & GO_THREONINE_TYPE_PEPTIDASE_ACTIVITY & 19 & -0.652 & -1.664 & 0.010183 & 0.041522 & 46 \\
\hline 3 & $\begin{array}{l}\text { GO_POSITIVE_REGULATION_OF_COAGULATIO } \\
\mathrm{N}\end{array}$ & 15 & -0.687 & -1.687 & 0.010965 & 0.034738 & 47 \\
\hline
\end{tabular}




\begin{tabular}{|c|c|c|c|c|c|c|c|}
\hline 3 & $\begin{array}{l}\text { GO_POSITIVE_REGULATION_OF_SEQUENCE_S } \\
\text { PECIFIC_DNA_BINDING_TRANSCRIPTION_FAC } \\
\text { TOR_ACTIVITY }\end{array}$ & 154 & -0.371 & -1.416 & 0.011299 & 0.186878 & 48 \\
\hline 3 & GO_RESPONSE_TO_BMP & 53 & -0.468 & -1.539 & 0.011976 & 0.099439 & 49 \\
\hline 3 & $\begin{array}{l}\text { GO_G_PROTEIN_COUPLED_RECEPTOR_SIGNA } \\
\text { LING_PATHWAY }\end{array}$ & 340 & -0.319 & -1.345 & 0.012324 & 0.248576 & 50 \\
\hline 3 & GO_RESPONSE_TO_GROWTH_HORMONE & 24 & -0.611 & -1.684 & 0.012605 & 0.035397 & 51 \\
\hline 3 & $\begin{array}{l}\text { REACTOME_PEPTIDE_LIGAND_BINDING_RECE } \\
\text { PTORS }\end{array}$ & 70 & -0.437 & -1.496 & 0.014085 & 0.127231 & 52 \\
\hline 3 & GO_PROTEIN_TYROSINE_KINASE_ACTIVITY & 114 & -0.39 & -1.432 & 0.014706 & 0.173519 & 53 \\
\hline 3 & $\begin{array}{l}\text { GO_REGULATION_OF_CALCIUM_ION_TRANSP } \\
\text { ORT }\end{array}$ & 127 & -0.371 & -1.412 & 0.014733 & 0.18997 & 54 \\
\hline 3 & GO_POSTSYNAPTIC_MEMBRANE & 102 & -0.387 & -1.423 & 0.014815 & 0.180585 & 55 \\
\hline 3 & GO_CELLULAR_RESPONSE_TO_ALCOHOL & 78 & -0.429 & -1.49 & 0.015385 & 0.131542 & 56 \\
\hline 3 & KEGG_TASTE_TRANSDUCTION & 15 & -0.66 & -1.612 & 0.015521 & 0.061411 & 57 \\
\hline 3 & $\begin{array}{l}\text { GO_NEGATIVE_REGULATION_OF_STRIATED_ } \\
\text { MUSCLE_CELL_DIFFERENTIATION }\end{array}$ & 18 & -0.638 & -1.619 & 0.016327 & 0.05833 & 58 \\
\hline 3 & GO_FEMALE_SEX_DIFFERENTIATION & 72 & -0.437 & -1.514 & 0.017928 & 0.114607 & 59 \\
\hline 3 & GO_RECEPTOR_REGULATOR_ACTIVITY & 26 & -0.546 & -1.56 & 0.019027 & 0.0871 & 60 \\
\hline 3 & GO_EPIDERMIS_DEVELOPMENT & 121 & -0.376 & -1.384 & 0.020147 & 0.214414 & 61 \\
\hline 3 & GO_TUBE_MORPHOGENESIS & 201 & -0.343 & -1.378 & 0.020561 & 0.217361 & 62 \\
\hline 3 & GO_PROTEIN_TRIMERIZATION & 25 & -0.555 & -1.555 & 0.021008 & 0.090123 & 63 \\
\hline 3 & GO_PROTEIN_MATURATION & 172 & -0.348 & -1.374 & 0.022388 & 0.220039 & 64 \\
\hline 3 & GO_SYNAPTIC_MEMBRANE & 141 & -0.356 & -1.366 & 0.023033 & 0.228839 & 65 \\
\hline 3 & GO_REGULATION_OF_CALCIUM_ION_IMPORT & 59 & -0.449 & -1.452 & 0.023077 & 0.158481 & 66 \\
\hline 3 & $\begin{array}{l}\text { GO_REGULATION_OF_RESPONSE_TO_BIOTIC_ } \\
\text { STIMULUS }\end{array}$ & 141 & -0.373 & -1.408 & 0.024621 & 0.193682 & 67 \\
\hline 3 & BIOCARTA_CASPASE_PATHWAY & 20 & -0.643 & -1.661 & 0.024691 & 0.042748 & 68 \\
\hline 3 & $\begin{array}{l}\text { GO_POSITIVE_REGULATION_OF_CELL_CELL_A } \\
\text { DHESION }\end{array}$ & 140 & -0.359 & -1.36 & 0.026217 & 0.234744 & 69 \\
\hline 3 & GO_RESPONSE_TO_VITAMIN_D & 26 & -0.571 & -1.612 & 0.026477 & 0.061176 & 70 \\
\hline 3 & $\begin{array}{l}\text { GO_POSITIVE_REGULATION_OF_WOUND_HEA } \\
\text { LING }\end{array}$ & 31 & -0.508 & -1.5 & 0.027723 & 0.124037 & 71 \\
\hline 3 & $\begin{array}{l}\text { GO_OXIDOREDUCTASE_ACTIVITY_ACTING_ON } \\
\text { _THE_CH_NH_GROUP_OF_DONORS }\end{array}$ & 18 & -0.619 & -1.613 & 0.028986 & 0.060919 & 72 \\
\hline
\end{tabular}




\begin{tabular}{|c|c|c|c|c|c|c|c|}
\hline 3 & $\begin{array}{l}\text { GO_PEPTIDYL_TYROSINE_AUTOPHOSPHORYLA } \\
\text { TION }\end{array}$ & 31 & -0.524 & -1.514 & 0.029787 & 0.114516 & 73 \\
\hline 3 & $\begin{array}{l}\text { GO_POSITIVE_REGULATION_OF_RELEASE_OF_- } \\
\text { SEQUESTERED_CALCIUM_ION_INTO_CYTOSOL }\end{array}$ & 21 & -0.574 & -1.558 & 0.03125 & 0.087933 & 74 \\
\hline 3 & $\begin{array}{l}\text { GO_ANCHORED_COMPONENT_OF_MEMBRA } \\
\mathrm{NE}\end{array}$ & 63 & -0.43 & -1.438 & 0.031434 & 0.168273 & 75 \\
\hline 3 & KEGG_CELL_ADHESION_MOLECULES_CAMS & 67 & -0.407 & -1.389 & 0.03263 & 0.209948 & 76 \\
\hline 3 & $\begin{array}{l}\text { GO_REGULATION_OF_CYSTEINE_TYPE_ENDOP } \\
\text { EPTIDASE_ACTIVITY_INVOLVED_IN_APOPTOTI } \\
\text { C_SIGNALING_PATHWAY }\end{array}$ & 17 & -0.611 & -1.549 & 0.037199 & 0.093531 & 77 \\
\hline 3 & $\begin{array}{l}\text { GO_PROTEIN_SERINE_THREONINE_PHOSPHAT } \\
\text { ASE_ACTIVITY }\end{array}$ & 46 & -0.45 & -1.436 & 0.037523 & 0.170187 & 78 \\
\hline 3 & $\begin{array}{l}\text { REACTOME_TRAF6_MEDIATED_NFKB_ACTIVA } \\
\text { TION }\end{array}$ & 18 & -0.61 & -1.538 & 0.038217 & 0.099583 & 79 \\
\hline 3 & $\begin{array}{l}\text { GO_NEUROTRANSMITTER_RECEPTOR_ACTIVIT } \\
\mathrm{Y}\end{array}$ & 23 & -0.524 & -1.451 & 0.038306 & 0.158575 & 80 \\
\hline 3 & $\begin{array}{l}\text { REACTOME_EXTRACELLULAR_MATRIX_ORGAN } \\
\text { IZATION }\end{array}$ & 49 & -0.447 & -1.437 & 0.039604 & 0.169763 & 81 \\
\hline 3 & GO_RESPONSE_TO_DSRNA & 42 & -0.479 & -1.474 & 0.040568 & 0.143423 & 82 \\
\hline 3 & $\begin{array}{l}\text { GO_PLASMA_MEMBRANE_RECEPTOR_COMPL } \\
\text { EX }\end{array}$ & 108 & -0.367 & -1.347 & 0.040665 & 0.2454 & 83 \\
\hline 3 & GO_GLIAL_CELL_MIGRATION & 25 & -0.56 & -1.527 & 0.041068 & 0.105567 & 84 \\
\hline 3 & $\begin{array}{l}\text { GO_POSITIVE_REGULATION_OF_PEPTIDASE_A } \\
\text { CTIVITY }\end{array}$ & 109 & -0.367 & -1.352 & 0.041746 & 0.241185 & 85 \\
\hline 3 & $\begin{array}{l}\text { REACTOME_N_GLYCAN_ANTENNAE_ELONGAT } \\
\text { ION_IN_THE_MEDIAL_TRANS_GOLGI }\end{array}$ & 16 & -0.609 & -1.513 & 0.042035 & 0.115264 & 86 \\
\hline 3 & $\begin{array}{l}\text { GO_POSITIVE_REGULATION_OF_CALCIUM_IO } \\
\text { N_IMPORT }\end{array}$ & 30 & -0.507 & -1.485 & 0.042339 & 0.135923 & 87 \\
\hline 3 & $\begin{array}{l}\text { GO_ALDEHYDE_DEHYDROGENASE_NAD_ACTI } \\
\text { VITY }\end{array}$ & 16 & -0.621 & -1.556 & 0.043299 & 0.089215 & 88 \\
\hline 3 & GO_NEGATIVE_REGULATION_OF_BINDING & 96 & -0.381 & -1.378 & 0.043478 & 0.217044 & 89 \\
\hline 3 & GO_RESPONSE_TO_ORGANOPHOSPHORUS & 94 & -0.379 & -1.364 & 0.044834 & 0.230599 & 90 \\
\hline 3 & $\begin{array}{l}\text { GO_REGULATION_OF_CALCIUM_ION_TRANSP } \\
\text { ORT_INTO_CYTOSOL }\end{array}$ & 52 & -0.443 & -1.435 & 0.046092 & 0.170155 & 91 \\
\hline 3 & GO_ANION_HOMEOSTASIS & 30 & -0.503 & -1.471 & 0.046908 & 0.144539 & 92 \\
\hline 3 & $\begin{array}{l}\text { GO_BRANCHING_MORPHOGENESIS_OF_AN_E } \\
\text { PITHELIAL_TUBE }\end{array}$ & 80 & -0.394 & -1.375 & 0.047131 & 0.219514 & 93 \\
\hline 3 & GO_LAMININ_BINDING & 16 & -0.627 & -1.562 & 0.048035 & 0.086292 & 94 \\
\hline
\end{tabular}




\section{Supplemental Table S2.4: A list of significantly altered gene sets identified by GSEA in the MDM dataset.}

\begin{tabular}{|c|c|c|c|c|c|c|}
\hline Filter & NAME & SIZE & ES & NES & $\begin{array}{l}\text { NOM p- } \\
\text { val }\end{array}$ & $\begin{array}{l}\text { FDR q- } \\
\text { val }\end{array}$ \\
\hline 1 & KEGG_JAK_STAT_SIGNALING_PATHWAY & 105 & -0.587 & -2.206 & 0 & $1.84 \mathrm{E}-05$ \\
\hline 1 & $\begin{array}{l}\text { GO_REGULATION_OF_INSULIN_RECEPTOR_SI } \\
\text { GNALING_PATHWAY }\end{array}$ & 27 & -0.707 & -2.075 & 0 & $2.16 \mathrm{E}-04$ \\
\hline 1 & $\begin{array}{l}\text { GO_REGULATION_OF_REACTIVE_OXYGEN_SPE } \\
\text { CIES_BIOSYNTHETIC_PROCESS }\end{array}$ & 54 & -0.597 & -2.039 & 0 & $3.58 \mathrm{E}-04$ \\
\hline 1 & $\begin{array}{l}\text { GO_POSITIVE_REGULATION_OF_MAPK_CASCA } \\
\text { DE }\end{array}$ & 300 & -0.467 & -2.018 & 0 & 4.86E-04 \\
\hline 1 & $\begin{array}{l}\text { GO_REGULATION_OF_NITRIC_OXIDE_BIOSYNT } \\
\text { HETIC_PROCESS }\end{array}$ & 42 & -0.628 & -2.004 & 0 & 5.99E-04 \\
\hline 1 & $\begin{array}{l}\text { GO_REGULATION_OF_REACTIVE_OXYGEN_SPE } \\
\text { CIES_METABOLIC_PROCESS }\end{array}$ & 113 & -0.514 & -1.95 & 0 & 0.001255 \\
\hline 1 & $\begin{array}{l}\text { GO_POSITIVE_REGULATION_OF_REACTIVE_OX } \\
\text { YGEN_SPECIES_METABOLIC_PROCESS }\end{array}$ & 67 & -0.527 & -1.857 & 0 & 0.003724 \\
\hline 1 & $\begin{array}{l}\text { GO_POSITIVE_REGULATION_OF_REACTIVE_OX } \\
\text { YGEN_SPECIES_BIOSYNTHETIC_PROCESS }\end{array}$ & 40 & -0.578 & -1.85 & 0 & 0.004018 \\
\hline 1 & GO_REGULATION_OF_MAPK_CASCADE & 437 & -0.411 & -1.838 & 0 & 0.004603 \\
\hline 1 & $\begin{array}{l}\text { GO_POSITIVE_REGULATION_OF_MAP_KINASE } \\
\text { _ACTIVITY }\end{array}$ & 140 & -0.451 & -1.754 & 0 & 0.010498 \\
\hline 1 & GO_PROTEIN_POLYUBIQUITINATION & 209 & -0.397 & -1.643 & 0 & 0.02578 \\
\hline 1 & GO_REGULATION_OF_MAP_KINASE_ACTIVITY & 227 & -0.369 & -1.56 & 0 & 0.044869 \\
\hline 1 & $\begin{array}{l}\text { GO_REGULATION_OF_GLYCOPROTEIN_METAB } \\
\text { OLIC_PROCESS }\end{array}$ & 32 & -0.725 & -2.237 & 0 & $4.32 \mathrm{E}-06$ \\
\hline 1 & GO_RESPONSE_TO_HYDROGEN_PEROXIDE & 88 & -0.563 & -2.039 & 0 & $3.59 \mathrm{E}-04$ \\
\hline 1 & GO_CELLULAR_RESPONSE_TO_LIPID & 299 & -0.471 & -2.029 & 0 & $3.98 \mathrm{E}-04$ \\
\hline 1 & $\begin{array}{l}\text { GO_POSITIVE_REGULATION_OF_GLYCOPROTE } \\
\text { IN_METABOLIC_PROCESS }\end{array}$ & 17 & -0.765 & -1.983 & 0 & $8.18 \mathrm{E}-04$ \\
\hline 1 & $\begin{array}{l}\text { GO_RELEASE_OF_CYTOCHROME_C_FROM_MI } \\
\text { TOCHONDRIA }\end{array}$ & 17 & -0.747 & -1.955 & 0 & 0.001168 \\
\hline 1 & $\begin{array}{l}\text { GO_RESPONSE_TO_REACTIVE_OXYGEN_SPECI } \\
\text { ES }\end{array}$ & 147 & -0.492 & -1.913 & 0 & 0.002041 \\
\hline 1 & GO_RESPONSE_TO_KETONE & 124 & -0.494 & -1.911 & 0 & 0.002073 \\
\hline 1 & GO_RESPONSE_TO_CORTICOSTEROID & 110 & -0.498 & -1.897 & 0 & 0.002411 \\
\hline 1 & BIOCARTA_CERAMIDE_PATHWAY & 20 & -0.685 & -1.82 & 0 & 0.005559 \\
\hline
\end{tabular}

\begin{tabular}{|c|c|}
\hline \multicolumn{2}{|r|}{ Legend } \\
\hline 1 & Immune signaling and function \\
\hline 2 & Cellular metabolism \\
\hline 3 & Other biological states and processes \\
\hline NAME & Name of gene set \\
\hline SIZE & Number of Genes within gene set \\
\hline ES & $\begin{array}{l}\text { Enrichment Score for gene set (Degree to which } \\
\text { this gene set is overrepresented at the top or } \\
\text { bottom of the ranked lit of genes) }\end{array}$ \\
\hline NES & Normalized enrichment score \\
\hline NOM p-val & Nominal $p$-value \\
\hline FDR q-val & $\begin{array}{l}\text { False discovery rate (corrected for multiple } \\
\text { testing) }\end{array}$ \\
\hline
\end{tabular}




\begin{tabular}{|c|c|c|c|c|c|c|c|}
\hline 1 & $\begin{array}{l}\text { KEGG_ADIPOCYTOKINE_SIGNALING_PATHWA } \\
\mathrm{Y}\end{array}$ & 55 & -0.527 & -1.791 & 0 & 0.00742 & 22 \\
\hline 1 & HALLMARK_HYPOXIA & 159 & -0.439 & -1.752 & 0 & 0.010636 & 23 \\
\hline 1 & GO_RESPONSE_TO_AMINO_ACID & 77 & -0.487 & -1.735 & 0 & 0.012492 & 24 \\
\hline 1 & $\begin{array}{l}\text { GO_CELLULAR_RESPONSE_TO_REACTIVE_OXY } \\
\text { GEN_SPECIES }\end{array}$ & 81 & -0.474 & -1.733 & 0 & 0.012733 & 25 \\
\hline 1 & GO_GUANYL_NUCLEOTIDE_BINDING & 267 & -0.401 & -1.714 & 0 & 0.015091 & 26 \\
\hline 1 & GO_RESPONSE_TO_OXYGEN_LEVELS & 226 & -0.404 & -1.684 & 0 & 0.019032 & 27 \\
\hline 1 & $\begin{array}{l}\text { GO_NEGATIVE_REGULATION_OF_HYDROLASE } \\
\text { _ACTIVITY }\end{array}$ & 235 & -0.394 & -1.666 & 0 & 0.021841 & 28 \\
\hline 1 & GO_ENZYME_INHIBITOR_ACTIVITY & 216 & -0.377 & -1.569 & 0 & 0.042438 & 29 \\
\hline 1 & GO_ION_HOMEOSTASIS & 351 & -0.33 & -1.437 & 0 & 0.093456 & 30 \\
\hline 1 & $\begin{array}{l}\text { GO_UBIQUITIN_LIKE_PROTEIN_LIGASE_BINDI } \\
\text { NG }\end{array}$ & 216 & -0.361 & -1.49 & 0.001672 & 0.068511 & 31 \\
\hline 1 & $\begin{array}{l}\text { GO_POSITIVE_REGULATION_OF_CATABOLIC_P } \\
\text { ROCESS }\end{array}$ & 319 & -0.337 & -1.47 & 0.001715 & 0.07719 & 32 \\
\hline 1 & GO_RESPONSE_TO_NUTRIENT & 147 & -0.417 & -1.652 & 0.001792 & 0.024083 & 33 \\
\hline 1 & $\begin{array}{l}\text { GO_REGULATION_OF_TYROSINE_PHOSPHORY } \\
\text { LATION_OF_STAT3_PROTEIN }\end{array}$ & 30 & -0.644 & -1.912 & 0.001992 & 0.002056 & 34 \\
\hline 1 & GO_RESPONSE_TO_RETINOIC_ACID & 62 & -0.462 & -1.59 & 0.001992 & 0.037086 & 35 \\
\hline 1 & GO_PROTEIN_PHOSPHATASE_2A_BINDING & 24 & -0.686 & -1.959 & 0.002041 & 0.001117 & 36 \\
\hline 1 & $\begin{array}{l}\text { GO_POSITIVE_REGULATION_OF_CYCLIC_NUCL } \\
\text { EOTIDE_METABOLIC_PROCESS }\end{array}$ & 51 & -0.513 & -1.694 & 0.003922 & 0.017498 & 37 \\
\hline 1 & GO_RESPONSE_TO_FATTY_ACID & 60 & -0.456 & -1.592 & 0.003937 & 0.036509 & 38 \\
\hline 1 & $\begin{array}{l}\text { GO_REGULATION_OF_PHOSPHATIDYLINOSITO } \\
\text { L_3_KINASE_SIGNALING }\end{array}$ & 91 & -0.437 & -1.609 & 0.003984 & 0.032427 & 39 \\
\hline 1 & GO_CELLULAR_RESPONSE_TO_NUTRIENT & 32 & -0.568 & -1.706 & 0.003992 & 0.01597 & 40 \\
\hline 1 & $\begin{array}{l}\text { GO_REGULATION_OF_PHOSPHOLIPID_METAB } \\
\text { OLIC_PROCESS }\end{array}$ & 45 & -0.533 & -1.74 & 0.004202 & 0.0119 & 41 \\
\hline 1 & GO_RESPONSE_TO_NICOTINE & 25 & -0.586 & -1.711 & 0.004255 & 0.015415 & 42 \\
\hline 1 & $\begin{array}{l}\text { GO_REGULATION_OF_CYCLIC_NUCLEOTIDE_ } \\
\text { METABOLIC_PROCESS }\end{array}$ & 70 & -0.46 & -1.628 & 0.005703 & 0.028739 & 43 \\
\hline 1 & $\begin{array}{l}\text { GO_POSITIVE_REGULATION_OF_CELLULAR_A } \\
\text { MIDE_METABOLIC_PROCESS }\end{array}$ & 84 & -0.429 & -1.55 & 0.005703 & 0.048073 & 44 \\
\hline 1 & GO_ACTIVATION_OF_MAPK_ACTIVITY & 92 & -0.414 & -1.524 & 0.005941 & 0.056371 & 45 \\
\hline 1 & $\begin{array}{l}\text { GO_RNA_PHOSPHODIESTER_BOND_HYDROLY } \\
\text { SIS_EXONUCLEOLYTIC }\end{array}$ & 26 & -0.632 & -1.835 & 0.006173 & 0.004793 & 46 \\
\hline
\end{tabular}




\begin{tabular}{|c|c|c|c|c|c|c|c|}
\hline 1 & $\begin{array}{l}\text { GO_REGULATION_OF_CALCIUM_ION_TRANSP } \\
\text { ORT }\end{array}$ & 124 & -0.402 & -1.556 & 0.007018 & 0.046058 & 47 \\
\hline 1 & $\begin{array}{l}\text { GO_POSITIVE_REGULATION_OF_NUCLEOTIDE } \\
\text { _METABOLIC_PROCESS }\end{array}$ & 67 & -0.467 & -1.652 & 0.007859 & 0.024041 & 48 \\
\hline 1 & $\begin{array}{l}\text { GO_REGULATION_OF_SEQUESTERING_OF_CA } \\
\text { LCIUM_ION }\end{array}$ & 71 & -0.448 & -1.571 & 0.008016 & 0.041983 & 49 \\
\hline 1 & $\begin{array}{l}\text { GO_MUCOPOLYSACCHARIDE_METABOLIC_PR } \\
\text { OCESS }\end{array}$ & 72 & -0.424 & -1.501 & 0.008214 & 0.064344 & 50 \\
\hline 1 & GO_REGULATION_OF_LIPID_KINASE_ACTIVITY & 36 & -0.543 & -1.682 & 0.008247 & 0.019424 & 51 \\
\hline 1 & $\begin{array}{l}\text { GO_POSITIVE_REGULATION_OF_PHOSPHATID } \\
\text { YLINOSITOL_3_KINASE_SIGNALING }\end{array}$ & 41 & -0.511 & -1.623 & 0.008658 & 0.029577 & 52 \\
\hline 1 & $\begin{array}{l}\text { GO_REGULATION_OF_PHOSPHATIDYLINOSITO } \\
\text { L_3_KINASE_ACTIVITY }\end{array}$ & 28 & -0.587 & -1.718 & 0.010309 & 0.014433 & 53 \\
\hline 1 & $\begin{array}{l}\text { GO_REGULATION_OF_NUCLEOTIDE_METABOL } \\
\text { IC_PROCESS }\end{array}$ & 110 & -0.394 & -1.476 & 0.011299 & 0.074384 & 54 \\
\hline 1 & $\begin{array}{l}\text { GO_REGULATION_OF_MITOCHONDRIAL_MEM } \\
\text { BRANE_POTENTIAL }\end{array}$ & 40 & -0.51 & -1.61 & 0.01232 & 0.032256 & 55 \\
\hline 1 & $\begin{array}{l}\text { GO_REGULATION_OF_CAMP_MEDIATED_SIG } \\
\text { NALING }\end{array}$ & 17 & -0.641 & -1.697 & 0.012685 & 0.017195 & 56 \\
\hline 1 & $\begin{array}{l}\text { GO_REGULATION_OF_RELEASE_OF_SEQUESTE } \\
\text { RED_CALCIUM_ION_INTO_CYTOSOL }\end{array}$ & 44 & -0.488 & -1.578 & 0.01378 & 0.039884 & 57 \\
\hline 1 & $\begin{array}{l}\text { GO_ORGANIC_CYCLIC_COMPOUND_CATABOLI } \\
\text { C_PROCESS }\end{array}$ & 331 & -0.307 & -1.328 & 0.013937 & 0.163159 & 58 \\
\hline 1 & KEGG_MAPK_SIGNALING_PATHWAY & 198 & -0.328 & -1.376 & 0.01476 & 0.129072 & 59 \\
\hline 1 & GO_SECONDARY_METABOLIC_PROCESS & 24 & -0.573 & -1.619 & 0.014894 & 0.030564 & 60 \\
\hline 1 & $\begin{array}{l}\text { GO_NEGATIVE_REGULATION_OF_FAT_CELL_D } \\
\text { IFFERENTIATION }\end{array}$ & 26 & -0.546 & -1.59 & 0.016529 & 0.036984 & 61 \\
\hline 1 & BIOCARTA_GSK3_PATHWAY & 25 & -0.561 & -1.602 & 0.017578 & 0.034212 & 62 \\
\hline 1 & GO_LIPID_PARTICLE & 38 & -0.483 & -1.528 & 0.018036 & 0.055139 & 63 \\
\hline 1 & $\begin{array}{l}\text { GO_REGULATION_OF_RESPONSE_TO_OXIDATI } \\
\text { VE_STRESS }\end{array}$ & 47 & -0.478 & -1.546 & 0.018293 & 0.049164 & 64 \\
\hline 1 & $\begin{array}{l}\text { GO_REGULATION_OF_FAT_CELL_DIFFERENTIA } \\
\text { TION }\end{array}$ & 61 & -0.451 & -1.541 & 0.019646 & 0.050824 & 65 \\
\hline 1 & $\begin{array}{l}\text { GO_REGULATION_OF_PROTEIN_CATABOLIC_P } \\
\text { ROCESS }\end{array}$ & 317 & -0.308 & -1.339 & 0.019769 & 0.154694 & 66 \\
\hline 1 & GO_CELLULAR_RESPONSE_TO_KETONE & 52 & -0.456 & -1.524 & 0.01992 & 0.056259 & 67 \\
\hline 1 & GO_COENZYME_BIOSYNTHETIC_PROCESS & 101 & -0.378 & -1.438 & 0.020913 & 0.092853 & 68 \\
\hline 1 & GO_RESPONSE_TO_HYDROPEROXIDE & 15 & -0.677 & -1.675 & 0.021413 & 0.020412 & 69 \\
\hline
\end{tabular}




\begin{tabular}{|c|c|c|c|c|c|c|c|}
\hline 1 & $\begin{array}{l}\text { GO_POSITIVE_REGULATION_OF_MITOCHOND } \\
\text { RION_ORGANIZATION }\end{array}$ & 122 & -0.362 & -1.407 & 0.021544 & 0.110315 & 70 \\
\hline 1 & $\begin{array}{l}\text { GO_POSITIVE_REGULATION_OF_PHOSPHOLIPI } \\
\text { D_METABOLIC_PROCESS }\end{array}$ & 30 & -0.539 & -1.61 & 0.022044 & 0.032382 & 71 \\
\hline 1 & $\begin{array}{l}\text { GO_POSITIVE_REGULATION_OF_PROTEIN_CA } \\
\text { TABOLIC_PROCESS }\end{array}$ & 215 & -0.324 & -1.331 & 0.022609 & 0.160612 & 72 \\
\hline 1 & GO_PROTEIN_UBIQUITINATION & 469 & -0.288 & -1.287 & 0.022989 & 0.198528 & 73 \\
\hline 1 & $\begin{array}{l}\text { GO_POSITIVE_REGULATION_OF_LIPID_KINASE } \\
\text { _ACTIVITY }\end{array}$ & 22 & -0.575 & -1.574 & 0.023109 & 0.041002 & 74 \\
\hline 1 & $\begin{array}{l}\text { GO_REGULATION_OF_CALCIUM_ION_TRANS } \\
\text { MEMBRANE_TRANSPORT }\end{array}$ & 67 & -0.418 & -1.475 & 0.02381 & 0.074861 & 75 \\
\hline 1 & GO_AMINOGLYCAN_METABOLIC_PROCESS & 111 & -0.373 & -1.41 & 0.024074 & 0.108554 & 76 \\
\hline 1 & $\begin{array}{l}\text { GO_POSITIVE_REGULATION_OF_MRNA_META } \\
\text { BOLIC_PROCESS }\end{array}$ & 37 & -0.508 & -1.579 & 0.025243 & 0.039799 & 77 \\
\hline 1 & $\begin{array}{l}\text { GO_POSITIVE_REGULATION_OF_CAMP_META } \\
\text { BOLIC_PROCESS }\end{array}$ & 44 & -0.468 & -1.52 & 0.025243 & 0.057426 & 78 \\
\hline 1 & $\begin{array}{l}\text { GO_CYCLIC_NUCLEOTIDE_METABOLIC_PROCE } \\
\text { SS }\end{array}$ & 31 & -0.501 & -1.501 & 0.02544 & 0.064303 & 79 \\
\hline 1 & $\begin{array}{l}\text { GO_CELLULAR_RESPONSE_TO_GLUCOSE_STA } \\
\text { RVATION }\end{array}$ & 25 & -0.571 & -1.664 & 0.026432 & 0.022224 & 80 \\
\hline 1 & GO_RNA_CATABOLIC_PROCESS & 194 & -0.323 & -1.327 & 0.027211 & 0.164254 & 81 \\
\hline 1 & $\begin{array}{l}\text { GO_POSITIVE_REGULATION_OF_LIPID_TRANS } \\
\text { PORT }\end{array}$ & 35 & -0.493 & -1.543 & 0.028169 & 0.049997 & 82 \\
\hline 1 & $\begin{array}{l}\text { GO_POSITIVE_REGULATION_OF_MONOOXYGE } \\
\text { NASE_ACTIVITY }\end{array}$ & 20 & -0.585 & -1.579 & 0.028698 & 0.039768 & 83 \\
\hline 1 & $\begin{array}{l}\text { GO_NEGATIVE_REGULATION_OF_PHOSPHOR } \\
\text { US_METABOLIC_PROCESS }\end{array}$ & 372 & -0.288 & -1.266 & 0.028765 & 0.218233 & 84 \\
\hline 1 & $\begin{array}{l}\text { GO_NAD_ADP_RIBOSYLTRANSFERASE_ACTIVIT } \\
\mathrm{Y}\end{array}$ & 24 & -0.563 & -1.621 & 0.031056 & 0.03015 & 85 \\
\hline 1 & $\begin{array}{l}\text { GO_REGULATION_OF_MRNA_CATABOLIC_PR } \\
\text { OCESS }\end{array}$ & 23 & -0.558 & -1.576 & 0.031373 & 0.040575 & 86 \\
\hline 1 & $\begin{array}{l}\text { GO_POSITIVE_REGULATION_OF_OXIDOREDUC } \\
\text { TASE_ACTIVITY }\end{array}$ & 30 & -0.504 & -1.509 & 0.031513 & 0.061401 & 87 \\
\hline 1 & $\begin{array}{l}\text { GO_REGULATION_OF_RESPONSE_TO_REACTI } \\
\text { VE_OXYGEN_SPECIES }\end{array}$ & 24 & -0.538 & -1.552 & 0.03373 & 0.047272 & 88 \\
\hline 1 & $\begin{array}{l}\text { GO_PURINE_NUCLEOBASE_METABOLIC_PROC } \\
\text { ESS }\end{array}$ & 20 & -0.558 & -1.527 & 0.034091 & 0.055346 & 89 \\
\hline 1 & $\begin{array}{l}\text { GO_POSITIVE_REGULATION_OF_RELEASE_OF_- } \\
\text { SEQUESTERED_CALCIUM_ION_INTO_CYTOSOL }\end{array}$ & 22 & -0.552 & -1.526 & 0.034557 & 0.055607 & 90 \\
\hline 1 & $\begin{array}{l}\text { GO_NEGATIVE_REGULATION_OF_PROTEIN_C } \\
\text { ATABOLIC_PROCESS }\end{array}$ & 87 & -0.384 & -1.41 & 0.03643 & 0.108565 & 91 \\
\hline
\end{tabular}




\begin{tabular}{|c|c|c|c|c|c|c|c|}
\hline 1 & $\begin{array}{l}\text { GO_POSITIVE_REGULATION_OF_LIPID_METAB } \\
\text { OLIC_PROCESS }\end{array}$ & 91 & -0.389 & -1.401 & 0.037383 & 0.113803 & 92 \\
\hline 1 & $\begin{array}{l}\text { GO_REGULATION_OF_STEROID_BIOSYNTHETI } \\
\text { C_PROCESS }\end{array}$ & 28 & -0.527 & -1.532 & 0.038462 & 0.053955 & 93 \\
\hline 1 & $\begin{array}{l}\text { GO_NEGATIVE_REGULATION_OF_CALCIUM_I } \\
\text { ON_TRANSPORT }\end{array}$ & 29 & -0.506 & -1.496 & 0.038855 & 0.066161 & 94 \\
\hline 1 & BIOCARTA_MITOCHONDRIA_PATHWAY & 17 & -0.597 & -1.521 & 0.039301 & 0.057329 & 95 \\
\hline 1 & GO_HYALURONAN_METABOLIC_PROCESS & 21 & -0.537 & -1.494 & 0.041322 & 0.067156 & 96 \\
\hline 1 & $\begin{array}{l}\text { GO_REGULATION_OF_MONOOXYGENASE_AC } \\
\text { TIVITY }\end{array}$ & 47 & -0.445 & -1.45 & 0.041667 & 0.086515 & 97 \\
\hline 1 & $\begin{array}{l}\text { KEGG_NICOTINATE_AND_NICOTINAMIDE_ME } \\
\text { TABOLISM }\end{array}$ & 17 & -0.577 & -1.522 & 0.042683 & 0.056795 & 98 \\
\hline 1 & $\begin{array}{l}\text { GO_REGULATION_OF_CELLULAR_AMINE_MET } \\
\text { ABOLIC_PROCESS }\end{array}$ & 68 & -0.41 & -1.452 & 0.042969 & 0.085666 & 99 \\
\hline 1 & GO_POLYUBIQUITIN_BINDING & 32 & -0.483 & -1.472 & 0.045098 & 0.076059 & 100 \\
\hline 1 & $\begin{array}{l}\text { GO_NEGATIVE_REGULATION_OF_REACTIVE_O } \\
\text { XYGEN_SPECIES_METABOLIC_PROCESS }\end{array}$ & 30 & -0.483 & -1.438 & 0.045455 & 0.093063 & 101 \\
\hline 1 & $\begin{array}{l}\text { GO_TETRAHYDROFOLATE_METABOLIC_PROCE } \\
\text { SS }\end{array}$ & 18 & -0.555 & -1.484 & 0.046512 & 0.07106 & 102 \\
\hline 1 & $\begin{array}{l}\text { GO_UBIQUITIN_LIKE_PROTEIN_SPECIFIC_PRO } \\
\text { TEASE_ACTIVITY }\end{array}$ & 82 & -0.371 & -1.384 & 0.046602 & 0.124368 & 103 \\
\hline 1 & BIOCARTA_ATM_PATHWAY & 20 & -0.543 & -1.481 & 0.047325 & 0.072358 & 104 \\
\hline 1 & $\begin{array}{l}\text { HALLMARK_REACTIVE_OXIGEN_SPECIES_PATH } \\
\text { WAY }\end{array}$ & 44 & -0.427 & -1.401 & 0.047521 & 0.113651 & 105 \\
\hline 1 & GO_RESPONSE_TO_ATP & 22 & -0.564 & -1.526 & 0.047817 & 0.055597 & 106 \\
\hline 1 & $\begin{array}{l}\text { GO_REGULATION_OF_MRNA_METABOLIC_PR } \\
\text { OCESS }\end{array}$ & 81 & -0.376 & -1.381 & 0.049541 & 0.125461 & 107 \\
\hline 2 & BIOCARTA_INFLAM_PATHWAY & 19 & -0.852 & -2.273 & 0 & 0 & 1 \\
\hline 2 & GO_CCR_CHEMOKINE_RECEPTOR_BINDING & 19 & -0.871 & -2.299 & 0 & 0 & 2 \\
\hline 2 & $\begin{array}{l}\text { GO_CELLULAR_RESPONSE_TO_BIOTIC_STIMUL } \\
\text { US }\end{array}$ & 134 & -0.588 & -2.309 & 0 & 0 & 3 \\
\hline 2 & $\begin{array}{l}\text { GO_CELLULAR_RESPONSE_TO_CYTOKINE_STI } \\
\text { MULUS }\end{array}$ & 431 & -0.652 & -2.904 & 0 & 0 & 4 \\
\hline 2 & $\begin{array}{l}\text { GO_CELLULAR_RESPONSE_TO_INTERFERON_G } \\
\text { AMMA }\end{array}$ & 93 & -0.784 & -2.93 & 0 & 0 & 5 \\
\hline 2 & $\begin{array}{l}\text { GO_CELLULAR_RESPONSE_TO_INTERLEUKIN_ } \\
1\end{array}$ & 63 & -0.679 & -2.354 & 0 & 0 & 6 \\
\hline 2 & GO_CHEMOKINE_ACTIVITY & 28 & -0.864 & -2.539 & 0 & 0 & 7 \\
\hline
\end{tabular}




\begin{tabular}{|c|c|c|c|c|c|c|c|}
\hline 2 & $\begin{array}{l}\text { GO_CHEMOKINE_MEDIATED_SIGNALING_PAT } \\
\text { HWAY }\end{array}$ & 38 & -0.788 & -2.418 & 0 & 0 & 8 \\
\hline 2 & GO_CHEMOKINE_RECEPTOR_BINDING & 35 & -0.843 & -2.649 & 0 & 0 & 9 \\
\hline 2 & GO_CYTOKINE_ACTIVITY & 114 & -0.716 & -2.748 & 0 & 0 & 10 \\
\hline 2 & $\begin{array}{l}\text { GO_CYTOKINE_MEDIATED_SIGNALING_PATH } \\
\text { WAY }\end{array}$ & 316 & -0.697 & -3.027 & 0 & 0 & 11 \\
\hline 2 & GO_CYTOKINE_RECEPTOR_BINDING & 171 & -0.623 & -2.53 & 0 & 0 & 12 \\
\hline 2 & $\begin{array}{l}\text { GO_DEFENSE_RESPONSE_TO_OTHER_ORGANI } \\
\text { SM }\end{array}$ & 297 & -0.612 & -2.643 & 0 & 0 & 13 \\
\hline 2 & GO_DEFENSE_RESPONSE_TO_VIRUS & 120 & -0.753 & -2.938 & 0 & 0 & 14 \\
\hline 2 & $\begin{array}{l}\text { GO_EXTERNAL_SIDE_OF_PLASMA_MEMBRAN } \\
\text { E }\end{array}$ & 137 & -0.615 & -2.408 & 0 & 0 & 15 \\
\hline 2 & $\begin{array}{l}\text { GO_G_PROTEIN_COUPLED_RECEPTOR_BINDI } \\
\text { NG }\end{array}$ & 138 & -0.591 & -2.306 & 0 & 0 & 16 \\
\hline 2 & $\begin{array}{l}\text { GO_I_KAPPAB_KINASE_NF_KAPPAB_SIGNALIN } \\
\text { G }\end{array}$ & 60 & -0.679 & -2.315 & 0 & 0 & 17 \\
\hline 2 & GO_IMMUNE_EFFECTOR_PROCESS & 336 & -0.603 & -2.629 & 0 & 0 & 18 \\
\hline 2 & GO_INFLAMMATORY_RESPONSE & 291 & -0.614 & -2.616 & 0 & 0 & 19 \\
\hline 2 & GO_INNATE_IMMUNE_RESPONSE & 388 & -0.626 & -2.748 & 0 & 0 & 20 \\
\hline 2 & $\begin{array}{l}\text { GO_INTERFERON_GAMMA_MEDIATED_SIGNA } \\
\text { LING_PATHWAY }\end{array}$ & 62 & -0.754 & -2.63 & 0 & 0 & 21 \\
\hline 2 & GO_LYMPHOCYTE_CHEMOTAXIS & 22 & -0.83 & -2.32 & 0 & 0 & 22 \\
\hline 2 & GO_LYMPHOCYTE_MIGRATION & 28 & -0.81 & -2.412 & 0 & 0 & 23 \\
\hline 2 & $\begin{array}{l}\text { GO_NEGATIVE_REGULATION_OF_DEFENSE_RE } \\
\text { SPONSE }\end{array}$ & 105 & -0.623 & -2.333 & 0 & 0 & 24 \\
\hline 2 & $\begin{array}{l}\text { GO_NEGATIVE_REGULATION_OF_EXTRINSIC_ } \\
\text { APOPTOTIC_SIGNALING_PATHWAY }\end{array}$ & 76 & -0.653 & -2.334 & 0 & 0 & 25 \\
\hline 2 & $\begin{array}{l}\text { GO_NEGATIVE_REGULATION_OF_MULTI_ORG } \\
\text { ANISM_PROCESS }\end{array}$ & 106 & -0.73 & -2.814 & 0 & 0 & 26 \\
\hline 2 & $\begin{array}{l}\text { GO_NEGATIVE_REGULATION_OF_TYPE_IIINTE } \\
\text { RFERON_PRODUCTION }\end{array}$ & 31 & -0.791 & -2.363 & 0 & 0 & 27 \\
\hline 2 & $\begin{array}{l}\text { GO_NEGATIVE_REGULATION_OF_VIRAL_GEN } \\
\text { OME_REPLICATION }\end{array}$ & 38 & -0.826 & -2.588 & 0 & 0 & 28 \\
\hline 2 & $\begin{array}{l}\text { GO_NEGATIVE_REGULATION_OF_VIRAL_PROC } \\
\text { ESS }\end{array}$ & 67 & -0.781 & -2.725 & 0 & 0 & 29 \\
\hline 2 & $\begin{array}{l}\text { GO_POSITIVE_REGULATION_OF_CELL_ACTIVA } \\
\text { TION }\end{array}$ & 214 & -0.563 & -2.349 & 0 & 0 & 30 \\
\hline 2 & $\begin{array}{l}\text { GO_POSITIVE_REGULATION_OF_CYTOKINE_BI } \\
\text { OSYNTHETIC_PROCESS }\end{array}$ & 45 & -0.737 & -2.427 & 0 & 0 & 31 \\
\hline
\end{tabular}




\begin{tabular}{|c|c|c|c|c|c|c|c|}
\hline 2 & $\begin{array}{l}\text { GO_POSITIVE_REGULATION_OF_CYTOKINE_PR } \\
\text { ODUCTION }\end{array}$ & 265 & -0.594 & -2.509 & 0 & 0 & 32 \\
\hline 2 & $\begin{array}{l}\text { GO_POSITIVE_REGULATION_OF_DEFENSE_RES } \\
\text { PONSE }\end{array}$ & 271 & -0.591 & -2.52 & 0 & 0 & 33 \\
\hline 2 & $\begin{array}{l}\text { GO_POSITIVE_REGULATION_OF_EXTRINSIC_A } \\
\text { POPTOTIC_SIGNALING_PATHWAY }\end{array}$ & 45 & -0.72 & -2.312 & 0 & 0 & 34 \\
\hline 2 & $\begin{array}{l}\text { GO_POSITIVE_REGULATION_OF_I_KAPPAB_KI } \\
\text { NASE_NF_KAPPAB_SIGNALING }\end{array}$ & 144 & -0.628 & -2.486 & 0 & 0 & 35 \\
\hline 2 & $\begin{array}{l}\text { GO_POSITIVE_REGULATION_OF_IMMUNE_RE } \\
\text { SPONSE }\end{array}$ & 404 & -0.518 & -2.306 & 0 & 0 & 36 \\
\hline 2 & $\begin{array}{l}\text { GO_POSITIVE_REGULATION_OF_INFLAMMAT } \\
\text { ORY_RESPONSE }\end{array}$ & 71 & -0.705 & -2.462 & 0 & 0 & 37 \\
\hline 2 & $\begin{array}{l}\text { GO_POSITIVE_REGULATION_OF_INNATE_IMM } \\
\text { UNE_RESPONSE }\end{array}$ & 203 & -0.582 & -2.406 & 0 & 0 & 38 \\
\hline 2 & $\begin{array}{l}\text { GO_POSITIVE_REGULATION_OF_INTERFERON } \\
\text { _GAMMA_PRODUCTION }\end{array}$ & 46 & -0.715 & -2.301 & 0 & 0 & 39 \\
\hline 2 & $\begin{array}{l}\text { GO_POSITIVE_REGULATION_OF_LEUKOCYTE_ } \\
\text { CHEMOTAXIS }\end{array}$ & 57 & -0.707 & -2.385 & 0 & 0 & 40 \\
\hline 2 & $\begin{array}{l}\text { GO_POSITIVE_REGULATION_OF_LEUKOCYTE_ } \\
\text { MIGRATION }\end{array}$ & 75 & -0.716 & -2.549 & 0 & 0 & 41 \\
\hline 2 & $\begin{array}{l}\text { GO_POSITIVE_REGULATION_OF_LEUKOCYTE_ } \\
\text { PROLIFERATION }\end{array}$ & 98 & -0.65 & -2.412 & 0 & 0 & 42 \\
\hline 2 & $\begin{array}{l}\text { GO_POSITIVE_REGULATION_OF_NF_KAPPAB_ } \\
\text { TRANSCRIPTION_FACTOR_ACTIVITY }\end{array}$ & 102 & -0.643 & -2.418 & 0 & 0 & 43 \\
\hline 2 & $\begin{array}{l}\text { GO_POSITIVE_REGULATION_OF_RESPONSE_T } \\
\text { O_WOUNDING }\end{array}$ & 103 & -0.631 & -2.39 & 0 & 0 & 44 \\
\hline 2 & $\begin{array}{l}\text { GO_POSITIVE_REGULATION_OF_STAT_CASCA } \\
\text { DE }\end{array}$ & 49 & -0.679 & -2.271 & 0 & 0 & 45 \\
\hline 2 & GO_REGULATION_OF_CHEMOTAXIS & 121 & -0.597 & -2.31 & 0 & 0 & 46 \\
\hline 2 & $\begin{array}{l}\text { GO_REGULATION_OF_CYTOKINE_BIOSYNTHET } \\
\text { IC_PROCESS }\end{array}$ & 71 & -0.665 & -2.341 & 0 & 0 & 47 \\
\hline 2 & $\begin{array}{l}\text { GO_REGULATION_OF_CYTOKINE_PRODUCTIO } \\
\mathrm{N}\end{array}$ & 398 & -0.57 & -2.513 & 0 & 0 & 48 \\
\hline 2 & $\begin{array}{l}\text { GO_REGULATION_OF_EXTRINSIC_APOPTOTIC } \\
\text { SIIGNALING_PATHWAY }\end{array}$ & 122 & -0.642 & -2.497 & 0 & 0 & 49 \\
\hline 2 & $\begin{array}{l}\text { GO_REGULATION_OF_EXTRINSIC_APOPTOTIC } \\
\text { SIGNALING_PATHWAY_IN_ABSENCE_OF_LIG } \\
\text { AND }\end{array}$ & 35 & -0.765 & -2.376 & 0 & 0 & 50 \\
\hline 2 & $\begin{array}{l}\text { GO_REGULATION_OF_GRANULOCYTE_CHEMO } \\
\text { TAXIS }\end{array}$ & 28 & -0.776 & -2.287 & 0 & 0 & 51 \\
\hline 2 & $\begin{array}{l}\text { GO_REGULATION_OF_I_KAPPAB_KINASE_NF_ } \\
\text { KAPPAB_SIGNALING }\end{array}$ & 188 & -0.621 & -2.563 & 0 & 0 & 52 \\
\hline
\end{tabular}




\begin{tabular}{|c|c|c|c|c|c|c|c|}
\hline 2 & $\begin{array}{l}\text { GO_REGULATION_OF_IMMUNE_EFFECTOR_P } \\
\text { ROCESS }\end{array}$ & 294 & -0.532 & -2.287 & 0 & 0 & 53 \\
\hline 2 & $\begin{array}{l}\text { GO_REGULATION_OF_INFLAMMATORY_RESP } \\
\text { ONSE }\end{array}$ & 192 & -0.624 & -2.562 & 0 & 0 & 54 \\
\hline 2 & $\begin{array}{l}\text { GO_REGULATION_OF_INNATE_IMMUNE_RES } \\
\text { PONSE }\end{array}$ & 280 & -0.601 & -2.568 & 0 & 0 & 55 \\
\hline 2 & $\begin{array}{l}\text { GO_REGULATION_OF_INTERFERON_GAMMA_ } \\
\text { PRODUCTION }\end{array}$ & 68 & -0.702 & -2.486 & 0 & 0 & 56 \\
\hline 2 & $\begin{array}{l}\text { GO_REGULATION_OF_INTERLEUKIN_1_BETA_ } \\
\text { PRODUCTION }\end{array}$ & 30 & -0.759 & -2.28 & 0 & 0 & 57 \\
\hline 2 & $\begin{array}{l}\text { GO_REGULATION_OF_INTERLEUKIN_1_PROD } \\
\text { UCTION }\end{array}$ & 38 & -0.758 & -2.387 & 0 & 0 & 58 \\
\hline 2 & $\begin{array}{l}\text { GO_REGULATION_OF_INTERLEUKIN_12_PROD } \\
\text { UCTION }\end{array}$ & 41 & -0.758 & -2.404 & 0 & 0 & 59 \\
\hline 2 & $\begin{array}{l}\text { GO_REGULATION_OF_LEUKOCYTE_CHEMOTA } \\
\text { XIS }\end{array}$ & 70 & -0.691 & -2.43 & 0 & 0 & 60 \\
\hline 2 & $\begin{array}{l}\text { GO_REGULATION_OF_LEUKOCYTE_MIGRATIO } \\
\mathrm{N}\end{array}$ & 107 & -0.658 & -2.513 & 0 & 0 & 61 \\
\hline 2 & $\begin{array}{l}\text { GO_REGULATION_OF_LEUKOCYTE_PROLIFERA } \\
\text { TION }\end{array}$ & 148 & -0.581 & -2.317 & 0 & 0 & 62 \\
\hline 2 & GO_REGULATION_OF_NECROTIC_CELL_DEATH & 22 & -0.826 & -2.301 & 0 & 0 & 63 \\
\hline 2 & $\begin{array}{l}\text { GO_REGULATION_OF_NF_KAPPAB_IMPORT_I } \\
\text { NTO_NUCLEUS }\end{array}$ & 41 & -0.755 & -2.397 & 0 & 0 & 64 \\
\hline 2 & $\begin{array}{l}\text { GO_REGULATION_OF_RESPONSE_TO_CYTOKI } \\
\text { NE_STIMULUS }\end{array}$ & 112 & -0.623 & -2.348 & 0 & 0 & 65 \\
\hline 2 & $\begin{array}{l}\text { GO_REGULATION_OF_T_CELL_DIFFERENTIATI } \\
\text { ON }\end{array}$ & 74 & -0.659 & -2.356 & 0 & 0 & 66 \\
\hline 2 & $\begin{array}{l}\text { GO_REGULATION_OF_T_CELL_PROLIFERATIO } \\
\mathrm{N}\end{array}$ & 105 & -0.595 & -2.275 & 0 & 0 & 67 \\
\hline 2 & $\begin{array}{l}\text { GO_REGULATION_OF_T_HELPER_CELL_DIFFER } \\
\text { ENTIATION }\end{array}$ & 20 & -0.829 & -2.277 & 0 & 0 & 68 \\
\hline 2 & $\begin{array}{l}\text { GO_REGULATION_OF_TYPE_I_INTERFERON_P } \\
\text { RODUCTION }\end{array}$ & 95 & -0.641 & -2.395 & 0 & 0 & 69 \\
\hline 2 & $\begin{array}{l}\text { GO_REGULATION_OF_VIRAL_GENOME_REPLI } \\
\text { CATION }\end{array}$ & 63 & -0.738 & -2.545 & 0 & 0 & 70 \\
\hline 2 & GO_RESPONSE_TO_BACTERIUM & 330 & -0.574 & -2.523 & 0 & 0 & 71 \\
\hline 2 & GO_RESPONSE_TO_INTERFERON_GAMMA & 109 & -0.781 & -2.934 & 0 & 0 & 72 \\
\hline 2 & GO_RESPONSE_TO_INTERLEUKIN_1 & 86 & -0.663 & -2.414 & 0 & 0 & 73 \\
\hline 2 & $\begin{array}{l}\text { GO_RESPONSE_TO_MOLECULE_OF_BACTERIA } \\
\text { L_ORIGIN }\end{array}$ & 243 & -0.589 & -2.486 & 0 & 0 & 74 \\
\hline
\end{tabular}




\begin{tabular}{|c|c|c|c|c|c|c|c|}
\hline 2 & $\begin{array}{l}\text { GO_RESPONSE_TO_TUMOR_NECROSIS_FACT } \\
\text { OR }\end{array}$ & 172 & -0.651 & -2.656 & 0 & 0 & 75 \\
\hline 2 & GO_RESPONSE_TO_TYPE_I_INTERFERON & 53 & -0.793 & -2.605 & 0 & 0 & 76 \\
\hline 2 & GO_RESPONSE_TO_VIRUS & 186 & -0.698 & -2.856 & 0 & 0 & 77 \\
\hline 2 & $\begin{array}{l}\text { GO_TUMOR_NECROSIS_FACTOR_MEDIATED_ } \\
\text { SIGNALING_PATHWAY }\end{array}$ & 93 & -0.643 & -2.332 & 0 & 0 & 78 \\
\hline 2 & HALLMARK_ALLOGRAFT_REJECTION & 162 & -0.637 & -2.558 & 0 & 0 & 79 \\
\hline 2 & HALLMARK_COMPLEMENT & 166 & -0.617 & -2.451 & 0 & 0 & 80 \\
\hline 2 & HALLMARK_IL2_STAT5_SIGNALING & 160 & -0.573 & -2.285 & 0 & 0 & 81 \\
\hline 2 & HALLMARK_IL6_JAK_STAT3_SIGNALING & 73 & -0.761 & -2.734 & 0 & 0 & 82 \\
\hline 2 & HALLMARK_INFLAMMATORY_RESPONSE & 167 & -0.765 & -3.09 & 0 & 0 & 83 \\
\hline 2 & HALLMARK_INTERFERON_ALPHA_RESPONSE & 87 & -0.889 & -3.209 & 0 & 0 & 84 \\
\hline 2 & HALLMARK_INTERFERON_GAMMA_RESPONSE & 182 & -0.864 & -3.55 & 0 & 0 & 85 \\
\hline 2 & HALLMARK_TNFA_SIGNALING_VIA_NFKB & 181 & -0.729 & -2.967 & 0 & 0 & 86 \\
\hline 2 & $\begin{array}{l}\text { KEGG_CYTOKINE_CYTOKINE_RECEPTOR_INTER } \\
\text { ACTION }\end{array}$ & 155 & -0.697 & -2.764 & 0 & 0 & 87 \\
\hline 2 & KEGG_CYTOSOLIC_DNA_SENSING_PATHWAY & 40 & -0.742 & -2.379 & 0 & 0 & 88 \\
\hline 2 & $\begin{array}{l}\text { KEGG_NOD_LIKE_RECEPTOR_SIGNALING_PAT } \\
\text { HWAY }\end{array}$ & 45 & -0.762 & -2.495 & 0 & 0 & 89 \\
\hline 2 & $\begin{array}{l}\text { KEGG_RIG_I_LIKE_RECEPTOR_SIGNALING_PAT } \\
\text { HWAY }\end{array}$ & 49 & -0.757 & -2.494 & 0 & 0 & 90 \\
\hline 2 & $\begin{array}{l}\text { KEGG_TOLL_LIKE_RECEPTOR_SIGNALING_PAT } \\
\text { HWAY }\end{array}$ & 84 & -0.625 & -2.281 & 0 & 0 & 91 \\
\hline 2 & GO_REGULATION_OF_CYTOKINE_SECRETION & 100 & -0.599 & -2.244 & 0 & $2.19 \mathrm{E}-06$ & 92 \\
\hline 2 & $\begin{array}{l}\text { GO_REGULATION_OF_ADAPTIVE_IMMUNE_R } \\
\text { ESPONSE }\end{array}$ & 91 & -0.61 & -2.251 & 0 & $2.24 \mathrm{E}-06$ & 93 \\
\hline 2 & $\begin{array}{l}\text { GO_REGULATION_OF_LYMPHOCYTE_DIFFERE } \\
\text { NTIATION }\end{array}$ & 94 & -0.608 & -2.252 & 0 & $2.26 \mathrm{E}-06$ & 94 \\
\hline 2 & $\begin{array}{l}\text { GO_ACTIVATION_OF_INNATE_IMMUNE_RESP } \\
\text { ONSE }\end{array}$ & 173 & -0.56 & -2.255 & 0 & $2.28 \mathrm{E}-06$ & 95 \\
\hline 2 & $\begin{array}{l}\text { GO_POSITIVE_REGULATION_OF_INTERLEUKIN } \\
\text { _12_PRODUCTION }\end{array}$ & 26 & -0.771 & -2.26 & 0 & $2.29 \mathrm{E}-06$ & 96 \\
\hline 2 & GO_MONOCYTE_CHEMOTAXIS & 25 & -0.796 & -2.261 & 0 & $2.30 \mathrm{E}-06$ & 97 \\
\hline 2 & GO_RESPONSE_TO_PROTOZOAN & 18 & -0.866 & -2.264 & 0 & $2.31 \mathrm{E}-06$ & 98 \\
\hline 2 & GO_POSITIVE_REGULATION_OF_CHEMOTAXIS & 82 & -0.632 & -2.266 & 0 & $2.32 \mathrm{E}-06$ & 99 \\
\hline 2 & $\begin{array}{l}\text { GO_REGULATION_OF_NEUTROPHIL_CHEMOT } \\
\text { AXIS }\end{array}$ & 22 & -0.799 & -2.269 & 0 & 2.34E-06 & 100 \\
\hline
\end{tabular}




\begin{tabular}{|c|c|c|c|c|c|c|c|}
\hline 2 & GO_RESPONSE_TO_INTERFERON_BETA & 17 & -0.851 & -2.214 & 0 & 1.65E-05 & 101 \\
\hline 2 & $\begin{array}{l}\text { GO_REGULATION_OF_INTERFERON_BETA_PR } \\
\text { ODUCTION }\end{array}$ & 38 & -0.69 & -2.195 & 0 & $1.81 \mathrm{E}-05$ & 102 \\
\hline 2 & KEGG_CHEMOKINE_SIGNALING_PATHWAY & 137 & -0.563 & -2.204 & 0 & $1.83 \mathrm{E}-05$ & 103 \\
\hline 2 & $\begin{array}{l}\text { GO_POSITIVE_REGULATION_OF_IMMUNE_EFF } \\
\text { ECTOR_PROCESS }\end{array}$ & 106 & -0.585 & -2.205 & 0 & 1.83E-05 & 104 \\
\hline 2 & BIOCARTA_IL22BP_PATHWAY & 15 & -0.887 & -2.212 & 0 & $1.85 \mathrm{E}-05$ & 105 \\
\hline 2 & $\begin{array}{l}\text { GO_REGULATION_OF_LYMPHOCYTE_APOPTO } \\
\text { TIC_PROCESS }\end{array}$ & 40 & -0.688 & -2.191 & 0 & 2.19E-05 & 106 \\
\hline 2 & GO_REGULATION_OF_CELL_ACTIVATION & 333 & -0.502 & -2.193 & 0 & $2.19 \mathrm{E}-05$ & 107 \\
\hline 2 & $\begin{array}{l}\text { GO_REGULATION_OF_TYROSINE_PHOSPHORY } \\
\text { LATION_OF_STAT_PROTEIN }\end{array}$ & 46 & -0.669 & -2.193 & 0 & $2.20 \mathrm{E}-05$ & 108 \\
\hline 2 & $\begin{array}{l}\text { GO_POSITIVE_REGULATION_OF_T_CELL_PROL } \\
\text { IFERATION }\end{array}$ & 69 & -0.625 & -2.185 & 0 & $2.56 \mathrm{E}-05$ & 109 \\
\hline 2 & $\begin{array}{l}\text { GO_POSITIVE_REGULATION_OF_INTERLEUKIN } \\
\text { 6_PRODUCTION }\end{array}$ & 48 & -0.672 & -2.172 & 0 & $3.25 \mathrm{E}-05$ & 110 \\
\hline 2 & $\begin{array}{l}\text { GO_POSITIVE_REGULATION_OF_NEUTROPHIL } \\
\text { _MIGRATION }\end{array}$ & 21 & -0.79 & -2.163 & 0 & 3.35E-05 & 111 \\
\hline 2 & GO_GRANULOCYTE_MIGRATION & 51 & -0.633 & -2.165 & 0 & 3.37E-05 & 112 \\
\hline 2 & $\begin{array}{l}\text { GO_REGULATION_OF_VIRAL_ENTRY_INTO_H } \\
\text { OST_CELL }\end{array}$ & 22 & -0.792 & -2.165 & 0 & $3.38 \mathrm{E}-05$ & 113 \\
\hline 2 & $\begin{array}{l}\text { GO_REGULATION_OF_NEUTROPHIL_MIGRATI } \\
\text { ON }\end{array}$ & 25 & -0.767 & -2.166 & 0 & $3.39 \mathrm{E}-05$ & 114 \\
\hline 2 & $\begin{array}{l}\text { GO_REGULATION_OF_CD4_POSITIVE_ALPHA_ } \\
\text { BETA_T_CELL_ACTIVATION }\end{array}$ & 29 & -0.744 & -2.166 & 0 & 3.40E-05 & 115 \\
\hline 2 & $\begin{array}{l}\text { GO_PATTERN_RECOGNITION_RECEPTOR_SIGN } \\
\text { ALING_PATHWAY }\end{array}$ & 91 & -0.584 & -2.16 & 0 & $3.68 \mathrm{E}-05$ & 116 \\
\hline 2 & GO_RESPONSE_TO_INTERFERON_ALPHA & 18 & -0.817 & -2.153 & 0 & 4.19E-05 & 117 \\
\hline 2 & GO_REGULATION_OF_STAT_CASCADE & 82 & -0.597 & -2.146 & 0 & $5.23 \mathrm{E}-05$ & 118 \\
\hline 2 & GO_INTERACTION_WITH_HOST & 114 & -0.562 & -2.147 & 0 & $5.24 \mathrm{E}-05$ & 119 \\
\hline 2 & BIOCARTA_IL10_PATHWAY & 17 & -0.828 & -2.141 & 0 & $5.86 \mathrm{E}-05$ & 120 \\
\hline 2 & GO_ZYMOGEN_ACTIVATION & 85 & -0.587 & -2.142 & 0 & 5.87E-05 & 121 \\
\hline 2 & $\begin{array}{l}\text { GO_REGULATION_OF_INTERLEUKIN_1_SECRE } \\
\text { TION }\end{array}$ & 21 & -0.787 & -2.137 & 0 & $6.02 \mathrm{E}-05$ & 122 \\
\hline 2 & $\begin{array}{l}\text { GO_POSITIVE_REGULATION_OF_INTERLEUKIN } \\
\text { _1_PRODUCTION }\end{array}$ & 22 & -0.782 & -2.13 & 0 & 7.00E-05 & 123 \\
\hline
\end{tabular}




\begin{tabular}{|c|c|c|c|c|c|c|c|}
\hline 2 & $\begin{array}{l}\text { GO_NEGATIVE_REGULATION_OF_EXTRINSIC_ } \\
\text { APOPTOTIC_SIGNALING_PATHWAY_VIA_DEAT } \\
\text { H_DOMAIN_RECEPTORS }\end{array}$ & 27 & -0.727 & -2.129 & 0 & 7.47E-05 & 124 \\
\hline 2 & $\begin{array}{l}\text { GO_POSITIVE_REGULATION_OF_INTERLEUKIN } \\
\text { _8_PRODUCTION }\end{array}$ & 31 & -0.713 & -2.129 & 0 & $7.48 \mathrm{E}-05$ & 125 \\
\hline 2 & $\begin{array}{l}\text { GO_POSITIVE_REGULATION_OF_INTERFERON } \\
\text { _BETA_PRODUCTION }\end{array}$ & 28 & -0.708 & -2.125 & 0 & 8.11E-05 & 126 \\
\hline 2 & $\begin{array}{l}\text { GO_MYD88_INDEPENDENT_TOLL_LIKE_RECEP } \\
\text { TOR_SIGNALING_PATHWAY }\end{array}$ & 30 & -0.726 & -2.125 & 0 & 8.27E-05 & 127 \\
\hline 2 & GO_REGULATION_OF_T_CELL_MIGRATION & 19 & -0.769 & -2.123 & 0 & 8.77E-05 & 128 \\
\hline 2 & $\begin{array}{l}\text { GO_POSITIVE_REGULATION_OF_LYMPHOCYTE } \\
\text { _MIGRATION }\end{array}$ & 19 & -0.792 & -2.122 & 0 & $8.93 \mathrm{E}-05$ & 129 \\
\hline 2 & BIOCARTA_TNFR2_PATHWAY & 16 & -0.794 & -2.12 & 0 & $9.06 \mathrm{E}-05$ & 130 \\
\hline 2 & BIOCARTA_HIVNEF_PATHWAY & 54 & -0.633 & -2.121 & 0 & 9.07E-05 & 131 \\
\hline 2 & $\begin{array}{l}\text { GO_NEGATIVE_REGULATION_OF_INFLAMMAT } \\
\text { ORY_RESPONSE }\end{array}$ & 67 & -0.607 & -2.116 & 0 & $9.84 \mathrm{E}-05$ & 132 \\
\hline 2 & $\begin{array}{l}\text { GO_CYTOPLASMIC_PATTERN_RECOGNITION_ } \\
\text { RECEPTOR_SIGNALING_PATHWAY }\end{array}$ & 26 & -0.734 & -2.113 & 0 & 1.02E-04 & 133 \\
\hline 2 & $\begin{array}{l}\text { GO_REGULATION_OF_LEUKOCYTE_APOPTOTI } \\
\text { C_PROCESS }\end{array}$ & 62 & -0.61 & -2.109 & 0 & 1.03E-04 & 134 \\
\hline 2 & $\begin{array}{l}\text { GO_TUMOR_NECROSIS_FACTOR_RECEPTOR_S } \\
\text { UPERFAMILY_BINDING }\end{array}$ & 38 & -0.661 & -2.107 & 0 & $1.08 \mathrm{E}-04$ & 135 \\
\hline 2 & GO_LEUKOCYTE_CELL_CELL_ADHESION & 193 & -0.521 & -2.105 & 0 & $1.08 \mathrm{E}-04$ & 136 \\
\hline 2 & $\begin{array}{l}\text { GO_REGULATION_OF_ERK1_AND_ERK2_CASC } \\
\text { ADE }\end{array}$ & 149 & -0.537 & -2.106 & 0 & 1.09E-04 & 137 \\
\hline 2 & $\begin{array}{l}\text { GO_REGULATION_OF_TYPE_I_INTERFERON_M } \\
\text { EDIATED_SIGNALING_PATHWAY }\end{array}$ & 24 & -0.763 & -2.107 & 0 & $1.09 \mathrm{E}-04$ & 138 \\
\hline 2 & $\begin{array}{l}\text { GO_POSITIVE_REGULATION_OF_B_CELL_PROL } \\
\text { IFERATION }\end{array}$ & 25 & -0.732 & -2.105 & 0 & $1.10 \mathrm{E}-04$ & 139 \\
\hline 2 & $\begin{array}{l}\text { GO_NEGATIVE_REGULATION_OF_IMMUNE_R } \\
\text { ESPONSE }\end{array}$ & 92 & -0.575 & -2.103 & 0 & $1.16 \mathrm{E}-04$ & 140 \\
\hline 2 & $\begin{array}{l}\text { GO_POSITIVE_REGULATION_OF_CYTOKINE_SE } \\
\text { CRETION }\end{array}$ & 65 & -0.613 & -2.102 & 0 & $1.24 \mathrm{E}-04$ & 141 \\
\hline 2 & $\begin{array}{l}\text { GO_REGULATION_OF_LYMPHOCYTE_MIGRATI } \\
\text { ON }\end{array}$ & 29 & -0.703 & -2.101 & 0 & $1.28 \mathrm{E}-04$ & 142 \\
\hline 2 & GO_DENDRITIC_CELL_DIFFERENTIATION & 24 & -0.738 & -2.098 & 0 & $1.35 \mathrm{E}-04$ & 143 \\
\hline 2 & $\begin{array}{l}\text { GO_POSITIVE_REGULATION_OF_LYMPHOCYTE } \\
\text { _DIFFERENTIATION }\end{array}$ & 59 & -0.609 & -2.089 & 0 & 1.73E-04 & 144 \\
\hline 2 & $\begin{array}{l}\text { GO_REGULATION_OF_CYTOKINE_PRODUCTIO } \\
\text { N_INVOLVED_IN_IMMUNE_RESPONSE }\end{array}$ & 40 & -0.653 & -2.088 & 0 & 1.76E-04 & 145 \\
\hline
\end{tabular}




\begin{tabular}{|c|c|c|c|c|c|c|c|}
\hline 2 & $\begin{array}{l}\text { GO_NEGATIVE_REGULATION_OF_CYTOKINE_P } \\
\text { RODUCTION }\end{array}$ & 142 & -0.536 & -2.086 & 0 & $1.83 \mathrm{E}-04$ & 146 \\
\hline 2 & GO_LEUKOCYTE_CHEMOTAXIS & 79 & -0.577 & -2.085 & 0 & 1.89E-04 & 147 \\
\hline 2 & GO_STAT_CASCADE & 39 & -0.665 & -2.083 & 0 & $1.91 \mathrm{E}-04$ & 148 \\
\hline 2 & BIOCARTA_NFKB_PATHWAY & 22 & -0.752 & -2.077 & 0 & $2.06 \mathrm{E}-04$ & 149 \\
\hline 2 & GO_REGULATION_OF_HEMOPOIESIS & 215 & -0.499 & -2.077 & 0 & 2.07E-04 & 150 \\
\hline 2 & GO_LEUKOCYTE_MEDIATED_IMMUNITY & 111 & -0.545 & -2.076 & 0 & $2.10 \mathrm{E}-04$ & 151 \\
\hline 2 & $\begin{array}{l}\text { GO_POSITIVE_REGULATION_OF_TYPE_I_INTER } \\
\text { FERON_PRODUCTION }\end{array}$ & 62 & -0.593 & -2.076 & 0 & $2.11 \mathrm{E}-04$ & 152 \\
\hline 2 & $\begin{array}{l}\text { GO_REGULATION_OF_CHEMOKINE_PRODUCTI } \\
\text { ON }\end{array}$ & 47 & -0.635 & -2.072 & 0 & $2.25 \mathrm{E}-04$ & 153 \\
\hline 2 & $\begin{array}{l}\text { GO_REGULATION_OF_PRODUCTION_OF_MOL } \\
\text { ECULAR_MEDIATOR_OF_IMMUNE_RESPONSE }\end{array}$ & 66 & -0.596 & -2.065 & 0 & $2.51 \mathrm{E}-04$ & 154 \\
\hline 2 & $\begin{array}{l}\text { GO_POSITIVE_REGULATION_OF_INTERLEUKIN } \\
\text { _1_BETA_PRODUCTION }\end{array}$ & 18 & -0.79 & -2.064 & 0 & 2.60E-04 & 155 \\
\hline 2 & $\begin{array}{l}\text { GO_REGULATION_OF_ACTIVATED_T_CELL_PR } \\
\text { OLIFERATION }\end{array}$ & 28 & -0.716 & -2.063 & 0 & 2.62E-04 & 156 \\
\hline 2 & GO_MYELOID_LEUKOCYTE_MIGRATION & 67 & -0.587 & -2.059 & 0 & $2.81 \mathrm{E}-04$ & 157 \\
\hline 2 & $\begin{array}{l}\text { GO_REGULATION_OF_INTERLEUKIN_8_PROD } \\
\text { UCTION }\end{array}$ & 43 & -0.637 & -2.053 & 0 & $3.14 \mathrm{E}-04$ & 158 \\
\hline 2 & GO_CELL_CHEMOTAXIS & 116 & -0.531 & -2.05 & 0 & $3.23 \mathrm{E}-04$ & 159 \\
\hline 2 & $\begin{array}{l}\text { GO_REGULATION_OF_LEUKOCYTE_MEDIATED } \\
\text { _IMMUNITY }\end{array}$ & 110 & -0.532 & -2.05 & 0 & 3.23E-04 & 160 \\
\hline 2 & GO_ACTIVATION_OF_IMMUNE_RESPONSE & 313 & -0.469 & -2.049 & 0 & 3.27E-04 & 161 \\
\hline 2 & $\begin{array}{l}\text { GO_POSITIVE_REGULATION_OF_NF_KAPPAB_I } \\
\text { MPORT_INTO_NUCLEUS }\end{array}$ & 23 & -0.744 & -2.048 & 0 & 3.32E-04 & 162 \\
\hline 2 & $\begin{array}{l}\text { GO_POSITIVE_REGULATION_OF_CHEMOKINE_ } \\
\text { PRODUCTION }\end{array}$ & 38 & -0.655 & -2.047 & 0 & 3.33E-04 & 163 \\
\hline 2 & $\begin{array}{l}\text { GO_REGULATION_OF_LEUKOCYTE_DIFFERENT } \\
\text { IATION }\end{array}$ & 166 & -0.514 & -2.046 & 0 & 3.35E-04 & 164 \\
\hline 2 & $\begin{array}{l}\text { GO_POSITIVE_REGULATION_OF_CD4_POSITIV } \\
\text { E_ALPHA_BETA_T_CELL_ACTIVATION }\end{array}$ & 21 & -0.756 & -2.036 & 0 & $3.64 \mathrm{E}-04$ & 165 \\
\hline 2 & $\begin{array}{l}\text { GO_POSITIVE_REGULATION_OF_PRODUCTION } \\
\text { _OF_MOLECULAR_MEDIATOR_OF_IMMUNE_ } \\
\text { RESPONSE }\end{array}$ & 42 & -0.633 & -2.031 & 0 & 3.86E-04 & 166 \\
\hline 2 & $\begin{array}{l}\text { GO_REGULATION_OF_B_CELL_PROLIFERATIO } \\
\mathrm{N}\end{array}$ & 40 & -0.632 & -2.031 & 0 & 3.86E-04 & 167 \\
\hline 2 & $\begin{array}{l}\text { GO_LIPOPOLYSACCHARIDE_MEDIATED_SIGNA } \\
\text { LING_PATHWAY }\end{array}$ & 28 & -0.693 & -2.03 & 0 & $3.90 \mathrm{E}-04$ & 168 \\
\hline
\end{tabular}




\begin{tabular}{|c|c|c|c|c|c|c|c|}
\hline 2 & $\begin{array}{l}\text { GO_ADAPTIVE_IMMUNE_RESPONSE_BASED_- } \\
\text { ON_SOMATIC_RECOMBINATION_OF_IMMUN } \\
\text { E_RECEPTORS_BUILT_FROM_IMMUNOGLOBU } \\
\text { LIN_SUPERFAMILY_DOMAINS }\end{array}$ & 97 & -0.547 & -2.03 & 0 & $3.91 \mathrm{E}-04$ & 169 \\
\hline 2 & GO_ALPHA_BETA_T_CELL_ACTIVATION & 41 & -0.639 & -2.028 & 0 & 3.97E-04 & 170 \\
\hline 2 & $\begin{array}{l}\text { GO_REGULATION_OF_T_HELPER_1_TYPE_IM } \\
\text { MUNE_RESPONSE }\end{array}$ & 18 & -0.779 & -2.029 & 0 & 3.97E-04 & 171 \\
\hline 2 & GO_CYTOKINE_RECEPTOR_ACTIVITY & 54 & -0.6 & -2.025 & 0 & 4.25E-04 & 172 \\
\hline 2 & $\begin{array}{l}\text { GO_REGULATION_OF_TUMOR_NECROSIS_FAC } \\
\text { TOR_SUPERFAMILY_CYTOKINE_PRODUCTION }\end{array}$ & 78 & -0.565 & -2.021 & 0 & 4.61E-04 & 173 \\
\hline 2 & $\begin{array}{l}\text { GO_REGULATION_OF_INTERFERON_ALPHA_P } \\
\text { RODUCTION }\end{array}$ & 17 & -0.775 & -2.02 & 0 & 4.70E-04 & 174 \\
\hline 2 & $\begin{array}{l}\text { GO_POSITIVE_REGULATION_OF_LEUKOCYTE_ } \\
\text { APOPTOTIC_PROCESS }\end{array}$ & 21 & -0.738 & -2.019 & 0 & 4.74E-04 & 175 \\
\hline 2 & KEGG_HEMATOPOIETIC_CELL_LINEAGE & 57 & -0.592 & -2.019 & 0 & 4.78E-04 & 176 \\
\hline 2 & $\begin{array}{l}\text { GO_REGULATION_OF_T_CELL_APOPTOTIC_PR } \\
\text { OCESS }\end{array}$ & 21 & -0.733 & -2.018 & 0 & $4.88 \mathrm{E}-04$ & 177 \\
\hline 2 & $\begin{array}{l}\text { GO_REGULATION_OF_ALPHA_BETA_T_CELL_D } \\
\text { IFFERENTIATION }\end{array}$ & 35 & -0.664 & -2.017 & 0 & 4.93E-04 & 178 \\
\hline 2 & $\begin{array}{l}\text { GO_NEGATIVE_REGULATION_OF_IMMUNE_SY } \\
\text { STEM_PROCESS }\end{array}$ & 272 & -0.478 & -2.017 & 0 & 4.96E-04 & 179 \\
\hline 2 & $\begin{array}{l}\text { GO_REGULATION_OF_T_CELL_MEDIATED_IM } \\
\text { MUNITY }\end{array}$ & 35 & -0.642 & -2.01 & 0 & $5.48 \mathrm{E}-04$ & 180 \\
\hline 2 & $\begin{array}{l}\text { GO_NEGATIVE_REGULATION_OF_IMMUNE_EF } \\
\text { FECTOR_PROCESS }\end{array}$ & 79 & -0.563 & -2.006 & 0 & $5.86 \mathrm{E}-04$ & 181 \\
\hline 2 & KEGG_GRAFT_VERSUS_HOST_DISEASE & 29 & -0.674 & -2.006 & 0 & 5.87E-04 & 182 \\
\hline 2 & $\begin{array}{l}\text { GO_POSITIVE_REGULATION_OF_ADAPTIVE_I } \\
\text { MMUNE_RESPONSE }\end{array}$ & 55 & -0.602 & -2.005 & 0 & $5.98 \mathrm{E}-04$ & 183 \\
\hline 2 & $\begin{array}{l}\text { GO_MYELOID_DENDRITIC_CELL_DIFFERENTIA } \\
\text { TION }\end{array}$ & 15 & -0.773 & -2.001 & 0 & $6.19 \mathrm{E}-04$ & 184 \\
\hline 2 & GO_MYELOID_LEUKOCYTE_ACTIVATION & 68 & -0.581 & -2.001 & 0 & $6.20 \mathrm{E}-04$ & 185 \\
\hline 2 & $\begin{array}{l}\text { KEGG_INTESTINAL_IMMUNE_NETWORK_FOR } \\
\text { _IGA_PRODUCTION }\end{array}$ & 32 & -0.662 & -1.996 & 0 & $6.66 \mathrm{E}-04$ & 186 \\
\hline 2 & GO_LYMPHOCYTE_MEDIATED_IMMUNITY & 83 & -0.553 & -1.996 & 0 & $6.71 \mathrm{E}-04$ & 187 \\
\hline 2 & GO_REGULATION_OF_B_CELL_ACTIVATION & 82 & -0.551 & -1.993 & 0 & 7.05E-04 & 188 \\
\hline 2 & $\begin{array}{l}\text { GO_REGULATION_OF_EPITHELIAL_CELL_APOP } \\
\text { TOTIC_PROCESS }\end{array}$ & 35 & -0.64 & -1.99 & 0 & $7.40 \mathrm{E}-04$ & 189 \\
\hline 2 & $\begin{array}{l}\text { GO_POSITIVE_REGULATION_OF_PEPTIDASE_A } \\
\text { CTIVITY }\end{array}$ & 114 & -0.52 & -1.987 & 0 & 7.83E-04 & 190 \\
\hline
\end{tabular}




\begin{tabular}{|c|c|c|c|c|c|c|c|}
\hline 2 & $\begin{array}{l}\text { GO_REGULATION_OF_TOLL_LIKE_RECEPTOR_ } \\
\text { SIGNALING_PATHWAY }\end{array}$ & 36 & -0.651 & -1.98 & 0 & $8.46 \mathrm{E}-04$ & 191 \\
\hline 2 & $\begin{array}{l}\text { GO_NEGATIVE_REGULATION_OF_VIRAL_ENTR } \\
\text { Y_INTO_HOST_CELL }\end{array}$ & 15 & -0.79 & -1.978 & 0 & 8.73E-04 & 192 \\
\hline 2 & $\begin{array}{l}\text { GO_REGULATION_OF_ALPHA_BETA_T_CELL_A } \\
\text { CTIVATION }\end{array}$ & 52 & -0.613 & -1.974 & 0 & $9.26 \mathrm{E}-04$ & 193 \\
\hline 2 & $\begin{array}{l}\text { GO_REGULATION_OF_DEFENSE_RESPONSE_T } \\
\text { O_VIRUS }\end{array}$ & 136 & -0.505 & -1.974 & 0 & 9.27E-04 & 194 \\
\hline 2 & $\begin{array}{l}\text { GO_REGULATION_OF_INTERLEUKIN_6_PROD } \\
\text { UCTION }\end{array}$ & 74 & -0.555 & -1.967 & 0 & 0.001018 & 195 \\
\hline 2 & KEGG_AUTOIMMUNE_THYROID_DISEASE & 31 & -0.645 & -1.966 & 0 & 0.001031 & 196 \\
\hline 2 & GO_DEFENSE_RESPONSE_TO_BACTERIUM & 108 & -0.524 & -1.965 & 0 & 0.001041 & 197 \\
\hline 2 & $\begin{array}{l}\text { GO_NEGATIVE_REGULATION_OF_I_KAPPAB_K } \\
\text { INASE_NF_KAPPAB_SIGNALING }\end{array}$ & 40 & -0.626 & -1.959 & 0 & 0.001118 & 198 \\
\hline 2 & $\begin{array}{l}\text { GO_POSITIVE_REGULATION_OF_HEMOPOIESI } \\
\mathrm{S}\end{array}$ & 117 & -0.511 & -1.958 & 0 & 0.001125 & 199 \\
\hline 2 & $\begin{array}{l}\text { GO_REGULATION_OF_LYMPHOCYTE_MEDIATE } \\
\text { D_IMMUNITY }\end{array}$ & 78 & -0.549 & -1.955 & 0 & 0.001174 & 200 \\
\hline 2 & GO_ALPHA_BETA_T_CELL_DIFFERENTIATION & 34 & -0.627 & -1.953 & 0 & 0.001204 & 201 \\
\hline 2 & GO_LEUKOCYTE_ACTIVATION & 305 & -0.453 & -1.95 & 0 & 0.001246 & 202 \\
\hline 2 & GO_CYTOKINE_PRODUCTION & 87 & -0.525 & -1.938 & 0 & 0.00146 & 203 \\
\hline 2 & GO_NIK_NF_KAPPAB_SIGNALING & 75 & -0.537 & -1.935 & 0 & 0.001539 & 204 \\
\hline 2 & GO_MYELOID_DENDRITIC_CELL_ACTIVATION & 19 & -0.716 & -1.935 & 0 & 0.001542 & 205 \\
\hline 2 & $\begin{array}{l}\text { GO_TOLL_LIKE_RECEPTOR_SIGNALING_PATH } \\
\text { WAY }\end{array}$ & 73 & -0.545 & -1.933 & 0 & 0.001561 & 206 \\
\hline 2 & $\begin{array}{l}\text { GO_NEGATIVE_REGULATION_OF_NF_KAPPAB } \\
\text { _IMPORT_INTO_NUCLEUS }\end{array}$ & 15 & -0.786 & -1.932 & 0 & 0.00158 & 207 \\
\hline 2 & $\begin{array}{l}\text { GO_NEGATIVE_REGULATION_OF_RESPONSE_ } \\
\text { TO_EXTERNAL_STIMULUS }\end{array}$ & 179 & -0.478 & -1.928 & 0 & 0.001683 & 208 \\
\hline 2 & KEGG_LEISHMANIA_INFECTION & 58 & -0.563 & -1.925 & 0 & 0.001747 & 209 \\
\hline 2 & $\begin{array}{l}\text { GO_NEGATIVE_REGULATION_OF_INNATE_IM } \\
\text { MUNE_RESPONSE }\end{array}$ & 29 & -0.648 & -1.92 & 0 & 0.001876 & 210 \\
\hline 2 & GO_REGULATION_OF_CELL_KILLING & 44 & -0.594 & -1.916 & 0 & 0.001973 & 211 \\
\hline 2 & GO_PROTEASE_BINDING & 84 & -0.529 & -1.91 & 0 & 0.002096 & 212 \\
\hline 2 & $\begin{array}{l}\text { GO_NEGATIVE_REGULATION_OF_T_CELL_DIFF } \\
\text { ERENTIATION }\end{array}$ & 19 & -0.717 & -1.909 & 0 & 0.002108 & 213 \\
\hline 2 & GO_T_CELL_HOMEOSTASIS & 24 & -0.66 & -1.899 & 0 & 0.002347 & 214 \\
\hline
\end{tabular}




\begin{tabular}{|c|c|c|c|c|c|c|c|}
\hline 2 & $\begin{array}{l}\text { GO_REGULATION_OF_LEUKOCYTE_MEDIATED } \\
\text { _CYTOTOXICITY }\end{array}$ & 38 & -0.596 & -1.896 & 0 & 0.00242 & 215 \\
\hline 2 & $\begin{array}{l}\text { GO_POSITIVE_REGULATION_OF_ALPHA_BETA } \\
\text { _T_CELL_ACTIVATION }\end{array}$ & 38 & -0.61 & -1.893 & 0 & 0.002491 & 216 \\
\hline 2 & $\begin{array}{l}\text { GO_REGULATION_OF_T_CELL_MEDIATED_CYT } \\
\text { OTOXICITY }\end{array}$ & 20 & -0.683 & -1.891 & 0 & 0.002573 & 217 \\
\hline 2 & BIOCARTA_IL7_PATHWAY & 17 & -0.733 & -1.89 & 0 & 0.002595 & 218 \\
\hline 2 & $\begin{array}{l}\text { GO_POSITIVE_REGULATION_OF_ACTIVATED_T } \\
\text { _CELL_PROLIFERATION }\end{array}$ & 20 & -0.683 & -1.888 & 0 & 0.002645 & 219 \\
\hline 2 & $\begin{array}{l}\text { GO_POSITIVE_REGULATION_OF_B_CELL_ACTI } \\
\text { VATION }\end{array}$ & 53 & -0.567 & -1.887 & 0 & 0.002667 & 220 \\
\hline 2 & GO_CELLULAR_RESPONSE_TO_VIRUS & 19 & -0.702 & -1.885 & 0 & 0.002726 & 221 \\
\hline 2 & GO_ADAPTIVE_IMMUNE_RESPONSE & 182 & -0.47 & -1.884 & 0 & 0.002761 & 222 \\
\hline 2 & $\begin{array}{l}\text { GO_POSITIVE_REGULATION_OF_TUMOR_NEC } \\
\text { ROSIS_FACTOR_SUPERFAMILY_CYTOKINE_PR } \\
\text { ODUCTION }\end{array}$ & 47 & -0.58 & -1.883 & 0 & 0.002783 & 223 \\
\hline 2 & $\begin{array}{l}\text { GO_REGULATION_OF_ALPHA_BETA_T_CELL_P } \\
\text { ROLIFERATION }\end{array}$ & 18 & -0.714 & -1.872 & 0 & 0.003165 & 224 \\
\hline 2 & GO_T_HELPER_1_TYPE_IMMUNE_RESPONSE & 18 & -0.712 & -1.864 & 0 & 0.003465 & 225 \\
\hline 2 & GO_LEUKOCYTE_HOMEOSTASIS & 41 & -0.574 & -1.86 & 0 & 0.003604 & 226 \\
\hline 2 & $\begin{array}{l}\text { GO_POSITIVE_REGULATION_OF_ALPHA_BETA } \\
\text { _T_CELL_DIFFERENTIATION }\end{array}$ & 27 & -0.647 & -1.86 & 0 & 0.003614 & 227 \\
\hline 2 & $\begin{array}{l}\text { GO_NEGATIVE_REGULATION_OF_NF_KAPPAB } \\
\text { _TRANSCRIPTION_FACTOR_ACTIVITY }\end{array}$ & 49 & -0.553 & -1.856 & 0 & 0.003784 & 228 \\
\hline 2 & $\begin{array}{l}\text { GO_POSITIVE_REGULATION_OF_INTERLEUKIN } \\
\text { 2_PRODUCTION }\end{array}$ & 25 & -0.641 & -1.855 & 0 & 0.003839 & 229 \\
\hline 2 & GO_T_CELL_MEDIATED_IMMUNITY & 23 & -0.663 & -1.854 & 0 & 0.003865 & 230 \\
\hline 2 & $\begin{array}{l}\text { GO_POSITIVE_REGULATION_OF_LEUKOCYTE_ } \\
\text { DIFFERENTIATION }\end{array}$ & 98 & -0.494 & -1.853 & 0 & 0.003912 & 231 \\
\hline 2 & $\begin{array}{l}\text { GO_REGULATION_OF_TUMOR_NECROSIS_FAC } \\
\text { TOR_MEDIATED_SIGNALING_PATHWAY }\end{array}$ & 42 & -0.593 & -1.852 & 0 & 0.003949 & 232 \\
\hline 2 & $\begin{array}{l}\text { GO_REGULATION_OF_INTERLEUKIN_2_PROD } \\
\text { UCTION }\end{array}$ & 33 & -0.625 & -1.852 & 0 & 0.003951 & 233 \\
\hline 2 & GO_HUMORAL_IMMUNE_RESPONSE & 89 & -0.507 & -1.846 & 0 & 0.004235 & 234 \\
\hline 2 & GO_LEUKOCYTE_PROLIFERATION & 61 & -0.545 & -1.846 & 0 & 0.004237 & 235 \\
\hline 2 & $\begin{array}{l}\text { GO_CD4_POSITIVE_ALPHA_BETA_T_CELL_ACT } \\
\text { IVATION }\end{array}$ & 24 & -0.647 & -1.842 & 0 & 0.004413 & 236 \\
\hline 2 & GO_RESPONSE_TO_OXIDATIVE_STRESS & 270 & -0.431 & -1.829 & 0 & 0.005067 & 237 \\
\hline
\end{tabular}




\begin{tabular}{|c|c|c|c|c|c|c|c|}
\hline 2 & $\begin{array}{l}\text { GO_INNATE_IMMUNE_RESPONSE_ACTIVATIN } \\
\text { G_CELL_SURFACE_RECEPTOR_SIGNALING_PAT } \\
\text { HWAY }\end{array}$ & 93 & -0.504 & -1.826 & 0 & 0.005204 & 238 \\
\hline 2 & $\begin{array}{l}\text { KEGG_EPITHELIAL_CELL_SIGNALING_IN_HELIC } \\
\text { OBACTER_PYLORI_INFECTION }\end{array}$ & 55 & -0.527 & -1.822 & 0 & 0.005483 & 239 \\
\hline 2 & $\begin{array}{l}\text { GO_CELLULAR_RESPONSE_TO_OXIDATIVE_ST } \\
\text { RESS }\end{array}$ & 144 & -0.462 & -1.821 & 0 & 0.005514 & 240 \\
\hline 2 & GO_LEUKOCYTE_APOPTOTIC_PROCESS & 17 & -0.704 & -1.818 & 0 & 0.00566 & 241 \\
\hline 2 & GO_DEATH_RECEPTOR_BINDING & 16 & -0.725 & -1.814 & 0 & 0.005901 & 242 \\
\hline 2 & $\begin{array}{l}\text { GO_POSITIVE_REGULATION_OF_CYTOKINE_PR } \\
\text { ODUCTION_INVOLVED_IN_IMMUNE_RESPON } \\
\text { SE }\end{array}$ & 21 & -0.664 & -1.793 & 0 & 0.007316 & 243 \\
\hline 2 & GO_LYMPHOCYTE_ACTIVATION & 256 & -0.426 & -1.786 & 0 & 0.007746 & 244 \\
\hline 2 & $\begin{array}{l}\text { KEGG_NATURAL_KILLER_CELL_MEDIATED_CYT } \\
\text { OTOXICITY }\end{array}$ & 94 & -0.478 & -1.766 & 0 & 0.00936 & 245 \\
\hline 2 & $\begin{array}{l}\text { GO_POSITIVE_REGULATION_OF_LEUKOCYTE_ } \\
\text { MEDIATED_IMMUNITY }\end{array}$ & 59 & -0.517 & -1.766 & 0 & 0.009399 & 246 \\
\hline 2 & $\begin{array}{l}\text { GO_POSITIVE_REGULATION_OF_LYMPHOCYTE } \\
\text { _MEDIATED_IMMUNITY }\end{array}$ & 49 & -0.54 & -1.747 & 0 & 0.011171 & 247 \\
\hline 2 & $\begin{array}{l}\text { GO_ANTIGEN_PROCESSING_AND_PRESENTATI } \\
\text { ON_OF_EXOGENOUS_PEPTIDE_ANTIGEN_VIA } \\
\text { _MHC_CLASS_I }\end{array}$ & 59 & -0.51 & -1.747 & 0 & 0.011208 & 248 \\
\hline 2 & KEGG_SYSTEMIC_LUPUS_ERYTHEMATOSUS & 68 & -0.493 & -1.733 & 0 & 0.012666 & 249 \\
\hline 2 & GO_T_CELL_DIFFERENTIATION & 95 & -0.457 & -1.706 & 0 & 0.015975 & 250 \\
\hline 2 & $\begin{array}{l}\text { GO_SIGNAL_TRANSDUCTION_BY_PROTEIN_PH } \\
\text { OSPHORYLATION }\end{array}$ & 290 & -0.401 & -1.707 & 0 & 0.015977 & 251 \\
\hline 2 & $\begin{array}{l}\text { GO_IMMUNE_RESPONSE_REGULATING_CELL_- } \\
\text { SURFACE_RECEPTOR_SIGNALING_PATHWAY }\end{array}$ & 245 & -0.404 & -1.694 & 0 & 0.01754 & 252 \\
\hline 2 & GO_LYMPHOCYTE_COSTIMULATION & 54 & -0.501 & -1.688 & 0 & 0.018505 & 253 \\
\hline 2 & GO_LEUKOCYTE_DIFFERENTIATION & 214 & -0.397 & -1.649 & 0 & 0.024558 & 254 \\
\hline 2 & GO_IMMUNE_SYSTEM_DEVELOPMENT & 412 & -0.361 & -1.6 & 0 & 0.034551 & 255 \\
\hline 2 & $\begin{array}{l}\text { GO_ANTIGEN_PROCESSING_AND_PRESENTATI } \\
\text { ON_OF_PEPTIDE_ANTIGEN_VIA_MHC_CLASS_ } \\
\text { I }\end{array}$ & 79 & -0.484 & -1.755 & 0.001789 & 0.010414 & 256 \\
\hline 2 & $\begin{array}{l}\text { GO_CELL_ACTIVATION_INVOLVED_IN_IMMUN } \\
\text { E_RESPONSE }\end{array}$ & 97 & -0.454 & -1.685 & 0.001828 & 0.018881 & 257 \\
\hline 2 & KEGG_ALLOGRAFT_REJECTION & 29 & -0.676 & -2.003 & 0.001876 & $6.03 \mathrm{E}-04$ & 258 \\
\hline 2 & $\begin{array}{l}\text { GO_ANTIGEN_RECEPTOR_MEDIATED_SIGNALI } \\
\text { NG_PATHWAY }\end{array}$ & 145 & -0.391 & -1.546 & 0.001905 & 0.049072 & 259 \\
\hline
\end{tabular}




\begin{tabular}{|c|c|c|c|c|c|c|c|}
\hline 2 & $\begin{array}{l}\text { GO_REGULATION_OF_MYELOID_CELL_DIFFER } \\
\text { ENTIATION }\end{array}$ & 127 & -0.401 & -1.58 & 0.001912 & 0.039399 & 260 \\
\hline 2 & $\begin{array}{l}\text { GO_T_CELL_ACTIVATION_INVOLVED_IN_IMM } \\
\text { UNE_RESPONSE }\end{array}$ & 41 & -0.581 & -1.811 & 0.001923 & 0.006045 & 261 \\
\hline 2 & $\begin{array}{l}\text { GO_NEGATIVE_REGULATION_OF_LEUKOCYTE } \\
\text { _PROLIFERATION }\end{array}$ & 50 & -0.526 & -1.739 & 0.001972 & 0.012088 & 262 \\
\hline 2 & $\begin{array}{l}\text { GO_CELLULAR_RESPONSE_TO_HYDROGEN_PE } \\
\text { ROXIDE }\end{array}$ & 50 & -0.53 & -1.773 & 0.001984 & 0.00882 & 263 \\
\hline 2 & $\begin{array}{l}\text { GO_REGULATION_OF_DEFENSE_RESPONSE_T } \\
\text { O_VIRUS_BY_HOST }\end{array}$ & 86 & -0.505 & -1.858 & 0.001988 & 0.003693 & 264 \\
\hline 2 & $\begin{array}{l}\text { GO_REGULATION_OF_INTERLEUKIN_17_PROD } \\
\text { UCTION }\end{array}$ & 16 & -0.685 & -1.778 & 0.002004 & 0.008395 & 265 \\
\hline 2 & $\begin{array}{l}\text { GO_TUMOR_NECROSIS_FACTOR_RECEPTOR_B } \\
\text { INDING }\end{array}$ & 24 & -0.651 & -1.83 & 0.002008 & 0.005007 & 266 \\
\hline 2 & BIOCARTA_IL1R_PATHWAY & 29 & -0.667 & -1.96 & 0.002092 & 0.001102 & 267 \\
\hline 2 & $\begin{array}{l}\text { GO_T_CELL_DIFFERENTIATION_INVOLVED_IN_ } \\
\text { IMMUNE_RESPONSE }\end{array}$ & 24 & -0.671 & -1.894 & 0.002146 & 0.002478 & 268 \\
\hline 2 & $\begin{array}{l}\text { GO_REGULATION_OF_ANTIGEN_PROCESSING } \\
\text { _AND_PRESENTATION }\end{array}$ & 17 & -0.745 & -1.943 & 0.002151 & 0.001365 & 269 \\
\hline 2 & GO_MYELOID_CELL_DIFFERENTIATION & 150 & -0.384 & -1.532 & 0.003591 & 0.053952 & 270 \\
\hline 2 & GO_FC_RECEPTOR_SIGNALING_PATHWAY & 163 & -0.392 & -1.569 & 0.00363 & 0.042445 & 271 \\
\hline 2 & GO_LYMPHOCYTE_DIFFERENTIATION & 148 & -0.396 & -1.565 & 0.003759 & 0.043417 & 272 \\
\hline 2 & GO_T_CELL_RECEPTOR_SIGNALING_PATHWAY & 120 & -0.424 & -1.622 & 0.003774 & 0.029895 & 273 \\
\hline 2 & GO_CELLULAR_DEFENSE_RESPONSE & 37 & -0.534 & -1.686 & 0.003846 & 0.018776 & 274 \\
\hline 2 & GO_B_CELL_MEDIATED_IMMUNITY & 50 & -0.505 & -1.669 & 0.003906 & 0.021337 & 275 \\
\hline 2 & $\begin{array}{l}\text { GO_NEGATIVE_REGULATION_OF_T_CELL_PRO } \\
\text { LIFERATION }\end{array}$ & 36 & -0.562 & -1.756 & 0.003992 & 0.010269 & 276 \\
\hline 2 & $\begin{array}{l}\text { GO_POSITIVE_REGULATION_OF_ALPHA_BETA } \\
\text { _T_CELL_PROLIFERATION }\end{array}$ & 15 & -0.687 & -1.734 & 0.004 & 0.012579 & 277 \\
\hline 2 & $\begin{array}{l}\text { GO_NEGATIVE_REGULATION_OF_LEUKOCYTE } \\
\text { _MEDIATED_IMMUNITY }\end{array}$ & 36 & -0.561 & -1.742 & 0.004008 & 0.011764 & 278 \\
\hline 2 & BIOCARTA_IL2RB_PATHWAY & 37 & -0.567 & -1.763 & 0.00404 & 0.009725 & 279 \\
\hline 2 & $\begin{array}{l}\text { GO_REGULATION_OF_MONOCYTE_CHEMOTA } \\
\text { XIS }\end{array}$ & 17 & -0.682 & -1.787 & 0.004107 & 0.007741 & 280 \\
\hline 2 & $\begin{array}{l}\text { GO_REGULATION_OF_ACUTE_INFLAMMATOR } \\
\text { Y_RESPONSE }\end{array}$ & 49 & -0.52 & -1.7 & 0.004141 & 0.016838 & 281 \\
\hline 2 & $\begin{array}{l}\text { GO_NEGATIVE_REGULATION_OF_STAT_CASCA } \\
\text { DE }\end{array}$ & 24 & -0.624 & -1.78 & 0.004211 & 0.008207 & 282 \\
\hline 2 & BIOCARTA_TH1TH2_PATHWAY & 15 & -0.705 & -1.782 & 0.004255 & 0.008051 & 283 \\
\hline
\end{tabular}




\begin{tabular}{|c|c|c|c|c|c|c|c|}
\hline 2 & $\begin{array}{l}\text { GO_NEGATIVE_REGULATION_OF_CHEMOTAXI } \\
\mathrm{S}\end{array}$ & 35 & -0.539 & -1.667 & 0.004283 & 0.021637 & 284 \\
\hline 2 & $\begin{array}{l}\text { GO_NEGATIVE_REGULATION_OF_TUMOR_NE } \\
\text { CROSIS_FACTOR_SUPERFAMILY_CYTOKINE_PR } \\
\text { ODUCTION }\end{array}$ & 30 & -0.554 & -1.655 & 0.004338 & 0.023703 & 285 \\
\hline 2 & $\begin{array}{l}\text { GO_POSITIVE_REGULATION_OF_T_CELL_MEDI } \\
\text { ATED_IMMUNITY }\end{array}$ & 23 & -0.645 & -1.783 & 0.004357 & 0.007989 & 286 \\
\hline 2 & BIOCARTA_IL12_PATHWAY & 18 & -0.68 & -1.804 & 0.004415 & 0.00648 & 287 \\
\hline 2 & $\begin{array}{l}\text { GO_DEFENSE_RESPONSE_TO_GRAM_POSITIV } \\
\text { E_BACTERIUM }\end{array}$ & 44 & -0.538 & -1.7 & 0.005618 & 0.016772 & 288 \\
\hline 2 & $\begin{array}{l}\text { GO_REGULATION_OF_RESPONSE_TO_INTERFE } \\
\text { RON_GAMMA }\end{array}$ & 18 & -0.709 & -1.883 & 0.005814 & 0.002782 & 289 \\
\hline 2 & $\begin{array}{l}\text { GO_NEGATIVE_REGULATION_OF_LYMPHOCYT } \\
\text { E_MEDIATED_IMMUNITY }\end{array}$ & 26 & -0.602 & -1.726 & 0.005848 & 0.013541 & 290 \\
\hline 2 & $\begin{array}{l}\text { GO_NEGATIVE_REGULATION_OF_LEUKOCYTE } \\
\text { _APOPTOTIC_PROCESS }\end{array}$ & 33 & -0.574 & -1.729 & 0.005906 & 0.01323 & 291 \\
\hline 2 & GO_VIRUS_RECEPTOR_ACTIVITY & 55 & -0.476 & -1.629 & 0.005917 & 0.028479 & 292 \\
\hline 2 & KEGG_VIRAL_MYOCARDITIS & 50 & -0.481 & -1.575 & 0.006073 & 0.040746 & 293 \\
\hline 2 & KEGG_PRIMARY_IMMUNODEFICIENCY & 24 & -0.648 & -1.841 & 0.006356 & 0.004463 & 294 \\
\hline 2 & $\begin{array}{l}\text { GO_NEGATIVE_REGULATION_OF_DEFENSE_RE } \\
\text { SPONSE_TO_VIRUS }\end{array}$ & 15 & -0.726 & -1.845 & 0.006424 & 0.004275 & 295 \\
\hline 2 & KEGG_ASTHMA & 18 & -0.639 & -1.698 & 0.006479 & 0.016993 & 296 \\
\hline 2 & $\begin{array}{l}\text { GO_PRODUCTION_OF_MOLECULAR_MEDIATO } \\
\text { R_OF_IMMUNE_RESPONSE }\end{array}$ & 46 & -0.483 & -1.58 & 0.00813 & 0.039423 & 297 \\
\hline 2 & $\begin{array}{l}\text { GO_NEGATIVE_REGULATION_OF_LYMPHOCYT } \\
\text { E_DIFFERENTIATION }\end{array}$ & 26 & -0.581 & -1.705 & 0.00818 & 0.016138 & 298 \\
\hline 2 & $\begin{array}{l}\text { GO_POSITIVE_REGULATION_OF_TYROSINE_P } \\
\text { HOSPHORYLATION_OF_STAT3_PROTEIN }\end{array}$ & 24 & -0.634 & -1.784 & 0.008197 & 0.00792 & 299 \\
\hline 2 & GO_T_CELL_PROLIFERATION & 32 & -0.563 & -1.706 & 0.009785 & 0.016021 & 300 \\
\hline 2 & BIOCARTA_PDGF_PATHWAY & 31 & -0.555 & -1.688 & 0.009785 & 0.018499 & 301 \\
\hline 2 & $\begin{array}{l}\text { GO_POSITIVE_REGULATION_OF_IMMUNOGLO } \\
\text { BULIN_PRODUCTION }\end{array}$ & 23 & -0.613 & -1.733 & 0.009804 & 0.012665 & 302 \\
\hline 2 & $\begin{array}{l}\text { GO_REGULATION_OF_INTERLEUKIN_10_PROD } \\
\text { UCTION }\end{array}$ & 29 & -0.565 & -1.673 & 0.009862 & 0.02076 & 303 \\
\hline 2 & $\begin{array}{l}\text { GO_LYMPHOCYTE_ACTIVATION_INVOLVED_IN } \\
\text { _IMMUNE_RESPONSE }\end{array}$ & 70 & -0.435 & -1.541 & 0.00996 & 0.050828 & 304 \\
\hline 2 & GO_MHC_CLASS_I_PROTEIN_BINDING & 16 & -0.681 & -1.759 & 0.010267 & 0.010003 & 305 \\
\hline 2 & $\begin{array}{l}\text { GO_NEGATIVE_REGULATION_OF_ADAPTIVE_I } \\
\text { MMUNE_RESPONSE }\end{array}$ & 25 & -0.594 & -1.695 & 0.010616 & 0.017342 & 306 \\
\hline
\end{tabular}




\begin{tabular}{|c|c|c|c|c|c|c|c|}
\hline 2 & $\begin{array}{l}\text { GO_NEGATIVE_REGULATION_OF_LEUKOCYTE } \\
\text { _DIFFERENTIATION }\end{array}$ & 56 & -0.465 & -1.58 & 0.011765 & 0.039398 & 307 \\
\hline 2 & $\begin{array}{l}\text { GO_REGULATION_OF_MACROPHAGE_ACTIVA } \\
\text { TION }\end{array}$ & 16 & -0.693 & -1.755 & 0.01232 & 0.010427 & 308 \\
\hline 2 & GO_CYTOKINE_SECRETION & 26 & -0.566 & -1.612 & 0.012685 & 0.031936 & 309 \\
\hline 2 & $\begin{array}{l}\text { GO_REGULATION_OF_TRANSFORMING_GRO } \\
\text { WTH_FACTOR_BETA_PRODUCTION }\end{array}$ & 17 & -0.639 & -1.678 & 0.013043 & 0.019889 & 310 \\
\hline 2 & GO_MYELOID_LEUKOCYTE_DIFFERENTIATION & 74 & -0.427 & -1.515 & 0.013462 & 0.059152 & 311 \\
\hline 2 & $\begin{array}{l}\text { KEGG_ANTIGEN_PROCESSING_AND_PRESENT } \\
\text { ATION }\end{array}$ & 58 & -0.477 & -1.597 & 0.014344 & 0.035413 & 312 \\
\hline 2 & $\begin{array}{l}\text { GO_NEGATIVE_REGULATION_OF_CYTOKINE_P } \\
\text { RODUCTION_INVOLVED_IN_IMMUNE_RESPO } \\
\text { NSE }\end{array}$ & 17 & -0.658 & -1.7 & 0.014553 & 0.016824 & 313 \\
\hline 2 & $\begin{array}{l}\text { GO_INFLAMMATORY_RESPONSE_TO_ANTIGE } \\
\text { NIC_STIMULUS }\end{array}$ & 19 & -0.632 & -1.697 & 0.014614 & 0.01709 & 314 \\
\hline 2 & $\begin{array}{l}\text { GO_NEGATIVE_REGULATION_OF_INTERFERO } \\
\text { N_GAMMA_PRODUCTION }\end{array}$ & 25 & -0.595 & -1.689 & 0.014768 & 0.018238 & 315 \\
\hline 2 & $\begin{array}{l}\text { GO_REGULATION_OF_NATURAL_KILLER_CELL } \\
\text { _MEDIATED_IMMUNITY }\end{array}$ & 25 & -0.58 & -1.649 & 0.015152 & 0.024534 & 316 \\
\hline 2 & GO_LYMPHOCYTE_HOMEOSTASIS & 34 & -0.539 & -1.655 & 0.015595 & 0.02366 & 317 \\
\hline 2 & $\begin{array}{l}\text { GO_REGULATION_OF_ANTIGEN_RECEPTOR_ } \\
\text { MEDIATED_SIGNALING_PATHWAY }\end{array}$ & 33 & -0.537 & -1.636 & 0.016393 & 0.027255 & 318 \\
\hline 2 & GO_ACUTE_INFLAMMATORY_RESPONSE & 44 & -0.499 & -1.613 & 0.016563 & 0.031855 & 319 \\
\hline 2 & $\begin{array}{l}\text { GO_POSITIVE_REGULATION_OF_ACUTE_INFLA } \\
\text { MMATORY_RESPONSE }\end{array}$ & 20 & -0.615 & -1.686 & 0.016598 & 0.018744 & 320 \\
\hline 2 & GO_NATURAL_KILLER_CELL_ACTIVATION & 31 & -0.523 & -1.563 & 0.016771 & 0.04408 & 321 \\
\hline 2 & BIOCARTA_IL2_PATHWAY & 21 & -0.589 & -1.624 & 0.016807 & 0.029401 & 322 \\
\hline 2 & $\begin{array}{l}\text { GO_ANTIGEN_PROCESSING_AND_PRESENTATI } \\
\text { ON }\end{array}$ & 180 & -0.343 & -1.388 & 0.017361 & 0.12172 & 323 \\
\hline 2 & $\begin{array}{l}\text { GO_NEGATIVE_REGULATION_OF_LYMPHOCYT } \\
\text { E_APOPTOTIC_PROCESS }\end{array}$ & 19 & -0.609 & -1.636 & 0.018036 & 0.027277 & 324 \\
\hline 2 & $\begin{array}{l}\text { KEGG_T_CELL_RECEPTOR_SIGNALING_PATHW } \\
\text { AY }\end{array}$ & 88 & -0.392 & -1.452 & 0.018315 & 0.085867 & 325 \\
\hline 2 & $\begin{array}{l}\text { GO_MODULATION_BY_SYMBIONT_OF_HOST_ } \\
\text { CELLULAR_PROCESS }\end{array}$ & 25 & -0.57 & -1.611 & 0.018634 & 0.032196 & 326 \\
\hline 2 & $\begin{array}{l}\text { GO_POSITIVE_REGULATION_OF_RESPONSE_T } \\
\text { O_CYTOKINE_STIMULUS }\end{array}$ & 23 & -0.582 & -1.615 & 0.018828 & 0.031446 & 327 \\
\hline 2 & $\begin{array}{l}\text { GO_REGULATION_OF_IMMUNOGLOBULIN_PR } \\
\text { ODUCTION }\end{array}$ & 33 & -0.544 & -1.695 & 0.018908 & 0.017366 & 328 \\
\hline
\end{tabular}




\begin{tabular}{|c|c|c|c|c|c|c|c|}
\hline 2 & $\begin{array}{l}\text { GO_REGULATION_OF_T_CELL_RECEPTOR_SIG } \\
\text { NALING_PATHWAY }\end{array}$ & 24 & -0.589 & -1.657 & 0.018947 & 0.023329 & 329 \\
\hline 2 & GO_PHAGOCYTOSIS & 133 & -0.375 & -1.472 & 0.020599 & 0.076193 & 330 \\
\hline 2 & $\begin{array}{l}\text { GO_REGULATION_OF_HUMORAL_IMMUNE_R } \\
\text { ESPONSE }\end{array}$ & 25 & -0.548 & -1.554 & 0.02079 & 0.046786 & 331 \\
\hline 2 & GO_T_CELL_SELECTION & 27 & -0.565 & -1.667 & 0.021053 & 0.021709 & 332 \\
\hline 2 & $\begin{array}{l}\text { GO_POSITIVE_REGULATION_OF_MYELOID_CE } \\
\text { LL_DIFFERENTIATION }\end{array}$ & 59 & -0.433 & -1.488 & 0.021318 & 0.069289 & 333 \\
\hline 2 & $\begin{array}{l}\text { GO_NEGATIVE_REGULATION_OF_VIRAL_TRAN } \\
\text { SCRIPTION }\end{array}$ & 20 & -0.595 & -1.618 & 0.021552 & 0.030692 & 334 \\
\hline 2 & GO_IMMUNOGLOBULIN_PRODUCTION & 33 & -0.511 & -1.575 & 0.022495 & 0.040839 & 335 \\
\hline 2 & GO_RESPONSE_TO_INTERLEUKIN_6 & 21 & -0.588 & -1.608 & 0.023013 & 0.032824 & 336 \\
\hline 2 & $\begin{array}{l}\text { KEGG_FC_GAMMA_R_MEDIATED_PHAGOCYT } \\
\text { OSIS }\end{array}$ & 81 & -0.402 & -1.428 & 0.023762 & 0.098617 & 337 \\
\hline 2 & $\begin{array}{l}\text { GO_REGULATION_OF_MAST_CELL_ACTIVATIO } \\
\mathrm{N}\end{array}$ & 30 & -0.525 & -1.602 & 0.025157 & 0.034154 & 338 \\
\hline 2 & $\begin{array}{l}\text { GO_NEGATIVE_REGULATION_OF_PRODUCTIO } \\
\text { N_OF_MOLECULAR_MEDIATOR_OF_IMMUNE } \\
\text { _RESPONSE }\end{array}$ & 20 & -0.547 & -1.486 & 0.028761 & 0.070152 & 339 \\
\hline 2 & BIOCARTA_IL6_PATHWAY & 22 & -0.555 & -1.542 & 0.028866 & 0.050526 & 340 \\
\hline 2 & $\begin{array}{l}\text { GO_POSITIVE_REGULATION_OF_INTERLEUKIN } \\
\text { _10_PRODUCTION }\end{array}$ & 19 & -0.61 & -1.59 & 0.029821 & 0.036982 & 341 \\
\hline 2 & $\begin{array}{l}\text { GO_REGULATION_OF_NATURAL_KILLER_CELL } \\
\text { _ACTIVATION }\end{array}$ & 20 & -0.57 & -1.531 & 0.030501 & 0.054031 & 342 \\
\hline 2 & $\begin{array}{l}\text { KEGG_AMYOTROPHIC_LATERAL_SCLEROSIS_A } \\
\text { LS }\end{array}$ & 40 & -0.485 & -1.527 & 0.030801 & 0.055345 & 343 \\
\hline 2 & $\begin{array}{l}\text { GO_MYELOID_LEUKOCYTE_MEDIATED_IMMU } \\
\text { NITY }\end{array}$ & 29 & -0.527 & -1.545 & 0.031873 & 0.049365 & 344 \\
\hline 2 & $\begin{array}{l}\text { GO_REGULATION_OF_MYELOID_LEUKOCYTE_ } \\
\text { DIFFERENTIATION }\end{array}$ & 79 & -0.398 & -1.435 & 0.032015 & 0.094317 & 345 \\
\hline 2 & GO_RESPONSE_TO_FUNGUS & 29 & -0.51 & -1.526 & 0.032609 & 0.055742 & 346 \\
\hline 2 & GO_ACUTE_PHASE_RESPONSE & 25 & -0.553 & -1.569 & 0.032854 & 0.042422 & 347 \\
\hline 2 & GO_ANTIGEN_BINDING & 68 & -0.409 & -1.434 & 0.035433 & 0.094721 & 348 \\
\hline 2 & GO_B_CELL_ACTIVATION & 90 & -0.373 & -1.377 & 0.037244 & 0.128049 & 349 \\
\hline 2 & $\begin{array}{l}\text { GO_NEGATIVE_REGULATION_OF_CYTOKINE_B } \\
\text { IOSYNTHETIC_PROCESS }\end{array}$ & 19 & -0.586 & -1.548 & 0.037422 & 0.04836 & 350 \\
\hline 2 & $\begin{array}{l}\text { GO_REGULATION_OF_VIRAL_RELEASE_FROM } \\
\text { _HOST_CELL }\end{array}$ & 29 & -0.509 & -1.502 & 0.040323 & 0.06421 & 351 \\
\hline
\end{tabular}




\begin{tabular}{|c|c|c|c|c|c|c|c|}
\hline 2 & $\begin{array}{l}\text { GO_FC_EPSILON_RECEPTOR_SIGNALING_PAT } \\
\text { HWAY }\end{array}$ & 113 & -0.35 & -1.34 & 0.042308 & 0.153951 & 352 \\
\hline 2 & KEGG_ACUTE_MYELOID_LEUKEMIA & 52 & -0.441 & -1.483 & 0.043233 & 0.071687 & 353 \\
\hline 2 & $\begin{array}{l}\text { GO_MYELOID_CELL_ACTIVATION_INVOLVED_I } \\
\text { N_IMMUNE_RESPONSE }\end{array}$ & 27 & -0.513 & -1.506 & 0.045161 & 0.062688 & 354 \\
\hline 2 & $\begin{array}{l}\text { GO_NEGATIVE_REGULATION_OF_TOLL_LIKE_ } \\
\text { RECEPTOR_SIGNALING_PATHWAY }\end{array}$ & 18 & -0.607 & -1.589 & 0.045738 & 0.037342 & 355 \\
\hline 2 & $\begin{array}{l}\text { GO_REGULATION_OF_B_CELL_APOPTOTIC_PR } \\
\text { OCESS }\end{array}$ & 17 & -0.603 & -1.575 & 0.045929 & 0.040791 & 356 \\
\hline 2 & $\begin{array}{l}\text { GO_CELLULAR_RESPONSE_TO_INTERLEUKIN_ } \\
6\end{array}$ & 18 & -0.583 & -1.54 & 0.046122 & 0.05098 & 357 \\
\hline 2 & GO_CYTOKINE_BINDING & 66 & -0.405 & -1.422 & 0.047431 & 0.101685 & 358 \\
\hline 2 & GO_POSITIVE_REGULATION_OF_AUTOPHAGY & 59 & -0.418 & -1.412 & 0.04888 & 0.107323 & 359 \\
\hline 2 & $\begin{array}{l}\text { GO_POSITIVE_REGULATION_OF_B_CELL_MEDI } \\
\text { ATED_IMMUNITY }\end{array}$ & 20 & -0.559 & -1.5 & 0.048936 & 0.064608 & 360 \\
\hline 3 & $\begin{array}{l}\text { GO_REGULATION_OF_MULTI_ORGANISM_PR } \\
\text { OCESS }\end{array}$ & 343 & -0.544 & -2.363 & 0 & 0 & 1 \\
\hline 3 & $\begin{array}{l}\text { GO_REGULATION_OF_RESPONSE_TO_WOUN } \\
\text { DING }\end{array}$ & 262 & -0.568 & -2.4 & 0 & 0 & 2 \\
\hline 3 & $\begin{array}{l}\text { GO_REGULATION_OF_SYMBIOSIS_ENCOMPAS } \\
\text { SING_MUTUALISM_THROUGH_PARASITISM }\end{array}$ & 168 & -0.645 & -2.603 & 0 & 0 & 3 \\
\hline 3 & KEGG_APOPTOSIS & 75 & -0.66 & -2.348 & 0 & 0 & 4 \\
\hline 3 & $\begin{array}{l}\text { GO_POSITIVE_REGULATION_OF_SEQUENCE_S } \\
\text { PECIFIC_DNA_BINDING_TRANSCRIPTION_FAC } \\
\text { TOR_ACTIVITY }\end{array}$ & 167 & -0.558 & -2.246 & 0 & $2.22 \mathrm{E}-06$ & 5 \\
\hline 3 & $\begin{array}{l}\text { GO_POSITIVE_REGULATION_OF_RESPONSE_T } \\
\text { O_EXTERNAL_STIMULUS }\end{array}$ & 196 & -0.552 & -2.267 & 0 & $2.33 \mathrm{E}-06$ & 6 \\
\hline 3 & $\begin{array}{l}\text { GO_NEGATIVE_REGULATION_OF_SIGNAL_TRA } \\
\text { NSDUCTION_IN_ABSENCE_OF_LIGAND }\end{array}$ & 21 & -0.847 & -2.268 & 0 & $2.34 \mathrm{E}-06$ & 7 \\
\hline 3 & $\begin{array}{l}\text { GO_POSITIVE_REGULATION_OF_PEPTIDYL_TY } \\
\text { ROSINE_PHOSPHORYLATION }\end{array}$ & 105 & -0.583 & -2.231 & 0 & $6.39 \mathrm{E}-06$ & 8 \\
\hline 3 & $\begin{array}{l}\text { GO_EXTRINSIC_APOPTOTIC_SIGNALING_PATH } \\
\text { WAY }\end{array}$ & 81 & -0.618 & -2.233 & 0 & $6.42 \mathrm{E}-06$ & 9 \\
\hline 3 & $\begin{array}{l}\text { GO_REGULATION_OF_CYSTEINE_TYPE_ENDOP } \\
\text { EPTIDASE_ACTIVITY }\end{array}$ & 160 & -0.555 & -2.223 & 0 & $1.05 \mathrm{E}-05$ & 10 \\
\hline 3 & $\begin{array}{l}\text { GO_REGULATION_OF_PEPTIDYL_TYROSINE_P } \\
\text { HOSPHORYLATION }\end{array}$ & 147 & -0.562 & -2.225 & 0 & $1.06 \mathrm{E}-05$ & 11 \\
\hline 3 & GO_REGULATION_OF_CELL_CELL_ADHESION & 264 & -0.52 & -2.227 & 0 & 1.06E-05 & 12 \\
\hline 3 & BIOCARTA_DEATH_PATHWAY & 30 & -0.748 & -2.227 & 0 & $1.06 \mathrm{E}-05$ & 13 \\
\hline
\end{tabular}




\begin{tabular}{|c|c|c|c|c|c|c|c|}
\hline 3 & $\begin{array}{l}\text { GO_POSITIVE_REGULATION_OF_CELL_CELL_A } \\
\text { DHESION }\end{array}$ & 173 & -0.551 & -2.217 & 0 & $1.25 \mathrm{E}-05$ & 14 \\
\hline 3 & GO_REGULATION_OF_PEPTIDASE_ACTIVITY & 247 & -0.512 & -2.176 & 0 & 3.11E-05 & 15 \\
\hline 3 & $\begin{array}{l}\text { GO_REGULATION_OF_HOMOTYPIC_CELL_CELL } \\
\text { _ADHESION }\end{array}$ & 216 & -0.524 & -2.176 & 0 & $3.11 \mathrm{E}-05$ & 16 \\
\hline 3 & $\begin{array}{l}\text { GO_REGULATION_OF_EXTRINSIC_APOPTOTIC } \\
\text { _SIGNALING_PATHWAY_VIA_DEATH_DOMAIN } \\
\text { _RECEPTORS }\end{array}$ & 45 & -0.666 & -2.166 & 0 & 3.39E-05 & 17 \\
\hline 3 & $\begin{array}{l}\text { GO_POSITIVE_REGULATION_OF_ERK1_AND_E } \\
\text { RK2_CASCADE }\end{array}$ & 100 & -0.579 & -2.167 & 0 & $3.41 \mathrm{E}-05$ & 18 \\
\hline 3 & GO_RESPONSE_TO_EXOGENOUS_DSRNA & 26 & -0.759 & -2.158 & 0 & 3.67E-05 & 19 \\
\hline 3 & $\begin{array}{l}\text { GO_POSITIVE_REGULATION_OF_LOCOMOTIO } \\
\mathrm{N}\end{array}$ & 285 & -0.501 & -2.152 & 0 & 4.72E-05 & 20 \\
\hline 3 & GO_APOPTOTIC_SIGNALING_PATHWAY & 229 & -0.514 & -2.15 & 0 & $5.08 \mathrm{E}-05$ & 21 \\
\hline 3 & HALLMARK_APOPTOSIS & 149 & -0.545 & -2.147 & 0 & $5.25 \mathrm{E}-05$ & 22 \\
\hline 3 & $\begin{array}{l}\text { GO_ESTABLISHMENT_OF_ENDOTHELIAL_BARR } \\
\text { IER }\end{array}$ & 20 & -0.774 & -2.13 & 0 & $7.52 \mathrm{E}-05$ & 23 \\
\hline 3 & $\begin{array}{l}\text { GO_REGULATION_OF_APOPTOTIC_SIGNALING } \\
\text { _PATHWAY }\end{array}$ & 284 & -0.491 & -2.124 & 0 & $8.26 \mathrm{E}-05$ & 24 \\
\hline 3 & $\begin{array}{l}\text { GO_POSITIVE_REGULATION_OF_APOPTOTIC_ } \\
\text { SIGNALING_PATHWAY }\end{array}$ & 146 & -0.532 & -2.117 & 0 & $9.52 \mathrm{E}-05$ & 25 \\
\hline 3 & GO_RESPONSE_TO_VITAMIN_D & 27 & -0.722 & -2.105 & 0 & $1.09 \mathrm{E}-04$ & 26 \\
\hline 3 & GO_CELLULAR_RESPONSE_TO_HEAT & 24 & -0.73 & -2.103 & 0 & $1.18 \mathrm{E}-04$ & 27 \\
\hline 3 & GO_RESPONSE_TO_DSRNA & 49 & -0.626 & -2.096 & 0 & $1.38 \mathrm{E}-04$ & 28 \\
\hline 3 & $\begin{array}{l}\text { GO_ACTIVATION_OF_CYSTEINE_TYPE_ENDOP } \\
\text { EPTIDASE_ACTIVITY }\end{array}$ & 74 & -0.589 & -2.094 & 0 & $1.54 \mathrm{E}-04$ & 29 \\
\hline 3 & GO_ENDOTHELIAL_CELL_DEVELOPMENT & 31 & -0.705 & -2.091 & 0 & 1.66E-04 & 30 \\
\hline 3 & GO_NECROPTOTIC_PROCESS & 21 & -0.761 & -2.085 & 0 & $1.91 \mathrm{E}-04$ & 31 \\
\hline 3 & GO_PROTEIN_TRIMERIZATION & 29 & -0.693 & -2.079 & 0 & 1.97E-04 & 32 \\
\hline 3 & $\begin{array}{l}\text { GO_NEGATIVE_REGULATION_OF_RESPONSE_ } \\
\text { TO_WOUNDING }\end{array}$ & 100 & -0.546 & -2.069 & 0 & $2.40 \mathrm{E}-04$ & 33 \\
\hline 3 & GO_KERATINOCYTE_DIFFERENTIATION & 42 & -0.635 & -2.067 & 0 & $2.45 \mathrm{E}-04$ & 34 \\
\hline 3 & GO_NECROTIC_CELL_DEATH & 27 & -0.71 & -2.061 & 0 & $2.69 \mathrm{E}-04$ & 35 \\
\hline 3 & $\begin{array}{l}\text { GO_POSITIVE_REGULATION_OF_CYSTEINE_TY } \\
\text { PE_ENDOPEPTIDASE_ACTIVITY_INVOLVED_IN_ } \\
\text { APOPTOTIC_SIGNALING_PATHWAY }\end{array}$ & 16 & -0.788 & -2.057 & 0 & 2.89E-04 & 36 \\
\hline 3 & $\begin{array}{l}\text { GO_REGULATION_OF_VASCULATURE_DEVELO } \\
\text { PMENT }\end{array}$ & 149 & -0.509 & -2.05 & 0 & $3.19 \mathrm{E}-04$ & 37 \\
\hline
\end{tabular}




\begin{tabular}{|c|c|c|c|c|c|c|c|}
\hline 3 & $\begin{array}{l}\text { HALLMARK_EPITHELIAL_MESENCHYMAL_TRA } \\
\text { NSITION }\end{array}$ & 141 & -0.521 & -2.046 & 0 & 3.34E-04 & 38 \\
\hline 3 & $\begin{array}{l}\text { GO_REGULATION_OF_ENDOTHELIAL_CELL_AP } \\
\text { OPTOTIC_PROCESS }\end{array}$ & 22 & -0.746 & -2.036 & 0 & $3.64 \mathrm{E}-04$ & 39 \\
\hline 3 & $\begin{array}{l}\text { GO_POSITIVE_REGULATION_OF_CELL_ADHESI } \\
\text { ON }\end{array}$ & 263 & -0.48 & -2.032 & 0 & 3.83E-04 & 40 \\
\hline 3 & $\begin{array}{l}\text { GO_NEGATIVE_REGULATION_OF_CYSTEINE_T } \\
\text { YPE_ENDOPEPTIDASE_ACTIVITY }\end{array}$ & 65 & -0.572 & -2.03 & 0 & 3.90E-04 & 41 \\
\hline 3 & GO_REGULATION_OF_PROTEIN_SECRETION & 253 & -0.476 & -2.023 & 0 & $4.52 \mathrm{E}-04$ & 42 \\
\hline 3 & GO_REGULATION_OF_CELL_ADHESION & 438 & -0.452 & -2.014 & 0 & 5.17E-04 & 43 \\
\hline 3 & $\begin{array}{l}\text { GO_POSITIVE_REGULATION_OF_TRANSCRIPTI } \\
\text { ON_FACTOR_IMPORT_INTO_NUCLEUS }\end{array}$ & 41 & -0.636 & -2.007 & 0 & $5.71 \mathrm{E}-04$ & 44 \\
\hline 3 & $\begin{array}{l}\text { GO_NEGATIVE_REGULATION_OF_CELLULAR_R } \\
\text { ESPONSE_TO_INSULIN_STIMULUS }\end{array}$ & 22 & -0.728 & -2.007 & 0 & 5.76E-04 & 45 \\
\hline 3 & GO_ENDOTHELIAL_CELL_DIFFERENTIATION & 46 & -0.62 & -2.004 & 0 & 5.99E-04 & 46 \\
\hline 3 & $\begin{array}{l}\text { GO_REGULATION_OF_CELLULAR_RESPONSE_T } \\
\text { O_INSULIN_STIMULUS }\end{array}$ & 38 & -0.634 & -2 & 0 & $6.30 \mathrm{E}-04$ & 47 \\
\hline 3 & GO_SIDE_OF_MEMBRANE & 271 & -0.472 & -1.997 & 0 & $6.63 \mathrm{E}-04$ & 48 \\
\hline 3 & GO_CELL_CELL_ADHESION & 353 & -0.455 & -1.991 & 0 & 7.17E-04 & 49 \\
\hline 3 & $\begin{array}{l}\text { GO_NEGATIVE_REGULATION_OF_CYTOKINE_S } \\
\text { ECRETION }\end{array}$ & 26 & -0.689 & -1.989 & 0 & $7.51 \mathrm{E}-04$ & 50 \\
\hline 3 & GO_LEUKOCYTE_MIGRATION & 180 & -0.49 & -1.987 & 0 & 7.84E-04 & 51 \\
\hline 3 & $\begin{array}{l}\text { GO_EXTRINSIC_APOPTOTIC_SIGNALING_PATH } \\
\text { WAY_VIA_DEATH_DOMAIN_RECEPTORS }\end{array}$ & 36 & -0.645 & -1.985 & 0 & 7.95E-04 & 52 \\
\hline 3 & GO_SINGLE_ORGANISM_CELL_ADHESION & 314 & -0.456 & -1.979 & 0 & $8.61 \mathrm{E}-04$ & 53 \\
\hline 3 & $\begin{array}{l}\text { GO_REGULATION_OF_TRANSCRIPTION_FACTO } \\
\text { R_IMPORT_INTO_NUCLEUS }\end{array}$ & 77 & -0.551 & -1.97 & 0 & $9.69 \mathrm{E}-04$ & 54 \\
\hline 3 & $\begin{array}{l}\text { GO_REGULATION_OF_SEQUENCE_SPECIFIC_D } \\
\text { NA_BINDING_TRANSCRIPTION_FACTOR_ACTIV } \\
\text { ITY }\end{array}$ & 263 & -0.461 & -1.964 & 0 & 0.001045 & 55 \\
\hline 3 & $\begin{array}{l}\text { GO_POSITIVE_REGULATION_OF_CARTILAGE_D } \\
\text { EVELOPMENT }\end{array}$ & 17 & -0.753 & -1.963 & 0 & 0.001063 & 56 \\
\hline 3 & GO_DEATH_RECEPTOR_ACTIVITY & 17 & -0.749 & -1.958 & 0 & 0.001131 & 57 \\
\hline 3 & $\begin{array}{l}\text { GO_REGULATION_OF_RESPONSE_TO_BIOTIC_ } \\
\text { STIMULUS }\end{array}$ & 165 & -0.484 & -1.957 & 0 & 0.001142 & 58 \\
\hline 3 & GO_PROTEIN_HETEROOLIGOMERIZATION & 68 & -0.558 & -1.955 & 0 & 0.001177 & 59 \\
\hline 3 & $\begin{array}{l}\text { GO_POSITIVE_REGULATION_OF_INTRINSIC_A } \\
\text { POPTOTIC_SIGNALING_PATHWAY }\end{array}$ & 46 & -0.6 & -1.952 & 0 & 0.001211 & 60 \\
\hline
\end{tabular}




\begin{tabular}{|c|c|c|c|c|c|c|c|}
\hline 3 & $\begin{array}{l}\text { GO_POSITIVE_REGULATION_OF_STRESS_ACTI } \\
\text { VATED_PROTEIN_KINASE_SIGNALING_CASCAD } \\
\text { E }\end{array}$ & 98 & -0.52 & -1.95 & 0 & 0.001256 & 61 \\
\hline 3 & $\begin{array}{l}\text { GO_POSITIVE_REGULATION_OF_PROTEIN_SEC } \\
\text { RETION }\end{array}$ & 140 & -0.5 & -1.948 & 0 & 0.001293 & 62 \\
\hline 3 & HALLMARK_KRAS_SIGNALING_UP & 145 & -0.494 & -1.946 & 0 & 0.001322 & 63 \\
\hline 3 & GO_CELL_SURFACE & 447 & -0.434 & -1.942 & 0 & 0.001388 & 64 \\
\hline 3 & $\begin{array}{l}\text { GO_NEGATIVE_REGULATION_OF_PEPTIDASE_ } \\
\text { ACTIVITY }\end{array}$ & 136 & -0.491 & -1.936 & 0 & 0.001521 & 65 \\
\hline 3 & KEGG_CELL_ADHESION_MOLECULES_CAMS & 82 & -0.539 & -1.936 & 0 & 0.001523 & 66 \\
\hline 3 & KEGG_PROTEASOME & 41 & -0.598 & -1.932 & 0 & 0.001601 & 67 \\
\hline 3 & $\begin{array}{l}\text { GO_NEGATIVE_REGULATION_OF_APOPTOTIC_ } \\
\text { SIGNALING_PATHWAY }\end{array}$ & 152 & -0.483 & -1.924 & 0 & 0.001769 & 68 \\
\hline 3 & GO_CELL_ACTIVATION & 409 & -0.434 & -1.922 & 0 & 0.001843 & 69 \\
\hline 3 & $\begin{array}{l}\text { GO_POSITIVE_REGULATION_OF_PROTEIN_IM } \\
\text { PORT }\end{array}$ & 77 & -0.537 & -1.913 & 0 & 0.002039 & 70 \\
\hline 3 & GO_POSITIVE_REGULATION_OF_CELL_DEATH & 462 & -0.425 & -1.911 & 0 & 0.002071 & 71 \\
\hline 3 & GO_NEGATIVE_REGULATION_OF_GROWTH & 160 & -0.475 & -1.909 & 0 & 0.002108 & 72 \\
\hline 3 & KEGG_TYPE_I_DIABETES_MELLITUS & 34 & -0.615 & -1.909 & 0 & 0.002117 & 73 \\
\hline 3 & BIOCARTA_RELA_PATHWAY & 16 & -0.745 & -1.908 & 0 & 0.002147 & 74 \\
\hline 3 & $\begin{array}{l}\text { GO_MODIFICATION_BY_SYMBIONT_OF_HOST } \\
\text { _MORPHOLOGY_OR_PHYSIOLOGY }\end{array}$ & 38 & -0.595 & -1.9 & 0 & 0.002326 & 75 \\
\hline 3 & GO_RESPONSE_TO_ESTROGEN & 142 & -0.478 & -1.888 & 0 & 0.002645 & 76 \\
\hline 3 & $\begin{array}{l}\text { GO_NEGATIVE_REGULATION_OF_PROTEIN_SE } \\
\text { CRETION }\end{array}$ & 63 & -0.552 & -1.887 & 0 & 0.002665 & 77 \\
\hline 3 & GO_RESPONSE_TO_ALCOHOL & 241 & -0.448 & -1.887 & 0 & 0.002673 & 78 \\
\hline 3 & GO_REGULATION_OF_PEPTIDE_TRANSPORT & 161 & -0.464 & -1.877 & 0 & 0.00302 & 79 \\
\hline 3 & GO_RESPONSE_TO_HEAT & 63 & -0.537 & -1.876 & 0 & 0.003035 & 80 \\
\hline 3 & $\begin{array}{l}\text { GO_MODIFICATION_OF_MORPHOLOGY_OR_P } \\
\text { HYSIOLOGY_OF_OTHER_ORGANISM }\end{array}$ & 71 & -0.536 & -1.875 & 0 & 0.003057 & 81 \\
\hline 3 & GO_GLAND_MORPHOGENESIS & 60 & -0.548 & -1.869 & 0 & 0.003293 & 82 \\
\hline 3 & $\begin{array}{l}\text { GO_NEGATIVE_REGULATION_OF_EPITHELIAL_ } \\
\text { CELL_APOPTOTIC_PROCESS }\end{array}$ & 20 & -0.695 & -1.868 & 0 & 0.003297 & 83 \\
\hline 3 & $\begin{array}{l}\text { GO_HYDROLASE_ACTIVITY_ACTING_ON_CARB } \\
\text { ON_NITROGEN_BUT_NOT_PEPTIDE_BONDS_I } \\
\text { N_CYCLIC_AMIDINES }\end{array}$ & 27 & -0.639 & -1.863 & 0 & 0.003469 & 84 \\
\hline
\end{tabular}




\begin{tabular}{|c|c|c|c|c|c|c|c|}
\hline 3 & $\begin{array}{l}\text { GO_REGULATION_OF_NUCLEOCYTOPLASMIC_ } \\
\text { TRANSPORT }\end{array}$ & 177 & -0.464 & -1.862 & 0 & 0.00355 & 85 \\
\hline 3 & BIOCARTA_NTHI_PATHWAY & 22 & -0.665 & -1.857 & 0 & 0.003762 & 86 \\
\hline 3 & $\begin{array}{l}\text { GO_POSITIVE_REGULATION_OF_LAMELLIPODI } \\
\text { UM_ORGANIZATION }\end{array}$ & 15 & -0.724 & -1.856 & 0 & 0.003806 & 87 \\
\hline 3 & $\begin{array}{l}\text { GO_NUCLEOTIDE_BINDING_DOMAIN_LEUCIN } \\
\text { E_RICH_REPEAT_CONTAINING_RECEPTOR_SIG } \\
\text { NALING_PATHWAY }\end{array}$ & 22 & -0.663 & -1.855 & 0 & 0.003852 & 88 \\
\hline 3 & $\begin{array}{l}\text { GO_REGULATION_OF_MITOCHONDRIAL_OUT } \\
\text { ER_MEMBRANE_PERMEABILIZATION_INVOLV } \\
\text { ED_IN_APOPTOTIC_SIGNALING_PATHWAY }\end{array}$ & 37 & -0.605 & -1.854 & 0 & 0.003884 & 89 \\
\hline 3 & $\begin{array}{l}\text { GO_POSITIVE_REGULATION_OF_CELL_PROLIF } \\
\text { ERATION }\end{array}$ & 489 & -0.411 & -1.848 & 0 & 0.004111 & 90 \\
\hline 3 & $\begin{array}{l}\text { GO_POSITIVE_REGULATION_OF_MITOCHOND } \\
\text { RIAL_OUTER_MEMBRANE_PERMEABILIZATIO } \\
\text { N_INVOLVED_IN_APOPTOTIC_SIGNALING_PAT } \\
\text { HWAY }\end{array}$ & 33 & -0.592 & -1.847 & 0 & 0.004174 & 91 \\
\hline 3 & $\begin{array}{l}\text { GO_POSITIVE_REGULATION_OF_NUCLEOCYTO } \\
\text { PLASMIC_TRANSPORT }\end{array}$ & 94 & -0.492 & -1.845 & 0 & 0.004265 & 92 \\
\hline 3 & GO_POSITIVE_REGULATION_OF_SECRETION & 224 & -0.437 & -1.844 & 0 & 0.004312 & 93 \\
\hline 3 & $\begin{array}{l}\text { GO_NEGATIVE_REGULATION_OF_ENDOTHELI } \\
\text { AL_CELL_APOPTOTIC_PROCESS }\end{array}$ & 15 & -0.742 & -1.843 & 0 & 0.004373 & 94 \\
\hline 3 & $\begin{array}{l}\text { GO_REGULATION_OF_INTRACELLULAR_TRANS } \\
\text { PORT }\end{array}$ & 448 & -0.412 & -1.842 & 0 & 0.004416 & 95 \\
\hline 3 & $\begin{array}{l}\text { GO_POSITIVE_REGULATION_OF_ESTABLISHM } \\
\text { ENT_OF_PROTEIN_LOCALIZATION }\end{array}$ & 359 & -0.42 & -1.841 & 0 & 0.004486 & 96 \\
\hline 3 & BIOCARTA_BIOPEPTIDES_PATHWAY & 34 & -0.604 & -1.835 & 0 & 0.004792 & 97 \\
\hline 3 & GO_MOLTING_CYCLE & 38 & -0.58 & -1.833 & 0 & 0.004875 & 98 \\
\hline 3 & GO_RESPONSE_TO_ESTRADIOL & 93 & -0.496 & -1.833 & 0 & 0.004917 & 99 \\
\hline 3 & $\begin{array}{l}\text { GO_POSITIVE_REGULATION_OF_VASCULATUR } \\
\text { E_DEVELOPMENT }\end{array}$ & 86 & -0.507 & -1.831 & 0 & 0.00496 & 100 \\
\hline 3 & GO_RESPONSE_TO_VITAMIN & 82 & -0.506 & -1.831 & 0 & 0.004978 & 101 \\
\hline 3 & $\begin{array}{l}\text { GO_NEGATIVE_REGULATION_OF_PROTEOLYSI } \\
\mathrm{S}\end{array}$ & 204 & -0.442 & -1.827 & 0 & 0.005171 & 102 \\
\hline 3 & GO_CELL_CELL_SIGNALING & 363 & -0.413 & -1.827 & 0 & 0.005177 & 103 \\
\hline 3 & GO_REGULATION_OF_PROTEIN_IMPORT & 143 & -0.463 & -1.827 & 0 & 0.00518 & 104 \\
\hline 3 & GO_HEPARIN_BINDING & 77 & -0.508 & -1.823 & 0 & 0.005405 & 105 \\
\hline 3 & GO GROWTH FACTOR RECEPTOR BINDING & 79 & -0.512 & -1.822 & 0 & 0.005436 & 106 \\
\hline
\end{tabular}




\begin{tabular}{|c|c|c|c|c|c|c|c|}
\hline 3 & $\begin{array}{l}\text { GO_REGULATION_OF_MEMBRANE_PERMEABI } \\
\text { LITY }\end{array}$ & 57 & -0.534 & -1.813 & 0 & 0.005948 & 107 \\
\hline 3 & $\begin{array}{l}\text { GO_REGULATION_OF_ENDOPLASMIC_RETICU } \\
\text { LUM_UNFOLDED_PROTEIN_RESPONSE }\end{array}$ & 24 & -0.639 & -1.811 & 0 & 0.00603 & 108 \\
\hline 3 & BIOCARTA_PROTEASOME_PATHWAY & 26 & -0.617 & -1.805 & 0 & 0.006437 & 109 \\
\hline 3 & $\begin{array}{l}\text { GO_CELLULAR_RESPONSE_TO_MECHANICAL_ } \\
\text { STIMULUS }\end{array}$ & 62 & -0.52 & -1.799 & 0 & 0.006855 & 110 \\
\hline 3 & GO_RESPONSE_TO_DRUG & 304 & -0.414 & -1.797 & 0 & 0.006985 & 111 \\
\hline 3 & $\begin{array}{l}\text { GO_POSITIVE_REGULATION_OF_PROTEIN_OLI } \\
\text { GOMERIZATION }\end{array}$ & 18 & -0.678 & -1.791 & 0 & 0.007472 & 112 \\
\hline 3 & GO_REGULATION_OF_SECRETION & 419 & -0.403 & -1.79 & 0 & 0.007508 & 113 \\
\hline 3 & GO_RESPONSE_TO_ACID_CHEMICAL & 205 & -0.432 & -1.785 & 0 & 0.007798 & 114 \\
\hline 3 & $\begin{array}{l}\text { GO_POSITIVE_REGULATION_OF_INTRACELLUL } \\
\text { AR_TRANSPORT }\end{array}$ & 268 & -0.417 & -1.78 & 0 & 0.008219 & 115 \\
\hline 3 & $\begin{array}{l}\text { GO_NEGATIVE_REGULATION_OF_CELL_ADHES } \\
\text { ION }\end{array}$ & 160 & -0.444 & -1.779 & 0 & 0.008305 & 116 \\
\hline 3 & GO_SKIN_DEVELOPMENT & 104 & -0.47 & -1.774 & 0 & 0.008683 & 117 \\
\hline 3 & GO_RESPONSE_TO_STEROID_HORMONE & 308 & -0.408 & -1.768 & 0 & 0.009263 & 118 \\
\hline 3 & $\begin{array}{l}\text { GO_REGULATION_OF_CYTOPLASMIC_TRANSP } \\
\text { ORT }\end{array}$ & 352 & -0.4 & -1.765 & 0 & 0.00947 & 119 \\
\hline 3 & GO_BLOOD_MICROPARTICLE & 65 & -0.506 & -1.759 & 0 & 0.010068 & 120 \\
\hline 3 & GO_RESPONSE_TO_TOXIC_SUBSTANCE & 168 & -0.436 & -1.756 & 0 & 0.010264 & 121 \\
\hline 3 & GO_GLYCOSAMINOGLYCAN_BINDING & 104 & -0.466 & -1.755 & 0 & 0.010412 & 122 \\
\hline 3 & HALLMARK_UV_RESPONSE_UP & 136 & -0.444 & -1.75 & 0 & 0.010862 & 123 \\
\hline 3 & $\begin{array}{l}\text { GO_CELLULAR_RESPONSE_TO_EXTERNAL_STI } \\
\text { MULUS }\end{array}$ & 198 & -0.429 & -1.747 & 0 & 0.011155 & 124 \\
\hline 3 & GO_REGULATION_OF_PROTEOLYSIS & 495 & -0.389 & -1.747 & 0 & 0.01121 & 125 \\
\hline 3 & GO_RESPONSE_TO_MECHANICAL_STIMULUS & 142 & -0.445 & -1.741 & 0 & 0.011806 & 126 \\
\hline 3 & GO_RESPONSE_TO_OSMOTIC_STRESS & 48 & -0.522 & -1.74 & 0 & 0.011893 & 127 \\
\hline 3 & GO_PROTEIN_MATURATION & 182 & -0.425 & -1.733 & 0 & 0.012725 & 128 \\
\hline 3 & $\begin{array}{l}\text { GO_REGULATION_OF_CELLULAR_AMINO_ACI } \\
\text { D_METABOLIC_PROCESS }\end{array}$ & 55 & -0.515 & -1.725 & 0 & 0.01364 & 129 \\
\hline 3 & $\begin{array}{l}\text { GO_REGULATION_OF_INTRINSIC_APOPTOTIC_ } \\
\text { SIGNALING_PATHWAY }\end{array}$ & 117 & -0.452 & -1.723 & 0 & 0.013877 & 130 \\
\hline 3 & $\begin{array}{l}\text { GO_INTRACELLULAR_RECEPTOR_SIGNALING_ } \\
\text { PATHWAY }\end{array}$ & 114 & -0.457 & -1.722 & 0 & 0.013982 & 131 \\
\hline
\end{tabular}




\begin{tabular}{|c|c|c|c|c|c|c|c|}
\hline 3 & $\begin{array}{l}\text { GO_G_PROTEIN_COUPLED_RECEPTOR_SIGNA } \\
\text { LING_PATHWAY }\end{array}$ & 347 & -0.395 & -1.719 & 0 & 0.014328 & 132 \\
\hline 3 & $\begin{array}{l}\text { GO_NEGATIVE_REGULATION_OF_ESTABLISHM } \\
\text { ENT_OF_PROTEIN_LOCALIZATION }\end{array}$ & 145 & -0.438 & -1.716 & 0 & 0.014695 & 133 \\
\hline 3 & $\begin{array}{l}\text { GO_NEGATIVE_REGULATION_OF_LOCOMOTIO } \\
\mathrm{N}\end{array}$ & 184 & -0.418 & -1.714 & 0 & 0.015098 & 134 \\
\hline 3 & GO_PEPTIDASE_INHIBITOR_ACTIVITY & 82 & -0.469 & -1.712 & 0 & 0.015273 & 135 \\
\hline 3 & GO_EPITHELIAL_CELL_DEVELOPMENT & 115 & -0.452 & -1.711 & 0 & 0.015415 & 136 \\
\hline 3 & GO_TAXIS & 286 & -0.401 & -1.711 & 0 & 0.01547 & 137 \\
\hline 3 & $\begin{array}{l}\text { GO_NEGATIVE_REGULATION_OF_CELL_ACTIV } \\
\text { ATION }\end{array}$ & 117 & -0.445 & -1.71 & 0 & 0.015546 & 138 \\
\hline 3 & GO_PEPTIDASE_REGULATOR_ACTIVITY & 113 & -0.453 & -1.707 & 0 & 0.015907 & 139 \\
\hline 3 & $\begin{array}{l}\text { GO_NEGATIVE_REGULATION_OF_CELL_PROLI } \\
\text { FERATION }\end{array}$ & 438 & -0.383 & -1.704 & 0 & 0.01624 & 140 \\
\hline 3 & GO_RESPONSE_TO_INORGANIC_SUBSTANCE & 356 & -0.388 & -1.699 & 0 & 0.016916 & 141 \\
\hline 3 & $\begin{array}{l}\text { GO_REGULATION_OF_MUSCLE_CONTRACTIO } \\
\mathrm{N}\end{array}$ & 87 & -0.463 & -1.686 & 0 & 0.018764 & 142 \\
\hline 3 & GO_REGULATION_OF_PROTEIN_BINDING & 130 & -0.431 & -1.685 & 0 & 0.018916 & 143 \\
\hline 3 & KEGG_PATHWAYS_IN_CANCER & 245 & -0.401 & -1.675 & 0 & 0.02045 & 144 \\
\hline 3 & $\begin{array}{l}\text { GO_POSITIVE_REGULATION_OF_KINASE_ACTI } \\
\text { VITY }\end{array}$ & 343 & -0.381 & -1.671 & 0 & 0.020936 & 145 \\
\hline 3 & GO_GROWTH_FACTOR_BINDING & 82 & -0.461 & -1.667 & 0 & 0.021634 & 146 \\
\hline 3 & $\begin{array}{l}\text { GO_VASCULAR_PROCESS_IN_CIRCULATORY_S } \\
\text { YSTEM }\end{array}$ & 82 & -0.457 & -1.661 & 0 & 0.02261 & 147 \\
\hline 3 & GO_NEGATIVE_REGULATION_OF_SECRETION & 118 & -0.432 & -1.659 & 0 & 0.023006 & 148 \\
\hline 3 & $\begin{array}{l}\text { GO_REGULATION_OF_CELLULAR_PROTEIN_LO } \\
\text { CALIZATION }\end{array}$ & 421 & -0.375 & -1.657 & 0 & 0.023317 & 149 \\
\hline 3 & GO_AGING & 199 & -0.406 & -1.657 & 0 & 0.023408 & 150 \\
\hline 3 & GO_REGULATION_OF_CELL_SHAPE & 100 & -0.442 & -1.655 & 0 & 0.023742 & 151 \\
\hline 3 & $\begin{array}{l}\text { GO_POSITIVE_REGULATION_OF_PROTEIN_SER } \\
\text { INE_THREONINE_KINASE_ACTIVITY }\end{array}$ & 204 & -0.399 & -1.653 & 0 & 0.023938 & 152 \\
\hline 3 & $\begin{array}{l}\text { GO_NEGATIVE_REGULATION_OF_INTRACELLU } \\
\text { LAR_TRANSPORT }\end{array}$ & 107 & -0.436 & -1.653 & 0 & 0.024001 & 153 \\
\hline 3 & $\begin{array}{l}\text { GO_DIVALENT_INORGANIC_CATION_HOMEOS } \\
\text { TASIS }\end{array}$ & 202 & -0.4 & -1.647 & 0 & 0.024963 & 154 \\
\hline 3 & $\begin{array}{l}\text { GO_REGULATION_OF_INTRACELLULAR_PROTE } \\
\text { IN_TRANSPORT }\end{array}$ & 282 & -0.381 & -1.641 & 0 & 0.026218 & 155 \\
\hline
\end{tabular}




\begin{tabular}{|c|c|c|c|c|c|c|c|}
\hline 3 & $\begin{array}{l}\text { GO_REGULATION_OF_PROTEIN_LOCALIZATIO } \\
\text { N_TO_NUCLEUS }\end{array}$ & 174 & -0.407 & -1.636 & 0 & 0.027271 & 156 \\
\hline 3 & $\begin{array}{l}\text { GO_POSITIVE_REGULATION_OF_CELLULAR_PR } \\
\text { OTEIN_LOCALIZATION }\end{array}$ & 267 & -0.385 & -1.635 & 0 & 0.027361 & 157 \\
\hline 3 & GO_HOMEOSTASIS_OF_NUMBER_OF_CELLS & 124 & -0.419 & -1.626 & 0 & 0.029033 & 158 \\
\hline 3 & HALLMARK_APICAL_JUNCTION & 138 & -0.412 & -1.624 & 0 & 0.029458 & 159 \\
\hline 3 & GO_REGULATION_OF_GROWTH & 424 & -0.363 & -1.621 & 0 & 0.030048 & 160 \\
\hline 3 & GO_GTPASE_ACTIVITY & 177 & -0.398 & -1.614 & 0 & 0.031581 & 161 \\
\hline 3 & GO_PROTEIN_OLIGOMERIZATION & 286 & -0.377 & -1.614 & 0 & 0.03161 & 162 \\
\hline 3 & GO_NEGATIVE_REGULATION_OF_TRANSPORT & 304 & -0.374 & -1.614 & 0 & 0.031659 & 163 \\
\hline 3 & GO_POSITIVE_REGULATION_OF_PROTEOLYSIS & 283 & -0.378 & -1.613 & 0 & 0.031785 & 164 \\
\hline 3 & $\begin{array}{l}\text { GO_POSITIVE_REGULATION_OF_CYTOPLASMI } \\
\text { C_TRANSPORT }\end{array}$ & 202 & -0.385 & -1.601 & 0 & 0.03434 & 165 \\
\hline 3 & $\begin{array}{l}\text { GO_POSITIVE_REGULATION_OF_TRANSFERAS } \\
\text { E_ACTIVITY }\end{array}$ & 457 & -0.356 & -1.597 & 0 & 0.035399 & 166 \\
\hline 3 & $\begin{array}{l}\text { GO_NEGATIVE_REGULATION_OF_CELL_GROW } \\
\text { TH }\end{array}$ & 115 & -0.409 & -1.596 & 0 & 0.035628 & 167 \\
\hline 3 & GO_CELL_PROLIFERATION & 449 & -0.354 & -1.58 & 0 & 0.039418 & 168 \\
\hline 3 & $\begin{array}{l}\text { GO_POSITIVE_REGULATION_OF_INTRACELLUL } \\
\text { AR_PROTEIN_TRANSPORT }\end{array}$ & 186 & -0.384 & -1.569 & 0 & 0.042454 & 169 \\
\hline 3 & GO_MEMBRANE_MICRODOMAIN & 205 & -0.378 & -1.568 & 0 & 0.042757 & 170 \\
\hline 3 & GO_WOUND_HEALING & 318 & -0.36 & -1.563 & 0 & 0.044205 & 171 \\
\hline 3 & GO_REGULATION_OF_PROTEIN_TARGETING & 225 & -0.37 & -1.549 & 0 & 0.048348 & 172 \\
\hline 3 & $\begin{array}{l}\text { GO_RESPONSE_TO_EXTRACELLULAR_STIMUL } \\
\text { US }\end{array}$ & 311 & -0.359 & -1.548 & 0 & 0.048375 & 173 \\
\hline 3 & $\begin{array}{l}\text { GO_REGULATION_OF_PROTEIN_MODIFICATIO } \\
\text { N_BY_SMALL_PROTEIN_CONJUGATION_OR_R } \\
\text { EMOVAL }\end{array}$ & 233 & -0.372 & -1.546 & 0 & 0.049161 & 174 \\
\hline 3 & $\begin{array}{l}\text { GO_NEGATIVE_REGULATION_OF_CELL_DIFFER } \\
\text { ENTIATION }\end{array}$ & 354 & -0.353 & -1.545 & 0 & 0.049415 & 175 \\
\hline 3 & $\begin{array}{l}\text { GO_EXTRACELLULAR_STRUCTURE_ORGANIZA } \\
\text { TION }\end{array}$ & 182 & -0.379 & -1.54 & 0 & 0.050981 & 176 \\
\hline 3 & $\begin{array}{l}\text { GO_NEGATIVE_REGULATION_OF_INTRACELLU } \\
\text { LAR_SIGNAL_TRANSDUCTION }\end{array}$ & 329 & -0.351 & -1.534 & 0 & 0.053027 & 177 \\
\hline 3 & GO_REGULATION_OF_CELL_GROWTH & 266 & -0.353 & -1.511 & 0 & 0.060864 & 178 \\
\hline 3 & GO_REGULATION_OF_SYSTEM_PROCESS & 281 & -0.353 & -1.505 & 0 & 0.062834 & 179 \\
\hline 3 & GO_RESPONSE_TO_WOUNDING & 370 & -0.34 & -1.499 & 0 & 0.065016 & 180 \\
\hline
\end{tabular}




\begin{tabular}{|c|c|c|c|c|c|c|c|}
\hline 3 & $\begin{array}{l}\text { GO_REGULATION_OF_PROTEIN_SERINE_THRE } \\
\text { ONINE_KINASE_ACTIVITY }\end{array}$ & 345 & -0.341 & -1.493 & 0 & 0.067518 & 181 \\
\hline 3 & GO_SIGNALING_RECEPTOR_ACTIVITY & 482 & -0.329 & -1.483 & 0 & 0.07165 & 182 \\
\hline 3 & GO_SECRETION & 363 & -0.326 & -1.431 & 0 & 0.096538 & 183 \\
\hline 3 & GO_REGULATION_OF_BODY_FLUID_LEVELS & 314 & -0.334 & -1.438 & 0.00165 & 0.092882 & 184 \\
\hline 3 & GO_CELLULAR_CHEMICAL_HOMEOSTASIS & 352 & -0.348 & -1.529 & 0.001661 & 0.054884 & 185 \\
\hline 3 & $\begin{array}{l}\text { GO_CELLULAR_RESPONSE_TO_ORGANIC_CYCL } \\
\text { IC_COMPOUND }\end{array}$ & 313 & -0.327 & -1.437 & 0.001672 & 0.09354 & 186 \\
\hline 3 & $\begin{array}{l}\text { GO_REGULATION_OF_HOMEOSTATIC_PROCES } \\
\mathrm{S}\end{array}$ & 306 & -0.349 & -1.529 & 0.001678 & 0.055004 & 187 \\
\hline 3 & GO_REGULATION_OF_GTPASE_ACTIVITY & 441 & -0.31 & -1.382 & 0.001684 & 0.125186 & 188 \\
\hline 3 & GO_CELLULAR_HOMEOSTASIS & 422 & -0.326 & -1.455 & 0.001686 & 0.08433 & 189 \\
\hline 3 & GO_SECRETION_BY_CELL & 301 & -0.327 & -1.422 & 0.001701 & 0.101206 & 190 \\
\hline 3 & GO_REPRODUCTIVE_SYSTEM_DEVELOPMENT & 263 & -0.341 & -1.461 & 0.001767 & 0.081171 & 191 \\
\hline 3 & GO_REGULATION_OF_BINDING & 217 & -0.361 & -1.508 & 0.001776 & 0.061965 & 192 \\
\hline 3 & GO_EPIDERMIS_DEVELOPMENT & 122 & -0.429 & -1.659 & 0.001779 & 0.022916 & 193 \\
\hline 3 & $\begin{array}{l}\text { GO_ACTIVATION_OF_PROTEIN_KINASE_ACTIV } \\
\text { ITY }\end{array}$ & 199 & -0.35 & -1.436 & 0.001786 & 0.094089 & 194 \\
\hline 3 & GO_PROTEIN_TYROSINE_KINASE_ACTIVITY & 113 & -0.424 & -1.626 & 0.001789 & 0.029136 & 195 \\
\hline 3 & $\begin{array}{l}\text { GO_REGULATION_OF_STRESS_ACTIVATED_PR } \\
\text { OTEIN_KINASE_SIGNALING_CASCADE }\end{array}$ & 144 & -0.392 & -1.549 & 0.001812 & 0.048237 & 196 \\
\hline 3 & $\begin{array}{l}\text { GO_POSITIVE_REGULATION_OF_CELL_PROJEC } \\
\text { TION_ORGANIZATION }\end{array}$ & 194 & -0.369 & -1.51 & 0.001842 & 0.061186 & 197 \\
\hline 3 & $\begin{array}{l}\text { GO_REGULATION_OF_STEM_CELL_DIFFERENT } \\
\text { IATION }\end{array}$ & 73 & -0.479 & -1.707 & 0.001848 & 0.015892 & 198 \\
\hline 3 & GO_RESPONSE_TO_TEMPERATURE_STIMULUS & 103 & -0.467 & -1.772 & 0.001855 & 0.008866 & 199 \\
\hline 3 & $\begin{array}{l}\text { GO_REGULATION_OF_ENDOTHELIAL_CELL_MI } \\
\text { GRATION }\end{array}$ & 79 & -0.463 & -1.677 & 0.001855 & 0.020113 & 200 \\
\hline 3 & GO_REGULATION_OF_JNK_CASCADE & 115 & -0.416 & -1.591 & 0.001862 & 0.03668 & 201 \\
\hline 3 & GO_EPIDERMAL_CELL_DIFFERENTIATION & 66 & -0.503 & -1.733 & 0.001873 & 0.012715 & 202 \\
\hline 3 & $\begin{array}{l}\text { GO_CYTOPLASMIC_MRNA_PROCESSING_BOD } \\
Y\end{array}$ & 55 & -0.481 & -1.636 & 0.00189 & 0.027129 & 203 \\
\hline 3 & GO_GROWTH_FACTOR_ACTIVITY & 76 & -0.488 & -1.759 & 0.001898 & 0.010046 & 204 \\
\hline 3 & GO_REGULATION_OF_ION_HOMEOSTASIS & 128 & -0.426 & -1.654 & 0.001908 & 0.023828 & 205 \\
\hline 3 & $\begin{array}{l}\text { GO_REGULATION_OF_STRIATED_MUSCLE_CEL } \\
\text { L_DIFFERENTIATION }\end{array}$ & 50 & -0.505 & -1.678 & 0.001916 & 0.019985 & 206 \\
\hline
\end{tabular}




\begin{tabular}{|c|c|c|c|c|c|c|c|}
\hline 3 & GO_MESODERM_DEVELOPMENT & 70 & -0.483 & -1.682 & 0.001923 & 0.019327 & 207 \\
\hline 3 & GO_DOUBLE_STRANDED_RNA_BINDING & 56 & -0.539 & -1.809 & 0.001927 & 0.006187 & 208 \\
\hline 3 & GO_ENDOTHELIUM_DEVELOPMENT & 58 & -0.499 & -1.698 & 0.001931 & 0.01698 & 209 \\
\hline 3 & $\begin{array}{l}\text { GO_POSITIVE_REGULATION_OF_PROTEIN_LO } \\
\text { CALIZATION_TO_NUCLEUS }\end{array}$ & 100 & -0.449 & -1.669 & 0.001934 & 0.021418 & 210 \\
\hline 3 & $\begin{array}{l}\text { GO_NEGATIVE_REGULATION_OF_CELL_CELL_ } \\
\text { ADHESION }\end{array}$ & 96 & -0.449 & -1.658 & 0.001934 & 0.023207 & 211 \\
\hline 3 & $\begin{array}{l}\text { GO_NEGATIVE_REGULATION_OF_HEMOPOIES } \\
\text { IS }\end{array}$ & 86 & -0.428 & -1.57 & 0.001934 & 0.042233 & 212 \\
\hline 3 & $\begin{array}{l}\text { GO_REGULATION_OF_MYOTUBE_DIFFERENTI } \\
\text { ATION }\end{array}$ & 31 & -0.605 & -1.82 & 0.001938 & 0.00556 & 213 \\
\hline 3 & GO_MICROVILLUS & 48 & -0.548 & -1.764 & 0.001949 & 0.009527 & 214 \\
\hline 3 & GO_LUNG_ALVEOLUS_DEVELOPMENT & 28 & -0.571 & -1.682 & 0.001969 & 0.019384 & 215 \\
\hline 3 & $\begin{array}{l}\text { GO_SERINE_TYPE_ENDOPEPTIDASE_INHIBITO } \\
\text { R_ACTIVITY }\end{array}$ & 41 & -0.55 & -1.747 & 0.001976 & 0.011136 & 216 \\
\hline 3 & $\begin{array}{l}\text { GO_NEGATIVE_REGULATION_OF_HOMOTYPIC } \\
\text { _CELL_CELL_ADHESION }\end{array}$ & 70 & -0.483 & -1.718 & 0.001992 & 0.01446 & 217 \\
\hline 3 & GO_RESPONSE_TO_GAMMA_RADIATION & 44 & -0.517 & -1.655 & 0.00202 & 0.023714 & 218 \\
\hline 3 & GO_REGULATION_OF_TISSUE_REMODELING & 43 & -0.549 & -1.748 & 0.002024 & 0.011067 & 219 \\
\hline 3 & $\begin{array}{l}\text { GO_REGULATION_OF_PROTEIN_INSERTION_I } \\
\text { NTO_MITOCHONDRIAL_MEMBRANE_INVOLVE } \\
\text { D_IN_APOPTOTIC_SIGNALING_PATHWAY }\end{array}$ & 28 & -0.647 & -1.916 & 0.002053 & 0.001981 & 220 \\
\hline 3 & $\begin{array}{l}\text { GO_CELLULAR_RESPONSE_TO_GROWTH_HOR } \\
\text { MONE_STIMULUS }\end{array}$ & 15 & -0.7 & -1.797 & 0.002096 & 0.006987 & 221 \\
\hline 3 & $\begin{array}{l}\text { GO_CYSTEINE_TYPE_ENDOPEPTIDASE_REGUL } \\
\text { ATOR_ACTIVITY_INVOLVED_IN_APOPTOTIC_P } \\
\text { ROCESS }\end{array}$ & 30 & -0.595 & -1.782 & 0.002105 & 0.008047 & 222 \\
\hline 3 & $\begin{array}{l}\text { GO_INTRINSIC_APOPTOTIC_SIGNALING_PATH } \\
\text { WAY_IN_RESPONSE_TO_DNA_DAMAGE_BY_P } \\
\text { 53_CLASS_MEDIATOR }\end{array}$ & 23 & -0.64 & -1.777 & 0.002105 & 0.00847 & 223 \\
\hline 3 & GO_PROSTATE_GLAND_DEVELOPMENT & 29 & -0.6 & -1.742 & 0.002105 & 0.011766 & 224 \\
\hline 3 & GO_RESPONSE_TO_COPPER_ION & 21 & -0.624 & -1.752 & 0.002128 & 0.010691 & 225 \\
\hline 3 & $\begin{array}{l}\text { GO_NEGATIVE_REGULATION_OF_RESPONSE_ } \\
\text { TO_ENDOPLASMIC_RETICULUM_STRESS }\end{array}$ & 29 & -0.613 & -1.811 & 0.002146 & 0.006087 & 226 \\
\hline 3 & $\begin{array}{l}\text { GO_NEGATIVE_REGULATION_OF_RESPONSE_ } \\
\text { TO_BIOTIC_STIMULUS }\end{array}$ & 21 & -0.665 & -1.812 & 0.002208 & 0.005989 & 227 \\
\hline 3 & $\begin{array}{l}\text { GO_NEGATIVE_REGULATION_OF_DEVELOPME } \\
\text { NTAL_PROCESS }\end{array}$ & 477 & -0.319 & -1.424 & 0.003344 & 0.100674 & 228 \\
\hline
\end{tabular}




\begin{tabular}{|c|c|c|c|c|c|c|c|}
\hline 3 & $\begin{array}{l}\text { GO_NEGATIVE_REGULATION_OF_PROTEIN_M } \\
\text { ODIFICATION_PROCESS }\end{array}$ & 459 & -0.3 & -1.346 & 0.00335 & 0.149165 & 229 \\
\hline 3 & GO_RESPONSE_TO_ALKALOID & 90 & -0.445 & -1.631 & 0.003597 & 0.028155 & 230 \\
\hline 3 & $\begin{array}{l}\text { GO_REGULATION_OF_MUSCLE_CELL_DIFFERE } \\
\text { NTIATION }\end{array}$ & 97 & -0.421 & -1.582 & 0.00363 & 0.038857 & 231 \\
\hline 3 & $\begin{array}{l}\text { GO_RESPONSE_TO_TRANSITION_METAL_NAN } \\
\text { OPARTICLE }\end{array}$ & 112 & -0.398 & -1.514 & 0.00363 & 0.059585 & 232 \\
\hline 3 & GO_DIGESTIVE_SYSTEM_DEVELOPMENT & 86 & -0.437 & -1.593 & 0.00369 & 0.03638 & 233 \\
\hline 3 & GO_REGULATION_OF_JUN_KINASE_ACTIVITY & 60 & -0.45 & -1.552 & 0.003697 & 0.04727 & 234 \\
\hline 3 & $\begin{array}{l}\text { GO_REGULATION_OF_EPITHELIAL_CELL_MIGR } \\
\text { ATION }\end{array}$ & 118 & -0.419 & -1.62 & 0.003711 & 0.030213 & 235 \\
\hline 3 & $\begin{array}{l}\text { GO_POSITIVE_REGULATION_OF_PROTEIN_CO } \\
\text { MPLEX_ASSEMBLY }\end{array}$ & 147 & -0.392 & -1.546 & 0.003731 & 0.049186 & 236 \\
\hline 3 & GO_REGULATION_OF_WOUND_HEALING & 77 & -0.462 & -1.655 & 0.003781 & 0.023692 & 237 \\
\hline 3 & GO_PROTEIN_KINASE_B_SIGNALING & 28 & -0.6 & -1.761 & 0.003795 & 0.00988 & 238 \\
\hline 3 & $\begin{array}{l}\text { GO_INTRINSIC_APOPTOTIC_SIGNALING_PATH } \\
\text { WAY }\end{array}$ & 118 & -0.417 & -1.611 & 0.003795 & 0.032224 & 239 \\
\hline 3 & $\begin{array}{l}\text { GO_NEGATIVE_REGULATION_OF_CYTOPLASM } \\
\text { IC_TRANSPORT }\end{array}$ & 90 & -0.433 & -1.591 & 0.003802 & 0.036801 & 240 \\
\hline 3 & $\begin{array}{l}\text { GO_NEGATIVE_REGULATION_OF_PROTEIN_M } \\
\text { ODIFICATION_BY_SMALL_PROTEIN_CONJUGA } \\
\text { TION_OR_REMOVAL }\end{array}$ & 120 & -0.422 & -1.615 & 0.003824 & 0.031384 & 241 \\
\hline 3 & GO_APOPTOTIC_MITOCHONDRIAL_CHANGES & 45 & -0.541 & -1.762 & 0.003839 & 0.00978 & 242 \\
\hline 3 & $\begin{array}{l}\text { GO_REGULATION_OF_CYSTEINE_TYPE_ENDOP } \\
\text { EPTIDASE_ACTIVITY_INVOLVED_IN_APOPTOTI } \\
\text { C_SIGNALING_PATHWAY }\end{array}$ & 19 & -0.765 & -2.072 & 0.003899 & $2.25 \mathrm{E}-04$ & 243 \\
\hline 3 & $\begin{array}{l}\text { GO_REGULATION_OF_SMOOTH_MUSCLE_CEL } \\
\text { L_PROLIFERATION }\end{array}$ & 71 & -0.452 & -1.599 & 0.003922 & 0.034886 & 244 \\
\hline 3 & GO_THREONINE_TYPE_PEPTIDASE_ACTIVITY & 18 & -0.664 & -1.775 & 0.00409 & 0.008603 & 245 \\
\hline 3 & GO_CARDIAC_CELL_DEVELOPMENT & 30 & -0.598 & -1.791 & 0.004141 & 0.007467 & 246 \\
\hline 3 & GO_RESPONSE_TO_PROGESTERONE & 34 & -0.557 & -1.701 & 0.004175 & 0.016697 & 247 \\
\hline 3 & $\begin{array}{l}\text { GO_POSITIVE_REGULATION_OF_PEPTIDYL_TH } \\
\text { REONINE_PHOSPHORYLATION }\end{array}$ & 16 & -0.675 & -1.747 & 0.004264 & 0.011211 & 248 \\
\hline 3 & $\begin{array}{l}\text { GO_INTRINSIC_APOPTOTIC_SIGNALING_PATH } \\
\text { WAY_IN_RESPONSE_TO_ENDOPLASMIC_RETIC } \\
\text { ULUM_STRESS }\end{array}$ & 23 & -0.646 & -1.865 & 0.004338 & 0.003405 & 249 \\
\hline 3 & GO_CALCIUM_ION_BINDING & 348 & -0.311 & -1.358 & 0.005008 & 0.14083 & 250 \\
\hline 3 & $\begin{array}{l}\text { GO_REGULATION_OF_METAL_ION_TRANSPOR } \\
T\end{array}$ & 195 & -0.356 & -1.464 & 0.005338 & 0.07971 & 251 \\
\hline
\end{tabular}




\begin{tabular}{|c|c|c|c|c|c|c|c|}
\hline 3 & $\begin{array}{l}\text { GO_CELLULAR_RESPONSE_TO_EXTRACELLULA } \\
\text { R_STIMULUS }\end{array}$ & 140 & -0.396 & -1.547 & 0.005415 & 0.048659 & 252 \\
\hline 3 & GO_REGULATION_OF_RNA_STABILITY & 119 & -0.393 & -1.512 & 0.005445 & 0.060268 & 253 \\
\hline 3 & $\begin{array}{l}\text { GO_STRESS_ACTIVATED_PROTEIN_KINASE_SI } \\
\text { GNALING_CASCADE }\end{array}$ & 81 & -0.442 & -1.586 & 0.005474 & 0.038108 & 254 \\
\hline 3 & $\begin{array}{l}\text { GO_NEGATIVE_REGULATION_OF_HOMEOSTA } \\
\text { TIC_PROCESS }\end{array}$ & 97 & -0.436 & -1.62 & 0.005484 & 0.030194 & 255 \\
\hline 3 & $\begin{array}{l}\text { GO_REGULATION_OF_CALCIUM_ION_TRANSP } \\
\text { ORT_INTO_CYTOSOL }\end{array}$ & 54 & -0.492 & -1.682 & 0.005576 & 0.019345 & 256 \\
\hline 3 & $\begin{array}{l}\text { GO_POSITIVE_REGULATION_OF_EPITHELIAL_C } \\
\text { ELL_MIGRATION }\end{array}$ & 76 & -0.466 & -1.663 & 0.005714 & 0.022361 & 257 \\
\hline 3 & $\begin{array}{l}\text { GO_POSITIVE_REGULATION_OF_JUN_KINASE_ } \\
\text { ACTIVITY }\end{array}$ & 49 & -0.493 & -1.586 & 0.005758 & 0.03799 & 258 \\
\hline 3 & GO_MESODERM_MORPHOGENESIS & 38 & -0.529 & -1.659 & 0.005769 & 0.023037 & 259 \\
\hline 3 & GO_REGULATION_OF_COAGULATION & 53 & -0.533 & -1.788 & 0.006 & 0.00761 & 260 \\
\hline 3 & $\begin{array}{l}\text { GO_POSITIVE_REGULATION_OF_ENDOTHELIA } \\
\text { L_CELL_MIGRATION }\end{array}$ & 49 & -0.511 & -1.674 & 0.00611 & 0.020551 & 261 \\
\hline 3 & GO_PERINUCLEAR_REGION_OF_CYTOPLASM & 462 & -0.3 & -1.349 & 0.006441 & 0.146829 & 262 \\
\hline 3 & GO_EPITHELIAL_CELL_DIFFERENTIATION & 280 & -0.335 & -1.433 & 0.006814 & 0.095422 & 263 \\
\hline 3 & $\begin{array}{l}\text { GO_PROTEIN_HETERODIMERIZATION_ACTIVIT } \\
\mathrm{Y}\end{array}$ & 286 & -0.316 & -1.363 & 0.00713 & 0.137328 & 264 \\
\hline 3 & $\begin{array}{l}\text { GO_NEGATIVE_REGULATION_OF_SEQUENCE_ } \\
\text { SPECIFIC_DNA_BINDING_TRANSCRIPTION_FAC } \\
\text { TOR_ACTIVITY }\end{array}$ & 98 & -0.406 & -1.52 & 0.007194 & 0.057548 & 265 \\
\hline 3 & GO_RIBONUCLEOPROTEIN_GRANULE & 108 & -0.42 & -1.598 & 0.007207 & 0.035212 & 266 \\
\hline 3 & $\begin{array}{l}\text { GO_NEGATIVE_REGULATION_OF_WNT_SIGNA } \\
\text { LING_PATHWAY }\end{array}$ & 134 & -0.363 & -1.423 & 0.007207 & 0.101101 & 267 \\
\hline 3 & $\begin{array}{l}\text { GO_REGULATION_OF_MUSCLE_SYSTEM_PRO } \\
\text { CESS }\end{array}$ & 121 & -0.404 & -1.563 & 0.007366 & 0.04406 & 268 \\
\hline 3 & $\begin{array}{l}\text { GO_REGULATION_OF_EPITHELIAL_CELL_PROLI } \\
\text { FERATION }\end{array}$ & 184 & -0.346 & -1.414 & 0.007394 & 0.106143 & 269 \\
\hline 3 & GO_REGULATION_OF_BLOOD_CIRCULATION & 157 & -0.366 & -1.474 & 0.007477 & 0.07507 & 270 \\
\hline 3 & $\begin{array}{l}\text { GO_CELLULAR_RESPONSE_TO_ACID_CHEMICA } \\
\mathrm{L}\end{array}$ & 113 & -0.424 & -1.618 & 0.007533 & 0.030759 & 271 \\
\hline 3 & $\begin{array}{l}\text { GO_NEGATIVE_REGULATION_OF_CELL_DEVEL } \\
\text { OPMENT }\end{array}$ & 184 & -0.354 & -1.441 & 0.00759 & 0.091381 & 272 \\
\hline 3 & $\begin{array}{l}\text { GO_NEGATIVE_REGULATION_OF_PROTEIN_BI } \\
\text { NDING }\end{array}$ & 61 & -0.475 & -1.653 & 0.007752 & 0.023892 & 273 \\
\hline 3 & GO_HEAT_SHOCK_PROTEIN_BINDING & 70 & -0.442 & -1.555 & 0.007874 & 0.046292 & 274 \\
\hline
\end{tabular}




\begin{tabular}{|c|c|c|c|c|c|c|c|}
\hline 3 & $\begin{array}{l}\text { GO_NEGATIVE_REGULATION_OF_NUCLEOCYT } \\
\text { OPLASMIC_TRANSPORT }\end{array}$ & 59 & -0.483 & -1.629 & 0.007984 & 0.02862 & 275 \\
\hline 3 & GO_RESPONSE_TO_ISOQUINOLINE_ALKALOID & 21 & -0.62 & -1.714 & 0.008 & 0.015088 & 276 \\
\hline 3 & GO_MESENCHYME_MORPHOGENESIS & 24 & -0.569 & -1.597 & 0.008097 & 0.035327 & 277 \\
\hline 3 & $\begin{array}{l}\text { GO_NEGATIVE_REGULATION_OF_PROTEIN_M } \\
\text { ATURATION }\end{array}$ & 26 & -0.572 & -1.652 & 0.00818 & 0.024075 & 278 \\
\hline 3 & GO_CELLULAR_RESPONSE_TO_VITAMIN & 22 & -0.663 & -1.804 & 0.008264 & 0.006516 & 279 \\
\hline 3 & GO_RESPONSE_TO_PEPTIDE & 280 & -0.324 & -1.389 & 0.008347 & 0.120975 & 280 \\
\hline 3 & GO_HIPPO_SIGNALING & 20 & -0.614 & -1.694 & 0.008403 & 0.017537 & 281 \\
\hline 3 & $\begin{array}{l}\text { GO_REGULATION_OF_PROTEIN_OLIGOMERIZ } \\
\text { ATION }\end{array}$ & 27 & -0.586 & -1.709 & 0.008421 & 0.015654 & 282 \\
\hline 3 & GO_ADENYLYLTRANSFERASE_ACTIVITY & 20 & -0.627 & -1.699 & 0.008475 & 0.016916 & 283 \\
\hline 3 & $\begin{array}{l}\text { GO_DEVELOPMENTAL_PROCESS_INVOLVED_I } \\
\text { N_REPRODUCTION }\end{array}$ & 365 & -0.308 & -1.347 & 0.008547 & 0.148952 & 284 \\
\hline 3 & $\begin{array}{l}\text { GO_REGULATION_OF_ENDOPLASMIC_RETICU } \\
\text { LUM_STRESS_INDUCED_INTRINSIC_APOPTOTI } \\
\text { C_SIGNALING_PATHWAY }\end{array}$ & 25 & -0.607 & -1.75 & 0.008677 & 0.010882 & 285 \\
\hline 3 & $\begin{array}{l}\text { GO_NEGATIVE_REGULATION_OF_STRIATED_ } \\
\text { MUSCLE_CELL_DIFFERENTIATION }\end{array}$ & 18 & -0.672 & -1.737 & 0.008753 & 0.012258 & 286 \\
\hline 3 & $\begin{array}{l}\text { GO_CYSTEINE_TYPE_ENDOPEPTIDASE_INHIBIT } \\
\text { OR_ACTIVITY_INVOLVED_IN_APOPTOTIC_PRO } \\
\text { CESS }\end{array}$ & 15 & -0.664 & -1.695 & 0.008753 & 0.017384 & 287 \\
\hline 3 & GO_CELL_JUNCTION_ORGANIZATION & 115 & -0.394 & -1.505 & 0.008945 & 0.062988 & 288 \\
\hline 3 & GO_FORMATION_OF_PRIMARY_GERM_LAYER & 70 & -0.452 & -1.585 & 0.009416 & 0.038163 & 289 \\
\hline 3 & GO_REGULATION_OF_HORMONE_SECRETION & 148 & -0.378 & -1.505 & 0.009615 & 0.062817 & 290 \\
\hline 3 & GO_SKIN_EPIDERMIS_DEVELOPMENT & 36 & -0.517 & -1.611 & 0.009653 & 0.032225 & 291 \\
\hline 3 & GO_CELL_CYCLE_ARREST & 116 & -0.391 & -1.496 & 0.009709 & 0.066296 & 292 \\
\hline 3 & $\begin{array}{l}\text { GO_REGULATION_OF_ANATOMICAL_STRUCT } \\
\text { URE_SIZE }\end{array}$ & 294 & -0.314 & -1.355 & 0.009788 & 0.142978 & 293 \\
\hline 3 & GO_PHOSPHATASE_COMPLEX & 36 & -0.523 & -1.583 & 0.00998 & 0.038763 & 294 \\
\hline 3 & $\begin{array}{l}\text { GO_NON_MEMBRANE_SPANNING_PROTEIN_ } \\
\text { TYROSINE_KINASE_ACTIVITY }\end{array}$ & 35 & -0.549 & -1.689 & 0.010204 & 0.018327 & 295 \\
\hline 3 & $\begin{array}{l}\text { GO_POSITIVE_REGULATION_OF_HOMEOSTATI } \\
\text { C_PROCESS }\end{array}$ & 144 & -0.377 & -1.479 & 0.010619 & 0.073014 & 296 \\
\hline 3 & $\begin{array}{l}\text { GO_NEGATIVE_REGULATION_OF_CELL_KILLIN } \\
\mathrm{G}\end{array}$ & 15 & -0.706 & -1.752 & 0.010776 & 0.010631 & 297 \\
\hline 3 & $\begin{array}{l}\text { GO_REGULATION_OF_ORGAN_MORPHOGENE } \\
\text { SIS }\end{array}$ & 168 & -0.356 & -1.442 & 0.011009 & 0.091011 & 298 \\
\hline
\end{tabular}




\begin{tabular}{|c|c|c|c|c|c|c|c|}
\hline 3 & GO_VASODILATION & 15 & -0.675 & -1.745 & 0.011013 & 0.011402 & 299 \\
\hline 3 & GO_PEPTIDYL_TYROSINE_MODIFICATION & 118 & -0.389 & -1.513 & 0.011173 & 0.059984 & 300 \\
\hline 3 & GO_CYCLIC_NUCLEOTIDE_BINDING & 17 & -0.659 & -1.737 & 0.011186 & 0.012333 & 301 \\
\hline 3 & KEGG_RIBOSOME & 77 & -0.425 & -1.529 & 0.011472 & 0.054864 & 302 \\
\hline 3 & $\begin{array}{l}\text { GO_NEGATIVE_REGULATION_OF_PHOSPHORY } \\
\text { LATION }\end{array}$ & 309 & -0.305 & -1.309 & 0.01157 & 0.178764 & 303 \\
\hline 3 & $\begin{array}{l}\text { GO_RNA_POLYMERASE_II_TRANSCRIPTION_F } \\
\text { ACTOR_ACTIVITY_SEQUENCE_SPECIFIC_DNA_- } \\
\text { BINDING }\end{array}$ & 351 & -0.297 & -1.308 & 0.011686 & 0.179638 & 304 \\
\hline 3 & $\begin{array}{l}\text { GO_NEGATIVE_REGULATION_OF_MUSCLE_CE } \\
\text { LL_DIFFERENTIATION }\end{array}$ & 36 & -0.542 & -1.662 & 0.011811 & 0.022389 & 305 \\
\hline 3 & $\begin{array}{l}\text { GO_REGULATION_OF_SMOOTH_MUSCLE_CO } \\
\text { NTRACTION }\end{array}$ & 31 & -0.549 & -1.651 & 0.012346 & 0.024182 & 306 \\
\hline 3 & $\begin{array}{l}\text { GO_REGULATION_OF_THYMOCYTE_AGGREGA } \\
\text { TION }\end{array}$ & 18 & -0.638 & -1.659 & 0.012552 & 0.022916 & 307 \\
\hline 3 & GO_ENDOCRINE_PANCREAS_DEVELOPMENT & 19 & -0.612 & -1.653 & 0.012766 & 0.023901 & 308 \\
\hline 3 & GO_MICROVILLUS_ORGANIZATION & 17 & -0.664 & -1.72 & 0.012793 & 0.014233 & 309 \\
\hline 3 & $\begin{array}{l}\text { GO_MOVEMENT_IN_ENVIRONMENT_OF_OTH } \\
\text { ER_ORGANISM_INVOLVED_IN_SYMBIOTIC_IN } \\
\text { TERACTION }\end{array}$ & 73 & -0.448 & -1.579 & 0.012868 & 0.03962 & 310 \\
\hline 3 & KEGG_TYPE_II_DIABETES_MELLITUS & 32 & -0.534 & -1.614 & 0.012987 & 0.031599 & 311 \\
\hline 3 & GO_FEMALE_GAMETE_GENERATION & 51 & -0.484 & -1.625 & 0.013109 & 0.029222 & 312 \\
\hline 3 & KEGG_P53_SIGNALING_PATHWAY & 60 & -0.436 & -1.513 & 0.013183 & 0.060097 & 313 \\
\hline 3 & $\begin{array}{l}\text { GO_REGULATION_OF_PROTEIN_COMPLEX_AS } \\
\text { SEMBLY }\end{array}$ & 269 & -0.317 & -1.339 & 0.013793 & 0.154672 & 314 \\
\hline 3 & GO_ADHERENS_JUNCTION_ORGANIZATION & 42 & -0.489 & -1.545 & 0.014056 & 0.049491 & 315 \\
\hline 3 & $\begin{array}{l}\text { GO_PLATELET_DERIVED_GROWTH_FACTOR_R } \\
\text { ECEPTOR_SIGNALING_PATHWAY }\end{array}$ & 32 & -0.554 & -1.669 & 0.01417 & 0.021418 & 316 \\
\hline 3 & $\begin{array}{l}\text { GO_MORPHOGENESIS_OF_A_BRANCHING_ST } \\
\text { RUCTURE }\end{array}$ & 98 & -0.38 & -1.428 & 0.014311 & 0.098376 & 317 \\
\hline 3 & GO_RESPONSE_TO_COLD & 32 & -0.517 & -1.55 & 0.014315 & 0.047791 & 318 \\
\hline 3 & $\begin{array}{l}\text { GO_MAMMARY_GLAND_EPITHELIUM_DEVEL } \\
\text { OPMENT }\end{array}$ & 33 & -0.533 & -1.612 & 0.014799 & 0.031922 & 319 \\
\hline 3 & GO_NEGATIVE_REGULATION_OF_BINDING & 100 & -0.397 & -1.488 & 0.014842 & 0.069446 & 320 \\
\hline 3 & HALLMARK_ANDROGEN_RESPONSE & 85 & -0.409 & -1.5 & 0.015209 & 0.064675 & 321 \\
\hline 3 & GO_ENDOCYTOSIS & 345 & -0.296 & -1.298 & 0.015679 & 0.18803 & 322 \\
\hline
\end{tabular}




\begin{tabular}{|c|c|c|c|c|c|c|c|}
\hline 3 & $\begin{array}{l}\text { GO_POSITIVE_REGULATION_OF_ORGANELLE_ } \\
\text { ASSEMBLY }\end{array}$ & 35 & -0.533 & -1.655 & 0.015717 & 0.023682 & 323 \\
\hline 3 & $\begin{array}{l}\text { GO_CELLULAR_RESPONSE_TO_AMINO_ACID_ } \\
\text { STIMULUS }\end{array}$ & 36 & -0.508 & -1.538 & 0.015905 & 0.051577 & 324 \\
\hline 3 & GO_INTERACTION_WITH_SYMBIONT & 38 & -0.502 & -1.587 & 0.015968 & 0.037605 & 325 \\
\hline 3 & GO_CELL_CELL_JUNCTION & 231 & -0.316 & -1.331 & 0.016043 & 0.160415 & 326 \\
\hline 3 & GO_REGULATION_OF_VASOCONSTRICTION & 32 & -0.531 & -1.612 & 0.016129 & 0.032024 & 327 \\
\hline 3 & GO_RESPONSE_TO_ZINC_ION & 39 & -0.5 & -1.604 & 0.016129 & 0.033741 & 328 \\
\hline 3 & $\begin{array}{l}\text { GO_POSITIVE_REGULATION_OF_VASOCONSTR } \\
\text { ICTION }\end{array}$ & 18 & -0.649 & -1.746 & 0.016393 & 0.011252 & 329 \\
\hline 3 & GO_SULFUR_COMPOUND_BINDING & 134 & -0.365 & -1.428 & 0.016453 & 0.098268 & 330 \\
\hline 3 & $\begin{array}{l}\text { GO_POSITIVE_REGULATION_OF_CELL_DEVELO } \\
\text { PMENT }\end{array}$ & 294 & -0.31 & -1.325 & 0.016892 & 0.16572 & 331 \\
\hline 3 & GO_HETEROTYPIC_CELL_CELL_ADHESION & 22 & -0.574 & -1.637 & 0.016949 & 0.027095 & 332 \\
\hline 3 & GO_PROTEIN_SECRETION & 73 & -0.418 & -1.498 & 0.016981 & 0.065452 & 333 \\
\hline 3 & GO_PROTEIN_SELF_ASSOCIATION & 32 & -0.545 & -1.653 & 0.017316 & 0.023928 & 334 \\
\hline 3 & GO_ER_NUCLEUS_SIGNALING_PATHWAY & 30 & -0.54 & -1.602 & 0.017341 & 0.034162 & 335 \\
\hline 3 & GO_PEPTIDE_HORMONE_PROCESSING & 17 & -0.632 & -1.646 & 0.017354 & 0.025014 & 336 \\
\hline 3 & GO_SEGMENTATION & 49 & -0.447 & -1.499 & 0.017787 & 0.065099 & 337 \\
\hline 3 & HALLMARK_COAGULATION & 94 & -0.389 & -1.436 & 0.017822 & 0.093795 & 338 \\
\hline 3 & $\begin{array}{l}\text { GO_REGULATION_OF_FILOPODIUM_ASSEMBL } \\
\mathrm{Y}\end{array}$ & 28 & -0.556 & -1.61 & 0.018405 & 0.032407 & 339 \\
\hline 3 & GO_PODOSOME & 20 & -0.562 & -1.553 & 0.01848 & 0.046864 & 340 \\
\hline 3 & GO_JNK_CASCADE & 66 & -0.444 & -1.532 & 0.018622 & 0.053659 & 341 \\
\hline 3 & GO_RESPONSE_TO_GROWTH_FACTOR & 323 & -0.298 & -1.292 & 0.018676 & 0.193757 & 342 \\
\hline 3 & BIOCARTA_CASPASE_PATHWAY & 22 & -0.589 & -1.602 & 0.019048 & 0.034205 & 343 \\
\hline 3 & GO_CELLULAR_RESPONSE_TO_ALCOHOL & 77 & -0.402 & -1.444 & 0.019084 & 0.089997 & 344 \\
\hline 3 & GO_VASCULATURE_DEVELOPMENT & 313 & -0.304 & -1.306 & 0.019164 & 0.181083 & 345 \\
\hline 3 & $\begin{array}{l}\text { GO_MAMMARY_GLAND_DUCT_MORPHOGEN } \\
\text { ESIS }\end{array}$ & 16 & -0.616 & -1.596 & 0.019272 & 0.035496 & 346 \\
\hline 3 & $\begin{array}{l}\text { GO_CYTOSOLIC_SMALL_RIBOSOMAL_SUBUNI } \\
\mathrm{T}\end{array}$ & 37 & -0.493 & -1.539 & 0.019313 & 0.051336 & 347 \\
\hline 3 & $\begin{array}{l}\text { GO_CELL_DEATH_IN_RESPONSE_TO_OXIDATI } \\
\text { VE_STRESS }\end{array}$ & 16 & -0.635 & -1.622 & 0.019438 & 0.029955 & 348 \\
\hline 3 & $\begin{array}{l}\text { GO_POSITIVE_REGULATION_OF_EPIDERMIS_D } \\
\text { EVELOPMENT }\end{array}$ & 18 & -0.585 & -1.561 & 0.019481 & 0.044805 & 349 \\
\hline
\end{tabular}




\begin{tabular}{|c|c|c|c|c|c|c|c|}
\hline 3 & $\begin{array}{l}\text { GO_POSITIVE_REGULATION_OF_STEM_CELL_ } \\
\text { DIFFERENTIATION }\end{array}$ & 33 & -0.514 & -1.528 & 0.019493 & 0.055054 & 350 \\
\hline 3 & HALLMARK_P53_PATHWAY & 163 & -0.341 & -1.366 & 0.019538 & 0.135675 & 351 \\
\hline 3 & GO_REGULATION_OF_HORMONE_LEVELS & 262 & -0.315 & -1.325 & 0.019643 & 0.165644 & 352 \\
\hline 3 & $\begin{array}{l}\text { GO_REGULATION_OF_PROTEIN_TYROSINE_KI } \\
\text { NASE_ACTIVITY }\end{array}$ & 46 & -0.475 & -1.54 & 0.020325 & 0.050983 & 353 \\
\hline 3 & $\begin{array}{l}\text { GO_POSITIVE_REGULATION_OF_CANONICAL_ } \\
\text { WNT_SIGNALING_PATHWAY }\end{array}$ & 85 & -0.422 & -1.535 & 0.020522 & 0.052536 & 354 \\
\hline 3 & $\begin{array}{l}\text { GO_REGULATION_OF_VESICLE_MEDIATED_TR } \\
\text { ANSPORT }\end{array}$ & 312 & -0.303 & -1.309 & 0.020548 & 0.178794 & 355 \\
\hline 3 & GO_NEGATIVE_REGULATION_OF_ANOIKIS & 16 & -0.646 & -1.677 & 0.02079 & 0.020061 & 356 \\
\hline 3 & $\begin{array}{l}\text { GO_POSITIVE_REGULATION_OF_PROTEIN_MO } \\
\text { DIFICATION_BY_SMALL_PROTEIN_CONJUGATI } \\
\text { ON_OR_REMOVAL }\end{array}$ & 165 & -0.336 & -1.367 & 0.020873 & 0.135071 & 357 \\
\hline 3 & $\begin{array}{l}\text { GO_CYSTEINE_TYPE_ENDOPEPTIDASE_INHIBIT } \\
\text { OR_ACTIVITY }\end{array}$ & 28 & -0.533 & -1.556 & 0.021142 & 0.045997 & 358 \\
\hline 3 & BIOCARTA_PML_PATHWAY & 17 & -0.615 & -1.614 & 0.021459 & 0.031586 & 359 \\
\hline 3 & $\begin{array}{l}\text { GO_NEGATIVE_REGULATION_OF_CANONICAL } \\
\text { _WNT_SIGNALING_PATHWAY }\end{array}$ & 116 & -0.372 & -1.435 & 0.021505 & 0.094266 & 360 \\
\hline 3 & GO_ACTIVATION_OF_JUN_KINASE_ACTIVITY & 30 & -0.525 & -1.558 & 0.021569 & 0.045397 & 361 \\
\hline 3 & $\begin{array}{l}\text { GO_NEGATIVE_REGULATION_OF_TELOMERE_ } \\
\text { MAINTENANCE }\end{array}$ & 20 & -0.571 & -1.584 & 0.021978 & 0.038532 & 362 \\
\hline 3 & $\begin{array}{l}\text { GO_NEGATIVE_REGULATION_OF_INTRACELLU } \\
\text { LAR_PROTEIN_TRANSPORT }\end{array}$ & 74 & -0.418 & -1.485 & 0.022059 & 0.070758 & 363 \\
\hline 3 & $\begin{array}{l}\text { GO_POSITIVE_REGULATION_OF_ACTIN_FILAM } \\
\text { ENT_POLYMERIZATION }\end{array}$ & 45 & -0.481 & -1.558 & 0.022133 & 0.04568 & 364 \\
\hline 3 & $\begin{array}{l}\text { GO_REGULATION_OF_OSTEOCLAST_DIFFEREN } \\
\text { TIATION }\end{array}$ & 43 & -0.47 & -1.496 & 0.022267 & 0.066442 & 365 \\
\hline 3 & $\begin{array}{l}\text { GO_POSITIVE_REGULATION_OF_TISSUE_REM } \\
\text { ODELING }\end{array}$ & 21 & -0.586 & -1.607 & 0.022321 & 0.032967 & 366 \\
\hline 3 & $\begin{array}{l}\text { GO_NEGATIVE_REGULATION_OF_SMOOTH_M } \\
\text { USCLE_CELL_PROLIFERATION }\end{array}$ & 25 & -0.543 & -1.563 & 0.022573 & 0.044056 & 367 \\
\hline 3 & GO_HORMONE_RECEPTOR_BINDING & 112 & -0.365 & -1.393 & 0.022599 & 0.11857 & 368 \\
\hline 3 & $\begin{array}{l}\text { GO_DEVELOPMENT_OF_PRIMARY_SEXUAL_C } \\
\text { HARACTERISTICS }\end{array}$ & 122 & -0.377 & -1.462 & 0.022684 & 0.081108 & 369 \\
\hline 3 & GO_PROTEIN_ACTIVATION_CASCADE & 39 & -0.488 & -1.528 & 0.022965 & 0.055153 & 370 \\
\hline 3 & $\begin{array}{l}\text { GO_REGULATION_OF_MEMBRANE_PROTEIN_ } \\
\text { ECTODOMAIN_PROTEOLYSIS }\end{array}$ & 16 & -0.632 & -1.611 & 0.023158 & 0.032252 & 371 \\
\hline 3 & GO_RESPONSE_TO_GONADOTROPIN & 20 & -0.625 & -1.654 & 0.023404 & 0.023884 & 372 \\
\hline
\end{tabular}




\begin{tabular}{|c|c|c|c|c|c|c|c|}
\hline 3 & GO_EXONUCLEASE_ACTIVITY & 58 & -0.442 & -1.497 & 0.023669 & 0.065916 & 373 \\
\hline 3 & $\begin{array}{l}\text { GO_POSITIVE_REGULATION_OF_ORGANELLE_ } \\
\text { ORGANIZATION }\end{array}$ & 414 & -0.287 & -1.281 & 0.023689 & 0.204445 & 374 \\
\hline 3 & $\begin{array}{l}\text { GO_TRANSCRIPTION_FROM_RNA_POLYMERA } \\
\text { SE_II_PROMOTER }\end{array}$ & 482 & -0.287 & -1.281 & 0.024077 & 0.203913 & 375 \\
\hline 3 & KEGG_PROSTATE_CANCER & 79 & -0.405 & -1.443 & 0.024254 & 0.090173 & 376 \\
\hline 3 & GO_RESPONSE_TO_GROWTH_HORMONE & 21 & -0.615 & -1.683 & 0.024336 & 0.019285 & 377 \\
\hline 3 & GO_SOMITOGENESIS & 33 & -0.501 & -1.507 & 0.024691 & 0.062071 & 378 \\
\hline 3 & GO_OVULATION_CYCLE & 70 & -0.426 & -1.52 & 0.025194 & 0.057556 & 379 \\
\hline 3 & BIOCARTA_STRESS_PATHWAY & 22 & -0.575 & -1.594 & 0.025478 & 0.036117 & 380 \\
\hline 3 & $\begin{array}{l}\text { GO_COLUMNAR_CUBOIDAL_EPITHELIAL_CELL } \\
\text { _DIFFERENTIATION }\end{array}$ & 63 & -0.421 & -1.464 & 0.025641 & 0.079972 & 381 \\
\hline 3 & GO_NEURAL_NUCLEUS_DEVELOPMENT & 49 & -0.465 & -1.52 & 0.025794 & 0.057627 & 382 \\
\hline 3 & $\begin{array}{l}\text { GO_NEGATIVE_REGULATION_OF_ADHERENS_ } \\
\text { JUNCTION_ORGANIZATION }\end{array}$ & 15 & -0.641 & -1.598 & 0.02603 & 0.03518 & 383 \\
\hline 3 & GO_CORE_PROMOTER_BINDING & 113 & -0.371 & -1.408 & 0.026168 & 0.109991 & 384 \\
\hline 3 & $\begin{array}{l}\text { GO_HETEROPHILIC_CELL_CELL_ADHESION_VI } \\
\text { A_PLASMA_MEMBRANE_CELL_ADHESION_M } \\
\text { OLECULES }\end{array}$ & 23 & -0.54 & -1.522 & 0.02621 & 0.056946 & 385 \\
\hline 3 & GO_REGULATION_OF_ENDOCRINE_PROCESS & 23 & -0.542 & -1.553 & 0.026423 & 0.046996 & 386 \\
\hline 3 & GO_REGENERATION & 115 & -0.362 & -1.393 & 0.026465 & 0.118485 & 387 \\
\hline 3 & GO_GROWTH & 246 & -0.316 & -1.329 & 0.026667 & 0.162137 & 388 \\
\hline 3 & GO_MEMBRANE_PROTEIN_PROTEOLYSIS & 28 & -0.513 & -1.508 & 0.02686 & 0.061887 & 389 \\
\hline 3 & GO_SOMITE_DEVELOPMENT & 39 & -0.47 & -1.497 & 0.026915 & 0.065837 & 390 \\
\hline 3 & $\begin{array}{l}\text { GO_NEGATIVE_REGULATION_OF_CELL_MORP } \\
\text { HOGENESIS_INVOLVED_IN_DIFFERENTIATION }\end{array}$ & 76 & -0.411 & -1.494 & 0.026923 & 0.067173 & 391 \\
\hline 3 & GO_OUTER_MEMBRANE & 142 & -0.348 & -1.363 & 0.027273 & 0.1379 & 392 \\
\hline 3 & GO_CIRCULATORY_SYSTEM_PROCESS & 192 & -0.33 & -1.351 & 0.027273 & 0.145684 & 393 \\
\hline 3 & $\begin{array}{l}\text { GO_TRANSLATION_ELONGATION_FACTOR_AC } \\
\text { TIVITY }\end{array}$ & 16 & -0.616 & -1.594 & 0.027368 & 0.036161 & 394 \\
\hline 3 & $\begin{array}{l}\text { GO_NEGATIVE_REGULATION_OF_TRANSFERA } \\
\text { SE_ACTIVITY }\end{array}$ & 273 & -0.309 & -1.322 & 0.027372 & 0.1674 & 395 \\
\hline 3 & GO_REGULATION_OF_PEPTIDE_SECRETION & 121 & -0.365 & -1.403 & 0.027473 & 0.11288 & 396 \\
\hline 3 & GO_PANCREAS_DEVELOPMENT & 36 & -0.498 & -1.526 & 0.027505 & 0.055527 & 397 \\
\hline 3 & GO_TISSUE_MORPHOGENESIS & 319 & -0.292 & -1.268 & 0.027539 & 0.215723 & 398 \\
\hline
\end{tabular}




\begin{tabular}{|c|c|c|c|c|c|c|c|}
\hline 3 & BIOCARTA_EGF_PATHWAY & 29 & -0.531 & -1.575 & 0.027833 & 0.040824 & 399 \\
\hline 3 & $\begin{array}{l}\text { GO_TRANSCRIPTIONAL_ACTIVATOR_ACTIVITY } \\
\text { _RNA_POLYMERASE_II_TRANSCRIPTION_REG } \\
\text { ULATORY_REGION_SEQUENCE_SPECIFIC_BIND } \\
\text { ING }\end{array}$ & 181 & -0.321 & -1.322 & 0.028169 & 0.167772 & 400 \\
\hline 3 & GO_RNA_POLYADENYLATION & 25 & -0.536 & -1.527 & 0.029014 & 0.055401 & 401 \\
\hline 3 & GO_CARDIAC_ATRIUM_DEVELOPMENT & 19 & -0.569 & -1.526 & 0.029213 & 0.055759 & 402 \\
\hline 3 & GO_SENSORY_ORGAN_DEVELOPMENT & 269 & -0.302 & -1.284 & 0.029463 & 0.20151 & 403 \\
\hline 3 & $\begin{array}{l}\text { GO_POSITIVE_REGULATION_OF_CALCIUM_IO } \\
\text { N_TRANSPORT }\end{array}$ & 64 & -0.419 & -1.46 & 0.029586 & 0.081666 & 404 \\
\hline 3 & $\begin{array}{l}\text { GO_REGULATION_OF_CATION_TRANSMEMBR } \\
\text { ANE_TRANSPORT }\end{array}$ & 126 & -0.353 & -1.361 & 0.03 & 0.139237 & 405 \\
\hline 3 & GO_CYSTEINE_TYPE_PEPTIDASE_ACTIVITY & 134 & -0.344 & -1.363 & 0.030075 & 0.137425 & 406 \\
\hline 3 & GO_KINASE_REGULATOR_ACTIVITY & 140 & -0.344 & -1.357 & 0.030189 & 0.141642 & 407 \\
\hline 3 & GO_RESPONSE_TO_METAL_ION & 246 & -0.313 & -1.318 & 0.030357 & 0.170743 & 408 \\
\hline 3 & GO_REGULATION_OF_CELL_MORPHOGENESIS & 374 & -0.287 & -1.256 & 0.030405 & 0.227779 & 409 \\
\hline 3 & BIOCARTA_KERATINOCYTE_PATHWAY & 41 & -0.484 & -1.54 & 0.030426 & 0.051057 & 410 \\
\hline 3 & $\begin{array}{l}\text { GO_REGULATION_OF_CANONICAL_WNT_SIG } \\
\text { NALING_PATHWAY }\end{array}$ & 157 & -0.348 & -1.39 & 0.030576 & 0.120748 & 411 \\
\hline 3 & GO_FRIZZLED_BINDING & 18 & -0.586 & -1.566 & 0.031683 & 0.043355 & 412 \\
\hline 3 & $\begin{array}{l}\text { GO_SIGNAL_TRANSDUCTION_BY_P53_CLASS_ } \\
\text { MEDIATOR }\end{array}$ & 101 & -0.377 & -1.418 & 0.031716 & 0.103513 & 413 \\
\hline 3 & $\begin{array}{l}\text { GO_POSITIVE_REGULATION_OF_HORMONE_S } \\
\text { ECRETION }\end{array}$ & 64 & -0.419 & -1.446 & 0.031776 & 0.088724 & 414 \\
\hline 3 & BIOCARTA_TID_PATHWAY & 18 & -0.634 & -1.645 & 0.031915 & 0.025218 & 415 \\
\hline 3 & GO_NEURON_PROJECTION_REGENERATION & 23 & -0.579 & -1.614 & 0.031983 & 0.031617 & 416 \\
\hline 3 & $\begin{array}{l}\text { GO_REGULATION_OF_OXIDATIVE_STRESS_IND } \\
\text { UCED_INTRINSIC_APOPTOTIC_SIGNALING_PA } \\
\text { THWAY }\end{array}$ & 23 & -0.531 & -1.519 & 0.03299 & 0.057988 & 417 \\
\hline 3 & $\begin{array}{l}\text { GO_REGULATION_OF_SMOOTH_MUSCLE_CEL } \\
\text { L_MIGRATION }\end{array}$ & 37 & -0.478 & -1.502 & 0.033333 & 0.06419 & 418 \\
\hline 3 & GO_EXTRACELLULAR_MATRIX & 211 & -0.325 & -1.347 & 0.033989 & 0.148222 & 419 \\
\hline 3 & $\begin{array}{l}\text { GO_REGULATION_OF_BMP_SIGNALING_PATH } \\
\text { WAY }\end{array}$ & 44 & -0.465 & -1.494 & 0.034115 & 0.067194 & 420 \\
\hline 3 & $\begin{array}{l}\text { GO_EMBRYONIC_PLACENTA_MORPHOGENESI } \\
\mathrm{S}\end{array}$ & 15 & -0.62 & -1.566 & 0.034274 & 0.043191 & 421 \\
\hline 3 & $\begin{array}{l}\text { GO_PEPTIDYL_TYROSINE_AUTOPHOSPHORYLA } \\
\text { TION }\end{array}$ & 30 & -0.51 & -1.539 & 0.034483 & 0.05114 & 422 \\
\hline
\end{tabular}




\begin{tabular}{|c|c|c|c|c|c|c|c|}
\hline 3 & $\begin{array}{l}\text { GO_POSITIVE_REGULATION_OF_PROTEIN_KIN } \\
\text { ASE_B_SIGNALING }\end{array}$ & 54 & -0.437 & -1.461 & 0.034483 & 0.081212 & 423 \\
\hline 3 & GO_MALE_SEX_DIFFERENTIATION & 90 & -0.389 & -1.426 & 0.034816 & 0.099716 & 424 \\
\hline 3 & $\begin{array}{l}\text { GO_NEGATIVE_REGULATION_OF_PEPTIDYL_T } \\
\text { YROSINE_PHOSPHORYLATION }\end{array}$ & 32 & -0.494 & -1.497 & 0.035294 & 0.065696 & 425 \\
\hline 3 & $\begin{array}{l}\text { GO_POSITIVE_REGULATION_OF_WNT_SIGNAL } \\
\text { ING_PATHWAY }\end{array}$ & 109 & -0.372 & -1.402 & 0.035336 & 0.112931 & 426 \\
\hline 3 & $\begin{array}{l}\text { GO_CELLULAR_RESPONSE_TO_NITROGEN_CO } \\
\text { MPOUND }\end{array}$ & 350 & -0.283 & -1.238 & 0.035889 & 0.24644 & 427 \\
\hline 3 & GO_EMBRYONIC_EYE_MORPHOGENESIS & 15 & -0.607 & -1.561 & 0.036 & 0.044781 & 428 \\
\hline 3 & $\begin{array}{l}\text { GO_REGULATION_OF_PROTEIN_EXPORT_FRO } \\
\text { M_NUCLEUS }\end{array}$ & 30 & -0.521 & -1.534 & 0.036217 & 0.053082 & 429 \\
\hline 3 & HALLMARK_KRAS_SIGNALING_DN & 85 & -0.379 & -1.388 & 0.036217 & 0.121575 & 430 \\
\hline 3 & $\begin{array}{l}\text { GO_POSITIVE_REGULATION_OF_ION_TRANSP } \\
\text { ORT }\end{array}$ & 134 & -0.341 & -1.336 & 0.03643 & 0.156903 & 431 \\
\hline 3 & GO_CELLULAR_RESPONSE_TO_DRUG & 43 & -0.469 & -1.498 & 0.036538 & 0.065418 & 432 \\
\hline 3 & $\begin{array}{l}\text { GO_POSITIVE_REGULATION_OF_OSSIFICATIO } \\
\mathrm{N}\end{array}$ & 56 & -0.428 & -1.436 & 0.03668 & 0.094003 & 433 \\
\hline 3 & GO_REGULATION_OF_ENDOCYTOSIS & 148 & -0.334 & -1.322 & 0.0369 & 0.168135 & 434 \\
\hline 3 & KEGG_PRION_DISEASES & 27 & -0.517 & -1.493 & 0.03719 & 0.067279 & 435 \\
\hline 3 & $\begin{array}{l}\text { GO_POSITIVE_REGULATION_OF_EPITHELIAL_C } \\
\text { ELL_PROLIFERATION }\end{array}$ & 90 & -0.38 & -1.399 & 0.037255 & 0.115103 & 436 \\
\hline 3 & $\begin{array}{l}\text { GO_POSITIVE_REGULATION_OF_EPITHELIAL_T } \\
\text { O_MESENCHYMAL_TRANSITION }\end{array}$ & 24 & -0.561 & -1.549 & 0.0375 & 0.048236 & 437 \\
\hline 3 & $\begin{array}{l}\text { GO_REGULATION_OF_CELL_SUBSTRATE_ADH } \\
\text { ESION }\end{array}$ & 120 & -0.357 & -1.357 & 0.038328 & 0.141677 & 438 \\
\hline 3 & $\begin{array}{l}\text { GO_POSITIVE_REGULATION_OF_PROTEIN_AU } \\
\text { TOPHOSPHORYLATION }\end{array}$ & 18 & -0.603 & -1.587 & 0.03956 & 0.037645 & 439 \\
\hline 3 & GO_REGULATION_OF_NUCLEASE_ACTIVITY & 20 & -0.573 & -1.553 & 0.039823 & 0.047111 & 440 \\
\hline 3 & $\begin{array}{l}\text { GO_REGULATION_OF_PHOSPHOPROTEIN_PH } \\
\text { OSPHATASE_ACTIVITY }\end{array}$ & 44 & -0.47 & -1.508 & 0.040404 & 0.061955 & 441 \\
\hline 3 & GO_MAMMARY_GLAND_MORPHOGENESIS & 22 & -0.545 & -1.503 & 0.040404 & 0.063687 & 442 \\
\hline 3 & $\begin{array}{l}\text { GO_REGULATION_OF_PROTEIN_PHOSPHATAS } \\
\text { E_TYPE_2A_ACTIVITY }\end{array}$ & 19 & -0.564 & -1.516 & 0.040984 & 0.059095 & 443 \\
\hline 3 & $\begin{array}{l}\text { GO_CELL_DIFFERENTIATION_INVOLVED_IN_E } \\
\text { MBRYONIC_PLACENTA_DEVELOPMENT }\end{array}$ & 16 & -0.589 & -1.523 & 0.041237 & 0.056632 & 444 \\
\hline 3 & BIOCARTA_FAS_PATHWAY & 30 & -0.507 & -1.517 & 0.041237 & 0.058815 & 445 \\
\hline 3 & $\begin{array}{l}\text { GO_EMBRYONIC_DIGESTIVE_TRACT_DEVELOP } \\
\text { MENT }\end{array}$ & 19 & -0.561 & -1.49 & 0.041322 & 0.068545 & 446 \\
\hline
\end{tabular}




\begin{tabular}{|c|c|c|c|c|c|c|c|}
\hline 3 & $\begin{array}{l}\text { GO_MODULATION_OF_TRANSCRIPTION_IN_O } \\
\text { THER_ORGANISM_INVOLVED_IN_SYMBIOTIC_ } \\
\text { INTERACTION }\end{array}$ & 17 & -0.591 & -1.542 & 0.041408 & 0.050441 & 447 \\
\hline 3 & GO_VESICLE_LUMEN & 58 & -0.43 & -1.47 & 0.041746 & 0.07716 & 448 \\
\hline 3 & GO_PROTEIN_KINASE_C_BINDING & 38 & -0.496 & -1.542 & 0.042105 & 0.050542 & 449 \\
\hline 3 & GO_PROTEIN_K48_LINKED_UBIQUITINATION & 42 & -0.451 & -1.44 & 0.042308 & 0.091902 & 450 \\
\hline 3 & $\begin{array}{l}\text { GO_MEMBRANE_PROTEIN_ECTODOMAIN_PR } \\
\text { OTEOLYSIS }\end{array}$ & 18 & -0.565 & -1.527 & 0.042463 & 0.055321 & 451 \\
\hline 3 & GO_ACTIN_BASED_CELL_PROJECTION & 117 & -0.358 & -1.358 & 0.042593 & 0.141463 & 452 \\
\hline 3 & $\begin{array}{l}\text { GO_POSITIVE_REGULATION_OF_TRANSLATIO } \\
\text { NAL_INITIATION }\end{array}$ & 20 & -0.538 & -1.468 & 0.042735 & 0.078064 & 453 \\
\hline 3 & GO_ENDOCARDIAL_CUSHION_DEVELOPMENT & 24 & -0.526 & -1.508 & 0.04277 & 0.061865 & 454 \\
\hline 3 & $\begin{array}{l}\text { GO_REGULATION_OF_CELL_PROJECTION_ASS } \\
\text { EMBLY }\end{array}$ & 109 & -0.354 & -1.336 & 0.04291 & 0.156631 & 455 \\
\hline 3 & $\begin{array}{l}\text { GO_NEGATIVE_REGULATION_OF_MYOBLAST_- } \\
\text { DIFFERENTIATION }\end{array}$ & 15 & -0.608 & -1.528 & 0.042945 & 0.055081 & 456 \\
\hline 3 & $\begin{array}{l}\text { GO_NEGATIVE_REGULATION_OF_CELLULAR_P } \\
\text { ROTEIN_LOCALIZATION }\end{array}$ & 106 & -0.368 & -1.39 & 0.042991 & 0.120411 & 457 \\
\hline 3 & GO_GLAND_DEVELOPMENT & 258 & -0.299 & -1.256 & 0.043029 & 0.228523 & 458 \\
\hline 3 & $\begin{array}{l}\text { GO_REGULATION_OF_MEMBRANE_DEPOLARI } \\
\text { ZATION }\end{array}$ & 30 & -0.497 & -1.49 & 0.043393 & 0.06852 & 459 \\
\hline 3 & GO_HEMOSTASIS & 207 & -0.311 & -1.275 & 0.04363 & 0.209081 & 460 \\
\hline 3 & GO_CALCIUM_ION_TRANSPORT & 125 & -0.355 & -1.375 & 0.044231 & 0.129401 & 461 \\
\hline 3 & $\begin{array}{l}\text { GO_POSITIVE_REGULATION_OF_ORGAN_GRO } \\
\text { WTH }\end{array}$ & 22 & -0.528 & -1.494 & 0.044534 & 0.067109 & 462 \\
\hline 3 & $\begin{array}{l}\text { GO_POSITIVE_REGULATION_OF_CELLULAR_C } \\
\text { OMPONENT_BIOGENESIS }\end{array}$ & 271 & -0.301 & -1.282 & 0.044983 & 0.202736 & 463 \\
\hline 3 & GO_MUSCLE_ADAPTATION & 18 & -0.578 & -1.538 & 0.045361 & 0.051526 & 464 \\
\hline 3 & GO_PITUITARY_GLAND_DEVELOPMENT & 17 & -0.57 & -1.516 & 0.046218 & 0.058938 & 465 \\
\hline 3 & $\begin{array}{l}\text { GO_ESTABLISHMENT_OR_MAINTENANCE_OF } \\
\text { _BIPOLAR_CELL_POLARITY }\end{array}$ & 26 & -0.511 & -1.485 & 0.046243 & 0.070801 & 466 \\
\hline 3 & $\begin{array}{l}\text { GO_TRANSCRIPTION_FACTOR_ACTIVITY_RNA_- } \\
\text { POLYMERASE_II_DISTAL_ENHANCER_SEQUEN } \\
\text { CE_SPECIFIC_BINDING }\end{array}$ & 51 & -0.424 & -1.407 & 0.046243 & 0.110503 & 467 \\
\hline 3 & $\begin{array}{l}\text { GO_SIGNAL_TRANSDUCTION_IN_ABSENCE_OF } \\
\text { _LIGAND }\end{array}$ & 23 & -0.551 & -1.55 & 0.046316 & 0.048086 & 468 \\
\hline 3 & GO_ANGIOGENESIS & 189 & -0.316 & -1.3 & 0.046346 & 0.18629 & 469 \\
\hline 3 & GO_ENDOCRINE_PROCESS & 20 & -0.563 & -1.517 & 0.046784 & 0.058711 & 470 \\
\hline
\end{tabular}




\begin{tabular}{|c|c|c|c|c|c|c|c|}
\hline 3 & $\begin{array}{l}\text { KEGG_NEUROTROPHIN_SIGNALING_PATHWA } \\
\mathrm{Y}\end{array}$ & 110 & -0.355 & -1.359 & 0.046904 & 0.140663 & 471 \\
\hline 3 & GO_FEMALE_SEX_DIFFERENTIATION & 67 & -0.409 & -1.435 & 0.047059 & 0.094241 & 472 \\
\hline 3 & $\begin{array}{l}\text { GO_POSITIVE_REGULATION_OF_BLOOD_CIRC } \\
\text { ULATION }\end{array}$ & 41 & -0.455 & -1.443 & 0.047131 & 0.090506 & 473 \\
\hline 3 & GO_REGULATION_OF_ANOIKIS & 21 & -0.549 & -1.529 & 0.048319 & 0.054933 & 474 \\
\hline 3 & GO_REGULATION_OF_PROTEIN_MATURATION & 54 & -0.421 & -1.4 & 0.04845 & 0.113937 & 475 \\
\hline 3 & $\begin{array}{l}\text { GO_DIVALENT_INORGANIC_CATION_TRANSP } \\
\text { ORT }\end{array}$ & 154 & -0.336 & -1.343 & 0.048913 & 0.151296 & 476 \\
\hline 3 & $\begin{array}{l}\text { GO_POSITIVE_REGULATION_OF_PROTEIN_TYR } \\
\text { OSINE_KINASE_ACTIVITY }\end{array}$ & 24 & -0.525 & -1.468 & 0.049462 & 0.077838 & 477 \\
\hline 3 & $\begin{array}{l}\text { GO_POSTTRANSCRIPTIONAL_REGULATION_OF } \\
\text { _GENE_EXPRESSION }\end{array}$ & 340 & -0.282 & -1.239 & 0.049488 & 0.245805 & 478 \\
\hline 3 & $\begin{array}{l}\text { GO_SIGNAL_TRANSDUCTION_IN_RESPONSE_T } \\
\text { O_DNA_DAMAGE }\end{array}$ & 83 & -0.386 & -1.408 & 0.049524 & 0.109547 & 479 \\
\hline 3 & GO_SEX_DIFFERENTIATION & 149 & -0.324 & -1.273 & 0.049904 & 0.211077 & 480 \\
\hline 3 & $\begin{array}{l}\text { GO_ENDOPLASMIC_RETICULUM_CALCIUM_IO } \\
\text { N_HOMEOSTASIS }\end{array}$ & 17 & -0.581 & -1.526 & 0.05 & 0.05559 & 481 \\
\hline
\end{tabular}




\section{Chapter 3. Differential remodeling of the electron transport chain is required to support TLR3 and TLR4 signaling and cytokine production in macrophages (Ahmed et al., 2019; Published in Scientific Reports)}

\subsection{Introduction}

The innate immune system, including tissue macrophages, represent the first line of defence against invading microbial pathogens. Early recognition depends on a variety of pattern recognition receptors (PRRs), which detect evolutionarily conserved structures termed pathogenassociated molecular patterns (PAMPs) $(1,2)$. Key players in this process are the Toll-like receptors (TLR), which are capable of detecting a range of PAMPs from viruses and bacteria (15). Among the best characterized are TLR3 and TLR4. TLR3 recognizes double stranded RNA (dsRNA), a common PAMP associated with viral infections (6). TLR4 primarily recognizes lipopolysaccharide (LPS), the core component of the outer membrane of Gram-negative bacteria (7). Both TLR3 and TLR4 differentially and dynamically modulate nuclear factor- $\mathrm{KB}(\mathrm{NF}-\mathrm{\kappa B})$ and interferon regulatory factory 3 (IRF3) signaling following receptor engagement. While TLR3 activates NF- $\mathrm{kB}$ and IRF3 signaling via TIR-domain-containing adapter-inducing interferon- $\beta$ protein (TRIF), TLR4 signals through both Myeloid differentiation primary response 88 (MyD88) and TRIF (1). Differential activation of these signaling pathways plays a critical role in fine-tuning pathogen specific antiviral and antibacterial responses (1).

Cellular metabolism has emerged as a key regulator of macrophage function. Metabolic reprogramming is required to meet the bioenergetic and biosynthetic demands of the cell and to drive effector functions (8-10). Alterations in metabolites and other bioactive metabolic products have also been shown to activate and regulate gene expression, signal transduction and epigenetic profiles $(9,11-14)$. Among the best characterized examples of macrophage reprogramming occurs following LPS stimulation $(9,14-18)$. Almost immediately after TLR4 engagement, macrophages 
downregulate oxidative phosphorylation (OXPHOS) activity and dramatically increase glycolysis to support rapid ATP production $(14,15,17-19)$. This repurposing of mitochondrial function also increases reactive oxygen species (ROS) production, which drives inflammatory cytokine production $(9,15,17)$. ROS production is driven by the combined effects of increased mitochondrial membrane potential and the oxidation of succinate by complex II of the electron transport chain (ETC) (15) suggesting flux through the ETC may play a central role in this process. However, it is still unclear if the dynamic modulation of ETC complexes and increased ROS production contributes to signaling events following engagement of other TLRs and if differential reprogramming of these processes contributes to ligand specific immune responses.

Mitochondria are known to play an important role in innate immune responses against RNA viruses (20-23). Recognition of cytosolic viral RNA by retinoic acid-inducible gene I (RIGI)-like receptors (RLR) and their downstream processes have been shown to require the participation of mitochondrial antiviral signaling (MAVS), a mitochondrial outer membrane adaptor protein $(20,24,25)$. MAVS acts as a scaffold and recruits effector molecules to the mitochondrial outer membrane leading to the activation of NF-kB and IRF3(20). More recent studies have shown that mitochondria also contribute to antiviral signaling via MAVS independent mechanisms. Tal et al. found that mitochondrial ROS (mtROS) potentiates RLR signaling. This signaling is regulated by autophagy and clearance of dysfunctional mitochondria (26). Alternatively, Yoshizumi et al. found that RLR-mediated antiviral responses are dependent on OXPHOS activity. This dependence is regulated by the mitochondrial fusion protein optic atrophy 1 (OPA1) (23). These findings suggest that other aspects of mitochondrial function, independent of MAVS mediated scaffolding, may play a central role in facilitating antiviral responses. 
While both TLR3 and RLR recognize and respond to virally derived RNAs, they signal through distinct transduction pathways to trigger antiviral immune responses $(27,28)$. To date, the role of the mitochondria in driving TLR3-mediated responses in macrophages is poorly understood. Previous studies have shown that engagement of TLR3 on hepatocytes and dendritic cells (DCs) causes a shift from OXPHOS towards aerobic glycolysis for energy production (2932). In DCs, this shift is driven predominately by the de novo production of type I interferons (IFN) and therefore is required to rapidly meet the increased energy demands of these activated cells (3032). Macrophages stimulated with high concentrations $(10 \mu \mathrm{g} / \mathrm{mL})$ of the synthetic TLR3 agonist polyinosinic-polycytidylic acid (Poly(I:C) or PIC) have been shown to downregulate Complex Iassociated ATP production under standard culture conditions (33). However, the functional consequences of this ETC reprogramming has yet to be elucidated.

In the current study, I used murine bone marrow-derived macrophages (BMM) to evaluate how reprogramming of mitochondrial function contributes to TLR3 and TLR4 signaling and cytokine production and how glucose availability affected these responses. I found that modulation of flux through the ETC and associated ROS production plays a critical role in cytokine production following TLR engagement. This reprogramming is ligand specific and may have differential effects on the expression of individual cytokines (e.g. accumulation of mitochondrial vs. cytosolic ROS). Further, low glucose conditions resulted in differential reprogramming of mitochondrial function following TLR3 engagement. This reprogramming upregulated complex III expression and associated mitochondrial ROS production, which amplified inflammatory and antiviral signaling and cytokine production. Collectively, these findings suggest that the ETC may act as a selective rheostat of innate immune responses that differentially regulates ligand specific responses based on nutrient availability. 


\subsection{Results}

\subsubsection{Differential production of pro-inflammatory and antiviral cytokines in PIC-and LPS- stimulated BMM.}

Despite activating the same transcription factors (e.g. NF- $\kappa B$ and IRF3), signaling through TLR3 and TLR4 are associated with distinct inflammatory and antiviral cytokine profiles. To evaluate these differences in our model system, I stimulated BMM with PIC (10ng/ml and $10 \mu \mathrm{g} / \mathrm{ml})$ or LPS $(100 \mathrm{ng} / \mathrm{ml})$ for 18 hours and assessed inflammatory (TNF- $\alpha$, IL-1 $\beta$, and IL-6) and antiviral (IFN- $\alpha$, IFN- $\beta$ and CXCL10) cytokine production in culture supernatants. PIC concentrations were selected to emulate responses in early (low levels of virus) and late stages of infection (high levels of virus), where Lin et al. found that only high $(\geq 10 \mu \mathrm{g} / \mathrm{ml})$ concentrations of PIC can induce robust inflammatory cytokine production (34). The LPS concentration was selected based on its ability to repurpose mitochondrial function to support ROS production (15). As previously reported (15), LPS induced a strong inflammatory response, produced intermediate levels of IFN- $\beta$ and CXCL10 and no IFN- $\alpha$ (Figure 3.1). Alternatively, stimulation with low concentrations of PIC induced low levels of antiviral and inflammatory production. Increasing the PIC concentration $(10 \mu \mathrm{g} / \mathrm{ml})$ significantly increased both inflammatory and antiviral cytokine production (Figure 3.1). 

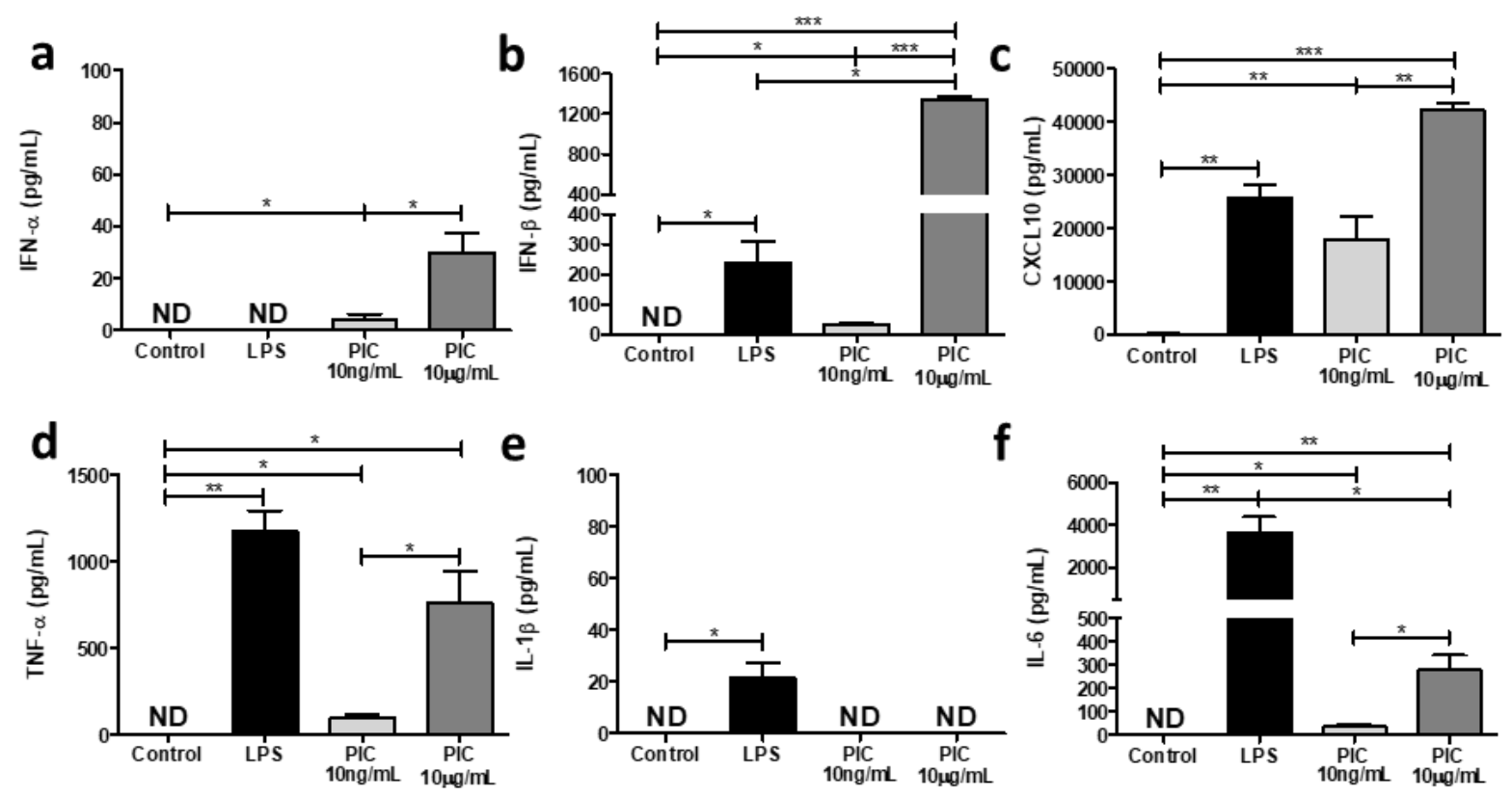

Figure 3.1: High, but not low, concentrations of Poly(I:C) are associated with proinflammatory cytokine production. Bone marrow-derived macrophages (BMDMs) were treated with either $100 \mathrm{ng} / \mathrm{mL}$ lipopolysaccharide (LPS), $10 \mathrm{ng} / \mathrm{mL}$ or $10 \mu \mathrm{g} / \mathrm{mL}$ Poly(I:C) (PIC) for 18 hours. Supernatant was collected and assessed for antiviral (IFN- $\alpha$, IFN- $\beta$, CXCL10) (a-c) and pro-inflammatory (TNF- $\alpha$, IL-1 $\beta$, IL-6) (d-f) cytokine expression. Data represents mean \pm SEM of four individual mice $(* \mathrm{p}<0.05, * * \mathrm{p}<0.01$, and $* * * \mathrm{p}<0.001)$. 


\subsubsection{BMM stimulated with low versus high concentrations of PIC differ in their ability to ramp up glycolytic activity under stress.}

Next, I evaluated the differential effects of LPS and PIC stimulation on cellular metabolism using the Seahorse extracellular efflux analyser. As described above, BMM were stimulated with LPS (100ng/ml), low (10ng/ml) or high $(10 \mu \mathrm{g} / \mathrm{ml})$ concentrations of PIC for 18 hours. Changes in proton efflux rate (PER) at baseline and in response to rotenone + antimycin (Rot/AA) and 2deoxyglucose (2-DG) injections were used to evaluate changes in glycolytic parameters. As previously described $(9,35)$, LPS stimulation increased PER levels at baseline, increased the \%PER derived from glycolysis and decreased the mitoOCR/glycoPER ratio suggesting a strong shift away from OXPHOS activity towards aerobic glycolysis (Figure 3.2). Stimulation with both concentrations of PIC also increased PER levels at baseline and increased the \%PER derived from glycolysis, but this increase was significantly lower than that observed following LPS-stimulation $(\mathrm{P}<0.001)$. Further, the reduction in the mitoOCR/glycoPER ratio was less pronounced suggesting that PIC-stimulated cells maintain higher levels of OXPHOS activity (Figure 3.2C). Despite similar basal PER levels (Figure 3.2B), low and high concentrations of PIC differentially affected the ability of BMM to ramp up glycolysis following stress with Rot/AA. While cells stimulated with lower concentrations maintained their ability to increase glycolytic activity following exposure to Rot/AA, cells stimulated with higher concentrations were unable to do so, suggesting they may be functioning at their maximum glycolytic capacity (Figure 3.2A). 

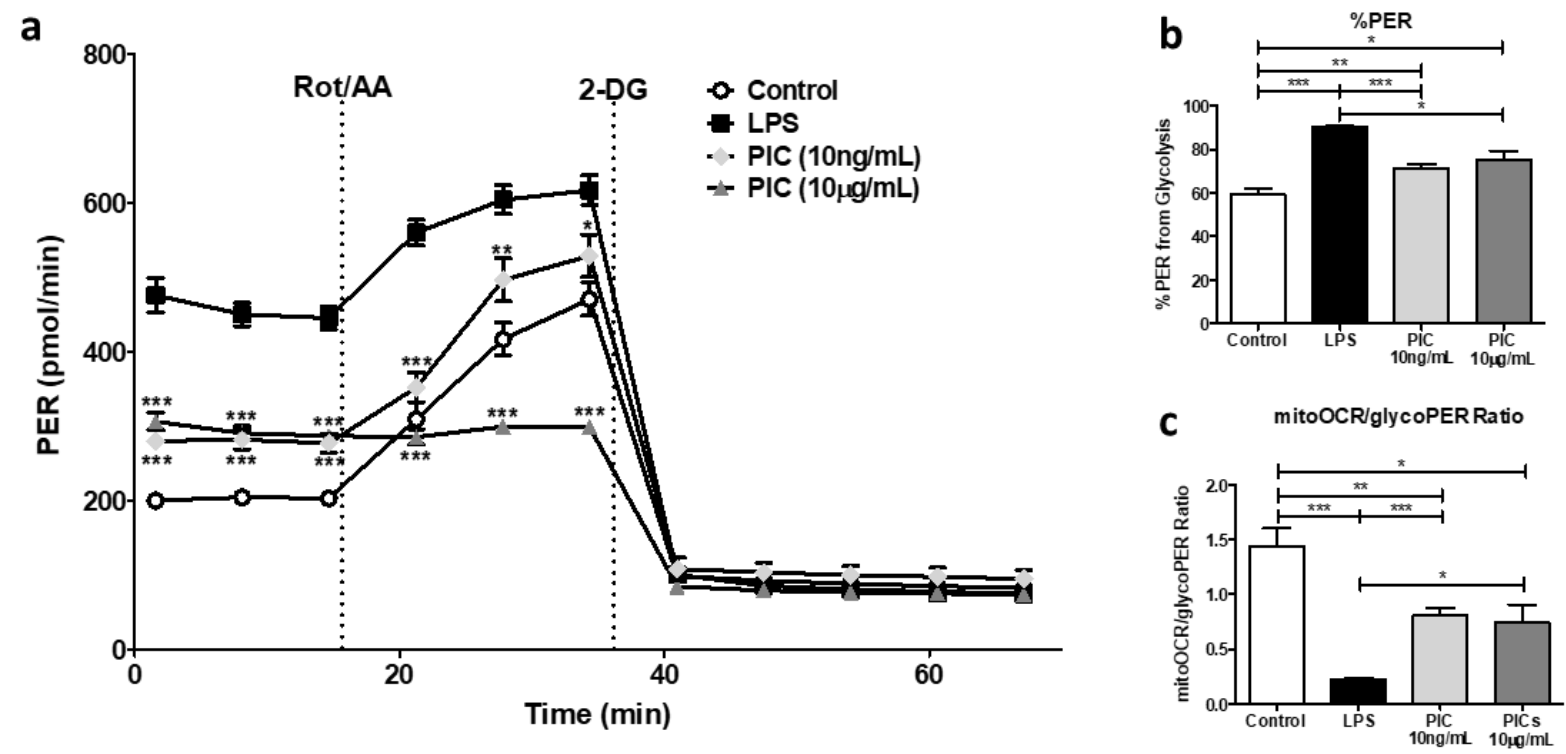

Figure 3.2: Macrophages activated using higher concentrations of $\mathrm{Poly}(\mathrm{I}: \mathrm{C})$ are functioning near their maximum glycolytic capacity. BMDMs were seeded onto Seahorse XFp miniplates and treated with $100 \mathrm{ng} / \mathrm{mL}$ LPS, $10 \mathrm{ng} / \mathrm{mL}$ or $10 \mu \mathrm{g} / \mathrm{mL}$ PIC for 18 hours. Glycolytic activity, indicated by the proton efflux rate (PER) was measured using sequential injections of rotenone plus antimycin A (Rot/AA) and 2-deoxyglucose (2-DG) (a), determining the \%PER dependent on glycolysis (b) and the ratio of mitochondrial oxygen consumption rate (mitoOCR) to glycolytic PER (c). Data represents mean \pm SEM of four individual mice. The levels of significance shown in Figure 2a represent pairwise comparisons against LPS-treated macrophages $\left({ }^{*} \mathrm{p}<0.05,{ }^{* *} \mathrm{p}<\right.$ 0.01 , and $* * * \mathrm{p}<0.001)$. 


\subsubsection{Maintenance of OXPHOS activity is an important for PIC but not LPS stimulation.}

To evaluate specific changes in OXPHOS activity, I used the Cell Mito Stress Test kit from Agilent. Features of OXPHOS activity were calculated based on changes in oxygen consumption rate (OCR) in response to successive injections of oligomycin (Oligo), carbonyl cyanide 4(trifluoromethoxy)phenylhydrazone (FCCP) and ROT/AA. Consistent with the literature (9, 19, 36), LPS stimulation dramatically reduced basal respiration, reduced mitochondrial ATP production, and reduced the ability of cells to increase oxygen consumption (e.g. spare respiratory capacity [SRC]) following FCCP treatment (Figure 3.3). High concentrations of PIC also reduced levels of basal respiration, ATP production and SRC compared to untreated cells but this impairment was less severe than that observed for LPS (basal respiration $\mathrm{p}<0.05$, SRC $\mathrm{p}<0.01$, ATP production $\mathrm{P}=0.08$ ). Interestingly, low concentrations of PIC did not alter basal respiration or ATP production but significantly reduced SRC suggesting these cells may have a reduced capacity to deal with stress (Figure $3.3 ; 37$ ). Given these differences in glycolysis and oxygen consumption the remaining experiments were performed using low concentration PIC (10ng/ml). 

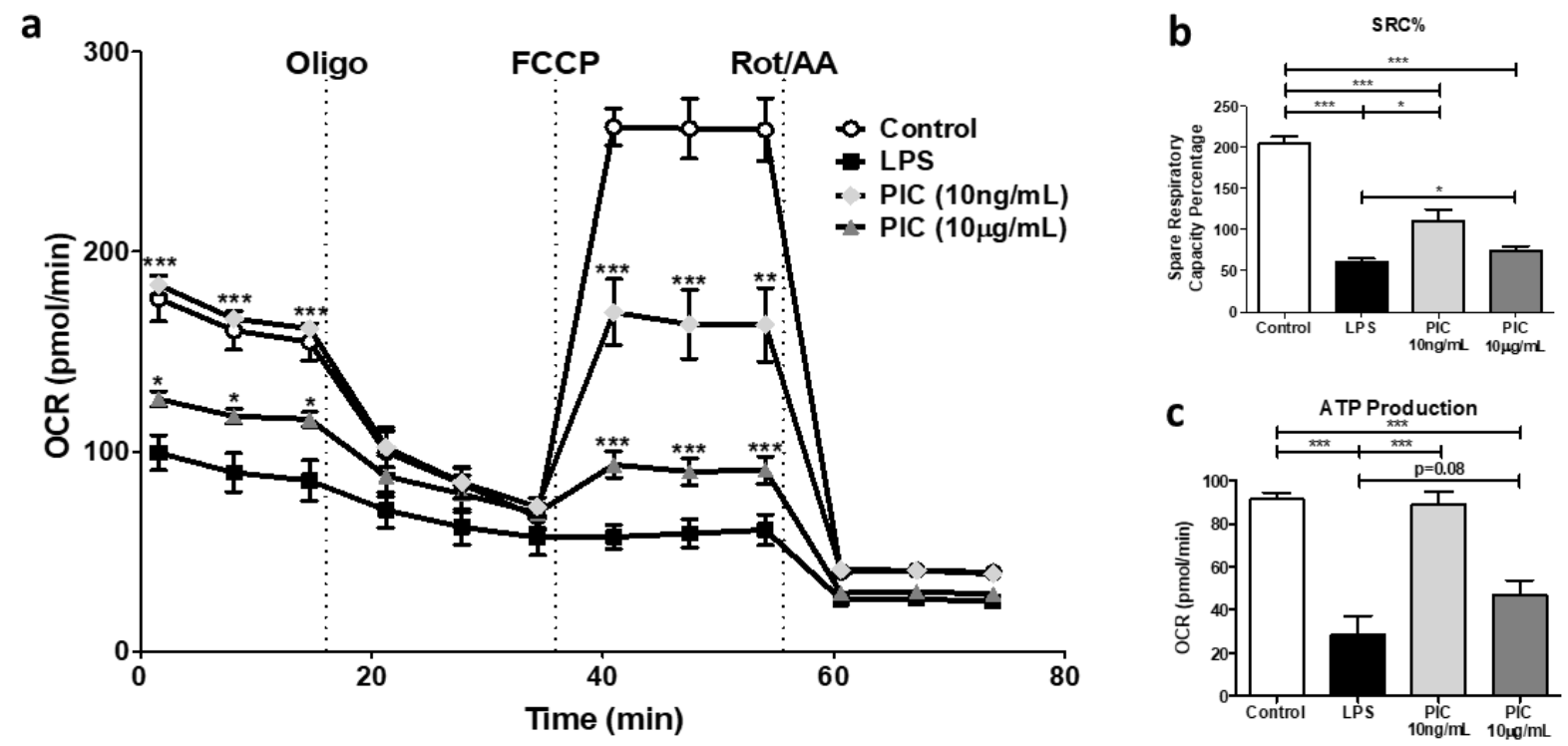

Figure 3.3: Poly(I:C) stimulation is linked to low sustained levels of oxidative phosphorylation (OXPHOS). Macrophages were plated onto Seahorse XFp miniplates and subsequently stimulated with $100 \mathrm{ng} / \mathrm{mL}$ LPS, $10 \mu \mathrm{g} / \mathrm{mL}$ or $10 \mathrm{ng} / \mathrm{mL}$ PIC for 18 hours. OXPHOS function was assessed via successive Oligomycin (Oligo), Carbonyl cyanide- $p$ trifluoromethoxyphenylhydrazone (FCCP), and Rot/AA injections (a), quantifying the spare respiratory capacity percentage (SRC\%) (b) and ATP production (c). Data represents mean $\pm \mathrm{SEM}$ of four individual mice. The levels of significance shown in Figure $3 \mathrm{a}$ is based on pairwise comparisons to LPS-treated macrophages $(* \mathrm{p}<0.05, * * \mathrm{p}<0.01$, and $* * * \mathrm{p}<0.001)$. 


\subsubsection{Reduced glucose availability is associated with increased inflammatory and antiviral cytokine production in PIC- but not LPS-stimulated BMM}

Macrophages are highly plastic cells whose responses are modified by environmental cues including nutrient availability (38-41). A recent study in BMM showed that cells are less dependent on OXPHOS activity under condition of high glucose $(>10 \mathrm{mM})$ and preferentially use aerobic glycolysis to rapidly produce ATP (23). Given the differential ability of BMM to reprogram OXPHOS activity based on glucose availability, I then evaluated how glucose levels affected TLR3 and TLR4 signaling and cytokine production. Most studies have been performed in standard culture conditions, which represent supra-physiological concentrations of glucose. For these studies, BMM were stimulated with LPS $(100 \mathrm{ng} / \mathrm{ml})$ and PIC $(10 \mathrm{ng} / \mathrm{ml})$ in standard $(25 \mathrm{mM})$ and low glucose $(0.5 \mathrm{mM})$ conditions. For the remainder of this paper, standard culture conditions will be referred as high glucose. Glucose levels had no effect on pro-inflammatory cytokine production in LPS-stimulated cells (Figure 3.4A-E) suggesting that even low glucose levels are sufficient to support TLR4 responses. Conversely, low glucose conditions increased proinflammatory (TNF- $\alpha$, IL-6) and IFN-associated cytokine production (IFN- $\alpha$, IFN- $\beta$, CXCL10) following PIC stimulation suggesting that glucose availability may fine tune the magnitude of the TLR3 response (Figure 3.4A-E). Interestingly, I found that high levels of glucose increase baseline OCR levels, spare respiratory capacity, and ATP production in untreated/resting cells. However, following PIC stimulation, high glucose further reduced basal OCR, spare respiratory capacity and ATP production suggesting these conditions may alter TLR3 associated mitochondrial reprogramming (Supplemental Figure S3.1).

To determine if this amplification was associated with altered signaling, I evaluated alterations in TLR3 and TLR4 adaptor protein expression and transcription factor phosphorylation following stimulation with LPS and PIC under high and low glucose conditions. While TRIF and 
TRAF6 expression was not affected by glucose levels (Supplemental Figure S3.2), low glucose was associated with a dramatic increase in total and phosphorylated IRF3 in PIC-stimulated BMM. Consistent with its more downstream role $(42,43)$, I also found IRF7 phosphorylation was delayed but sustained at high levels in low glucose conditions (Figures 3.4F and 3.4G). Furthermore, I found that levels of phosphorylated IкB $\alpha$ were increased in low glucose conditions in PIC but not LPS-stimulated cells, which may explain the increased TNF- $\alpha$ and IL-6 production following TLR3 engagement (Figure 3.4H). Collectively, these results suggest that high glucose may limit mitochondrial reprogramming and associated antiviral and pro-inflammatory signaling and cytokine production in a TLR3 specific manner. 

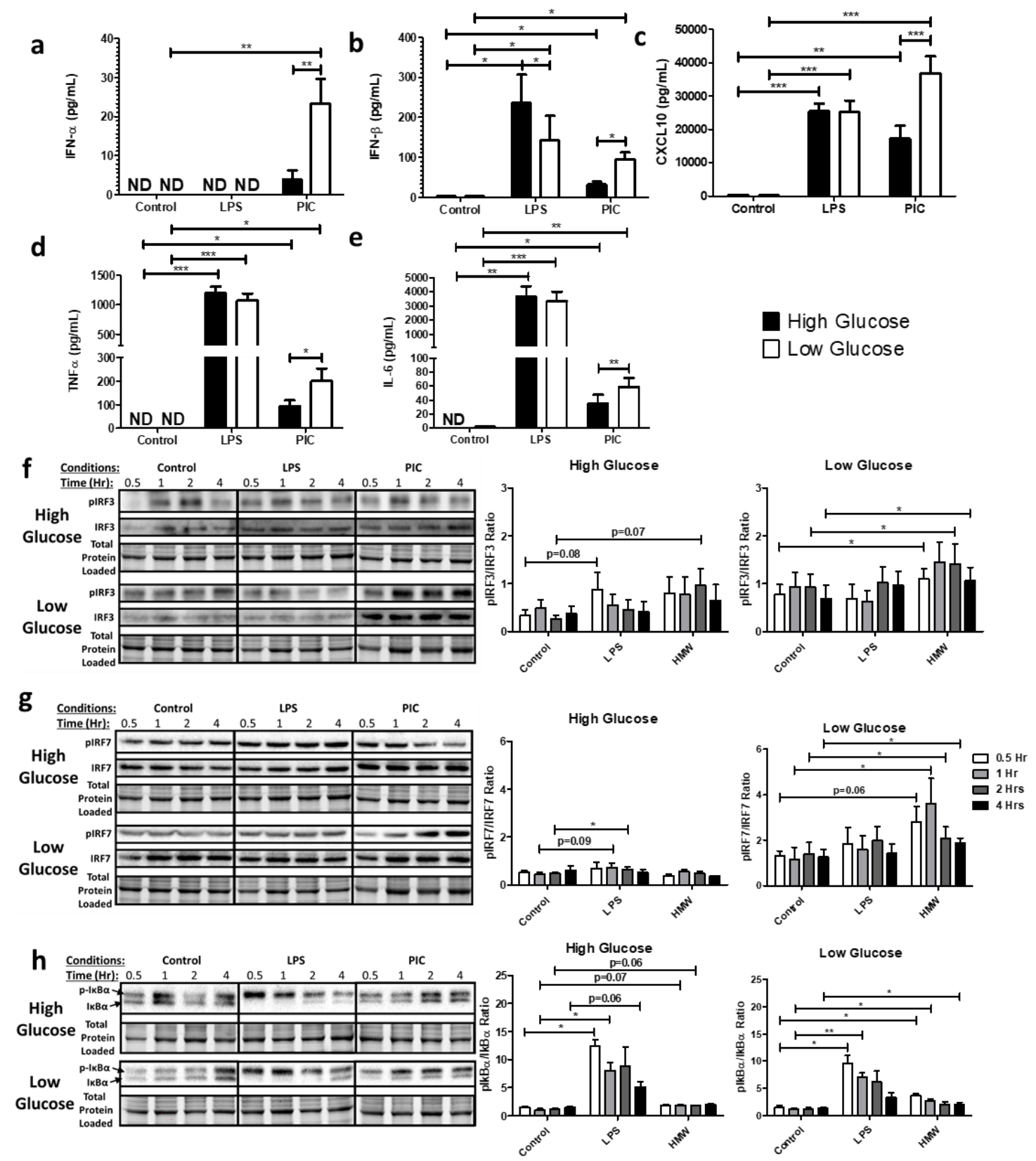

Figure 3.4: Low glucose conditions are associated with increased IRF activation and increased type I IFN production. Macrophages were stimulated with either $100 \mathrm{ng} / \mathrm{mL}$ LPS or $10 \mathrm{ng} / \mathrm{mL}$ PIC for 18 hours under high glucose $(25 \mathrm{mM})$ or low glucose $(0.5 \mathrm{mM})$ media conditions. Supernatant was collected for assessing antiviral (IFN- $\alpha$, IFN- $\beta$, CXCL10) (a-c) and pro-inflammatory (TNF- $\alpha$, IL-6) cytokine (d-e) expression. Cell lysates were harvested to quantify IRF3 (f), IRF7 (g) and Ikb $\alpha$ (h) expression via immunoblotting. Data represents mean \pm SEM of four individual mice $(* \mathrm{p}<0.05, * * \mathrm{p}<0.01$, and $* * * \mathrm{p}<0.001)$. 
a

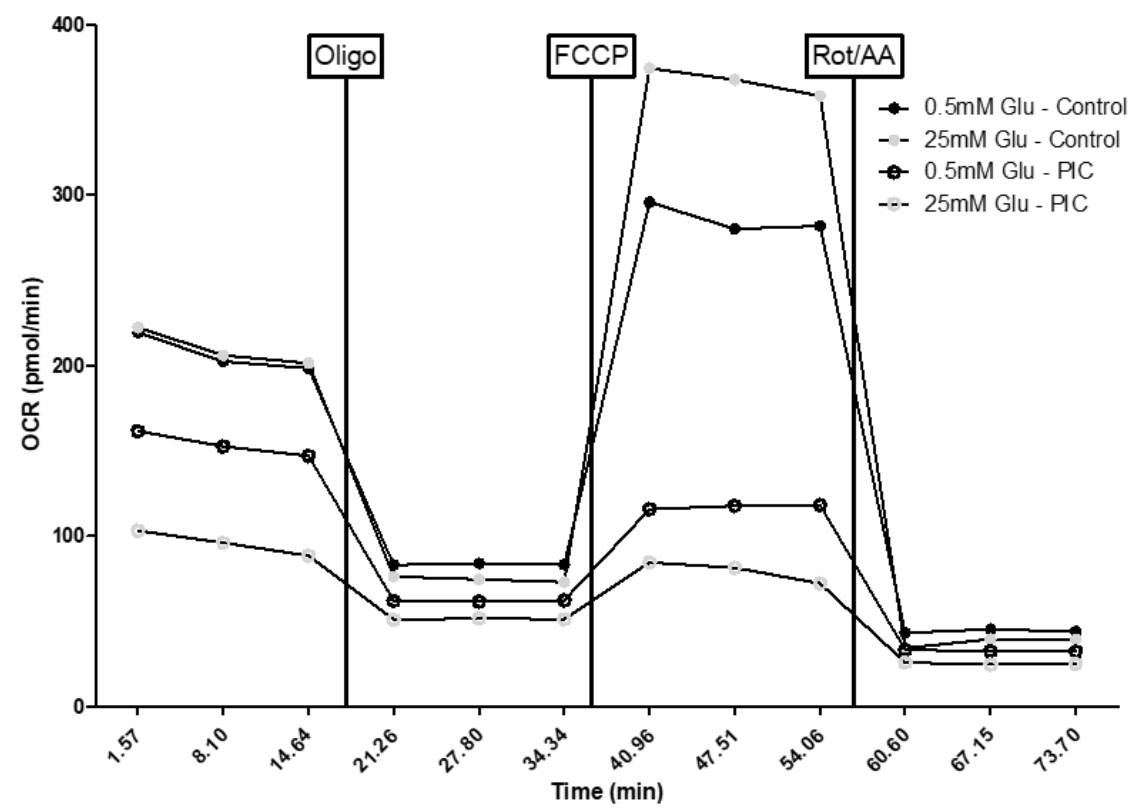

b

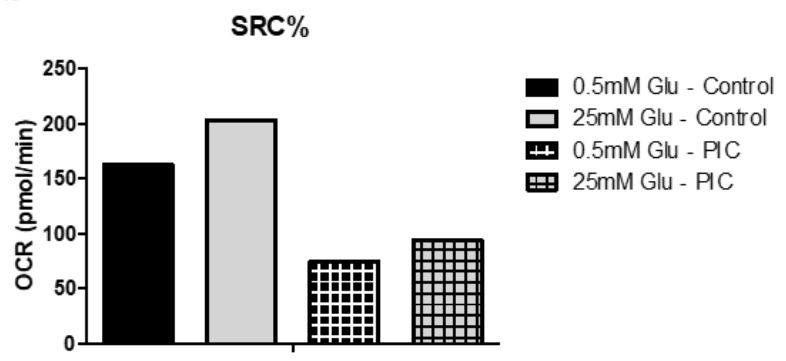

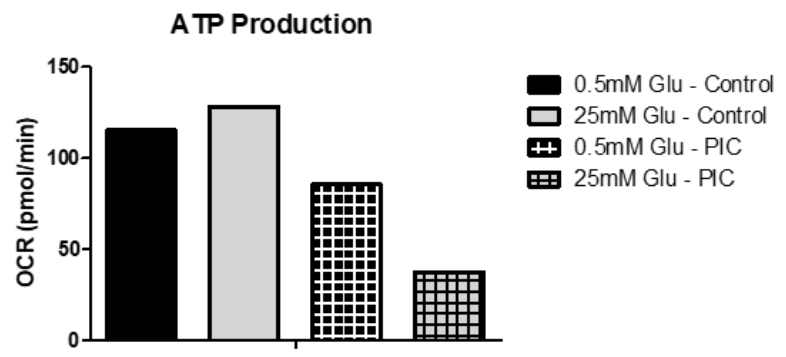

Supplemental Figure S3.1: PIC activation is associated with increased OXPHOS function under low glucose conditions. BMDMs were plated onto Seahorse XFp miniplates and treated with $10 \mu \mathrm{g} / \mathrm{mL}$ PIC for 18 hours. OXPHOS function was assessed via successive Oligomycin (Oligo), Carbonyl cyanide-p-trifluoromethoxyphenylhydrazone (FCCP), and Rot/AA injections (a). Quantification of the spare respiratory capacity percentage (SRC\%) (b) and ATP production (c). Data shown represents a test run using one representative animal. 
a
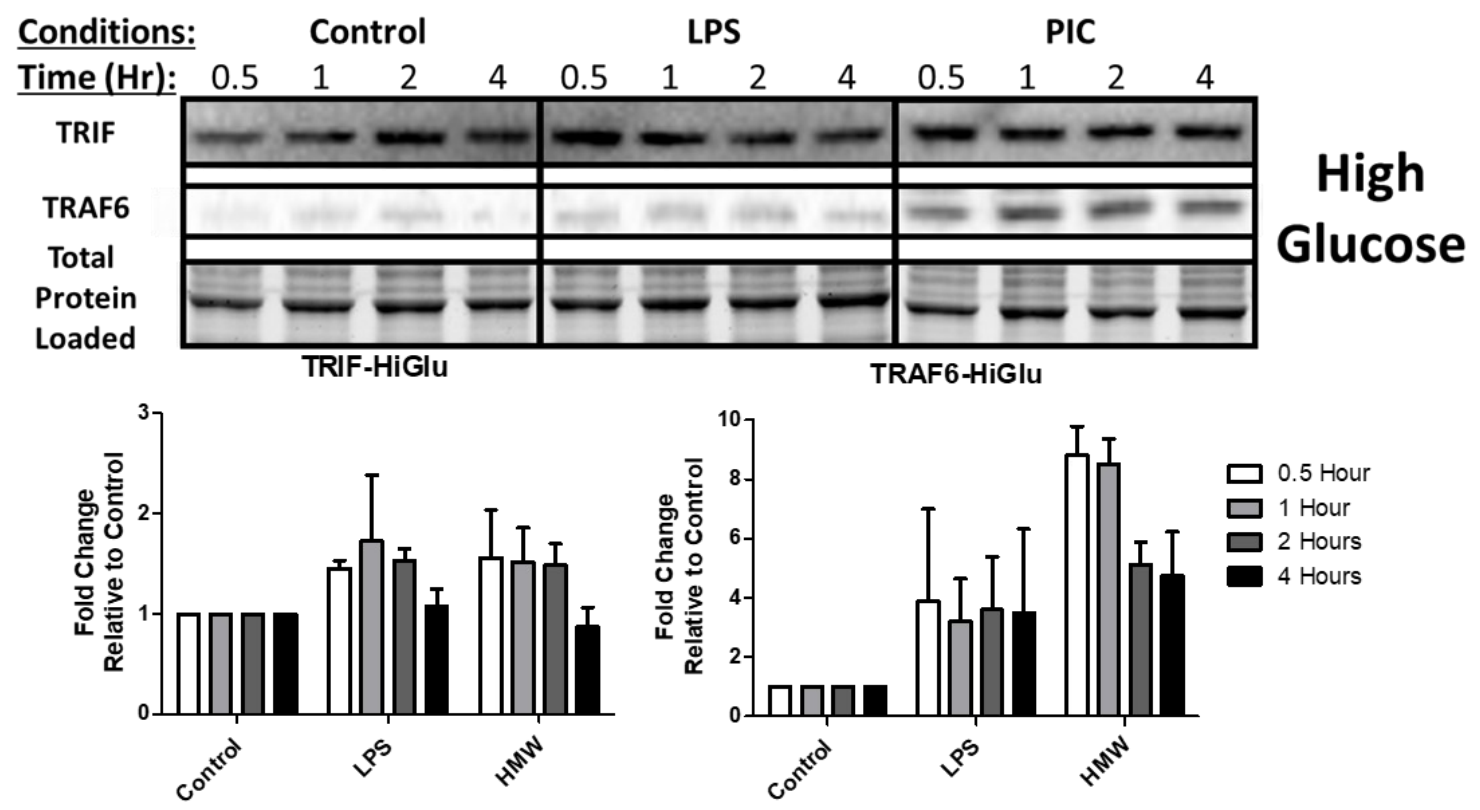

b

\begin{tabular}{llllllllllllll} 
Conditions: & \multicolumn{3}{c}{ Control } & \multicolumn{4}{c}{ LPS } & \multicolumn{4}{c}{ PIC } \\
Time (Hr): & 0.5 & 1 & 2 & 4 & 0.5 & 1 & 2 & 4 & 0.5 & 1 & 2 & 4
\end{tabular}
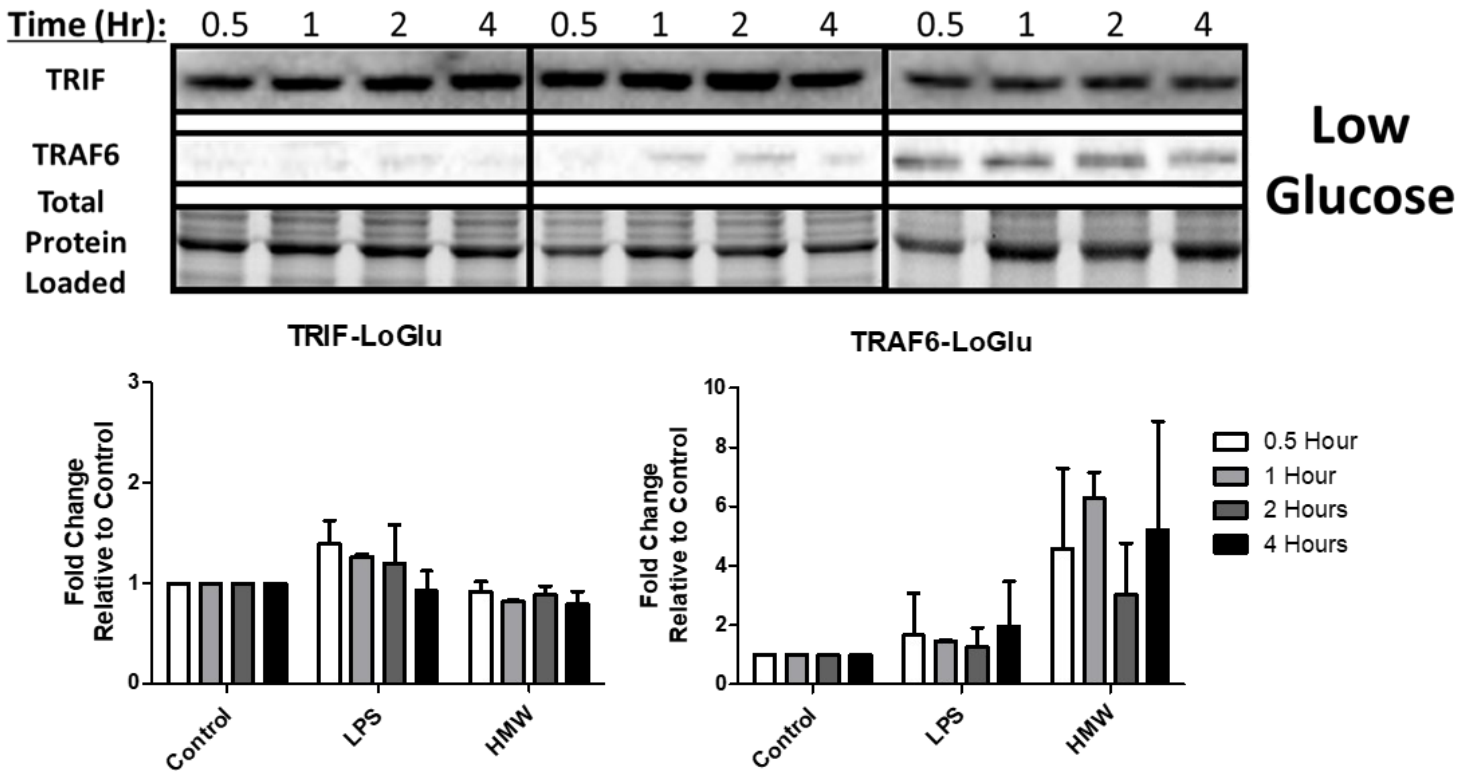

Supplemental Figure S3.2: TRIF and TRAF6 expression during PIC activation is not affected by glucose levels. Macrophages stimulated either with LPS or PIC for 18 hours under high glucose (a) or low glucose (b) media conditions were examined for differences in TLR signaling. Protein levels of TRIF and TRAF6 were measured via immunoblotting. Data shown represents blots from a representative animal while data shown represents mean \pm SEM of two individual mice. 


\subsubsection{Reduced glucose availability is associated with altered mitochondrial membrane potential and ETC complex expression following TLR engagement.}

To better understand how glucose levels affect TLR3 associated alterations in OXPHOS activity, I examined alterations in mitochondrial membrane potential (MMP) and ETC complex expression in high and low glucose conditions. MMP was assessed using the fluorescent dye Tetramethylrhodamine (TMRM). As previously described (15), LPS stimulation was associated with increased sequestration of TMRM by activated mitochondria (TMRM Mean Fluorescence Intensity $[\mathrm{MFI}]$ ) in both high and low glucose conditions (Figure 3.5A). Conversely, PICstimulation did not significantly increase levels of TMRM sequestration in positive cells. Instead, PIC was associated with a significant increase in the number of cells expressing low levels of TMRM, which further increased under low glucose conditions ( $24 \%$ vs. $38 \%, p=0.09)$. To evaluate if altered ETC flux contributes to altered membrane potential, I examined ETC complex expression following LPS and PIC stimulation in high versus low glucose conditions. In high glucose conditions, alterations in expression were highly variable across animals. LPS-stimulation moderately decreased complex II (SDHB) expression whereas PIC increased complex IV (COX4) (Figure 3.5B). Alterations in ETC complex expression were more pronounced in low glucose conditions. Specifically, both LPS and PIC were associated with decreased expression of complexes I and IV. The only alteration unique to PIC in the low glucose condition was the significant increase in complex III (Figure 3.5B). In addition to its role as a proton pump, complex III is a major generator of mtROS (44) and may contribute to the amplification of inflammatory and antiviral cytokine production following TLR3 engagement under low glucose conditions. 
a
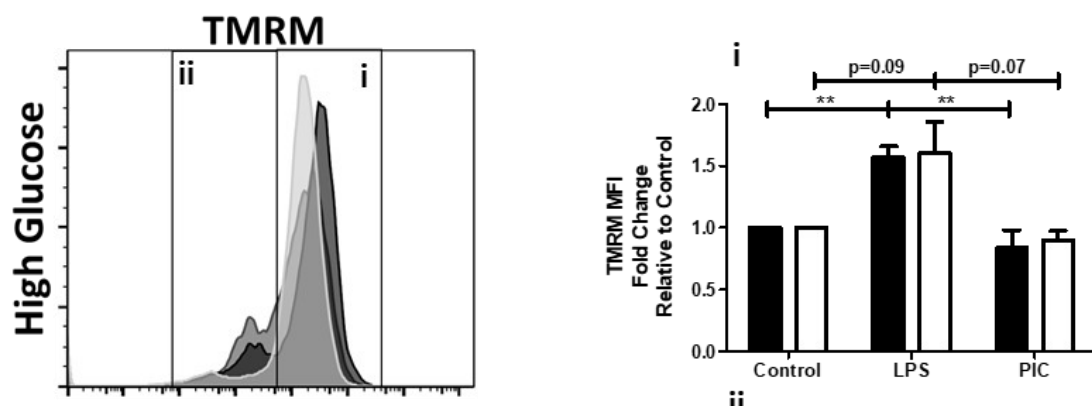

High Glucose
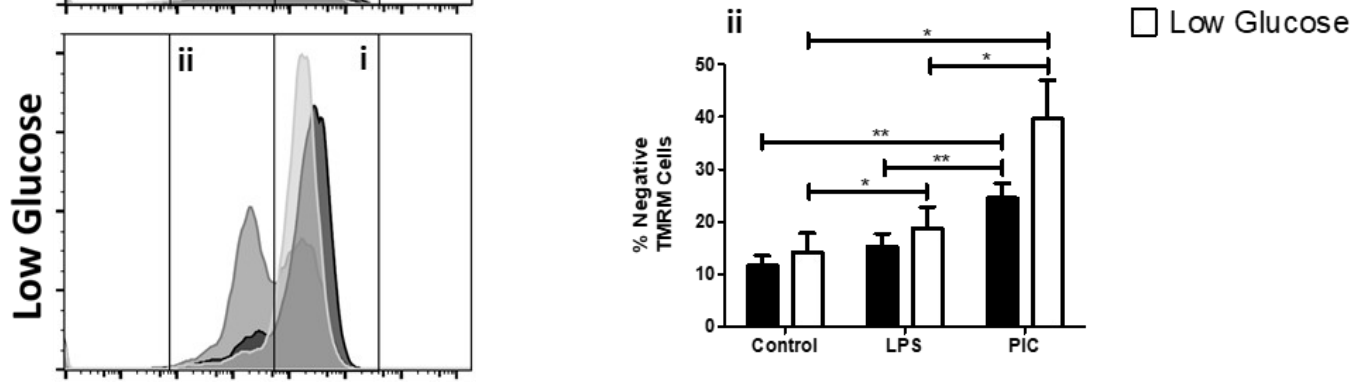

b
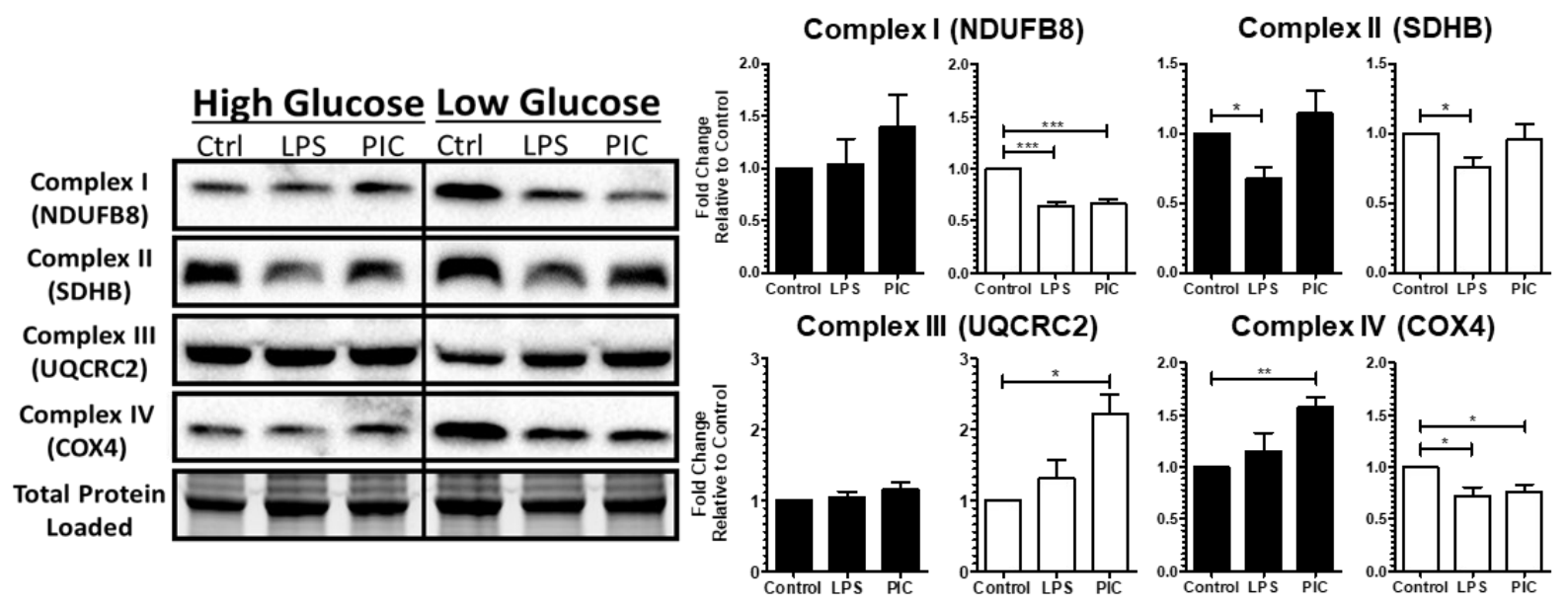

Figure 3.5: Poly(I:C) activation is linked to altered mitochondrial activity under low glucose conditions. BMDMs treated with LPS or PIC for 18 hours under high glucose or low glucose media conditions were characterized for differences in mitochondrial function. Tetramethylrhodamine (TMRM) staining was used to measure, via flow cytometry, mitochondrial membrane potential (a). Core protein levels of Complexes I-IV of the electron transport chain was quantified via immunoblotting (b). Data represents mean \pm SEM of four individual mice $(* \mathrm{p}<$ $0.05, * * \mathrm{p}<0.01$, and $* * * \mathrm{p}<0.001)$. 


\subsubsection{Flux through the ETC chain is required for inflammatory and antiviral cytokine production following TLR engagement.}

To evaluate the relative contribution of specific ETC complexes to cytokine production, BMM were stimulated with LPS $(100 \mathrm{ng} / \mathrm{ml})$ and PIC $(10 \mathrm{ng} / \mathrm{ml})$ under high and low glucose conditions in the presence or absence of Rotenone (Complex I inhibitor), Antimycin (Complex III inhibitor) and Cyanide (Complex IV inhibitor). Inhibition of Complex III and IV significantly

reduced CXCL10 (III: $\downarrow 51 \%$; IV: $\downarrow 72 \%$ ) and TNF production (III: $\downarrow 54 \%$; IV: $\downarrow 77 \%$ ) following LPS stimulation (Figure 3.6A). Under low glucose conditions, inhibition of Complex I also limited LPS-associated cytokine production (CXCL10: $\downarrow 57 \%$; TNF: $\downarrow 55 \%$ ). Similarly, inhibition of Complexes I, III and IV significantly reduced inflammatory (TNF) and antiviral cytokine production (IFN- $\alpha$, IFN- $\beta$, CXCL10) following PIC stimulation (Figure 3.6B, Supplemental Figure S3.3). The magnitude of this inhibition was further amplified under low glucose conditions, particularly for complex III (CXCL10: $\downarrow 70 \%$ vs $\downarrow 92 \%$; TNF- $\alpha$ : $\downarrow 78 \%$ vs. $\downarrow 89 \%$; IFN- $\beta$ : $\downarrow 79 \%$ vs. $\downarrow 88 \%$ ). These results suggest that ETC flux is required for inflammatory and antiviral cytokine production and that under low glucose conditions, alterations in complex III expression may play a central role in the amplification of these responses following TLR3 engagement. 

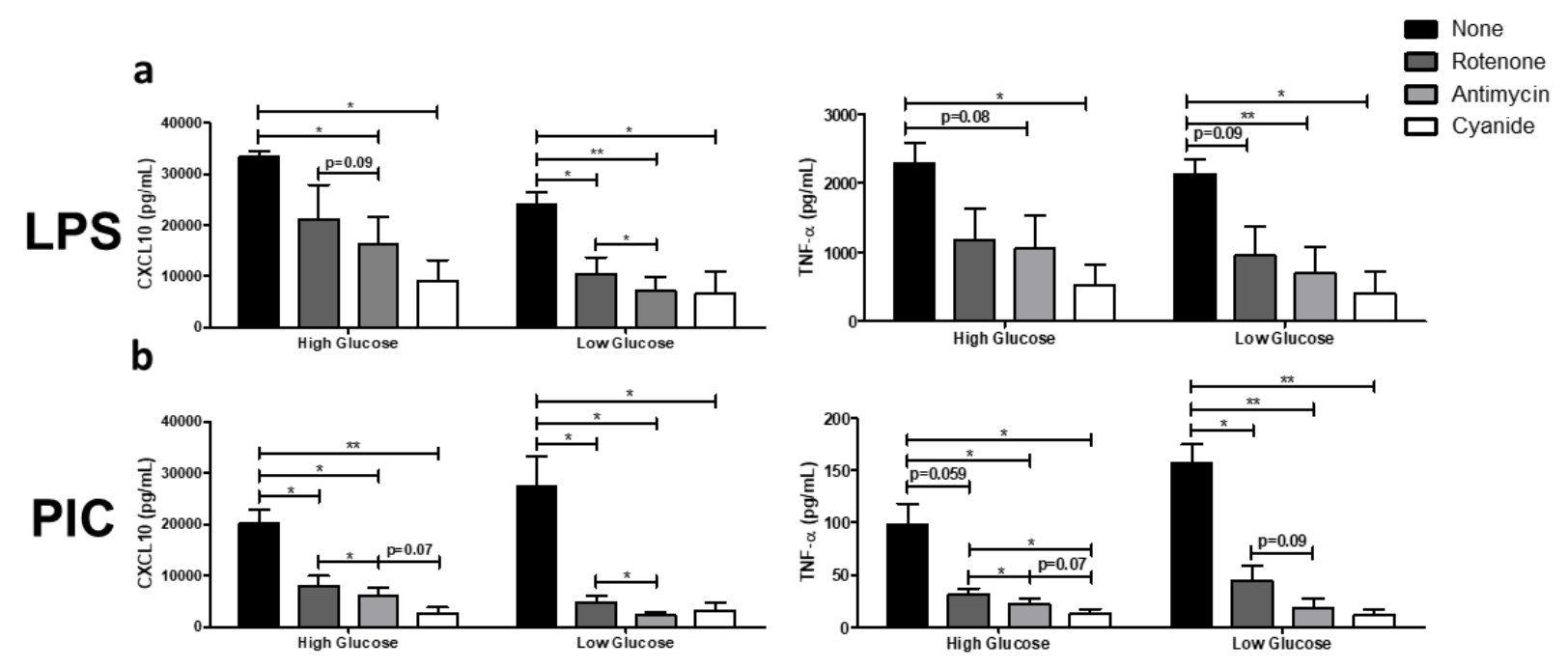

Figure 3.6: Targeting ETC activity reduces type I IFN-mediated responses during Poly(I:C) activation. LPS- (a) or PIC- (b) stimulated BMDMs were co-treated with a panel of ETC inhibitors (Rotenone, Antimycin, Cyanide) to assess the importance of mitochondrial function for antiviral responses. CXCL10 and TNF- $\alpha$ cytokine secretion was measured after 18 hours in high glucose or low glucose media conditions. Data represents mean $\pm \mathrm{SEM}$ of three individual mice $\left({ }^{*} \mathrm{p}<0.05,{ }^{* *} \mathrm{p}<0.01\right.$, and $\left.{ }^{* * *} \mathrm{p}<0.001\right)$. 

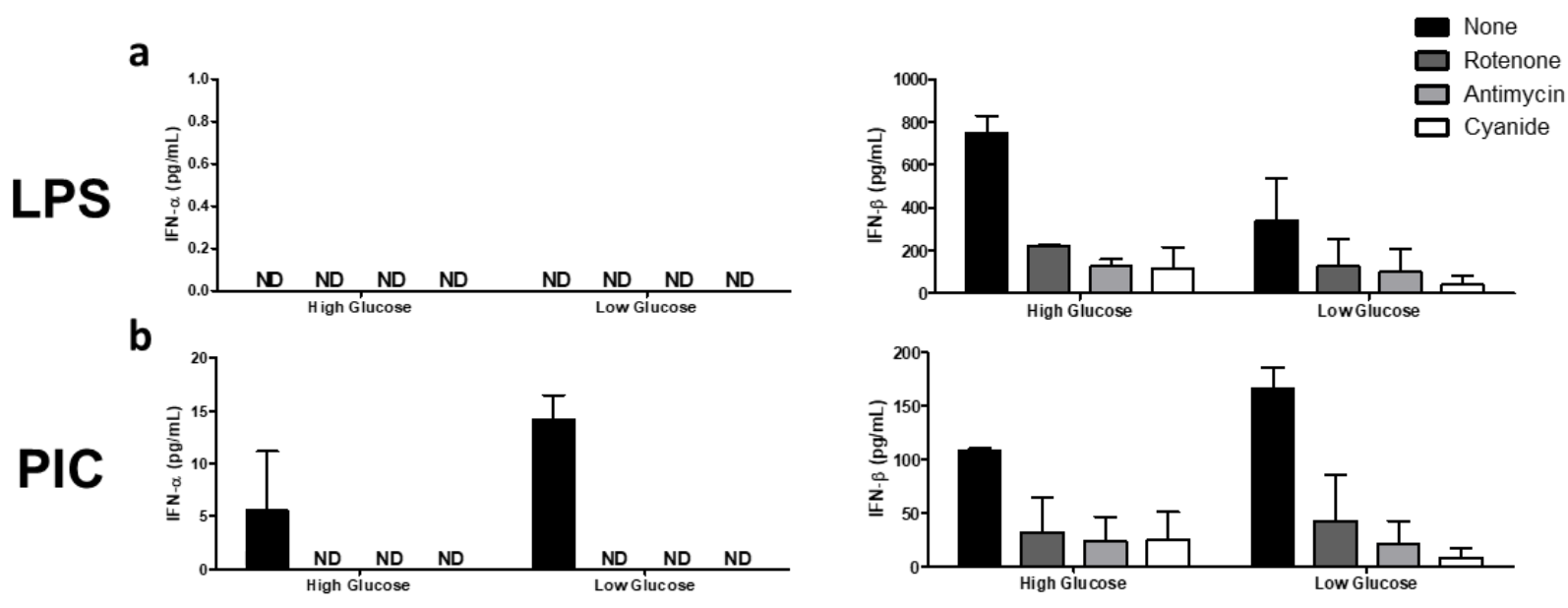

Supplemental Figure S3.3: Targeting ETC function in BMM leads to complete loss of type I IFN responses. LPS- (a) or PIC- (b) stimulated BMDMs were co-treated with a panel of ETC (Rotenone, Antimycin, Cyanide) inhibitors to assess the importance of mitochondrial function to antiviral responses. CXCL10 and TNF- $\alpha$ cytokine secretion was measured after 18 hours in high glucose or low glucose media conditions. Data shown represents a mean of two individual animals. 


\subsubsection{Mitochondrial and cytosolic ROS accumulate in PIC stimulated BMM under low glucose conditions.}

Given the central role of complexes I and III in driving mitochondrial ROS production, I next quantified mitochondrial superoxide production using the fluorescent probe MitoSOX ${ }^{\mathrm{TM}}$ Red. As previously reported (45), LPS stimulation was associated with increased mitochondrial superoxide production compared to untreated BMM. This increase was unaffected by glucose availability (Figure 3.7A). PIC stimulation was also associated with increased mitochondrial superoxide production (Figure 3.7A); however, its production was further increased under low glucose conditions. To determine if this superoxide accumulation was associated with altered antioxidant expression, cellular levels of superoxide dismutase 2 (SOD2) and mitochondrial glutathione peroxidase 4 (mtGPX4) were evaluated via western blots. In high glucose conditions, both LPS and PIC stimulated cells significantly increased SOD2 (LPS-FC=2.56 \pm 0.44 ; PIC$\mathrm{FC}=1.75 \pm 0.40)$ and $\mathrm{mtGPX} 4$ expression (LPS-FC $=1.40 \pm 0.07 ; \mathrm{PIC}-\mathrm{FC}=1.48 \pm 0.22$ ) levels (Figure 3.7B). Alternatively, while LPS upregulated both antioxidant proteins under low glucose conditions (SOD2-FC=2.11 $\pm 0.40 ;$ mtGPX4-FC $=2.09 \pm 0.41)$, levels of SOD2 $(\mathrm{FC}=0.83 \pm 0.22)$ and mtGPX4 (FC=1.35 \pm 0.38$)$ were not altered following PIC. This may contribute to the accumulation of superoxide in the mitochondria. To evaluate if these alterations affect the accumulation of cytosolic ROS, I used CellROX ${ }^{\mathrm{TM}}$ Orange and quantified $\mathrm{H}_{2} \mathrm{O}_{2}$ levels in cell lysates. CellROX ${ }^{\mathrm{TM}}$ Orange has a high affinity for hydroxyl radicals, $\mathrm{H}_{2} \mathrm{O}_{2}$ and superoxide. BMM treated with LPS exhibit increased levels of cytosolic ROS in both high and low glucose conditions (Figure 3.7C). Conversely, increased cytosolic ROS was only observed in low glucose conditions following PIC stimulation (Figure 3.7C and 3.7D). These results suggest that low glucose conditions are associated with increased mitochondrial and cytosolic ROS accumulation following PIC stimulation, which may contribute to the amplification of the cytokine response. 

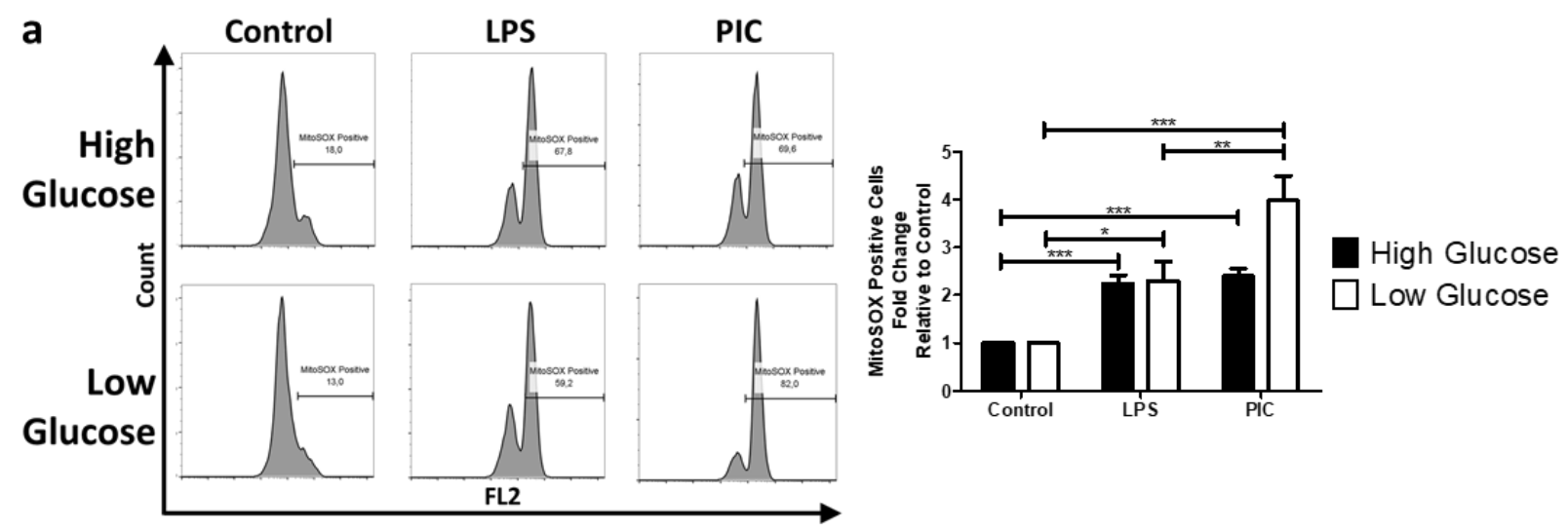

b
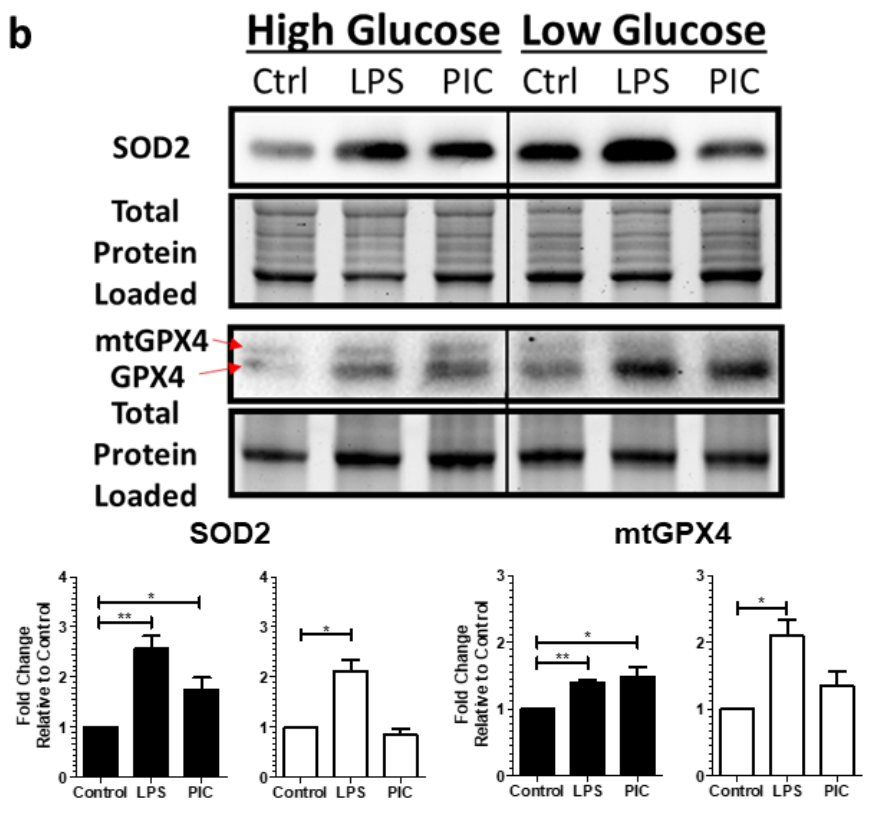

C

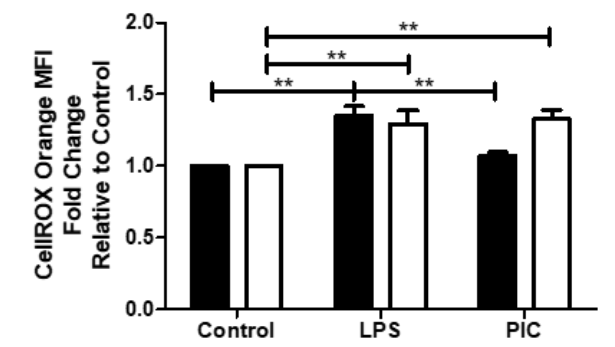

d

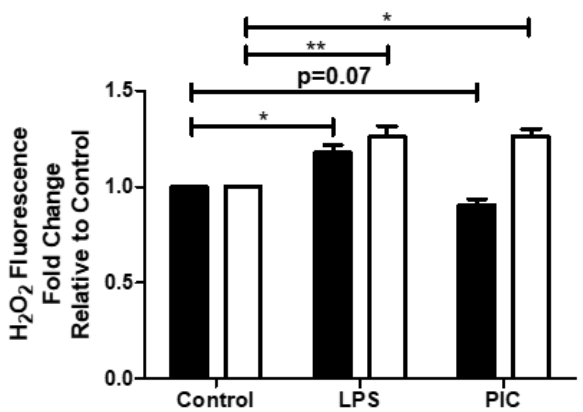

Figure 3.7: Poly(I:C) activation promotes mitochondrial ROS production and accumulation. Macrophages treated either with LPS or PIC for 18 hours under high glucose or low glucose media conditions were examined for differences in redox metabolism. Mitochondrial superoxide production was measured using MitoSOX ${ }^{\mathrm{TM}}$ Red (a). Protein levels of antioxidant proteins superoxide dismutase (SOD2) and mitochondrial glutathione peroxidase 4 (mtGPX4) were measured via immunoblotting (b). Cytosolic ROS production was measured using CellROX ${ }^{\mathrm{TM}}$ Orange (c). Hydrogen peroxide levels were quantified using the Cell-based Hydrogen Peroxide Assay kit (d). Data represents mean \pm SEM of four individual mice $(* p<0.05, * * p<0.01$, and $* * * \mathrm{p}<0.001)$. 


\subsubsection{Mitochondrial and cellular ROS play a central role in TLR3 and TLR4 associated cytokine production.}

To evaluate if mtROS contributes to cytokine production following TLR3 and TLR4 engagement, cells stimulated with either LPS $(100 \mathrm{ng} / \mathrm{ml})$ or PIC $(10 \mathrm{ng} / \mathrm{ml})$ under high and low glucose conditions were co-treated with either antioxidants (MitoTEMPO, N-acetylcysteine) or an inhibitor of superoxide production (S3QEL). MitoTEMPO (MT) is a mitochondria-specific antioxidant that selectively scavenges mitochondrial superoxide. Alternatively, N-acetylcysteine (NAC) boosts glutathione synthesis reducing total overall cellular ROS production. S3QEL selectively inhibits superoxide production from the outer Q-binding site of the ETC complex III without altering OXPHOS. Inhibition of mitochondrial ROS had differential effects on cytokine production following LPS stimulation (Figure 3.8A; Supplemental Figure S3.4). Whereas TNF- $\alpha$ (MT: $\downarrow 50 \%$; S3QEL: $\downarrow 48 \%$ ) and IFN- $\beta$ levels (MT: $\downarrow 60 \%$; S3QEL: $\downarrow 61 \%$ ) were decreased following MT and S3QEL production, CXCL10 levels were unaffected. CXCL10 production was only reduced following NAC treatment (CXCL10: $\downarrow 71 \%$ ) suggesting cytosolic ROS may play a more important role in its production following LPS stimulation. Alternatively, inhibition of mitochondrial ROS significantly reduced TNF- $\alpha$, CXCL10, IFN- $\alpha$ and IFN- $\beta$ production in PICactivated macrophages (Figure 3.8B; Supplemental Figure S3.4). The magnitude of S3QEL inhibition was further amplified under low glucose conditions (TNF- $\alpha$ : $\downarrow 54 \%$ vs. $\downarrow 66 \%$; CXCL10: not significant vs $\downarrow 66 \%$, IFN- $\beta$ : $\downarrow 61 \%$ vs. $\downarrow 73 \%$ ) suggesting complex III plays an important role in the amplification of the TLR3 responses. Similar to LPS, NAC treatment had the most pronounced effects on cytokine production in both and high low glucose conditions suggesting that both mitochondrial and cytosolic ROS contribute to TLR3 mediate cytokine production. 

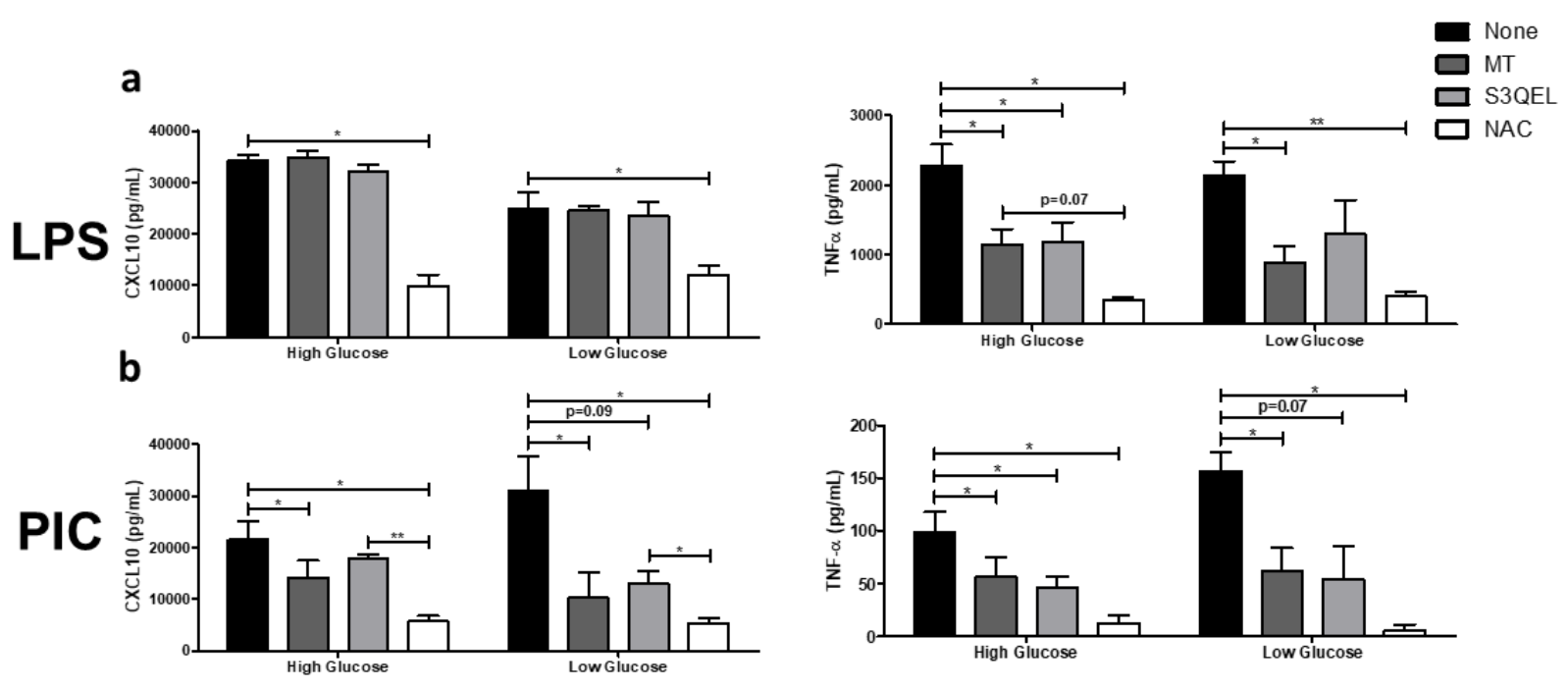

Figure 3.8: Type I IFN production can be inhibited by altering mtROS generation during Poly(I:C) activation. LPS- (a) or PIC- (b) stimulated BMDMs were co-treated with a panel of mtROS (MT, S3QEL, NAC) modulators to assess the importance of mitochondrial function for antiviral responses. CXCL10 and TNF- $\alpha$ cytokine secretion was measured after 18 hours in high glucose or low glucose media conditions. Data represents mean $\pm \mathrm{SEM}$ of three individual mice $\left({ }^{*} \mathrm{p}<0.05,{ }^{* *} \mathrm{p}<0.01\right.$, and $\left.{ }^{* * *} \mathrm{p}<0.001\right)$. 

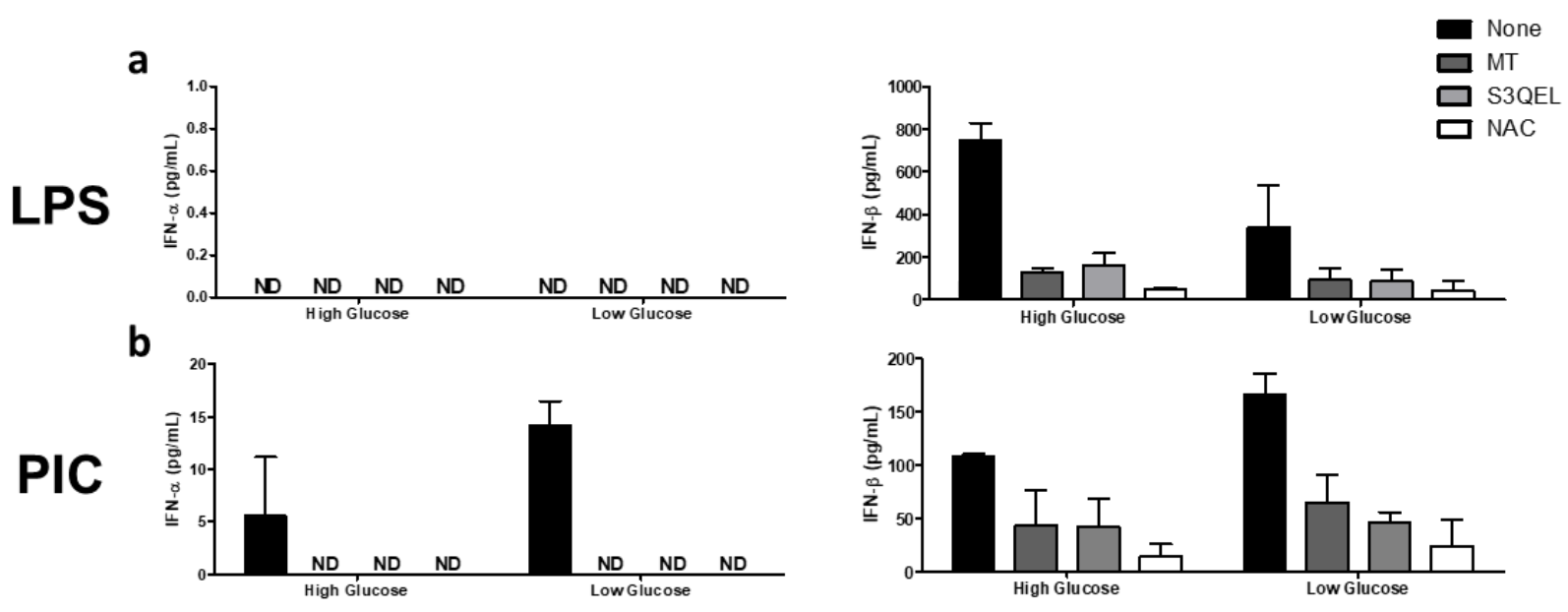

Supplemental Figure S3.4: Targeting mtROS leads to loss of type I IFN production. LPS- (a) or PIC- (b) stimulated BMDMs were co-treated with a panel of mtROS (MT, S3QEL, NAC) modulators to assess the importance of mitochondrial function to antiviral responses. CXCL10 and TNF- $\alpha$ cytokine secretion was measured after 18 hours in high glucose or low glucose media conditions. Data shown represents a mean of two individual animals. 


\subsection{Discussion}

Increasing evidence suggests that mitochondria play a critical role in driving innate immune responses against bacteria and viruses $(15,16,20-23,33)$. However, it is unclear if specific features of mitochondrial reprogramming contribute to pathogen specific immune responses or how nutrient availability may affect these processes. In the current study, I found that TLR3 and TLR4 engagement uniquely remodeled ETC complex expression, resulting in differential accumulation of mitochondrial and cytosolic ROS. This differential ROS production is required to support ligand specific inflammatory and antiviral cytokine profiles. I also found that the magnitude of TLR3 but not TLR4 responses were modulated by glucose availability. Under conditions of low glucose, TLR3 engagement was associated with increased ETC complex III expression, increased mitochondrial and cytosolic ROS, and increased inflammatory and antiviral cytokine production. This increased cytokine production was selectively reversed by targeting superoxide production from the outer Q-binding site of the ETC complex III. Collectively, these findings suggest that the ETC may act as a selective rheostat of macrophage function that regulates not only the nature (antibacterial vs. antiviral) but the magnitude of the response, which may depend on nutrient availability.

It is widely accepted that inflammatory macrophages undergo metabolic reprogramming to support cytokine production and effector functions. In LPS-stimulated cells, reprogramming is associated with a near complete inhibition of OXPHOS and an increased reliance on aerobic glycolysis to support rapid energy production $(9,15,17,36,46)$. This switch is driven by altered flux through the tricarboxylic acid (TCA), which repurposes mitochondrial function to support superoxide production and drive intracellular anti-bacterial responses $(15,17)$. While it was initially assumed all "inflammatory" stimuli induce similar responses, increasing evidence 
suggests this may not be the case $(22,23,47)$. In the current study, I found that PIC stimulation inhibited OXPHOS activity in a dose dependent manner. However, even at its highest concentrations $(10 \mu \mathrm{g} / \mathrm{ml})$, PIC did not completely inhibit OXPHOS and some level of cellular respiration was maintained. Consistent with these findings, Yoshizumi et al. found that RLRmediated responses in macrophages are dependent on OXPHOS both in vitro and in vivo. In BMM, disruption of cellular respiration severely impaired RLR induced interferon and proinflammatory cytokine production (23). In mice, inhibition of OXPHOS was found to increase susceptibility to viral infection and induce significant inflammation in the lung (23). Wu et al. found that TLR9 engagement and type I IFN production in plasmacytoid dendritic cells was associated with increased OXPHOS activity. This increase was fueled by fatty acid oxidation (FAO) and was required for full cellular activation (48). In vivo, inhibition of FAO resulted in a diminished capacity to control lymphocytic choriomeningitis virus (48). Several IFN-stimulated genes, such as ISG15, have also been linked to the regulation of mitochondrial function during viral infection suggesting a secondary wave of mitochondrial reprogramming may occur following the TLR engagement and the induction of type I IFN responses (49). Collectively, these studies suggest that some level of OXPHOS activity may be required to mount functional antiviral immune responses but that these responses may vary by ligand and cell type.

In addition to altered cellular respiration, LPS and PIC stimulation were associated with alterations in mitochondrial membrane potential (MMP). MMP is generated by the proton pumps of the ETC (Complexes I, III and IV) to support mitochondrial ATP production (50). Various studies have reported altered MMP following macrophage activation. Mills et al. found that LPS stimulation was associated with increased MMP via reverse electron flow (RET). This RET was required to drive electrons back towards Complex I in order to support mitochondrial ROS 
production and antimicrobial effector functions (15). Koshiba et al, have found MMP is required for MAVS-mediated antiviral signaling. Specifically, they found that inhibition of mitochondrial fusion resulted in a widespread loss of MMP. This loss in MMP correlated with the level of inhibition of RLR-induced antiviral responses (22). Unlike LPS, I found that PIC stimulation was associated with decreased MMP in a subset of cells. While it is unclear what exactly these cells represent, Tal et al. reported that when autophagy is inhibited, increased accumulation of dysfunctional mitochondria and increased mitochondrial ROS production drives excess RLR signaling (26). In this study, it is unclear if this subset of cells with decreased MMP are the main producers of ROS in our model system.

Recent studies have provided evidence that changes in the ETC, particularly in complex I and II, contribute to the regulation of antibacterial immune responses $(15,33)$ however, it unclear is if similar remodeling occurs during antiviral responses. Here, I found that PIC stimulated cells undergo differential remodeling of the ETC, particularly with limited glucose availability. Standard cell culture conditions represent supra-physiological levels of glucose $(25 \mathrm{mM}$ vs. $5-7 \mathrm{mM}$ in fasting blood from non-diabetic individuals (51)) and may alter mitochondrial reprogramming in vitro (52). Under low glucose conditions, both LPS and PIC were found to downregulate complex I and IV expression. Interestingly, in the low glucose condition PIC also increased complex III expression. Importantly, this increased expression was associated with the amplification of the TLR3 cytokine production, which was reversed by the selective inhibition of ROS production by complex III. In support of our results, others have linked complex III to immune activation and function. In $\mathrm{T}$ cells, Sena et al. demonstrated that specific deletion of Rieske iron-sulfur protein (RISP), an essential component of Complex III, reduced mtROS production, nuclear factor of activated T cells (NFAT) activation and IL-2-mediated T cell activation and 
antigen-specific expansion in vivo (53). Alternatively, ablation of complex III in regulatory T cells has been shown to reduce their inhibitory capacity without altering cell proliferation and survival (54). In macrophages, listeria infection has been shown to increase ROS production via complex III, which drives NF-kappa-B essential modulator (NEMO) dimerization, increasing the inhibitor of nuclear factor kappa-B kinase (IKK) activation, NF- $\mathrm{kB}$ signaling and cytokine production (47) Our study is among the first to identify associations between complex III mediated ROS production and TLR3 signaling and antiviral immune responses.

While ROS are generally considered toxic and damaging, increasing evidence suggests they also influence cellular signaling $(55,56)$. Superoxide and its more stable derivative hydrogen peroxide have been shown to regulate a variety of biological responses such as cell proliferation, differentiation, and migration (55). In the current study, I found that both mitochondrial superoxide and cytosolic ROS contribute to inflammatory and antiviral cytokine production following TLR engagement and that differential accumulation of ROS across these compartments may contribute to pathogen specific responses. Furthermore, under low glucose conditions, I found PIC but not LPS was associated with increased mitochondrial superoxide and cytosolic ROS production, which consequentially amplified cytokine production in these cells. These results suggest that the dynamic regulation of ROS production, likely through the modulation of complex III, may act as a rheostat that regulates the magnitude of antiviral immune responses. A central role for mitochondrial ROS in antiviral responses has been previously reported. Agod et al. found that mitochondrial superoxide drives increased MAVS protein expression in plasmacytoid DCs, increasing Akt and IRF3 activation and subsequent type I IFN production (57). Wang et al. showed shRNA knockout of SOD2 in cell lines increased viral replication and reduced antiviral responses (58), likely a result of decreased mitochondrial $\mathrm{H}_{2} \mathrm{O}_{2}$ production, a known redox-sensitive activator 
of NF-kB and IRF signalling (47, 59-61). While I believe that mitochondrial superoxide and associated hydrogen peroxide production are the main drivers of cytokine production in our system, I cannot exclude the possibility that alternative cytosolic sources of ROS may also contribute. NADPH oxidase (NOX)-generated ROS during respiratory syncytial virus (RSV) and herpes simplex virus (HSV) infections can activate both NF-kB and IRF signalling $(60,61)$. Similarly, Yang et al. reported that high concentrations of PIC increase NOX2 activity and ROS production in BMMs, which was required for signal transducer and activator of transcription 1 (STAT1)-mediated signalling (62). A similar phenomenon was observed by To et al. using TLR7 ligands (63). Further studies are required to determine the specific contribution of mitochondrial vs. cytosolic derived ROS in driving these processes and the distinct roles of superoxide vs. hydrogen peroxide on signaling and effector function.

Taken together, our results suggest that dynamic remodeling of the ETC complex expression represents a mechanism by which macrophages modulate cytokine production following TLR engagement. I found that this remodeling was associated with differential accumulation of mitochondrial vs. cytosolic ROS, which may drive ligand specific cytokine profiles. I hypothesize that this differential accumulation may be driven by a dependence on complex I (LPS) versus complex III (PIC) for ROS production. Interestingly, I also found that TLR3 but not TLR4 associated mitochondrial reprogramming was dependent on glucose availability in the microenvironment. Supra-physiological levels of glucose have been shown to decrease a cell's dependence on mitochondria for energy production $(52,64)$. Similarly, our results suggest high glucose conditions may also alter mitochondrial reprogramming associated with TLR3 engagement. Accordingly, it is important to develop a detailed understanding of these 
processes in a variety of bacterial and viral infections to identify new therapeutic approaches to help boost specific and functional effector functions.

\subsection{Methods}

\subsubsection{Reagents}

Lipopolysaccharide (LPS) and high molecular weight Poly(I:C) (PIC) were purchased from InvivoGen. MitoTEMPO (MT), N-acetylcysteine (NAC), antimycin A (AA), rotenone (ROT), potassium cyanide, 2-deoxyglucose (2-DG), oligomycin (OM) and carbonyl cyanide-ptrifluoromethoxyphenylhydrazone (FCCP) were acquired from Sigma-Aldrich while S3QEL-2 was purchased from Cedarlane. IL-1 $\beta$, IL-6, IL-10, TNF- $\alpha$, and CXCL10 ELISA kits were purchased from R\&D Systems. The IFN- $\alpha /$ IFN- $\beta$ 2-Plex Mouse ProcartaPlex ${ }^{\mathrm{TM}}$ Luminex Panel kit used was from Invitrogen. Tetramethylrhodamine, methyl ester (TMRM), MitoSOX Red and CellROX Orange probes were from ThermoFisher. All antibodies used in this study can be found in Table S1. Antibodies against Complex II (SDHB) was from Abcam while antibodies recognizing SOD2 was purchased from Cell Signalling Technology. Antibodies targeting IRF3, pIRF3 (Ser385), IRF7, pIRF7 (Ser477), GPX4, Ikb $\alpha$, Complexes I (NDUFB8), III (UQCRC2) and IV (COX4) were purchased from ThermoFisher.

\subsubsection{BMM culturing and stimulation}

Total bone marrow cells were collected from the tibias and femurs of 6-13-week-old C57BL/6 mice, cryopreserved in a 90\% FBS/10\% DMSO solution, and frozen until use. Cells were cultured for ten days in DMEM media with $10 \%$ fetal bovine serum, $1 \%$ penicillin/streptomycin (Life Technologies), and 15\% L929 fibroblast cell-conditioned medium on a $100 \mathrm{~mm}$ Petri dish as previously described (65). On day 10 , differentiated bone marrowderived macrophages (BMM) were detached, counted, and plated into tissue-culture treated plates 
at $1 \times 106$ cells $/ \mathrm{mL}$. BMMs were stimulated with $100 \mathrm{ng} / \mathrm{mL}$ LPS, $10 \mathrm{ng} / \mathrm{mL}$ or $10 \mu \mathrm{g} / \mathrm{mL}$ PIC under high (DMEM medium supplemented with $25 \mathrm{mM}$ glucose) or low glucose conditions (DMEM medium supplemented with $0.5 \mathrm{mM}$ glucose). The relative contribution of ETC-derived ROS on BMM inflammatory and antiviral cytokine production were assessed by co-treating stimulated cells with $1 \mu \mathrm{M}$ ROT, $5 \mu \mathrm{M}$ AA, $5 \mathrm{mM}$ cyanide, $500 \mu \mathrm{M}$ MT, $5 \mathrm{mM}$ NAC or $5 \mu \mathrm{M}$ S3QEL-2.

\subsubsection{Cytokine quantification}

After the 18-hour stimulation, cytokine production was assessed in culture supernatants. IL-1 $\beta$, IL-6, IL-10, TNF- $\alpha$, and CXCL10 levels were assessed by ELISAs according to the manufactures instructions (R\&D Systems). IFN- $\alpha$ and IFN- $\beta$ levels were measured using IFN$\alpha /$ IFN- $\beta$ 2-Plex Mouse ProcartaPlex ${ }^{\mathrm{TM}}$ Luminex Panel (Invitrogen).

\subsubsection{Western blot analysis}

Untreated and stimulated BMMs $\left(1 \times 10^{6}\right.$ cells $)$ were lysed directly in the cell culture vessel using Pierce RIPA buffer (ThermoFisher) supplemented with HALT ${ }^{\mathrm{TM}}$ Protease and Phosphatase Inhibitor (ThermoFisher). Total protein was quantified using the DC assay (Bio-Rad.) and resolved on a TGX ${ }^{\mathrm{TM}}$ FastCast ${ }^{\mathrm{TM}}$ Acrylamide gels (Bio-Rad). Gels were imaged directly using the StainFree application of a ChemiDoc XR (Bio-Rad) prior to transferring onto a PVDF membrane. Membranes were blocked overnight in 5\% non-fat dry milk (w/v), washed and incubated overnight with the appropriate primary antibody. Horseradish peroxidase-conjugated secondary antibodies and Clarity ${ }^{\mathrm{TM}}$ Western ECL Blotting Substrate (Bio-Rad) were used to visualize specified protein bands. Protein densitometry was analyzed according to previously described methodology (66).

\subsubsection{Assessment of mitochondrial function by flow cytometry}

BMMs were plated on $100 \mathrm{~mm}$ Petri dishes and stimulated with $100 \mathrm{ng} / \mathrm{mL}$ LPS or $10 \mathrm{ng} / \mathrm{mL}$ and $10 \mu \mathrm{g} / \mathrm{mL}$ PIC for 18 hours. Cells were then washed and stained with fluorescent probes 
according to the manufacturer's instructions (30mins treatment at $37^{\circ} \mathrm{C}$ in select solutions). Mitochondrial membrane potential was measured using 10nM TMRM. Mitochondrial and Cellular ROS were monitored using $2.5 \mu \mathrm{M}$ MitoSOX Red in PBS and $5 \mu \mathrm{M}$ CellROX Orange in normal high glucose media, respectively. Cellular levels of fluorescence were quantified using an Attune NxT Flow Cytometer (ThermoFisher) and the results were analyzed using FlowJo Software. Results are reported as the percentage of positive cells and as mean fluorescence intensity (MFI), the latter being used to describe the level of expression on a population of positive cells.

\subsubsection{Quantification of cellular hydrogen peroxide production}

BMMs were plated onto $96-$ well black plates at 50,000 cells/well and stimulated with $100 \mathrm{ng} / \mathrm{mL}$ LPS or $10 \mathrm{ng} / \mathrm{mL}$ PIC under high or low glucose conditions for 1 hour. Cells were then washed before using the Cell-based Hydrogen Peroxide Assay Kit (Abcam) to measure $\mathrm{H} 2 \mathrm{O} 2$ production. Cells were incubated with the AbGreen $\mathrm{H}_{2} \mathrm{O}_{2}$ indicator for 30 minutes before monitoring the relative difference in fluorescence using a fluorescence microplate reader $(490 \mathrm{~nm}$ $\mathrm{Ex} / 520 \mathrm{~nm} \mathrm{Em})$

\subsubsection{Metabolic extracellular flux analysis}

BMMs were plated onto Seahorse XFp cell culture miniplates at 50,000 cells/well (Seahorse Bioscience) and stimulated with 100ng/mL LPS or $10 \mathrm{ng} / \mathrm{mL}$ and $10 \mu \mathrm{g} / \mathrm{mL}$ PIC for 18 hours. Extracellular acidification rate (ECAR) and oxygen consumption rate (OCR) were evaluated using a XFp Flux Analyzer (Seahorse Bioscience). Baseline ECAR and changes in glycolytic rate were assessed using the Seahorse XFp Glycolytic Rate Assay Kit (Agilent) according to the manufacturer's instructions. Basal respiration, ATP production-coupled respiration, maximal and reserve capacities and non-mitochondrial respiration were assessed using the Seahorse XFp Cell Mito Stress Test Kit (Agilent). 


\subsubsection{Statistical Analysis}

Data used in this study was analyzed using GraphPad Prism software. Values shown represent the mean $\pm \mathrm{SEM}$ of biological replicates, where the number of replicates are reported in the figure legends. Statistical significance was calculated using a paired Student's t-test $\left({ }^{*} \mathrm{p}<0.05\right.$, $* * \mathrm{p}<0.01$, and $* * * \mathrm{p}<0.001)$ 


\subsection{References}

1. $\quad$ Kawai, T., and S. Akira. 2006. TLR signaling. Cell Death Differ. 13: 816-825.

2. Takeda, K., and S. Akira. 2005. Toll-like receptors in innate immunity. Int Immunol. 17: $1-14$.

3. Heil, F., H. Hemmi, H. Hochrein, F. Ampenberger, C. Kirschning, S. Akira, G. Lipford, H. Wagner, and S. Bauer. 2004. Species-Specific Recognition of Single-Stranded RNA via Toll-like Receptor 7 and 8. Science 303: 1526-1529.

4. Hemmi, H., O. Takeuchi, T. Kawai, T. Kaisho, S. Sato, H. Sanjo, M. Matsumoto, K. Hoshino, H. Wagner, K. Takeda, and S. Akira. 2000. A Toll-like receptor recognizes bacterial DNA. Nature 408: 740-745.

5. Lund, J. M., L. Alexopoulou, A. Sato, M. Karow, N. C. Adams, N. W. Gale, A. Iwasaki, and R. A. Flavell. 2004. Recognition of single-stranded RNA viruses by Toll-like receptor 7. Proc Natl Acad Sci U S A 101: 5598-5603.

6. Alexopoulou, L., A. C. Holt, R. Medzhitov, and R. A. Flavell. 2001. Recognition of double-stranded RNA and activation of NF-[kappa]B by Toll-like receptor 3. Nature 413: $732-738$.

7. Chow, J. C., D. W. Young, D. T. Golenbock, W. J. Christ, and F. Gusovsky. 1999. Tolllike Receptor-4 Mediates Lipopolysaccharide-induced Signal Transduction. J Biol Chem 274: 10689-10692.

8. Infantino, V., V. Iacobazzi, F. Palmieri, and A. Menga. 2013. ATP-citrate lyase is essential for macrophage inflammatory response. Biochem. Biophys. Res. Commun. 440: 105-111.

9. Tannahill, G. M., A. M. Curtis, J. Adamik, E. M. Palsson-McDermott, A. F. McGettrick, G. Goel, C. Frezza, N. J. Bernard, B. Kelly, N. H. Foley, L. Zheng, A. Gardet, Z. Tong, S. S. Jany, S. C. Corr, M. Haneklaus, B. E. Caffrey, K. Pierce, S. Walmsley, F. C. Beasley, E. Cummins, V. Nizet, M. Whyte, C. T. Taylor, H. Lin, S. L. Masters, E. Gottlieb, V. P. Kelly, C. Clish, P. E. Auron, R. J. Xavier, and L. A. J. O/'Neill. 2013. Succinate is an inflammatory signal that induces IL-1 $\beta$ through HIF-1 $\alpha$. Nature 496: 238-242.

10. Errea, A., D. Cayet, P. Marchetti, C. Tang, J. Kluza, S. Offermanns, J.-C. Sirard, and M. Rumbo. 2016. Lactate Inhibits the Pro-Inflammatory Response and Metabolic Reprogramming in Murine Macrophages in a GPR81-Independent Manner. PLoS ONE 11: e0163694.

11. Kesarwani, P., A. K. Murali, A. A. Al-Khami, and S. Mehrotra. 2013. Redox regulation of T-cell function: from molecular mechanisms to significance in human health and disease. Antioxid. Redox Signal. 18: 1497-1534.

12. Wang, H., H. Flach, M. Onizawa, L. Wei, M. T. McManus, and A. Weiss. 2014. Negative regulation of Hifla expression and TH17 differentiation by the hypoxia-regulated microRNA miR-210. Nat. Immunol. 15: 393-401.

13. Shirai, T., R. R. Nazarewicz, B. B. Wallis, R. E. Yanes, R. Watanabe, M. Hilhorst, L. Tian, D. G. Harrison, J. C. Giacomini, T. L. Assimes, J. J. Goronzy, and C. M. Weyand. 2016. The glycolytic enzyme PKM2 bridges metabolic and inflammatory dysfunction in coronary artery disease. J. Exp. Med. 213: 337-354.

14. Palsson-McDermott, E. M., A. M. Curtis, G. Goel, M. A. R. Lauterbach, F. J. Sheedy, L. E. Gleeson, M. W. M. van den Bosch, S. R. Quinn, R. Domingo-Fernandez, D. G. W. Johnston, J.-k. Jiang, W. J. Israelsen, J. Keane, C. Thomas, C. Clish, M. Vander Heiden, R. J. Xavier, and L. A. J. O’Neill. 2015. Pyruvate Kinase M2 Regulates Hif-1a Activity 
and IL-1 $\beta$ Induction and Is a Critical Determinant of the Warburg Effect in LPS-Activated Macrophages. Cell Metab. 21: 65-80.

15. Mills, E. L., B. Kelly, A. Logan, A. S. H. Costa, M. Varma, C. E. Bryant, P. Tourlomousis, J. H. M. Däbritz, E. Gottlieb, I. Latorre, S. C. Corr, G. McManus, D. Ryan, H. T. Jacobs, M. Szibor, R. J. Xavier, T. Braun, C. Frezza, M. P. Murphy, and L. A. O’Neill. 2016. Succinate Dehydrogenase Supports Metabolic Repurposing of Mitochondria to Drive Inflammatory Macrophages. Cell 167: 457-470.e413.

16. Kelly, B., G. M. Tannahill, M. P. Murphy, and L. A. J. O'Neill. 2015. Metformin Inhibits the Production of Reactive Oxygen Species from NADH:Ubiquinone Oxidoreductase to Limit Induction of Interleukin-1 $\beta$ (IL-1 $\beta$ ) and Boosts Interleukin-10 (IL-10) in Lipopolysaccharide (LPS)-activated Macrophages. J Biol Chem 290: 20348-20359.

17. Jha, A. K., S. C.-C. Huang, A. Sergushichev, V. Lampropoulou, Y. Ivanova, E. Loginicheva, K. Chmielewski, K. M. Stewart, J. Ashall, B. Everts, E. J. Pearce, E. M. Driggers, and M. N. Artyomov. 2015. Network Integration of Parallel Metabolic and Transcriptional Data Reveals Metabolic Modules that Regulate Macrophage Polarization. Immunity 42: 419-430.

18. Lampropoulou, V., A. Sergushichev, M. Bambouskova, S. Nair, E. E. Vincent, E. Loginicheva, L. Cervantes-Barragan, X. Ma, S. C.-C. Huang, T. Griss, C. J. Weinheimer, S. Khader, G. J. Randolph, E. J. Pearce, R. G. Jones, A. Diwan, M. S. Diamond, and M. N. Artyomov. 2016. Itaconate Links Inhibition of Succinate Dehydrogenase with Macrophage Metabolic Remodeling and Regulation of Inflammation. Cell Metab. 24: 158-166.

19. Rodríguez-Prados, J., P. G. Través, J. Cuenca, D. Rico, J. Aragonés, P. Martín-Sanz, M. Cascante, and L. Boscá. 2010. Substrate Fate in Activated Macrophages: A Comparison between Innate, Classic, and Alternative Activation. J. Immunol. 185: 605-614.

20. Seth, R. B., L. Sun, C.-K. Ea, and Z. J. Chen. 2005. Identification and Characterization of MAVS, a Mitochondrial Antiviral Signaling Protein that Activates NF- $\kappa$ B and IRF3. Cell 122: 669-682.

21. Castanier, C., D. Garcin, A. Vazquez, and D. Arnoult. 2010. Mitochondrial dynamics regulate the RIG-I-like receptor antiviral pathway. EMBO Rep 11: 133.

22. Koshiba, T. 2013. Mitochondrial-mediated antiviral immunity. Biochim Biophys Acta 1833: 225-232.

23. Yoshizumi, T., H. Imamura, T. Taku, T. Kuroki, A. Kawaguchi, K. Ishikawa, K. Nakada, and T. Koshiba. 2017. RLR-mediated antiviral innate immunity requires oxidative phosphorylation activity. Sci Rep 7: 5379.

24. Kawai, T., K. Takahashi, S. Sato, C. Coban, H. Kumar, H. Kato, K. J. Ishii, O. Takeuchi, and S. Akira. 2005. IPS-1, an adaptor triggering RIG-I- and Mda5-mediated type I interferon induction. Nat Immunol 6: 981-988.

25. Xu, L.-G., Y.-Y. Wang, K.-J. Han, L.-Y. Li, Z. Zhai, and H.-B. Shu. 2005. VISA Is an Adapter Protein Required for Virus-Triggered IFN- $\beta$ Signaling. Mol Cell 19: 727-740.

26. Tal, M. C., M. Sasai, H. K. Lee, B. Yordy, G. S. Shadel, and A. Iwasaki. 2009. Absence of autophagy results in reactive oxygen species-dependent amplification of RLR signaling. Proc Natl Acad Sci U S A 106: 2770.

27. Kawai, T., and S. Akira. 2006. Innate immune recognition of viral infection. Nat Immunol 7: 131-137.

28. Meylan, E., and J. Tschopp. 2006. Toll-Like Receptors and RNA Helicases: Two Parallel Ways to Trigger Antiviral Responses. Mol Cell 22: 561-569. 
29. Djafarzadeh, S., M. Vuda, J. Takala, M. Ochs, and S. M. Jakob. 2011. Toll-like receptor3 -induced mitochondrial dysfunction in cultured human hepatocytes. Mitochondrion 11: 83-88.

30. Pantel, A., A. Teixeira, E. Haddad, E. G. Wood, R. M. Steinman, and M. P. Longhi. 2014. Direct Type I IFN but Not MDA5/TLR3 Activation of Dendritic Cells Is Required for Maturation and Metabolic Shift to Glycolysis after Poly IC Stimulation. PLoS Biol. 12: e1001759.

31. Everts, B., E. Amiel, S. C.-C. Huang, A. M. Smith, C.-H. Chang, W. Y. Lam, V. Redmann, T. C. Freitas, J. Blagih, G. J. W. van der Windt, M. N. Artyomov, R. G. Jones, E. L. Pearce, and E. J. Pearce. 2014. TLR-driven early glycolytic reprogramming via the kinases TBK1IKK[epsiv] supports the anabolic demands of dendritic cell activation. Nat Immunol 15: 323-332.

32. Hu, W., A. Jain, Y. Gao, I. M. Dozmorov, R. Mandraju, E. K. Wakeland, and C. Pasare. 2015. Differential outcome of TRIF-mediated signaling in TLR4 and TLR3 induced DC maturation. Proc Natl Acad Sci U S A 112: 13994.

33. Garaude, J., R. Acín-Pérez, S. Martínez-Cano, M. Enamorado, M. Ugolini, E. NistalVillán, S. Hervás-Stubbs, P. Pelegrín, L. E. Sander, J. A. Enríquez, and D. Sancho. 2016. Mitochondrial respiratory-chain adaptations in macrophages contribute to antibacterial host defense. Nature immunology 17: 1037-1045.

34. Lin, B., B. Dutta, and I. D. C. Fraser. 2017. Systematic Investigation of Multi-TLR Sensing Identifies Regulators of Sustained Gene Activation in Macrophages. Cell Syst 5: 25-37.e23.

35. Krawczyk, C. M., T. Holowka, J. Sun, J. Blagih, E. Amiel, R. J. DeBerardinis, J. R. Cross, E. Jung, C. B. Thompson, R. G. Jones, and E. J. Pearce. 2010. Toll-like receptor-induced changes in glycolytic metabolism regulate dendritic cell activation. Blood 115: 4742.

36. Van den Bossche, J., J. Baardman, Natasja A. Otto, S. van der Velden, Annette E. Neele, Susan M. van den Berg, R. Luque-Martin, H.-J. Chen, Marieke C. S. Boshuizen, M. Ahmed, Marten A. Hoeksema, Alex F. de Vos, and Menno P. J. de Winther. 2016. Mitochondrial Dysfunction Prevents Repolarization of Inflammatory Macrophages. Cell Rep 17: 684-696.

37. Sriskanthadevan, S., D. V. Jeyaraju, T. E. Chung, S. Prabha, W. Xu, M. Skrtic, B. Jhas, R. Hurren, M. Gronda, X. Wang, Y. Jitkova, M. A. Sukhai, F.-H. Lin, N. Maclean, R. Laister, C. A. Goard, P. J. Mullen, S. Xie, L. Z. Penn, I. M. Rogers, J. E. Dick, M. D. Minden, and A. D. Schimmer. 2015. AML cells have low spare reserve capacity in their respiratory chain that renders them susceptible to oxidative metabolic stress. Blood: blood-2014-2008594408.

38. O'Neill, L. A. J., and E. J. Pearce. 2016. Immunometabolism governs dendritic cell and macrophage function. J. Exp. Med. 213: 15-23.

39. Wei, J., J. Raynor, T.-L. M. Nguyen, and H. Chi. 2017. Nutrient and Metabolic Sensing in T Cell Responses. Front Immunol 8: 247.

40. Langston, P. K., M. Shibata, and T. Horng. 2017. Metabolism Supports Macrophage Activation. Front Immunol 8: 61.

41. Covarrubias, A. J., H. I. Aksoylar, and T. Horng. 2015. Control of macrophage metabolism and activation by mTOR and Akt signaling. Semin Immunol 27: 286-296.

42. Honda, K., H. Yanai, H. Negishi, M. Asagiri, M. Sato, T. Mizutani, N. Shimada, Y. Ohba, A. Takaoka, N. Yoshida, and T. Taniguchi. 2005. IRF-7 is the master regulator of type-I interferon-dependent immune responses. Nature 434: 772-777. 
43. Honda, K., A. Takaoka, and T. Taniguchi. 2006. Type I Inteferon Gene Induction by the Interferon Regulatory Factor Family of Transcription Factors. Immunity 25: 349-360.

44. Li, X., P. Fang, J. Mai, E. T. Choi, H. Wang, and X.-f. Yang. 2013. Targeting mitochondrial reactive oxygen species as novel therapy for inflammatory diseases and cancers. $J$ Hematol Oncol 6: 19.

45. West, A. P., I. E. Brodsky, C. Rahner, D. K. Woo, H. Erdjument-Bromage, P. Tempst, M. C. Walsh, Y. Choi, G. S. Shadel, and S. Ghosh. 2011. TLR signalling augments macrophage bactericidal activity through mitochondrial ROS. Nature 472: 476-480.

46. Tan, Z., N. Xie, H. Cui, D. R. Moellering, E. Abraham, V. J. Thannickal, and G. Liu. 2015. Pyruvate Dehydrogenase Kinase 1 Participates in Macrophage Polarization via Regulating Glucose Metabolism. J Immunol 194: 6082.

47. Herb, M., A. Gluschko, K. Wiegmann, A. Farid, A. Wolf, O. Utermöhlen, O. Krut, M. Krönke, and M. Schramm. 2019. Mitochondrial reactive oxygen species enable proinflammatory signaling through disulfide linkage of NEMO. Sci Signal 12: eaar5926.

48. Wu, D., David E. Sanin, B. Everts, Q. Chen, J. Qiu, Michael D. Buck, A. Patterson, Amber M. Smith, C.-H. Chang, Z. Liu, Maxim N. Artyomov, Erika L. Pearce, M. Cella, and Edward J. Pearce. 2016. Type 1 Interferons Induce Changes in Core Metabolism that Are Critical for Immune Function. Immunity 44: 1325-1336.

49. Baldanta, S., M. Fernández-Escobar, R. Acín-Perez, M. Albert, E. Camafeita, I. Jorge, J. Vázquez, J. A. Enríquez, and S. Guerra. 2017. ISG15 governs mitochondrial function in macrophages following vaccinia virus infection. PLOS Pathog 13: e1006651.

50. Zorova, L. D., V. A. Popkov, E. Y. Plotnikov, D. N. Silachev, I. B. Pevzner, S. S. Jankauskas, V. A. Babenko, S. D. Zorov, A. V. Balakireva, M. Juhaszova, S. J. Sollott, and D. B. Zorov. 2018. Mitochondrial membrane potential. Analytical biochemistry 552: 5059.

51. American Diabetes, A. 2009. Diagnosis and classification of diabetes mellitus. Diabetes Care 32 Suppl 1: S62-S67.

52. McKee, T. J., and S. V. Komarova. 2017. Is it time to reinvent basic cell culture medium? Am J Physiol Cell Physiol 312: C624-C626.

53. Sena, Laura A., S. Li, A. Jairaman, M. Prakriya, T. Ezponda, David A. Hildeman, C.-R. Wang, Paul T. Schumacker, Jonathan D. Licht, H. Perlman, Paul J. Bryce, and Navdeep S. Chandel. 2013. Mitochondria Are Required for Antigen-Specific T Cell Activation through Reactive Oxygen Species Signaling. Immunity 38: 225-236.

54. Weinberg, S. E., B. D. Singer, E. M. Steinert, C. A. Martinez, M. M. Mehta, I. MartínezReyes, P. Gao, K. A. Helmin, H. Abdala-Valencia, L. A. Sena, P. T. Schumacker, L. A. Turka, and N. S. Chandel. 2019. Mitochondrial complex III is essential for suppressive function of regulatory T cells. Nature 565: 495-499.

55. Holmström, K. M., and T. Finkel. 2014. Cellular mechanisms and physiological consequences of redox-dependent signalling. Nature Reviews Molecular Cell Biology 15: 411.

56. Nathan, C., and A. Cunningham-Bussel. 2013. Beyond oxidative stress: an immunologist's guide to reactive oxygen species. Nature Reviews Immunology 13: 349.

57. Agod, Z., T. Fekete, M. M. Budai, A. Varga, A. Szabo, H. Moon, I. Boldogh, T. Biro, A. Lanyi, A. Bacsi, and K. Pazmandi. 2017. Regulation of type I interferon responses by mitochondria-derived reactive oxygen species in plasmacytoid dendritic cells. Redox Biol 13: 633-645. 
58. Wang, W., Y. Jin, N. Zeng, Q. Ruan, and F. Qian. 2017. SOD2 Facilitates the Antiviral Innate Immune Response by Scavenging Reactive Oxygen Species. Viral Immunol 30: 582-589.

59. Takada, Y., A. Mukhopadhyay, G. C. Kundu, G. H. Mahabeleshwar, S. Singh, and B. B. Aggarwal. 2003. Hydrogen Peroxide Activates NF- $\mathrm{kB}$ through Tyrosine Phosphorylation

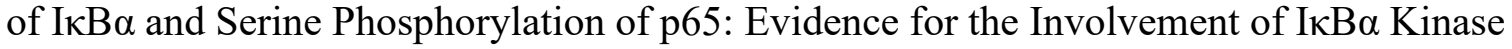
and Syk Protein-Tyrosine Kinase. J Biol Chem 278: 24233-24241.

60. Indukuri, H., S. M. Castro, S.-M. Liao, L. A. Feeney, M. Dorsch, A. J. Coyle, R. P. Garofalo, A. R. Brasier, and A. Casola. 2006. Ikkepsilon regulates viral-induced interferon regulatory factor-3 activation via a redox-sensitive pathway. Virology 353: 155-165.

61. Gonzalez-Dosal, R., K. A. Horan, S. H. Rahbek, H. Ichijo, Z. J. Chen, J. J. Mieyal, R. Hartmann, and S. R. Paludan. 2011. HSV Infection Induces Production of ROS, which Potentiate Signaling from Pattern Recognition Receptors: Role for S-glutathionylation of TRAF3 and 6. PLOS Pathog 7: e1002250.

62. Yang, C.-S., J.-J. Kim, S. J. Lee, J. H. Hwang, C.-H. Lee, M.-S. Lee, and E.-K. Jo. 2013. TLR3-Triggered Reactive Oxygen Species Contribute to Inflammatory Responses by Activating Signal Transducer and Activator of Transcription-1. The Journal of Immunology 190: 6368.

63. To, E. E., R. Vlahos, R. Luong, M. L. Halls, P. C. Reading, P. T. King, C. Chan, G. R. Drummond, C. G. Sobey, B. R. S. Broughton, M. R. Starkey, R. van der Sluis, S. R. Lewin, S. Bozinovski, L. A. J. O’Neill, T. Quach, C. J. H. Porter, D. A. Brooks, J. J. O’Leary, and S. Selemidis. 2017. Endosomal NOX2 oxidase exacerbates virus pathogenicity and is a target for antiviral therapy. Nature Communications 8: 69.

64. Ackermann, T., and S. Tardito. 2019. Cell Culture Medium Formulation and Its Implications in Cancer Metabolism. Trends in Cancer 5: 329-332.

65. Weischenfeldt, J., and B. Porse. 2008. Bone Marrow-Derived Macrophages (BMM): Isolation and Applications. CSH Protoc 2008: pdb.prot5080.

66. Taylor, S. C., T. Berkelman, G. Yadav, and M. Hammond. 2013. A Defined Methodology for Reliable Quantification of Western Blot Data. Mol Biotechnol 55: 217-226. 


\section{Chapter 4. HIF-1a regulation of pro-inflammatory cytokine production following TLR3 engagement is dependent on viral nucleic acid length and glucose availability (Ahmed et al. under revision, submitted to Journal of Immunology)}

\subsection{Introduction}

Macrophages serve as sentinels of the innate immune response, surveying tissues and quickly removing pathogens and apoptotic debris to re-establish and maintain homeostasis (1-3). Emerging evidence suggests cellular metabolism is an important regulator of macrophage function, however the mechanisms underlying these processes are not fully understood. Macrophages require a dynamic metabolism to satisfy their bioenergetic and biosynthetic demands, it but also to modulate their gene expression, signal transduction and epigenetic profiles (4-11). As the metabolic hub of the cell, mitochondria play a central role in these processes $(5,6$, $10,11)$. They serve as a scaffold for innate immune signaling, producing bioactive molecules and metabolites that amplify inflammatory and antiviral signaling through pattern recognition receptors (PRR) (6, 11-15). For example, reactive oxygen species (ROS) produced by mitochondria act as rheostats to modulate the magnitude of inflammatory and antiviral signaling in macrophages based on their surrounding nutrient environment (11, 16-18).

Hypoxia-inducible factor-1 $\alpha$ (HIF-1 $\alpha)$ is a transcription factor that has been shown to regulate mitochondrial function, oxygen consumption and substrate choices to support energy metabolism (19-23). Originally discovered as part of the cellular hypoxic response, it is now recognized that HIF-1 $\alpha$ is induced by a wide range of cellular stressors, including bacterial and viral infection (24-26). It modulates cellular migration, phagocytosis and increases intracellular killing of bacteria $(23,25,27,28)$. It has also been shown to upregulate inflammatory cytokines (IL-1 $\beta$, TNF- $\alpha$, IL-6) and glycolytic enzyme (GLUT1, PKM2, PDK1) gene expression (19, 20, 25 , $27,29,30)$ to support macrophage activation during bacterial infections $(19,20,25,27)$. In viral 
infections, HIF-1 $\alpha$ expression promote pathogenesis. For example, the interplay between HIF-1 $\alpha$ and the HIV accessory protein Vpr induces viral gene expression and dysregulates multiple host pathways to support infection $(31,32)$. HIF- $1 \alpha$ also drives the release of extracellular vesicles, which increase inflammation and contribute to HIV-associated immune dysfunction (33). In Hepatitis C, viral proteins activate HIF-1 $\alpha$ by normoxic stabilization $(34,35)$. This stabilization is required to increase the expression of glycolytic enzymes, which promotes survival of infected cells via nonoxidative glucose utilization (34). Consistent with these findings, HIF-1 $\alpha$ has also been shown to promote survival of infected cells in Hepatitis B, Kaposi's sarcoma-associated herpesvirus and Epstein-Barr infections (36-38). While certain viruses modulate HIF-1 $\alpha$ for their own benefit, it is unclear if this transcription factor is also required to initiate or support aspects of an antiviral immune response.

A variety of PRRs, including Retinoic acid-inducible gene-I (RIG-I)-like receptors (RLR) and Toll-like receptors (TLR; TLR3/7/9), sense and recognize viral nucleic acids to initiate antiviral immune responses $(13,14,39-43)$. This recognition results in the phosphorylation and homo- or heterodimerization of the interferon regulatory factor (IRF) transcription factors IRF3 and IRF7, which drive type I interferon (IFN) production (44). It also activates NF-kB through TRIF, TRAF3 or TRAF6, all of which stimulates inflammatory cytokine production (45). Polyinosinic:polycytidylic acid or Poly(I:C) is a double-stranded homopolymer commonly used to model antiviral immune responses to viral double stranded RNA (dsRNA). In the cytosol, Poly(I:C) interacts with either RIG-I or melanoma differentiation-associated gene 5 (MDA5), depending on the length of the dsRNA. RIG-I preferentially interacts with shorter dsRNA $(<1 \mathrm{~kb})$, whereas MDA5 interacts with longer strands $(>2 \mathrm{~kb})(43,46)$. In the endosome, Poly(I:C) interacts with TLR3 irrespective of length (41). However, Mian et al. (47) found that long strands of 
Poly(I:C) $(10 \mu \mathrm{g} / \mathrm{mL})(2-8 \mathrm{~kb})$ induced strong inflammatory responses in fibroblasts, whereas shorter lengths (0.5-1.5 kb) were more potent in activating macrophage-like cell lines (RAW264.7) and human PBMCs. Furthermore, the magnitude of antiviral and inflammatory responses in human monocyte-derived dendritic cells was found to depend on the form (ssRNA versus dsRNA) and length of the RNA as well as the level of 5' phosphorylation (48). While further investigation is required, these differences are likely related to differential engagement of TLR3. Stable TLR3 dimerization requires a minimum of 40-50 bp, and dsRNA binding affinity can be altered depending on strand length (49-51).

Here, I show that the magnitude of the inflammatory response following TLR3 engagement on mouse bone marrow-derived macrophages (BMM) differs based on the length of Poly(I:C) (HMW; $1.5-8 \mathrm{~kb}$ vs. LMW; $0.2-1 \mathrm{~kb}$ ) and is linked to differential accumulation of HIF-1 $\alpha$, which is strengthened under conditions of stress, including glucose deprivation. I also show that HIF-1 $\alpha$ is differentially regulated by ROS (mitochondrial and cytosolic) and pyruvate kinase M2 (PKM2), depending on the length of Poly(I:C) used. Collectively, these results suggest HIF-1 $\alpha$ may be used to fine-tune TLR3-specific inflammatory responses under conditions of stress. 


\subsection{Materials and Methods}

\subsubsection{Reagents}

High glucose Dulbecco's Modified Eagle Media (DMEM) (with 4 mM L-glutamine and 1 $\mathrm{mM}$ sodium pyruvate), DMEM without glucose/glutamine/pyruvate/phenol red, fetal bovine serum (FBS), penicillin/streptomycin (PenStrep), glucose, sodium pyruvate and L-glutamine were purchased from Life Technologies. Lipopolysaccharide (LPS), high molecular weight Poly(I:C) (HMW) and low molecular weight Poly(I:C) (LMW) were obtained from InvivoGen. Acriflavine (ACR), N-acetylcysteine (NAC), antimycin A (AA), rotenone (ROT), 2-deoxyglucose (2-DG), oligomycin (OM) and carbonyl cyanide-p-trifluoromethoxyphenylhydrazone (FCCP) were acquired from Sigma-Aldrich, while DASA-58 was from Cayman Chemical. Tetramethylrhodamine, methyl ester (TMRM), MitoSOX ${ }^{\mathbf{T M}}$ Red and CellROX ${ }^{\mathbf{T M}}$ Orange probes were purchased from ThermoFisher. IL-6, IL-10, and TNF- $\alpha$ ELISA kits were attained from R\&D Systems. Antibodies against HIF-1 $\alpha$ (D2U3T) and PKM2 (D78A4) were obtained from Cell Signalling Technology. Polyclonal antibodies targeting I $\mathrm{B} \alpha$ were from ThermoFisher, whereas the GAPDH (6C5) antibody was purchased from Advanced ImmunoChemical Inc.

\subsubsection{Isolation, differentiation, and treatment of bone marrow-derived macrophages (BMM)}

All animal procedures were approved by the Carleton University Animal Care Committee and were conducted in accordance with the guidelines provided by the Canadian Council for Animal Care. Bone marrow progenitor cells were isolated from the tibias and femurs of 6-13week-old C57BL/6 mice and cryopreserved until use. Progenitors were cultured on a $100 \mathrm{~mm}$ Petri dish for 10 days in high glucose DMEM media with 10\% FBS, 1\% PenStrep, and 15\% L929 fibroblast cell-conditioned medium $(11,52)$. L929 conditioned media was prepared as previously described (52). Once differentiated, BMM were collected, counted, and plated onto tissue-culture treated plates at a density of $1 \times 10^{6}$ cells $/ \mathrm{mL}$ and allowed to rest overnight. BMMs were then 
stimulated with $10 \mathrm{ng} / \mathrm{mL}$ high molecular weight (HMW; $1.5-8 \mathrm{~kb}$ ) or $10 \mathrm{ng} / \mathrm{mL}$ low molecular weight Poly(I:C) (LMW; 0.2-1 kb) (Sigma-Aldrich) in complete DMEM media with either high $(25 \mathrm{mM})$ or low glucose $(0.5 \mathrm{mM})$. Low glucose media was prepared using the no glucose/glutamine/pyruvate/phenol red DMEM media that was subsequently supplemented with $0.5 \mathrm{mM}$ glucose and matching concentrations of glutamine $(4 \mathrm{mM})$ and sodium pyruvate $(1 \mathrm{mM})$. Cells were stimulated for a range of 0.5 to 18 hours depending on the endpoint analysis. To better understand if oxygen levels can further alter HIF-1 $\alpha$ accumulation, treatments were done under normoxic (atmospheric $\mathrm{O}_{2}$ levels) and hypoxic (1\%) conditions using a hypoxic incubator. To evaluate the role of HIF-1 $\alpha$, PKM2 and ROS in inflammatory signaling and cytokine production, BMM cells were stimulated with HMW- or LMW-Poly(I:C) in the presence or absence of inhibitor (1 $\mu \mathrm{M}$ ACR, $20 \mu \mathrm{M}$ DASA-58, or $5 \mathrm{mM}$ NAC respectively).

\subsubsection{Quantification of Cytokine Production in Culture Supernatants}

Cell culture supernatants were collected from BMM after 18 hours of stimulation with HMW or LMW Poly(I:C) and spun at 1,800 RPM for 10 minutes to remove cellular debris. TNF$\alpha$, IL-6 and IL-10 levels were evaluated using ELISAs according to the manufacturer's instructions (R\&D Systems).

\subsubsection{Evaluation of protein expression in total BMM lysates}

Following the stimulation of BMM with HMW or LMW Poly(I:C) in high or low glucose media, cells were lysed using Pierce RIPA buffer supplemented with HALT ${ }^{\mathrm{TM}}$ Protease and Phosphatase Inhibitor (ThermoFisher). Total protein content in each sample was quantified using the DC assay (Bio-Rad). For each protein tested, $30 \mu \mathrm{g}$ of total protein was loaded onto $12 \%$ TGX $^{\mathrm{TM}}$ FastCast ${ }^{\mathrm{TM}}$ Acrylamide gels (Bio-Rad) and imaged using the ChemiDoc XR's Stain-Free program (Bio-Rad). Resolved proteins were transferred onto PVDF membranes using the Trans- 
Blot $^{\circledR}$ Turbo $^{\mathrm{TM}}$ Transfer System and blocked overnight in 5\% non-fat powdered milk (w/v) prior to overnight incubation with the appropriate primary antibody in TBS with 5\% BSA (w/v) and $0.1 \%$ Tween-20. Horseradish peroxidase-conjugated secondary antibodies and Clarity ${ }^{\mathrm{TM}}$ Western ECL Blotting Substrate (Bio-Rad) were used to visualize protein bands of interest. Protein band densitometry was analyzed using methods previously described (53). In short, the chemiluminescence intensity of target bands was normalized to the amount of total protein loaded in its respective lane, as determined by the Stain-Free application on the ChemiDoc ${ }^{\mathrm{TM}} \mathrm{XR}$ imager (Bio-Rad). The levels of target protein expression were further normalized relative to its respective control samples and presented as a fold change value. For $\mathrm{I} \kappa \mathrm{B} \alpha$, following normalization described above, the protein expression data was presented as the ratio between phosphorylated I $\mathrm{B} \alpha$ levels divided by total I $\kappa \mathrm{B} \alpha$ levels.

\subsubsection{Evaluation of Mitochondrial Membrane Potential, Mitochondrial ROS and Total Cytosolic ROS using Flow Cytometry}

Untreated and stimulated BMMs were detached and stained with fluorescent probes according to the manufacturer's instructions. Differences in mitochondrial membrane potential (MMP) were evaluated using $10 \mathrm{nM}$ TMRM (ThermoFisher), which is a lipophilic cationic dye that accumulates in the mitochondrial matrix in proportion to the magnitude of MMP across the inner mitochondrial membrane. Mitochondrial and cellular ROS levels were monitored using 2.5 $\mu \mathrm{M}$ MitoSOX ${ }^{\mathrm{TM}}$ Red (ThermoFisher) in PBS and $5 \mu \mathrm{M}$ CellROX ${ }^{\mathrm{TM}}$ Orange (ThermoFisher). MitoSOX ${ }^{\mathrm{TM}}$ Red is a fluorogenic probe that specifically localizes to active mitochondria via MMP detection and selectively reacts with superoxide in the mitochondrial matrix to create a highly florescent red product. CellROX ${ }^{\mathrm{TM}}$ Orange is a cell-permeable fluorogenic dye that localizes to the cytoplasm and strongly fluoresces upon oxidation by various ROS. Single cell analysis was measured using an Attune NxT Flow Cytometer (ThermoFisher). A minimum of 10,000 events 
were captured per sample. Data was analyzed using Flow Jo Software (version 10.0.7). After gating upon the macrophage population based on size (via FSC and SSC), events were characterized based on their fluorescent profiles of the respective probes. For experiments where the TMRM and MitoSOX ${ }^{\mathrm{TM}}$ Red stains were used, sub-populations were categorized as either "positive" cells (high fluorescence) or "negative" cells (low fluorescence) for their respective stain. TMRM data was presented as the percentage of "TMRM negative" cells. MitoSOX ${ }^{\mathrm{TM}}$ Red data was presented as the percentage of "MitoSOX Red positive" cells. For experiments where the CellROX Orange probe was used, samples were characterized based on changes in the median fluorescence intensity (MFI) of the total population. The MFI value of each sample was normalized to its respective control sample and presented as a fold change value.

\subsubsection{Characterization of Energy Metabolism of BMM}

Harvested BMMs were plated onto Seahorse XFp cell culture miniplates (Agilent) at a density of 50,000 cells/well before stimulation. Cells were then stimulated with either $10 \mathrm{ng} / \mathrm{mL}$ HMW or LMW Poly(I:C) for 18 hours. Measurement of extracellular acidification rate (ECAR) and oxygen consumption rate (OCR) were done using a XFp Flux Analyzer (Agilent). Changes in glycolytic rate were assessed using the Seahorse XFp Glycolytic Rate Assay Kit (Agilent) according to the manufacturer's instructions. BMMs treated under high or low glucose conditions were exposed to sequential rotenone plus antimycin A (ROT/AA) and 2-deoxyglucose (2-DG) injections, and ECAR measurements were recorded. ECAR measurements were converted to proton efflux rate (PER) using the Wave Desktop software (Seahorse Bioscience) to better represent extracellular acidification. PER measurements were used to determine differences in basal glycolytic rate, \%PER dependent on glycolysis and the ratio of mitochondrial oxygen consumption rate (mitoOCR) to glycolytic PER (glycoPER) across different samples. 
Mitochondrial function was evaluated using the Seahorse XFp Cell Mito Stress Test Kit (Agilent). OCR measurements were recorded after HMW- and LMW-activated BMM were exposed to successive injections of OM, FCCP, and ROT/AA. Changes in OCR were used to measure the spare respiratory capacity percentage (SRC\%) and ATP production of activated BMM.

Glucose consumption of BMM treated with HMW or LMW was done using a Contour ${ }^{\circledR}$ Next glucometer (54). After 18 hours of HMW or LMW treatment, cell culture supernatants were collected and spun down (as described above) to remove cellular debris. Afterwards, $5 \square \mathrm{L}$ of supernatant was loaded onto a test strip attached to a glucometer, and the amount of glucose remaining in sample after treatment was recorded. Prior to treatment, glucose levels in both high and low glucose media were recorded, and the percent glucose consumption was calculated for each sample by dividing the amount of glucose that remained after $18 \mathrm{hrs}$ by the amount of glucose present before treatment. As a positive control, BMM were also treated with $100 \mathrm{ng} / \mathrm{mL} \mathrm{LPS}$, a TLR ligand known to cause BMM to heavily depend on glucose as a primary energy source to mount a proper effector response $(19,21,55,56)$.

\subsubsection{Statistical Analyses}

Data used in this study was analyzed using GraphPad Prism software. Values shown represent the means $\pm \mathrm{SEM}$ of biological replicates, where the number of replicates is reported in the figure legends. Statistical significance was calculated using a paired Student's t-test $\left({ }^{*} \mathrm{p}<0.05\right.$, $* * \mathrm{p}<0.01$, and $* * * \mathrm{p}<0.001)$. 


\subsection{Results:}

\subsubsection{HIF-1 $\alpha$ is induced following TLR3 engagement and is regulated by glucose availability}

Emerging evidence suggests HIF-1 $\alpha$ is regulated by certain viruses to promote viral replication and survival of infected cells $(31,32,34,35)$; however, it is unclear if its accumulation is required to support antiviral immune responses. To evaluate if HIF-1 $\alpha$ is modulated during early antiviral immune responses, HIF- $\alpha$ protein expression was quantified via western blot after BMM were stimulated with HMW or LMW Poly(I:C) (10 ng/mL each) for 18 hours. Under standard cell culture conditions (high glucose; $25 \mathrm{mM}$ ), HMW but not LMW Poly(I:C) stimulation was associated with HIF-1 $\alpha$ accumulation, compared to unstimulated control cells $(\mathrm{FC}=1.72, \mathrm{p}=0.042)$ (Figure 4.1). However, in glucose deprived conditions (0.5 mM), both HMW and LMW Poly(I:C) stimulation increased HIF-1 $\alpha$ (HMW FC=2.91, $\mathrm{p}=0.039$; LMW FC=2.24, $\mathrm{p}=0.004)$. Given its established role in regulating gene expression under hypoxic conditions (57), I investigated if HIF$1 \alpha$ expression levels were further modified by oxygen levels. To do this, BMMs were stimulated with HMW and LMW Poly(I:C) in high or low glucose and under hypoxic conditions $\left(1 \% \mathrm{O}_{2}\right)$. In high glucose conditions, hypoxia increased HIF-1 $\alpha$ levels in control cells and further increased accumulation of HIF-1 $\alpha$ following stimulation with HMW and LMW Poly(I:C) (Supplemental Figure S4.1). In contrast, under conditions of glucose and oxygen deprivation, HMW and LMW Poly(I:C) stimulation were not associated with any further increase in HIF-1 $\alpha$ compared to control cells. Collectively, these data suggest that, while HIF-1 $\alpha$ may be important in antiviral responses in HMW-stimulated cells, it only contributes to LMW Poly(I:C) responses under conditions of stress. Furthermore, they suggest that macrophages may have a maximal capacity to respond to stress. 

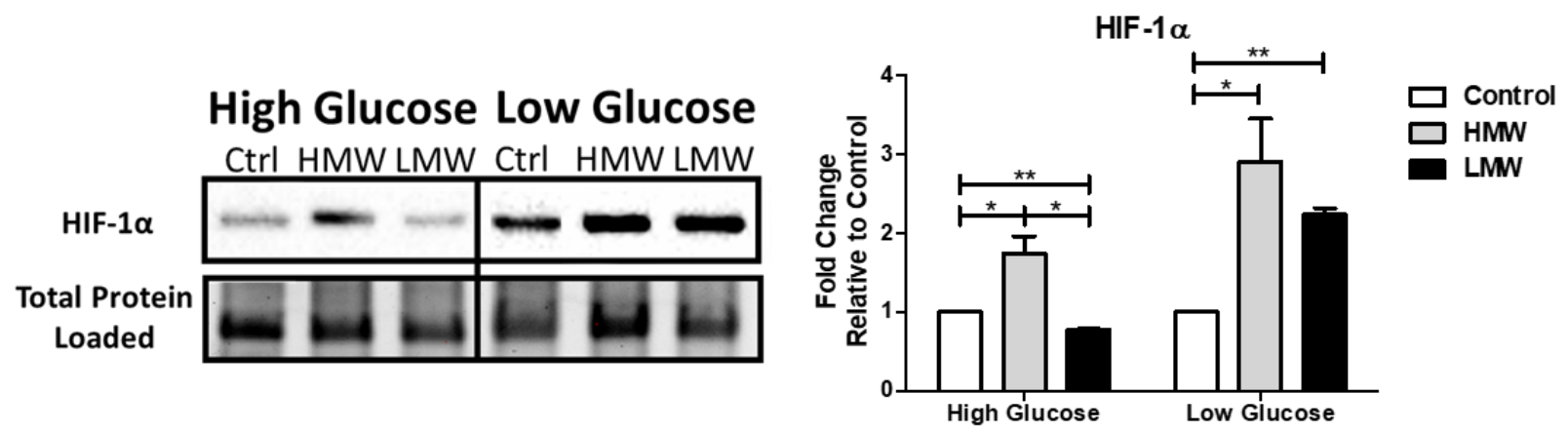

Figure 4.1: HIF-1 $\alpha$ differentially accumulates in BMM following stimulation with HMW vs. LMW Poly(I:C) in low and high glucose. BMM were stimulated with either $10 \mathrm{ng} / \mathrm{mL}$ HMW- or $10 \mathrm{ng} / \mathrm{mL}$ LMW Poly(I:C) for 18 hours under high glucose $(25 \mathrm{mM})$ or low glucose $(0.5 \mathrm{mM})$ conditions. Total cell lysates were used to quantify HIF-1 $\alpha$ expression via immunoblotting. Band intensity was normalized to the total protein levels in each of their respective lanes using the Bio-Rad Stain-Free Application. Afterwards, the expression of HIF-1 $\alpha$ in each glucose condition was normalized to its respective control sample and presented as a fold change value. Data represents means \pm SEM of four individual mice $(* p<0.05, * * p<0.01$, and $* * * \mathrm{p}<0.001)$. 

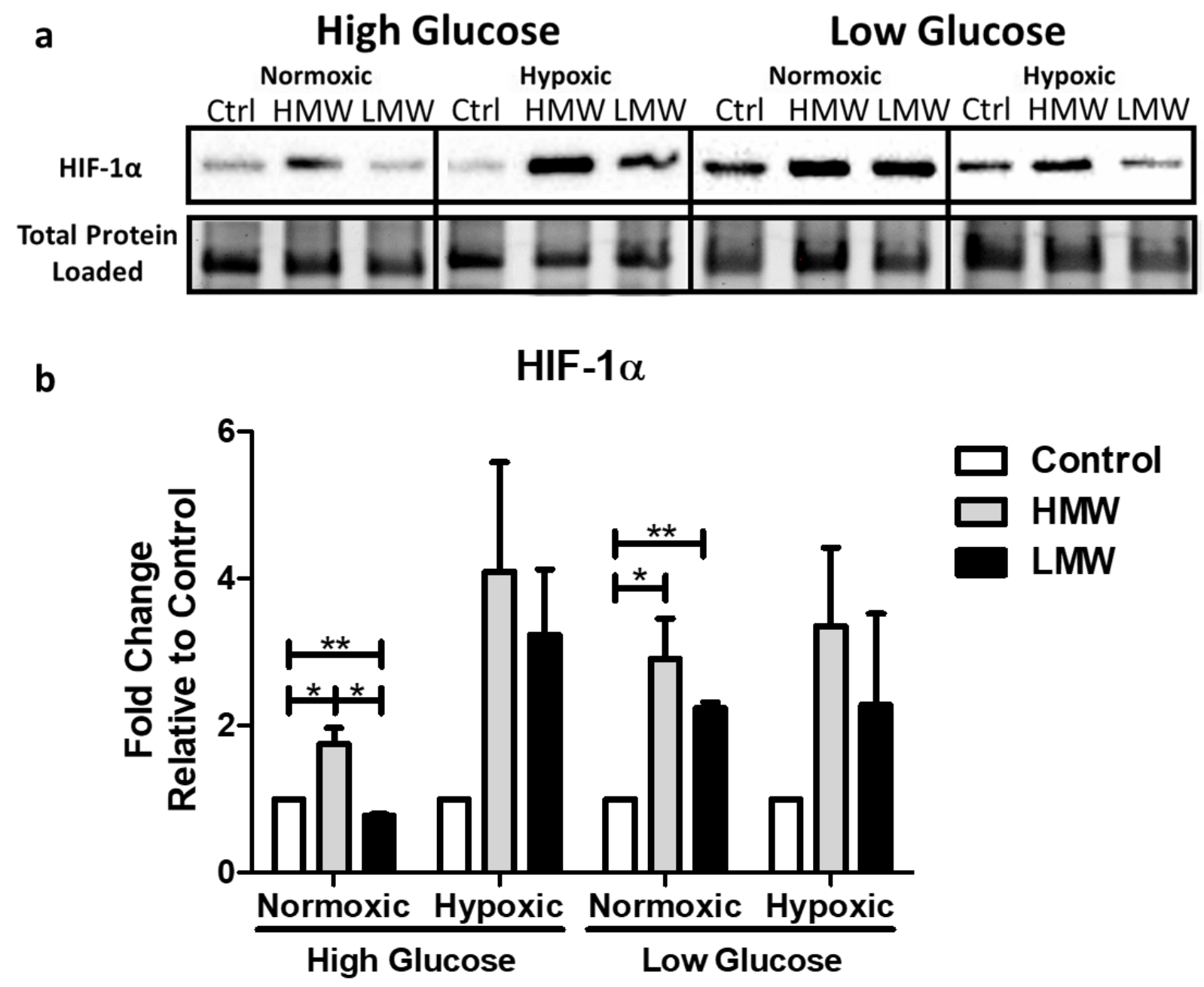

Supplemental Figure S4.1: HIF-1a expression is activated under glucose and/or oxygen deprivation conditions. BMM were stimulated with either HMW or LMW Poly(I:C) for 18 hours under high glucose or low glucose conditions. Total cell lysates were used to quantify HIF-1 $\alpha$ expression via immunoblotting. Band intensity was normalized to the total protein levels in each of their respective lanes using the Bio-Rad Stain-Free Application. Afterwards, the expression of HIF-1 $\alpha$ in each glucose condition was normalized to their respective control sample and presented as a fold change value. Data represents mean \pm SEM of four individual mice $(* p<0.05, * * p<$ 0.01 , and $* * * \mathrm{p}<0.001)$. 


\subsubsection{Stimulation of BMM with LMW Poly(I:C) does not alter glycolytic activity}

Given the role of HIF-1 $\alpha$ in regulating glycolytic gene expression, I then investigated if TLR3 engagement was associated with differential modulation of glycolysis. I have previously shown that stimulation with HMW Poly(I:C) is associated with increased glycolytic activity, but this increase is significantly lower than that observed following LPS stimulation (11). To determine if similar changes are observed with LMW Poly(I:C), BMM were stimulated with HMW and LMW Poly(I:C) in high and low glucose media. Changes in glycolytic rate were evaluated using Agilent's Glycolytic Rate Assay and are reported as changes in proton efflux rate (PER) at baseline and in response to treatments of rotenone and antimycin A (ROT/AA) and 2deoxyglucose (2-DG). As previously reported (11), HMW stimulation in high and low glucose conditions increased PER levels following stimulation, increased \%PER derived from glycolysis and decreased the mitoOCR/glycoPER ratio (Figure 4.2A-C). However, LMW stimulation was not associated with any significant changes in glycolytic function despite the marked upregulation of HIF- $1 \alpha$ in low glucose conditions (Figure 4.2A-C). To verify these results, I examined if HMW and LMW Poly(I:C) stimulations were associated with altered glucose uptake from the culture supernatant. In high glucose media, HMW but not LMW Poly(I:C) stimulation increased glucose consumption. As seen in the glycolytic rate assay, this increase was less pronounced than that observed following LPS stimulation (Figure 4.2D). In low glucose media, however, neither HMW nor LMW Poly(I:C) stimulation were associated with significant glucose uptake, suggesting these cells may adapt to the environment and use alternative energy sources under stressed conditions. In contrast, LPS stimulated cells further increased its glucose uptake to compensate for the reduced glucose in the environment (Figure 4.2D). These results suggest that cells stimulated with HMW and LMW Poly(I:C) may have differential dependencies on glycolysis to support their activation 
status. Moreover, it appears that HIF-1 $\alpha$ accumulation following glucose deprivation has a limited effect on glycolytic activity.

\subsubsection{Preventing HIF-1 dimerization blocks pro-inflammatory cytokine production via inhibition of $N F-\kappa B$ activation}

To determine if the differential accumulation of HIF-1 $\alpha$ is associated with altered cytokine production, BMM were stimulated with HMW and LMW Poly(I:C) for 18 hours under high and low glucose conditions. Cytokine production in the culture supernatant was evaluated. Under high glucose conditions, HMW stimulated cells produced significantly higher levels of TNF- $\alpha$ (HMW: 93.1 pg/mL, p=0.013 vs. LMW: 25.0 pg/mL, p=0.048), IL-6 (HMW: 35.3 pg/mL, p=0.034 vs. LMW: $13.9 \mathrm{pg} / \mathrm{mL}, \mathrm{p}=0.076$ ) and IL-10 (HMW: $128.4 \mathrm{pg} / \mathrm{mL}, \mathrm{p}=0.024$ vs. LMW: $47.9 \mathrm{pg} / \mathrm{mL}$, $\mathrm{p}=0.592)$ (Figure 4.3A-C). IL-1 $\beta$ was not detected in any condition following TLR3 engagement (data not shown). Consistent with this increase in TNF- $\alpha$ and IL-6, levels of p-IкB $\alpha$ in HMW stimulated cells remained elevated at 2- and 4-hours post-stimulation relative to controls (Figure 4.3D). Interestingly, LMW stimulation transiently increased $\mathrm{p}-\mathrm{I} \kappa \mathrm{B} \alpha$ levels at 0.5 hours before returning to control levels 2- and 4-hours after stimulation (Figure 4.3D). Low glucose conditions were associated with a significant increase in cytokine production in both HMW and LMW stimulated cells (TNF- $\alpha$ [HMW: FC=2.16, p=0.045 vs. LMW: $F C=1.61, p=0.012]$, IL-6 [HMW: $\mathrm{FC}=1.68, \mathrm{p}=0.003$ vs. LMW: $\mathrm{FC}=2.51, \mathrm{p}=0.017]$ and IL-10 [HMW: $\mathrm{FC}=1.33, \mathrm{p}=0.014$ vs. LMW: $\mathrm{FC}=1.91, \mathrm{p}=0.008]$ ) (Figure 4.3A-C). Additionally, both stimuli maintained and demonstrated sustained levels of $\mathrm{p}-\mathrm{I} \kappa \mathrm{B} \alpha$ for up to 4 hours post-stimulation (Figure $3 \mathrm{~d}$ ).

To determine if HIF-1 $\alpha$ plays a direct role in regulating inflammatory cytokine production, cells were then stimulated for 18 hours with HMW or LMW under high or low glucose conditions in the presence or absence of ACR, which directly binds to HIF-1 $\alpha$ and block HIF complex dimerization and limits its transcriptional activity without preventing its accumulation (58; 
Supplemental Figure S4.2). Under high glucose conditions, preventing dimerization slightly reduced TNF- $\alpha$ (HMW: $\downarrow 31.6 \%, p=0.417$ vs. LMW: $\downarrow 37.8 \%, p=0.08)$ and IL-6 production (HMW: $\downarrow 49.6 \%, \mathrm{p}=0.049$ vs. LMW: $\downarrow 100 \%, \mathrm{p}=0.021$ ) but did not reduce IL-10 levels (Figure 4.4A-D; Supplemental Figure S4.3A-B). Under low glucose conditions, ACR was associated with a more dramatic decrease in pro-inflammatory cytokine production following HMW Poly(I:C) stimulation (TNF- $\alpha: 57.1 \%, \mathrm{p}=0.024$, IL-6: $57.6 \%, \mathrm{p}=0.043$ ) and an almost complete inhibition of production following LMW Poly(I:C) (TNF- $\alpha$ : 93.7\%, p<0.001, IL-6: 100\%, p=0.004) (Figure 4.4A-D). Consistent with these findings, ACR had a more pronounced effect on reducing $\mathrm{p}$-I $\kappa \mathrm{B} \alpha$ levels and the $\mathrm{p}-\mathrm{I} \kappa \mathrm{B} \alpha / \mathrm{I} \kappa \mathrm{B} \alpha$ ratio in low glucose conditions (Figure 4.4E). These results suggest that HIF-1 $\alpha$, through the modulation of NF- $\mathrm{kB}$, contributes to inflammatory cytokine production following TLR3 engagement. They also suggest that HIF-1 $\alpha$ 's role in regulating inflammatory cytokine production, but not glycolysis, may become more important under conditions of stress. 

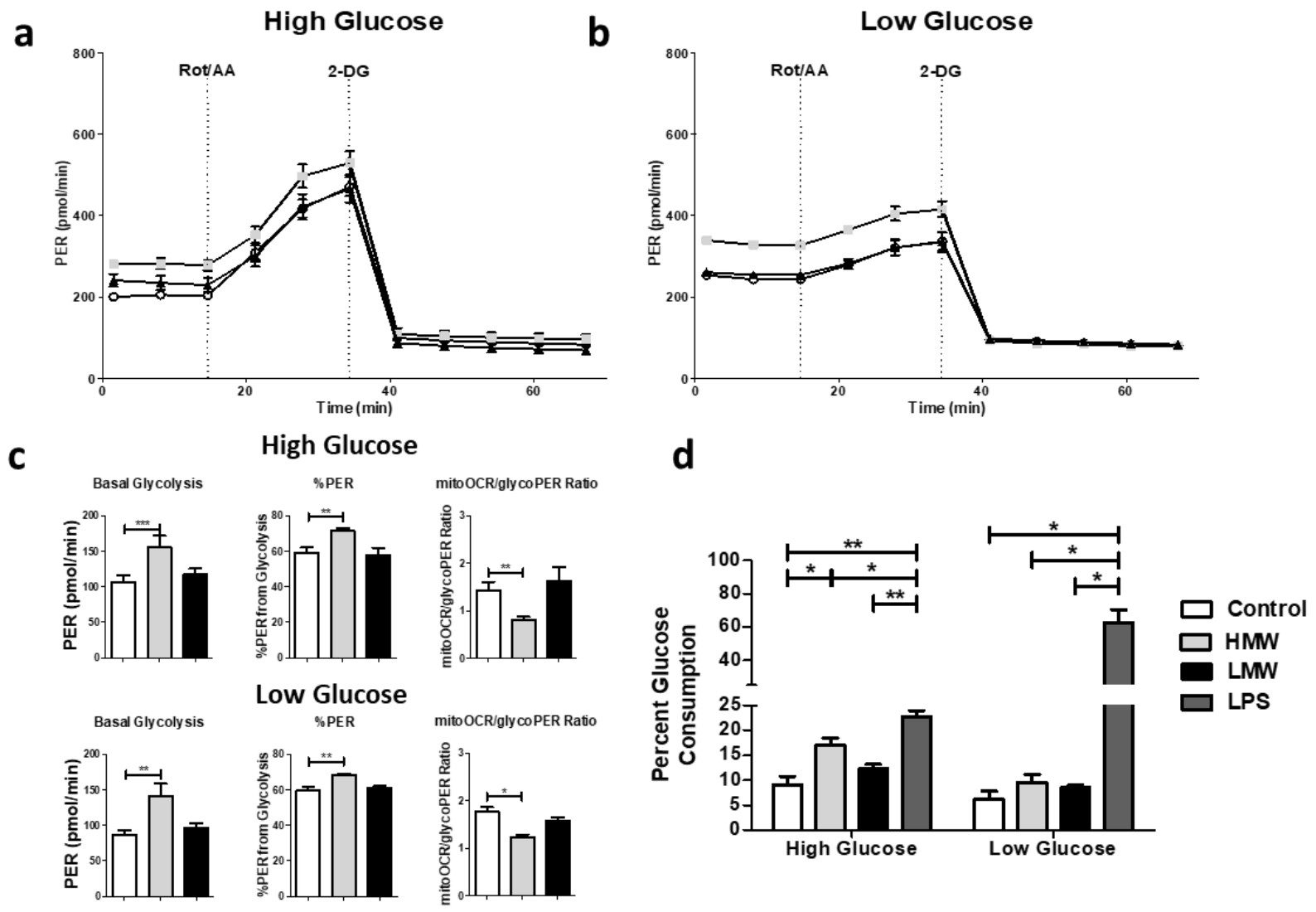

Figure 4.2: HMW Poly(I:C) stimulation is associated with increased glycolytic demands compared to LMW. Macrophages were stimulated with $10 \mathrm{ng} / \mathrm{mL}$ HMW Poly(I:C) or $10 \mathrm{ng} / \mathrm{mL}$ LMW Poly(I:C) for 18 hours. Glycolytic activity, as measured by Proton efflux rate (PER), was determined using a Seahorse efflux analyzer. Cells treated under high or low glucose conditions were exposed to sequential rotenone plus antimycin A (Rot/AA) and 2-deoxyglucose (2-DG) injections $(\mathbf{a}, \mathbf{b})$ in order to determine basal glycolytic rate, \%PER dependent on glycolysis and the ratio of mitochondrial oxygen consumption rate (mitoOCR) to glycolytic PER (c). Glucose consumption by BMM after stimulation with HMW and LMW Poly(I:C) and LPS was determined using a glucometer $(\mathbf{d})$. Data represents means \pm SEM of four individual mice $\left({ }^{*} p<\right.$ $0.05, * * \mathrm{p}<0.01$, and $* * * \mathrm{p}<0.001)$. 
a

Conditions: No ACR ACR

Treatment: Ctrl HMW LMW Ctrl HMW LMW

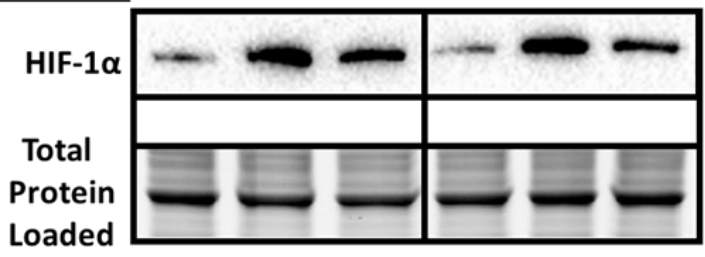

High Glucose

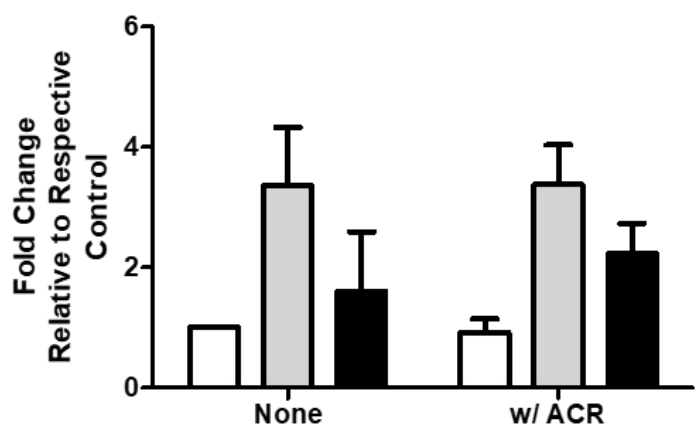

b Conditions: No ACR ACR

Treatment: Ctrl HMW LMW Ctrl HMW LMW

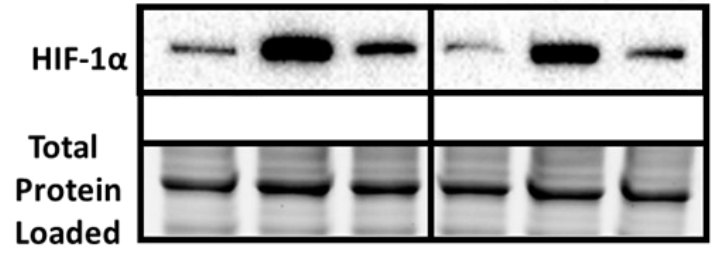

Low Glucose

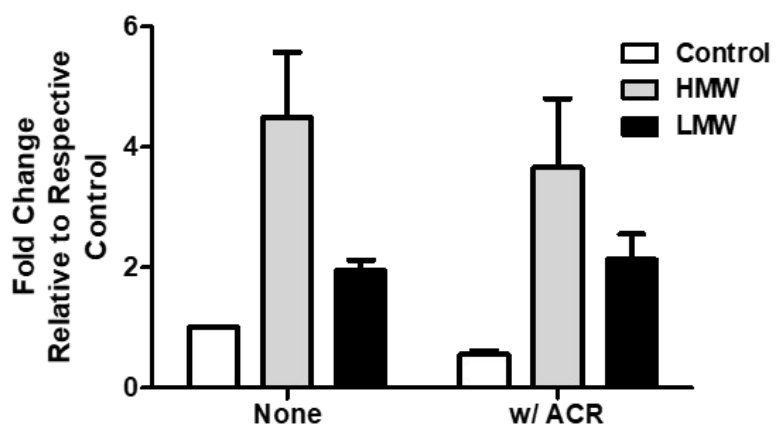

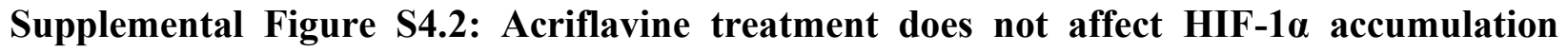
during HMW or LMW activation. HMW- or LMW-Poly(I:C) stimulated macrophages were cotreated with acriflavine (ACR) under high glucose (a) or low glucose (b) conditions for 18 hours. Total cell lysates were used to quantify HIF-1 $\alpha$ expression via immunoblotting. Band intensity was normalized to the total protein levels in each of their respective lanes using the Bio-Rad StainFree Application. HIF-1 $\alpha$ levels in each glucose condition was normalized to their respective control sample and presented as a fold change value. Data represents mean \pm SEM of two individual mice. 

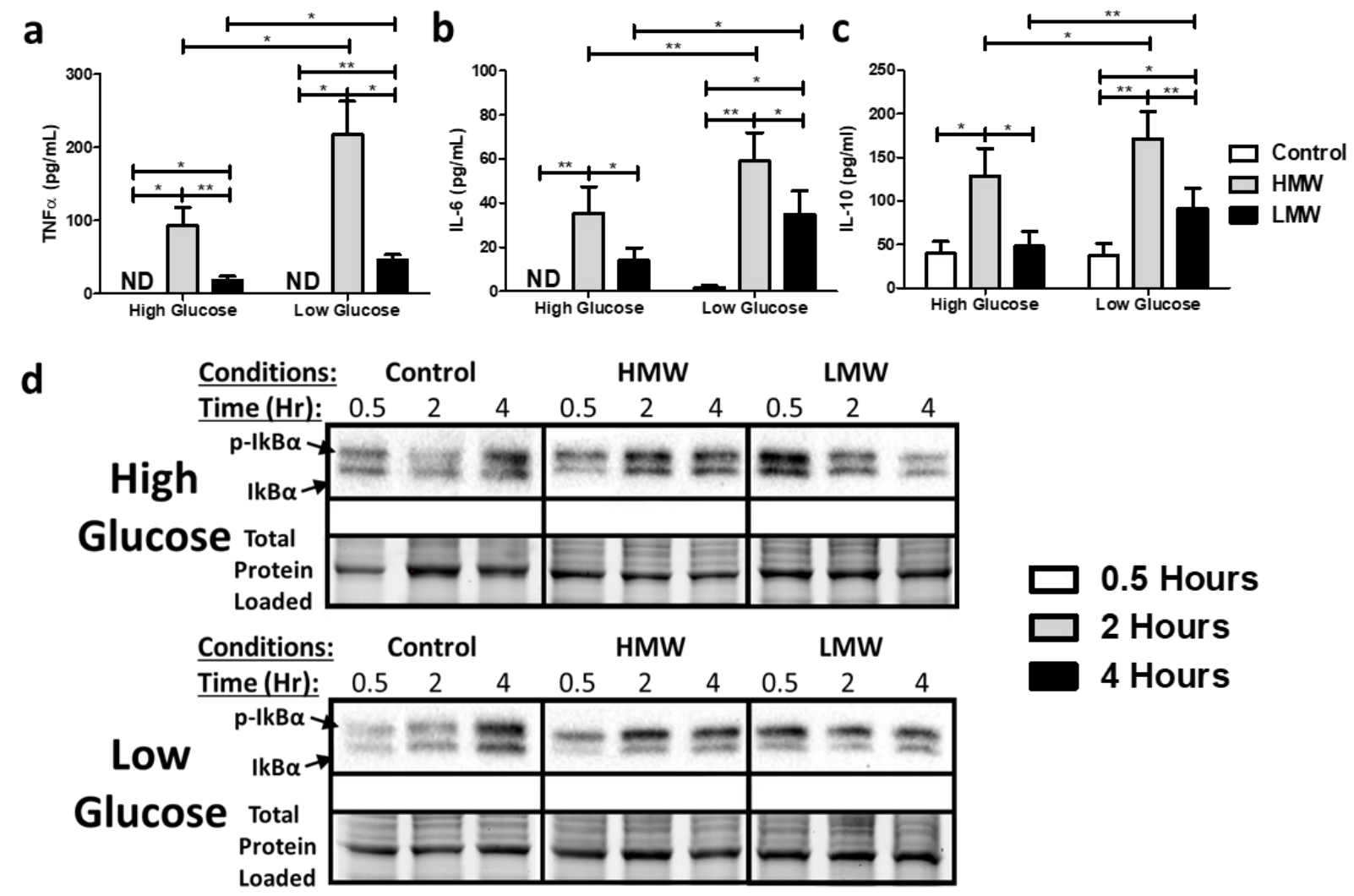

Figure 4.3: Low glucose conditions increased NF- $\mathrm{KB}$ activation and pro-inflammatory cytokine production. BMM were treated with $10 \mathrm{ng} / \mathrm{mL}$ HMW or $10 \mathrm{ng} / \mathrm{mL}$ LMW Poly(I:C) for 18 hours under high or low glucose media conditions. Pro-inflammatory cytokine production (TNF- $\alpha$ (a), IL-6 (b) and IL-10 (c)) was evaluated in culture supernatant. Total cell lysates were harvested to measure total and phosphorylated I $\mathrm{B} \alpha$ expression using immunoblotting at 0.5-, 2and 4-hours post-stimulation (d). Band intensity was normalized to the total protein levels in each of their respective lanes using the Bio-Rad Stain-Free Application. Afterwards, the expression of $\mathrm{I} \kappa \mathrm{B} \alpha$ in each glucose condition was normalized to its respective control sample collected at the same time point and presented as a fold change value. Data represents means \pm SEM of four individual mice $(* \mathrm{p}<0.05, * * \mathrm{p}<0.01$, and $* * * \mathrm{p}<0.001)$. 


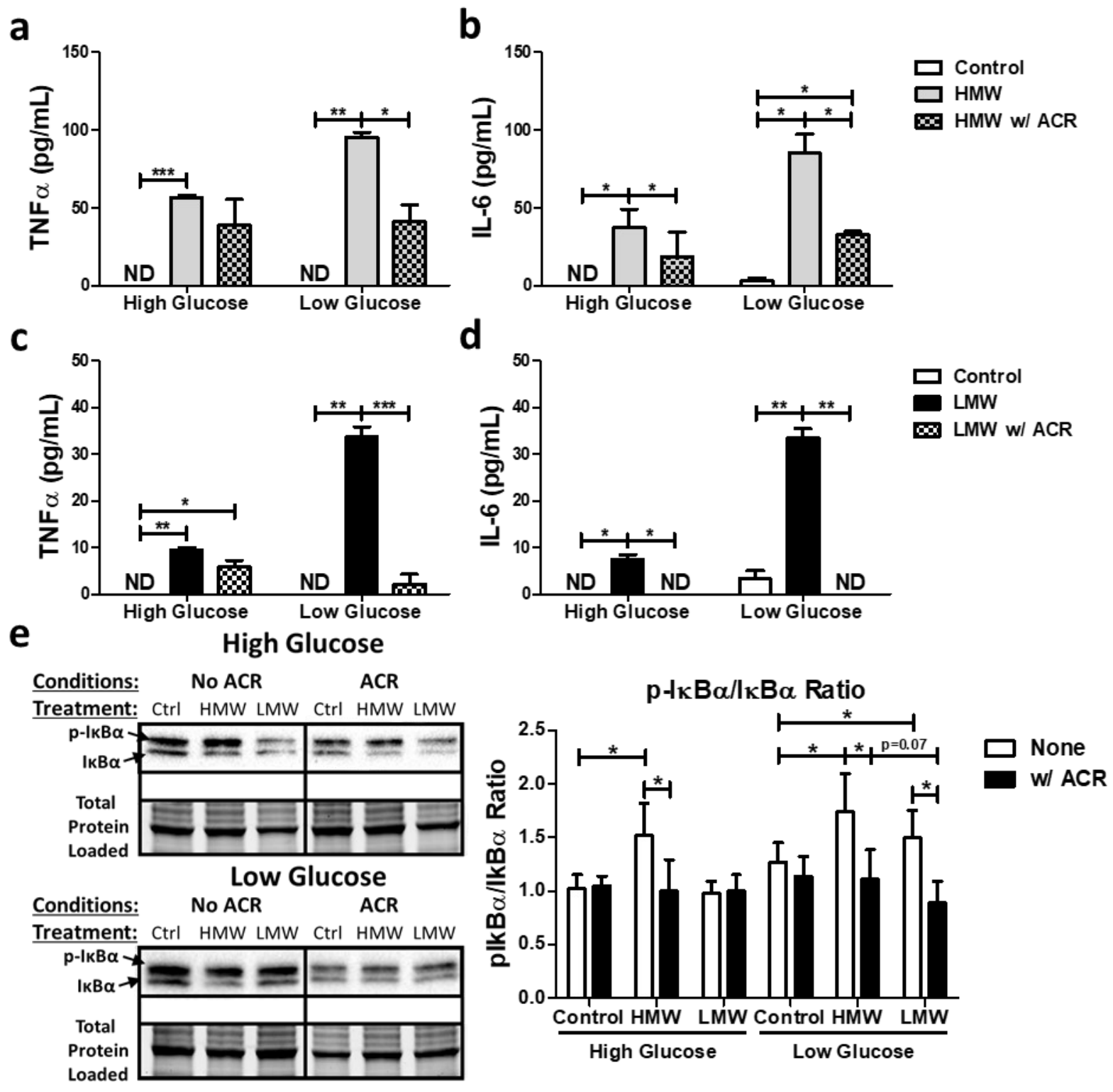

Figure 4.4: Targeting HIF-1 dimerization blunts pro-inflammatory but not antiinflammatory cytokine expression. HMW or LMW Poly(I:C) stimulated macrophages were co-treated with acriflavine (ACR) under high glucose or low glucose conditions for 18 hours. Production of TNF- $\alpha(\mathbf{a}, \mathbf{c})$ and IL-6 $(\mathbf{b}, \mathbf{d})$ in culture supernatant after HMW (a-b) or LMW activation (c-d) was measured using ELISA. Total protein and phosphorylation levels of IкB $\alpha$ from total cell lysates were quantified using immunoblotting after 18 hours (e). Band intensity was normalized to the total protein levels in each of their respective lanes using the Bio-Rad Stain-Free Application. Afterwards, the expression of IкB $\alpha$ in each glucose condition was normalized to its respective control sample and presented as a fold change value. Data represents means \pm SEM of three individual mice $\left(* p<0.05,{ }^{* *} \mathrm{p}<0.01\right.$, and $\left.{ }^{* * *} \mathrm{p}<0.001\right)$. 
a

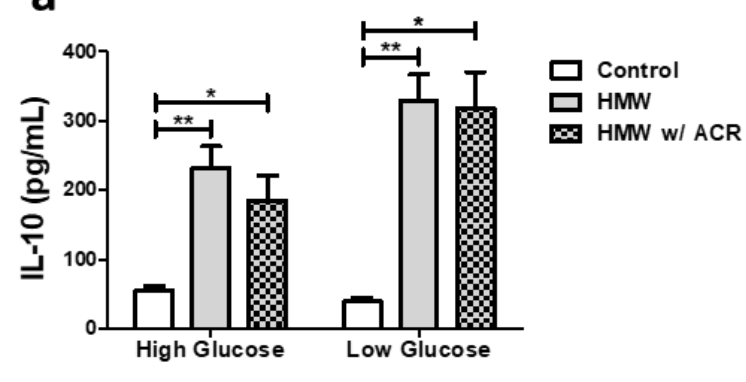

C

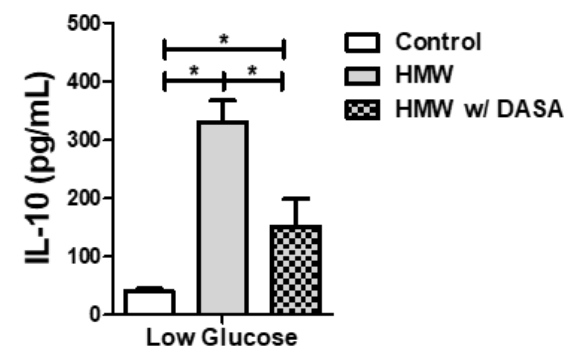

e

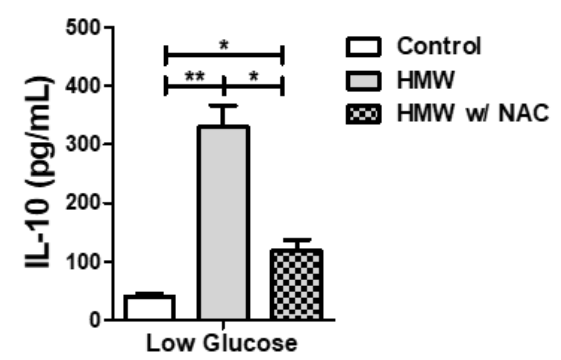

g

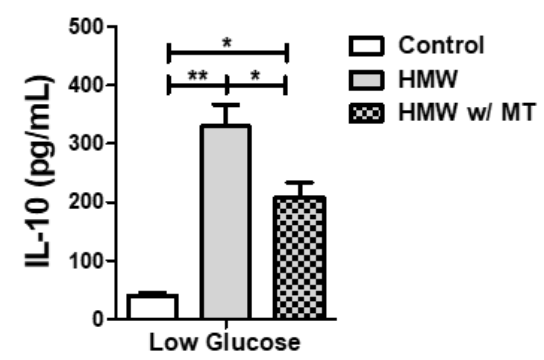

b

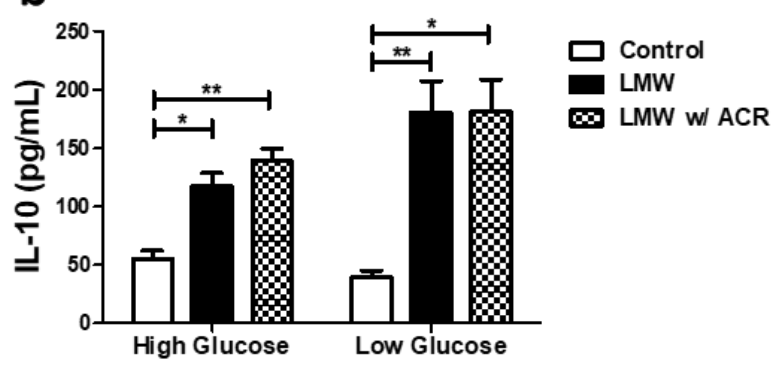

d

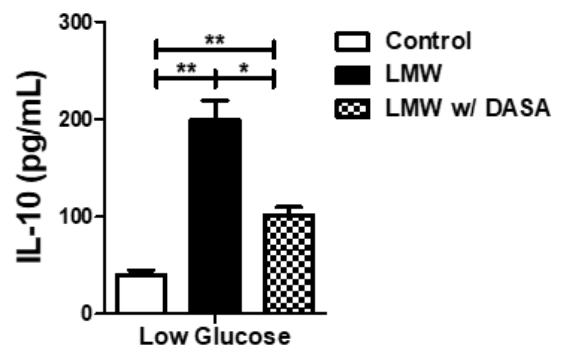

f

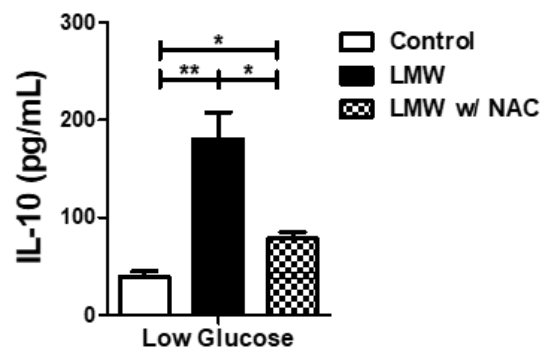

h

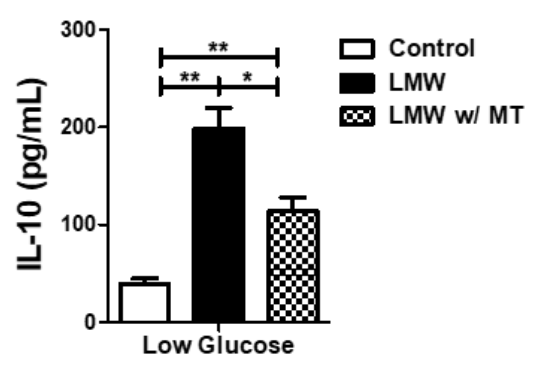

Supplemental Figure S4.3: HIF-1a does not directly regulate IL-10 expression. HMW- or LMW-Poly(I:C) stimulated macrophages were co-treated with either acriflavine (ACR) (a, b), DASA-58 (c, d), N-acetylcysteine (NAC) (e, f) or MitoTEMPO (MT) (g, h) under high glucose (a, c, e, g) or low glucose (b, d, f, h) conditions for 18 hours. IL-10 expression levels in culture supernatant were measured via ELISA. Data represents mean \pm SEM of three individual mice $\left({ }^{*} p\right.$ $<0.05, * * \mathrm{p}<0.01$, and $* * * \mathrm{p}<0.001)$. 


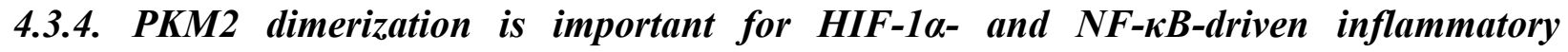 responses following TLR3 engagement}

The stability and accumulation of HIF- $1 \alpha$ is highly regulated at various levels by inhibitory proteins and metabolites $(20,59,60)$, redox status $(30,61,62)$ and transcriptional co-activators $(19,21)$. In particular, pyruvate kinase M2 (PKM2) has been shown to co-activate HIF-1 $\alpha$ under inflammatory conditions $(19,21,62)$. To evaluate if PKM2 is required for the co-activation of HIF-1 $\alpha$ following TLR3 engagement, BMM were stimulated with HMW and LMW Poly(I:C) under low glucose conditions in the presence or absence of DASA-58, which is a PKM2 "activator" that locks the protein in an active glycolytic tetrameric state, preventing its interactions with HIF-1 $\alpha$. These experiments were conducted in low glucose conditions as this condition potently encourages HIF-1 $\alpha$ accumulation. As seen with LPS (19), co-treatment with DASA-58 significantly reduced inflammatory cytokine production in cells stimulated with HMW and LMW Poly(I:C) (Figure 4.5A-D). However, as observed for ACR, the effects of DASA-58 on TNF- $\alpha$ (HMW: $\downarrow 54 \%, p=0.026$ vs. LMW: $\downarrow 93 \%, p=0.017$ ) and IL-6 (HMW: $\downarrow 80 \%, p=0.032$ vs. LMW: $\downarrow 100 \%, \mathrm{p}=0.004)$ production were more pronounced in LMW compared to HMW Poly(I:C) stimulated cells. Consistent with these findings, maintaining PKM2 in its tetrameric state

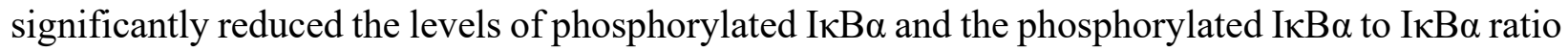
(HMW: $\downarrow 37.4 \%, p=0.048$ vs. LMW: $\downarrow 50.1 \%, p=0.038$ ) (Figure 4.5F). Interestingly, unlike ACR, DASA-58 also reduced IL-10 production (Supplemental Figure S4.3C-D). This is likely related to the ability of PKM2 to modulate STAT3 $(21,63)$. Collectively, these data suggest that the effects of PKM2 on the co-activation (and potentially accumulation [Figure 4.5E]) of HIF-1 $\alpha$, and not its own role in glycolysis, contribute to the regulation of inflammatory cytokine production during antiviral immune responses. Further, while PKM2 and HIF-1 $\alpha$ may be the central regulators of 
inflammatory cytokine production following LMW Poly(I:C) stimulation, other factors may contribute to the regulation following HMW.

\subsubsection{Cytosolic and mitochondrial ROS differentially regulate HMW and LMW inflammatory cytokine production}

Previous studies have shown that ROS, whether generated in the cytosol or by mitochondria, are key modulators of HIF-1 $\alpha$ stability during stress responses $(21,30,61,64)$. As such, I examined if both types of ROS might play a role in regulating HIF-1 $\alpha$ accumulation following 18-hour HMW and LMW Poly(I:C) stimulation under low glucose conditions. MitoSOX $^{\mathrm{TM}}$ Red was used to measure mitochondrial superoxide production and CellROX ${ }^{\mathrm{TM}}$ Orange was used to measure cytosolic ROS (Figures 4.6A and 4.7A). Both HMW and LMW Poly(I:C) stimulations were associated with increased mitochondrial superoxide accumulation (HMW FC=4.02, $\mathrm{p}<0.001$; LMW FC=2.97, $\mathrm{p}=0.007$ ) (Figure 4.6A). To evaluate if this increase in mitochondrial superoxide contributes to the stabilization of HIF-1 $\alpha$, I used MitoTEMPO to scavenge superoxide from active mitochondria. This co-treatment significantly reduced HIF-1 $\alpha$ levels in both HMW and LMW stimulated cells (HMW: $\downarrow 49 \%$, $\mathrm{p}=0.014$ vs. LMW: $\downarrow 56 \%$, $\mathrm{p}=0.013$ ) (Figure 4.6B). Consistent with these findings, levels of I $\mathrm{KB} \alpha$ phosphorylation (HMW: $\downarrow 35 \%, p=0.042$ vs. LMW: $\downarrow 30 \%, p=0.019$ ) (Figure $4.6 \mathrm{G}$ ) and cytokine production were decreased following MT co-treatment (TNF- $\alpha$ [HMW: $\downarrow 33 \%, p=0.023$ vs. LMW: $\downarrow 45 \%, p=0.049]$ and IL-6 [HMW: $\downarrow 75 \%, p=0.048$ vs. LMW: $\downarrow 45 \%, p=0.045]$ ) (Figure 4.6C-F). These findings suggest that mitochondrial ROS play a role in stabilizing HIF-1 $\alpha$ following TLR3 engagement.

Next, I wanted to see if cytosolic ROS also contribute to the accumulation of HIF-1 $\alpha$ following TLR3 engagement. Only HMW-treated cells were associated with a detectable increase in cytosolic ROS (HMW FC=1.33, $\mathrm{p}=0.003$; $\mathrm{LMW} \mathrm{FC=1.09,} \mathrm{p}=0.096$ ) (Figure 4.7A). Consistent with these findings, the antioxidant $N$-acetylcysteine (NAC) which targets total cellular ROS 
production, only reduced HIF-1 $\alpha$ accumulation in HMW stimulated cells (Figure 4.7B). Interestingly, NAC resulted in a near complete loss in TNF- $\alpha$ (HMW: $\downarrow 93 \%, p=0.006$ vs. LMW: $\downarrow 100 \%, \mathrm{p}=0.004$ ) and IL-6 secretion (HMW: $\downarrow 81 \%, \mathrm{p}=0.022$ vs. LMW: $\downarrow 100 \%, \mathrm{p}=0.004$ ) (Figure 4.7C-F) following both HMW and LMW stimulation. NAC was also associated with significant loss in both forms of $\mathrm{I} \kappa \mathrm{B} \alpha$ as well as the $\mathrm{p}$-I $\kappa \mathrm{B} \alpha / \mathrm{I} \kappa \mathrm{B} \alpha$ ratio (HMW: $\downarrow 29 \%, \mathrm{p}=0.040$ vs. LMW: $\downarrow 19 \%, \mathrm{p}=0.049$ ) (Figure $4.7 \mathrm{G}$ ). In addition to the scavenging of cellular ROS, NAC has been shown to increase the levels of cysteine, which is used as a substrate to generate glutathione (GSH), the principal antioxidant produced by the cell (65). GSH can also mediate post-translational modifications to signaling proteins such as IKK- $\beta$, which may reduce NF- $\kappa B$ activity (66). Collectively, these data suggest cytosolic ROS may only contribute to HIF-1 $\alpha$ accumulation (and potentially associated cytokine production) following HMW-mediated engagement of TLR3. 

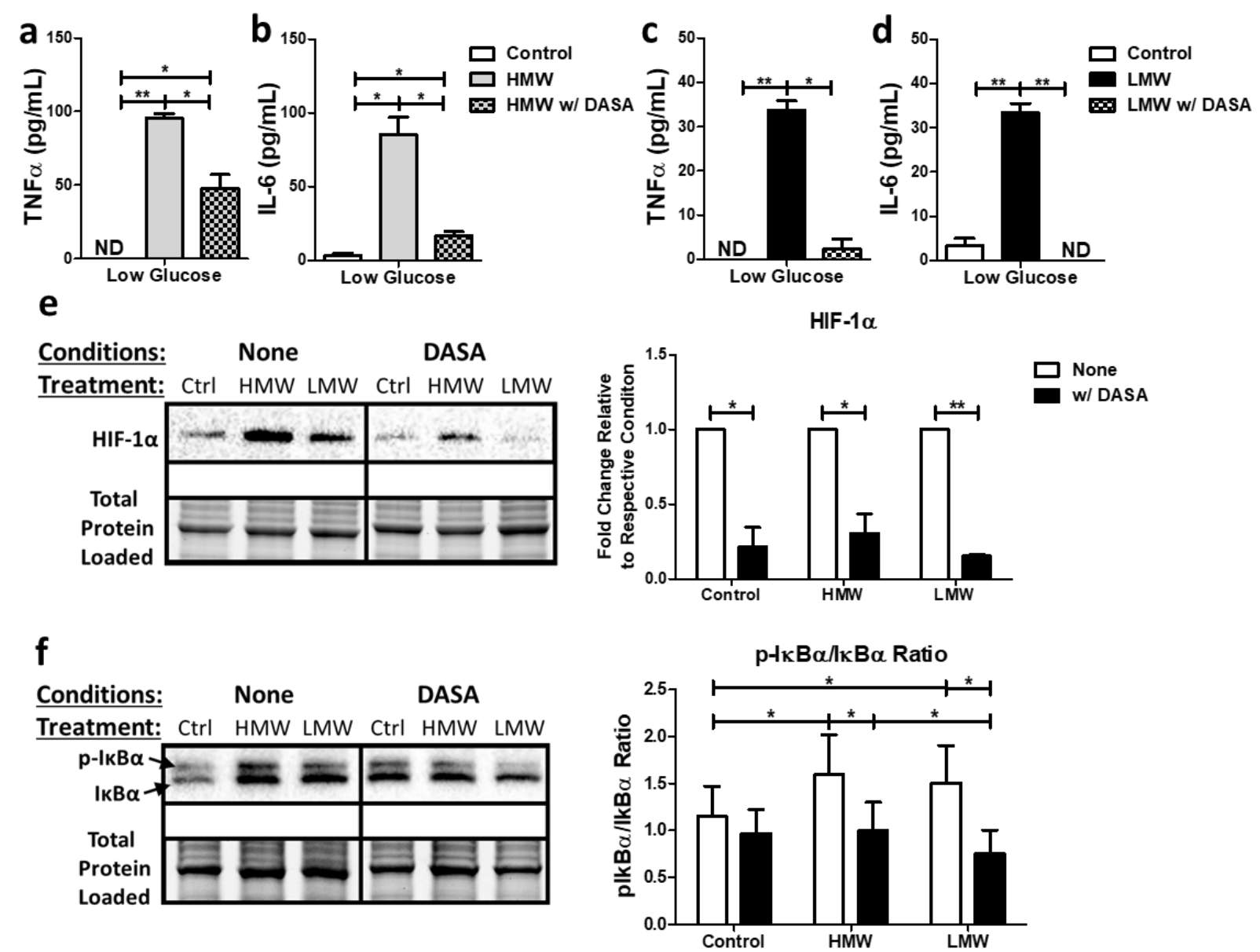

Figure 4.5: Maintenance of PKM2 in tetramer form reduces inflammatory cytokine production under low glucose conditions by reducing HIF-1 $\alpha$ accumulation and NF-KB activation. Inflammatory cytokine production was measured in culture supernatant from cells stimulated with HMW or LMW Poly(I:C) for 18 hours in the presence of DASA-58. These experiments were performed under low glucose conditions. Secretion (a-d) of TNF- $\alpha(\mathbf{a}, \mathbf{c})$ and IL-6 (b, d) under low glucose conditions after HMW (a-b) or LMW activation (c-d) were quantified via ELISA. Expression of HIF-1 $\alpha(\mathbf{e})$ and I $\mathrm{KB} \alpha(\mathbf{f})$ under low glucose conditions were assessed using immunoblotting. Band intensity was normalized to the total protein levels in each of their respective lanes using the Bio-Rad Stain-Free Application. Afterwards, the expression of I $\mathrm{B} \alpha$ in each glucose condition was normalized to its respective control sample and presented as a fold change value. Data represents means \pm SEM of three individual mice $\left({ }^{*} \mathrm{p}<0.05,{ }^{* *} \mathrm{p}<\right.$ 0.01 , and $* * * p<0.001)$. 

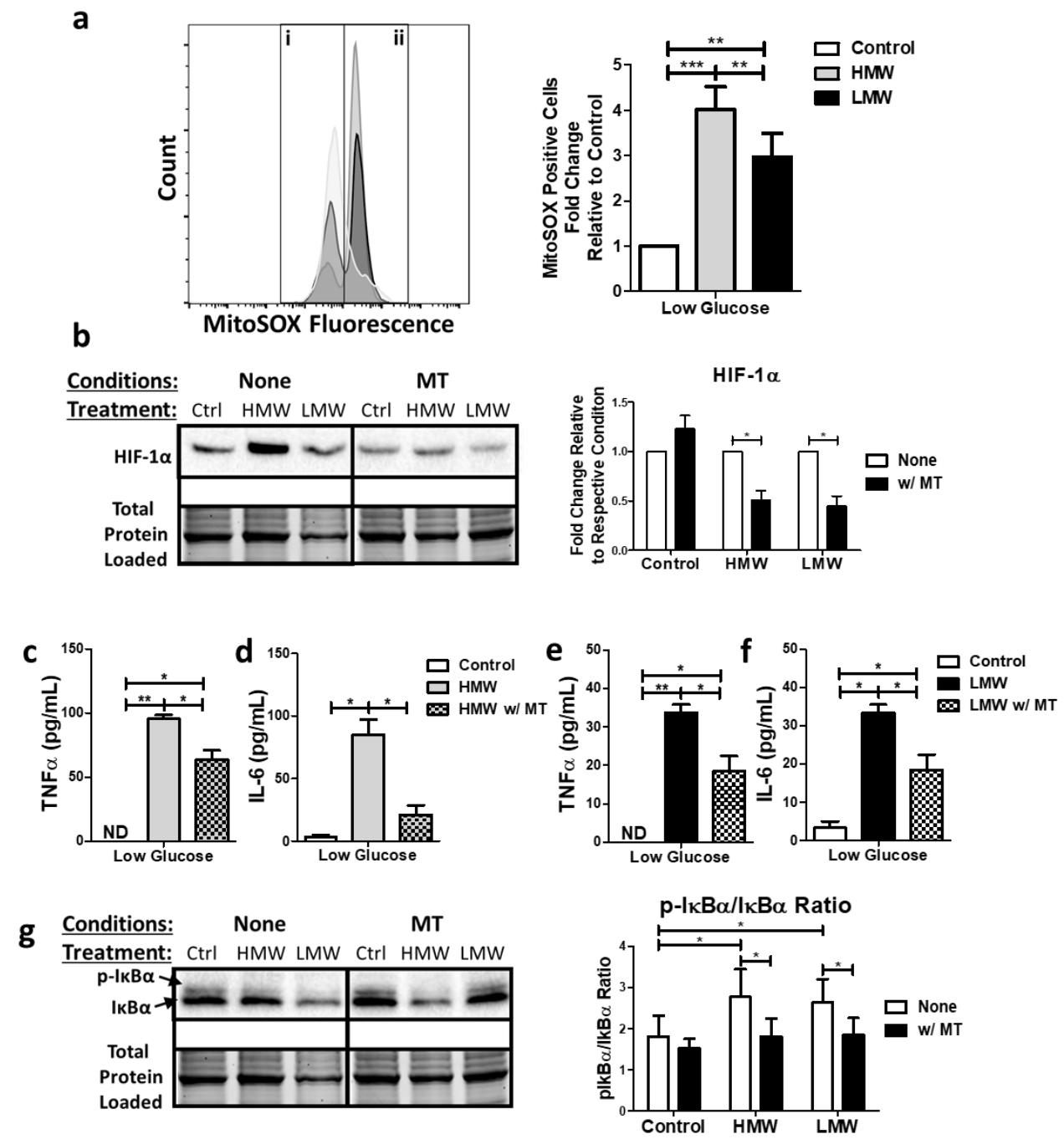

Figure 4.6: HMW- and LMW-mediated inflammatory cytokine production is blunted by mitochondrial ROS scavenging. HMW- and LMW-stimulated macrophages were examined for their differences in mitochondrial ROS production after 18 hours. Mitochondrial superoxide production was quantified using MitoSOX ${ }^{\mathrm{TM}}$ Red staining. Cells stained with MitoSOX ${ }^{\mathrm{TM}}$ Red were categorized into either a "negative" cell population $(i)$ or a "positive" population ( $i i)$, where fold change difference in the percentage of positive cells relative to the control sample was shown in the bar graph to the right of the histogram (a). Next, BMM were treated with HMW or LMW in the presence or absence of MitoTEMPO (MT) for 18 hours. Expression of HIF-1 $\alpha$ (b) and $\mathrm{I} \kappa \mathrm{B} \alpha(\mathbf{g})$ under low glucose conditions was assessed using immunoblotting. Band intensity was normalized to the total protein levels in each of their respective lanes using the Bio-Rad Stain-Free Application. Afterwards, the expression of $\mathrm{I} \kappa \mathrm{B} \alpha$ in each glucose condition was normalized to its respective control sample and presented as a fold change value. The effects of mitochondrial superoxide loss on inflammatory responses were assessed my measuring cytokine production (TNF- $\alpha(\mathbf{c}, \mathbf{e}) \&$ IL-6 $(\mathbf{d}, \mathbf{f}))$ using ELISA. Data represents means \pm SEM of three individual mice $(* \mathrm{p}<0.05, * * \mathrm{p}<0.01$, and $* * * \mathrm{p}<0.001)$. 

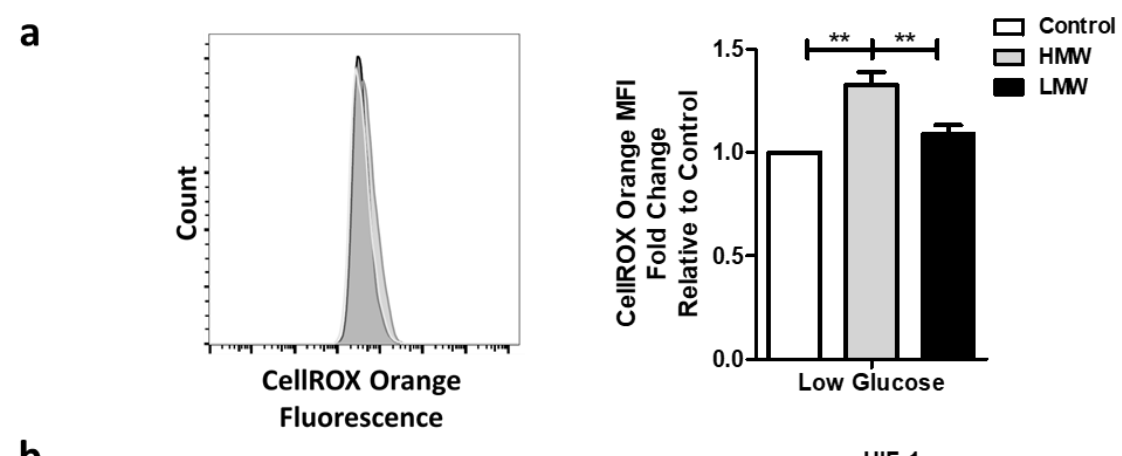

b
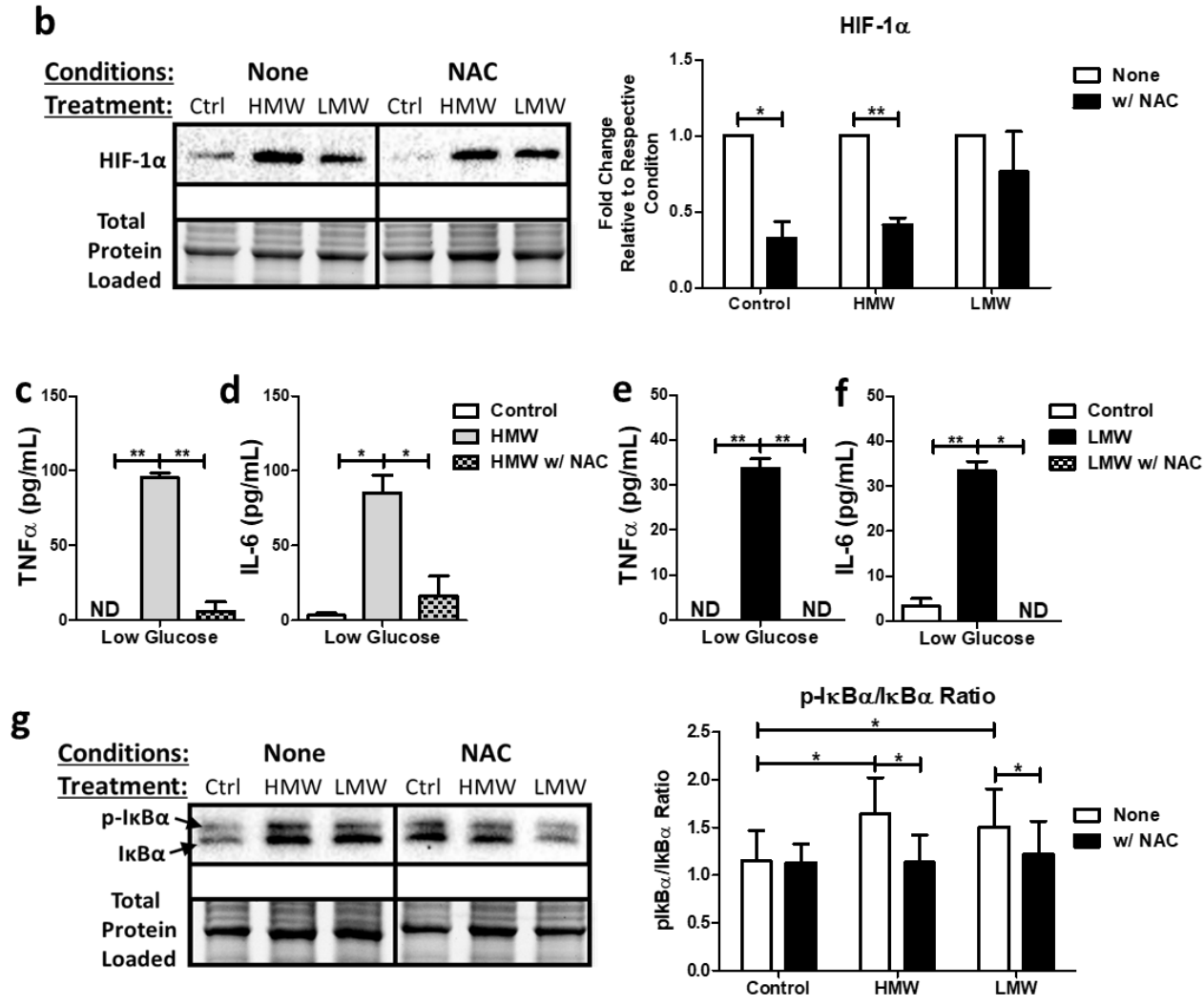

Figure 4.7: NAC differentially alters HIF-1 $\alpha$ accumulation and completely inhibits inflammatory responses following HMW and LMW. HMW- and LMW-stimulated macrophages were examined for their differences in ROS production after 18 hours. Cytosolic ROS production was measured using CellROX ${ }^{\mathrm{TM}}$ Orange staining (a). For CellROX ${ }^{\mathrm{TM}}$ Orange staining, cells were analyzed based on changes in MFI. Next, to evaluate the effects of total cellular ROS on inflammatory responses, BMM were stimulated with HMW and LMW Poly(I:C) in the presence or absence of N-acetylcysteine (NAC). Expression of HIF-1 $\alpha$ (b) and $\mathrm{I} \kappa \mathrm{B} \alpha(\mathbf{g})$ under low glucose conditions was assessed using immunoblotting. Band intensity was normalized to the total protein levels in each of their respective lanes using the Bio-Rad StainFree Application. Afterwards, the expression of $\mathrm{I} \kappa \mathrm{B} \alpha$ in each glucose condition was normalized to its respective control sample and presented as a fold change value. Cytokine production (TNF$\alpha(\mathbf{c}, \mathbf{e}) \&$ IL-6 $(\mathbf{d}, \mathbf{f}))$ was measured using ELISA. Data represents mean \pm SEM of three individual mice $(* \mathrm{p}<0.05, * * \mathrm{p}<0.01$, and $* * * \mathrm{p}<0.001)$. 


\subsection{Discussion:}

The role of HIF-1 $\alpha$ in regulating cellular metabolism and inflammation during antibacterial immune responses has been widely investigated $(19,20,25,27,29,30)$. However, its potential role in supporting antiviral immune responses is not fully understood. Here, I show that TLR3 activation and inflammatory cytokine production are differentially modulated by short and long dsRNA and that this process is dependent on HIF-1 $\alpha$ accumulation, which is modified by glucose availability. Engagement of TLR3 by long dsRNAs (e.g., HMW Poly(I:C)) increased glycolytic activity and pro-inflammatory cytokine production in both high and low glucose conditions. This cytokine production is regulated by HIF-1 $\alpha$ accumulation and is stabilized by PKM2, mitochondrial ROS, and cytosolic ROS. Conversely, engagement of TLR3 by short dsRNAs (e.g., LMW Poly(I:C)) did not alter glycolysis but rather induce a subdued inflammatory response in high glucose conditions. In contrast, stress (low glucose) conditions, increased HIF-1 $\alpha$ expression and enhanced inflammatory cytokine production. In these activated cells, HIF-1 $\alpha$ was stabilized by PKM2 and mitochondrial ROS, but not cytosolic ROS. Collectively, these data suggest that HIF-l $\alpha$ seems to fine tune the level of inflammatory cytokine production in antiviral immune responses and may act to drive differential responses to diverse activating ligands as well as nutrient availability in tissue microenvironments. These findings also suggest that HIF-1 $\alpha$ and the proteins/molecules that stabilize its accumulation may represent potential targets to reduce proinflammatory cytokine production in antiviral immune responses.

While HIF-1 $\alpha$ was originally identified under hypoxic conditions, studies have shown that nonhypoxic stimuli can also activate this transcription factor in a cell specific manner(24). In LPSstimulated cells, over one hundred HIF-1 $\alpha$-related target genes are upregulated, including those associated with glucose metabolism (e.g. PFKL, GAPDH, LDHA, PDK1), inflammation (e.g. 
IL1B, iNOS) and redox homeostasis (e.g. GPX3, HMOX1, SOD2) $(19,67,68)$. HIF-1 $\alpha$ plays a central role in inducing the Warburg effect in these inflammatory cells $(21,24,25,62,69)$. This inflammatory phenotype can be reversed by genetic deletion of HIF-1 $\alpha$ or by inhibiting glycolysis (e.g., 2-DG) $(60,70,71)$. Furthermore, HIF-1 $\alpha$ overexpression in macrophages induce a hyperinflammatory state with heightened IL-1 $\beta$, IL-6 and TNF- $\alpha$ expression (62). Taken together, these studies suggest HIF- $1 \alpha$-induced glycolysis is required to support inflammatory cytokine production. Here, I found that HMW but not LMW Poly(I:C) was associated with increased glycolysis in standard culture conditions (high glucose). In stark contrast to LPS stimulated cells, neither HMW nor LMW Poly(I:C) stimulation increased glucose uptake under low glucose conditions. Interestingly, while HMW was associated with a more pronounced inflammatory response, potentially linked to increased glycolysis, inflammatory cytokine production in LMW stimulated cells depended more on HIF-1 $\alpha$ accumulation (Figure 4). Collectively, our results suggest HIF-1 $\alpha$ may regulate antiviral inflammatory responses, at least in part, using mechanisms independent of glycolysis.

HIF-1 $\alpha /$ NF-kB crosstalk has been shown to play a critical role in mounting effective immune responses $(26,72,73)$. Traditionally, NF- $\mathrm{kB}$ is thought to regulate HIF-1 $\alpha$ function. Bonello et al. (74) found an NF- $\mathrm{kB}$ binding element in the HIF-1 $\alpha$ promoter and showed that hydrogen peroxide $\left(\mathrm{H}_{2} \mathrm{O}_{2}\right)$, and the activation of NOX4-containing NADPH oxidase, increased NF-kB-mediated HIF-1 $\alpha$ expression. In bacterial infections, IKK- $\beta$ has been shown to regulate HIF-1 $\alpha$ accumulation $(26,73)$ Further, Hepatitis C infection has been shown to boost HIF-1 $\alpha$ expression in a NF-kB- and MAPK-dependent manner (35). In this study, I found that HIF-1 $\alpha$ may also regulate NF- $\kappa B$ activity. Consistent with these findings, HIF-1 $\alpha$ has been shown to drive the expression of NF-kB-regulated inflammatory mediators in LPS-stimulated macrophages $(25,75)$, 
and knocking out HIF-1 $\alpha$ has been shown to protect against LPS-induced mortality (75). HIF-1 $\alpha$ mediated activation of NF-KB and IL-1 $\beta$ is also required for neutrophil survival and function (76). While further studies are required to better understand the specific mechanisms by which HIF-1 $\alpha$ regulates NF- $\mathrm{KB}$, our studies suggest there may be bidirectional communication between HIF-1 $\alpha$ and NF- $\mathrm{kB}$, creating a feed-forward loop to activate inflammatory cells.

Several studies show that short and long cytosolic dsRNA differentially interact with RIGI and MDA5, respectively (43, 46). I have shown that extracellular short (LMW: 200-1000 bp) vs. long (HMW: 1500-8000 bp) Poly(I:C) differentially engage TLR3, inducing distinct inflammatory and metabolic responses. Leonard et al. (51) found that dsRNA binds to TLR3 dimers in a saturable, specific, and reversible manner. To induce these dimers, dsRNA strands must be long enough (40-50 bp) to weakly interact with three separate contact sites on each monomer $(49,50)$. Binding affinity increases with strand length, with longer dsRNAs binding to multiple TLR3 dimers to induce differential signaling (51). Emerging evidence suggests that TLR3 responsiveness may differ between cell types $(47,48,77)$. While MEF responsiveness is greater with longer dsRNA strands, RAW264.7 cells are more responsive to shorter dsRNA strands (47). Similarly, Jiang et al. (48) found human monocyte-derived dendritic cells produce more robust antiviral and pro-inflammatory responses to short synthetic and influenza A specific-dsRNA strands. It is unclear what drives these cell-specific responses, but dsRNA has been shown to interact with other cellular receptors, including CCR5 (78). The idea of functional sensing is not specific to TLR3. Engagement of TLR4 with increasing concentrations of LPS selectively activates intracellular adaptor molecules. For example, picogram concentrations activate TRAM/TRIF signaling, whereas nanogram concentrations are required to also activate MyD88 
signaling and induce robust inflammatory responses (79-81). This highlights the remarkable ability of TLRs to sense stimuli and respond based on ligand type, length, and concentration.

HIF-1 $\alpha$ expression and function are regulated at multiple levels: post-translationally, metabolically, and at the level of complex formation. Under normoxic conditions, prolyl hydroxylases (PHDs) and the Factor Inhibiting HIF-I (FIH-I) hydroxylate proline and asparagine residues respectively along HIF- $1 \alpha$, leading to the destabilization and degradation of HIF- $1 \alpha$ (8286). These hydroxylation reactions are impeded in the absence of oxygen, resulting in HIF-1 $\alpha$ stabilization and accumulation (84). In this study, I found that HIF-1 $\alpha$ was stabilized by several factors including PKM2, which exists in tetramer and dimer forms in the cytosol. In its tetramer form, PKM2 serves as the final enzyme of glycolysis. As a dimer, it translocates into the nucleus and co-activates HIF-1 $\alpha(19,87,88)$, which promotes an inflammatory state by increasing the expression of lactose dehydrogenase (LDH) and pyruvate dehydrogenase (PDH) kinase (PDK). This inactivates the conversion of pyruvate to acetyl-CoA by PDH and forces pyruvate towards LDH to produce lactate (88-91). This, consequentially, increases the expression of IL-1 $\beta$, IL-6 and TNF- $\alpha(20,21,75,92)$. The PKM2 dimer also functions as a protein tyrosine kinase. It phosphorylates STAT3 in the nucleus leading to IL-6 and IL-1 $\beta$ expression $(21,63)$. Small molecule activators, which lock PKM2 into its glycolytically active tetramer form, decrease glycolytic-associated inflammation $(19,21)$. This secondary "moonlighting" function of PKM2 is not limited to inflammatory macrophages, as it is also important for proper Th1 and Th17 differentiation. Silencing, inhibiting, or locking PKM2 resulted in reduced $\mathrm{T}$ cell activation, proliferation, and associated cytokine production $(93,94)$. Here, I found that locking PKM2 into its tetramer using DASA-58 resulted in decreased accumulation of HIF- $1 \alpha$, NF- $\kappa B$ activation, and inflammatory cytokine production in low glucose conditions. Interestingly, locking PKM2 into its 
tetramer form produced a more pronounced effect on LMW responses, suggesting other factors may also contribute to HIF-1 $\alpha$ stabilization following stimulation with HMW Poly(I:C).

Exogenous or endogenous $\mathrm{H}_{2} \mathrm{O}_{2}$ and siRNA-mediated inhibition of mitochondrial superoxide dismutase 2 (SOD2) have also been shown to stabilize HIF-1 $\alpha$ protein levels under normoxic conditions, suggesting ROS may help stabilize HIF-1 $\alpha$ levels (95-98). Both superoxide and $\mathrm{H}_{2} \mathrm{O}_{2}$ have also been shown to directly inhibit PHD activity by oxidizing the iron cofactor of these enzymes $(99,100)$. Furthermore, $\mathrm{H}_{2} \mathrm{O}_{2}$ can induce disulfide bond-mediated PHD2 dimerization, which inactivates the protein and results in HIF-1 $\alpha$ stabilization (101). Mitochondrial ROS have also been shown to stabilize HIF-1 $\alpha$ by possibly oxidizing the iron cofactor of PHDs (102). Additionally, targeting superoxide production from Complex III of the ETC without the loss of OXPHOS has been shown to reduce HIF-1 $\alpha$ accumulation (103). Here, I found that mitochondrial and cytosolic ROS differentially contribute to HIF-1 $\alpha$ stabilization following HMW vs. LMW Poly(I:C) stimulations. In HMW-stimulated macrophages, both cellular and mitochondrial ROS contributed to HIF-1 $\alpha$ accumulation, NF- $\mathrm{kB}$ activation and the secretion of inflammatory cytokines. However, in LMW-activated macrophages only mitochondrial ROS were found to be critical in driving a pro-inflammatory state. Interestingly, Li et al. (104) found that a small amount of HIF-1 $\alpha$ localizes to the outer mitochondrial membrane under hypoxic conditions or after $\mathrm{H}_{2} \mathrm{O}_{2}$ treatment. In addition, the authors developed a mitochondrial-targeted form of HIF$1 \alpha$. This ectopic form of HIF-1 $\alpha$ attenuates apoptosis, reduces mtDNA release, and increases MMP (104). Cells with depleted mtDNA have diminished respiration, TCA cycle activity, and MMP leading to reduced HIF-1 $\alpha$ levels (105). Genetic reconstitution of MMP restored ROS levels in these cells, which was critical for HIF-1 $\alpha$ accumulation (105). This highlights the importance of ROS and mitochondrial function to HIF-1 $\alpha$ transcriptional activity. 
Interestingly, I found that co-treatment with NAC resulted in a near complete reduction of inflammatory cytokine production in both HMW and LMW stimulated cells, irrespective of cytosolic ROS accumulation. NAC functions by replenishing the cysteine supply in the cells, which is used to produce GSH (106). While GSH is an important ROS scavenger, it has also been shown to post-translationally modify proteins (glutathionylation) associated with TLR3 signaling $(17,66,107,108)$. Reynaert et al. (107) found that glutathionylation reduces IKK- $\beta$ activity,

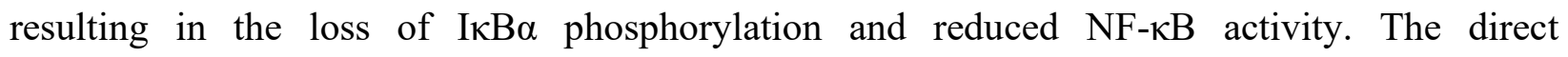
glutathionylation of I $\mathrm{I} B \alpha$ has also been shown to prevent its phosphorylation, and therefore preventing NF- $\mathrm{kB}$ activation (66). Furthermore, the DNA-binding site of the p50 subunit of NF$\kappa \mathrm{B}$ can be S-glutathionylated, leading to reduced pro-inflammatory cytokine expression (108).

In summary, I show that HIF-1 $\alpha$ plays an important role in regulating the magnitude of inflammatory responses in mouse BMM following TLR3 engagement. HIF-1 $\alpha$ amplifies these inflammatory responses following strong engagement of TLR3 and under stress conditions, such as glucose deprivation. This may play a role in a macrophage's ability to survey tissues for pathogens and shape its response based on the pathogen and the tissue microenvironment (109). I also show that this amplifying effect is largely mediated by increasing I $\mathrm{K} B \alpha$ phosphorylation and NF- $\mathrm{kB}$ activity. Based on these findings, it is tempting to speculate that targeting HIF-1 $\alpha$ or its stabilizing factors may represent a potential therapeutic strategy to reduce high levels of damaging inflammation during acute and chronic viral infections. 


\subsection{References}

1. Ross, J. A., and M. J. Auger. 2002. Biology of the Macrophage. In The Macrophage, 2nd ed. B. Burke, and C. E. Lewis, eds. Oxford University Press, New York, New York. 1-57.

2. Pearce, Erika L., and Edward J. Pearce. 2013. Metabolic pathways in immune cell activation and quiescence. Immunity 38: 633-643.

3. Mosser, D. M., and J. P. Edwards. 2008. Exploring the full spectrum of macrophage activation. Nat Rev Immunol 8: 958-969.

4. Djafarzadeh, S., M. Vuda, J. Takala, M. Ochs, and S. M. Jakob. 2011. Toll-like receptor3 -induced mitochondrial dysfunction in cultured human hepatocytes. Mitochondrion 11: 83-88.

5. Koshiba, T. 2013. Mitochondrial-mediated antiviral immunity. Biochim Biophys Acta 1833: 225-232.

6. Yoshizumi, T., H. Imamura, T. Taku, T. Kuroki, A. Kawaguchi, K. Ishikawa, K. Nakada, and T. Koshiba. 2017. RLR-mediated antiviral innate immunity requires oxidative phosphorylation activity. Sci Rep 7: 5379.

7. Pantel, A., A. Teixeira, E. Haddad, E. G. Wood, R. M. Steinman, and M. P. Longhi. 2014. Direct type I IFN but not MDA5/TLR3 activation of dendritic cells is required for maturation and metabolic shift to glycolysis after Poly IC stimulation. PLoS Biol. 12: e1001759.

8. Everts, B., E. Amiel, S. C.-C. Huang, A. M. Smith, C.-H. Chang, W. Y. Lam, V. Redmann, T. C. Freitas, J. Blagih, G. J. W. van der Windt, M. N. Artyomov, R. G. Jones, E. L. Pearce, and E. J. Pearce. 2014. TLR-driven early glycolytic reprogramming via the kinases TBK1IKKe supports the anabolic demands of dendritic cell activation. Nat Immunol 15: 323332.

9. Garaude, J., R. Acín-Pérez, S. Martínez-Cano, M. Enamorado, M. Ugolini, E. NistalVillán, S. Hervás-Stubbs, P. Pelegrín, L. E. Sander, J. A. Enríquez, and D. Sancho. 2016. Mitochondrial respiratory-chain adaptations in macrophages contribute to antibacterial host defense. Nat Immunol 17: 1037-1045.

10. Wu, D., David E. Sanin, B. Everts, Q. Chen, J. Qiu, Michael D. Buck, A. Patterson, Amber M. Smith, C.-H. Chang, Z. Liu, Maxim N. Artyomov, Erika L. Pearce, M. Cella, and Edward J. Pearce. 2016. Type 1 Interferons induce changes in core metabolism that are critical for immune function. Immunity 44: 1325-1336.

11. Ahmed, D., D. Roy, A. Jaworski, A. Edwards, A. Abizaid, A. Kumar, A. Golshani, and E. Cassol. 2019. Differential remodeling of the electron transport chain is required to support TLR3 and TLR4 signaling and cytokine production in macrophages. Sci Rep 9: 18801.

12. Seth, R. B., L. Sun, C.-K. Ea, and Z. J. Chen. 2005. Identification and characterization of MAVS, a mitochondrial antiviral signaling protein that a ctivates NF- $\kappa \mathrm{B}$ and IRF3. Cell 122: 669-682.

13. Kawai, T., K. Takahashi, S. Sato, C. Coban, H. Kumar, H. Kato, K. J. Ishii, O. Takeuchi, and S. Akira. 2005. IPS-1, an adaptor triggering RIG-I- and MDA5-mediated type I interferon induction. Nat Immunol 6: 981-988.

14. Xu, L.-G., Y.-Y. Wang, K.-J. Han, L.-Y. Li, Z. Zhai, and H.-B. Shu. 2005. VISA is an adapter protein required for virus-triggered IFN- $\beta$ Ssgnaling. Mol Cell 19: 727-740.

15. Tal, M. C., M. Sasai, H. K. Lee, B. Yordy, G. S. Shadel, and A. Iwasaki. 2009. Absence of autophagy results in reactive oxygen species-dependent amplification of RLR signaling. Proc Natl Acad Sci U S A 106: 2770. 
16. Indukuri, H., S. M. Castro, S.-M. Liao, L. A. Feeney, M. Dorsch, A. J. Coyle, R. P. Garofalo, A. R. Brasier, and A. Casola. 2006. Ikke regulates viral-induced interferon regulatory factor-3 activation via a redox-sensitive pathway. Virology 353: 155-165.

17. Gonzalez-Dosal, R., K. A. Horan, S. H. Rahbek, H. Ichijo, Z. J. Chen, J. J. Mieyal, R. Hartmann, and S. R. Paludan. 2011. HSV infection induces production of ROS, which potentiate signaling from pattern recognition receptors: Role for S-glutathionylation of TRAF3 and 6. PLOS Pathog 7: e1002250.

18. Yang, C.-S., J.-J. Kim, S. J. Lee, J. H. Hwang, C.-H. Lee, M.-S. Lee, and E.-K. Jo. 2013. TLR3-triggered reactive oxygen species contribute to inflammatory responses by activating Signal transducer and activator of transcription-1. J Immunol 190: 6368.

19. Palsson-McDermott, E. M., A. M. Curtis, G. Goel, M. A. R. Lauterbach, F. J. Sheedy, L. E. Gleeson, M. W. M. van den Bosch, S. R. Quinn, R. Domingo-Fernandez, D. G. W. Johnston, J.-k. Jiang, W. J. Israelsen, J. Keane, C. Thomas, C. Clish, M. Vander Heiden, R. J. Xavier, and L. A. J. O’Neill. 2015. Pyruvate kinase M2 regulates Hif-1 $\alpha$ activity and IL-1 $\beta$ induction and is a critical determinant of the warburg effect in LPS-activated macrophages. Cell Metab. 21: 65-80.

20. Tannahill, G. M., A. M. Curtis, J. Adamik, E. M. Palsson-McDermott, A. F. McGettrick, G. Goel, C. Frezza, N. J. Bernard, B. Kelly, N. H. Foley, L. Zheng, A. Gardet, Z. Tong, S. S. Jany, S. C. Corr, M. Haneklaus, B. E. Caffrey, K. Pierce, S. Walmsley, F. C. Beasley, E. Cummins, V. Nizet, M. Whyte, C. T. Taylor, H. Lin, S. L. Masters, E. Gottlieb, V. P. Kelly, C. Clish, P. E. Auron, R. J. Xavier, and L. A. J. O/'Neill. 2013. Succinate is an inflammatory signal that induces IL-1 $\beta$ through HIF-1 $\alpha$. Nature 496: 238-242.

21. Shirai, T., R. R. Nazarewicz, B. B. Wallis, R. E. Yanes, R. Watanabe, M. Hilhorst, L. Tian, D. G. Harrison, J. C. Giacomini, T. L. Assimes, J. J. Goronzy, and C. M. Weyand. 2016. The glycolytic enzyme PKM2 bridges metabolic and inflammatory dysfunction in coronary artery disease. J. Exp. Med. 213: 337-354.

22. Karshovska, E., Y. Wei, P. Subramanian, R. Mohibullah, C. Geißler, I. Baatsch, A. Popal, J. Corbalán Campos, N. Exner, and A. Schober. 2020. HIF-1 $\alpha$ (Hypoxia-inducible factor$1 \alpha$ ) promotes macrophage necroptosis by regulating miR-210 an d miR-383. Arterioscler Thromb Vasc Biol 40: 583-596.

23. Palazon, A., A. W. Goldrath, V. Nizet, and R. S. Johnson. 2014. HIF transcription factors, inflammation, and immunity. Immunity 41: 518-528.

24. Blouin, C. C., E. L. Pagé, G. M. Soucy, and D. E. Richard. 2004. Hypoxic gene activation by lipopolysaccharide in macrophages: Implication of Hypoxia-inducible factor $1 \alpha$. Blood 103: 1124-1130.

25. Peyssonnaux, C., V. Datta, T. Cramer, A. Doedens, E. A. Theodorakis, R. L. Gallo, N. Hurtado-Ziola, V. Nizet, and R. S. Johnson. 2005. HIF-1 $\alpha$ expression regulates the bactericidal capacity of phagocytes. J. Clin. Invest. 115: 1806-1815.

26. Rius, J., M. Guma, C. Schachtrup, K. Akassoglou, A. S. Zinkernagel, V. Nizet, R. S. Johnson, G. G. Haddad, and M. Karin. 2008. NF- $\mathrm{BB}$ links innate immunity to the hypoxic response through transcriptional regulation of HIF-1 $\alpha$. Nature 453: 807-811.

27. Cramer, T., Y. Yamanishi, B. E. Clausen, I. Förster, R. Pawlinski, N. Mackman, V. H. Haase, R. Jaenisch, M. Corr, V. Nizet, G. S. Firestein, H.-P. Gerber, N. Ferrara, and R. S. Johnson. 2003. HIF-1 $\alpha$ is essential for myeloid cell-mediated inflammation. Cell 112: 645657. 
28. Anand, R., S. Gribar, J. Li, J. W. Kohler, M. F. Branca, T. D. Dubowski, C. Sodhi, and D. Hackam. 2007. Hypoxia causes an increase in phagocytosis by macrophages in a Hif-1 $\alpha-$ dependent manner. J Leukoc Biol 82.

29. Frede, S., C. Stockmann, P. Freitag, and J. Fandrey. 2006. Bacterial lipopolysaccharide induces HIF-1 activation in human monocytes via p44/42 MAPK and NF-kappaB. Biochem J 396: 517-527.

30. Mills, E. L., B. Kelly, A. Logan, A. S. H. Costa, M. Varma, C. E. Bryant, P. Tourlomousis, J. H. M. Däbritz, E. Gottlieb, I. Latorre, S. C. Corr, G. McManus, D. Ryan, H. T. Jacobs, M. Szibor, R. J. Xavier, T. Braun, C. Frezza, M. P. Murphy, and L. A. O’Neill. 2016. Succinate dehydrogenase supports metabolic repurposing of mitochondria to drive inflammatory macrophages. Cell 167: 457-470.e413.

31. Deshmane, S. L., R. Mukerjee, S. Fan, L. Del Valle, C. Michiels, T. Sweet, I. Rom, K. Khalili, J. Rappaport, S. Amini, and B. E. Sawaya. 2009. Activation of the oxidative stress pathway by HIV-1 Vpr leads to induction of hypoxia-inducible factor 1alpha expression. $J$ Biol Chem 284.

32. Deshmane, S. L., S. Amini, S. Sen, K. Khalili, and B. E. Sawaya. 2011. Regulation of the HIV-1 promoter by HIF-1 $\alpha$ and Vpr proteins. Virol J 8: 477.

33. Duette, G., P. Pereyra Gerber, J. Rubione, P. S. Perez, A. L. Landay, S. M. Crowe, Z. Liao, K. W. Witwer, M. P. Holgado, J. Salido, J. Geffner, O. Sued, C. S. Palmer, and M. Ostrowski. 2018. Induction of HIF-1 $\alpha$ by HIV-1 infection in CD4(+) T cells promotes viral replication and drives extracellular vesicle-mediated inflammation. mBio 9: e00757-00718.

34. Ripoli, M., A. D'Aprile, G. Quarato, M. Sarasin-Filipowicz, J. Gouttenoire, R. Scrima, O. Cela, D. Boffoli, M. H. Heim, D. Moradpour, N. Capitanio, and C. Piccoli. 2010. Hepatitis $\mathrm{C}$ virus-linked mitochondrial dysfunction promotes Hypoxia-inducible factor $1 \alpha$-mediated glycolytic adaptation. $J$ Virol 84: 647-660.

35. Nasimuzzaman, M., G. Waris, D. Mikolon, D. G. Stupack, and A. Siddiqui. 2007. Hepatitis $\mathrm{C}$ virus stabilizes Hypoxia-inducible factor $1 \alpha$ and stimulates the synthesis of Vascular endothelial growth factor. J Virol 81: 10249.

36. Yoo, Y.-G., S. H. Oh, E. S. Park, H. Cho, N. Lee, H. Park, D. K. Kim, D.-Y. Yu, J. K. Seong, and M.-O. Lee. 2003. Hepatitis B virus X protein enhances transcriptional activity of Hypoxia-inducible factor- $1 \alpha$ through activation of Mitogen-activated protein kinase pathway. J Biol Chem 278: 39076-39084.

37. Cai, Q.-L., J. S. Knight, S. C. Verma, P. Zald, and E. S. Robertson. 2006. EC5S ubiquitin complex is recruited by KSHV latent antigen LANA for degradation of the VHL and p53 tumor suppressors. PLOS Pathog 2: e116.

38. Kondo, S., S. Y. Seo, T. Yoshizaki, N. Wakisaka, M. Furukawa, I. Joab, K. L. Jang, and J. S. Pagano. 2006. EBV latent membrane protein 1 up-regulates Hypoxia-inducible factor $1 \alpha$ through Siah1-mediated down-regulation of Prolyl hydroxylases 1 and 3 in nasopharyngeal epithelial cells. Cancer Res 66: 9870.

39. Heil, F., H. Hemmi, H. Hochrein, F. Ampenberger, C. Kirschning, S. Akira, G. Lipford, H. Wagner, and S. Bauer. 2004. Species-specific recognition of single-stranded RNA via Toll-like receptor 7 and 8. Science 303: 1526-1529.

40. Lund, J., A. Sato, S. Akira, R. Medzhitov, and A. Iwasaki. 2003. Toll-like receptor 9mediated recognition of herpes simplex virus-2 by plasmacytoid dendritic cells. $J$ Exp Med 198: 513-520. 
41. Alexopoulou, L., A. C. Holt, R. Medzhitov, and R. A. Flavell. 2001. Recognition of double-stranded RNA and activation of NF-[kappa]B by Toll-like receptor 3. Nature 413: 732-738.

42. Yoneyama, M., M. Kikuchi, T. Natsukawa, N. Shinobu, T. Imaizumi, M. Miyagishi, K. Taira, S. Akira, and T. Fujita. 2004. The RNA helicase RIG-I has an essential function in double-stranded RNA-induced innate antiviral responses. Nat Immunol 5: 730-737.

43. Kato, H., O. Takeuchi, S. Sato, M. Yoneyama, M. Yamamoto, K. Matsui, S. Uematsu, A. Jung, T. Kawai, K. J. Ishii, O. Yamaguchi, K. Otsu, T. Tsujimura, C.-S. Koh, C. Reis e Sousa, Y. Matsuura, T. Fujita, and S. Akira. 2006. Differential roles of MDA5 and RIG-I helicases in the recognition of RNA viruses. Nature 441: 101-105.

44. Takeuchi, O., and S. Akira. 2009. Innate immunity to virus infection. Immunol Rev 227: 75-86.

45. Kawai, T., and S. Akira. 2007. Signaling to NF-кB by Toll-like receptors. Trends Mol Med 13: 460-469.

46. Kato, H., O. Takeuchi, E. Mikamo-Satoh, R. Hirai, T. Kawai, K. Matsushita, A. Hiiragi, T. S. Dermody, T. Fujita, and S. Akira. 2008. Length-dependent recognition of doublestranded ribonucleic acids by Retinoic acid-inducible gene-I and Melanoma differentiation-associated gene 5. J Exp Med 205: 1601-1610.

47. Mian, M. F., A. N. Ahmed, M. Rad, A. Babaian, D. Bowdish, and A. A. Ashkar. 2013. Length of dsRNA (poly I:C) drives distinct innate immune responses, depending on the cell type. J Leukoc Biol 94: 1025-1036.

48. Jiang, M., P. Österlund, L. P. Sarin, M. M. Poranen, D. H. Bamford, D. Guo, and I. Julkunen. 2011. Innate immune responses in human Monocyte-derived dendritic cells are highly dependent on the size and the $5^{\prime}$ phosphorylation of RNA molecules. J Immunol 187: 1713.

49. Wang, Y., L. Liu, D. R. Davies, and D. M. Segal. 2010. Dimerization of Toll-like receptor 3 (TLR3) is required for ligand binding. J Biol Chem 285: 36836-36841.

50. Liu, L., I. Botos, Y. Wang, J. N. Leonard, J. Shiloach, D. M. Segal, and D. R. Davies. 2008. Structural basis of Toll-like receptor 3 signaling with double-stranded RNA. Science 320: 379-381.

51. Leonard, J. N., R. Ghirlando, J. Askins, J. K. Bell, D. H. Margulies, D. R. Davies, and D. M. Segal. 2008. The TLR3 signaling complex forms by cooperative receptor dimerization. Proc Natl Acad Sci U S A 105: 258-263.

52. Weischenfeldt, J., and B. Porse. 2008. Bone marrow-derived macrophages (BMM): Isolation and applications. CSH Protoc 2008: pdb.prot5080.

53. Taylor, S. C., T. Berkelman, G. Yadav, and M. Hammond. 2013. A defined methodology for reliable quantification of Western blot data. Mol Biotechnol 55: 217-226.

54. Achari, A. E., and S. K. Jain. 2016. L-Cysteine supplementation increases adiponectin synthesis and secretion, and GLUT4 and glucose utilization by upregulating disulfide bond A-like protein expression mediated by MCP-1 inhibition in 3T3-L1 adipocytes exposed to high glucose. Mol Cell Biochem 414: 105-113.

55. Rodríguez-Prados, J., P. G. Través, J. Cuenca, D. Rico, J. Aragonés, P. Martín-Sanz, M. Cascante, and L. Boscá. 2010. Substrate fate in activated macrophages: A comparison between innate, classic, and alternative activation. J Immunol 185: 605-614.

56. Freemerman, A. J., A. R. Johnson, G. N. Sacks, J. J. Milner, E. L. Kirk, M. A. Troester, A. N. Macintyre, P. Goraksha-Hicks, J. C. Rathmell, and L. Makowski. 2014. Metabolic 
reprogramming of macrophages: Glucose transporter 1 (GLUT1)-mediated glucose metabolism drives a proinflammatory phenotype. J Biol Chem 289: 7884-7896.

57. Semenza, G. L. 1998. Hypoxia-inducible factor 1 and the molecular physiology of oxygen homeostasis. J Lab Clin Med 131: 207-214.

58. Lee, K., H. Zhang, D. Z. Qian, S. Rey, J. O. Liu, and G. L. Semenza. 2009. Acriflavine inhibits HIF-1 dimerization, tumor growth, and vascularization. Proc Natl Acad Sci US A 106: 17910-17915.

59. Swain, L., M. Wottawa, A. Hillemann, A. Beneke, H. Odagiri, K. Terada, M. Endo, Y. Oike, K. Farhat, and D. M. Katschinski. 2014. Prolyl-4-hydroxylase domain 3 (PHD3) is a critical terminator for cell survival of macrophages under stress conditions. J Leukoc Biol 96: 365-375.

60. Semba, H., N. Takeda, T. Isagawa, Y. Sugiura, K. Honda, M. Wake, H. Miyazawa, Y. Yamaguchi, M. Miura, D. M. R. Jenkins, H. Choi, J.-w. Kim, M. Asagiri, A. S. Cowburn, H. Abe, K. Soma, K. Koyama, M. Katoh, K. Sayama, N. Goda, R. S. Johnson, I. Manabe, R. Nagai, and I. Komuro. 2016. HIF-1 $\alpha$-PDK1 axis-induced active glycolysis plays an essential role in macrophage migratory capacity. Nat Commun 7: 11635.

61. Shatrov, V. A., V. V. Sumbayev, J. Zhou, and B. Brüne. 2003. Oxidized low-density lipoprotein (oxLDL) triggers hypoxia-inducible factor-1 $\alpha$ (HIF-1 $\alpha$ ) accumulation via redox-dependent mechanisms. Blood 101: 4847-4849.

62. Wang, T., H. Liu, G. Lian, S.-Y. Zhang, X. Wang, and C. Jiang. 2017. HIF1 $\alpha$-induced glycolysis metabolism is essential to the activation of inflammatory macrophages. Mediators Inflamm 2017: 10.

63. Gao, X., H. Wang, Jenny J. Yang, X. Liu, and Z.-R. Liu. 2012. Pyruvate kinase M2 regulates gene transcription by acting as a protein kinase. Mol Cell 45: 598-609.

64. Wang, D., D. Malo, and S. Hekimi. 2010. Elevated mitochondrial reactive oxygen species generation affects the immune response via hypoxia-inducible factor- $1 \alpha$ in long-lived Mclk1+/- mouse mutants. J Immunol 184: 582.

65. De Rosa, S. C., M. D. Zaretsky, J. G. Dubs, M. Roederer, M. Anderson, A. Green, D. Mitra, N. Watanabe, H. Nakamura, I. Tjioe, S. C. Deresinski, W. A. Moore, S. W. Ela, D. Parks, and L. A. Herzenberg. 2000. N-acetylcysteine replenishes glutathione in HIV infection. Eur J Clin Invest 30: 915-929.

66. Kil, I. S., S. Y. Kim, and J.-W. Park. 2008. Glutathionylation regulates IкB. Biochem Biophys Res Commun 373: 169-173.

67. Wenger, R. H., D. P. Stiehl, and G. Camenisch. 2005. Integration of oxygen signaling at the consensus HRE. Sci STKE 2005: re12.

68. Dengler, V. L., M. Galbraith, and J. M. Espinosa. 2014. Transcriptional regulation by hypoxia inducible factors. Crit Rev Biochem Mol Biol 49: 1-15.

69. Everts, B., and E. J. Pearce. 2014. Metabolic control of dendritic cell activation and function: recent advances and clinical implications. Front Immunol 5: 203.

70. Li, C., Y. Wang, Y. Li, Q. Yu, X. Jin, X. Wang, A. Jia, Y. Hu, L. Han, J. Wang, H. Yang, D. Yan, Y. Bi, and G. Liu. 2018. HIF1 $\alpha$-dependent glycolysis promotes macrophage functional activities in protecting against bacterial and fungal infection. Sci Rep 8: 3603.

71. Liu, L., Y. Lu, J. Martinez, Y. Bi, G. Lian, T. Wang, S. Milasta, J. Wang, M. Yang, G. Liu, D. R. Green, and R. Wang. 2016. Proinflammatory signal suppresses proliferation and shifts macrophage metabolism from Myc-dependent to HIF1 $\alpha$-dependent. Proc Natl Acad Sci U S A 113: 1564. 
72. D'Ignazio, L., D. Bandarra, and S. Rocha. 2016. NF- $\kappa$ B and HIF crosstalk in immune responses. FEBS J 283: 413-424.

73. Fitzpatrick, S. F., M. M. Tambuwala, U. Bruning, B. Schaible, C. C. Scholz, A. Byrne, A. O'Connor, W. M. Gallagher, C. R. Lenihan, J. F. Garvey, K. Howell, P. G. Fallon, E. P. Cummins, and C. T. Taylor. 2011. An intact canonical NF- $\kappa B$ pathway is required for inflammatory gene expression in response to hypoxia. In J Immunol, United States. 10911096.

74. Bonello, S., C. Zähringer, S. BelAiba Rachida, T. Djordjevic, J. Hess, C. Michiels, T. Kietzmann, and A. Görlach. 2007. Reactive oxygen species activate the HIF-1 $\alpha$ promoter via a functional NFאB site. Arterioscler Thromb Vasc Biol 27: 755-761.

75. Peyssonnaux, C., P. Cejudo-Martin, A. Doedens, A. S. Zinkernagel, R. S. Johnson, and V. Nizet. 2007. Cutting edge: Essential role of Hypoxia inducible factor-1 $\alpha$ in development of Lipopolysaccharide-induced sepsis. J Immunol 178: 7516-7519.

76. Walmsley, S. R., C. Print, N. Farahi, C. Peyssonnaux, R. S. Johnson, T. Cramer, A. Sobolewski, A. M. Condliffe, A. S. Cowburn, N. Johnson, and E. R. Chilvers. 2005. Hypoxia-induced neutrophil survival is mediated by HIF-1alpha-dependent NF-kappaB activity. J Exp Med 201: 105-115.

77. Zhou, Y., M. Guo, X. Wang, J. Li, Y. Wang, L. Ye, M. Dai, L. Zhou, Y. Persidsky, and W. Ho. 2013. TLR3 activation efficiency by high or low molecular mass poly I:C. Innate Immun 19: 184-192.

78. Shaheen, Z. R., B. S. Christmann, J. D. Stafford, J. M. Moran, R. M. L. Buller, and J. A. Corbett. 2019. CCR5 is a required signaling receptor for macrophage expression of inflammatory genes in response to viral double-stranded RNA. Am J Physiol Regul Integr Comp Physiol 316: R525-R534.

79. Maitra, U., L. Gan, S. Chang, and L. Li. 2011. Low-dose Endotoxin induces inflammation by selectively removing nuclear receptors and activating CCAAT/enhancer-binding protein $\delta$. J Immunol 186: 4467-4473.

80. Maitra, U., H. Deng, T. Glaros, B. Baker, D. G. S. Capelluto, Z. Li, and L. Li. 2012. Molecular mechanisms responsible for the selective and low-grade induction of proinflammatory mediators in Murine macrophages by Lipopolysaccharide. J Immunol 189: 1014-1023.

81. Yuan, R., S. Geng, and L. Li. 2016. Molecular mechanisms that underlie the dynamic adaptation of innate monocyte memory to varying stimulant strength of TLR ligands. Front Immunol 7: 497.

82. Ohh, M., C. W. Park, M. Ivan, M. A. Hoffman, T.-Y. Kim, L. E. Huang, N. Pavletich, V. Chau, and W. G. Kaelin. 2000. Ubiquitination of hypoxia-inducible factor requires direct binding to the $\beta$-domain of the von Hippel-Lindau protein. Nat Cell Biol 2: 423-427.

83. Maxwell, P. H., M. S. Wiesener, G.-W. Chang, S. C. Clifford, E. C. Vaux, M. E. Cockman, C. C. Wykoff, C. W. Pugh, E. R. Maher, and P. J. Ratcliffe. 1999. The tumour suppressor protein VHL targets hypoxia-inducible factors for oxygen-dependent proteolysis. Nature 399: 271-275.

84. Brahimi-Horn, C., N. Mazure, and J. Pouysségur. 2005. Signalling via the hypoxiainducible factor- $1 \alpha$ requires multiple posttranslational modifications. Cell Signal 17: 1-9.

85. Mahon, P. C., K. Hirota, and G. L. Semenza. 2001. FIH-1: A novel protein that interacts with HIF-1 $\alpha$ and VHL to mediate repression of HIF-1 transcriptional activity. Genes Dev 15: $2675-2686$. 
86. Lando, D., D. J. Peet, J. J. Gorman, D. A. Whelan, M. L. Whitelaw, and R. K. Bruick. 2002. FIH-1 is an asparaginyl hydroxylase enzyme that regulates the transcriptional activity of Hypoxia-inducible factor. Genes Dev 16: 1466-1471.

87. Luo, W., H. Hu, R. Chang, J. Zhong, M. Knabel, R. O'Meally, R. N. Cole, A. Pandey, and G. L. Semenza. 2011. Pyruvate kinase M2 Is a PHD3-stimulated coactivator for Hypoxiainducible factor 1. Cell 145: 732-744.

88. Luo, W., and G. L. Semenza. 2011. Pyruvate kinase M2 regulates glucose metabolism by functioning as a coactivator for Hypoxia-inducible factor 1 in cancer cells. Oncotarget 2: 551-556.

89. Imtiyaz, H. Z., and M. C. Simon. 2010. Hypoxia-inducible factors as essential regulators of inflammation. Curr. Top. Microbiol. Immunol. 345: 105-120.

90. Jantsch, J., D. Chakravortty, N. Turza, A. T. Prechtel, B. Buchholz, R. G. Gerlach, M. Volke, J. Gläsner, C. Warnecke, M. S. Wiesener, K.-U. Eckardt, A. Steinkasserer, M. Hensel, and C. Willam. 2008. Hypoxia and Hypoxia-inducible factor-1 $\alpha$ modulate Lipopolysaccharide-induced dendritic cell activation and function. J Immunol 180: 46974705.

91. Papandreou, I., R. A. Cairns, L. Fontana, A. L. Lim, and N. C. Denko. 2006. HIF-1 mediates adaptation to hypoxia by actively downregulating mitochondrial oxygen consumption. Cell Metab 3: 187-197.

92. Das Gupta, K., M. R. Shakespear, J. E. B. Curson, A. M. V. Murthy, A. Iyer, M. P. Hodson, D. Ramnath, V. A. Tillu, J. B. von Pein, R. C. Reid, K. Tunny, D. M. Hohenhaus, S. V. Moradi, G. M. Kelly, T. Kobayashi, J. H. Gunter, A. J. Stevenson, W. Xu, L. Luo, A. Jones, W. A. Johnston, A. Blumenthal, K. Alexandrov, B. M. Collins, J. L. Stow, D. P. Fairlie, and M. J. Sweet. 2020. Class IIa Histone deacetylases drive Toll-like receptor-inducible glycolysis and macrophage inflammatory responses via Pyruvate kinase M2. Cell Rep 30: 2712-2728.e2718.

93. Kono, M., K. Maeda, I. Stocton-Gavanescu, W. Pan, M. Umeda, E. Katsuyama, C. Burbano, S. Y. K. Orite, M. Vukelic, M. G. Tsokos, N. Yoshida, and G. C. Tsokos. 2019. Pyruvate kinase M2 is requisite for Th1 and Th17 differentiation. JCI Insight 4: e127395.

94. Angiari, S., M. C. Runtsch, C. E. Sutton, E. M. Palsson-McDermott, B. Kelly, N. Rana, H. Kane, G. Papadopoulou, E. L. Pearce, K. H. G. Mills, and L. A. J. O'Neill. 2020. Pharmacological activation of Pyruvate kinase M2 inhibits CD4(+) T cell pathogenicity and suppresses autoimmunity. Cell Metab 31: 391-405.e398.

95. Guzy, R. D., B. Hoyos, E. Robin, H. Chen, L. Liu, K. D. Mansfield, M. C. Simon, U. Hammerling, and P. T. Schumacker. 2005. Mitochondrial complex III is required for hypoxia-induced ROS production and cellular oxygen sensing. Cell Metab 1: 401-408.

96. Brunelle, J. K., E. L. Bell, N. M. Quesada, K. Vercauteren, V. Tiranti, M. Zeviani, R. C. Scarpulla, and N. S. Chandel. 2005. Oxygen sensing requires mitochondrial ROS but not oxidative phosphorylation. Cell Metab 1: 409-414.

97. Chandel, N. S., D. S. McClintock, C. E. Feliciano, T. M. Wood, J. A. Melendez, A. M. Rodriguez, and P. T. Schumacker. 2000. Reactive oxygen species generated at mitochondrial Complex III stabilize Hypoxia-inducible factor-1 $\alpha$ during hypoxia: A mechanism of $\mathrm{O}_{2}$ sensing. J Biol Chem 275: 25130-25138.

98. Kaewpila, S., S. Venkataraman, G. R. Buettner, and L. W. Oberley. 2008. Manganese Superoxide dismutase modulates Hypoxia-inducible factor-1 $\alpha$ induction via superoxide. Cancer Res 68: 2781. 
99. Chua, Y. L., E. Dufour, E. P. Dassa, P. Rustin, H. T. Jacobs, C. T. Taylor, and T. Hagen. 2010. Stabilization of Hypoxia-inducible factor- $1 \alpha$ protein in hypoxia occurs independently of mitochondrial reactive oxygen species production. $J$ Biol Chem 285: 31277-31284.

100. Brüne, B., and J. Zhou. 2007. Hypoxia-inducible factor-1 $\alpha$ under the control of Nitric oxide. Methods Enzymol 435: 463-478.

101. Lee, G., H.-S. Won, Y.-M. Lee, J.-W. Choi, T.-I. Oh, J.-H. Jang, D.-K. Choi, B.-O. Lim, Y. J. Kim, J.-W. Park, P. Puigserver, and J.-H. Lim. 2016. Oxidative dimerization of PHD2 is responsible for its inactivation and contributes to metabolic reprogramming via HIF-1 $\alpha$ activation. Sci Rep 6: 18928.

102. Bell, E. L., T. A. Klimova, J. Eisenbart, C. T. Moraes, M. P. Murphy, G. R. S. Budinger, and N. S. Chandel. 2007. The Qo site of the mitochondrial complex III is required for the transduction of hypoxic signaling via reactive oxygen species production. J Cell Biol 177: 1029-1036.

103. Orr, A. L., L. Vargas, C. N. Turk, J. E. Baaten, J. T. Matzen, V. J. Dardov, S. J. Attle, J. Li, D. C. Quackenbush, R. L. S. Goncalves, I. V. Perevoshchikova, H. M. Petrassi, S. L. Meeusen, E. K. Ainscow, and M. D. Brand. 2015. Suppressors of superoxide production from mitochondrial complex III. Nat Chem Biol 11: 834-836.

104. Li, H.-S., Y.-N. Zhou, L. Li, S.-F. Li, D. Long, X.-L. Chen, J.-B. Zhang, L. Feng, and Y.P. Li. 2019. HIF-1 $\alpha$ protects against oxidative stress by directly targeting mitochondria. Redox Biol 25: 101109.

105. Martínez-Reyes, I., Lauren P. Diebold, H. Kong, M. Schieber, H. Huang, Christopher T. Hensley, Manan M. Mehta, T. Wang, Janine H. Santos, R. Woychik, E. Dufour, Johannes N. Spelbrink, Samuel E. Weinberg, Y. Zhao, Ralph J. DeBerardinis, and Navdeep S. Chandel. 2016. TCA cycle and mitochondrial membrane potential are necessary for diverse biological functions. Mol Cell 61: 199-209.

106. Haddad, J. J., and H. L. Harb. 2005. L-gamma-Glutamyl-L-cysteinyl-glycine (glutathione; GSH) and GSH-related enzymes in the regulation of pro- and anti-inflammatory cytokines: a signaling transcriptional scenario for redox(y) immunologic sensor(s)? Mol Immunol 42: 987-1014.

107. Reynaert, N. L., A. van der Vliet, A. S. Guala, T. McGovern, M. Hristova, C. Pantano, N. H. Heintz, J. Heim, Y.-S. Ho, D. E. Matthews, E. F. M. Wouters, and Y. M. W. JanssenHeininger. 2006. Dynamic redox control of NF-kB through glutaredoxin-regulated Sglutathionylation of inhibitory KB kinase $\beta$. Proc Natl Acad Sci U S A 103: 13086.

108. Pineda-Molina, E., P. Klatt, J. Vázquez, A. Marina, M. García de Lacoba, D. Pérez-Sala, and S. Lamas. 2001. Glutathionylation of the p50 subunit of NF-kB: A mechanism for redox-induced inhibition of DNA binding. Biochemistry 40: 14134-14142.

109. Takenaka, S., S. McCormick, E. Safroneeva, Z. Xing, and J. Gauldie. 2009. Influence of the Tissue Microenvironment on Toll-Like Receptor Expression by CD11 $\mathrm{c}^{+}$AntigenPresenting Cells Isolated from Mucosal Tissues. Clin Vaccine Immunol 16: 1615. 


\section{Chapter 5. dsRNA strand length dictates the manner of mitochondrial reprogramming and $\mathrm{ROS}$-activated antiviral response during TLR3 engagement (Ahmed et al. in preparation, to be submitted to Frontiers Immunology)}

\subsection{Introduction}

Macrophages are highly dynamic cells that are very responsive to their local microenvironment (1-3). They develop region-specific transcriptional identities, which drive tissue specific immune responses (4-8). During viral infections, macrophages play an important role in initiating antiviral responses to inhibit viral dissemination and activate the adaptive immune response. To do this, they surveil the microenvironment using pattern-recognition receptors (PRRs) such as Toll-like receptors (TLR3/7/8/9) and Retinoic acid-inducible gene-I (RIG-I)-like receptors (RLRs) (9-12). Recognition of viral ligands (e.g, viral nucleic acids) and engagement of their respective PRR activates downstream signaling pathways that upregulates type I interferon (IFN) production, which promotes antiviral programming in infected and uninfected bystander cells (13-16). Emerging evidence suggests these responses are modulated by nutrient availability and the metabolic status of the cell but the mechanisms underlying these processes remain incompletely understood (17-22).

It is increasingly recognized that mitochondria many represent a rheostat of the cell, regulating macrophage activation and function $(19,22-27)$. First, they support cellular bioenergetics and biosynthetic demands by metabolizing glucose $(18,28-31)$, glutamine $(32,33)$, branched-chain amino acids $(34-36)$, and fatty acids $(37,38)$. The intermediate metabolites and bioactive molecules derived from these pathways (e.g., TCA metabolites, reactive oxygen species (ROS)) also regulate cellular signaling, transcription and epigenetics and have been shown to control cell fate decision and in regulate immune responses $(19,21,24,39-43)$. In antiviral immune responses, the mitochondria has been shown to act as a scaffold to support cytosolic RLR 
activation through the aggregation of the mitochondrial antiviral signaling (MAVS) protein (26, 44-47). Oxidative phosphorylation (OXPHOS) $(22,48)$ and mitochondrial fusion/fission dynamics $(49,50)$ have also been shown to modulate RLR-mediated antiviral responses, but it is unclear if other PRR engage similar mitochondrial processes.

TLR3 is an endosomal PRR, which is activated by viral dsRNA resulting in the phosphorylation and homo- or heterodimerization of the interferon regulatory factor (IRF) transcription factors IRF3 and IRF7 and the production type I IFN (51). This PRR has been shown to recognize a wide range of dsRNA lengths (min: 40-50bp; max: $>8 \mathrm{~kb}$ ) in order to mount responses against a variety of viruses, which can possess segmented genomes of various lengths (52-56). Recent studies suggest that short and long dsRNA differentially engage TLR3 to induce cell and pathogen specific immune responses $(57,58)$. Consistent with these findings, I recently reported that engagement of TLR3 by high molecular weight (HMW; 1.5-8 kb) vs. low molecular weight (LMW; 0.2-1 kb) Poly(I:C) differentially activates NF-kB transcription and inflammatory cytokine production in a HIF-1 $\alpha$ dependent manner (59). However, it is unclear if differential engagement of TLR3 also modulates type I IFN responses.

I have recently shown that TLR3-mediated responses require both OXPHOS activity and Complex III-derived mtROS to support antiviral signaling in macrophages (21). However, it is unknown if TLR3 engagement with short vs. long dsRNA dictates the manner of mitochondrial reprogramming that occurs. In this study, I investigated the differential effects of short and long dsRNA (i.e., HMW vs. LMW Poly(I:C)) on type I IFN responses and mitochondrial function in murine bone marrow derived macrophages (BMM). I found that responses to both HMW and LMW Poly(I:C) requires sustained OXPHOS to drive mtROS-mediated antiviral responses. Further, I found the reliance of TLR3 on mitochondrial OXPHOS activity and mtROS production 
is amplified in conditions of low glucose suggesting nutrient availability plays a critical in defining local immune responses. Importantly, this study also showed that engagement of TLR3 by HMW and LMW Poly(I:C) is associated with differential production of mtROS by Complex I and Complex III of the electron transport chain (ETC). This differential production of mtROS regulated the magnitude of the type I IFN responses and suggests the ETC may act as a rheostat to regulate pathogen specific immune responses.

\subsection{Materials and Methods}

\subsubsection{Reagents}

High glucose Dulbecco's Modified Eagle Media (DMEM) (with 4 mM L-glutamine and 1 $\mathrm{mM}$ sodium pyruvate), DMEM without glucose/glutamine/pyruvate/phenol red, fetal bovine serum (FBS), penicillin/streptomycin (PenStrep), glucose, sodium pyruvate and L-glutamine were purchased from Life Technologies. High molecular weight Poly(I:C) (HMW) and low molecular weight Poly(I:C) (LMW) were obtained from InvivoGen. N-acetylcysteine (NAC), antimycin A (AA), rotenone (ROT), oligomycin (OM), carbonyl cyanide-p-trifluoromethoxyphenylhydrazone (FCCP), and SQ1EL-1 were acquired from Sigma-Aldrich, while S3QEL-2 was from Cedarlane. Tetramethylrhodamine, methyl ester (TMRM) and MitoSOX ${ }^{\mathrm{TM}}$ Red probes were purchased from ThermoFisher. CXCL10 ELISA kit were attained from R\&D Systems. The IFN- $\alpha /$ IFN- $\beta$ 2-Plex Mouse ProcartaPlex ${ }^{\mathrm{TM}}$ Luminex Panel kit used was from Invitrogen. The Cell-based Hydrogen Peroxide Assay Kit and antibodies detecting Complex II (SDHB) was from Abcam while antibodies recognizing SOD2 was purchased from Cell Signaling Technology. Antibodies against IRF3, pIRF3 (Ser385), IRF7, pIRF7 (Ser477), GPX4, Ikb $\alpha$, Complexes I (NDUFB8), III (UQCRC2) and IV (COX4) were from ThermoFisher. 


\subsubsection{Animals}

All animal procedures were approved by the Carleton University Animal Care Committee and were conducted in accordance with the guidelines provided by the Canadian Council for Animal Care. C57BL/6 mice colonies are maintained at the Carleton University Vivarium. The bone marrow cells from the tibias and femurs of 6-13-week-old mice were isolated and cryopreserved in a 90\% FBS/10\% dimethylsulfoxide (DMSO) mixture until use.

\subsubsection{Culture and Treatment of Mouse Bone Marrow-derived Macrophages (BMMs)}

As previously described $(21,60)$, mouse bone marrow progenitors were differentiated for 10 days in high glucose DMEM media supplemented with 10\% FBS, 1\% PenStrep and 15\% L929 fibroblast cell-conditioned medium on a 100mm Petri dish. L929-conditional media was prepared as previously described (21). On day 10, the differentiated BMMs were harvested, counted, and seeded onto tissue culture-treated plates at a concentration of $1 \times 10^{6}$ cells $/ \mathrm{mL}$ and allowed to rest overnight.

BMMs were stimulated with $10 \mathrm{ng} / \mathrm{mL}$ high molecular weight (HMW) or low molecular weight Poly(I:C) (LMW) in complete DMEM media with either high $(25 \mathrm{mM})$ or low glucose $(0.5 \mathrm{mM})$. Low glucose media was prepared by supplementing DMEM media without glucose/glutamine/pyruvate/phenol red with $0.5 \mathrm{mM}$ glucose and matching concentrations of glutamine $(4 \mathrm{mM})$ and sodium pyruvate $(1 \mathrm{mM})$ present in high glucose DMEM media. Cells were stimulated for 0.5 to 18 hours depending on the endpoint assay. To evaluate the role of Complex I and III activity and ROS production on antiviral signaling, BMMs were co-treated with $500 \mu \mathrm{M}$ MT, $5 \mathrm{mM}$ NAC, $5 \mu \mathrm{M}$ S1QEL or $5 \mu \mathrm{M}$ S3QEL-2. 


\subsubsection{Measurement of Cytokine Secretion}

Cell culture supernatants were collected from activated BMM after 18 hours of stimulation and then centrifuged to remove cellular debris. CXCL10 levels were assessed by ELISAs according to the manufacturer's instructions. IFN- $\alpha$ and IFN- $\beta$ levels were measured using IFN$\alpha /$ IFN- $\beta$ 2-Plex Mouse ProcartaPlex ${ }^{\mathrm{TM}}$ Luminex Panel.

\subsubsection{Western Blot Analysis}

Following stimulations, BMMs were lysed with Pierce RIPA buffer supplemented with HALT $^{\mathrm{TM}}$ Protease and Phosphatase Inhibitor (ThermoFisher). Total protein levels in each sample were measured using the DC assay (Bio-Rad). A total of 30ug of protein from each sample was loaded onto $12 \%$ TGX $^{\mathrm{TM}}$ FastCast ${ }^{\mathrm{TM}}$ Acrylamide gels (Bio-Rad) and imaged using the ChemiDoc $^{\mathrm{TM}}$ XR's Stain-Free program (Bio-Rad). Resolved proteins were transferred onto PVDF membranes using the Trans-Blot ${ }^{\circledR}$ Turbo $^{\mathrm{TM}}$ Transfer System and blocked overnight in 5\% non-fat dry milk (w/v) prior to overnight incubation with the appropriate primary antibody in 5\% BSA $(w / v)$ in TBS with $0.1 \%$ Tween-20. Horseradish peroxidase-conjugated secondary antibodies and Clarity $^{\mathrm{TM}}$ Western ECL Blotting Substrate (Bio-Rad) were used to visualize protein bands of interest. The protein band chemiluminescence intensity of target proteins were normalized to the amount of total protein loaded in its respective lane, as determined by the Stain-Free application on the ChemiDoc ${ }^{\mathrm{TM}} \mathrm{XR}$ imager (Bio-Rad). For I $\kappa \mathrm{B} \alpha$, IRF3 and IRF7, following normalization described above, the protein expression data was presented as the ratio between phosphorylated protein levels divided by total protein levels.

\subsubsection{Assessment of Mitochondrial Function using Flow Cytometry}

BMMs were plated onto $60 \mathrm{~mm}$ Petri dishes (at a concentration of $1 \times 10^{6}$ cells $/ \mathrm{mL}$ ) and allowed to rest overnight before treatment with either 100ng/mL LPS, 10ng/mL HMW Poly(I:C) 
or 10ng/mL LMW Poly(I:C) for 18 hours. Cells were then collected, washed, and stained with fluorescent probes according to manufacturer's instructions. Mitochondrial abundance was measured using 150nM MitoTracker Green (ThermoFisher) in serum free DMEM media. Differences in mitochondrial membrane potential (MMP) was evaluated using 10nM TMRM (ThermoFisher) in complete media. Mitochondrial and Cellular ROS levels were monitored using 2.5 $\mu \mathrm{M}$ MitoSOX ${ }^{\mathrm{TM}}$ Red (ThermoFisher) in PBS and $5 \mu \mathrm{M}$ CellROX ${ }^{\mathrm{TM}}$ Orange (ThermoFisher) in complete media. Cellular levels of fluorescence were quantified using an Attune NxT Flow Cytometer (ThermoFisher) and the results were analyzed using FlowJo Software. Results are reported as the percentage of positive cells and as mean fluorescence intensity (MFI), the latter being used to describe the level of expression on positive cells.

\subsubsection{Measurement of Cellular $\mathrm{H}_{2} \mathrm{O}_{2}$ Production by fluorescence spectrometry}

BMMs were plated onto 96-well black plates at 50,000 cells/well and stimulated with 100ng/mL LPS, 10ng/mL HMW Poly(I:C) or 10ng/mL LMW Poly(I:C) for 1 hour. Cells were then washed before using the Cell-based Hydrogen Peroxide Assay Kit (Abcam) to measure $\mathrm{H} 2 \mathrm{O} 2$ production. Cells were incubated with the AbGreen $\mathrm{H}_{2} \mathrm{O}_{2}$ indicator for 30 minutes before monitoring the relative difference in fluorescence using a fluorescence microplate reader $(490 \mathrm{~nm}$ Ex/520nm Em).

\subsubsection{Characterization of Energy Metabolism of BMMs}

Harvested BMMs were plated onto Seahorse XFp cell culture miniplates (Seahorse Bioscience) at a density of 50,000 cells/well before stimulation. Cells were then stimulated with either 10ng/mL HMW Poly(I:C) or 10ng/mL LMW Poly(I:C) for 18 hours. Measurement of extracellular acidification rate (ECAR) and oxygen consumption rate (OCR) were evaluated using a XFp Flux Analyzer (Seahorse Bioscience). Alterations in mitochondrial function was assessed 
using the Seahorse XFp Cell Mito Stress Test Kit (Agilent). BMM were exposed to successive injections of Oligomycin (OM), Carbonyl cyanide-p-trifluoromethoxyphenylhydrazone (FCCP), and Rot/AA and OCR levels were used to evaluate changes in spare respiratory capacity percentage (SRC\%) and ATP production across stimulations and culture conditions.

\subsubsection{Statistical Analyses}

Data used in this study was analyzed using GraphPad Prism software. Values shown represent the mean \pm SEM of biological replicates, where the number of replicates is reported in the figure legends. Statistical significance was calculated using a paired Student's t-test $\left({ }^{*} \mathrm{p}<0.05\right.$, $* * \mathrm{p}<0.01$, and $* * * \mathrm{p}<0.001)$.

\subsection{Results}

\subsubsection{TLR3 associated type I IFN responses are amplified under low glucose conditions}

It is increasingly recognized that the composition of cell culture media can alter intracellular metabolic responses in vitro (61). For example, high glucose has been shown to alter metabolic responses following RLR engagement by reducing cellular dependence on OXPHOS and increasing reliance on glycolysis (22). Given this finding, I first wanted to evaluate if HMW vs. LMW Poly(I:C) induce differential activation of type I IFN responses and if these responses were affected by glucose availability (high $[25 \mathrm{mM}]$ vs. low $[0.5 \mathrm{mM}]$ ). Irrespective of the glucose concentration, I found that HMW stimulation induced higher levels of IFN- $\alpha$, IFN- $\beta$ and CXCL10 production compared to LMW Poly(I:C) (Figure 5.1A-C). I also found that low glucose resulted in an amplification of type I IFN responses in both HMW- (IFN $\alpha$ FC: 5.935 , $p=0.008$; IFN- $\beta$ FC: 2.972, $\mathrm{p}=0.006$; CXCL10 FC: 2.195, $\mathrm{p}=0.005$ ) and LMW-stimulated cells (IFN $\alpha$ FC: 2.081, $\mathrm{p}=0.036$; IFN- $\beta$ FC: $3.349, \mathrm{p}=0.003$; CXCL10 FC: 4.866, $\mathrm{p}=0.025)$. Consistent with these findings, low glucose was also associated with a dramatic upregulation in both total and 
phosphorylated IRF3 (pIRF3) expression (Figure 5.1D) and a sustained increased in pIRF7 following TLR3 engagement (Figure 5.1E), which may explain the increase in type I IFN production under these conditions.

\subsubsection{Low glucose conditions are associated with increased cellular respiration to compensate for increased proton leak}

Given the central role of the mitochondria is regulating TLR3 responses (21), I wanted to see if HMW and LMW Poly(I:C) stimulations differentially reprogram OXPHOS activity. I also wanted to see if these processes were altered by glucose availability. To do this, I used Agilent's Cell Mito Stress Test kit and the Seahorse XF Analyzer. Unlike LPS (21), HMW and LMW Poly(I:C) stimulation did not alter levels of basal respiration or ATP production compared to unstimulated controls (Figure 5.2A-D). However, both stimuli were associated with a significant decrease in the spare respiratory capacity, with HMW having a more pronounced effect compared to LMW Poly(I:C). Interestingly, glucose availability did not alter the effects of HMW vs. LMW Poly(I:C) on \%SRC. However, it did result in an increase in cellular respiration and proton leak in both control and stimulated BMMs (Figure 5.2A, B, E). Given that changes in proton leak can directly alter mitochondrial membrane potential (MMP) across the inner mitochondrial membrane (68), I evaluated MMP in stimulated cells using the fluorescent dye Tetramethylrhodamine (TMRM). I found that TLR3 engagement was associated with a significant increase in the proportion of cells with low TMRM sequestration but not in overall decrease in the frequency of mitochondria (Figure 5.2F and Supplemental Figure S5.1). Consistent with observed increase in proton leak, I also found the portion of TMRM negative cells further increased in low glucose conditions. Collectively, these results suggest that limited glucose availability may cause BMM to increase their respiratory activity and compensate with proton leak to limit mitochondrial ROS accumulation. 

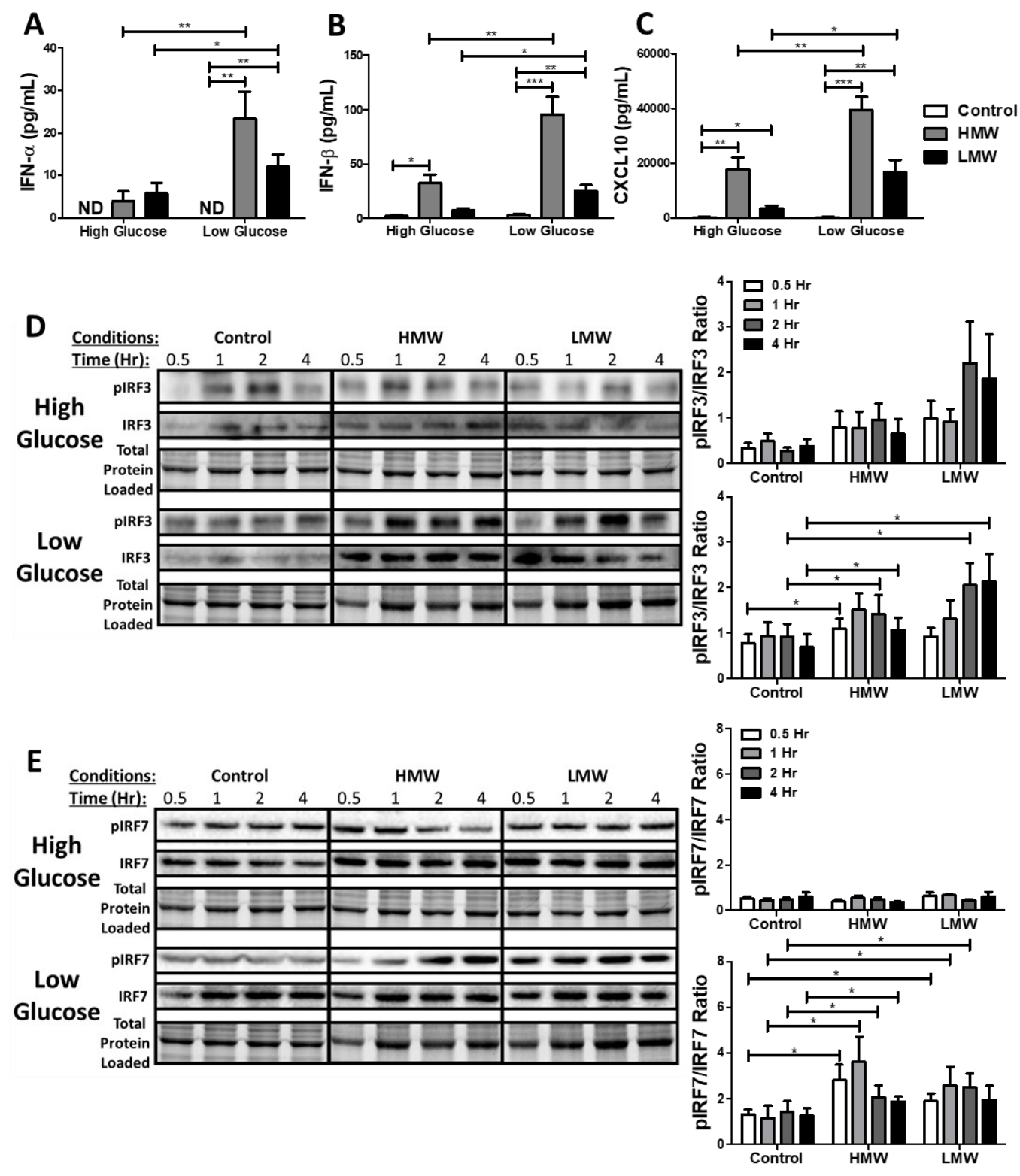

Figure 5.1: Low glucose conditions are associated with amplified antiviral signalling and subsequent cytokine production. BMMs were treated with $10 \mathrm{ng} / \mathrm{mL} \mathrm{HMW}$ or $10 \mathrm{ng} / \mathrm{mL} \mathrm{LMW}$ Poly(I:C) for 18 hours under high or low glucose media conditions. Cytokine expression (IFN- $\alpha$ (A), IFN- $\beta$ (B) and CXCL10 (C)) was evaluated in culture supernatant. Total cell lysates were harvested to measure total and phosphorylated IRF3 (D) and IRF7 (E) expression using immunoblotting at $0.5,1,2 \& 4$ hours after treatment. Band intensity was normalized to the total protein levels in each of their respective lanes using the Bio-Rad Stain-Free Application. Afterwards, the expression of IRF3 and IRF7 in each glucose condition was normalized respective to the intensity of their phosphorylated forms (pIRF3 and pIRF7) to their respective control sample collected at the same time point and presented as a fold change value. Data represents mean \pm SEM of four individual mice $(* \mathrm{p}<0.05, * * \mathrm{p}<0.01$, and $* * * \mathrm{p}<0.001)$. 
A

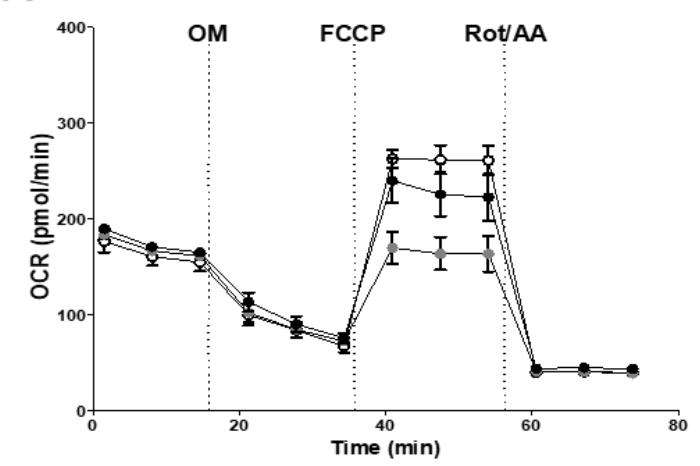

C

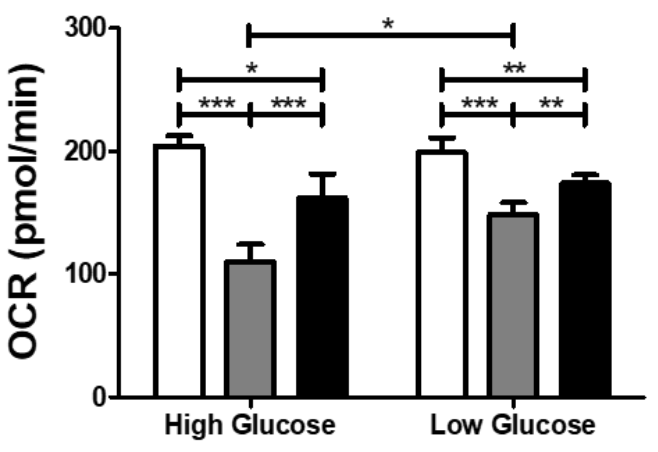

E

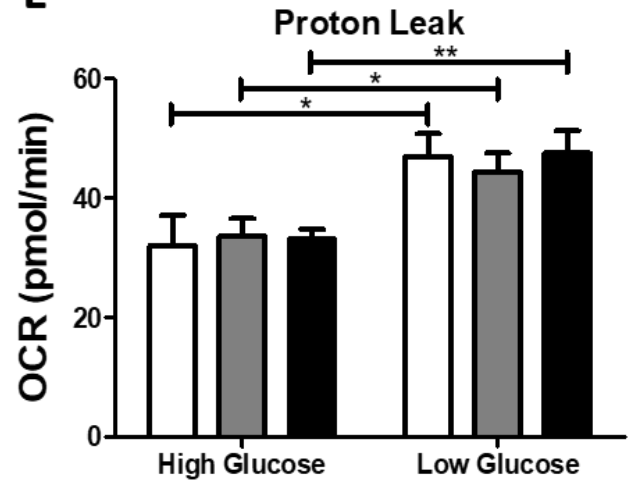

B

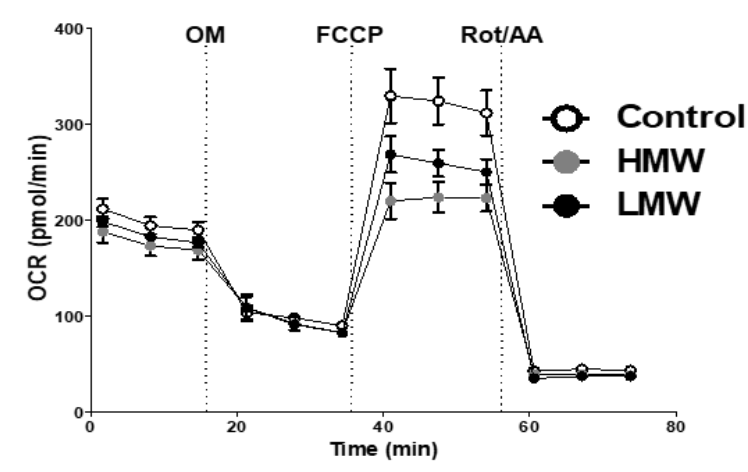

D

ATP Production

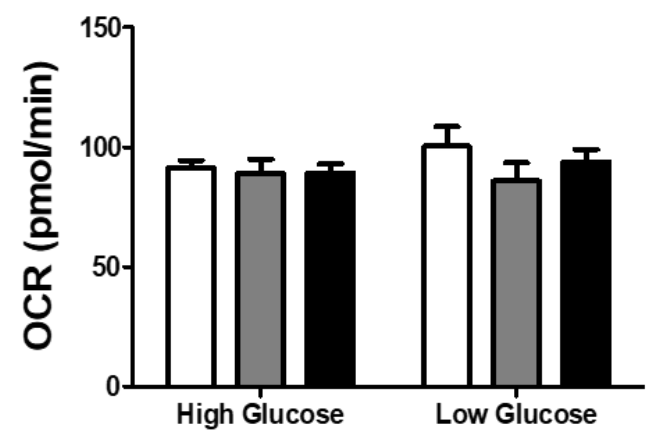

$\mathbf{F}$

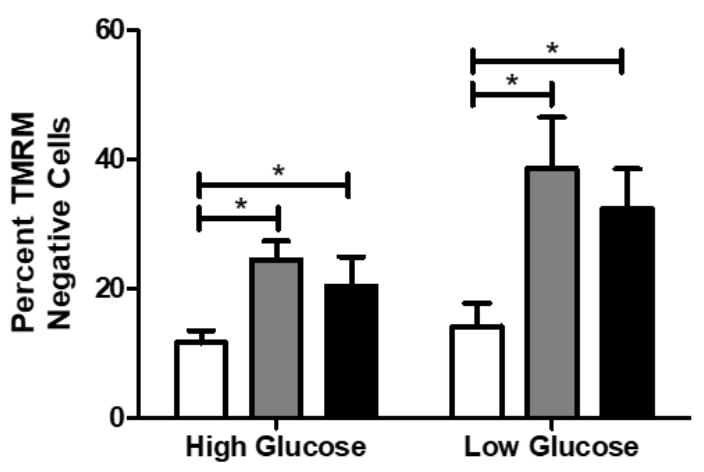

Figure 5.2: Increased proton leak drives boost in respiration under low glucose conditions. BMMs were plated onto Seahorse XFp miniplates and subsequently stimulated with HMW or LMW for 18 hours under high (A) and low (B) glucose conditions prior to assessing OXPHOS activity via consecutive Oligomycin (Oligo), Carbonyl cyanide-ptrifluoromethoxyphenylhydrazone (FCCP), and Rot/AA injection. This allowed for the quantification of OXPHOS features such as spare respiratory capacity percentage (SRC\%; C), ATP production (D) and proton leak (E). Tetramethylrhodamine (TMRM) staining was used to measure changes in mitochondrial membrane potential $(\mathbf{F})$ and reported as the percentage of TMRM negative cells. Data represents mean \pm SEM of four individual mice. $\left(* p<0.05,{ }^{* *} p<\right.$ 0.01 , and $* * * \mathrm{p}<0.001)$. 


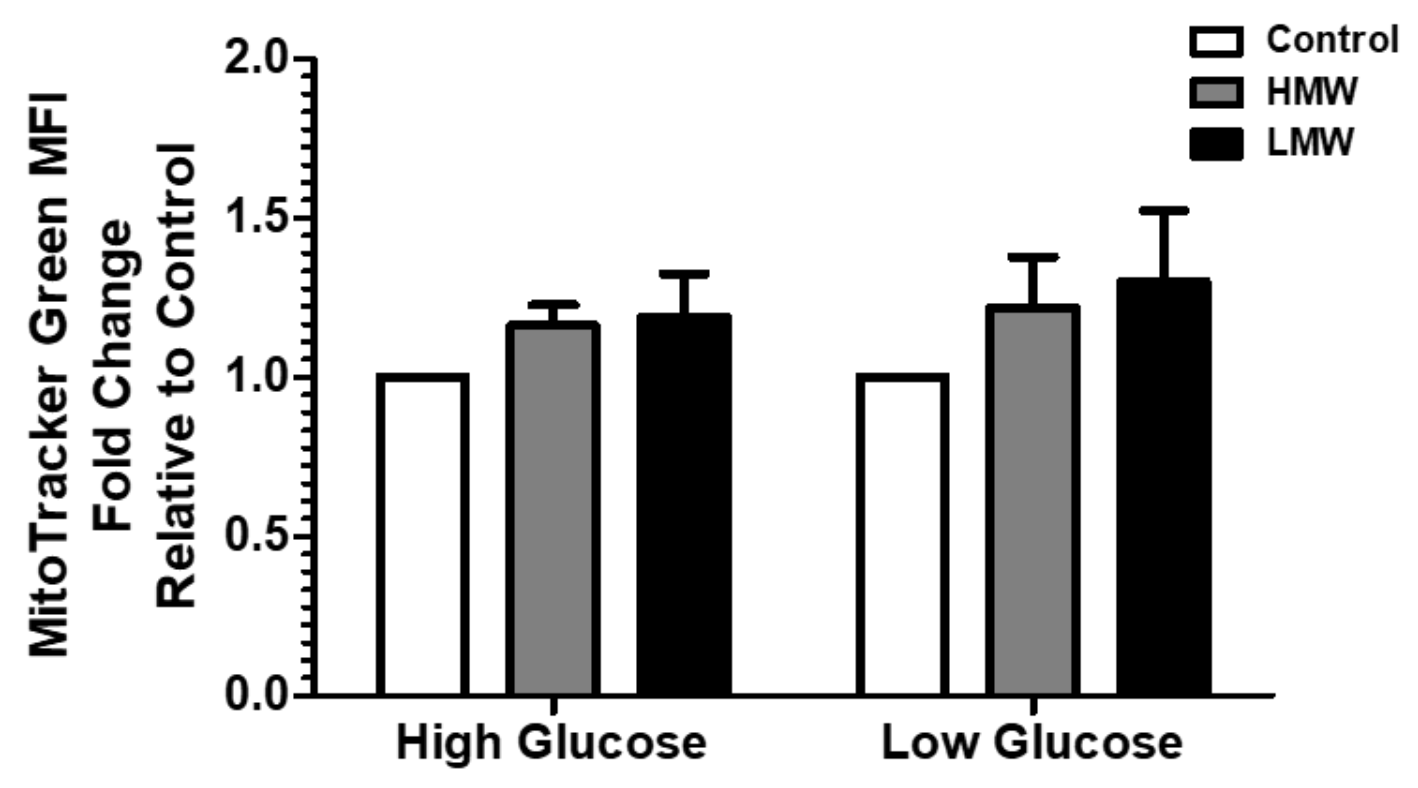

Supplemental Figure S5.1: TLR3 engagement does not alter mitochondrial mass. BMMs treated with HMW or LMW for 18 hours in high or low glucose media were evaluated for changes in mitochondrial abundance. MitoTracker Green staining was used to measure mitochondrial abundance. Data represents mean \pm SEM of four individual mice $(* p<0.05, * * p$ $<0.01$, and $* * * \mathrm{p}<0.001)$. 


\subsubsection{The amplification of type I IFN responses in low glucose conditions is driven by increased mitochondrial ROS accumulation}

I have previously shown that HMW Poly(I:C) is associated with increased mitochondrial and cytosolic ROS accumulation, which regulates cytokine production (21). Here, I wanted to investigate if differential accumulation of mitochondrial ROS (mtROS) contributes to the observed differences in type I IFN cytokine production following HMW vs. LMW Poly(I:C) stimulation. For these studies, mitochondrial superoxide production was measured using the fluorescent probe MitoSOX ${ }^{\mathrm{TM}}$ Red. As expected, TLR3 engagement increased mitochondrial superoxide production in stimulated cells compared to unstimulated controls (Figure 5.3A). However, mtROS accumulation was more pronounced in cells stimulated with HMW compared to LMW Poly(I:C) (HMW FC: 2.396, LMW FC: 1.370, p<0.001) and was further increased under low glucose conditions (HMW: 2.396 vs. 4.020, LMW: 1.370 vs 2.974). Interestingly, I also found that low glucose conditions were associated with increased hydrogen peroxide levels, which was absent in high glucose suggesting a more global change in cellular redox status (Figure 5.3B). To determine if this accumulation was associated with altered mitochondrial antioxidant responses, I measured changes in SOD2 and mtGPX4 levels using western blot. Unlike the increased levels of antioxidants observed in high glucose conditions, Poly(I:C) stimulations in low glucose did not altered antioxidant expression (Figure 5.3C and D). This lack of induction may further potentiate ROS accumulation in these cells.

Next, to determine if mtROS accumulation contributes to antiviral signaling and cytokine production, BMM were stimulated with HMW or LMW Poly(I:C) in the presence or absence of antioxidants (MitoTEMPO [MT], N-acetylcysteine [NAC]). MT scavenges superoxide from active mitochondria whereas NAC targets total cellular ROS production. Both MT and NAC treatments resulted in a near complete loss of type I IFN (IFN- $\alpha$, IFN- $\beta$ ) production following 
TLR3 engagement across conditions (Figure 5.4). This ROS scavenging also dramatically reduced CXCL10 levels (Figure 5.4). The complete loss in CXCL10 production in NAC but not MT treated cells (HMW HG: $\downarrow 92 \%$, LG: $\downarrow 94 \%$; LMW HG: $\downarrow 83 \%$, LG: $\downarrow 96 \%$ ) suggests non-mitochondrially derived ROS may also potentiate this signaling.

\subsubsection{Complex I expression is differentially modulated by HMW vs. LMW Poly(I:C)}

To better understand what may be driving the increased accumulation of mtROS, I evaluated changes in ETC protein expression using western blot. Again, I found that glucose availability dramatically affected the ability of the mitochondria function. In high glucose conditions, alterations in ETC expression were highly variable across animals. The only difference that was significant was an increase in Complex IV (COX4) (Figure 5.5). However, under low glucose conditions, both HMW and LMW Poly(I:C) were associated with decreased Complex I (NDUFB8) (HMW FC: 0.669, LMW FC: 0.540) and Complex IV expression (HMW FC: 0.783, LMW FC: 0.711) as well as an increase Complex III (UQCRC2) expression (HMW FC: 2.219, LMW FC: 2.293) (Figure 5.5). Importantly, the downregulation of Complex I was more pronounced in LMW compared to HMW Poly(I:C) $(\mathrm{p}=0.038)$ (Figure 5.5). 
A

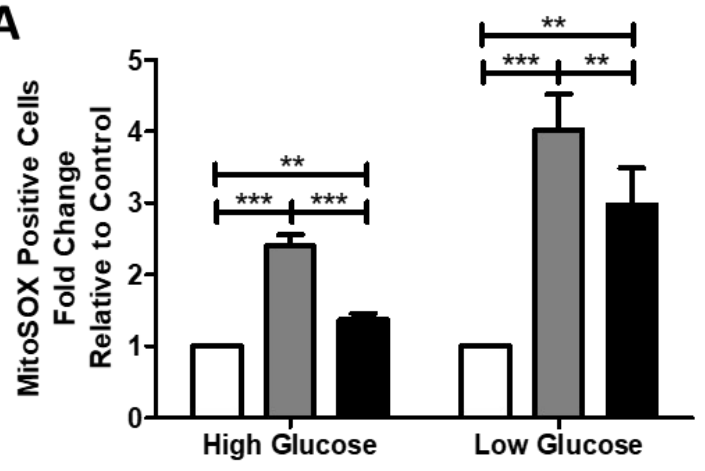

C

Total

Protein

Loaded

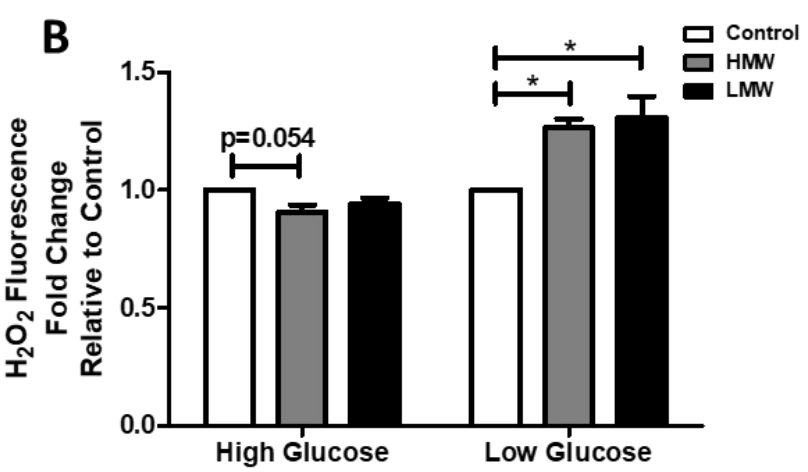

SOD2

D
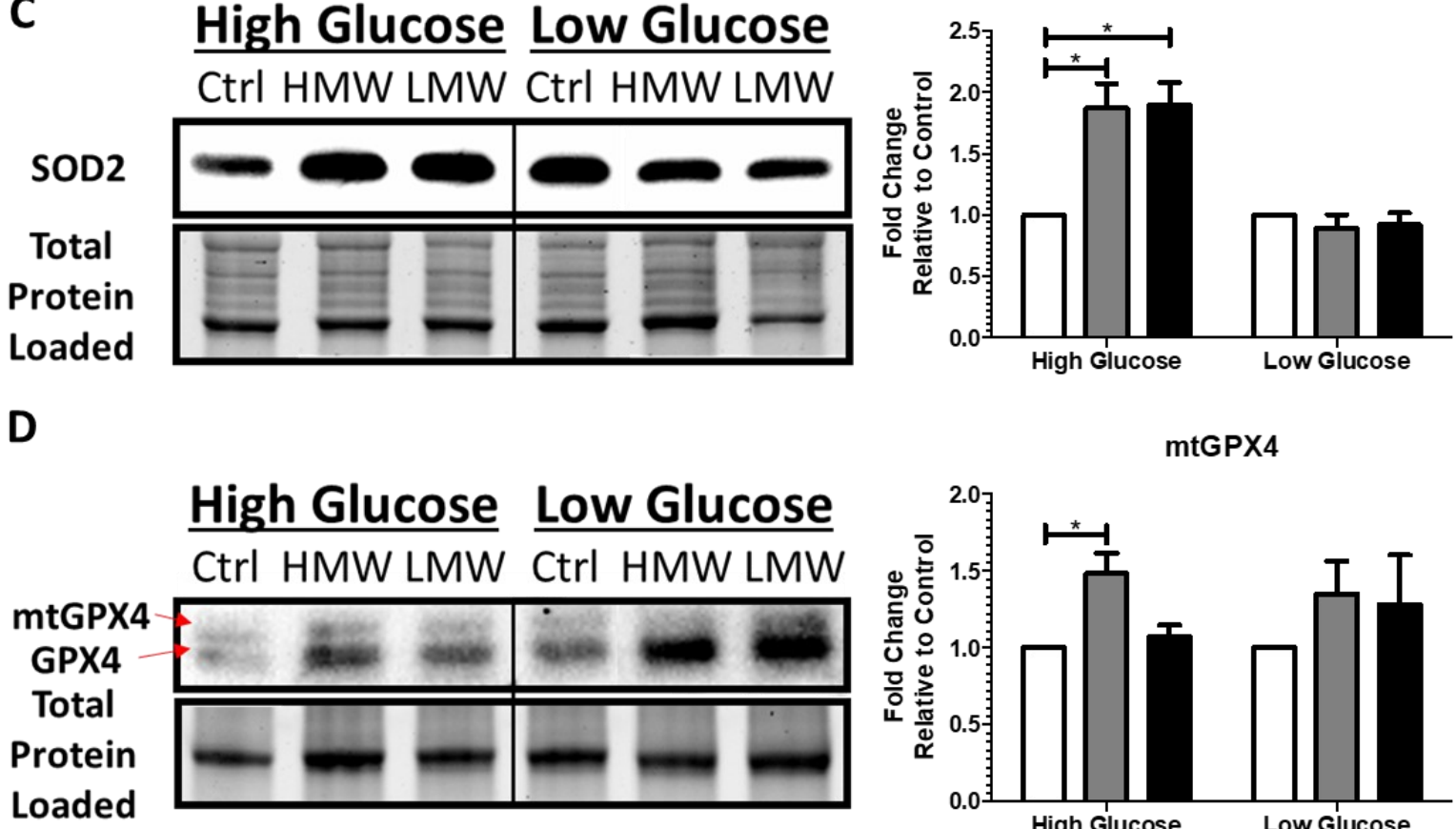

mtGPX4

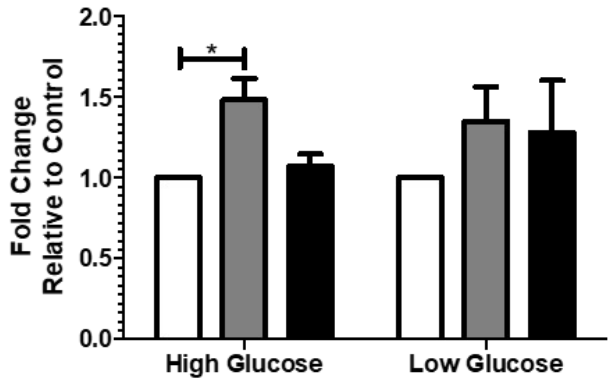

Figure 5.3: HMW and LMW activation is linked to elevated mitochondrial ROS and loss of mitochondrial antioxidant response under low glucose conditions. HMW- and LMWstimulated BMMs were probed for changes in redox metabolism under high or low glucose conditions. Mitochondrial superoxide levels were measured using MitoSOX ${ }^{\mathrm{TM}} \operatorname{Red}(\mathbf{A})$. Hydrogen peroxide levels were quantified using the Cell-based Hydrogen Peroxide Assay kit (B). Expression levels of antioxidant proteins superoxide dismutase 2 (SOD2) (C) and mitochondrial glutathione peroxidase 4 (mtGPX4) (D) were quantified using immunoblotting. Data represents mean \pm SEM of four individual mice $\left({ }^{*} \mathrm{p}<0.05,{ }^{* *} \mathrm{p}<0.01\right.$, and $\left.{ }^{* * *} \mathrm{p}<0.001\right)$. 
A
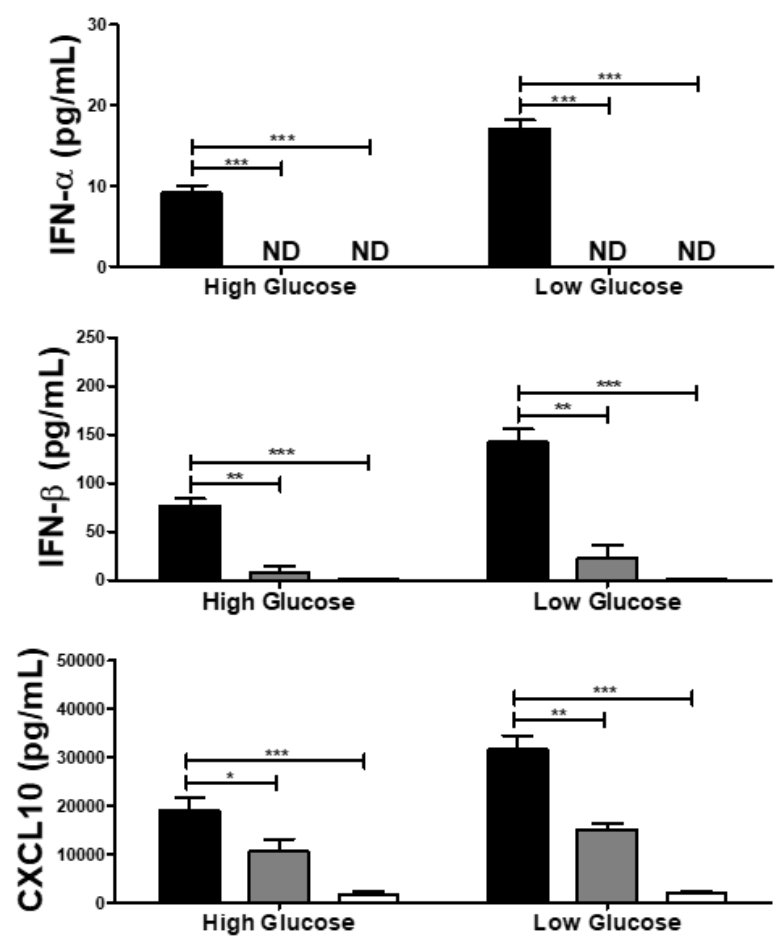

B

LMW
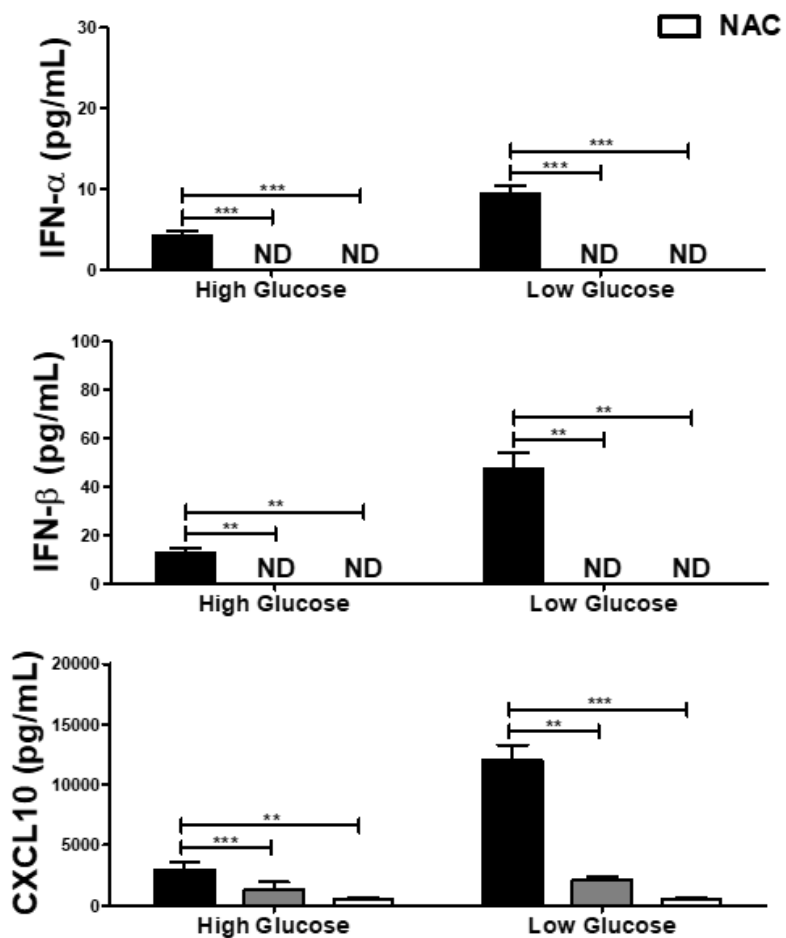

Figure 5.4: Scavenging ROS blunts Poly(I:C)-mediated type I IFN production. HMW- (A) or LMW-activated BMMs (B) were co-treated with two different ROS scavengers (MT, NAC) under high or low glucose conditions to better understand the importance of ROS to drive antiviral responses. IFN- $\alpha$, IFN- $\beta$ and CXCL10 cytokine production were quantified after 18 hours via ELISA. Data represents mean \pm SEM of four individual mice $\left({ }^{*} \mathrm{p}<0.05, * * \mathrm{p}<0.01\right.$, and ${ }^{* * *} \mathrm{p}<$ $0.001)$. 

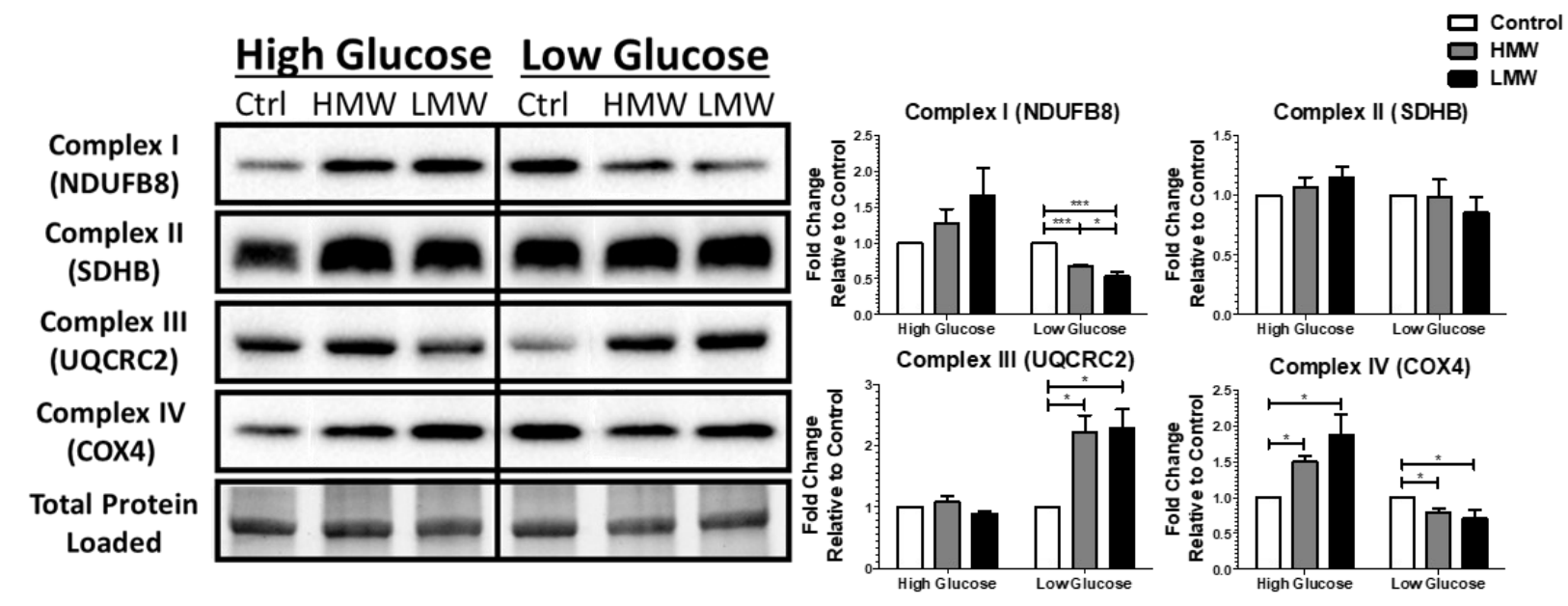

Figure 5.5: dsRNA length-dependent TLR3 engagement determines the degree of Complex I expression under low glucose conditions. Macrophages treated with HMW or LMW for 18 hours in high or low glucose media were evaluated for differences in mitochondrial function. Protein levels of Complexes I-IV of the ETC was quantified via immunoblotting. Band intensity was normalized to the total protein levels in each of their respective lanes using the Bio-Rad StainFree Application. Data represents mean \pm SEM of four individual mice $\left({ }^{*} p<0.05,{ }^{* *} p<0.01\right.$, and $* * * \mathrm{p}<0.001)$. 


\subsubsection{Mitochondrial ROS from Complexes I and III differentially drive type I IFN responses following stimulation with $\mathrm{HMW}$ or LMW Poly(I:C)}

Mitochondrial superoxide production is a by-product of cellular respiration that occurs because of electron leakage across the ETC (62-64). Given complexes I and III are the major producers of mtROS, I wondered if their altered expression following HMW and LMW stimulation contributed to increased mtROS production and type I IFN responses in low glucose conditions. For these experiments BMM were stimulated with HMW or LMW Poly (I:C) in the presence or absence of S1QEL or S3QEL. These inhibitors prevent superoxide production from Complex I (S1QEL) or Complex III (S3QEL) without impairing OXPHOS activity. Under these low glucose conditions S1QEL and S3QEL were found to have differing effects on HMW vs. LMW Poly(I:C) responses. Whereas ROS production by complexes I and III was required to drive type I IFN and CXCL10 production in LMW Poly (I:C) stimulated cells (Figure 5.6B), complex I ROS production was not required for IFN- $\beta$ production following HMW Poly (I:C) (Figure 5.6A). In fact, it appears that high levels of mtROS derived from complex I may inhibit the production IFN- $\beta$ under these conditions $(\uparrow 21 \%, \mathrm{p}=0.038)$. Consistent with these findings, S1QEL and S3QEL were found to dramatically reduced the levels of pIRF3/7 following LMW. However, treatment with S1QEL did not affect pIRF3 levels in HMW treated cells (Figures 5.6C and D). Interestingly, Honda et al. (13) have shown that pIRF3 plays a central role in driving early type I IFN responses including IFN- $\beta$ production. Alternatively, IRF7 phosphorylation is required to support later phases of these responses, which include IFN- $\alpha$, CXCL10 and further IFN- $\beta$ production. 
A

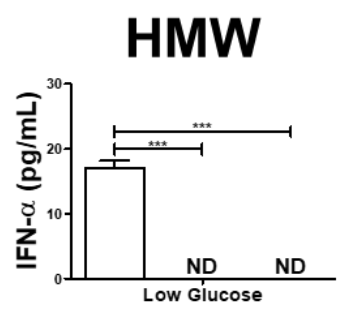

C
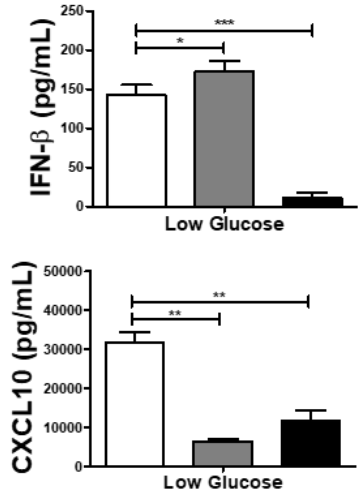

Conditions:

None

S1 Treatment: Ctrl HMW LMW Ctrl HMW LMW Ctrl HMW LMW

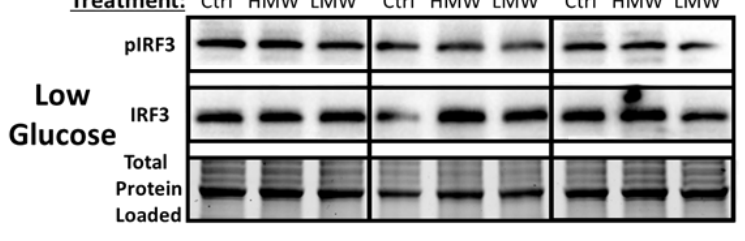

D

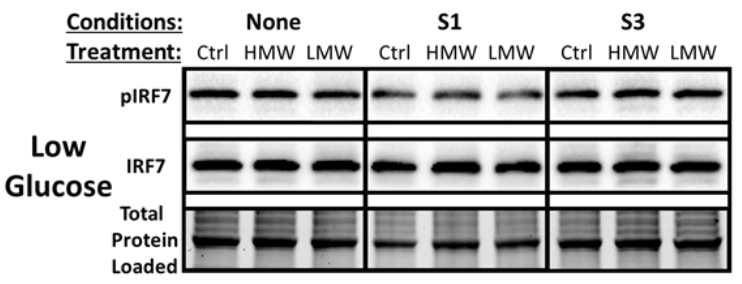

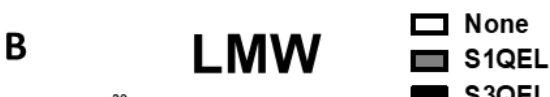
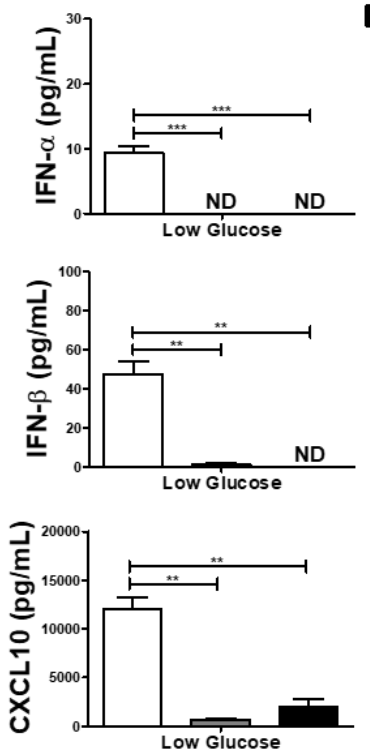
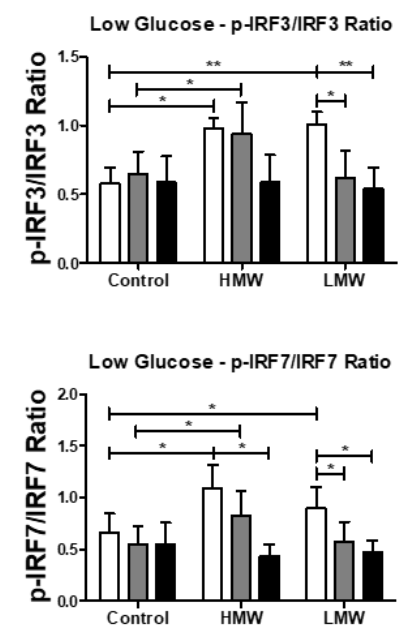

Figure 5.6: Preventing the production of Complex I generated mtROS can unlock HMWmediated IFN production via increased IRF3/7 activation. BMMs treated with either HMW (A) or LMW (B) were co-treated with specific suppressor of electron leak from Complex I (S1QEL) or Complex III (S3QEL) under high or low glucose conditions to better understand the importance of mtROS to proper antiviral response. IFN- $\alpha$, IFN- $\beta$ and CXCL1 0 cytokine production were quantified after 18 hours. Total cell lysates were harvested to measure total and phosphorylated IRF3 (C) and IRF7 (D) after S1QEL and S3QEL co-treatment using immunoblotting. Band intensity was normalized to the total protein levels in each of their respective lanes using the Bio-Rad Stain-Free Application. Afterwards, the expression of IRF3 and IRF7 in each glucose condition was normalized respective to the intensity of their phosphorylated forms (pIRF3 and pIRF7) to their respective control sample collected at the same time point and presented as a fold change value. Data represents mean $\pm \mathrm{SEM}$ of four individual mice $(* \mathrm{p}<0.05, * * \mathrm{p}<0.01$, and $* * * \mathrm{p}<0.001)$. 


\subsection{Discussion}

dsRNA viruses are a diverse family of microorganisms capable of infecting everything from bacteria to animals $(53,55,56)$. The genomes of these viruses are highly diverse and can be either monopartite or segmented in structure (up to 12 dsRNA fragments of varying sizes) $(53,54$, 56). For example, the genome of the Megabirnaviridae family consist of two segments between 7.0-9.0 kb in length, the Partitiviridae family have between $2-3$ segments of $1.4-2.3 \mathrm{~kb}$ in length and the Reoviridae family possess 10-12 genomic segments between $0.7-5.0 \mathrm{~kb}$ in length (55). To effectively respond to these viruses, macrophages must recognize both long and short dsRNA. While cytosolic PRR differentially recognize short vs. long nucleic acids (e.g., RIG-I $(<1 \mathrm{~kb})$ and MDA5 $(>2 \mathrm{~kb}))(65,66)$, endosomal TLR3 must recognize all forms and lengths of dsRNA $>40$ $50 \mathrm{bp}(51,52)$. In the current study, I found that TLR3 engagement by long vs. short dsRNA differentially reprograms mitochondrial function to support early antiviral responses and that these processes are tightly regulated by glucose availability. I show that strong type I IFN responses, as seen following stimulation with HMW Poly(I:C) and in low glucose conditions, require significant accumulation of mtROS to drive IRF3 and IRF7 phosphorylation and cytokine production. Further, this accumulation is regulated by the modulation complex I ( $\downarrow)$ and III ( $\uparrow)$ of the ETC. I also show there may be a maximal capacity of mtROS to drive type I IFN responses. When these levels exceed a certain threshold, for example following HMW stimulation in low glucose conditions, mtROS derived from Complex I may suppress type I IFN responses.

Previous work has identified an intimate link between mitochondrial function and antiviral responses. Central to this relationship is the requirement of sustained OXPHOS activity and MMP across the inner mitochondrial membrane $(19,22-27)$. Yoshimuzi et al. (22) demonstrated the importance of OXPHOS activity to RLR-mediated responses of macrophages. Targeting 
OXPHOS led to increased viral susceptibility and the induction of robust inflammatory responses in the lung (22). Furthermore, complete dissipation of MMP was shown to blunt RLR-mediated antiviral signaling due to reduced MAVS aggregation (50). This dependency on OXPHOS is directly associated with the inhibitory effects of glucose metabolism (48). The rate-limiting glycolytic protein hexokinase 2 (HK2) directly interacts with MAVS along the outer mitochondrial membrane natively, which is lost during RIG-I activation leading to reduced glycolytic activity and lactate production (48). Further, lactate is a natural suppressor of MAVS signaling and can limiting the magnitude of an antiviral immune response (48). Unlike LPS $(18,19,25,32)$, I found that irrespective of the length of dsRNA, some level of cellular respiration is required to support TLR3-mediated response. Further, while I did not specifically evaluate the inhibitory effects of glycolysis on these processes, I found that low glucose conditions supported higher levels of cellular respiration, proton leak and mtROS production, IRF3/7 signaling and type I IFN production. Interestingly, $\mathrm{Wu}$ et al. have shown that increased OXPHOS activity in TLR9activated plasmacytoid dendritic cells is linked to an autocrine type I IFN-mediated boost in fatty acid oxidation (FAO) (67). Future studies are required to see if FAO plays a role in these TLR3 responses.

One key factor that influences MMP and ultimately OXPHOS function is passive or active proton leak, which facilitates the transport of protons back into the matrix independent of OXPHOS (68). I have found that the amplification of TLR3-mediated antiviral responses under low glucose conditions was associated with increased mitochondrial respiration due to elevated proton leak. This elevation in proton leak is also likely responsible for the increase in the proportion of BMM with low MMP. This contrasts with RIG-I signaling, where overexpression of uncoupling protein 2 (UCP2) in MEFs resulted in increased active proton leak, inactivating 
MAVS-mediated signaling (50). There has been some debate as to the effect of proton leak on mtROS production. Koshiba et al. (50) show that the over expression of UCP2 caused attenuated mtROS generation which is critical to driving its antiviral response (42). In our study, I show that the increase in proton leak during both HMW and LMW stimulation under low glucose conditions resulted in augmented mtROS production, which was also vital to facilitating increased IRF3/7 phosphorylation. Despite these differences, both RIG-I- and TLR3-mediated antiviral signaling is driven in part by mtROS $(21,42,69)$.

Our previous work was among the first to link complex III-mediated mtROS to increased antiviral signaling in HMW-activated macrophages (21). In this study, I show that complex IIIassociated ROS production is also required to induce early (IFN- $\beta$ ) and later (IFN- $\alpha$ and CXCL10) type I IFN responses following stimulation with HMW and LMW Poly(I:C). I also show that mtROS from Complex I contributes to TLR3 signaling. However, unlike complex III, I found that complex I is downregulated by HMW and LMW Poly(I:C) stimulation in low glucose conditions. I speculate this downregulation, which is more pronounced in LMW- vs. HMW-stimulated cells, may represent a protective mechanism to limit the accumulation of mtROS and the detrimental effects of these oxidizing species. Consistent with these findings, HMW-stimulated cells in low glucose support the highest levels of mtROS. Further, I found that inhibition of complex I ROS production increased IFN- $\beta$ production. This may be related to the dependence of early antiviral immune responses on OXPHOS activity and their ability of these cells to form supercomplexes. ETC supercomplex formation reduces the travel path of electrons between individual complexes and limit the chance of electron leakage and subsequent mitochondrial superoxide production ( 70 , 71). During antibacterial responses, macrophages undergo ETC supercomplex disassembly to mount a robust inflammatory response, mediated by mtROS production and subsequent NLRP3 
inflammasome activation $(24,72)$. Yet, studies that have investigated the role of ETC supercomplexes during viral infections is limited. Champagne et al. (73) show that during Influenza infection, methylation-controlled $\mathrm{J}$ protein $(\mathrm{MCJ}) \mathrm{KO} \mathrm{CD} 8+\mathrm{T}$ cells have improved immunity due to increased ETC supercomplex assembly. The resulting increase in OXPHOS activity and MMP led to increased IFN- $\gamma$ and IL-2 secretion (73). Bladanta et al. (74) conducted studies using vaccinia-infected BMM and found that knocking out the mitochondrial-associated interferon-stimulating gene (ISG) ISG15 caused a reduction of free Complex I and more supercomplex formation. But while the loss of ISG15 resulted in reduced mtROS, ISG15 ${ }^{-/}$BMM were more susceptible to vaccinia infection (74). Further studies are required to better understand how the dynamics of ETC supercomplexes affect the antiviral capabilities of macrophages.

In summary, I have shown that ETC complex architecture during TLR3 engagement is influenced by the dsRNA strand length. TLR3 engagement requires OXPHOS to mount mtROSdriven antiviral responses regardless of strand length and this dependency is exaggerated under low glucose conditions. Yet, Complex I-derived mtROS dampen type I IFNs production during HMW responses while LMW activation requires mtROS from complexes I and III for a proper response. Interestingly, blocking superoxide production from complex I without hampering electron flow significantly improved HMW-derived type I IFN production. This demonstrates the functional plasticity of macrophages and their capability to sufficiently shape its response to various viruses and in different tissue microenvironments. These findings not only highlight the role of the ETC as a modulator that is capable of fine-tuning antiviral response based on the microenvironment but provides a potential new approach for enhancing antiviral therapies in patients with acute viral infections. 


\subsection{References}

1. Ross, J. A., and M. J. Auger. 2002. Biology of the macrophage. In The macrophage, 2nd ed. B. Burke, and C. E. Lewis, eds. Oxford University Press, New York, New York. 1-57.

2. Varol, C., A. Mildner, and S. Jung. 2015. Macrophages: Development and Tissue Specialization. Annual Review of Immunology 33: 643-675.

3. Mosser, D. M., and J. P. Edwards. 2008. Exploring the full spectrum of macrophage activation. Nat Rev Immunol 8: 958-969.

4. Gordon, S., and P. R. Taylor. 2005. Monocyte and macrophage heterogeneity. Nat Rev Immunol 5: 953-964.

5. Yu, X., A. Buttgereit, I. Lelios, S. G. Utz, D. Cansever, B. Becher, and M. Greter. 2017. The cytokine TGF- $\beta$ promotes the development and homeostasis of alveolar macrophages. Immunity 47: 903-912 e904.

6. Naito, M., G. Hasegawa, Y. Ebe, and T. Yamamoto. 2004. Differentiation and function of Kupffer cells. Medical Electron Microscopy 37: 16-28.

7. Biswas, S. K., and E. Lopez-Collazo. 2009. Endotoxin tolerance: new mechanisms, molecules and clinical significance. Trends in Immunology 30: 475-487.

8. Smythies, L. E., R. Shen, D. Bimczok, L. Novak, R. H. Clements, D. E. Eckhoff, P. Bouchard, M. D. George, W. K. Hu, S. Dandekar, and P. D. Smith. 2010. Inflammation Anergy in Human Intestinal Macrophages Is Due to Smad-induced I $\mathrm{B} \alpha$ Expression and NF-кB Inactivation. Journal of Biological Chemistry 285: 19593-19604.

9. $\quad$ Murphy, K. M. 2011. Janeway's Immunobiology. Taylor \& Francis Group.

10. Bianchi, M. E. 2007. DAMPs, PAMPs and alarmins: all we need to know about danger. $J$. Leukoc. Biol. 81: 1-5.

11. Fitzgerald, K. A., and J. C. Kagan. 2020. Toll-like receptors and the control of immunity. Cell 180: 1044-1066.

12. Reikine, S., J. B. Nguyen, and Y. Modis. 2014. Pattern recognition and signaling mechanisms of RIG-I and MDA5. Front Immunol 5: 342-342.

13. Honda, K., A. Takaoka, and T. Taniguchi. 2006. Type I Inteferon Gene Induction by the Interferon Regulatory Factor Family of Transcription Factors. Immunity 25: 349-360.

14. Ivashkiv, L. B., and L. T. Donlin. 2014. Regulation of type I interferon responses. Nat. Rev. Immunol. 14: 36-49.

15. Kawai, T., and S. Akira. 2006. Innate immune recognition of viral infection. Nat Immunol 7: 131-137.

16. Schneider, W. M., M. D. Chevillotte, and C. M. Rice. 2014. Interferon-Stimulated Genes: A Complex Web of Host Defenses. Annu. Rev. Immunol. 32: 513-545.

17. Zhu, L., T. Yang, L. Li, L. Sun, Y. Hou, X. Hu, L. Zhang, H. Tian, Q. Zhao, J. Peng, H. Zhang, R. Wang, Z. Yang, L. Zhang, and Y. Zhao. 2014. TSC1 controls macrophage polarization to prevent inflammatory disease. Nat Commun 5.

18. Rodríguez-Prados, J., P. G. Través, J. Cuenca, D. Rico, J. Aragonés, P. Martín-Sanz, M. Cascante, and L. Boscá. 2010. Substrate fate in activated macrophages: A comparison between innate, classic, and alternative activation. $J$ Immunol 185: 605-614.

19. Mills, E. L., B. Kelly, A. Logan, A. S. H. Costa, M. Varma, C. E. Bryant, P. Tourlomousis, J. H. M. Däbritz, E. Gottlieb, I. Latorre, S. C. Corr, G. McManus, D. Ryan, H. T. Jacobs, M. Szibor, R. J. Xavier, T. Braun, C. Frezza, M. P. Murphy, and L. A. O’Neill. 2016. Succinate dehydrogenase supports metabolic repurposing of mitochondria to drive inflammatory macrophages. Cell 167: 457-470.e413. 
20. Van den Bossche, J., J. Baardman, Natasja A. Otto, S. van der Velden, Annette E. Neele, Susan M. van den Berg, R. Luque-Martin, H.-J. Chen, Marieke C. S. Boshuizen, M. Ahmed, Marten A. Hoeksema, Alex F. de Vos, and Menno P. J. de Winther. 2016. Mitochondrial Dysfunction Prevents Repolarization of Inflammatory Macrophages. Cell Rep 17: 684-696.

21. Ahmed, D., D. Roy, A. Jaworski, A. Edwards, A. Abizaid, A. Kumar, A. Golshani, and E. Cassol. 2019. Differential remodeling of the electron transport chain is required to support TLR3 and TLR4 signaling and cytokine production in macrophages. Sci Rep 9: 18801.

22. Yoshizumi, T., H. Imamura, T. Taku, T. Kuroki, A. Kawaguchi, K. Ishikawa, K. Nakada, and T. Koshiba. 2017. RLR-mediated antiviral innate immunity requires oxidative phosphorylation activity. Sci Rep 7: 5379.

23. Koshiba, T. 2013. Mitochondrial-mediated antiviral immunity. Biochim Biophys Acta 1833: 225-232.

24. Garaude, J., R. Acín-Pérez, S. Martínez-Cano, M. Enamorado, M. Ugolini, E. NistalVillán, S. Hervás-Stubbs, P. Pelegrín, L. E. Sander, J. A. Enríquez, and D. Sancho. 2016. Mitochondrial respiratory-chain adaptations in macrophages contribute to antibacterial host defense. Nat Immunol 17: 1037-1045.

25. Kelly, B., G. M. Tannahill, M. P. Murphy, and L. A. J. O'Neill. 2015. Metformin Inhibits the Production of Reactive Oxygen Species from NADH:Ubiquinone Oxidoreductase to Limit Induction of Interleukin-1 $\beta$ (IL-1 $\beta$ ) and Boosts Interleukin-10 (IL-10) in Lipopolysaccharide (LPS)-activated Macrophages. J Biol Chem 290: 20348-20359.

26. Seth, R. B., L. Sun, C.-K. Ea, and Z. J. Chen. 2005. Identification and characterization of MAVS, a mitochondrial antiviral signaling protein that a ctivates NF-KB and IRF3. Cell 122: 669-682.

27. Castanier, C., D. Garcin, A. Vazquez, and D. Arnoult. 2010. Mitochondrial dynamics regulate the RIG-I-like receptor antiviral pathway. EMBO Rep 11: 133.

28. Jiang, H., H. Shi, M. Sun, Y. Wang, Q. Meng, P. Guo, Y. Cao, J. Chen, X. Gao, E. Li, and J. Liu. 2016. PFKFB3-Driven Macrophage Glycolytic Metabolism Is a Crucial Component of Innate Antiviral Defense. J. Immunol. 197: 2880-2890.

29. Semba, H., N. Takeda, T. Isagawa, Y. Sugiura, K. Honda, M. Wake, H. Miyazawa, Y. Yamaguchi, M. Miura, D. M. R. Jenkins, H. Choi, J.-w. Kim, M. Asagiri, A. S. Cowburn, H. Abe, K. Soma, K. Koyama, M. Katoh, K. Sayama, N. Goda, R. S. Johnson, I. Manabe, R. Nagai, and I. Komuro. 2016. HIF-1 $\alpha$-PDK1 axis-induced active glycolysis plays an essential role in macrophage migratory capacity. Nat Commun 7: 11635.

30. Wang, T., H. Liu, G. Lian, S.-Y. Zhang, X. Wang, and C. Jiang. 2017. HIF1 $\alpha$-induced glycolysis metabolism is essential to the activation of inflammatory macrophages. Mediators Inflamm 2017: 10.

31. Aki, T., T. Funakoshi, K. Noritake, K. Unuma, and K. Uemura. 2020. Extracellular glucose is crucially involved in the fate decision of LPS-stimulated RAW264.7 murine macrophage cells. Scientific Reports 10: 10581.

32. Jha, A. K., S. C.-C. Huang, A. Sergushichev, V. Lampropoulou, Y. Ivanova, E. Loginicheva, K. Chmielewski, K. M. Stewart, J. Ashall, B. Everts, E. J. Pearce, E. M. Driggers, and M. N. Artyomov. 2015. Network Integration of Parallel Metabolic and Transcriptional Data Reveals Metabolic Modules that Regulate Macrophage Polarization. Immunity 42: 419-430. 
33. Liu, M., R. S. O’Connor, S. Trefely, K. Graham, N. W. Snyder, and G. L. Beatty. 2019. Metabolic rewiring of macrophages by $\mathrm{CpG}$ potentiates clearance of cancer cells and overcomes tumor-expressed CD47-mediated 'don't-eat-me' signal. Nature Immunology 20: 265-275.

34. Ko, J. H., A. Olona, A. E. Papathanassiu, N. Buang, K. S. Park, A. S. H. Costa, C. Mauro, C. Frezza, and J. Behmoaras. 2020. BCAT1 affects mitochondrial metabolism independently of leucine transamination in activated human macrophages. J Cell Sci 2020: jcs.247957.

35. Papathanassiu, A. E., J.-H. Ko, M. Imprialou, M. Bagnati, P. K. Srivastava, H. A. Vu, D. Cucchi, S. P. McAdoo, E. A. Ananieva, C. Mauro, and J. Behmoaras. 2017. BCAT1 controls metabolic reprogramming in activated human macrophages and is associated with inflammatory diseases. Nat Commun 8: 16040.

36. Yoon, B. R., Y.-J. Oh, S. W. Kang, E. B. Lee, and W.-W. Lee. 2018. Role of SLC7A5 in metabolic reprogramming of Human monocyte/macrophage immune responses. Front Immunol 9: 53.

37. Huang, S. C.-C., B. Everts, Y. Ivanova, D. O'Sullivan, M. Nascimento, A. M. Smith, W. Beatty, L. Love-Gregory, W. Y. Lam, C. M. O'Neill, C. Yan, H. Du, N. A. Abumrad, J. F. Urban Jr, M. N. Artyomov, E. L. Pearce, and E. J. Pearce. 2014. Cell-intrinsic lysosomal lipolysis is essential for alternative activation of macrophages. Nat Immunol 15: 846-855.

38. Malandrino, M. I., R. Fucho, M. Weber, M. Calderon-Dominguez, J. F. Mir, L. Valcarcel, X. Escoté, M. Gómez-Serrano, B. Peral, L. Salvadó, S. Fernández-Veledo, N. Casals, M. Vázquez-Carrera, F. Villarroya, J. J. Vendrell, D. Serra, and L. Herrero. 2015. Enhanced fatty acid oxidation in adipocytes and macrophages reduces lipid-induced triglyceride accumulation and inflammation. Am J Physiol Endocrinol Metab 308: E756-769.

39. Tannahill, G. M., A. M. Curtis, J. Adamik, E. M. Palsson-McDermott, A. F. McGettrick, G. Goel, C. Frezza, N. J. Bernard, B. Kelly, N. H. Foley, L. Zheng, A. Gardet, Z. Tong, S. S. Jany, S. C. Corr, M. Haneklaus, B. E. Caffrey, K. Pierce, S. Walmsley, F. C. Beasley, E. Cummins, V. Nizet, M. Whyte, C. T. Taylor, H. Lin, S. L. Masters, E. Gottlieb, V. P. Kelly, C. Clish, P. E. Auron, R. J. Xavier, and L. A. J. O/'Neill. 2013. Succinate is an inflammatory signal that induces IL-1 $\beta$ through HIF-1 $\alpha$. Nature 496: 238-242.

40. Palsson-McDermott, E. M., A. M. Curtis, G. Goel, M. A. R. Lauterbach, F. J. Sheedy, L. E. Gleeson, M. W. M. van den Bosch, S. R. Quinn, R. Domingo-Fernandez, D. G. W. Johnston, J.-k. Jiang, W. J. Israelsen, J. Keane, C. Thomas, C. Clish, M. Vander Heiden, R. J. Xavier, and L. A. J. O'Neill. 2015. Pyruvate kinase M2 regulates Hif- $1 \alpha$ activity and IL-1 $\beta$ induction and is a critical determinant of the warburg effect in LPS-activated macrophages. Cell Metab. 21: 65-80.

41. West, A. P., I. E. Brodsky, C. Rahner, D. K. Woo, H. Erdjument-Bromage, P. Tempst, M. C. Walsh, Y. Choi, G. S. Shadel, and S. Ghosh. 2011. TLR signalling augments macrophage bactericidal activity through mitochondrial ROS. Nature 472: 476-480.

42. Tal, M. C., M. Sasai, H. K. Lee, B. Yordy, G. S. Shadel, and A. Iwasaki. 2009. Absence of autophagy results in reactive oxygen species-dependent amplification of RLR signaling. Proc Natl Acad Sci U S A 106: 2770.

43. Herb, M., A. Gluschko, K. Wiegmann, A. Farid, A. Wolf, O. Utermöhlen, O. Krut, M. Krönke, and M. Schramm. 2019. Mitochondrial reactive oxygen species enable proinflammatory signaling through disulfide linkage of NEMO. Sci Signal 12: eaar5926. 
44. Yoneyama, M., M. Kikuchi, T. Natsukawa, N. Shinobu, T. Imaizumi, M. Miyagishi, K. Taira, S. Akira, and T. Fujita. 2004. The RNA helicase RIG-I has an essential function in double-stranded RNA-induced innate antiviral responses. Nat Immunol 5: 730-737.

45. Yoneyama, M., M. Kikuchi, K. Matsumoto, T. Imaizumi, M. Miyagishi, K. Taira, E. Foy, Y.-M. Loo, M. Gale, and S. Akira. 2005. Shared and unique functions of the DExD/H-box helicases RIG-I, MDA5, and LGP2 in antiviral innate immunity. $J$ Immunol 175: 28512858.

46. Kawai, T., K. Takahashi, S. Sato, C. Coban, H. Kumar, H. Kato, K. J. Ishii, O. Takeuchi, and S. Akira. 2005. IPS-1, an adaptor triggering RIG-I- and MDA5-mediated type I interferon induction. Nat Immunol 6: 981-988.

47. Hou, F., L. Sun, H. Zheng, B. Skaug, Q.-X. Jiang, and Z. J. Chen. 2011. MAVS forms functional prion-like aggregates to activate and propagate antiviral innate immune response. Cell 146: 448-461.

48. Zhang, W., G. Wang, Z. G. Xu, H. Tu, F. Hu, J. Dai, Y. Chang, Y. Chen, Y. Lu, H. Zeng, Z. Cai, F. Han, C. Xu, G. Jin, L. Sun, B. S. Pan, S. W. Lai, C. C. Hsu, J. Xu, Z. Z. Chen, H. Y. Li, P. Seth, J. Hu, X. Zhang, H. Li, and H. K. Lin. 2019. Lactate is a natural suppressor of RLR signaling by targeting MAVS. Cell 178: 176-189.e115.

49. Yu, C.-Y., J.-J. Liang, J.-K. Li, Y.-L. Lee, B.-L. Chang, C.-I. Su, W.-J. Huang, M. M. C. Lai, and Y.-L. Lin. 2015. Dengue virus impairs mitochondrial fusion by cleaving mitofusins. PLoS pathog 11: e1005350.

50. Koshiba, T., K. Yasukawa, Y. Yanagi, and S.-i. Kawabata. 2011. Mitochondrial membrane potential is required for MAVS-mediated antiviral signaling. Sci Signal 4: ra7.

51. Alexopoulou, L., A. C. Holt, R. Medzhitov, and R. A. Flavell. 2001. Recognition of doublestranded RNA and activation of NF-[kappa]B by Toll-like receptor 3. Nature 413: 732738.

52. Liu, L., I. Botos, Y. Wang, J. N. Leonard, J. Shiloach, D. M. Segal, and D. R. Davies. 2008. Structural basis of Toll-like receptor 3 signaling with double-stranded RNA. Science 320: 379-381.

53. Wickner, R. 1993. Double-stranded RNA virus replication and packaging. J Biol Chem 268: 3797-3800.

54. Roldão, A., A. C. Silva, M. C. M. Mellado, P. M. Alves, and M. J. T. Carrondo. 2011. 1.47 - Viruses and Virus-Like Particles in Biotechnology: Fundamentals and Applications. In Comprehensive Biotechnology (Second Edition). M. Moo-Young, ed. Academic Press, Burlington. 625-649.

55. Jia, H., K. Dong, L. Zhou, G. Wang, N. Hong, D. Jiang, and W. Xu. 2017. A dsRNA virus with filamentous viral particles. Nature Commun 8: 168.

56. King, A. M. Q., E. Lefkowitz, M. J. Adams, and E. B. Carstens. 2011. Virus taxonomy: ninth report of the International Committee on Taxonomy of Viruses. Elsevier.

57. Mian, M. F., A. N. Ahmed, M. Rad, A. Babaian, D. Bowdish, and A. A. Ashkar. 2013. Length of dsRNA (poly I:C) drives distinct innate immune responses, depending on the cell type. J Leukoc Biol 94: 1025-1036.

58. Jiang, M., P. Österlund, L. P. Sarin, M. M. Poranen, D. H. Bamford, D. Guo, and I. Julkunen. 2011. Innate immune responses in human Monocyte-derived dendritic cells are highly dependent on the size and the 5 ' phosphorylation of RNA molecules. J Immunol 187: 1713. 
59. Ahmed, D., A. Humphrey, D. Roy, M.-E. Sheridan, A. Jaworski, A. Edwards, J. Donner, A. Abizaid, W. Willmore, A. Kumar, A. Golshani, and E. Cassol. 2020. HIF-1 $\alpha$ regulation of pro-inflammatory cytokine production following TLR3 engagement is dependent on viral nucleic acid length and glucose availability [Manuscript submitted for publication]. $J$ Immunol: 1-20.

60. Weischenfeldt, J., and B. Porse. 2008. Bone marrow-derived macrophages (BMM): Isolation and applications. CSH Protoc 2008: pdb.prot5080.

61. Lagziel, S., E. Gottlieb, and T. Shlomi. 2020. Mind your media. Nat Metab.

62. Voet, D., J. G. Voet, and C. W. Pratt. 2016. Fundamentals of biochemistry: life at the molecular level. John Wiley \& Sons.

63. Berg, J. M., J. L. Tymoczko, and L. Stryer. 2008. Biochemistry. Macmillan.

64. Spinelli, J. B., and M. C. Haigis. 2018. The multifaceted contributions of mitochondria to cellular metabolism. Nat Cell Biol 20: 745-754.

65. Kato, H., O. Takeuchi, S. Sato, M. Yoneyama, M. Yamamoto, K. Matsui, S. Uematsu, A. Jung, T. Kawai, K. J. Ishii, O. Yamaguchi, K. Otsu, T. Tsujimura, C.-S. Koh, C. Reis e Sousa, Y. Matsuura, T. Fujita, and S. Akira. 2006. Differential roles of MDA5 and RIG-I helicases in the recognition of RNA viruses. Nature 441: 101-105.

66. Kato, H., O. Takeuchi, E. Mikamo-Satoh, R. Hirai, T. Kawai, K. Matsushita, A. Hiiragi, T. S. Dermody, T. Fujita, and S. Akira. 2008. Length-dependent recognition of doublestranded ribonucleic acids by Retinoic acid-inducible gene-I and Melanoma differentiation-associated gene 5. J Exp Med 205: 1601-1610.

67. Wu, D., David E. Sanin, B. Everts, Q. Chen, J. Qiu, Michael D. Buck, A. Patterson, Amber M. Smith, C.-H. Chang, Z. Liu, Maxim N. Artyomov, Erika L. Pearce, M. Cella, and Edward J. Pearce. 2016. Type 1 Interferons induce changes in core metabolism that are critical for immune function. Immunity 44: 1325-1336.

68. Jastroch, M., A. S. Divakaruni, S. Mookerjee, J. R. Treberg, and M. D. Brand. 2010. Mitochondrial proton and electron leaks. Essays Biochem 47: 53-67.

69. Agod, Z., T. Fekete, M. M. Budai, A. Varga, A. Szabo, H. Moon, I. Boldogh, T. Biro, A. Lanyi, A. Bacsi, and K. Pazmandi. 2017. Regulation of type I IFN responses by mitochondria-derived ROS in plasmacytoid dendritic cells. Redox Biol 13: 633-645.

70. Acín-Pérez, R., P. Fernández-Silva, M. L. Peleato, A. Pérez-Martos, and J. A. Enriquez. 2008. Respiratory active mitochondrial supercomplexes. Mol Cell 32: 529-539.

71. Garlid, K. D., M. Jabůrek, P. Ježek, and M. Vařecha. 2000. How do uncoupling proteins uncouple? Biochim Biophys Acta 1459: 383-389.

72. Su, Y., T. P. Miettinen, L. Mu, E. Mirek, S. R. Manalis, T. G. Anthony, H. Sesaki, and J. Chen. 2020. Disassembly of ETC Complexes Drives Macrophage Inflammatory Responses by Reprogramming Cellular Metabolism and Translation [Manuscirpt submitted for publication]. Cell Rep Available at SSRN: https://ssrn.com/abstract=3611881: 1-54.

73. Champagne, Devin P., Ketki M. Hatle, Karen A. Fortner, A. D’Alessandro, Tina M. Thornton, R. Yang, D. Torralba, J. Tomás-Cortázar, Yong W. Jun, Kyo H. Ahn, Kirk C. Hansen, L. Haynes, J. Anguita, and M. Rincon. 2016. Fine-tuning of CD8+ T cell mitochondrial metabolism by the respiratory chain repressor MCJ dictates protection to Influenza virus. Immunity 44: 1299-1311.

74. Baldanta, S., M. Fernández-Escobar, R. Acín-Perez, M. Albert, E. Camafeita, I. Jorge, J. Vázquez, J. A. Enríquez, and S. Guerra. 2017. ISG15 governs mitochondrial function in macrophages following vaccinia virus infection. PLOS Pathog 13: e1006651. 


\section{Chapter 6. CONCLUSIONS AND PRESPECTIVES}

A host's response to a viral infection is a complex and multifaceted set of processes that attempts to overcome the virus' ability to exploit host cellular machinery and circumvent the immunological countermeasures designed to prevent such a takeover (1). On the front lines against infection, macrophages are responsible for sensing and immediately respond to invading viruses in almost every tissue in the body $(2,3)$. To initiate antiviral immune responses, macrophages possess a series of PRRs that can detect conserved viral PAMPs such as genomic DNA and RNA (2-5). They also play an important role in attracting and activating other immune cells to help in the fight against viral invasion $(2,6,7)$. If the immune system is successful, they also assist in reestablishing tissue homeostasis in the host at the end of the infection (6-9). As a result, this versatility of functions demonstrates the macrophage's role as a bridge between the innate and adaptive immune systems.

Recent evidence suggests cellular metabolism is a central regulator of macrophage effector functions. Macrophages undergo profound metabolic changes to not only meet the biosynthetic and bioenergetic needs of the cell but to regulate various cellular processes including transcription factor activity, cytokine production and cell differentiation (10-12). To date, the majority of work conducted in the field of immunometabolism concerning macrophages centers on the dichotomy of metabolic profiles between pro-inflammatory M1 macrophages (i.e. LPS/TLR4 engagement) and anti-inflammatory M2 macrophages (i.e. IL-4/IL-10 activation) $(13,14)$. The prevailing theory over the last half decade has been that the metabolic reprogramming associated with LPS activation was consistently observed across the other inflammatory states. However, more recent work has slowly started to erode this notion and has generated a hypothesis that PRR engagement induces a diverse spectrum of metabolic and activation states. To better elucidate the metabolic 
mechanisms that drive and support TLR-mediated responses further studies are required. Interestingly, as more data is generated, it is becoming increasingly clear that the mitochondria is at the center at regulating the dynamic nature of macrophage function and activation.

In this thesis, I endeavored to development a better understanding of the mechanics surrounding the metabolic regulation of macrophages during antiviral responses. In particular, I was interested in understanding how cellular metabolism contributes to the regulation of TLRmediated antiviral responses. I hypothesized that mitochondrial function was a central regulator of these responses in macrophages and that dynamic modulation of mitochondrial function could serve to drive ligand specific immune responses. To perform these studies, I employed a combination of immunological techniques and systems biology approaches to systematically evaluate the metabolic pathways associated with antiviral responses in macrophages. Using these profiles, I then functionally evaluated the roles of select metabolic pathways and networks on antiviral immune responses using a primary macrophage model system. These studies evaluated associations between bioenergetic profiles and immune function.

In Chapter 2, I conducted one of the first studies to systematically evaluate how type I IFNs modulate cellular metabolism in macrophages using transcriptional profiling. Using publicly available microarray datasets available through the Gene Expression Omnibus (GEO) database at the NCBI, I developed metabolic gene signatures associated with early IFN- $\alpha$ responses in mouse BMMs and human MDMs. I found that in both model systems, short-term treatments with IFN- $\alpha$ were associated with an extensive metabolic reprogramming, with $>500$ metabolic genes linked to bioenergetic pathways, cellular redox balance, branched chain amino acid catabolism, cell membrane composition, FA synthesis and FAO. By conducting this untargeted comparative study, 
my work provided novel insights into the metabolic processes required to support and facilitate type I IFN responses in mouse and human macrophages.

With this understanding, I then focused my in vitro functional studies on two wellcharacterized inducers of type I IFN responses (LPS, Poly(I:C)). The purpose of these studies was to evaluate differences in metabolic reprogramming between TLR3- and TLR4-mediated responses in mouse BMMs. In Chapter 3, I show that differences in mitochondrial reprogramming between TLR3 and TLR4 engagement is directly linked to changes in the expression of ETC complexes. These changes resulted in differential production of mitochondrial vs. cytosolic ROS, which drove their ligand specific inflammatory and antiviral programs. Interestingly, TLR3assocaited responses were found to be associated with increased mtROS production by Complex III and that this accumulation of mtROS played a central role in driving cytokine production in these cells. This work is among the first to suggest that targeting complex III mtROS production may provide a novel means of modulating macrophage antiviral responses. Another key insight from this study was the role of glucose availability in manipulating these TLR3-mediated responses. I showed that in low glucose conditions, TLR3, but not TLR4 responses had amplified pro-inflammatory and antiviral cytokine production. This was linked to increased Complex III expression and subsequent elevated mitochondrial and cytosolic ROS.

These studies also showcased the preferred energy sources for TLR3 vs. TLR4 responses. While LPS activation primarily relies on glycolysis for energy, maintaining some OXPHOS activity is a requisite feature of Poly(I:C) activation. Standard culture conditions contain supraphysiological levels of most nutrients in order to maintain cells ex vivo (15-17). For example, glucose levels in standard DMEM media $(25 \mathrm{mM})$ are more than five times found in the fasting blood of non-diabetic individuals and more than twice as high as found in hyperglycemic patients 
$(15,18)$. Moreover, levels of glucose found in the tissues are a small percentage compared to the levels found in blood under homeostasis, which drops further during infection as a result of increased consumption $(19,20)$. My findings are consistent with another study that demonstrated that macrophages were less dependent on OXPHOS activity in high glucose media, skewing antiviral immune responses (21). Collectively, these results suggest that assessing immune function under more oxidative conditions by reducing the concentrations of glucose in the media may provide a better representation of effector function in vivo.

The latter two studies in this thesis were performed to evaluate how metabolic reprogramming contributes to differential engagement TLR3 and activation of antiviral immune responses using short and long dsRNA. I also conducted these experiments under high and low glucose conditions to evaluate how these responses differed under standard in vitro conditions and conditions more conducive to in vivo comparisons. In Chapter 4 , I found that HIF-1 $\alpha$ could modulate the inflammatory response of TLR3-activated macrophages and that this modulation differed based on the length of the Poly(I:C) ligand and the nutrient environment, where low glucose conditions resulted in a more robust inflammatory response. Given the fact that dsRNA viruses possess segmented genomes with variable lengths, macrophages must retain plasticity to proper respond to all ligands. Differential engagement of TLR3 and distinct metabolic profiles may play a central role is driving ligand specific immune functions. Further, this study highlights the possibility of differentially targeting HIF-1 $\alpha$ function depending on the specific viral infection to diminish potentially damaging inflammation during antiviral responses.

In Chapter 5, I further examined the relationship between Poly(I:C) ligand length, differential mitochondrial reprogramming, and the regulation of type I IFN responses. Under low glucose conditions, I found this dependency was further exaggerated leading to an amplification 
in mtROS production. The increase in mtROS resulted in increased IRF-3 and -7 signalling and increased type I IFN production. Interestingly, I also found that the length of Poly(I:C) strand directly influences the manner of ETC restructuring required to support antiviral responses. While mtROS accumulation drives IRF3/7 activation and subsequent antiviral responses during TLR3 engagement, Complex I-derived mtROS diminish type I IFNs production during HMW responses and blocking superoxide production from Complex I without hindering electron flow through the complex, improves HMW-mediated type I IFN production. This suggests that a dependence on OXPHOS activity during the early phase of the antiviral response is more important to LMWmediated response, which requires mtROS from complexes I and III for a proper response. This highlights the role of the ETC as a key modulator of antiviral responses and potentially positions the complexes of the ETC as new targets to improve antiviral therapies by targeting components that are harmful to macrophage antiviral function during acute viral infections.

Further investigation from this chapter suggests a role for ETC supercomplexes in facilitating macrophage antiviral responses. Recent studies have investigated ETC supercomplexes in the context of macrophage antibacterial responses and linked its disassembly to an increase in mtROS production which enables its robust inflammatory responses and NLRP3 inflammasome activation (22, 23). Su et al. (23) also examined if ETC supercomplex disassembly was conserved across TLR stimuli. Using a high concentration of Poly(I:C) $(10 \mu \mathrm{g} / \mathrm{mL})$, which in Chapter 3 was found to caused severe OXPHOS impairment and boosted pro-inflammatory cytokine production, TLR3-activated macrophages underwent a similar disassembly to LPS-activated macrophages including a loss of Complex I-associated ETC supercomplex structures. It is possible that the effects of Complex I-derived mtROS observed in HMW-stimulated BMM is due to increased free Complex I in mitochondria and subsequent mtROS production from that site. Thus, developing 
mechanisms to maintain Complex I-associated ETC supercomplex structures may be one possibility to augment macrophage antiviral responses. But more work is required to elucidate whether this possibility is a reality.

Taken together, this thesis brings together a plethora of investigative techniques to probe the importance of cellular metabolism in driving the antiviral response in macrophages. The research completed in this thesis provides the foundational work required to better understand the complicated relationship between metabolic networks and effector function. A systematic understanding of these processes non-diseased models can be translated to provide critical insights into the dysregulation of these networks in diseased states such as live acute and chronic viral infections. In addition, I identified potential metabolic targets that can modulate the magnitude of inflammatory and antiviral responses and thus represent new prospective targets for the improvement of antiviral therapies. 


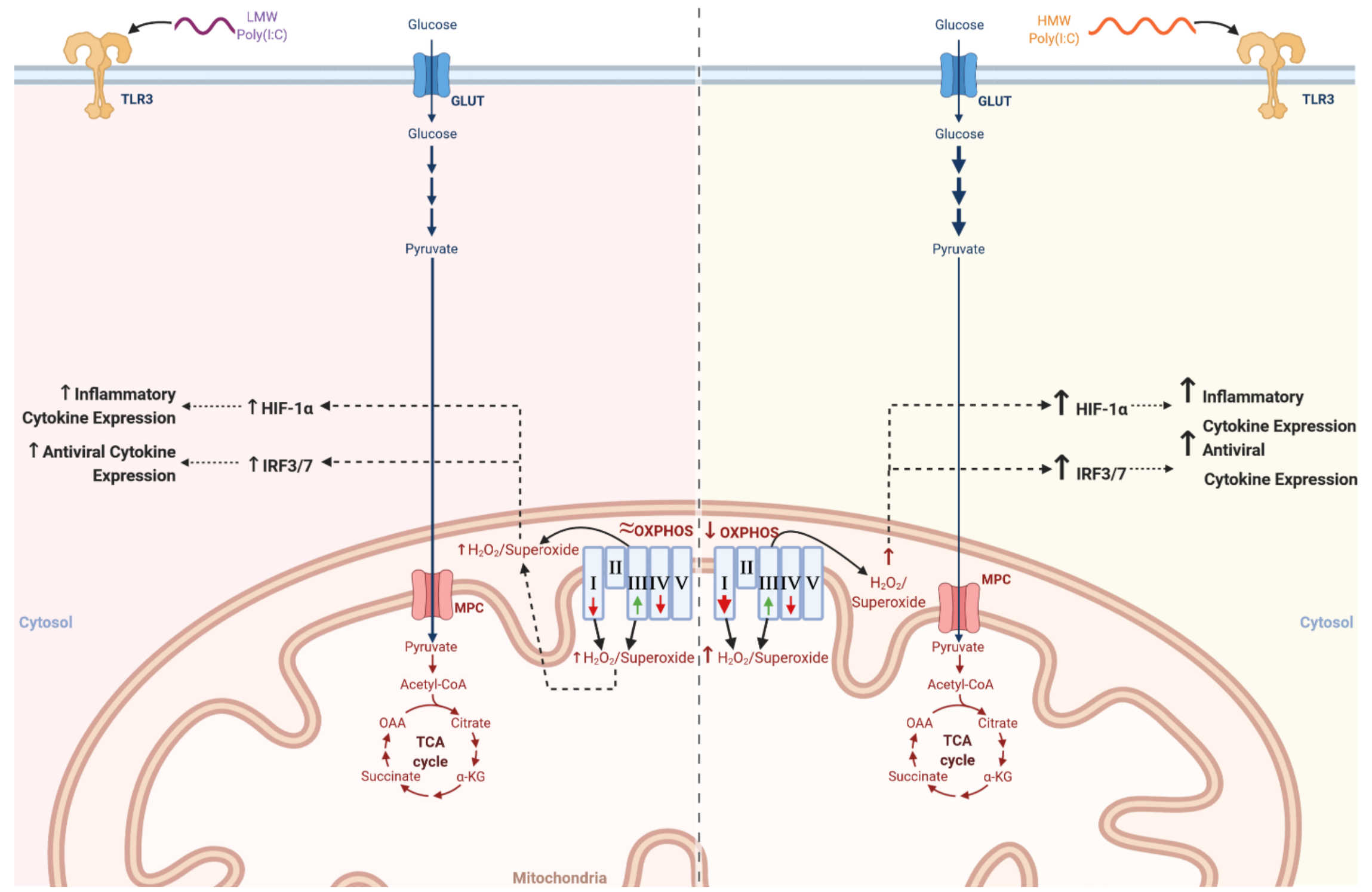

Figure 6.1: A summary of the findings pertaining to the role of cellular metabolism in TLR3-mediated responses. 


\subsection{References}

1. Fritsch, S. D., and T. Weichhart. 2016. Effects of Interferons and Viruses on Metabolism. Front. Immunol. 7: 630.

2. Murphy, K. M. 2011. Janeway's Immunobiology. Taylor \& Francis Group.

3. Bianchi, M. E. 2007. DAMPs, PAMPs and alarmins: all we need to know about danger. $J$. Leukoc. Biol. 81: 1-5.

4. Fitzgerald, K. A., and J. C. Kagan. 2020. Toll-like receptors and the control of immunity. Cell 180: 1044-1066.

5. Reikine, S., J. B. Nguyen, and Y. Modis. 2014. Pattern recognition and signaling mechanisms of RIG-I and MDA5. Front Immunol 5: 342-342.

6. Ross, J. A., and M. J. Auger. 2002. The biology of the macrophage. In The macrophage, 2nd ed. B. Burke, and C. E. Lewis, eds. Oxford University Press, New York, New York. $1-57$.

7. Mosser, D. M., and J. P. Edwards. 2008. Exploring the full spectrum of macrophage activation. Nat Rev Immunol 8: 958-969.

8. Fadok, V. A., D. L. Bratton, A. Konowal, P. W. Freed, J. Y. Westcott, and P. M. Henson. 1998. Macrophages that have ingested apoptotic cells in vitro inhibit proinflammatory cytokine production through autocrine/paracrine mechanisms involving TGF-beta, PGE2, and PAF. Journal of Clinical Investigation 101: 890-898.

9. Fadok, V. A., P. P. McDonald, D. L. Bratton, and P. M. Henson. 1998. Regulation of macrophage cytokine production by phagocytosis of apoptotic and post-apoptotic cells. Biochem Soc Trans 26: 653-656.

10. Kesarwani, P., A. K. Murali, A. A. Al-Khami, and S. Mehrotra. 2013. Redox regulation of T-cell function: from molecular mechanisms to significance in human health and disease. Antioxid. Redox Signal. 18: 1497-1534.

11. Tannahill, G. M., A. M. Curtis, J. Adamik, E. M. Palsson-McDermott, A. F. McGettrick, G. Goel, C. Frezza, N. J. Bernard, B. Kelly, N. H. Foley, L. Zheng, A. Gardet, Z. Tong, S. S. Jany, S. C. Corr, M. Haneklaus, B. E. Caffrey, K. Pierce, S. Walmsley, F. C. Beasley, E. Cummins, V. Nizet, M. Whyte, C. T. Taylor, H. Lin, S. L. Masters, E. Gottlieb, V. P. Kelly, C. Clish, P. E. Auron, R. J. Xavier, and L. A. J. O/'Neill. 2013. Succinate is an inflammatory signal that induces IL-1 $\beta$ through HIF-1 $\alpha$. Nature 496: 238-242.

12. Wang, H., H. Flach, M. Onizawa, L. Wei, M. T. McManus, and A. Weiss. 2014. Negative regulation of Hifla expression and TH17 differentiation by the hypoxia-regulated microRNA miR-210. Nat. Immunol. 15: 393-401.

13. Zhu, L., Q. Zhao, T. Yang, W. Ding, and Y. Zhao. 2015. Cellular metabolism and macrophage functional polarization. Int Rev Immunol 34: 82-100.

14. Wynn, T. A., A. Chawla, and J. W. Pollard. 2013. Origins and Hallmarks of Macrophages: Development, Homeostasis, and Disease. Nature 496: 445-455.

15. McKee, T. J., and S. V. Komarova. 2017. Is it time to reinvent basic cell culture medium? Am J Physiol Cell Physiol 312: C624-C626.

16. Ackermann, T., and S. Tardito. 2019. Cell Culture Medium Formulation and Its Implications in Cancer Metabolism. Trends in Cancer 5: 329-332.

17. Kedia-Mehta, N., and D. K. Finlay. 2019. Competition for nutrients and its role in controlling immune responses. Nat Commun 10: 2123.

18. American Diabetes, A. 2009. Diagnosis and classification of diabetes mellitus. Diabetes Care 32 Suppl 1: S62-S67. 
19. Gey, K. F. 1956. The concentration of glucose in rat tissues. Biochem J 64: 145-150.

20. Varanasi, S. K., D. Donohoe, U. Jaggi, and B. T. Rouse. 2017. Manipulating Glucose Metabolism during Different Stages of Viral Pathogenesis Can Have either Detrimental or Beneficial Effects. J Immunol.

21. Yoshizumi, T., H. Imamura, T. Taku, T. Kuroki, A. Kawaguchi, K. Ishikawa, K. Nakada, and T. Koshiba. 2017. RLR-mediated antiviral innate immunity requires oxidative phosphorylation activity. Sci Rep 7: 5379.

22. Garaude, J., R. Acín-Pérez, S. Martínez-Cano, M. Enamorado, M. Ugolini, E. NistalVillán, S. Hervás-Stubbs, P. Pelegrín, L. E. Sander, J. A. Enríquez, and D. Sancho. 2016. Mitochondrial respiratory-chain adaptations in macrophages contribute to antibacterial host defense. Nat Immunol 17: 1037-1045.

23. Su, Y., T. P. Miettinen, L. Mu, E. Mirek, S. R. Manalis, T. G. Anthony, H. Sesaki, and J. Chen. 2020. Disassembly of ETC Complexes Drives Macrophage Inflammatory Responses by Reprogramming Cellular Metabolism and Translation [Manuscirpt submitted for publication]. Cell Rep Available at SSRN: https://ssrn.com/abstract=3611881: 1-54. 